\title{
Targeted Therapeutics in
}

\section{Inflammatory Atherosclerosis}


Targeted Therapeutics in Inflammatory Atherosclerosis 


\section{The Graduation Committee}

\begin{tabular}{|c|c|c|}
\hline Chairperson/Secretary & Prof. Dr. Ir. J.W.M. Hilgenkamp & University of Twente \\
\hline Promoter (Supervisor) & Prof. Dr. G. Storm & University of Twente \\
\hline Promoter (Supervisor) & Prof. Dr. W.J.M. Mulder & $\begin{array}{l}\text { Ichan School of Medicine } \\
\text { at Mount Sinai }\end{array}$ \\
\hline $\begin{array}{l}\text { Co-promoter } \\
\text { (Co-supervisor) }\end{array}$ & Dr. J.M. Metselaar & University of Twente \\
\hline Referent (Referee) & Dr. R.J. Kok & Utrecht University \\
\hline External Member & Prof. Dr. Ir. W.E. Hennink & Utrecht University \\
\hline External Member & Prof. Dr. M.P.J. de Winther & $\begin{array}{l}\text { University of Amsterdam, } \\
\text { The Academic Medical Center }\end{array}$ \\
\hline External Member & Prof. Dr. R.M. Schiffelers & $\begin{array}{l}\text { Univeristy Medical Center } \\
\text { Utrecht }\end{array}$ \\
\hline Internal Member & Prof. Dr. H.B.J. Karperien & University of Twente \\
\hline Internal Member & Dr. J. Prakash & University of Twente \\
\hline
\end{tabular}


The research in this thesis has received funding from the European Union's Seventh Framework Programme for research, technological development and demonstration ('NanoAthero' project FP7-NMP-2012-LARGE-6-309820) as well as the Netherlands Organisation for Scientific Research Vidi grant (OND1352797).
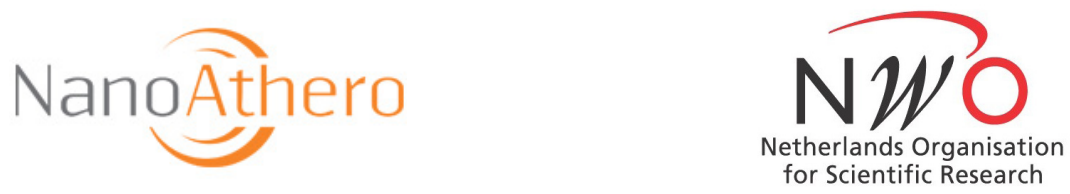

The studies in this thesis have been carried out in five institutes:
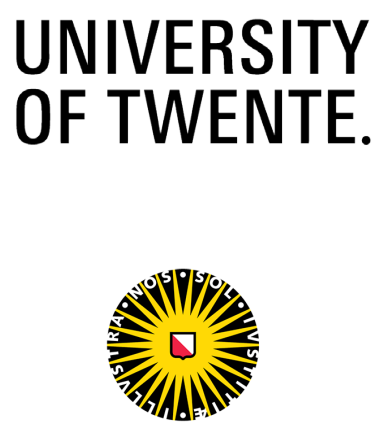

Universiteit Utrecht
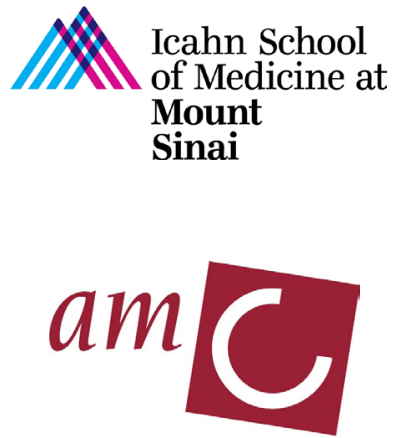

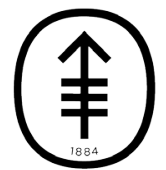

Memorial Sloan Kettering Cancer Center

The printing of this thesis was financially supported by Faculty of Science and Technology, University of Twente, Enschede, the Netherlands

Title: Targeted Therapeutics in Inflammatory Atherosclerosis

Author: Amr Alaarg

ISBN: 978-90-365-4383-5

DOI: $10.3990 / 1.9789036543835$

(C) 2017 Amr Alaarg. All rights reserved.

Cover idea and design by Amr Alaarg, Jun Tang, and Proefschriftmaken

Printed by Proefschriftmaken 



\title{
TARgeted THERAPEUtics IN \\ INFLAMMATORY ATHEROSCLEROSIS
}

\author{
DISSERTATION \\ to obtain \\ the doctoral degree at the University of Twente, \\ under the authority of the Rector Magnificus \\ Prof. Dr. T.T.M. Palstra, \\ on account of the decision of the graduation committee, \\ to be publicly defended \\ on Friday, September 08, 2017 at 14:45 \\ by \\ Amr Muhmed Sabry Abdelhakeem Alaarg \\ born on April 14, 1985, in Buraydah, Saudi Arabia
}


This dissertation has been approved by:

Promoter (Supervisor)

Prof. Dr. G. Storm

Promoter (Supervisor)

Prof. Dr. W.J.M. Mulder

Co-promoter (Co-supervisor) Dr. J.M. Metselaar

Referent (Referee) Dr. R.J. Kok 
"You will learn by reading, but you will understand with Love." Shams Tabrizi

To Ahmed You will never be forgotten 



\section{Contents}

$\begin{array}{lll}\text { Chapter } 1 \quad \text { General Introduction } & 11\end{array}$

Chapter 2 Applying Nanomedicine in Maladaptive Inflammation and 19 Angiogenesis

Published in Advanced Drug Delivery Reviews

Chapter 3 Docosahexaenoic Acid Liposomes for Targeting Chronic Inflammatory Diseases and Cancer: an In Vitro Assessment Published in International Journal of Nanomedicine

Chapter 4 Multiple Pathway Assessment to Predict Anti-Atherogenic Efficacy of Drugs Targeting Macrophages in Atherosclerotic Plaques

Published in Vascular Pharmacology

Chapter 5 Immune Cell Screening of a Nanoparticle Library Improves Atherosclerosis Therapy

Published in Proceedings of the National Academy of Sciences of the United States of America

Chapter 6 A Systematic Comparison of Clinically Viable Nanomedicines

Targeting HMG-CoA Reductase in Inflammatory

Atherosclerosis

Published in Journal of Controlled Release

Chapter 7 Hyaluronan Nanoparticles Selectively Target Plaque-

Associated Macrophages and Improve Plaque Phenotype in Atherosclerosis

Published in ACS NANO

Chapter 8 Summary and Perspectives 237

Appendices Nederlandse Samenvatting 250

Acknowledgements 252

List of Publications 



\section{CHAPTER 1}

$\mathrm{M}$

ore effective medicines are needed for the treatment of cardiovascular diseases. The objective of this thesis was to rationally explore, develop, and apply novel targeted therapeutics in experimental atherosclerosis based on the current understanding of the disease pathophysiology.

\section{ATHEROSCLEROSIS AND INFLAMMATION}

Atherosclerosis is a chronic progressive vascular disease [1]. The major complications of atherosclerosis are acute myocardial infarction, stroke, and ischemic limbs, the three top cardiovascular diseases which account for most noncommunicable deaths globally (17.7 million people/year, according to the World Health Organization) [2]. Despite the advancements in diagnosis and medical therapies, the incidence of atherosclerosis-associated life-threating conditions remains high under the current standard of care. Thus, the arena of atherosclerosis aspires for clinically viable novel therapeutics.

For the most of the $20^{\text {th }}$ century, atherosclerosis was considered as merely a lipiddriven disorder, characterized by cholesterol accumulation and thrombotic debris in the arterial wall [3]. Due to recent insights in immunology, the inflammatory component of atherosclerosis is now more appreciated as a primary driving factor in the initiation, progression, and complication of the disease [4,5]. At early stages of atherosclerosis, risk factors such as hypertension and elevated levels of apolipoprotein B-containing lipoproteins activate endothelial NF- $\kappa \mathrm{B}$ and induce focal expression of adhesion molecules, including vascular cell adhesion molecule 1 (VCAM-1) and intercellular adhesion molecules (ICAMs) [6,7]. The focal expression of these adhesion molecules in areas that are predisposed to develop atherosclerotic lesions (e.g. aortic root and arches) facilitates the engagement of circulating immune cells, in particular, monocytes which differentiate into lesion-associated macrophages, one of the most abundant immune cells in atherosclerotic lesions [8-10]. The progression and instability of atherosclerotic plaques - the focal buildup results from the accumulation of lipoproteins, calcium and other immune cells in the vessel wall - are primarily affected by plaque-associated macrophages $[11,12]$. In the early stages of atherosclerosis, plaque macrophages, derived from recruited monocytes, secrete apoB-lipoprotein binding proteoglycans increasing apoB and lipid retention in the subendothelial space [13]. Furthermore, macrophages produce reactive oxygen and nitrogen species inducing lipoprotein modifications, mainly through peroxidation [14]. These changes cause additional inflammatory processes that result in recruitment of more monocytes into the arterial intima. Additionally, macrophages engulf oxidized lipoproteins leading to the formation of foam cells, a hallmark of early atherosclerotic lesions [15]. Over the course of the disease, a complex interplay of maladaptive responses, including secretion of proinflammatory 
cytokines, unbalanced oxidative stress, impaired cholesterol efflux and local macrophage proliferation, contributes to atherosclerotic plaque remodeling and instability [16-18]. Therefore, designing and applying novel therapeutics targeting macrophage activity in atherosclerotic plaques may improve disease outcome and reduce its deadly consequences.

\section{NANOMEDICINES FOR ATHEROSCLEROSIS}

Targeted nanomedicines are nanosized drug delivery systems designed to enhance the accumulation of therapeutic molecules at pathological lesions, and thereby therapeutic efficacy. The preferential extravasation of nanoparticles, upon their systemic administration, at diseased tissue is mainly driven by associated pathophysiological changes, such as locally increased vascular permeability. Such a phenomenon, commonly denoted the enhanced permeability and retention (EPR) effect, results in the passive accumulation of drugs associated with nanocarriers and other macromolecules. Also important, nanocarriers can lower the exposure of non-target tissues to the drug as the local blood vessels do not show increased permeability (i.e. improving safety).

A myriad of nanoparticles, such as liposomes, polymeric micelles, and lipidbased nanoparticles, have been employed for the development of nanomedicines, especially for targeting highly toxic antitumor drugs to solid tumors $[19,20]$. A classic example is the PEGylated liposomal formulation of doxorubicin (Doxil $\left.{ }^{\circledR}\right)$, which demonstrated an improved safety profile (i.e. lower cardiotoxicity) in comparison to the free drug regimen in multiple oncological indications (e.g. breast cancer, ovarian cancer, and multiple myeloma) [21,22]. Despite a growing list of clinically available examples, the majority of nanomedicines under development remain at the preclinical stage. Furthermore, research in the nanomedicine field primarily focuses on oncological applications $[23,24]$.

Although cancer and atherosclerosis have different phenotypic characteristics, both diseases possess several common pathophysiological features in which inflammation and enhanced vascular permeability play critical roles [25-28]. Thus, tailoring nanomedicines for drug targeting in atherosclerosis, learning from the lessons in oncological applications, is a valid strategy. Moreover, due to monocytes / macrophages' high uptake capacity, nanomedicines are attractive candidates for targeted drug delivery to these critical effector cells to modulate their function in atherosclerosis (discussed above). 


\section{CHALLENGES IN DEVELOPING NANOMEDICINES FOR A THEROSCLEROSIS}

Several challenges and opportunities need to be carefully considered to optimally exploit nanomedicines and promote their clinical translation in atherosclerosis, So far, the field of nanomedicines has witnessed an increase in the number of nanomaterials of different compositions, shapes, and sizes. However, these nanomaterials are usually not fully characterized in vivo [29]. Moreover, sufficient investment and focus on understanding the disease itself, in which nanomedicines will be applied, are lacking.

Assessment of drug candidates' effects on the different pathways in atherosclerosis can improve our selection of the drug to target. An example of the importance of selecting the right therapeutic molecules is manifested in a pioneering clinical trial in atherosclerosis [37, Clinical Trials Identifier: NCT01601106]. The trial has been conducted using liposomal prednisolone phosphate, a potent anti-inflammatory glucocorticoid, to silence plaque inflammation. Through analyzing the isolated iliofemoral plaques, the study has demonstrated that nanomedicines can accumulate in atherosclerotic plaque macrophages in humans. However, the treatment did not reduce the arterial wall inflammation. Moreover, a follow-up study showed that prednisolone might accelerate atherosclerosis by promoting macrophage lipotoxicity and may not be an ideal candidate for targeted drug delivery [31]. Hence, careful selection of the therapeutic molecules is crucial.

Also, the selection of a proper nanoparticle type is a major factor. Beyond the size, shape, and composition of nanoparticles, which are important factors, assessment of the nanomaterial's biological performance (e.g. pharmacokinetics, tissue distribution, atherosclerotic plaque targeting and efficacy) is essential. Systematic approaches for assessing the behavior of different types of nanoparticles under (patho-)physiological conditions is often lacking. Such assessment can yield valuable information facilitating the further development of clinically relevant and efficacious nanotherapeutics. Also, adopting head-to-head approaches by comparing nanoparticles to each other can facilitate repurposing of nanomaterials, instead of inventing more new ones, to deliver compounds in maladaptive inflammatory diseases (e.g. atherosclerosis) beyond cancer. 


\section{OBJECTIVE AND OUTLINE}

The aim of this thesis is to investigate the utility of different types of nanomedicines for targeting macrophages in inflammatory atherosclerotic plaques. Following this introduction, in Chapter 2, this thesis continues with a literature overview on the systematic application of nanomedicines to target monocytes and macrophages in maladaptive inflammatory diseases, including atherosclerosis. In Chapter 3, the characteristics and in vitro evaluation of a naturally-inspired strategy to incorporate $\omega 3$ polyunsaturated fatty acids in PEGylated liposomes were studied. Such $\omega 3$ liposomes are expected to exert anti-inflammatory, antioxidant, and antiproliferative activities which were evaluated using macrophages in vitro. Since the majority of nanomedicine-mediated drug delivery strategies exploit small molecules, Chapter 4 is focused on the screening and selection of a range of potential drug candidates for targeted delivery in atherosclerosis. The effects of drug molecules were assessed using macrophages in vitro cultures macrophages, rating their overall performance with respect to proinflammatory cytokine release, oxidative stress, lipid handling, endoplasmic reticulum (ER) stress, and cell proliferation, which are critical processes in plaque macrophages. Following the selection of drug candidates, Chapter $\mathbf{5}$ deals with the implementation and evaluation of a combinatorial library of nanomaterials, based on endogenous high-density lipoprotein, in the apolipoprotein E-deficient $\left(\right.$ Apoe $\left.^{-l-}\right)$ mouse model of atherosclerosis. We quantitatively evaluated nanoparticle uptake by immune cells. Based on the outcome of this immune cell screening, we incorporated a liver $\mathrm{X}$ receptor agonist (LXR), a drug category that was rated highly anti-atherogenic in Chapter 4, into two nanocarriers: one showing favorable uptake by plaque-associated macrophages (HDL) whereas the other does not (PLGA-HDL). The therapeutic effects of the LXR nanomedicines and their ability to modulate the liver toxicity of the compound were further explored in vivo. Chapter 6 investigates the pharmaceutical characteristics and the in vivo performance of three different types of targeted nanomedicines loaded with simvastatin, a clinically available drug, which was also rated high in our in vitro screening in Chapter 4 . Drug stability/association with the nanoparticles, the biodistribution, target cell uptake, and efficacy to reduce the number of plaque macrophages of the simvastatinloaded nanocarriers were evaluated in $A p o e^{-1-}$ mice with advanced atherosclerosis. Chapter 7 presents an alternative and innovative strategy in which nanoparticles were prepared from hyaluronan, a biologically active polymer, which can target hyaluronan-binding receptors expressed on macrophages in atherosclerotic plaques. The in vivo performance of the hyaluronan nanoparticles was investigated in atherosclerotic mouse and rabbit models. Chapter 8 summarizes the results of the thesis and provides perspectives for the future application of nanomedicines to treat chronic inflammatory diseases, with particular emphasis on atherosclerosis. 


\section{REFERENCES}

[1] P. Libby, P.M. Ridker, G.K. Hansson, Progress and challenges in translating the biology of atherosclerosis, Nature. 473 (2011) 317-325. doi:10.1038/nature10146 [doi].

[2] The World Health Organization, Cardiovascular diseases (CVDs)-Fact sheet, (n.d.). http:/ / www.who.int/mediacentre/factsheets/fs317/en/.

[3] P. Libby, History of Discovery : Inflammation in Atherosclerosis, Arter. Thromb Vasc Biol. 32 (2012) 2045-2051. doi:10.1161 / ATVBAHA.108.179705.History.

[4] A.R. Tall, L. Yvan-Charvet, Cholesterol, inflammation and innate immunity, Nat. Rev. 15 (2015) 104-116. doi:10.1038/nri3793 [doi].

[5] P. Libby, Y. Okamoto, V.Z. Rocha, E. Folco, Inflammation in atherosclerosis: transition from theory to practice, Circ. J. 74 (2010) 213-220. doi:JST.JSTAGE/circj/CJ-09-0706 [pii].

[6] R. Gareus, E. Kotsaki, S. Xanthoulea, I. van der Made, M.J. Gijbels, R. Kardakaris, A. Polykratis, G. Kollias, M.P. de Winther, M. Pasparakis, Endothelial cell-specific NF-kappaB inhibition protects mice from atherosclerosis, Cell Metab. 8 (2008) 372-383. doi:10.1016/j. cmet.2008.08.016 [doi].

[7] P. Libby, M. DiCarli, R. Weissleder, The vascular biology of atherosclerosis and imaging targets, J Nucl Med. 51 Suppl 1 (2010) 33S-37S. doi:jnumed.109.069633 [pii] \r10.2967/ jnumed.109.069633.

[8] Z. Mallat, S. Taleb, H. Ait-Oufella, A. Tedgui, The role of adaptive T cell immunity in atherosclerosis., J. Lipid Res. 50 Suppl (2009) S364-9. doi:10.1194/jlr.R800092-JLR200.

[9] K.J. Moore, F.J. Sheedy, E.A. Fisher, Macrophages in atherosclerosis: a dynamic balance, Nat. Rev. 13 (2013) 709-721. doi:10.1038/nri3520 [doi].

[10] M. Nahrendorf, F.K. Swirski, Immunology. Neutrophil-macrophage communication in inflammation and atherosclerosis, Immunol. Commun. Inflamm. Atheroscler. 349 (2015) 237-238. doi:10.1126/science.aac7801 [doi].

[11] A.C. Van Der Wal, A.E. Becker, Atherosclerotic plaque rupture - Pathologic basis of plaque stability and instability, Cardiovasc. Res. 41 (1999) 334-344. doi:10.1016/S00086363(98)00276-4.

[12] C.L. Lendon, M.J. Davies, G.V.R. Born, P.D. Richardson, Atherosclerotic plaque caps are locally weakened when macrophages density is increased, Atherosclerosis. 87 (1991) 8790. doi:10.1016/0021- 9150(91)90235-U.

[13] K.J. Moore, I. Tabas, Macrophages in the pathogenesis of atherosclerosis, Cell. 145 (2011) 341-355. doi:10.1016/j.cell.2011.04.005.

[14] H. Li, S. Horke, U. Forstermann, Vascular oxidative stress, nitric oxide and atherosclerosis, Atherosclerosis. 237 (2014) 208-219. doi:10.1016/j.atherosclerosis.2014.09.001 [doi].

[15] I. Hilgendorf, F.K. Swirski, C.S. Robbins, Monocyte fate in atherosclerosis, Arterioscler. Thromb. Vasc. Biol. 35 (2015) 272-279. doi:10.1161 / ATVBAHA.114.303565.

[16] C.S. Robbins, I. Hilgendorf, G.F. Weber, I. Theurl, Y. Iwamoto, J.L. Figueiredo, R. Gorbatov, G.K. Sukhova, L.M. Gerhardt, D. Smyth, C.C. Zavitz, E.A. Shikatani, M. Parsons, N. van Rooijen, H.Y. Lin, M. Husain, P. Libby, M. Nahrendorf, R. Weissleder, F.K. Swirski, Local proliferation dominates lesional macrophage accumulation in atherosclerosis, Nat. Med. 19 (2013) 1166-1172. doi:10.1038/nm.3258 [doi].

[17] I. Tabas, Macrophage death and defective inflammation resolution in atherosclerosis., Nat. Rev. Immunol. 10 (2010) 36-46. doi:10.1038/nri2675.

[18] W.V. Brown, A.T. Remaley, P.M. Ridker, JCL Roundtable: Is inflammation a future target in preventing arteriosclerotic cardiovascular disease, J. Clin. Lipidol. 9 (2015) 119-128. doi:10.1016/j.jacl.2015.01.007. 
[19] V.P. Torchilin, Targeted pharmaceutical nanocarriers for cancer therapy and imaging., AAPS J. 9 (2007) E128-47. doi:10.1208/aapsj0902015.

[20] E.K. Chow, D. Ho, Cancer nanomedicine: from drug delivery to imaging, Sci. Transl. Med. 5 (2013) 216rv4. doi:10.1126/ scitranslmed.3005872 [doi].

[21] S.M. Moghimi, A.C. Hunter, J.C. Murray, Nanomedicine: current status and future prospects, FASEB J. 19 (2005) 311-330. doi:19/3/311 [pii].

[22] T. Lammers, F. Kiessling, W.E. Hennink, G. Storm, Drug targeting to tumors: principles, pitfalls and (pre-) clinical progress, J. Control. Release. 161 (2012) 175-187. doi:10.1016/j. jconrel.2011.09.063 [doi].

[23] J.I. Hare, T. Lammers, M.B. Ashford, S. Puri, G. Storm, S.T. Barry, Challenges and strategies in anti-cancer nanomedicine development: An industry perspective, Adv. Drug Deliv. Rev. 108 (2017) 25-38. doi:10.1016/j.addr.2016.04.025.

[24] R. van der Meel, T. Lammers, W.E. Hennink, Cancer nanomedicines: oversold or underappreciated?, Expert Opin. Drug Deliv. 14 (2017) 1-5. doi:10.1080/17425247.2017. 1262346.

[25] J.J. Li, R.L. Gao, Should atherosclerosis be considered a cancer of the vascular wall?, Med. Hypotheses. 64 (2005) 694-698. doi:10.1016/j.mehy.2004.11.043.

[26] J.S. Ross, N.E. Stagliano, M.J. Donovan, R.E. Breitbart, G.S. Ginsburg, Atherosclerosis: a cancer of the blood vessels?, Am. J. Clin. Pathol. 116 Suppl (2001) 97-107. doi:10.1309/ YNCK-9R19-5JA3-K2K9.

[27] M. Morganti, A. Carpi, A. Nicolini, I. Gorini, B. Glaviano, M. Fini, G. Giavaresi, C. Mittermayer, R. Giardino, Atherosclerosis and cancer: Common pathways on the vascular endothelium, Biomed. Pharmacother. 56 (2002)317-324. doi:10.1016/S0753-3322(02)002421.

[28] M.E. Lobatto, V. Fuster, Z.A. Fayad, W.J. Mulder, Perspectives and opportunities for nanomedicine in the management of atherosclerosis, Nat. Rev. Discov. 10 (2011) 835-852. doi:10.1038/nrd3578 [doi].

[29] S. Mitragotri, T. Lammers, Y.H. Bae, S. Schwendeman, S. De Smedt, J.-C. Leroux, D. Peer, I.C. Kwon, H. Harashima, A. Kikuchi, Y.-K. Oh, V. Torchilin, W. Hennink, J. Hanes, K. Park, Drug Delivery Research for the Future: Expanding the Nano Horizons and Beyond, J. Control. Release. 246 (2017) 183-184. doi:10.1016/j.jconrel.2017.01.011.

[30] F.M. van der Valk, D.F. van Wijk, M.E. Lobatto, H.J. Verberne, G. Storm, M.C.M. Willems, D.A. Legemate, A.J. Nederveen, C. Calcagno, V. Mani, S. Ramachandran, M.P.M. Paridaans, M.J. Otten, G.M. Dallinga-Thie, Z.A. Fayad, M. Nieuwdorp, D.M. Schulte, J.M. Metselaar, W.J.M. Mulder, E.S. Stroes, Prednisolone-containing liposomes accumulate in human atherosclerotic macrophages upon intravenous administration., Nanomedicine. 11 (2015) 1039-46. doi:10.1016/j.nano.2015.02.021.

[31] F.M. van der Valk, D.M. Schulte, S. Meiler, J. Tang, K.H. Zheng, J. Van den Bossche, T. Seijkens, M. Laudes, M. de Winther, E. Lutgens, A. Alaarg, J.M. Metselaar, G.M. DallingaThie, W.J.M. Mulder, E.S.G. Stroes, A.A.J. Hamers, Liposomal prednisolone promotes macrophage lipotoxicity in experimental atherosclerosis, Nanomedicine Nanotechnology, Biol. Med. 12 (2016) 1463-1470. doi:10.1016/j.nano.2016.02.022. 




\section{CHAPTER 2}

\section{Applying Nanomedicine in Maladaptive Inflammation and Angiogenesis*}

\section{ABSTRACT}

I

nflammation and angiogenesis drive the development and progression of multiple devastating diseases such as atherosclerosis, cancer, rheumatoid arthritis, and inflammatory bowel disease. Though these diseases have very different phenotypic consequences, they possess several common pathophysiological features in which monocyte recruitment, macrophage polarization, and enhanced vascular permeability play critical roles. Thus, developing rational targeting strategies tailored to the different stages of the journey of monocytes, from bone marrow to local lesions, and their extravasation from the vasculature in diseased tissues will advance nanomedicine. The integration of in vivo imaging uniquely allows studying nanoparticle kinetics, accumulation, clearance, and biological activity, at levels ranging from subcellular to an entire organism, and will shed light on the fate of intravenously administered nanomedicines. We anticipate that convergence of nanomedicines, biomedical engineering, and life sciences will help to advance clinically relevant therapeutics and diagnostic agents for patients with chronic inflammatory diseases. 


\section{INTRODUCTION}

Inflammation is a key underlying process in several disorders, including cancer, cardiovascular diseases, rheumatoid arthritis, and inflammatory bowel disease (IBD). The aforementioned maladies are leading causes of morbidity and mortality worldwide and impose a substantial socioeconomic burden [1,2]. Although new therapeutics are being developed, the incidence of complications remains high under the current standard of care [3,4]. This is in part because conventional therapies are not specific to the diseased tissue, and therefore cause considerable offtarget adverse effects [5]. Equally important, disease heterogeneity is not taken into account. Current diagnostic approaches do not allow identification of those patients who would benefit the most from a given therapy or those who would likely suffer from complications [6-8]. Additionally, therapeutic strategies are often diseasespecific, disregarding the common denominator: inflammation. Seemingly diverse, the diseases mentioned above possess common pathophysiological features driven - or aggravated - by inflammation, including leukocytosis, tissue remodeling, local cell proliferation and angiogenesis [9-11].

Extensive research of different pathophysiological processes has unveiled the role of multiple immune cells in diseased tissues that drive the development and progression of inflammatory disorders. One of the most effector cells in inflammatory lesions is a monocyte-derived macrophage, which is a key component of innate immunity [12-16]. Monocytes and macrophages not only contribute to the local inflammatory milieu of chronic inflammatory diseases but also modulate endothelial permeability and recruitment of supplementary immune cells, thereby driving disease progression $[17,18]$. Elucidating and understanding macrophage dynamics over the course of inflammatory disease progression offer unique opportunities for designing more specific and efficacious diagnostic and therapeutic agents. For example, our increased knowledge of the inflammatory process has yielded powerful immunotherapies [19], including anti-tumor necrosis factor alpha (TNF- $\alpha$ ) antibodies [20], interleukin 6 (IL-6) inhibitors [21], and bifunctional antibodies [22], which modulate the immune response to achieve remission of chronic inflammation.

Simultaneously, nanomedicine - defined as "the application of nanotechnology for treatment, diagnosis, monitoring, and control of biological systems" [23] - has experienced unprecedented growth in the past decade. In addition to improving the therapeutic index of drugs, monocyte/macrophage-targeted nanomedicine can be implemented as a cell-specific drug delivery strategy to pharmacologically modulate inflammation. Such 'immunomodulatory nanoplatforms' can also be adopted for diagnostic and imaging purposes, not only at the level of local inflammatory lesions and organs involved in monocytosis but also to visualize macrophage dynamics systemically $[24,25]$. 
This review discusses the interplay between advances in nanomedicine and our understanding of chronic inflammatory disorders. This vantage point allows exploiting the commonalities of monocyte / macrophage dynamics across diseases for targeted therapy and diagnosis. We will highlight how nanomedicine can be applied at the inflammatory process' different stages, in progressing disease. In addition, we will share our perspective on inflammation-oriented nanomedicine design and how it will facilitate developing improved therapies and diagnostic tools for patients. Although this review focuses on monocytes and macrophages as key effector cells in chronic inflammation and most prone to take up nanomedicines, other immune cells, such as T and B cells, have vital roles in complex paracrine signaling between macrophages and other cells in the inflammatory lesions, discussed in detail in other reviews [26-29].

\section{INFLAMMATION AND ANGIOGENESIS IN DISEASE}

Inflammation is a vital protective process in host defense against infections and injuries, which ultimately - through a resolution process - restores homeostatic conditions in the affected tissue [30]. However, persistent unresolved inflammation can contribute to the initiation and progression of several chronic diseases, including cancer [27], atherosclerosis [31], rheumatoid arthritis [29], and IBD [32]. The inflammatory response consists of interplay between innate and adaptive immunity $[33,34]$. This cross-talk modifies the function of other cells, such as epithelial cells (e.g. endothelial cells) and mesenchymal cells (e.g. smooth muscle cells) over time, leading to different disease stages [35,36]. Macrophages are key players that intercommunicate with adaptive immune $\mathrm{T}$ and $\mathrm{B}$ cells, and also directly affect the inflammatory milieu through secretion of inflammatory mediators [10,37]. Inflammatory macrophages differentiate from lesion infiltrating monocytes and express a set of pattern recognition receptors (e.g. Toll-like receptors and scavenger receptors) that can sense pathogen-associated molecular patterns (PAMPs) and endogenous tissue damage-associated molecular patterns (DAMPs) [38]. Engagement of these receptors results in activation of nuclear factorkappa B (NF-kB), and production of proinflammatory cytokines, reactive oxygen and nitrogen species, and other mediators that can intensify the inflammatory response $[39,40]$. Moreover, due to elevated metabolic activity, inflamed tissues are notoriously hypoxic, activating macrophages, fibroblasts, and endothelial cells to produce hypoxia-inducible factors (HIFs) [41-43] and vascular endothelial growth factor (VEGF). Note that VEGF was originally denoted vascular permeability factor (VPF). Chronic exposure to HIF 1 and 2, VEGF, and inflammatory cytokines results in degradation of endothelial cell adherens junctions, dissolution of the basement membrane, and dramatic destabilization of existing microvessels [44-46]. Moreover, these factors facilitate endothelial cells' proliferation and migration, inducing the 
growth of new blood vessels from existing ones, a process known as angiogenesis. Angiogenesis and chronic inflammation, long treated as two distinct processes, are thus codependent [47-49]. In the next sections, we will provide an overview of the involvement of monocytes and macrophages in the pathogenesis of several chronic inflammatory diseases, and discuss the common aberrant endothelial function and pathological angiogenesis, which are affected by the local inflammatory milieu.

\subsection{Cancer}

Cancer is a multifaceted malady in which cells are no longer completely responsive to the signals that regulate cellular differentiation, growth, proliferation, and death. As a result, these cancerous cells accumulate within the tissue, causing local damage and inflammation [27]. Rudolf Virchow established the first link between cancer and inflammation in the $19^{\text {th }}$ century when he observed the infiltration of leukocytes in neoplastic tissues [50]. In the last twenty years, our understanding of carcinogenesis and the tumor microenvironment has supported Virchow's hypothesis, substantiating the role of inflammation in the initiation, promotion, and metastatic progression of cancer [51,52]. Moreover, increasing epidemiological evidence suggests that several chronic inflammatory diseases (e.g. IBD and hepatitis), associated or not with infectious agents, predispose individuals to several types of cancer $[51,53]$. Tumor-associated macrophages (TAMs) are the most abundant immune cells in the tumor microenvironment [54]. They are derived from circulating inflammatory monocytes, which are preferentially attracted to lesions by tumor-derived chemotactic factors, like C-C motif chemokine ligand 2 (CCL-2; also known as monocyte chemoattractant protein 1, MCP-1) [55]. TAM density is positively correlated with the levels of tumor-derived CCL-2, and usually associated with poor prognosis in various types of cancer [56-58]. The protumorigenic activities of TAMs can be appreciated in cancer initiation, tumor cell invasion and migration, and metastasis [59]. Inflammatory macrophages are key producers of reactive nitrogen and oxygen species that can generate oncogenic mutations and lead to tumor initiation [60]. These reactive species also cause activation of epithelial cells and the consequent recruitment of more monocytes [61]. In addition to the initiation phase, most invasive tumors demonstrate collective intravasation, in which groups of tumor cells invade the blood vessels while maintaining cell-cell contacts $[62,63]$. Tumor cell intravasation is shown to occur upon physical contact with macrophages, which induces RhoA GTPase activity in tumor cells, regulates actin cytoskeleton, and triggers the formation of invadopodia [64,65]. The combined effect of improved RhoA GTPase activity and proteolytic enzymes (e.g. MMPs), secreted by TAMs and cancer-associated fibroblasts (CAFs), enables tumor cells to degrade and break through matrix barriers in the course of tumor cell transendothelial migration [66-68]. Moreover, the paracrine signaling between tumor cells and stromal cells (e.g. CAFs and TAMs), through epidermal growth factor (EGF) and colony stimulating factor-1 (CSF-1), can activate tumor cell growth, and enhance migration and extravasation $[69,70]$. In most tumors, malignant switch and tumor propagation require an 
adequate supply of oxygen and nutrients, and an effective way to remove waste products, which is achieved through the development of a dense vessel network that connects the tumor to host circulation. Tumor vasculature development, also known as the angiogenic switch, is profoundly dependent on TAMs and other stromal cells (e.g. endothelial cells, smooth muscle cells, fibroblasts), which secrete several angiogenic growth factors and proteinases, including VEGFs [71,72] and matrix metalloproteinases [73]. The developed tumor vasculature is usually chaotic and leaky (i.e. with large inter-endothelial cell fenestrations), facilitating tumor cell intravasation and metastatic dissemination [74]. These migrating cells establish themselves at metastatic niches where they recruit monocytes and macrophages, mainly via CCL-2. Metastasis-associated macrophages (MAMs) secrete another chemokine, CCL-3, which affects MAM retention and interaction with tumor cells, enhancing metastatic seeding and growth [57].

\subsection{Atherosclerotic cardiovascular diseases}

Atherosclerosis is a chronic inflammatory disease of the large and medium-sized arteries [37]. Although atherosclerotic cardiovascular diseases are as old as ancient Egyptian mummies [75], they remain the leading cause of mortality worldwide, accounting for most deaths among non-communicable diseases. According to the World Health Organization, 17.7 million deaths per year, an estimated 31\% of all deaths worldwide, can be attributed to atherosclerotic diseases [76]. Due to recent advances in immunology, the inflammatory component of atherosclerosis is now more appreciated, and the disease is no longer thought to be only due to aberrant lipid deposition. Inflammation plays a prominent role at different stages of atherosclerosis and contributes to its complications [40,77]. At early stages, risk factors such as smoking, hypertension, and elevated levels of apolipoprotein B-containing lipoproteins can induce, partially via activating NF- $\kappa \mathrm{B}$, focal expression of endothelial adhesion molecules, including vascular cell adhesion molecule 1 (VCAM-1) and intercellular adhesion molecules (ICAMs) [78,79]. The focal expression of these adhesion molecules in areas that are prone to develop atherosclerotic lesions (e.g. aortic root and arches) facilitates the binding and adherence of circulating immune cells, such as monocytes. Once adhered, monocytes transmigrate to the subendothelial space and mature into resident macrophages under the influence of a complex mix of vascular wall- derived chemokines, such as macrophage colonystimulating factor (M-CSF) [80]. The accumulation of lipoproteins, calcium, and immune cells within the vessel wall leads to the development of focal lesions, known as atherosclerotic plaques. This buildup is mainly affected by plaqueresident macrophages, which secrete apoB-lipoprotein binding proteoglycans increasing apoB retention in the subendothelial space [81]. Moreover, macrophages produce reactive oxygen and nitrogen species inducing lipoprotein modifications, mainly through peroxidation. These modifications fuel further inflammatory processes that result in recruitment of more monocytes into the arterial intima [82]. Additionally, macrophages engulf the native and modified lipoproteins leading to 
the formation of foam cells, which further amplify lipoprotein oxidation, uptake, retention, and modification through expression of scavenger receptors, such as type A scavenger receptor (SRA) and a member of the type B family, namely CD36 $[83,84]$. These receptors can also cooperate with toll-like receptors and promote inflammasome activation and secretion of proinflammatory cytokines in response to modified lipoproteins $[85,86]$. Moreover, macrophages secrete proteolytic enzymes, including MMPs, which may contribute to atherosclerotic plaque remodeling and instability [87]. Similar to tumor angiogenesis, atherosclerotic plaques also undergo neovascularization under the influence of macrophage- derived angiogenic factors [88]. Neovascularization contributes to advanced plaques instability by facilitating the infiltration of additional inflammatory immune cells and/or acting as a source of intraplaque hemorrhage [89,90]. Furthermore, perturbed lipid efflux may increase macrophage cell death in advanced stages, aggravating the inflammatory burden and decreasing plaque stability [91].

\subsection{Rheumatoid arthritis}

Rheumatoid arthritis (RA) is an immune-mediated, chronic inflammatory disease that primarily affects synovial membranes, cartilages, and bones [29]. The prevalence of the disease is around 1\% globally and usually associated with progressive disability, severe morbidity and systemic complications, such as accelerated atherosclerosis [92]. The cause of RA is unknown [93]. However, the increasing understanding of RA pathophysiology underscores the role of immune cells in the initiation and progression of the disease [94]. The cross-talk between immune cells and skeletal systems, the two key components of RA osteoimmunology, results in the production of autoantibodies, infiltration of immune cells into the affected joints, and ultimately joint destruction [95]. Macrophages are key effectors in these processes, as evidenced by their abundance in the synovial lining and the strong correlation between macrophage number and the extent of joint destruction $[96,97]$. The crosstalk between macrophages and T cells plays a crucial role in disease initiation. Macrophages activate naïve T cells, likely by acting as antigen presenting cells and/or through $\mathrm{T}$ cell-monocyte/macrophage interaction [98,99]. Once activated, naïve $\mathrm{T}$ cells enter a proliferative state and secrete cytokines, including interferon-gamma (IFN- $\gamma$ ) and IL-17, which further skew macrophages towards an inflammatory state [100]. Immunohistological studies of isolated synovium have shown that activated macrophages are the main producers of proinflammatory cytokines, including IL-1, IL-6, and TNF- $\alpha$ [101,102]. These cytokines trigger synovial fibroblast activation, leading to hypertrophied synovium (also called pannus) which is a tumor-like tissue that invades and destroys the local articular tissue [102,103]. Furthermore, macrophage-derived granulocyte-macrophage colony stimulating factor (GM-CSF) and other cytokines stimulate the maturation of innate immune cells, their efflux from bone marrow, and their migration into the synovium [104]. In addition to the inflammatory component, RA progression is usually associated with pathophysiological angiogenesis. The infiltration of immune cells and the 
highly inflammatory milieu results in local hypoxia, secretion of HIFs and growth factors (mainly by macrophages and fibroblasts), increased vascular permeability, and formation of new blood vessels to supply nutrients and oxygen [105,106]. Such a complex microenvironment of proinflammatory cytokines and immune cells stimulate osteoclasts - multinucleated cells of the monocytic lineage - to degrade bone matrix and solubilize bone calcium, leading to inflammation-driven bone erosion in advanced stages $[107,108]$.

\subsection{Inflammatory bowel disease}

Inflammatory bowel disease (IBD), which includes Crohn's disease (CD) and ulcerative colitis (UC), are chronic, relapsing inflammatory diseases of the gastrointestinal (GI) tract [109]. In these diseases, inflammation impairs the ability of the affected GI to function properly, resulting in persistent diarrhea, abdominal pain, cramping, and rectal bleeding. As most chronic inflammatory diseases, genetic predispositions, environmental triggers, and immune cells contribute to IBD pathogenesis and progression $[109,110]$. Under normal physiological conditions, the main function of intestinal immune cells is to maintain the integrity of the epithelial barrier along the GI tract against external stimuli - e.g. food and the microflora - without eliciting a strong inflammatory reaction [111]. Intestinal macrophages, strategically located in the intestinal lamina propria, represent the largest macrophage population in the body [112]. During an IBD flare, this tolerance is lost either by a change in the microflora or a defect in the immune response to the existing flora $[113,114]$. Such a change leads to imbalance in the number of $T$ cells and recruitment of blood monocyte and their differentiation into activated macrophages, which are phenotypically different from resident macrophages [115]. The activated macrophages secrete proinflammatory cytokines, including IL-23 and TNF- $\alpha$ [116,117]. In addition to their inflammatory actions, these cytokines modulate epithelial cell growth, $\mathrm{T}$ cell activity, intestinal and vascular permeability, and the production of reactive oxygen species $[118,119]$. The active phase of IBD is usually accompanied by increased microvascular density and permeability, which facilitate the infiltration of more proinflammatory immune cells [120]. Ultimately, these inflammation-driven changes lead to barrier dysfunction, tissue damage, and fibrosis $[121,122]$. 


\section{APPLYING NANOMEDICINE IN INFLAMMATION DYNAMICS}

There are strong associations and comorbidities between different types of chronic inflammatory disorders [123-125]. Also, striking similarities in the dynamics of the aforementioned diseases and other chronic inflammatory disorders exist. The diseases are usually initiated by a persistent local tissue insult or injury that activates the (extra) medullar hematopoiesis and attracts inflammatory monocytes to the lesions. Infiltrating monocytes and lesion-associated macrophages affect other cells in the microenvironment and induce pathological angiogenesis and local tissue remodeling. Such holistic understanding of chronic inflammation dynamics offers opportunities to develop therapeutics and diagnostic agents that can be applied to multiple diseases. Employing nanomedicines in chronic inflammatory diseases can advance, revive, and repurpose efficacious therapeutics and diagnostic agents. Nanomaterials are highly tunable and can be designed to exhibit different sizes, shapes, and surface chemistry, which can be exploited to modulate nanoparticles' in vivo behavior (e.g. circulation kinetics, cell uptake, and tissue penetration) [126]. Moreover, nanoparticles can be used as carriers for different therapeutic cargos such as small molecule drugs (hydrophilic and hydrophobic) [127], peptides [128], and nucleic acids [129]. Nanoparticles can also be used to solubilize poorly water-soluble compounds that are intended for parental use [130]. Additionally, nanomedicine approaches may lead to a drug's improved therapeutic index through increasing the on-target efficacy, while reducing the off-target toxicity [131]. As diagnostic probes, nanoparticles are highly amenable and can be labeled for optical, magnetic resonance imaging (MRI), computed tomography (CT) and nuclear imaging approaches $[24,132,133]$. In addition, due to the high phagocytic activities of monocytes and macrophages, nanoparticles are suitable for imaging not only inflammatory lesions, but also the dynamics of inflammatory cells in disease [24,134]. Thus, applying nanomedicine at the different stages of monocyte/macrophage dynamics can foster our understanding of inflammation and can be used to modulate these processes. An overview of these processes that can be tackled by nanomedicine on a systemic level is depicted in Figure 1. In subsequent sections, we will zoom in the exploitation of nanomedicine and the processes that monocytes and macrophages modulate during chronic inflammation in their corresponding compartments. In this context, we will share lessons learned and challenges faced, followed by our outlook on the future. 
A
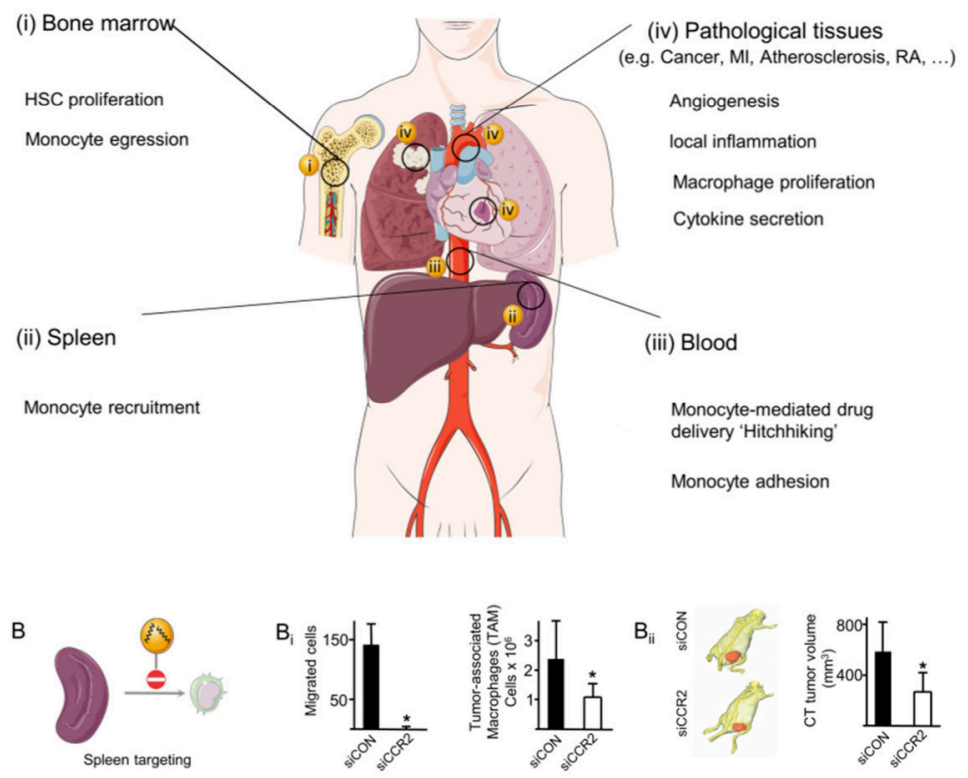

$B_{\text {ii }}$ 五

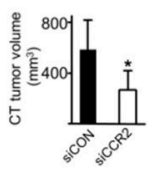

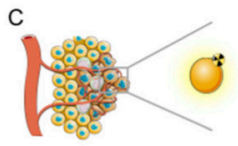

TAM targeting

D

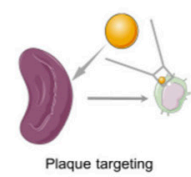

E

Other applications
$\mathrm{C}_{\mathrm{i}}$

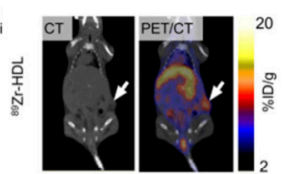

$D_{i}$

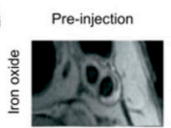

$E_{i}$

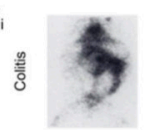

$\mathrm{C}_{\text {ii }}$

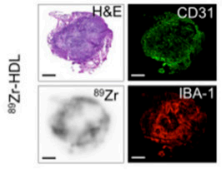

$\mathrm{D}_{\text {ii }}$

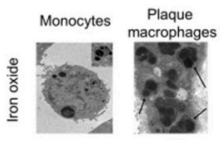

$\mathrm{E}_{\mathrm{ii}}$

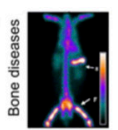

Figure 1I Applying nanomedicine along the journey of monocytes in inflammatory disorders. (A) Several processes that contribute to monocyte/macrophage dynamics in maladaptive inflammation and angiogenesis can be exploited for imaging and therapeutic purposes at the systems level. In (i) and (ii), tackling HSC proliferation and monocyte migration from the bone marrow and spleen by nanomedicines can be an upstream approach to control inflammation. In (iii), trafficking of inflammatory monocytes in the circulation and their adhesion to activated endothelial cells can be exploited for cell-mediated therapies and diagnosis. (iv) Applying nanomedicines at the lesion level can be realized by tackling the enhanced vascular permeability, local macrophage proliferation and activity, and the secretion of proteases or cytokines. Examples of nanomedicines targeting (B) the spleen, (C) tumor-associated macrophages, (D) monocyte migration, and (E) other diseased tissues. Graphs and images in $B_{i}$ and $B_{i i}$ are adapted, with permission, from [1]. PET/CT in $\mathrm{C}_{\mathrm{i}}$ and histological images in $\mathrm{C}_{\mathrm{ii}}$ are reproduced, with permission, from [2]. MRI images in $\mathrm{D}_{i}$ were adapted, with permission, from [3] while histological images in $\mathrm{D}_{\mathrm{ii}}$ are adapted, with permission, from [4] (left) and [5] (right). Scintigraphic images of rabbits in $\mathrm{E}_{\mathrm{i}}$ and $\mathrm{E}_{\mathrm{ii}}$ are reproduced, with permission, from [6] and [7], respectively. HSC: hematopoietic stem cell, MI: myocardial infarction, RA: rheumatoid arthritis, TAM: tumor-associated macrophages. 


\subsection{Bone marrow activation}

Under homeostatic conditions, the bone marrow contains self-renewing, quiescent hematopoietic stem cells (HSCs), which maintain the blood levels of monocytes and other immune cells within normal physiological values. However, chronic inflammatory diseases are associated with elevated systemic levels of proinflammatory cytokines, such as IL-1, IL-6, and TNF- $\alpha$ [142-144], and TLR agonists, like heat-shock proteins, and saturated and unsaturated fatty acids [145]. Furthermore, stress, which results in increased sympathetic activity and release of noradrenaline, is often associated with chronic inflammatory diseases [146-148]. Such biochemical changes can lead to HSC proliferation and overproduction of inflammatory monocytes, which ultimately accumulate in lesions [146,149]. The mobilization/ egression of inflammatory monocytes from the bone marrow is enhanced by the CCL-2/CCR-2 interaction and the increased permeability of blood vessels in the bone marrow [150]. Moreover, bone marrow components and inflammation contribute to the local angiogenic processes. A classic example is bone marrow angiogenesis in patients with multiple myeloma [151] and breast cancer [152]. Metastatic breast cancer and myeloma plasma cells induce activation of inflammatory cells, including macrophages, to secrete angiogenic factors such as VEGF, fibroblast growth factor-2, and granulocyte macrophage-colony stimulating factor [153], which activate endothelial cells and accelerate angiogenesis in the bone marrow. Moreover, bone marrow macrophages, under the influence of fibroblast and plasma cells-secreted factors, can acquire endothelial cell markers and transform into cells that are functionally and phenotypically similar to bone marrow endothelial cells [154]. Thus, they participate in the development of the bone marrow microvascular system (i.e. vasculogenic mimicry). Bone marrow also is the main source of endothelial progenitor cells (EPCs) and other myeloid cells which contribute to the angiogenic processes at distant inflammatory lesions [155].

Nanomedicine's application at different stages of bone marrow activation holds promise for the diagnosis and treatment of inflammatory diseases (Figure 2). Nanoparticle delivery of anti-inflammatory drugs (e.g. corticosteroids) [156] or CCR-2 siRNA [135] can modulate the bone marrow response to circulating proinflammatory cytokines. The enhanced permeability of bone marrow blood vessels and the increased nanoparticle uptake by activated macrophages can facilitate their accumulation and retention [157-159]. Nanoparticles, including liposomes and reconstituted high-density lipoprotein (rHDL), have been shown to accumulate in the bone marrow of animals with inflammatory conditions, such as osteomyelitis [160] and atherosclerosis [161], to a greater extent than in nondiseased counterparts. Moreover, nanoparticle accumulation in the bone marrow can be further enhanced through active targeting. For example, conjugation of bisphosphonates (or alendronate) to poly(lactic-co-glycolic acid) (PLGA) polymeric nanoparticles increases their affinity to hydroxyapatite, a major mineral component of the bone $[162,163]$. Alternatively, incorporation of certain synthetic substances 
has been shown to relatively reduce nanoparticle accumulation in the liver while, concomitantly, increase their accumulation in the bone. Two of such materials are the hydrophilic non-ionic surfactant poloxamer 407 and the anionic amphiphilic lipid L-glutamic acid N-(3-carboxy-1- oxopropyl)-1,5-dihexadecyl ester which have been used as integral components of bone marrow-targeted nanospheres [164] and liposomes [141], respectively. The use of bone marrow-targeted nanomedicines can improve the therapeutic efficacy of small molecules, such as bortezomib in myeloma [162]. Tackling the hyper-proliferative state and/or the egression of HSCs and inflammatory monocytes has been shown to be an upstream approach for managing inflammatory diseases [135]. In addition, combining bone marrowtargeted nanomedicines with molecular imaging can be used to assess the effect of this therapeutic approach on bone marrow activation. For instance, the proliferation of bone marrow macrophages and HSCs can be quantified noninvasively with molecular imaging using ${ }^{18} \mathrm{~F}-3^{\prime}$-fluoro-3'-deoxythymidine $\left({ }^{18} \mathrm{~F}-\mathrm{FLT}\right)$ positron emission tomography-computed tomography (PET/CT) [165-167]. Furthermore, their activation can be evaluated by ${ }^{18} \mathrm{~F}$ - fluorodeoxyglucose $\left({ }^{18} \mathrm{~F}-\mathrm{FDG}\right) \mathrm{PET} / \mathrm{CT}$ [168]. 


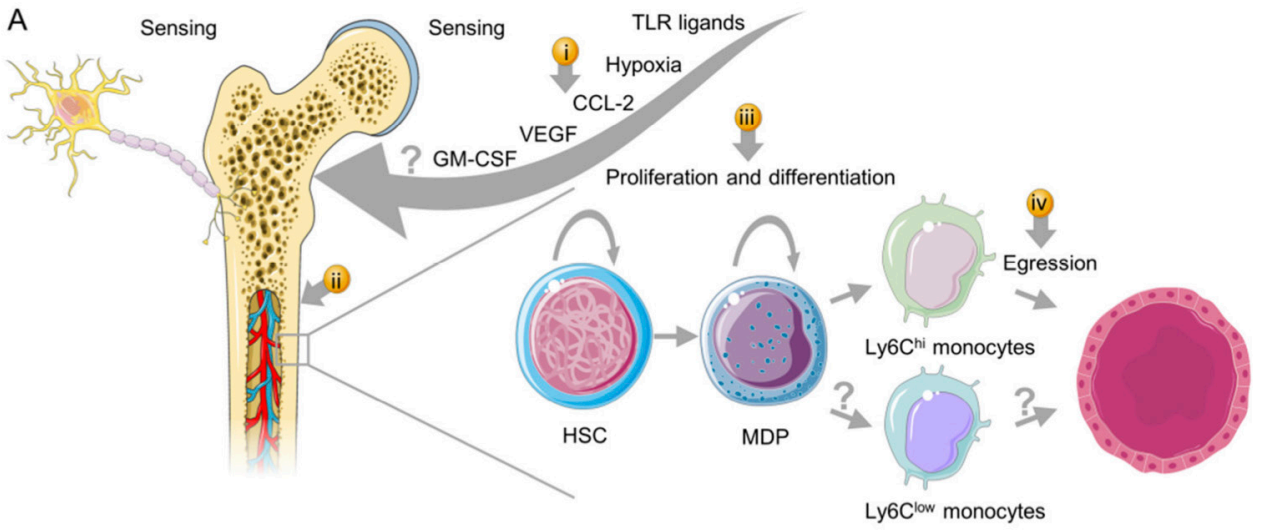

B

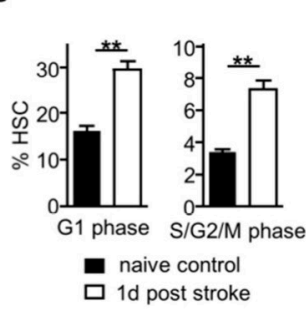

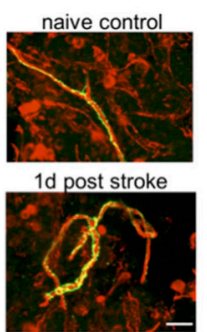

C

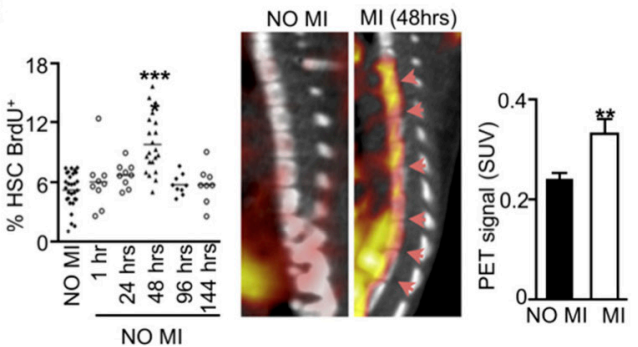

Figure 2 I Bone marrow activation. Inflammatory disorders are characterized by elevated levels of circulating cytokines, growth factors, and damage-associated molecules. The stress and pain associated with the disease increase sympathetic nervous activity. Both biochemical and neuronal changes increase proliferation and migration of both HSCs and inflammatory monocytes (Ly6Chi) from bone marrow niches. (A) Nanomedicine can be used to target different features of bone marrow activation, including (i) circulating bone marrow activators, (ii) bone marrow permeability, (iii) HSC proliferation, and (iv) monocyte egress. Combining nanomedicines with molecular imaging at the medullar level can advance our understanding of disease progress. For example, (B) ischemic stroke increases the sympathetic nervous activity, which regulates the proliferation and cell cycle of HSCs, as shown by immunofluorescence staining of tyrosine hydroxylase rich nerve fibers of the sternal bone marrow. (C) Myocardial infarction (MI) increases HSC proliferation in the bone marrow, a process that can be quantified by BrdU staining, and imaged by ${ }^{18}$ F-FLT positron emission tomography/computed tomography (PET/CT). Panel B is modified, with permission, from [8]. Panel C is modified, with permission, from [9]. TLR: toll-like receptor, CCL-2: C-C motif chemokine 2, VEGF: vascular endothelial growth factor, GM-CSF: granulocytemacrophage colony-stimulating factor, HSC: hematopoietic stem cell, MDP: monocyte and dendritic cell progenitor, SUV: standardized uptake value, BrdU: bromodeoxyuridine. 


\subsection{Monocyte mobilization and recruitment}

In the course of chronic inflammation, recruitment and trafficking of inflammatory monocytes are driven by a gradient of circulating chemokines. Although bone marrow is the major source of blood monocytes, extramedullar hematopoiesis (i.e. outside the bone marrow), especially in the spleen, is also driven by inflammation $[170,171]$ (Figure 3). After fetal development, splenic hematopoiesis remains dormant, and the spleen acts as a reservoir seeded with HSCs mobilized from the bone marrow. However, these spleen-resident HSCs are highly responsive, and they regain their proliferative capacity upon exposure to hormonal and inflammatory cues $[170,172,173]$. For example, inflammatory conditions, such as atherosclerosistriggered ischemic events, can activate splenic hematopoiesis and result in overproduction and deployment of splenic inflammatory monocytes into the circulation [172]. Hence, imaging splenic activity in such pathological conditions, for example by ${ }^{18} \mathrm{~F}$-FDG-PET, can be useful to monitor therapeutic response and has been shown to serve as a good predictor of future complications by Tawakol and colleagues [174]. The accelerated hematopoiesis and rapid egress of inflammatory monocytes from splenic niches rely in part on CCL-2/CCR-2 interaction and angiotensin II (Ang II)-Ang type I and II receptors $[14,175,176]$. Applying nanomedicine to target splenic inflammatory monocytes is compelling due to the high accumulation of systemically administered nanoparticles in the spleen [177]. Leuschner et al. have used CCR-2 siRNA-loaded nanoparticles to selectively knock down the expression of CCR-2 in splenic inflammatory monocytes without affecting the patrolling, noninflammatory subset [135]. Such a treatment led to a monocyte reduction in lesions and was shown a viable therapeutic approach in multiple conditions such as acute myocardial infarction (AMI), pancreatic islet transplantation, and cancer [135]. Similarly, irbesartan-loaded PLGA nanoparticles have been used to block Ang II type 1 receptor, inhibiting the recruitment of inflammatory monocytes and reducing infarct size in a myocardial ischemia-reperfusion (IR) injury model [178].

Noninvasive monitoring of the journey of inflammatory monocytes through the circulation could provide insights into their homing, tissue adhesion, and tissue penetration characteristics. Such understanding would not only allow evaluating disease progression and response to treatment but could also help to design immunomodulatory therapeutics which have monocyte-like features to increase their tissue/lesion penetration. Several imaging techniques combined with or without nanomedicines have been used to monitor monocytes on the move. For example, intravital microscopy (IVM) can be used to detect fluorescent cells (e.g. $\mathrm{GFP}^{+}$monocytes) in live organisms and to study their behavior in the circulation, in hematopoietic tissues, and in healthy and diseased tissues with a single-cell resolution $[175,181-183]$. Moreover, the exclusive uptake of certain fluorescently labeled nanoparticles, such as single-walled carbon nanotube (SWNT), by inflammatory monocytes allows studying both nanoparticle and monocyte trafficking in tumor models using IVM [179]. Alternatively, monocyte dynamics can be studied in 
deeper tissues with excellent spatial resolution using magnetic resonance imaging (MRI). Contrast MRI uses exogenous cell labels, such as superparamagnetic iron oxide (SPIOs) nanoparticles or Fluorine-19 $\left({ }^{19} \mathrm{~F}\right)$-based perfluorocarbon (PFC) nanoemulsions [184]. Iron oxide nanoparticles have already been used to image monocyte dynamics in a rodent model of glioblastoma [185] and in patients with myocardial infarction [186]. Alternatively, ${ }^{19} \mathrm{~F}-\mathrm{MRI}$ allows direct detection and imaging of labeled cells for unambiguous identification and quantification unlike iron oxide- based MRI [187]. Therefore, the detected signal can be derived directly from the injected PFC nanoemulsion engulfed by monocytes, for example after myocardial infarction [188]. 
A

A

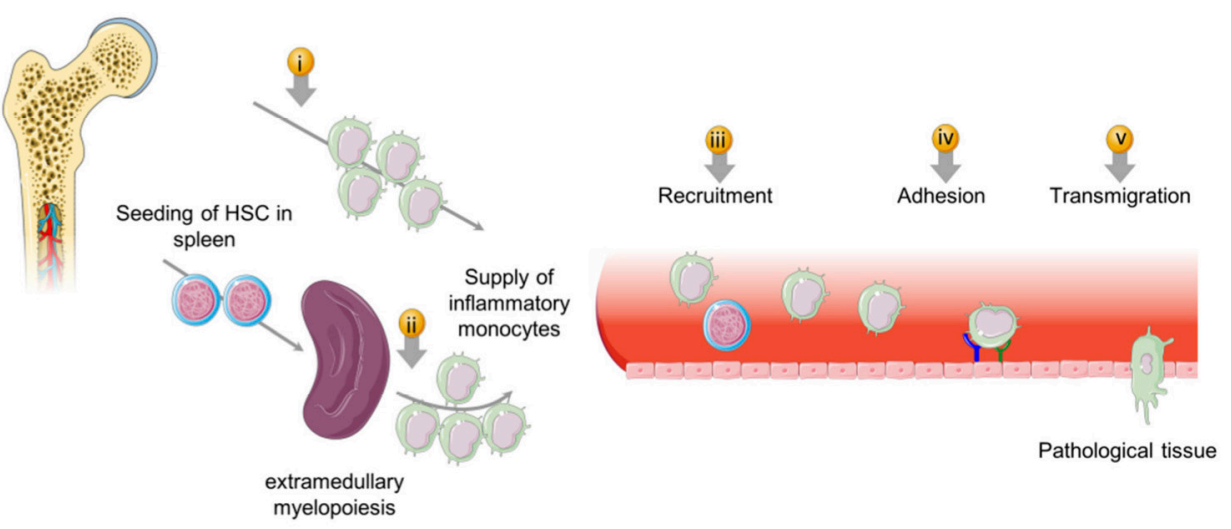

B

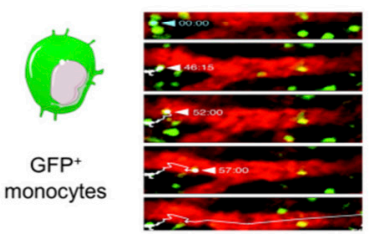

C

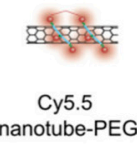

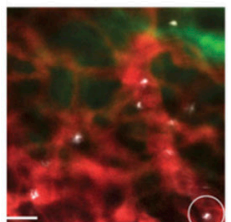

D
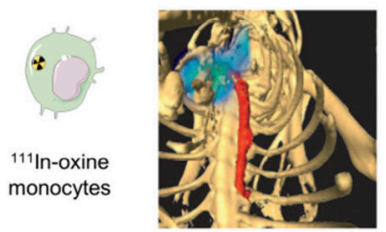

Figure 3 | Monocyte mobilization and recruitment (A) In response to inflammation, the spleen, in addition to the bone marrow, overproduces monocytes that enter the circulation. The inflammatory monocytes are guided by a gradient of chemokines in their journey to the inflamed lesions. The adhesion and the preferential accumulation of monocytes in lesions are driven by overexpression of certain receptors by the inflamed endothelium, including vascular cell adhesion molecule 1 (VCAM-1) and intercellular adhesion molecule 1 (ICAM-1). Nanomedicines can be exploited for imaging and therapeutic purposes at different stages of the monocyte journey in the circulation, starting from (i) monocyte egress from bone marrow, (ii) monocyte production and release from the spleen, (iii) monocyte trafficking in the blood stream, (iv) adhesion of monocytes to the inflamed endothelium, and (v) monocyte accumulation in the lesions. (B) Ischemic myocardial injury induces rapid deployment of splenic monocytes, which can be imaged by intravital microscopy of green fluorescent protein $\left(\mathrm{GFP}^{+}\right)$monocytes. $(\mathrm{C})$ The preferential uptake of nanoparticles by Ly6Chi monocytes allows studying both monocyte and nanoparticle trafficking in the circulation using intravital microscopy. (D) Labeling monocytes with a radiotracer (e.g. [111Indium] oxyquinoline, ${ }^{111}$ In-oxine) enables noninvasive tracking and visualization of monocyte accumulation in atherosclerotic plaques and other inflammatory lesions using single photon emission/computed tomography (SPECT/CT). Panel B is adapted, with permission, from [10]. Panel C is reproduced, with permission, from [11]. Panel D is adapted, with permission, from [12]. 
Nuclear imaging with PET or single-photon-emission-computed-tomography (SPECT), which are highly sensitive and quantitative methods, could be another method to evaluate monocyte trafficking in vivo. SPECT with Technetium-99m tagged autologous monocytes - radiolabeled ex vivo and reinjected in the same patients - has been used to visualize the continuous migration of monocytes into the inflamed synovial tissue of RA patients [189]. The same approach has been used to monitor monocyte recruitment in live atherosclerotic apolipoprotein E knockout $\left(\right.$ Apoe $\left.{ }^{-/-}\right)$mice for up to 7 days with Indium-111 (111In)-tagged monocytes and microSPECT / CT [180]. Moreover, the use of Zirconium-89 (89Zr)-feraheme nanoparticles, which are specifically taken up by blood monocytes, can enable studying monocyte dynamics by PET [190].

The homing features of circulating monocytes can be exploited to advance targeted therapeutics and diagnostic agents in inflammatory diseases. Such an approach has been realized by designing monocyte-mimicking nanoparticles as drug delivery systems to prolong their circulation times and achieve specific tissue targeting. Nanoporous silicon nanoparticles coated with leukocyte membranes possess celllike functions and exert their targeting through receptor-ligand interaction, evading opsonization and clearance by the immune system [191]. In a similar approach, Cao et al. have prepared camouflaged liposomes with isolated macrophage membranes, which homed to lung metastasis and improved the delivery of an anti-cancer drug [192]. Furthermore, incorporating lipid and protein components extracted from leukocytes into a liposomal formulation has been shown to improve their targeting to inflamed vasculature, enabling selective and effective delivery of dexamethasone [193].

An alternative approach is hitchhiking/backpacking with monocytes, which can be achieved by designing polymeric nanoparticles that strongly attach to the surface of monocytes [194]. These cellular backpacks were shown to evade monocyte phagocytosis (due to size, disk-like shape, and flexibility) without affecting monocytes' ability to target inflamed tissues in vivo [195,196].

The interactions between circulating inflammatory monocytes and the adhesion molecules expressed on the activated endothelium are inherently linked to lesion inflammation, and, therefore, also represent a potential therapeutic target. Knocking down multiple cell adhesion molecules using targeted delivery of siRNA-loaded nanoparticles has been shown to decrease monocyte recruitment in atherosclerotic plaques and ischemic myocardium, thereby reducing inflammation of infarcted myocardium and improving recovery after ischemia in mice [197]. Additionally, targeting adhesion molecules, like VCAM-1, P-selectin, or ICAM-1, with nanoparticles can be used for molecular imaging of the activated endothelium in atherosclerosis [198,199], cancer [200], and arthritis [201]. For example, echogenic nanoparticles can be applied for molecular ultrasound imaging of vascular markers in inflammatory lesions. Lee et al. demonstrated a positive correlation between in vivo ultrasound imaging of VEGF receptor-2 (VEGFR-2)-targeted microbubbles and the 
VEGFR-2 expression on endothelial cells of breast cancer tumors [202]. Furthermore, echogenic immunoliposomes targeting ICAM-1, VCAM-1, and tissue factor have been applied for atherosclerosis imaging [203].

\subsection{Enhanced microvascular permeability}

Local macrophage activity and lesion growth (e.g. tumors, atherosclerotic plaque, or RA pannus) drive the generation of blood vessels to meet the increased demand for oxygen and nutrients. Angiogenic neovessels in chronic inflammation are phenotypically heterogeneous and characterized by excessive branching, chaotic patterns and enhanced permeability to macromolecules and nanomedicines [45]. Therefore, applying nanomedicine to target the processes governing angiogenesis is an attractive approach to delivering therapeutic and imaging agents [204]. The formation of neovessels is intricately controlled by angiogenic factors, including VEGFs and VEGFRs (Figure 4). Inhibition of angiogenesis using polymeric nanoparticles loaded with angiogenesis inhibitors such as the fumagillin analog TNP-470 has been shown to 1) suppress tumor growth in melanoma [205], Lewis lung carcinoma [205], and ovarian cancer mouse models [206]; 2) reduce plaque angiogenesis and inhibit advanced atherosclerosis in Apoe $e^{-1-}$ mice $[207,208]$; and 3) suppress arthritis and protect from bone destruction in mice [209]. Alternatively, inhibiting the secretion of proinflammatory cytokines and proteases, which affect the proliferation and migration behavior of the preexisting endothelial cells, can be a complementary strategy to direct anti- angiogenic therapies. For example, liposomal delivery of anti-inflammatory compounds such as glucocorticoids has been shown to suppress tumor growth in mice, partially through inhibition of tumor angiogenesis [210].

Angiogenic neovessels are also characterized by overexpression of certain receptors like $\alpha \mathrm{v} \beta 3$ integrin adhesion receptors compared to quiescent vessels [214-217]. Attaching ligands (e.g. antibodies, nanobodies, and peptides) to the nanoparticles can allow better distribution in the vascular bed of the lesion and increase their internalization by target endothelial cells [218,219]. For example, $\alpha v \beta 3$ integrin-targeted delivery of an anti-angiogenic gene has been shown to selectively increase apoptosis of tumor endothelial cells, which subsequently led to tumor cell apoptosis and regression of primary and metastatic tumors [220]. Additionally, $\alpha \mathrm{v} \beta 3$ integrin-targeted ligands and nanoparticles have been used to image angiogenesis in cancer [221-223], atherosclerosis [224,225], and arthritis [226], using different imaging modalities such as ultrasound, MRI, PET, and SPECT. These $\alpha \mathrm{v} \beta 3$-targeted imaging strategies can also be used to monitor the therapeutic response $[207,208]$. 


\subsection{The proinflammatory milieu and local cell proliferation}

Tackling the adhesion of circulating monocytes to the lesion vasculature can yield favorable therapeutic outcomes in chronic inflammatory diseases as mentioned before. However, once monocytes adhere, they infiltrate into the lesions and differentiate into macrophages, which possess an inflammatory phenotype and secrete large amounts of proteases, inflammatory cytokines, reactive radicals, and auto- and paracrine signaling molecules [227-229]. These "flaring" molecules drive lesion remodeling, invasiveness (and metastasis in the case of cancer), and can induce a phenotypical change in preexisting macrophages and other cells [230232]. Thus, preventing macrophage activation and dampening local inflammation by using nanomedicines to deliver anti-inflammatory drugs can induce a favorable phenotype [233]. Glucocorticoids are potent anti-inflammatory drugs, which, however, generally come with severe side effects upon prolonged use [234]. Alternatively, glucocorticoid nanomedicines have been shown to silence local inflammation and halt the progression of several chronic inflammatory diseases, including atherosclerosis [235], cancer [236], and RA [212,237] while minimizing side effects. Another approach is to activate inflammation-resolution pathways through delivering proresolving mediators to lesions (e.g. annexin A1 and resolving D1). Such an approach can result not only in anti-inflammatory effects but also in tissue repair and restoration of homeostatic conditions [238]. Nanoparticle delivery of an annexin A1-mimicking peptide to atherosclerotic lesions results in a marked improvement in key advanced plaque features, including an increase in the protective collagen layer, a decrease in protease activity, suppression of oxidative stress, and reduced plaque necrosis [239]. 
A

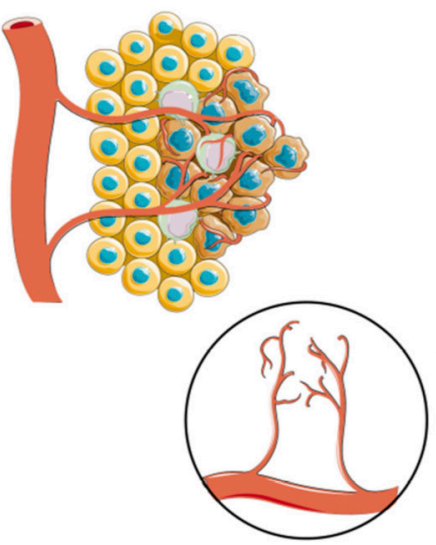

B

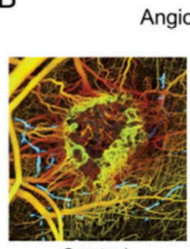

Control
Angiogenesis

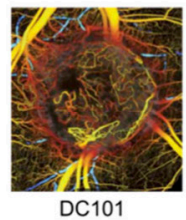

C

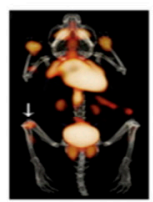

Control

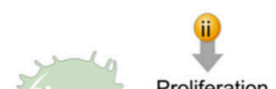

Proliferation iii)

Differentiation

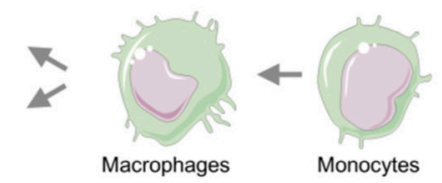

Macrophages

Monocytes

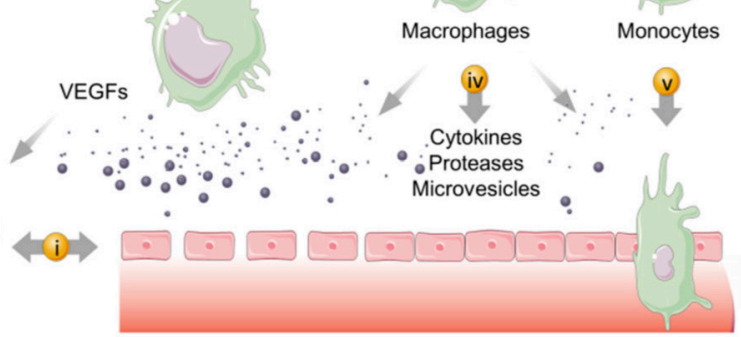

D

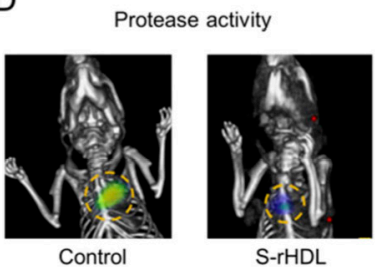

Figure 4 I Enhanced microvascular permeability and local inflammation. (A) Inflammatory monocytes accumulate in inflamed lesions and differentiate into tissue resident macrophages. A cascade of events ensues such as local macrophage proliferation, release of proinflammatory cytokines, proteases and cellular vesicles, which can aggravate the inflammatory condition and recruit more inflammatory cells. In addition, the release of vascular endothelial growth factors and other cytokines induce angiogenesis and increase microvascular permeability to macromolecules. Diagnostic and therapeutic nanoparticles can be used to tackle (i) pathological angiogenesis and enhanced permeability, (ii) local cell proliferation, (iii) monocyte differentiation, (iv) cytokine and chemokine release (v) and monocyte infiltration. (B) Cancerassociated angiogenesis and response to anti-angiogenic therapies can be monitored in vivo using optical frequency domain imaging. (C) Anti-inflammatory effect of prednisolone phosphate (PLP) liposomes in rheumatoid arthritis can noninvasively be assessed by ${ }^{18}$ F-FDG PET /CT. (D) Protease activity in response to simvastatin rHDL nanoparticles can be monitored in vivo using fluorescence molecular tomography / computed tomography (FMT/CT). Panel B is adapted, with permission, from [13]. Panel C is reproduced, with permission, from [14]. Panel D is adapted, with permission, from [15]. 
Although monocyte recruitment is a key process driving the settlement of lesionassociated macrophages, the expansion of the macrophage population can also be driven by local proliferation [240-243]. Tackling monocyte recruitment alone may not be sufficient to reduce local inflammation, especially in advanced stages [244246]. Consequently, restricting the expansion of the macrophage population can reduce the burden of local inflammation. For example, depletion of TAMs using clodronate liposomes slows down tumor growth in vivo and increases the efficacy of other anti-cancer drugs [247,248]. Additionally, clodronate liposomes have been used to deplete synovial macrophages, which resulted in reduced inflammation and prevented joint destruction [249,250]. Furthermore, simvastatin reconstituted HDL nanoparticles have been applied to reduce atherosclerotic plaque inflammation [213] by inhibiting local macrophage proliferation [251] in Apoe $e^{-1-}$ mice with advanced atherosclerotic plaques. Methotrexate, a folate inhibitor with anti-proliferative activities, conjugated to dendrimer nanoparticles has been shown to reduce arthritisinduced inflammatory parameters such as ankle swelling, paw volume, cartilage damage, and bone resorption in a rat model of collagen-induced arthritis [252]. The same group has also demonstrated that methotrexate nanoparticles improve the therapeutic response in an animal model of human epithelial cancer [253]. Of note, low-dose of methotrexate is currently being studied in the Cardiovascular Inflammation Reduction Trial (CIRT) to test if it can reduce vascular inflammation and decrease rates of myocardial infarction, stroke, and cardiovascular death (Clinical Trial Identifier: NCT01594333).

The increased macrophage activity drives changes in the inflammatory milieu, which include elevated protease activity, abnormal glucose metabolism, and decreased $\mathrm{pH}[227,254,255]$. Such changes can be exploited to develop "smart" probes and nanomedicines that are responsive to the changes inherent to inflammatory lesions. An attractive approach is to design nanomedicines with bioactive domains that can be activated by the increased activity of proteases like caspases, MMPs, and proteinases [256-259]. For example, Boeneman et al. have developed caspase 3 -sensitive quantum dot-fluorescent protein nanoparticles [260]. Similarly, cathepsin B-sensitive fluorogenic chitosan nanoparticles have been used to discriminate metastases in vivo in three metastatic mouse models [261]. Also, Ferber et al. have adopted a nanotheranostic approach by developing two cathepsin B-sensitive $\mathrm{N}$-(2- hydroxypropyl)methacrylamide (HPMA) copolymer-based systems for noninvasive imaging of breast cancer progression and drug release [262]. MMPsensitive dual ${ }^{64} \mathrm{Cu}$-labeled fluorescent chitosan nanoparticles have also been used to visualize tumors in vivo by PET and near-infrared fluorescence imaging [263]. Moreover, active targeting of MMP sensitive dendrimer nanoparticles designed for dual MRI/optical imaging improves MRI-guided clinical staging, presurgical planning, and intraoperative fluorescence-guided surgery [264]. In addition to the biochemical changes, lesion-associated macrophages overexpress certain receptors such as major histocompatibility complex class II (Class II MHC) and surface integrin CD11b, which can also be exploited for imaging purposes [265]. Rashidian et al. have developed nanobodies labeled with $18 \mathrm{~F}$ targeting MHC II+ and CD11b+cells, which 
allow specific, noninvasive imaging of immune responses by PET/CT, showing higher specificity over the clinically used ${ }^{18}$ F-FDG PET/CT [266]. Alternatively, we have developed ${ }^{89} \mathrm{Zr}$-labeled rHDL to image TAM by PET [136] specifically.

\section{LESSONS, CHALLENGES, AND PERSPECTIVES}

Chronic inflammatory diseases and associated angiogenesis share common pathophysiological features in which monocytes and macrophages are key effector cells. Understanding and exploiting monocyte dynamics and functions can lead to the development of improved therapeutic and diagnostic agents that are clinically useful in multiple diseases and disease settings. Nanomedicines can be engineered, developed, and customized in numerous ways to tackle the different features of maladaptive inflammation and angiogenesis, for therapeutic and diagnostics endeavors. Additionally, due to monocytes and macrophages' high uptake capacity, nanomedicines can also enable us to study these cells' dynamics in disease and decipher different pathophysiological features. Therefore, it stands out as a promising approach to modulate monocyte/macrophage function and to also visualize and quantitate their dynamics. However, to broaden our exploitation of nanomedicine and reinvigorate clinical translation, several challenges and opportunities need to be taken into consideration:

- First, the application of nanomedicine, especially for drug delivery, needs to be rigorously assessed. Traditionally, nanomedicine's main application was improving a drug's therapeutic index by increasing the concentration in the target tissue, while simultaneously minimizing the off-site effects. Today, the community is concerned about the small quantities of injected nanomaterial that reaches target lesions (e.g. tumor) in patients, and if nanomedicines provide real benefits [267-269]. These debates are mainly fueled by limited clinical experience with nanomedicines and undermine a rational discourse.

- Second, research groups are often inclined to apply their nanomedicine in a single disease, such as cancer, overlooking the potential application in other diseases. A holistic understanding of the pathophysiological processes in chronic inflammation would allow repurposing, reviving and reapplying nanomedicines across a range of maladies without the need to create novel nanomedicines. Patient/ disease-centric approaches for applying nanomedicines are more valuable than material-based approaches [270,271].

- Additionally, wehave witnessed an expansion in the development of new materials for nanomedicine, but these are rarely fully evaluated under biologically relevant conditions. Implementing screening procedures to evaluate new and preexisting 
nanomaterials with regards to their compatibility with different drugs, their biological behavior and tissue/ cell specificity and their toxicity profile is the key to developing clinically relevant nanotherapeutics and diagnostic agents [272274].

- Applying nanomedicines in maladaptive inflammation and angiogenesis can be more challenging due to the inherent heterogeneity of the disease and the dynamic changes in lesion phenotype over time. Furthermore, heterogeneity and plasticity are hallmarks of monocytes and macrophages [59,275]. Growing evidence demonstrates the need to liberate our research from the dichotomous concept of macrophage activation: classical vs. alternative, i.e. M1 and M2, respectively [276,277]. These facts have implications on how nanomedicines can be best applied. Firstly, lesion heterogeneity can be manifested in the remarkable heterogeneity of the enhanced permeability and retention (EPR) effect, which impacts nanomedicine accumulation and, hence, their efficacy. Implementation of screening methods to identify patients who can benefit from nanomedicine should be a key factor for further developments [278]. Secondly, developing nanomedicines based on the M1/M2 macrophage paradigm is a shorthand approach that will probably not lead to clinically viable products. Understanding the function, dynamics, and memory of monocytes and macrophages, in their systemic and local milieu, is a rather more enabling and clinically relevant strategy.

Given the impact of chronic inflammatory diseases, successful translation of nanomedicines into the clinic can be socioeconomically rewarding, yet challenging. Convergence of nanomedicine, life and physical sciences, bioinformatics, bioengineering, and pharma perspectives can help to reduce development costs, close the gap between preclinical research and clinical translation, and ultimately bring more efficacious nanomedicines to patients (Figure 5). 


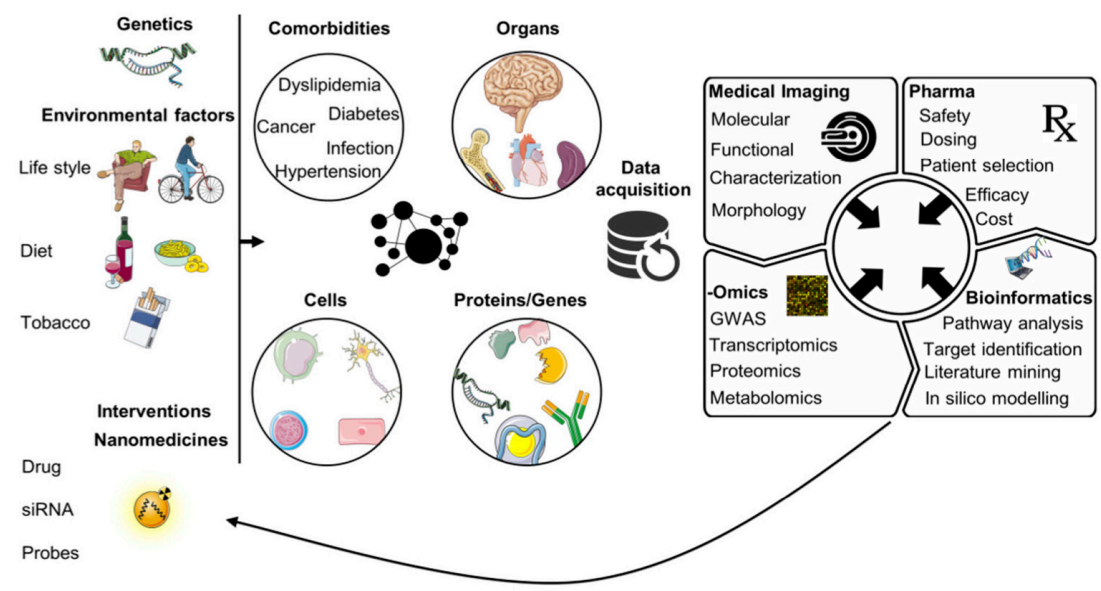

Figure 5 I Considerations for applying and developing nanomedicines for chronic inflammatory diseases. Chronic inflammatory diseases are multifactorial disorders in which genetic background and environmental factors interact and affect different dynamic systems, including genes, signaling pathways, cells, and organs. Nanomedicine should be approached in a holistic way, in which nanodrugs' systemic interactions are investigated, and can be used to visualize and/or modulate multiple processes. Data acquisition and convergence of nanomedicine with the different biomedical fields and big data (e.g. transcriptomics, proteomics, and genomics) can not only contribute to deciphering these complex diseases but also help to predict the efficacy of nanomedicines and to develop clinically relevant products. 


\section{REFERENCES}

[1] C.D. Mathers, D. Loncar, Projections of global mortality and burden of disease from 2002 to 2030, PLoS Med. 3 (2006) e442 (doi:06-PLME-RA-0071R2).

[2] P. Jacobs, R. Bissonnette, L.C. Guenther, Socioeconomic burden of immune-mediated inflammatory diseases focusing on work productivity and disability, J. Rheumatol. 88 (2011) 55 61, http:/ / dx.doi.org/10.3899/jrheum.110901.

[3] K. Strong, C. Mathers, S. Leeder, R. Beaglehole, Preventing chronic diseases: how many lives can we save?, Lancet 366 (2005) 15781582 (doi:S0140 6736(05)67341 2).

[4] M. Mitka, New basic care goals seek to rein in global rise in cardiovascular disease, JAMA 308 (2012) 1725 1726, http: / / dx.doi.org/10.3201/ eid1811.111850.

[5] I. Tabas, C.K. Glass, Anti-inflammatory therapy in chronic disease: challenges and opportunities, Science 339 (2013) 166 172, http: / / dx.doi.org / 10.1126/ science.1230720.

[6] M.A. Hamburg, F.S. Collins, The path to personalized medicine, N. Engl. J.Med. 363 (2010) 301 304, http: / / dx.doi.org/10.1056/ NEJMp1006304.

[7] D.R. Parkinson, B.E. Johnson, G.W. Sledge, Making personalized cancer medicine a reality: challenges and opportunities in the development of biomarkers and companion diagnostics, Clin. Cancer Res. 18 (2012) 619 624, http:/ / dx.doi.org/10.1158/1078-0432. CCR-11-2017.

[8] B. Godman, A.E. Finlayson, P.K. Cheema, E. Zebedin-Brandl, I. Gutierrez-Ibarluzea, J. Jones, R.E. Malmstrom, E. Asola, C. Baumgartel, M. Bennie, I. Bishop, A. Bucsics, S. Campbell, E. Diogene, A. Ferrario, J. Furst, K. Garuoliene, M. Gomes, K. Harris, A. Haycox, H. Herholz, K. Hviding, S. Jan, M. Kalaba, C. Kvalheim, O. Laius, S.A. Loov, K. Malinowska, A. Martin, L. McCullagh, F. Nilsson, K. Paterson, U. Schwabe, G. Selke, C. Sermet, S. Simoens, D. Tomek, V. Vlahovic-Palcevski, L. Voncina, M. Wladysiuk, M. van Woerkom, D. Wong- Rieger, C. Zara, R. Ali, L.L. Gustafsson, Personalizing health care: feasibility and future implications, BMC Med. 11 (2013) 179 (doi:1741 7015 11-179).

[9] J. Folkman, Angiogenesis: an organizing principle for drug discovery?, Nat. Rev. Discov. 6 (2007) 273286 (doi:nrd2115).

[10] S.I. Grivennikov, F.R. Greten, M. Karin, Immunity, inflammation, and cancer, Cell 140 (2010) 883 899, http: / / dx.doi.org/10.1016/j.cell.2010.01.025.

[11] J.C. Mason, P. Libby, Cardiovascular disease in patients with chronic inflammation: mechanisms underlying premature cardiovascular events in rheumatologic conditions, Eur. Heart J. 36 (2015) 482-9c, http: / / dx.doi.org/10.1093/eurheartj/ehu403.

[12] I.A. Udalova, A. Mantovani, M. Feldmann, Macrophage heterogeneity in the context of rheumatoid arthritis., Nat. Rev. Rheumatol. 12 (2016) 472-85.doi:10.1038/ nrrheum.2016.91.

[13] G.K. Hansson, A. Hermansson, The immune system in atherosclerosis, Nat. Immunol. 12 (2011) 204 212, http: / / dx.doi.org/10.1038/ni.2001.

[14] M.A. Ingersoll, A.M. Platt, S. Potteaux, G.J. Randolph, Monocyte trafficking in acute and chronic inflammation, Trends Immunol. 32 (2011) 470 477, http: / / dx.doi.org/10.1016/j. it.2011.05.001.

[15] M.J. Pittet, F.K. Swirski, Monocytes link atherosclerosis and cancer, Eur. J. Immunol. 41 (2011) 2519 2522, http:/ / dx.doi.org/10.1002/eji.201141727.

[16] E.C. Steinbach, S.E. Plevy, The role of macrophages and dendritic cells in the initiation of inflammation in IBD, Inflamm. Bowel Dis. 20 (2014) 166 175, http: / / dx.doi.org / 10.1097/ MIB.0b013e3182a69dca.

[17] T.A. Wynn, A. Chawla, J.W. Pollard, Macrophage biology in development, homeostasis and disease, Nature 496 (2013) 445 455, http: / / dx.doi.org/10.1038/ nature12034. 
[18] M. Nahrendorf, F.K. Swirski, Immunology. Neutrophil-macrophage communication in inflammation and atherosclerosis, Science 349 (2015) 237238, http: / / dx.doi.org / 10.1126/ science.aac7801.

[19] A.C. Chan, P.J. Carter, Therapeutic antibodies for autoimmunity and inflammation, Nat. Rev. 10 (2010) 301 316, http: / / dx.doi.org/10.1038/nri2761.

[20] M. Wong, D. Ziring, Y. Korin, S. Desai, S. Kim, J. Lin, D. Gjertson, J. Braun, E. Reed, R.R. Singh, TNFalpha blockade in human diseases: mechanisms and future directions, Clin. Immunol. 126 (2008) 121136 (doi:S1521 6616(07)01327 7).

[21] J.S. Smolen, A. Beaulieu, A. Rubbert-Roth, C. Ramos-Remus, J. Rovensky, E. Alecock, T. Woodworth, R. Alten, O. Investigators, Effect of interleukin-6 receptor inhibition with tocilizumab in patients with rheumatoid arthritis (OPTION study): a double-blind, placebo-controlled, randomised trial, Lancet 371 (2008) 987997 (doi:10.1016/S0140 6736(08)60453-5).

[22] P. Chames, D. Baty, Bispecific antibodies for cancer therapy: the light at the end of the tunnel?, MAbs 1 (2009) 539547.

[23] B.Y. Kim, J.T. Rutka, W.C. Chan, Nanomedicine, N. Engl. J. Med. 363 (2010) 2434 2443, http: / / dx.doi.org/10.1056/NEJMra0912273.

[24] R. Weissleder, M. Nahrendorf, M.J. Pittet, Imaging macrophages with nanoparticles, Nat. Mater. 13 (2014) 125 138, http:/ / dx.doi.org/10.1038/nmat3780.

[25] W.J. Mulder, F.A. Jaffer, Z.A. Fayad, M. Nahrendorf, Imaging and nanomedicine in inflammatory atherosclerosis, Sci. Transl. Med. 6 (2014) 239sr1, http://dx.doi. org/10.1126/ scitranslmed.3005101.

[26] M.M. Gottesman, O. Lavi, M.D. Hall, J.P. Gillet, Toward a better understanding of the complexity of cancer drug resistance, Annu. Rev. Pharmacol. Toxicol. 56 (2016) 85 102, http: / / dx.doi.org/10.1146/ annurev-pharmtox- 010715-103111.

[27] L.M. Coussens, Z. Werb, Inflammation and cancer, Nature 420 (2002) 860 867, http:/ / dx.doi.org/10.1038/nature01322.

[28] C. Lahoute, O. Herbin, Z. Mallat, A. Tedgui, Adaptive immunity in atherosclerosis: mechanisms and future therapeutic targets, Nat. Rev. 8 (2011) 348 358, http:/ / dx.doi. org $/ 10.1038 /$ nrcardio.2011.62.

[29] I.B. McInnes, G. Schett, The pathogenesis of rheumatoid arthritis, N. Engl. J. Med. 365 (2011) 2205 2219, http: / / dx.doi.org/10.1056/NEJMra1004965.

[30] R. Medzhitov, Origin and physiological roles of inflammation, Nature 454 (2008) 428 435, http: / / dx.doi.org/10.1038/nature07201.

[31] R. Ross, Atherosclerosis an inflammatory disease, N. Engl. J.Med. 340 (1999) 115126 , http: / / dx.doi.org/10.1056/NEJM199901143400207.

[32] S.B. Hanauer, Inflammatory bowel disease: epidemiology, pathogenesis, and therapeutic opportunities, Inflamm. Bowel Dis. 12 (Suppl. 1) (2006) S3 S9 (doi:00054725 20060100100002).

[33] R.R. Packard, A.H. Lichtman, P. Libby, Innate and adaptive immunity in atherosclerosis, Semin. Immunopathol. 31 (2009) 5 22, http: / / dx.doi.org/10.1007/ s00281-009-0153-8.

[34] T.F. Gajewski, H. Schreiber, Y.X. Fu, Innate and adaptive immune cells in the tumor microenvironment, Nat. Immunol. 14 (2013) 1014 1022, http://dx.doi.org/10.1038/ ni.2703.

[35] M. Karin, F.R. Greten, NF-kappaB: linking inflammation and immunity to cancer development and progression, Nat. Rev. 5 (2005) 749759 (doi:nri1703).

[36] I.F. Charo, R.M. Ransohoff, The many roles of chemokines and chemokine receptors in inflammation, N. Engl. J. Med. 354 (2006) 610621 (doi:354/6/610).

[37] G.K. Hansson, Inflammation, atherosclerosis, and coronary artery disease, N. Engl. J. Med. 352 (2005) 1685 1695, http:/ / dx.doi.org/10.1056/ NEJMra043430. 
[38] S. Gordon, Pattern recognition receptors: doubling up for the innate immune response, Cell 111 (2002) 927930 (doi:S0092867402012011).

[39] O. Takeuchi, S. Akira, Pattern recognition receptors and inflammation, Cell 140 (2010) 805 820, http:/ / dx.doi.org/10.1016/j.cell.2010.01.022.

[40] A.R. Tall, L. Yvan-Charvet, Cholesterol, inflammation and innate immunity, Nat. Rev. 15 (2015) 104 116, http:/ / dx.doi.org/10.1038/nri3793.

[41] C. Murdoch, M. Muthana, C.E. Lewis, Hypoxia regulates macrophage functions in inflammation, J. Immunol. 175 (2005) 62576263 (doi:175/10/6257).

[42] H.Z. Imtiyaz, E.P. Williams, M.M. Hickey, S.A. Patel, A.C. Durham, L.J.Yuan, R. Hammond, P.A. Gimotty, B. Keith, M.C. Simon, Hypoxia-inducible factor 2alpha regulates macrophage function in mouse models of acute and tumor inflammation, $\mathrm{J}$. Clin. Invest. 120 (2010) 2699 2714, http: / / dx.doi.org/10. 1172/JCI39506.

[43] C. Strehl, M. Fangradt, U. Fearon, T. Gaber, F. Buttgereit, D.J. Veale, Hypoxia: how does the monocyte- macrophage system respond to changes in oxygen availability?, J. Leukoc. Biol. 95 (2014) 233 241, http:/ / dx.doi.org/10. 1189/jlb.1212627.

[44] D. Bates, S. Harper, Regulation of vascular permeability by vascular endothelial growth factors, Vasc. Pharmacol. 39 (2002) 225 237, http:/ / dx.doi.org/10.1016/S15371891(03)00011-9.

[45] J.A. Nagy, L. Benjamin, H. Zeng, A.M. Dvorak, H.F. Dvorak, Vascular permeability, vascular hyperpermeability and angiogenesis, Angiogenesis 11 (2008) 109 119, http:// dx.doi.org/10.1007/ s10456-008- 9099-z.

[46] L. Claesson-Welsh, Vascular permeability the essentials, Ups. J. Med. Sci. 120 (2015) 135 143, http:/ / dx.doi.org/10.3109/03009734.2015.1064501.

[47] J.R. Jackson, M.P. Seed, C.H. Kircher, D.A. Willoughby, J.D. Winkler, The codependence of angiogenesis and chronic inflammation, FASEB J. 11 (1997) 457465.

[48] A. Naldini, F. Carraro, Role of inflammatory mediators in angiogenesis, Curr. Drug Targets Inflamm Allergy 4 (2005) 38.

[49] D.A. Walsh, C.I. Pearson, Angiogenesis in the pathogenesis of inflammatory joint and lung diseases, Arthritis Res. 3 (2001) 147 153, http: / / dx.doi.org/10.1186/ar292.

[50] F. Balkwill, A. Mantovani, Inflammation and cancer: back to Virchow?, Lancet 357 (2001) 539545 (doi:S0140 6736(00)04046 0).

[51] A. Mantovani, P. Allavena, A. Sica, F. Balkwill, Cancer-related inflammation, Nature 454 (2008) 436 444, http: / / dx.doi.org/10.1038/nature07205.

[52] Y. Ben-Neriah, M. Karin, Inflammation meets cancer, with NF-kappaB as the matchmaker, Nat. Immunol. 12 (2011) 715 723, http:/ / dx.doi.org/10.1038/ ni.2060.

[53] H.B. El-Serag, Epidemiology of viral hepatitis and hepatocellular carcinoma, Gastroenterology 142 (2012) http:/ / dx.doi.org/10.1053/j.gastro.2011.12.061 (1264-1273. e1).

[54] R. Noy, J.W. Pollard, Tumor-associated macrophages: from mechanisms to therapy, Immunity 41 (2014) 49- 61, http:/ / dx.doi.org/10.1016/j.immuni.2014.06.010.

[55] R.A. Franklin, M.O. Li, Ontogeny of tumor-associated macrophages and its implication in cancer regulation, Trends Cancer 2 (2016) 20-34, http://dx.doi.org/10.1016/j. trecan.2015.11.004.

[56] Q.W. Zhang, L. Liu, C.Y. Gong, H.S. Shi, Y.H. Zeng, X.Z. Wang, Y.W. Zhao, Y.Q. Wei, Prognostic significance of tumor-associated macrophages in solid tumor: a metaanalysis of the literature, PLoS One 7 (2012)e50946http:/ / dx.doi.org/10.1371/journal. pone.0050946.

[57] T. Kitamura, B.Z. Qian, D. Soong, L. Cassetta, R. Noy, G. Sugano, Y. Kato, J. Li, J.W. Pollard, CCL2- induced chemokine cascade promotes breast cancer metastasis by enhancing retention of metastasis-associated macrophages, J. Exp. Med. 212 (2015) 1043- 
1059, http: / / dx.doi.org/10.1084/jem.20141836.

[58] S.Y. Lim, A.E. Yuzhalin, A.N. Gordon-Weeks, R.J. Muschel, Targeting the CCL2-CCR2 signaling axis in cancer metastasis, Oncotarget 7 (2016) 28697-28710, http://dx.doi. org/10.18632/ oncotarget.7376.

[59] B.Z. Qian, J.W. Pollard, Macrophage diversity enhances tumor progression and metastasis, Cell 141 (2010) 39-51, http: / / dx.doi.org/10.1016/j.cell.2010.03.014.

[60] G.Y. Liou, P. Storz, Reactive oxygen species in cancer, Free Radic. Res. 44 (2010) 479-496, http: / / dx.doi.org/10.3109/10715761003667554.

[61] M. Mittal, M.R. Siddiqui, K. Tran, S.P. Reddy, A.B. Malik, Reactive oxygen species in inflammation and tissue injury, Antioxid. Redox Signal. 20 (2014) 1126-1167, http:/ / dx.doi.org/10.1089/ars.2012.5149.

[62] K. Wolf, Y.I. Wu, Y. Liu, J. Geiger, E. Tam, C. Overall, M.S. Stack, P. Friedl, Multi-step pericellular proteolysis controls the transition from individual to collective cancer cell invasion, Nat. Cell Biol. 9 (2007) 893- 904 (doi:ncb1616).

[63] P. Friedl, D. Gilmour, Collective cell migration in morphogenesis, regeneration and cancer, Nat. Rev. Mol. Cell Biol. 10 (2009) 445-457, http: / / dx.doi.org/10.1038/nrm2720.

[64] M. Roh-Johnson, J. Bravo-Cordero, A. Patsialou, V. Sharma, P. Guo, H. Liu, L. Hodgson, J. Condeelis, Macrophage contact induces RhoA GTPase signaling to trigger tumor cell intravasation, Oncogene 33 (2013) 4203-4212, http: / / dx.doi.org/10.1038/ onc.2013.377.

[65] J.B. Wyckoff, Y. Wang, E.Y. Lin, J.-F. Li, S. Goswami, E.R. Stanley, J.E. Segall, J.W. Pollard, J. Condeelis, Direct visualization of macrophage-assisted tumor cell Intravasation in mammary tumors, Cancer Res. 67 (2007) 2649-2656, http:/ / dx.doi.org/10.1158/00085472.CAN-06-1823.

[66] P. Friedl, K. Wolf, Tube travel: the role of proteases in individual and collective cancer cell invasion, Cancer Res. 68 (2008) 7247-7249, http:/ / dx.doi.org/10.1158/0008-5472.CAN08-0784.

[67] K. Kessenbrock, V. Plaks, Z. Werb, Matrix metalloproteinases: regulators of the tumor microenvironment, Cell 141 (2010) 52-67, http:/ / dx.doi.org/10.1016/j.cell.2010.03.015.

[68] R. Guiet, E. Van Goethem, C. Cougoule, S. Balor, A. Valette, T. Al Saati, C.A. Lowell, V. Le Cabec, I. Maridonneau-Parini, The process of macrophage migration promotes matrix metalloproteinase-independent invasion by tumor cells, J. Immunol. 187 (2011) 38063814, http: / / dx.doi.org/10.4049/jimmunol.1101245.

[69] J. Wyckoff, W. Wang, E.Y. Lin, Y. Wang, F. Pixley, E.R. Stanley, T. Graf, J.W. Pollard, J. Segall, J. Condeelis, A Paracrine Loop between Tumor Cells and Macrophages is Required for Tumor Cell Migration in Mammary Tumors, 20047022-7029, http:/ / dx.doi. org/10.1158/0008-5472.CAN-04-1449.

[70] S. Goswami, E. Sahai, J.B. Wyckoff, M. Cammer, D. Cox, F.J. Pixley, E.R. Stanley, J.E. Segall, J.S. Condeelis, Macrophages promote the invasion of breast carcinoma cells via a colony-stimulating factor- 1/epidermal growth factor paracrine loop, Cancer Res. 65 (2005) 5278-5283 (doi:65/12/5278).

[71] E.Y. Lin, J.W. Pollard, Tumor-associated macrophages press the angiogenic switch in breast cancer, Cancer Res. 67 (2007) 5064-5066 (doi:67 / 11/5064).

[72] J.L. Owen, M. Mohamadzadeh, Macrophages and chemokines as mediators of angiogenesis, Front. Physiol. 4 (2013) 159, http:/ / dx.doi.org/10.3389/ fphys.2013.00159.

[73] J.E. Rundhaug, Matrix metalloproteinases and angiogenesis, J. Cell. Mol. Med. 9 (2005) 267-285 (doi:009.002.05).

[74] N. McCarthy, Leaky effect, Nat. Rev. 12 (2012) 157, http: / / dx.doi.org/10.1038/nrc3227.

[75] A.H. Allam, R.C. Thompson, L.S. Wann, M.I. Miyamoto, A.-H. Nur El-Din, G.A. ElMaksoud, M. Al- Tohamy Soliman, I. Badr, H.A. El-Rahman Amer, M.L. Sutherland, J.D. Sutherland, G.S. Thomas, Atherosclerosis in ancient Egyptian mummies: the 
Horus study, JACC Cardiovasc. Imaging 4 (2011) 315-327, http: / / dx.doi.org/10.1016/j. jcmg.2011.02.002.

[76] World Health Organization, Noncommunicable diseases mortality and morbidity, 2015, in: http:/ / www.who.int/gho/ncd/mortality-morbidity/en/, 2015.

[77] P. Libby, Y. Okamoto, V.Z. Rocha, E. Folco, Inflammation in atherosclerosis: transition from theory to practice, Circ. J. 74 (2010) 213-220 (doi:JST.JSTAGE/ circj/CJ-09-0706).

[78] S. Blankenberg, S. Barbaux, L. Tiret, Adhesion molecules and atherosclerosis, Atherosclerosis 170 (2003) 191-203 (doi:S0021-9150(03)00097-2).

[79] R. Gareus, E. Kotsaki, S. Xanthoulea, I. van der Made, M.J. Gijbels, R. Kardakaris, A. Polykratis, G. Kollias, M.P . de Winther, M. Pasparakis, Endothelial cell-specific NFkappaB inhibition protects mice from atherosclerosis, Cell Metab. 8 (2008) 372-383, http: / / dx.doi.org/10.1016/j.cmet.2008.08.016.

[80] J.H. Qiao, J. Tripathi, N.K. Mishra, Y. Cai, S. Tripathi, X.P. Wang, S. Imes, M.C. Fishbein, S.K. Clinton, P. Libby, A.J. Lusis, T.B. Rajavashisth, Role of macrophage colonystimulating factor in atherosclerosis: studies of osteopetrotic mice, Am. J. Pathol. 150 (1997) 1687-1699.

[81] I. Tabas, K.J. Williams, J. Boren, Subendothelial lipoprotein retention as the initiating process in atherosclerosis: update and therapeutic implications, Circulation 116 (2007) 1832-1844 (doi:116/16/1832).

[82] Y.S. Bae, J.H. Lee, S.H. Choi, S. Kim, F. Almazan, J.L. Witztum, Y.I. Miller, Macrophages generate reactive oxygen species in response to minimally oxidized low-density lipoprotein: toll-like receptor 4- and spleen tyrosine kinase-dependent activation of NADPH oxidase 2, Circ. Res. 104 (2009) 210-218, http://dx.doi.org/10.1161/ CIRCRESAHA.108.181040 (21p following 218).

[83] E.A. Podrez, M. Febbraio, N. Sheibani, D. Schmitt, R.L. Silverstein, D.P. Hajjar, P.A. Cohen, W.A. Frazier, H.F. Hoff, S.L. Hazen, Macrophage scavenger receptor CD36 is the major receptor for LDL modified by monocyte-generated reactive nitrogen species, J. Clin. Invest. 105 (2000) 1095-1108, http: / / dx.doi.org/10.1172 / jci8574.

[84] K.J. Moore, M.W. Freeman, Scavenger receptors in atherosclerosis: beyond lipid uptake, Arterioscler. Thromb. Vasc. Biol. 26 (2006) 1702-1711 (doi:01.ATV.0000229218.97976.43).

[85] P. Duewell, H. Kono, K.J. Rayner, C.M. Sirois, G. Vladimer, F.G. Bauernfeind, G.S. Abela, L. Franchi, G. Nunez, M. Schnurr, T. Espevik, E. Lien, K.A. Fitzgerald, K.L. Rock, K.J. Moore, S.D. Wright, V. Hornung, E. Latz, NLRP3 inflammasomes are required for atherogenesis and activated by cholesterol crystals, Nature 464 (2010) 1357-1361, http:/ / dx.doi.org/10.1038/nature08938.

[86] F.J. Sheedy, A. Grebe, K.J. Rayner, P. Kalantari, B. Ramkhelawon, S.B. Carpenter, C.E. Becker, H.N. Ediriweera, A.E. Mullick, D.T. Golenbock, L.M. Stuart, E. Latz, K.A. Fitzgerald, K.J. Moore, CD36 coordinates NLRP3 inflammasome activation by facilitating intracellular nucleation of soluble ligands into particulate ligands in sterile inflammation, Nat. Immunol. 14 (2013) 812-820, http: / / dx.doi.org/10.1038/ni.2639.

[87] P.K. Shah, E. Falk, J.J. Badimon, A. Fernandez-Ortiz, A. Mailhac, G. Villareal-Levy, J.T. Fallon, J. Regnstrom, V. Fuster, Human monocyte-derived macrophages induce collagen breakdown in fibrous caps of atherosclerotic plaques. Potential role of matrix-degrading metalloproteinases and implications for plaque rupture, Circulation 92 (1995) 1565-1569.

[88] J.M. Isner, Cancer and atherosclerosis: the broad mandate of angiogenesis, Circulation 99 (1999) 1653-1655.

[89] P .R. Moreno, K.R. Purushothaman, M. Sirol, A.P . Levy, V . Fuster, Neovascularization in human atherosclerosis, Circulation 113 (2006) 2245-2252 (doi:113/18/2245).

[90] R. Hutter, W.S.Speidl, C. Valdiviezo, B. Sauter, R. Corti, V. Fuster, J.J. Badimon, Macrophages transmit potent proangiogenic effects of oxLDL in vitro and in vivo involving HIF-1alpha 
activation: a novel aspect of angiogenesis in atherosclerosis, J. Cardiovasc. Transl. Res. 6 (2013) 558-569, http: / / dx.doi.org/10.1007/s12265- 013-9469-9.

[91] K.J. Moore, F.J. Sheedy, E.A. Fisher, Macrophages in atherosclerosis: a dynamic balance, Nat. Rev. 13 (2013) 709-721, http: / / dx.doi.org/10.1038/ nri3520.

[92] M. Cross, E. Smith, D. Hoy, L. Carmona, F. Wolfe, T. Vos, B. Williams, S. Gabriel, M. Lassere, N. Johns, R. Buchbinder, A. Woolf, L. March, The global burden of rheumatoid arthritis: estimates from the global burden of disease 2010 study, Ann. Rheum. Dis. 73 (2014) 1316-1322, http: / / dx.doi.org/10.1136/ annrheumdis-2013- 204627.

[93] I.B. McInnes, J.R. O'Dell, State-of-the-art: rheumatoid arthritis, Ann. Rheum. Dis. 69 (2010) 1898-1906, http:/ / dx.doi.org/10.1136/ard.2010.134684.

[94] A. Gierut, H. Perlman, R.M. Pope, Innate immunity and rheumatoid arthritis, Rheum. Dis. Clin. N. Am. 36 (2010) 271-296, http: / / dx.doi.org/10.1016/j.rdc.2010.03.004.

[95] H. Takayanagi, Osteoimmunology in 2014: two-faced immunology-from osteogenesis to bone resorption, Nat. Rev. 11 (2015) 74-76, http: / / dx.doi.org/10.1038/nrrheum.2014.219.

[96] D. Mulherin, O. Fitzgerald, B. Bresnihan, Synovial tissue macrophage populations and articular damage in rheumatoid arthritis, Arthritis Rheum. 39 (1996)115-124.

[97] J.J. Haringman, D.M. Gerlag, A.H. Zwinderman, T.J.M. Smeets, M.C. Kraan, D. Baeten, I.B. McInnes, B. Bresnihan, P.P. Tak, Synovial tissue macrophages: a sensitive biomarker for response to treatment in patients with rheumatoid arthritis, Ann. Rheum. Dis. 64 (2005) 834-838 (doi:ard.2004.029751).

[98] J.M. Li, P. Isler, J.M. Dayer, D. Burger, Contact-dependent stimulation of monocytic cells and neutrophils by stimulated human T-cell clones, Immunology 84 (1995) 571-576.

[99] D.M. Frucht, M. Aringer, J. Galon, C. Danning, M. Brown, S. Fan, M. Centola, C.Y. Wu, N. Yamada, H. El Gabalawy, J.J. O'Shea, Stat4 is expressed in activated peripheral blood monocytes, dendritic cells, and macrophages at sites of Th1-mediated inflammation, J. Immunol. 164 (2000) 4659-4664 (doi:ji v164n9p4659).

[100] C.A. Roberts, A.K. Dickinson, L.S. Taams, The interplay between monocytes/ macrophages and CD4(+) T cell subsets in rheumatoid arthritis, Front. Immunol. 6 (2015) 571, http:/ / dx.doi.org/10.3389/ fimmu.2015.00571.

[101] J. Bondeson, S.D. Wainwright, S. Lauder, N. Amos, C.E. Hughes, The role of synovial macrophages and macrophage-produced cytokines in driving aggrecanases, matrix metalloproteinases, and other destructive and inflammatory responses in osteoarthritis, Arthritis Res. Ther. 8 (2006) R187 (doi:ar2099).

[102] R.W. Kinne, B. Stuhlmuller, G.R. Burmester, Cells of the synovium in rheumatoid arthritis. Macrophages, Arthritis Res. Ther. 9 (2007) 224, http://dx.doi.org/10.1186/ ar2333.

[103] H. Ishikawa, S. Hirata, Y. Andoh, H. Kubo, N. Nakagawa, Y. Nishibayashi, K. Mizuno, An immunohistochemical and immunoelectron microscopic study of adhesion molecules in synovial pannus formation in rheumatoid arthritis, Rheumatol. Int. 16 (1996) 53-60.

[104] I.B. McInnes, G. Schett, Cytokines in the pathogenesis of rheumatoid arthritis, Nat. Rev. 7 (2007) 429-442 (doi:nri2094).

[105] T. Kasama, F. Shiozawa, K. Kobayashi, N. Yajima, M. Hanyuda, H.T. Takeuchi, Y. Mori, M. Negishi, H. Ide, M. Adachi, Vascular endothelial growth factor expression by activated synovial leukocytes in rheumatoid arthritis: critical involvement of the interaction with synovial fibroblasts, Arthritis Rheum. 44 (2001) 2512-2524.

[106] E.M. Paleolog, Angiogenesis in rheumatoid arthritis, Arthritis Res. 4 (Suppl. 3) (2002) S81-S90, http:/ / dx.doi.org/10.1186/ar575.

[107] J.F. Charles, L.Y. Hsu, E.C. Niemi, A. Weiss, A.O. Aliprantis, M.C. Nakamura, Inflammatory arthritis increases mouse osteoclast precursors with myeloid suppressor function, J. Clin. Invest. 122 (2012) 4592-4605, http: / / dx.doi.org/10.1172 / JCI60920. 
[108] I.E. Adamopoulos, E.D. Mellins, Alternative pathways of osteoclastogenesis in inflammatory arthritis, Nat. Rev. 11 (2015) 189-194, http://dx.doi.org/10.1038/ nrrheum.2014.198.

[109] R.J. Xavier, D.K. Podolsky, Unravelling the pathogenesis of inflammatory bowel disease, Nature 448 (2007) 427-434 (doi:nature06005).

[110] J.K. Yamamoto-Furusho, D.K. Podolsky, Innate immunity in inflammatory bowel disease, World J. Gastroenterol. 13 (2007) 5577-5580.

[111] A.M. Mowat, Anatomical basis of tolerance and immunity to intestinal antigens, Nat. Rev. 3 (2003) 331-341, http: / / dx.doi.org/10.1038/nri1057.

[112] Y.R. Mahida, The key role of macrophages in the immunopathogenesis of inflammatory bowel disease, Inflamm. Bowel Dis. 6 (2000) 21-33.

[113] C. Neut, P. Bulois, P. Desreumaux, J.M. Membre, E. Lederman, L. Gambiez, A. Cortot, P. Quandalle, H. van Kruiningen, J.F. Colombel, Changes in the bacterial flora of the neoterminal ileum after ileocolonic resection for Crohn's disease, Am. J. Gastroenterol. 97 (2002) 939-946, http: / / dx.doi.org/10.1111/j. 1572- 0241.2002.05613.x.

[114] B. Khor, A. Gardet, R.J. Xavier, Genetics and pathogenesis of inflammatory bowel disease, Nature 474 (2011) 307-317, http: / / dx.doi.org/10.1038/nature10209.

[115] C.C. Bain, C.L. Scott, H. Uronen-Hansson, S. Gudjonsson, O. Jansson, O. Grip, M. Guilliams, B. Malissen, W.W. Agace, A.M. Mowat, Resident and pro-inflammatory macrophages in the colon represent alternative context-dependent fates of the same Ly6Chi monocyte precursors, Mucosal Immunol. 6 (2013) 498-510, http://dx.doi. org $/ 10.1038 / \mathrm{mi} .2012 .89$.

[116] S.H. Murch, C.P. Braegger, J.A. Walker-Smith, T.T. MacDonald, Location of tumour necrosis factor alpha by immunohistochemistry in chronic inflammatory bowel disease, Gut 34 (1993) 1705-1709.

[117] G.H. Waetzig, D. Seegert, P. Rosenstiel, S. Nikolaus, S. Schreiber, p38 Mitogen-activated protein kinase is activated and linked to TNF-alpha signaling in inflammatory bowel disease, J. Immunol. 168 (2002) 5342-5351.

[118] M.F. Neurath, Cytokines in inflammatory bowel disease, Nat. Rev. 14 (2014) 329-342, http: / / dx.doi.org/10.1038/nri3661.

[119] D. McGovern, F. Powrie, The IL23 axis plays a key role in the pathogenesis of IBD, Gut 56 (2007) 1333- 1336 (doi:gt115402).

[120] T. Griga, B. May, O. Pfisterer, K.M. Muller, F. Brasch, Immunohistochemical localization of vascular endothelial growth factor in colonic mucosa of patients with inflammatory bowel disease, Hepato- Gastroenterology 49 (2002) 116-123.

[121] W. Strober, I. Fuss, P. Mannon, The fundamental basis of inflammatory bowel disease, J. Clin. Invest. 117 (2007) 514-521, http:/ / dx.doi.org/10.1172/JCI30587.

[122] M.F. Neurath, New targets for mucosal healing and therapy in inflammatory bowel diseases, Mucosal Immunol. 7 (2014) 6-19, http:/ / dx.doi.org/10.1038/mi.2013.73.

[123] C. Han, D.W. Robinson Jr, M.V. Hackett, L.C. Paramore, K.H. Fraeman, M.V. Bala, Cardiovascular disease and risk factors in patients with rheumatoid arthritis, psoriatic arthritis, and ankylosing spondylitis, J. Rheumatol. 33 (2006) 2167-2172 (doi:06/13/0912).

[124] S.L. Arvikar, M.C. Fisher, Inflammatory bowel disease associated arthropathy, Curr. Rev. Muscoskelet. Med. 4 (2011) 123-131, http: / / dx.doi.org/10.1007/ s12178-011-9085-8.

[125] M.C. Whitlock, J. Yeboah, G.L. Burke, H. Chen, H.D. Klepin, W.G. Hundley, Cancer and its association with the development of coronary artery calcification: an assessment from the multi-ethnic study of atherosclerosis, J. Am. Heart Assoc. 4 (2015) http://dx.doi. org/10.1161/JAHA.115.002533.

[126] B. Pelaz, C. Alexiou, R.A. Alvarez-Puebla, F. Alves, A.M. Andrews, S. Ashraf, L.P. Balogh, L. Ballerini, A. Bestetti, C. Brendel, S. Bosi, M. Carril, W.C.W. Chan, C. Chen, 
X. Chen, X. Chen, Z. Cheng, D. Cui, J. Du, C. Dullin, A. Escudero, N. Feliu, M. Gao, M. George, Y. Gogotsi, A. Grünweller, Z. Gu, N.J. Halas, N. Hampp, R.K. Hartmann, M.C. Hersam, P . Hunziker, J. Jian, X. Jiang, P . Jungebluth, P. Kadhiresan, K. Kataoka, A. Khademhosseini, J. Kopeček, N.A. Kotov, H.F. Krug, D.S. Lee, C.-M. Lehr, K.W. Leong, X.-J. Liang, M. Ling Lim, L.M. Liz-Marzán, X. Ma, P. Macchiarini, H. Meng, H. Möhwald, P. Mulvaney, A.E. Nel, S. Nie, P. Nordlander, T. Okano, J. Oliveira, T.H. Park, R.M. Penner, M. Prato, V. Puntes, V.M. Rotello, A. Samarakoon, R.E. Schaak, Y. Shen, S. Sjöqvist, A.G. Skirtach, M.G. Soliman, M.M. Stevens, H.-W. Sung, B.Z. Tang, R. Tietze, B.N. Udugama, J.S. VanEpps, T. Weil, P.S. Weiss, I. Willner, Y. Wu, L. Yang, Z. Yue, Q. Zhang, Q. Zhang, X.-E. Zhang, Y. Zhao, X. Zhou, W.J. Parak, Diverse applications of Nanomedicine, ACS Nano 11 (2017) 2313-2381, http: / / dx.doi.org/10.1021/acsnano.6b06040.

[127] J.I. Hare, T. Lammers, M.B. Ashford, S. Puri, G. Storm, S.T. Barry, Challenges and strategies in anti-cancer nanomedicine development: an industry perspective, Adv. Drug Deliv. Rev. 108 (2017) 25-38, http: / / dx.doi.org/10.1016/j.addr.2016.04.025.

[128] Z. Niu, I. Conejos-Sanchez, B.T. Griffin, C.M. O’Driscoll, M.J. Alonso, Lipid-based nanocarriers for oral peptide delivery, Adv. Drug Deliv. Rev. 106 (2016) 337-354 (doi:S0169-409X(16)30100-4).

[129] P. Ofek, G. Tiram, R. Satchi-Fainaro, Angiogenesis regulation by nanocarriers bearing RNA interference, Adv. Drug Deliv. Rev. (2017) (doi:S0169-409X(17)30027-3).

[130] J.E. Kipp, The role of solid nanoparticle technology in the parenteral delivery of poorly water-soluble drugs, Int. J. Pharm. 284 (2004) 109-122, http://dx.doi.org/10.1016/j. ijpharm.2004.07.019.

[131] A. Gabizon, M. Bradbury, U. Prabhakar, W. Zamboni, S. Libutti, P. Grodzinski, Cancer nanomedicines: closing the translational gap, Lancet 384 (2014) 2175-2176, http:// dx.doi.org/10.1016/S0140-6736(14)61457-4.

[132] T. Ojha, L. Rizzo, G. Storm, F. Kiessling, T. Lammers, Image-guided drug delivery: preclinical applications and clinical translation, Expert Opin. Drug Deliv. 12 (2015) 12031207, http: / / dx.doi.org/10.1517/17425247.2015.1059420.

[133] E.K. Chow, D. Ho, Cancer nanomedicine: from drug delivery to imaging, Sci. Transl. Med. 5 (2013) 216rv4, http:/ / dx.doi.org/10.1126/ scitranslmed.3005872.

[134] F. Kiessling, M.E. Mertens, J. Grimm, T. Lammers, Nanoparticles for imaging: top or flop?, Radiology 273 (2014) 10-28, http: / / dx.doi.org/10.1148/ radiol.14131520.

[135] F. Leuschner, P. Dutta, R. Gorbatov, T.I. Novobrantseva, J.S. Donahoe, G. Courties, K.M. Lee, J.I. Kim, J.F. Markmann, B. Marinelli, P. Panizzi, W.W. Lee, Y. Iwamoto, S. Milstein, H. Epstein-Barash, W. Cantley, J. Wong, V. Cortez-Retamozo, A. Newton, K. Love, P. Libby, M.J. Pittet, F.K. Swirski, V. Koteliansky, R. Langer, R. Weissleder, D.G. Anderson, M. Nahrendorf, Therapeutic siRNA silencing in inflammatory monocytes in mice, Nat. Biotechnol. 29 (2011) 1005-1010, http: / / dx.doi.org/10.1038/ nbt.1989.

[136] C. Pérez-Medina, J. Tang, D. Abdel-Atti, B. Hogstad, M. Merad, E.A. Fisher, Z.A. Fayad, J.S. Lewis, W.J.M. Mulder, T. Reiner, C. Perez-Medina, J. Tang, D. Abdel-Atti, B. Hogstad, M. Merad, E.A. Fisher, Z.A. Fayad, J.S. Lewis, W.J.M. Mulder, T. Reiner, PET imaging of tumor-associated macrophages with ${ }^{89} \mathrm{Zr}$-labeled high-density lipoprotein nanoparticles, J. Nucl. Med. 56 (2015) 1272-1277, http: / / dx.doi.org/10.2967 / jnumed.115.158956.

[137] T.Y. Tang, S.P. Howarth, S.R. Miller, M.J. Graves, A.J. Patterson, J.M. U-King-Im, Z.Y. Li, S.R. Walsh, A.P. Brown, P.J. Kirkpatrick, E.A. Warburton, P.D. Hayes, K. Varty, J.R. Boyle, M.E. Gaunt, A. Zalewski, J.H. Gillard, The ATHEROMA (atorvastatin therapy: effects on reduction of macrophage activity) study. Evaluation using ultrasmall superparamagnetic iron oxide-enhanced magnetic resonance imaging in carotid disease, J. Am. Coll. Cardiol. 53 (2009) 2039-2050, http: / / dx.doi.org/10.1016/j.jacc.2009.03.018.

[138] S.R. Alam, C. Stirrat, J. Richards, S. Mirsadraee, S.I. Semple, G. Tse, P. Henriksen, D.E. 
Newby, Vascular and plaque imaging with ultrasmall superparamagnetic particles of iron oxide, J. Cardiovasc. Magn. Reson. 17 (2015) 83-84, http://dx.doi.org/10.1186/ s12968-015-0183-4.

[139] M.E. Kooi, V.C. Cappendijk, K.B. Cleutjens, A.G. Kessels, P.J. Kitslaar, M. Borgers, P.M. Frederik, M.J. Daemen, J.M. van Engelshoven, Accumulation of ultrasmall superparamagnetic particles of iron oxide in human atherosclerotic plaques can be detected by in vivo magnetic resonance imaging, Circulation 107 (2003) 2453- 2458, http: / / dx.doi.org/10.1161/01.CIR.0000068315. 98705.CC.

[140] A.H. Brouwers, D.J.DeJong, E.T.Dams, W.J.Oyen, O.C. Boerman, P.Laverman, T.H. Naber, G. Storm, F.H. Corstens, Tc-99m-PEG-liposomes for the evaluation of colitis in Crohn's disease, J. Drug Target. 8 (2000) 225-233, http: / / dx.doi.org/10.3109/10611860008997901.

[141] K. Sou, B. Goins, S. Takeoka, E. Tsuchida, W.T. Phillips, Selective uptake of surfacemodified phospholipid vesicles by bone marrow macrophages in vivo, Biomaterials 28 (2007) 2655-2666 (doi:S0142-9612(07)00127-5).

[142] S.I. Grivennikov, M. Karin, Inflammatory cytokines in cancer: tumour necrosis factor and interleukin 6 take the stage, Ann. Rheum. Dis. 70 (Suppl. 1) (2011) i104-i108, http:/ / dx.doi.org/10.1136/ard.2010.140145.

[143] E.M. Pietras, C. Mirantes-Barbeito, S. Fong, D. Loeffler, L.V . Kovtonyuk, S. Zhang, R. Lakshminarasimhan, C.P. Chin, J.-M.M. Techner, B. Will, C. Nerlov, U. Steidl, M.G. Manz, T. Schroeder, E. Passegue, E. Passegué, Chronic interleukin-1 exposure drives haematopoietic stem cells towards precocious myeloid differentiation at the expense of self-renewal, Nat. Cell Biol. 18 (2016) 607-618, http:/ / dx.doi.org/10.1038/ncb3346.

[144] W. Strober, I.J. Fuss, Pro-inflammatory cytokines in the pathogenesis of IBD, Gastroenterology 140 (2011) 1756-1767, http: / / dx.doi.org/10.1053/j.gastro.2011.02.016.

[145] C. Zuany-Amorim, J. Hastewell, C. Walker, Toll-like receptors as potential therapeutic targets for multiple diseases, Nat. Rev. Discov. 1 (2002) 797-807, http://dx.doi. org $/ 10.1038 / \mathrm{nrd} 914$.

[146] T. Heidt, H.B. Sager, G. Courties, P. Dutta, Y. Iwamoto, A. Zaltsman, C. von Zur Muhlen, C. Bode, G.L. Fricchione, J. Denninger, C.P. Lin, C. Vinegoni, P. Libby, F.K. Swirski, R. Weissleder, M. Nahrendorf, Chronic variable stress activates hematopoietic stem cells, Nat. Med. 20 (2014) 754-758, http: / / dx.doi. org/10.1038/nm.3589.

[147] B.F. Reader, B.L. Jarrett, D.B. McKim, E.S. Wohleb, J.P. Godbout, J.F. Sheridan, Peripheral and central effects of repeated social defeat stress: monocyte trafficking, microglial activation, and anxiety, Neuroscience 289 (2015) 429-442, http:/ / dx.doi.org/10.1016/j. neuroscience.2015.01.001.

[148] A. Tawakol, A. Ishai, R.A. Takx, A.L. Figueroa, A. Ali, Y. Kaiser, Q.A. Truong, C.J. Solomon, C. Calcagno, V. Mani, C.Y. Tang, W.J. Mulder, J.W. Murrough, U. Hoffmann, M. Nahrendorf, L.M. Shin, Z.A. Fayad, R.K. Pitman, Relation between resting amygdalar activity and cardiovascular events: a longitudinal and cohort study, Lancet 389 (2017) 834-845, http: / / dx.doi.org/10.1016/S0140-6736(16)31714-7.

[149] A. Mendelson, P.S. Frenette, Hematopoietic stem cell niche maintenance during homeostasis and regeneration, Nat. Med. 20 (2014) 833-846, http: / / dx.doi.org / 10.1038/ nm.3647.

[150] C. Shi, T. Jia, S. Mendez-Ferrer, T.M. Hohl, N.V. Serbina, L. Lipuma, I. Leiner, M.O. Li, P.S. Frenette, E.G. Pamer, Bone marrow mesenchymal stem and progenitor cells induce monocyte emigration in response to circulating toll-like receptor ligands, Immunity 34 (2011) 590-601, http:/ / dx.doi.org/10.1016/j.immuni.2011.02.016.

[151] A. Vacca, D. Ribatti, Bone marrow angiogenesis in multiple myeloma, Leukemia 20 (2006) 193-199 (doi:2404067).

[152] M. Chavez-Macgregor, A. Aviles-Salas, D. Green, A. Fuentes-Alburo, C. Gomez-Ruiz, A. 
Aguayo, Angiogenesis in the bone marrow of patients with breast cancer, Clin. Cancer Res. 11 (2005) 5396-5400 (doi:11/15/5396).

[153] R. Ria, A. Reale, A. De Luisi, A. Ferrucci, M. Moschetta, A. Vacca, Bone marrow angiogenesis and progression in multiple myeloma, Am. J. Blood Res. 1 (2011) 76-89.

[154] C. Scavelli, B. Nico, T. Cirulli, R. Ria, G. Di Pietro, D. Mangieri, A. Bacigalupo, G. Mangialardi, A.M. Coluccia, T. Caravita, S. Molica, D. Ribatti, F. Dammacco, A. Vacca, Vasculogenic mimicry by bone marrow macrophages in patients with multiple myeloma, Oncogene 27 (2008) 663-674 (doi:1210691).

[155] R.K. Jain, D.G. Duda, Role of bone marrow-derived cells in tumor angiogenesis and treatment, Cancer Cell 3 (2003) 515-516 (doi:S1535610803001387).

[156] B. Ozbakir, B.J. Crielaard, J.M. Metselaar, G. Storm, T. Lammers, Liposomal corticosteroids for the treatment of inflammatory disorders and cancer, J. Control. Release 190 (2014) 624-636, http: / / dx.doi.org/10.1016/j.jconrel.2014.05.039.

[157] S.T. Proulx, E. Kwok, Z. You, M.O. Papuga, C.A. Beck, D.J. Shealy, L.M. Calvi, C.T. Ritchlin, H.A. Awad, B.F. Boyce, L. Xing, E.M. Schwarz, Elucidating bone marrow edema and myelopoiesis in murine arthritis using contrast-enhanced magnetic resonance imaging, Arthritis Rheum. 58 (2008) 2019-2029, http:/ / dx.doi.org/10.1002/ art.23546.

[158] H.E. Daldrup-Link, T.M. Link, E.J. Rummeny, C. August, S. Konemann, H. Jurgens, W. Heindel, Assessing permeability alterations of the blood-bone marrow barrier due to total body irradiation: in vivo quantification with contrast enhanced magnetic resonance imaging, Bone Marrow Transplant. 25 (2000) 71-78, http://dx.doi.org/10.1038/ sj.bmt.1702087.

[159] A. Ziadloo, S.R. Burks, E.M. Gold, B.K. Lewis, A. Chaudhry, M.J. Merino, V. Frenkel, J.A. Frank, Enhanced homing permeability and retention of bone marrow stromal cells by noninvasive pulsed focused ultrasound, Stem Cells 30 (2012) 1216-1227, http:/ / dx.doi. org/10.1002/stem.1099.

[160] V. Awasthi, B. Goins, R. Klipper, R. Loredo, D. Korvick, W.T. Phillips, Imaging experimental osteomyelitis using radiolabeled liposomes, J. Nucl. Med. 39 (1998) 10891094.

[161] C. Pérez-Medina, T. Binderup, M.E. Lobatto, J. Tang, C. Calcagno, L. Giesen, C.H. Wessel, J. Witjes, S. Ishino, S. Baxter, Y. Zhao, S. Ramachandran, M. Eldib, B.L. SánchezGaytán, P.M. Robson, J. Bini, J.F. Granada, K.M. Fish, E.S.G. Stroes, R. Duivenvoorden, S. Tsimikas, J.S. Lewis, T. Reiner, V. Fuster, A. Kjær, E.A. Fisher, Z.A. Fayad, W.J.M. Mulder, In vivo PET imaging of HDL in multiple atherosclerosis models, JACC Cardiovasc. Imaging 9 (2016) 950-961, http: / / dx.doi.org/10.1016/j.jcmg.2016.01.020.

[162] A. Swami, M.R. Reagan, P. Basto, Y. Mishima, N. Kamaly, S. Glavey, S. Zhang, M. Moschetta, D. Seevaratnam, Y. Zhang, J. Liu, M. Memarzadeh, J. Wu, S. Manier, J. Shi, N. Bertrand, Z.N. Lu, K. Nagano, R. Baron, A. Sacco, A.M. Roccaro, O.C. Farokhzad, I.M. Ghobrial, Engineered nanomedicine for myeloma and bone microenvironment targeting, Proc. Natl. Acad. Sci. U. S. A. 111 (2014) 10287-10292, http://dx.doi.org/10.1073/ pnas.1401337111.

[163] V. Hengst, C. Oussoren, T. Kissel, G. Storm, Bone targeting potential of bisphosphonatetargeted liposomes. Preparation, characterization and hydroxyapatite binding in vitro, Int. J. Pharm. 331 (2007) 224-227 (doi:S0378-5173(06)00981-1).

[164] C.J. Porter, S.M. Moghimi, L. Illum, S.S. Davis, The polyoxyethylene/ polyoxypropylene block co-polymer poloxamer-407 selectively redirects intravenously injected microspheres to sinusoidal endothelial cells of rabbit bone marrow, FEBS Lett. 305 (1992) 62-66 (doi:0014-5793(92)80655-Z).

[165] A. Agool, R.H. Slart, K.K. Thorp, A.W. Glaudemans, D.C. Cobben, L.B. Been, F.R. Burlage, P.H. Elsinga, R.A. Dierckx, E. Vellenga, J.L. Holter, Effect of radiotherapy and 
chemotherapy on bone marrow activity: a 18F- FLT-PET study, Nucl. Med. Commun. 32 (2011) 17-22.

[166] K. Fuchs, U. Kohlhofer, L. Quintanilla-Martinez, D. Lamparter, I. Kotter, G. Reischl, M. Rocken, B.J. Pichler, M. Kneilling, In vivo imaging of cell proliferation enables the detection of the extent of experimental rheumatoid arthritis by $3^{\prime}$-deoxy-3'-18ffluorothymidine and small-animal PET, J. Nucl. Med. 54 (2013) 151-158, http:/ / dx.doi. org/10.2967/jnumed.112.106740.

[167] Y.X. Ye, C. Calcagno, T. Binderup, G. Courties, E.J. Keliher, G.R. Wojtkiewicz, Y. Iwamoto, J. Tang, C. Pérez-Medina, V. Mani, S. Ishino, C.B. Johnbeck, U. Knigge, Z.A. Fayad, P. Libby, R. Weissleder, A. Tawakol, S. Dubey, A.P. Belanger, M.F. Di Carli, F.K. Swirski, A. Kjaer, W.J.M. Mulder, M. Nahrendorf, Imaging macrophage and hematopoietic progenitor proliferation in atherosclerosis, Circ. Res. 117 (2015) 835-845, http:/ / dx.doi. org/10.1161/CIRCRESAHA.115.307024.

[168] E.J. Kim, S. Kim, D.O. Kang, H.S. Seo, Metabolic activity of the spleen and bone marrow in patients with acute myocardial infarction evaluated by 18f-fluorodeoxyglucose positron emission tomograpic imaging, Circ. Imaging 7 (2014) 454-460, http:/ / dx.doi. org/10.1161/CIRCIMAGING.113.001093.

[169] G. Courties, F. Herisson, H.B. Sager, T. Heidt, Y. Ye, Y. Wei, Y. Sun, N. Severe, P. Dutta, J. Scharff, D.T. Scadden, R. Weissleder, F.K. Swirski, M.A. Moskowitz, M. Nahrendorf, Ischemic stroke activates hematopoietic bone marrow stem cells, Circ. Res. 116 (2015) 407-417, http: / / dx.doi.org/10.1161 / CIRCRESAHA.116.305207.

[170] P. Dutta, H.B. Sager, K.R. Stengel, K. Naxerova, G. Courties, B. Saez, L. Silberstein, T. Heidt, M. Sebas, Y. Sun, G. Wojtkiewicz, P.F. Feruglio, K. King, J.N. Baker, A.M. van der Laan, A. Borodovsky, K. Fitzgerald, M. Hulsmans, F. Hoyer, Y. Iwamoto, C. Vinegoni, D. Brown, M. Di Carli, P. Libby, S.W. Hiebert, D.T. Scadden, F.K. Swirski, R. Weissleder, M. Nahrendorf, Myocardial infarction activates CCR2(+) hematopoietic stem and progenitor cells, Cell Stem Cell 16 (2015) 477-487, http: / / dx.doi.org/10.1016/j.stem.2015.04.008.

[171] P. Dutta, M. Nahrendorf, Regulation and consequences of monocytosis, Immunol. Rev. 262 (2014) 167-178, http:/ / dx.doi.org/10.1111/imr.12219.

[172] C.S. Robbins, A. Chudnovskiy, P.J. Rauch, J.L. Figueiredo, Y. Iwamoto, R. Gorbatov, M. Etzrodt, G.F. Weber, T. Ueno, N. van Rooijen, M.J. Mulligan-Kehoe, P. Libby, M. Nahrendorf, M.J. Pittet, R. Weissleder, F.K. Swirski, Extramedullary hematopoiesis generates Ly-6C(high) monocytes that infiltrate atherosclerotic lesions, Circulation 125 (2012) 364-374, http: / / dx.doi.org/10.1161/ CIRCULATIONAHA.111.061986.

[173] V. Cortez-Retamozo, M. Etzrodt, A. Newton, R. Ryan, F. Pucci, S.W. Sio, W. Kuswanto, P.J. Rauch, A. Chudnovskiy, Y. Iwamoto, R. Kohler, B. Marinelli, R. Gorbatov, G. Wojtkiewicz, P. Panizzi, M. Mino-Kenudson, R. Forghani, J.L. Figueiredo, J.W. Chen, R. Xavier, F.K. Swirski, M. Nahrendorf, R. Weissleder, M.J. Pittet, Angiotensin II drives the production of tumor-promoting macrophages, Immunity 38 (2013) 296-308, http:/ / dx.doi.org/10.1016/j.immuni.2012.10.015.

[174] H. Emami, P. Singh, M. MacNabb, E. Vucic, Z. Lavender, J.H.F. Rudd, Z.A. Fayad, J. Lehrer-Graiwer, M. Korsgren, A.L. Figueroa, J. Fredrickson, B. Rubin, U. Hoffmann, Q.A. Truong, J.K. Min, A. Baruch, K. Nasir, M. Nahrendorf, A. Tawakol, Splenic metabolic activity predicts risk of future cardiovascular events, JACC Cardiovasc. Imaging 8 (2015) 121-130, http: / / dx.doi.org/10.1016/j.jcmg.2014.10.009.

[175] F.K. Swirski, M. Nahrendorf, M. Etzrodt, M. Wildgruber, V. Cortez-Retamozo, P. Panizzi, J.-L.L. Figueiredo, R.H. Kohler, A. Chudnovskiy, P. Waterman, E. Aikawa, T.R. Mempel, P. Libby, R. Weissleder, M.J. Pittet, Identification of splenic reservoir monocytes and their deployment to inflammatory sites, Science 325 (2009) 612-616, http://dx.doi. org/10.1126/science.1175202. 
[176] S. Mellak, H. Ait-Oufella, B. Esposito, X. Loyer, M. Poirier, T.F. Tedder, A. Tedgui, Z. Mallat, S. Potteaux, Angiotensin II mobilizes spleen monocytes to promote the development of abdominal aortic aneurysm in Apoe ${ }^{-1-}$ mice, Arterioscler. Thromb. Vasc. Biol. 35 (2015) 378-388, http: / / dx.doi.org/10.1161 / ATVBAHA.114.304389.

[177] E. Blanco, H. Shen, M. Ferrari, Principles of nanoparticle design for overcoming biological barriers to drug delivery, Nat. Biotechnol. 33 (2015) 941-951, http:/ / dx.doi. org / 10.1038/nbt.3330.

[178] Y. Nakano, T. Matoba, M. Tokutome, D. Funamoto, S. Katsuki, G. Ikeda, K. Nagaoka, A. Ishikita, K. Nakano, J. Koga, K. Sunagawa, K. Egashira, Nanoparticle-mediated delivery of Irbesartan induces cardioprotection from myocardial ischemia-reperfusion injury by antagonizing monocyte-mediated inflammation, Sci. Rep. 6 (2016) 29601, http:/ / dx.doi. org/10.1038/ srep29601.

[179] B.R. Smith, E.E.B. Ghosn, H. Rallapalli, J.A. Prescher, T. Larson, L.A. Herzenberg, S.S. Gambhir, Selective uptake of single walled carbon nanotubes by circulating monocytes for enhanced tumour delivery, Nat. Nanotechnol. 9 (2014) 481-487, http://dx.doi. org/10.1038/nnano.2014.62.

[180] M.F. Kircher, J. Grimm, F.K. Swirski, P. Libby, R.E. Gerszten, J.R. Allport, R. Weissleder, Noninvasive in vivo imaging of monocyte trafficking to atherosclerotic lesions, Circulation 117 (2008) 388-395, http: / / dx.doi.org/10.1161 / CIRCULA TIONAHA.107.719765.

[181] L.M. Carlin, C. Auffray, F. Geissmann, Measuring intravascular migration of mouse Ly6C(low) monocytes in vivo using intravital microscopy, Curr. Protoc. Immunol. (2013) http: / / dx.doi.org/10.1002/0471142735.im1433s101 (Chapter 14, Unit 14.33.1-16).

[182] R. Rua, D.B. McGavern, Elucidation of monocyte/macrophage dynamics and function by intravital imaging, J. Leukoc. Biol. 98 (2015) 319-332, http://dx.doi.org/10.1189/ jlb.4RI0115-006RR.

[183] S. McArdle, Z. Mikulski, K. Ley, Live cell imaging to understand monocyte, macrophage, and dendritic cell function in atherosclerosis, J. Exp. Med. 213 (2016) 1117-1131, http:/ / dx.doi.org/10.1084/jem.20151885.

[184] E.T. Ahrens, J.W.M. Bulte, Tracking immune cells in vivo using magnetic resonance imaging, Nat. Rev. 13 (2013) 755-763, http:/ / dx.doi.org/10.1038/nri3531.

[185] R. Yang, S. Sarkar, D.J. Korchinski, Y. Wu, V.W. Yong, J.F. Dunn, MRI monitoring of monocytes to detect immune stimulating treatment response in brain tumor, NeuroOncology (2016) (doi:now180).

[186] J.M. Richards, C.A. Shaw, N.N. Lang, M.C. Williams, S.I. Semple, T.J. MacGillivray, C. Gray, J.H. Crawford, S.R. Alam, A.P. Atkinson, E.K. Forrest, C. Bienek, N.L. Mills, A. Burdess, K. Dhaliwal, A.J. Simpson, W.A. Wallace, A.T. Hill, P.H. Roddie, G. McKillop, T.A. Connolly, G.Z. Feuerstein, G.R. Barclay, M.L. Turner, D.E. Newby, In vivo mononuclear cell tracking using superparamagnetic particles of iron oxide: feasibility and safety in humans, Circ. Imaging 5 (2012) 509-517, http://dx.doi.org/10.1161/ CIRCIMAGING.112.972596.

[187] J. Ruiz-Cabello, B.P. Barnett, P.A. Bottomley, J.W. Bulte, Fluorine (19F) MRS and MRI in biomedicine, NMR Biomed. 24 (2011) 114-129, http:/ / dx.doi.org/10.1002/nbm.1570.

[188] F. Bonner, M.W. Merx, K. Klingel, P. Begovatz, U. Flogel, M. Sager, S. Temme, C. Jacoby, M. Salehi Ravesh, C. Grapentin, R. Schubert, J. Bunke, M. Roden, M. Kelm, J. Schrader, Monocyte imaging after myocardial infarction with $19 \mathrm{~F}$ MRI at $3 \mathrm{~T}$ : a pilot study in explanted porcine hearts, Eur. Heart J.Cardiovasc. Imaging 16 (2015) 612-620, http:/ / dx.doi.org/10.1093/ehjci/jev008.

[189] R.M. Thurlings, C.A. Wijbrandts, R.J. Bennink, S.E. Dohmen, C. Voermans, D. Wouters, E.S. Izmailova, D.M. Gerlag, B.L.F. van Eck-Smit, P.P. Tak, Monocyte scintigraphy in rheumatoid arthritis: the dynamics of monocyte migration in immune-mediated 
inflammatory disease, PLoS One 4 (2009) e7865, http://dx.doi.org/10.1371/journal. pone.0007865 (doi:09-PONE-RA-09911).

[190] M.D. Normandin, H. Yuan, M.Q. Wilks, H.H. Chen, J.M. Kinsella, H. Cho, N.J. Guehl, N. Absi-Halabi, S.M. Hosseini, G. El Fakhri, D.E. Sosnovik, L. Josephson, Heat-induced radiolabeling of nanoparticles for monocyte tracking by PET, Angew. Chem. Int. Ed. Engl. 54 (2015) 13002-13006, http: / / dx.doi.org/10.1002/anie.201505525.

[191] A. Parodi, N. Quattrocchi, A.L. van de Ven, C. Chiappini, M. Evangelopoulos, J.O. Martinez, B.S. Brown, S.Z. Khaled, I.K. Yazdi, M.V. Enzo, L. Isenhart, M. Ferrari, E. Tasciotti, Synthetic nanoparticles functionalized with biomimetic leukocyte membranes possess cell-like functions, Nat. Nanotechnol. 8 (2013) 61-68, http: / / dx.doi.org / 10.1038/ nnano.2012.212.

[192] H. Cao, Z. Dan, X. He, Z. Zhang, H. Yu, Q. Yin, Y. Li, Liposomes coated with isolated macrophage membrane can target lung metastasis of breast cancer, ACS Nano 10 (2016) 7738-7748, http: / / dx.doi.org/10.1021/acsnano.6b03148.

[193] R. Molinaro, C. Corbo, J.O. Martinez, F. Taraballi, M. Evangelopoulos, S. Minardi, I.K. Yazdi, P. Zhao, E. De Rosa, M.B. Sherman, A. De Vita, N.E. Toledano Furman, X. Wang, A. Parodi, E. Tasciotti, Biomimetic proteolipid vesicles for targeting inflamed tissues, Nat. Mater. 15 (2016) 1037-1046, http: / / dx.doi.org/10.1038/nmat4644.

[194] R. Polak, R.M. Lim, M.M. Beppu, R.N. Pitombo, R.E. Cohen, M.F. Rubner, Liposomeloaded cell backpacks, Adv. Healthc. Mater. 4 (2015) 2832-2841,http://dx.doi. org/10.1002/adhm.201500604.

[195] A.C. Anselmo, S. Kumar, V. Gupta, A.M. Pearce, A. Ragusa, V. Muzykantov, S. Mitragotri, Exploiting shape, cellular-hitchhiking and antibodies to target nanoparticles to lung endothelium: synergy between physical, chemical and biological approaches, Biomaterials 68 (2015) 1-8, http: / / dx.doi.org/10.1016/j. biomaterials.2015.07.043.

[196] A.C. Anselmo, S. Mitragotri, Cell-mediated delivery of nanoparticles: taking advantage of circulatory cells to target nanoparticles, J. Control. Release 190 (2014) 531-541, http:/ / dx.doi.org/10.1016/j.jconrel.2014.03.050.

[197] H.B. Sager, P. Dutta, J.E. Dahlman, M. Hulsmans, G. Courties, Y. Sun, T. Heidt, C. Vinegoni, A. Borodovsky, K. Fitzgerald, G.R. Wojtkiewicz, Y. Iwamoto, B. Tricot, O.F. Khan, K.J. Kauffman, Y. Xing, T.E. Shaw, P. Libby, R. Langer, R. Weissleder, F.K. Swirski, D.G. Anderson, M. Nahrendorf, RNAi targeting multiple cell adhesion molecules reduces immune cell recruitment and vascular inflammation after myocardial infarction, Sci. Transl. Med. 8 (2016) 342ra80, http: / / dx.doi.org/10.1126/ scitranslmed.aaf1435.

[198] M.A. McAteer, J.E. Schneider, Z.A. Ali, N. Warrick, C.A. Bursill, C. von zur Muhlen, D.R. Greaves, S. Neubauer, K.M. Channon, R.P. Choudhury, Magnetic resonance imaging of endothelial adhesion molecules in mouse atherosclerosis using dualtargeted microparticles of iron oxide, Arterioscler. Thromb. Vasc. Biol. 28 (2008) 77-83 (doi:ATVBAHA.107.145466).

[199] K.A. Kelly, J.R. Allport, A. Tsourkas, V.R. Shinde-Patil, L. Josephson, R. Weissleder, Detection of vascular adhesion molecule-1 expression using a novel multimodal nanoparticle, Circ. Res. 96 (2005) 327-336 (doi:01.RES.0000155722.17881.dd).

[200] S. Gosk, T. Moos, C. Gottstein, G. Bendas, VCAM-1 directed immunoliposomes selectively target tumor vasculature in vivo, Biochim. Biophys. Acta 1778 (2008) 854-863, http: / / dx.doi.org/10.1016/j.bbamem.2007.12.021.

[201] X. Shao, H. Zhang, J.R. Rajian, D.L. Chamberland, P.S. Sherman, C.A. Quesada, A.E. Koch, N.A. Kotov, X. Wang, 125I-labeled gold nanorods for targeted imaging of inflammation, ACS Nano 5 (2011) 8967-8973, http:/ / dx.doi.org/10.1021/ nn203138t.

[202] D.J. Lee, A. Lyshchik, J. Huamani, D.E. Hallahan, A.C. Fleischer, Relationship between retention of a vascular endothelial growth factor receptor 2 (VEGFR2)-targeted 
ultrasonographic contrast agent and the level of VEGFR2 expression in an in vivo breast cancer model, J. Ultrasound Med. 27 (2008) 855-866.

[203] A.J. Hamilton, S.-L. Huang, D. Warnick, M. Rabbat, B. Kane, A. Nagaraj, M. Klegerman, D.D. McPherson, Intravascular ultrasound molecular imaging of atheroma components in vivo, J. Am. Coll. Cardiol. 43 (2004) 453- 460, http://dx.doi.org/10.1016/j. jacc.2003.07.048.

[204] P.A. Jarzyna, L.H. Deddens, B.H. Kann, S. Ramachandran, C. Calcagno, W. Chen, A. Gianella, R.M. Dijkhuizen, A.W. Griffioen, Z.A. Fayad, W.J.M. Mulder, Tumor angiogenesis phenotyping by nanoparticle- facilitated magnetic resonance and nearinfrared fluorescence molecular imaging, Neoplasia 14 (2012) 964-973 (doi:121148).

[205] R. Satchi-Fainaro, M. Puder, J.W. Davies, H.T. Tran, D.A. Sampson, A.K. Greene, G. Corfas, J. Folkman, Targeting angiogenesis with a conjugate of HPMA copolymer and TNP-470, Nat. Med. 10 (2004) 255-261, http: / / dx.doi.org / 10.1038/nm1002.

[206] Y. Wang, P. Liu, Y. Duan, X. Yin, Q. Wang, X. Liu, X. Wang, J. Zhou, W. Wang, L. Qiu, W. Di, Specific cell targeting with APRPG conjugated PEG-PLGA nanoparticles for treating ovarian cancer, Biomaterials 35 (2014) 983-992, http://dx.doi.org/10.1016/j. biomaterials.2013.09.062.

[207] P.M. Winter, A.M. Neubauer, S.D. Caruthers, T.D. Harris, J.D. Robertson, T.A. Williams, A.H. Schmieder, G. Hu, J.S. Allen, E.K. Lacy, H. Zhang, S.A. Wickline, G.M. Lanza, Endothelial $\alpha v \beta 3$ integrin-targeted fumagillin nanoparticles inhibit angiogenesis in atherosclerosis, Arterioscler. Thromb. Vasc. Biol. 26 (2006) 2103-2109 (doi:01.A TV .0000235724.11299.76).

[208] P.M. Winter, S.D. Caruthers, H. Zhang, T.A. Williams, S.A. Wickline, G.M. Lanza, Antiangiogenic synergism of integrin-targeted fumagillin nanoparticles and atorvastatin in atherosclerosis, JACC Cardiovasc. Imaging 1 (2008) 624-634, http://dx.doi. org/10.1016/j.jcmg.2008.06.003.

[209] H. Zhou, H.W. Chan, S.A. Wickline, G.M. Lanza, C.T.N. Pham, $\alpha v \beta 3$-Targeted nanotherapy suppresses inflammatory arthritis in mice, FASEB J. 23 (2009) 2978-2985 (doi:09-129874 [pii).

[210] M. Banciu, J.M. Metselaar, R.M. Schiffelers, G. Storm, Liposomal glucocorticoids as tumor-targeted anti- angiogenic nanomedicine in B16 melanoma-bearing mice, J. Steroid Biochem. Mol. Biol. 111 (2008) 101-110, http: / / dx.doi.org/10.1016/j.jsbmb.2008.05.004.

[211] B.J. Vakoc, R.M. Lanning, J.A. Tyrrell, T.P. Padera, L.A. Bartlett, T. Stylianopoulos, L.L. Munn, G.J. Tearney, D. Fukumura, R.K. Jain, B.E. Bouma, Three-dimensional microscopy of the tumor microenvironment in vivo using optical frequency domain imaging, Nat. Med. 15 (2009) 1219-1223, http: / / dx.doi.org / 10.1038/nm.1971.

[212] T. van der Geest, J.M. Metselaar, D. Gerrits, P.L. van Lent, G. Storm, P. Laverman, O.C. Boerman, [(18)]F FDG PET/CT imaging to monitor the therapeutic effect of liposomeencapsulated prednisolone in experimental rheumatoid arthritis, J. Control. Release 209 (2015) 20-26, http: / / dx.doi.org/10.1016/j.jconrel.2015.04.019.

[213] R. Duivenvoorden, J. Tang, D.P. Cormode, A.J. Mieszawska, D. Izquierdo-Garcia, C. Ozcan, M.J. Otten, N. Zaidi, M.E. Lobatto, S.M. van Rijs, B. Priem, E.L. Kuan, C. Martel, B. Hewing, H. Sager, M. Nahrendorf, G.J. Randolph, E.S.G. Stroes, V. Fuster, E.A. Fisher, Z.A. Fayad, W.J.M. Mulder, A statin-loaded reconstituted high- density lipoprotein nanoparticle inhibits atherosclerotic plaque inflammation., Nat. Commun. 5 (2014) 3065. doi:10.1038/ncomms4065.

[214] L.A. Maile, W.H. Busby, T.C. Nichols, D.A. Bellinger, E.P. Merricks, M. Rowland, U. Veluvolu, D.R. Clemmons, A monoclonal antibody against $\alpha \mathrm{v} \beta 3$ integrin inhibits development of atherosclerotic lesions in diabetic pigs, Sci. Transl. Med. 2 (2010) 18ra11, http: / / dx.doi.org/10.1126/ scitranslmed.3000476. 
[215] S. Dixon, J. Bashutski, M. Rütz, H. Goldberg, S. Sims, A. Gramoun, S. Shorey, M. Manolson, J. Heersche, H. Zhang, J. Suzich, S. Mao, The $\alpha v \beta 3$ integrin as a therapeutic target for inhibition of bone resorption, Arthritis Res. Ther. 6 (2004) 23 (doi:ar1358).

[216] S. Danese, M. Sans, C. de la Motte, C. Graziani, G. West, M.H. Phillips, R. Pola, S. Rutella, J. Willis, A. Gasbarrini, C. Fiocchi, Angiogenesis as a novel component of inflammatory bowel disease pathogenesis, Gastroenterology 130 (2006) 2060-2073 (doi:S0016-5085(06)00739-6).

[217] E.K. Sloan, N. Pouliot, K.L. Stanley, J. Chia, J.M. Moseley, D.K. Hards, R.L. Anderson, Tumor-specific expression of $\alpha \mathrm{v} \beta 3$ integrin promotes spontaneous metastasis of breast cancer to bone, Breast Cancer Res. 8 (2006) R20 (doi:bcr1398).

[218] D.B. Kirpotin, D.C. Drummond, Y. Shao, M.R. Shalaby, K. Hong, U.B. Nielsen, J.D. Marks, C.C. Benz, J.W. Park, Antibody targeting of long-circulating lipidic nanoparticles does not increase tumor localization but does increase internalization in animal models, Cancer Res. 66 (2006) 6732-6740 (doi:66/13/6732).

[219] D.W. Bartlett, H. Su, I.J. Hildebrandt, W.A. Weber, M.E. Davis, Impact of tumor-specific targeting on the biodistribution and efficacy of siRNA nanoparticles measured by multimodality in vivo imaging, Proc. Natl. Acad. Sci. U. S. A. 104 (2007) 15549-15554 (doi:0707461104).

[220] J.D. Hood, M. Bednarski, R. Frausto, S. Guccione, R.A. Reisfeld, R. Xiang, D.A. Cheresh, Tumor regression by targeted gene delivery to the neovasculature, Science 296 (2002) 2404-2407, http: / / dx.doi.org/10.1126/ science.1070200.

[221] X. Montet, K. Montet-Abou, F. Reynolds, R. Weissleder, L. Josephson, Nanoparticle imaging of integrins on tumor cells, Neoplasia 8 (2006) 214-222, http://dx.doi. org / $10.1593 /$ neo.05769.

[222] W.J. Mulder, K. Castermans, J.R. van Beijnum, M.G. Oude Egbrink, P.T. Chin, Z.A. Fayad, C.W. Lowik, E.L. Kaijzel, I. Que, G. Storm, G.J. Strijkers, A.W. Griffioen, K. Nicolay, Molecular imaging of tumor angiogenesis using $\alpha \mathrm{v} \beta 3$-integrin targeted multimodal quantum dots, Angiogenesis 12 (2009) 17-24, http://dx.doi.org/10.1007/s10456-0089124-2.

[223] C.R. Anderson, X. Hu, H. Zhang, J. Tlaxca, A.E. Decleves, R. Houghtaling, K. Sharma, M. Lawrence, K.W. Ferrara, J.J. Rychak, Ultrasound molecular imaging of tumor angiogenesis with an integrin targeted microbubble contrast agent, Investig. Radiol. 46 (2011) 215-224, http:/ / dx.doi.org/10.1097/ RLI.0b013e3182034fed.

[224] P.M. Winter, A.M. Morawski, S.D. Caruthers, R.W. Fuhrhop, H. Zhang, T.A. Williams, J.S. Allen, E.K. Lacy, J.D. Robertson, G.M. Lanza, S.A. Wickline, Molecular imaging of angiogenesis in early-stage atherosclerosis with alpha(v)beta3-integrin-targeted nanoparticles, Circulation 108 (2003) 2270-2274, http://dx.doi.org/10.1161/01. CIR.0000093185.16083.95.

[225] A.J. Beer, J. Pelisek, P. Heider, A. Saraste, C. Reeps, S. Metz, S. Seidl, H. Kessler, H.J. Wester, H.H. Eckstein, M. Schwaiger, PET/CT imaging of integrin alphavbeta3 expression in human carotid atherosclerosis, JACC Cardiovasc. Imaging 7 (2014) 178-187, http:/ / dx.doi.org/10.1016/j.jcmg.2013.12.003.

[226] A. Zheleznyak, T.J. Wadas, C.D. Sherman, J.M. Wilson, P.J. Kostenuik, K.N. Weilbaecher, C.J. Anderson, Integrin $\alpha v \beta 3$ as a PET imaging biomarker for osteoclast number in mouse models of negative and positive osteoclast regulation, Mol. Imaging Biol. 14 (2012) 500-508, http: / / dx.doi.org/10.1007/-011-0512-4.

[227] Z. Szekanecz, A.E. Koch, Macrophages and their products in rheumatoid arthritis, Curr. Opin. Rheumatol. 19 (2007) 289-295, http: / / dx.doi.org/10.1097 / BOR.0b013e32805e87ae.

[228] T. Quillard, K. Croce, F.A. Jaffer, R. Weissleder, P. Libby, Molecular imaging of macrophage protease activity in cardiovascular inflammation in vivo, Thromb. Haemost. 105 (2011) 
828-836, http: / / dx.doi.org/10.1160/ TH10-09-0589.

[229] K.Y. Park, G. Li, M.O. Platt, Monocyte-derived macrophage assisted breast cancer cell invasion as a personalized, predictive metric to score metastatic risk, Sci. Rep. 5 (2015) 13855, http: / / dx.doi.org/10.1038/ srep13855.

[230] R.W. Kinne, R. Brauer, B. Stuhlmuller, E. Palombo-Kinne, G.R. Burmester, Macrophages in rheumatoid arthritis, Arthritis Res. 2 (2000) 189-202, http:/ / dx.doi.org/10.1186/ar86.

[231] C. Verollet, G.M. Charriere, A. Labrousse, C. Cougoule, V. Le Cabec, I. MaridonneauParini, Extracellular proteolysis in macrophage migration: losing grip for a breakthrough, Eur. J. Immunol. 41 (2011) 2805-2813, http: / / dx.doi.org/10.1002/ eji.201141538.

[232] A.B. Caldwell, Z. Cheng, J.D. Vargas, H.A. Birnbaum, A. Hoffmann, Network dynamics determine the autocrine and paracrine signaling functions of TNF, Genes Dev. 28 (2014) 2120-2133, http: / / dx.doi.org/10.1101/gad.244749.114.

[233] B.J. Crielaard, T. Lammers, R.M. Schiffelers, G. Storm, Drug targeting systems for inflammatory disease: one for all, all for one, J. Control. Release 161 (2012) 225-234, http: / / dx.doi.org/10.1016/j.jconrel.2011.12.014.

[234] A.E. Coutinho, K.E. Chapman, The anti-inflammatory and immunosuppressive effects of glucocorticoids, recent developments and mechanistic insights, Mol. Cell. Endocrinol. 335 (2011) 2-13 (doi:S0303- 7207(10)00210-8).

[235] M.E. Lobatto, Z.A. Fayad, S. Silvera, E. Vucic, C. Calcagno, V. Mani, S.D. Dickson, K. Nicolay, M. Banciu, R.M. Schiffelers, J.M. Metselaar, L. van Bloois, H.-S.S. Wu, J.T. Fallon, J.H. Rudd, V. Fuster, E.A. Fisher, G. Storm, W.J.M. Mulder, Multimodal clinical imaging to longitudinally assess a nanomedical anti- inflammatory treatment in experimental atherosclerosis, Mol. Pharm. 7 (2010) 2020-2029, http: / / dx.doi.org/10.1021/mp100309y.

[236] A.K. Deshantri, S.A. Kooijmans, S.A. Kuijpers, M. Coimbra, A. Hoeppener, G. Storm, M.H. Fens, R.M. Schiffelers, Liposomal prednisolone inhibits tumor growth in a spontaneous mouse mammary carcinoma model, J. Control. Release 243 (2016) 243-249 (doi:S0168-3659(16)30658-7).

[237] L. Quan, Y. Zhang, B.J. Crielaard, A. Dusad, S.M. Lele, C.J. Rijcken, J.M. Metselaar, H. Kostkova, T. Etrych, K. Ulbrich, F. Kiessling, T.R. Mikuls, W.E. Hennink, G. Storm, T. Lammers, D. Wang, Nanomedicines for inflammatory arthritis: head-to-head comparison of glucocorticoid-containing polymers, micelles, and liposomes, ACS Nano 8 (2014) 458466, http:/ / dx.doi.org/10.1021/nn4048205.

[238] C.N. Serhan, N. Chiang, T.E. Van Dyke, Resolving inflammation: dual anti-inflammatory and pro- resolution lipid mediators, Nat. Rev. 8 (2008) 349-361,http://dx.doi. org / 10.1038/nri2294.

[239] G. Fredman, N. Kamaly, S. Spolitu, J. Milton, D. Ghorpade, R. Chiasson, G. Kuriakose, M. Perretti, O. Farokhzad, I. Tabas, Targeted nanoparticles containing the proresolving peptide Ac2-26 protect against advanced atherosclerosis in hypercholesterolemic mice, Sci. Transl. Med. 7 (2015) 275ra20, http: / / dx.doi.org/10.1126/ scitranslmed.aaa1065.

[240] S.J. Jenkins, D. Ruckerl, P.C. Cook, L.H. Jones, F.D. Finkelman, N. van Rooijen, A.S. MacDonald, J.E. Allen, Local macrophage proliferation, rather than recruitment from the blood, is a signature of Th2 inflammation, Science 332 (2011) 1284-1288, http:/ / dx.doi. org / 10.1126/science.1204351.

[241] D. Hashimoto, A. Chow, C. Noizat, P. Teo, M.B. Beasley, M. Leboeuf, C.D. Becker, P. See, J. Price, D. Lucas, M. Greter, A. Mortha, S.W. Boyer, E.C. Forsberg, M. Tanaka, N. van Rooijen, A. García-Sastre, E.R. Stanley, F. Ginhoux, P.S. Frenette, M. Merad, Tissue resident macrophages self-maintain locally throughout adult life with minimal contribution from circulating monocytes, Immunity 38 (2013) http:/ /dx.doi. org/10.1016/j.immuni.2013.04.004.

[242] F.K. Swirski, I. Hilgendorf, C.S. Robbins, From proliferation to proliferation: monocyte 
lineage comes full circle, Semin. Immunopathol. 36 (2014) 137-148, http://dx.doi. org/10.1007/ s00281-013-0409-1.

[243] S.U. Amano, J.L. Cohen, P. Vangala, M. Tencerova, S.M. Nicoloro, J.C. Yawe, Y. Shen, M.P. Czech, M. Aouadi, Local proliferation of macrophages contributes to obesityassociated adipose tissue inflammation, Cell Metab. 19 (2014) 162-171, http:/ / dx.doi. org/10.1016/j.cmet.2013.11.017.

[244] M.J. Campbell, N.Y. Tonlaar, E.R. Garwood, D. Huo, D.H. Moore, A.I. Khramtsov, A. Au, F. Baehner, Y. Chen, D.O. Malaka, A. Lin, O.O. Adeyanju, S. Li, C. Gong, M. McGrath, O.I. Olopade, L.J. Esserman, Proliferating macrophages associated with high grade, hormone receptor negative breast cancer and poor clinical outcome, Breast Cancer Res. Treat. 128 (2011) 703-711, http: / / dx.doi.org/10.1007/ s10549-010-1154-y.

[245] C.S. Robbins, I. Hilgendorf, G.F. Weber, I. Theurl, Y. Iwamoto, J.-L.L. Figueiredo, R. Gorbatov, G.K. Sukhova, L.M.S. Gerhardt, D. Smyth, C.C.J. Zavitz, E.A. Shikatani, M. Parsons, N. van Rooijen, H.Y. Lin, M. Husain, P. Libby, M. Nahrendorf, R. Weissleder, F.K. Swirski, Local proliferation dominates lesional macrophage accumulation in atherosclerosis, Nat. Med. 19 (2013) 1166-1172, http:/ / dx.doi.org/10.1038/nm.3258.

[246] A. Lindau, C. Härdtner, S.P. Hergeth, K.D. Blanz, B. Dufner, N. Hoppe, N. Anto-Michel, J. Kornemann, J. Zou, L.M.S. Gerhardt, T. Heidt, F. Willecke, S. Geis, P. Stachon, D. Wolf, P. Libby, F.K. Swirski, C.S. Robbins, W. McPheat, S. Hawley, M. Braddock, R. Gilsbach, L. Hein, C. von zur Mühlen, C. Bode, A. Zirlik, I. Hilgendorf, C. Hardtner, S.P. Hergeth, K.D. Blanz, B. Dufner, N. Hoppe, N. Anto-Michel, J. Kornemann, J. Zou, L.M.S. Gerhardt, T. Heidt, F. Willecke, S. Geis, P. Stachon, D. Wolf, P. Libby, F.K. Swirski, C.S. Robbins, W. McPheat, S. Hawley, M. Braddock, R. Gilsbach, L. Hein, C. von zur Muhlen, C. Bode, A. Zirlik, I. Hilgendorf, Atheroprotection through SYK inhibition fails in established disease when local macrophage proliferation dominates lesion progression, Basic Res. Cardiol. 111 (2016) http: / / dx.doi.org/10.1007/ s00395-016-0535-8 (20- 016-0535-8. Epub 2016 Feb 18).

[247] S.M. Zeisberger, B. Odermatt, C. Marty, A.H. Zehnder-Fjallman, K. Ballmer-Hofer, R.A. Schwendener, Clodronate-liposome-mediated depletion of tumour-associated macrophages: a new and highly effective antiangiogenic therapy approach, Br. J. Cancer 95 (2006) 272-281 (doi:6603240).

[248] J.M. Fritz, M.A. Tennis, D.J. Orlicky, H. Yin, C. Ju, E.F. Redente, K.S. Choo, T.A. Staab, R.J. Bouchard, D.T. Merrick, A.M. Malkinson, L.D. Dwyer-Nield, Depletion of tumor-associated macrophages slows the growth of chemically induced mouse lung adenocarcinomas, Front. Immunol. 5 (2014) http://dx.doi.org/10.3389/ fimmu.2014.00587.

[249] P.J. Richards, A.S. Williams, R.M. Goodfellow, B.D. Williams, Liposomal clodronate eliminates synovial macrophages, reduces inflammation and ameliorates joint destruction in antigen-induced arthritis, Rheumatology (Oxford) 38 (1999) 818-825.

[250] P. Barrera, A. Blom, P.L. van Lent, L. van Bloois, J.H. Beijnen, N. van Rooijen, M.C. de Waal Malefijt, L.B. van de Putte, G. Storm, W.B. van den Berg, Synovial macrophage depletion with clodronate-containing liposomes in rheumatoid arthritis, Arthritis Rheum. 43 (2000) 1951-1959, http://dx.doi.org/10.1002/1529- 0131(200009)43:9<1951::AIDANR5>3.0.CO;2-K.

[251] J. Tang, M.E. Lobatto, L. Hassing, S. van der Staay, S.M. van Rijs, C. Calcagno, M.S. Braza, S. Baxter, F. Fay, B.L. Sanchez-Gaytan, R. Duivenvoorden, H.B. Sager, Y.M. Astudillo, W. Leong, S. Ramachandran, G. Storm, C. Perez-Medina, T. Reiner, D.P. Cormode, G.J. Strijkers, E.S.G. Stroes, F.K. Swirski, M. Nahrendorf, E.A. Fisher, Z.A. Fayad, W.J.M. Mulder, Inhibiting macrophage proliferation suppresses atherosclerotic plaque inflammation, Sci. Adv. 1 (2015) e1400223 (doi:e1400223). 
[252] T.P. Thomas, S.N. Goonewardena, I.J. Majoros, A. Kotlyar, Z. Cao, P.R. Leroueil, J.R. Baker Jr, Folate- targeted nanoparticles show efficacy in the treatment of inflammatory arthritis, Arthritis Rheum. 63 (2011) 2671- 2680, http:/ / dx.doi.org/10.1002/art.30459.

[253] J.F. Kukowska-Latallo, K.A. Candido, Z. Cao, S.S. Nigavekar, I.J. Majoros, T.P. Thomas, L.P. Balogh, M.K. Khan, J.R. Baker Jr, Nanoparticle targeting of anticancer drug improves therapeutic response in animal model of human epithelial cancer, Cancer Res. 65 (2005) 5317-5324 (doi:65/12/5317).

[254] A. Bellocq, S. Suberville, C. Philippe, F. Bertrand, J. Perez, B. Fouqueray, G. Cherqui, L. Baud, Low environmental $\mathrm{pH}$ is responsible for the induction of nitric-oxide synthase in macrophages. Evidence for involvement of nuclear factor-kappaB activation, J. Biol. Chem. 273 (1998) 5086-5092.

[255] T. Shirai, R.R. Nazarewicz, B.B. Wallis, R.E. Yanes, R. Watanabe, M. Hilhorst, L. Tian, D.G. Harrison, J.C. Giacomini, T.L. Assimes, J.J. Goronzy, C.M. Weyand, The glycolytic enzyme PKM2 bridges metabolic and inflammatory dysfunction in coronary artery disease, J. Exp. Med. 213 (2016) 337-354, http:/ / dx.doi.org/10.1084/jem.20150900.

[256] T.J. Harris, G. von Maltzahn, M.E. Lord, J.H. Park, A. Agrawal, D.H. Min, M.J. Sailor, S.N. Bhatia, Protease-triggered unveiling of bioactive nanoparticles, Small 4 (2008) 13071312, http:/ / dx.doi.org/10.1002/ smll.200701319.

[257] E. Kisin-Finfer, S. Ferber, R. Blau, R. Satchi-Fainaro, D. Shabat, Synthesis and evaluation of new NIR- fluorescent probes for cathepsin B: ICT versus FRET as a turn-ON modeof-action, Bioorg. Med. Chem. Lett. 24 (2014) 2453-2458, http://dx.doi.org/10.1016/j. bmcl.2014.04.022.

[258] T.H. Witney, A. Hoehne, R.E. Reeves, O. Ilovich, M. Namavari, B. Shen, F.T. Chin, J. Rao, S.S. Gambhir, A systematic comparison of 18F-C-SNAT to established radiotracer imaging agents for the detection of tumor response to treatment, Clin. Cancer Res. 21 (2015) 3896-3905, http: / / dx.doi.org/10.1158/1078-0432.CCR-14- 3176.

[259] S. Gandhi, H. Arami, K.M. Krishnan, Detection of cancer-specific proteases using magnetic relaxation of peptide-conjugated nanoparticles in biological environment, Nano Lett. 16 (2016) 3668-3674, http: / / dx.doi.org/10.1021/acs.nanolett.6b00867.

[260] K. Boeneman, B.C. Mei, A.M. Dennis, G. Bao, J.R. Deschamps, H. Mattoussi, I.L. Medintz, Sensing caspase 3 activity with quantum dot-fluorescent protein assemblies, J. Am. Chem. Soc. 131 (2009) 3828-3829, http: / / dx.doi.org/10.1021/ja809721j.

[261] J.H. Ryu, J.H. Na, H.K. Ko, D.G. You, S. Park, E. Jun, H.J. Yeom, D.H. Seo, J.H. Park, S.Y. Jeong, I.S. Kim, B.S. Kim, I.C. Kwon, K. Choi, K. Kim, Non-invasive optical imaging of cathepsin B with activatable fluorogenic nanoprobes in various metastatic models, Biomaterials 35 (2014) 2302-2311, http:/ / dx.doi.org/10.1016/j.biomaterials.2013.11.080.

[262] S. Ferber, H. Baabur-Cohen, R. Blau, Y. Epshtein, E. Kisin-Finfer, O. Redy, D. Shabat, R. Satchi-Fainaro, Polymeric nanotheranostics for real-time non-invasive optical imaging of breast cancer progression and drug release, Cancer Lett. 352 (2014) 81-89, http:/ / dx.doi.org/10.1016/j.canlet.2014.02.022.

[263] S. Lee, S.W. Kang, J.H. Ryu, J.H. Na, D.E. Lee, S.J. Han, C.M. Kang, Y.S. Choe, K.C. Lee, J.F. Leary, K. Choi, K.H. Lee, K. Kim, Tumor-homing glycol chitosan-based optical/ PET dual imaging nanoprobe for cancer diagnosis, Bioconjug. Chem. 25 (2014) 601-610, http: / / dx.doi.org/10.1021/bc500020g.

[264] E.S. Olson, T. Jiang, T.A. Aguilera, Q.T. Nguyen, L.G. Ellies, M. Scadeng, R.Y. Tsien, Activatable cell penetrating peptides linked to nanoparticles as dual probes for in vivo fluorescence and MR imaging of proteases, Proc. Natl. Acad. Sci. U. S. A. 107 (2010) 4311-4316, http:/ / dx.doi.org/10.1073/pnas.0910283107.

[265] P.J. Murray, T.A. Wynn, Protective and pathogenic functions of macrophage subsets, Nat. Rev. 11 (2011) 723-737, http: / / dx.doi.org/10.1038/ nri3073. 
[266] M. Rashidian, E.J. Keliher, A.M. Bilate, J.N. Duarte, G.R. Wojtkiewicz, J.T. Jacobsen, J. Cragnolini, L.K. Swee, G.D. Victora, R. Weissleder, H.L. Ploegh, Noninvasive imaging of immune responses, Proc. Natl. Acad. Sci. U. S. A. 112 (2015) 6146-6151, http:/ / dx.doi. org/10.1073/pnas.1502609112.

[267] S. Wilhelm, A.J. Tavares, Q. Dai, S. Ohta, J. Audet, H.F. Dvorak, W.C.W. Chan, Analysis of nanoparticle delivery to tumours, Nat. Rev. Mater. 1 (2016) 16014, http://dx.doi. org / 10.1038/natrevmats.2016.14.

[268] S.E. McNeil, S. Verwoert, G.J. Charrois, T.M. Allen, Evaluation of nanomedicines: stick to the basics, Nat. Rev. Mater. 1 (2016) 16073, http://dx.doi.org/10.1038/ natrevmats.2016.73.

[269] T. Lammers, F. Kiessling, M. Ashford, W. Hennink, D. Crommelin, G. Strom, Cancer nanomedicine: is targeting our target?, Nat. Rev. Mater. 1 (2016) 16069, http:/ / dx.doi. org/10.1038/natrevmats.2016.69.

[270] W.R. Sanhai, J.H. Sakamoto, R. Canady, M. Ferrari, Seven challenges for nanomedicine, Nat. Nanotechnol. 3 (2008) 242-244, http: / / dx.doi.org/10.1038/nnano.2008.114.

[271] R. van der Meel, T. Lammers, W.E. Hennink, Cancer nanomedicines: oversold or underappreciated?, Expert Opin. Drug Deliv. 14 (2017) 1-5, http:/ / dx.doi.org/10.1080 /17425247.2017.1262346.

[272] A. Akinc, A. Zumbuehl, M. Goldberg, E.S. Leshchiner, V. Busini, N. Hossain, S.A. Bacallado, D.N. Nguyen, J. Fuller, R. Alvarez, A. Borodovsky, T. Borland, R. Constien, A. de Fougerolles, J.R. Dorkin, K. Narayanannair Jayaprakash, M. Jayaraman, M. John, V. Koteliansky, M. Manoharan, L. Nechev, J. Qin, T. Racie, D. Raitcheva, K.G. Rajeev, D.W. Sah, J. Soutschek, I. Toudjarska, H.P. Vornlocher, T.S. Zimmermann, R. Langer, D.G. Anderson, A combinatorial library of lipid-like materials for delivery of RNAi therapeutics, Nat. Biotechnol. 26 (2008) 561-569, http: / / dx.doi.org/10.1038/nbt1402.

[273] Y. Zhao, F. Fay, S. Hak, J. Manuel Perez-Aguilar, B.L. Sanchez-Gaytan, B. Goode, R. Duivenvoorden, C. de Lange Davies, A. Bjorkoy, H. Weinstein, Z.A. Fayad, C. PerezMedina, W.J.M. Mulder, A. Bjørkøy, H. Weinstein, Z.A. Fayad, C. Pérez-Medina, W.J.M. Mulder, Augmenting drug-carrier compatibility improves tumour nanotherapy efficacy, Nat. Commun. 7 (2016) 11221, http: / / dx.doi.org/10.1038/ncomms11221.

[274] J. Tang, S. Baxter, A. Menon, A. Alaarg, B.L. Sanchez-Gaytan, F. Fay, Y. Zhao, M. Ouimet, M.S. Braza, V.A. Longo, D. Abdel-Atti, R. Duivenvoorden, C. Calcagno, G. Storm, S. Tsimikas, K.J. Moore, F.K. Swirski, M. Nahrendorf, E.A. Fisher, C. Pérez-Medina, Z.A. Fayad, T. Reiner, W.J.M. Mulder, Immune cell screening of a nanoparticle library improves atherosclerosis therapy, Proc. Natl. Acad. Sci. U. S. A. 113 (2016) http:/ / dx.doi. org/10.1073/pnas.1609629113.

[275] A. Sica, A. Mantovani, Macrophage plasticity and polarization: in vivo veritas, J. Clin. Invest. 122 (2012) 787-795, http: / / dx.doi.org/10.1172/JCI59643.

[276] F.O. Martinez, S. Gordon, The M1 and M2 paradigm of macrophage activation: time for reassessment, F1000Prime Rep. 6 (2014) http: / / dx.doi.org/10.12703/ P6-13.

[277] M. Nahrendorf, F.K. Swirski, Abandoning M1/M2 for a network model of macrophage function, Circ. Res. 119 (2016) 414-417, http://dx.doi.org/10.1161/ CIRCRESAHA.116.309194.

[278] C. Pérez-Medina, D. Abdel-Atti, J. Tang, Y. Zhao, Z.A. Fayad, J.S. Lewis, W.J.M. Mulder, T. Reiner, C. Perez-Medina, D. Abdel-Atti, J. Tang, Y. Zhao, Z.A. Fayad, J.S. Lewis, W.J.M. Mulder, T. Reiner, Nanoreporter PET predicts the efficacy of anti-cancer nanotherapy, Nat. Commun. 7 (2016) 11838, http: / / dx.doi.org/10.1038/ncomms11838. 




\section{CHAPTER 3}

\section{Docosahexaenoic Acid Liposomes for Targeting Chronic Inflammatory Diseases and Cancer: an In Vitro Assessment*}

\section{ABSTRACT}

I

nflammation, oxidative stress, and uncontrolled cell proliferation are common key features of chronic inflammatory diseases, such as atherosclerosis and cancer. $1 \omega 3$ polyunsaturated fatty acids (PUFAs; also known as omega3 fatty acids or fish oil) have beneficial effects against inflammation upon dietary consumption. However, these effects cannot be fully exploited unless diets are enriched with high concentrations of fish oil supplements over long periods of time. Here, a nanomedicine-based approach is presented for delivering effective levels of PUFAs to inflammatory cells. Nanoparticles are internalized by immune cells and hence can adequately deliver bioactive lipids into these target cells. The $\omega 3$ FA docosahexaenoic acid was formulated into liposomes ( $\omega$-liposomes) and evaluated for antiinflammatory effects in different types of immune cells. $\omega$-Liposomes strongly inhibited the release of reactive oxygen species and reactive nitrogen species from human neutrophils and murine macrophages, and also inhibited the production of the proinflammatory cytokines TNF $\alpha$ and MCP1. Moreover, $\omega$-liposomes inhibited tumor cell proliferation when evaluated in FaDu head and neck squamous carcinoma and $4 \mathrm{~T} 1$ breast cancer cells in in vitro cultures. We propose that $\omega$-liposomes are a promising nanonutraceutical formulation for intravenous delivery of fish oil FAs, which may be beneficial in the treatment of inflammatory disorders and cancer. 


\section{INTRODUCTION}

Dietary $\omega 3$ polyunsaturated fatty acids (PUFAs), including docosahexaenoic acid (DHA), have been reported to be beneficial in the treatment of cardiovascular diseases and cancer [1-4]. Such diseases share common pathophysiological features, including unbalanced oxidative stress, an inflammatory microenvironment, and aberrant cell proliferation, which are potential therapeutic targets for PUFAs [59]. Several clinical studies have been conducted to exploit the beneficial effects of PUFAs. However, such effects on the aforementioned targets are typically observed after longterm oral consumption of PUFAs in high amounts $[10,11]$. The oral dosage of DHA intake varies, ranging from 0.4 to $4 \mathrm{~g} /$ day for at least 4 months (Clinical Trials Identifier: NCT01078909 and NCT01865448). Bouwens et al. used a combination of DHA and eicosapentaenoic acid (EPA; $1.8 \mathrm{~g} /$ day for 26 weeks) to demonstrate antiinflammatory effects in humans [12].

To benefit fully from the effects of PUFAs, we propose a novel approach to enrich their content specifically in target cells that are involved in inflammatory disorders. Instead of oral intake of high doses of fish oil, we propose to specifically increase the levels of PUFAs in inflamed lesions through the use of nanomedicine. Nanoparticles can accumulate in inflamed tissues and are efficiently taken up, mainly by macrophages and to a lesser extent other immune cells [13]. The accumulation of nanoparticles in pathological tissues was first described for tumors and occurs via their enhanced extravasation through leaky blood-vessel walls [14]. This phenomenon has been named the enhanced permeability and retention (EPR) effect and has also been described for chronic inflammatory disorders, such as rheumatoid arthritis and atherosclerosis $[15,16]$. An example of a clinically available nanomedicine that exploits the EPR effect is the liposomal formulation of doxorubicin, branded Doxil, indicated for breast cancer, ovarian cancer, multiple myeloma, and AIDS-related Kaposi's sarcoma $[17,18]$.

We propose a novel liposomal formulation of docosahexaenoic acid-loaded liposomes ( $\omega$-liposomes), by incorporating DHA into polyethylene glycol (PEG) ylated liposomes. Such long-circulating PEGylated liposomes can accumulate in inflamed tissues and deliver their PUFA cargo into macrophages and other immune cells. In the present study, we report the physicochemical characteristics of $\omega$-liposomes and their anti-inflammatory effects in different types of immune cells (Figure 1). In addition, to demonstrate their potential benefit in the treatment of cancer, their growth-arresting effects in human and murine cancer cell lines were also evaluated. 


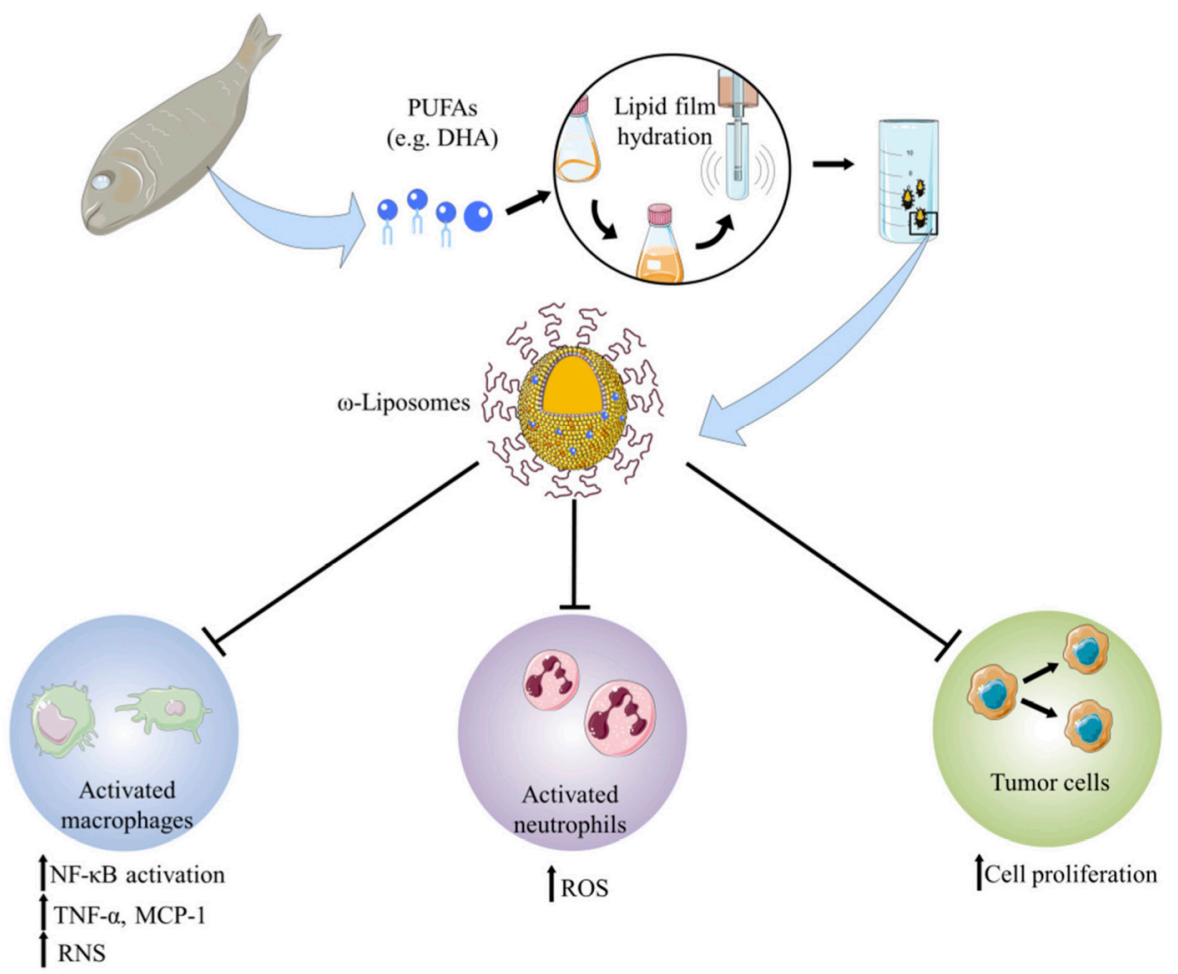

Figure 1। Preparation of $\omega$-liposomes and their cellular targets in disease. Fish oil and oily fish are rich in $\omega 3$ polyunsaturated fatty acids (PUFAs) such as docosahexaenoic acid (DHA). We formulated DHA into $\omega$-liposomes by lipid-film hydration followed by downsizing using extrusion. PEGylated long-circulating liposomes have been shown to accumulate in inflammatory lesions and tumors via the enhanced retention and permeability effect, and thus facilitate local delivery of the PUFAs in $\omega$-liposomes. Multiple pathophysiological processes can be tackled with $\omega$-liposomes. Tissue-associated immune cells, such as plaque macrophages or tumor-associated macrophages, accelerate the progression and invasiveness of the disease by releasing proinflammatory cytokines, such as TNF $\alpha$ and MCP1, and reactive nitrogen species (RNS), which are usually mediated by increased activity of NFKB. Neutrophils can also have significant effects on destabilizing atherosclerotic plaques or on tumor progression via the release of reactive oxygen species (ROS) and via intercommunication with macrophages. A third target for $\omega$ liposomes is the abnormal cellular proliferation of tumor cells. PEG, polyethylene glycol; $\omega$-liposomes, docosahexaenoic acid-loaded liposomes. 


\section{MATERIALS AND METHODS}

\subsection{Chemicals}

1,2-Dipalmitoyl-sn-glycero-3-phosphocholine (DPPC) and N-(carbonylmethoxy-PEG 2,000 )-1,2-distearoyl-sn-glycero-3-phosphoethanolamine (DSPE$\mathrm{PEG}_{2,000}$ ) were purchased from Lipoid AG (Steinhausen, Switzerland). L- $\alpha$ Phosphatidylethanolamine-N-(lissamine rhodamine B sulfonyl) (rhodamine PE) was obtained from Avanti Polar Lipids (Alabaster, AL, USA). All other materials were purchased from Sigma-Aldrich Co. (St Louis, MO, USA) unless otherwise stated.

\subsection{Liposomal Preparation and DHA Loading into Liposomes}

DHA-loaded liposomes ( $\omega$-liposomes) and control liposomes (C-liposomes) were prepared with DPPC, cholesterol, DSPE-PEG2,000, and DHA ( $\omega$-liposomes only) in molar ratios indicated in Table 1 . Rhodamine PE was added at $0.2 \mathrm{~mol} \%$ for fluorescent labeling when applicable. Typical batch sizes were prepared with 20 $\mathrm{mM}$ total lipid (TL). Liposomes were prepared using lipid film/hydration. Lipids, including DHA, were dissolved in chloroform-methanol $(1: 1 \mathrm{v} / \mathrm{v}$, total $4 \mathrm{~mL})$ in a round-bottom flask, and a lipid film was prepared by rotary evaporation (Büchi Labortechnik AG, Flawil, Switzerland), followed by an additional drying step under a stream of nitrogen for 1 hour. Subsequently, the lipid film was hydrated with 5 $\mathrm{mL}$ 4-(2-hydroxyethyl)-1-piperazineethanesulfonic acid (HEPES)-buffered saline (HBS) solution ( $\mathrm{pH}$ 7.4) to form a lipid dispersion. To downsize the lipid dispersion and to form uniform-size liposomes, the lipid particles were reduced by multiple sequential extrusion steps using a Lipex extruder (Northern Lipids, Burnaby, BC, Canada) through polycarbonate membranes (Nuclepore, Pleasanton, CA, USA) with final filters of pore size $100 \mathrm{~nm}$. The resulting liposomes were purified by either sequential centrifugation steps (as per Eckert et al.) [19] or by ultrafiltration. Briefly, liposomes were centrifuged at $8,000 \times \mathrm{g}$ for 20 minutes to remove the debris. The supernatant was removed and centrifuged again at $15,000 \times \mathrm{g}$ for 20 minutes or subjected to ultrafiltration using filtration units with a $100 \mathrm{kDa}$ molecular weight cutoff (Sartorius Stedim Biotech SA, Aubagne, France). Liposomes were then filtered through a $0.22 \mu \mathrm{m}$ nylon filter (CellTreat Scientific Products, Pepperell, MA, USA) 


\subsection{Characterization of Liposomes}

The mean particle-size distribution and polydispersity index (PDI) of the liposomes were determined by dynamic light scattering (DLS) using a Malvern CGS3 multiangle goniometer (Malvern Instruments, Malvern, UK) with a JDS Uniphase $22 \mathrm{~mW}$ He-Ne laser operating at $632 \mathrm{~nm}$, an optical fiber-based detector and a digital LV/LSE-5003 correlator. All measurements were performed at a $90^{\circ}$ angle. The $\zeta$-potential of the liposomes was determined by laser Doppler electrophoresis using a Zetasizer Nano-Z (Malvern Instruments). Liposomes were diluted in $10 \mathrm{mM}$ HEPES buffer ( $\mathrm{pH}$ 7.4) prior to measurements. The phospholipid content of liposomes was determined with a phosphate assay, in accordance with Rouser et al. [20]. The DHA content of liposomes was determined after their disruption in acetonitrile by highperformance liquid chromatography on a Shimadzu system equipped with a C18 column, two LC-10AT pumps, and an SPD-M10AVP photodiode array detector at a wavelength of $237 \mathrm{~nm}$. The absence of free DHA and the homogeneity of particle size were confirmed by separation of free DHA from liposomes by size-exclusion chromatography on a Superdex 10/300 column (GE Healthcare UK Ltd, Little Chalfont, UK) using phosphate-buffered saline as eluent at a flow rate of $1 \mathrm{~mL} / \mathrm{min}$. Liposome colloidal stability under storage conditions was studied by monitoring their mean size and size distribution with DLS every 4-5 days for 30 days upon storage in $\mathrm{HBS}$ at $4^{\circ} \mathrm{C}$.

\subsection{Experiments with Immune Cells}

Murine RAW264.7 macrophages and human THP1 monocytes were obtained from the American Type Culture Collection (ATCC). RAW264.7 macrophages were cultured in Dulbecco's Modified Eagle's Medium (DMEM) high glucose, while THP1 cells were cultured in Roswell Park Memorial Institute 1640 medium. Both media were supplemented with $10 \%$ (v/v) fetal bovine serum, penicillin (100 IU/ $\mathrm{mL})$, streptomycin $(100 \mu \mathrm{g} / \mathrm{mL})$, and amphotericin B $(0.25 \mu \mathrm{g} / \mathrm{mL})$ and incubated at $37{ }^{\circ} \mathrm{C}$ under $5 \% \mathrm{CO}_{2}$ atmosphere. THP1 monocytes were differentiated into macrophages by stimulation with $50 \mathrm{ng} / \mathrm{mL}$ of phorbol-12-myristate-13-acetate (PMA) for 24 hours. Afterward, PMA was washed and cells incubated in fresh medium for another 24 hours. The RAW264.7 NFkB Luc-reporter cell line, stably transfected with $3 x-N F \kappa B$ Luc plasmid, was kindly provided by Professor MPJ de Winther (Academic Medical Centre, Amsterdam), and cultured as RAW264.7 cells. Human polymorphonuclear neutrophils (PMNs) were freshly isolated from a human buffy coat (Sanquin Blood Supply, Amsterdam) and suspended in Hank's balanced salt solution (HBSS) supplemented with 1\% gelatin. HEK-Blue hTLR4 cells were purchased from InvivoGen (San Diego, CA, USA), and were subcultured in DMEM high glucose supplemented with $10 \%(\mathrm{v} / \mathrm{v})$ fetal bovine serum, $2-4 \mathrm{mM}$ L-glutamine, penicillin (50 IU / mL), streptomycin $(50 \mu \mathrm{g} / \mathrm{mL})$, and amphotericin B $(0.25 \mu \mathrm{g} / \mathrm{mL})$, and incubated at $37^{\circ} \mathrm{C}$ under a $5 \% \mathrm{CO}_{2}$ atmosphere. 


\subsubsection{Liposome-Uptake Study}

RAW264.7 (40,000 cells) was seeded into each well of a $\mu$-Slide VI 0.4 IbiTreat Microscopy Chamber (Ibidi GmbH, Planegg, Germany) and incubated overnight. Cells were treated with $0.125 \mathrm{mM}$ TL of rhodamine-labeled $\omega$-liposomes or rhodamine-labeled C-liposomes for 4 hours at $37{ }^{\circ} \mathrm{C}$. Afterward, cell nuclei were stained with Hoechst for 30 minutes, followed by thorough washing steps. Cells were visualized on a BZ-9000 fluorescence microscope (Keyence, Osaka, Japan) or on a confocal SPE-II microscope (Leica Microsystems, Wetzlar, Germany).

\subsubsection{Inhibition of Production of Reactive Nitrogen Species (NO Assay)}

To evaluate the effect of liposomal formulations on the release of reactive nitrogen species, RAW264.7 cells were seeded at 100,000 cells per well in a 96-well plate. After 24 hours of incubation, cell culture medium was removed from all wells and replaced with fresh medium spiked with the respective treatments - $\omega$-liposomes or C-liposomes - at the indicated concentrations. After 2 hours, lipopolysaccharide (LPS) was added at a final concentration of $100 \mathrm{ng} / \mathrm{mL}$, followed by incubation at 37 ${ }^{\circ} \mathrm{C}$ for another 22 hours. In another setup, we evaluated the effect of $\omega$-liposomes or C-liposomes on RAW264.7 cells prestimulated with LPS. Briefly, we first stimulated RAW264.7 cells with LPS (100 ng/mL) for 2 or 4 hours, after which cells were washed with medium or not, and subsequently treated with the liposomes for another 22 hours. In either the pretreatment or the prestimulation setup, the supernatant was collected for a nitric oxide assay with Griess reagents. Absorbance was measured at $550 \mathrm{~nm}$ on a SpectroStar Nano (BMG LabTech, Ortenberg, Germany). The effect of liposome formulations on cell viability was assessed under similar conditions of the assay (ie, LPS stimulation, seeding density, and exposure time) to assure that the noticed antioxidant effects were not due to significant cytotoxic effects of the formulations. A CellTiter 96 $\AA$ Aqueous One solution viability assay (Promega Corporation, Fitchburg, WI, USA) was performed as per the supplier's protocol, and the absorbance was measured using EZ Read 400 (Biochrom, Cambridge, UK).

RAW264.7 NFkB Luc cells were seeded at 80,000 per well in a 96-well plate. After 24 hours of incubation, the medium was removed from all wells and replaced with either fresh medium or the respective $\omega$-liposomes or C-liposomes formulations at the indicated concentrations. This medium was removed and cells washed with fresh medium twice after 4 hours of treatment. Cells were stimulated with LPS at a final concentration of $100 \mathrm{ng} / \mathrm{mL}$ for 2 hours. Luciferase activity was determined using a One-Glo luciferase assay system (Promega Corporation) according to the supplier's instructions. Cell viability was assessed under the same exposure time of LPS treatment and LPS stimulation as described earlier. 


\subsubsection{Inhibition of Production of Proinflammatory Cytokines TNF $\alpha$ and MCP1}

THP1 cells, a commonly used model for mimicking macrophages in the vasculature [21] were seeded at 250,000 cells per well in a 24-well plate and differentiated into macrophages as described earlier. The medium was replaced by a medium containing liposomal formulations for 2 hours, then stimulated with LPS for an additional 22 hours. The supernatant was collected and stored at $-80{ }^{\circ} \mathrm{C}$ until further analysis. TNF $\alpha$ and MCP1 were measured by enzyme-linked immunosorbent assay (ELISA) according to the manufacturer's instructions (ELISA Max ${ }^{\mathrm{TM}}$ deluxe set human TNF $\alpha$ and human MCP1/CCL2 kits; BioLegend, San Diego, CA, US). Cell viability was determined as described earlier.

\subsubsection{Inhibition of Production of Reactive Oxygen Species (ROS Assay)}

PMNs, freshly obtained from human buffy coat, were diluted to a final concentration of 250,000 cells / $\mathrm{mL}$ and treated with $\omega$-liposomes and C-liposomes formulations at indicated concentrations in a white 96-well plate for 30 minutes. Subsequently, luminol, as luminescence enhancer, and zymosan, as reactive oxygen species (ROS) inducer, were pipetted into each well. The chemiluminescence resulting from ROS production was measured immediately using a Titertek Luminoskan (TechGen International, Zellik, Belgium).

\subsubsection{Inhibition of Toll-Like Receptor 4 (TLR4) Activation}

HEK-Blue hTLR4 cells were seeded at 25,000 cells per well in a 96-well plate and treated with liposomes for 2 hours, followed by 16 hours of LPS stimulation. A Quanti-Blue assay (InvivoGen) was performed according to the manufacturer's instructions to determine the level of secreted alkaline phosphatase as a reporter for TLR4 activation. Cell viability was assessed as mentioned earlier.

\subsection{Experiments with Cancer Cell Lines}

Murine 4T1 breast cancer cells and human FaDu squamous cell carcinoma cells were obtained from the ATCC. Cells were cultured in DMEM supplemented with $10 \%(\mathrm{v} / \mathrm{v})$ fetal bovine serum, penicillin $(100 \mathrm{IU} / \mathrm{mL})$, streptomycin $(100 \mu \mathrm{g} / \mathrm{mL})$, and amphotericin B $(0.25 \mu \mathrm{g} / \mathrm{mL})$. Normal human umbilical vein endothelial cells (HUVECs) were obtained from Lonza (Basel, Switzerland) and cultured in EBM basal medium supplemented with EGM-2 SingleQuot kit supplements and growth factors (Lonza). 
FaDu squamous cell carcinoma cells or 4T1 metastatic breast cancer cells were seeded at a density of 3,000 cells per well in a 96-well plate. HUVECs, which served as control (normal cells), were seeded at a density of 4,000 cells per well in a 96-well plate. After 24 hours, cells were treated with $\omega$-liposomes and C-liposomes at the indicated concentrations for 24 hours. To determine the number of dividing cells, bromodeoxyuridine (BrdU) reagent was added to the cells for 4-6 hours and an ELISA BrdU colorimetric immunoassay (Hoffman-La Roche Ltd, Basel, Switzerland) was performed, according to the manufacturer's protocol.

\subsection{Statistical Analysis}

Data are expressed as mean \pm standard error of mean. Statistical analyses by Student's t-test were performed with GraphPad Prism; values of $\mathrm{P}<0.05$ were considered statistically significant.

\section{RESULTS AND DISCUSSION}

\subsection{Preparation and Characterization of $\omega$-Liposomes}

The physicochemical characteristics of PUFA-loaded PEGylated liposomes ( $\omega$-liposomes) and control PEGylated liposomes (C-liposomes) are summarized in Table 1. Hydration of the lipid film with HBS resulted in a heterogeneous, milky dispersion for both $\omega$-liposomes and C-liposomes. The cumulative size distribution at 90\% (D90) of this lipid dispersion before extrusion was $2 \mu \mathrm{m}$ and $3 \mu \mathrm{m}$ for C-liposomes and $\omega$-liposomes, respectively (Figure S1A). After extrusion, both liposomal formulations were translucent and displayed comparable mean sizes (around $100 \mathrm{~nm}$ ), D90 (around $120 \mathrm{~nm}$ ), and narrow size distributions (polydispersity $<0.1$ ) (Table 1 and Figure S1B). The narrow size distribution is also demonstrated in the size distribution histograms obtained from DLS (Figure S2). The slightly negativeclose to neutral $\zeta$-potentials indicated that the negative charge of the DHA lipids was shielded by the PEG corona on the surface of liposomes (Table 1). TL recovery after extrusion was $69 \% \pm 8 \%$ and $70 \% \pm 10 \%$ for $\omega$-liposomes and C-liposomes, respectively, as determined by Rouser phosphate assay. The entrapment efficiency and DHA-loading content in $\omega$-liposomes were $81 \% \pm 3 \%$ and $15 \% \pm 1 \%$, respectively (Table 1). Separation of liposomes and free DHA by size-exclusion chromatography showed that DHA was effectively incorporated into the liposomes (Figure 2A). Changes in mean size and size distribution of both liposomal formulations were minimal under liposome-storage conditions at $4{ }^{\circ} \mathrm{C}$ over a period of 5 weeks (Figure $2 \mathrm{~B}$ and $\mathrm{C}$ ). Liposome-size distribution was relatively narrow and remained $<0.1$ on the PDI. In addition, there were no significant changes in particle size or PDI values between C-liposomes and $\omega$-liposomes in a stability study at room temperature and 
$37^{\circ} \mathrm{C}$ at physiological $\mathrm{pH}$ (ie, $\mathrm{pH} 7.4$ ) (Figure S3). Furthermore, differential scanning calorimetry showed no differences in the thermograms of the formulations, likely due to the stabilizing effect of cholesterol (Figure S4).

\subsection{Cell-Uptake/Interaction Studies of Liposomes}

Lipid-based nanocarriers can be efficiently internalized by phagocytic cells, and hence are an attractive system to deliver PUFAs to immune cells. Macrophages are known to be the most efficient in uptake of nanoparticles among the different cell types and may act as a reservoir for nanotherapeutics [13,22]. Figure 3 shows that RAW264.7 macrophages internalized both $\omega$-liposomes and C-liposomes to a similar extent. Fluorescence microscopy was used to visualize the association and the uptake of rhodamine-labeled liposomes. We further utilized the power of confocal microscopy to visualize a focal intracellular plane, minimizing any extracellular signal. The accumulation of labeled liposomes in perinuclear vesicles suggests an accumulation of either formulation in the endosomal/lysosomal compartment (Figure 3A-D). 
Table 1 I Characteristics of liposomes

\begin{tabular}{llllllll}
\hline $\begin{array}{l}\text { Liposomal } \\
\text { formulation }\end{array}$ & $\begin{array}{l}\text { Molar ratio } \\
\text { (DHA:DPPC: } \\
\text { Chol: DSPE- } \begin{array}{l}\text { Mean } \\
(\mathrm{nm})\end{array}\end{array}$ & $\begin{array}{l}\text { Polydispersity } \\
\text { PEG }\end{array}$ index & $\begin{array}{l}\zeta \text {-Potential } \\
(\mathrm{mV})\end{array}$ & $\begin{array}{l}\text { Total } \\
\text { lipid } \\
\text { yield } \\
(\%)\end{array}$ & $\begin{array}{l}\text { DHA- } \\
\text { loading } \\
\text { content }(\%)\end{array}$ & $\begin{array}{l}\text { DHA- } \\
\text { entrapment } \\
\text { efficiency }(\%)\end{array}$ \\
\hline c-liposomes & $1: 0.85: 1: 0.15$ & $99 \pm 16$ & $0.06 \pm 0.01$ & $-15.7 \pm 2.5$ & $69 \pm 8$ & $14.6 \pm 1.2$ & $81.35 \pm 3.24$ \\
C-liposomes & $0: 1.85: 1: 0.15$ & $100 \pm 17$ & $0.08 \pm 0.03$ & $-15.0 \pm 0.6$ & $70 \pm 10 \begin{array}{l}\text { Not } \\
\text { applicable }\end{array}$ & $\begin{array}{l}\text { Not } \\
\text { applicable }\end{array}$ \\
\hline
\end{tabular}

Loading content $(\%)=$ Amount of DHA (mg) / Amount of total lipid $(\mathrm{mg}) \times 100 \%$ Entrapment efficiency $(\%)=($ Final drug $/$ lipid ratio $) /($ Initial drug $/$ lipid ratio $) \times 100 \%$ Total lipid yield $(\%)=$ Total lipid recovered $(\mu \mathrm{mol}) /$ Initial lipid used $(\mu \mathrm{mo}) \times 100 \%$

Abbreviations: DHA, docosahexaenoic acid; DPPC, 1,2-dipalmitoyl-sn-glycero-3-phosphocholine; Chol, cholesterol; DSPE, distearoyl phosphatidylethanolamine; PEG, polyethylene glycol; $\omega$-liposomes, docosahexaenoic acid-loaded liposomes. yield (\%) Initial lipid used

A

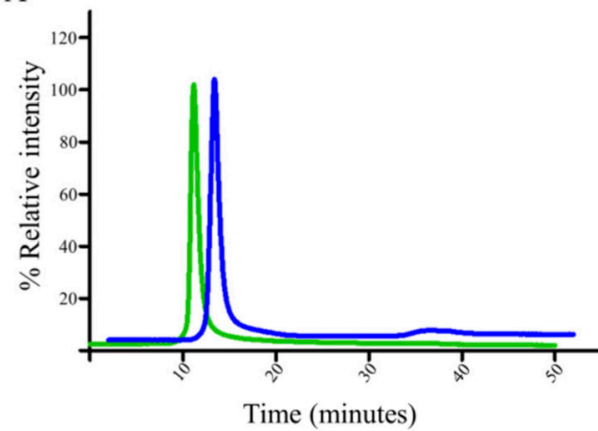

B

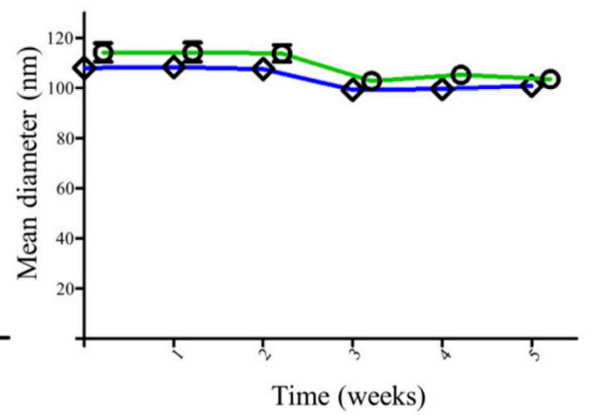

$\mathrm{C}$

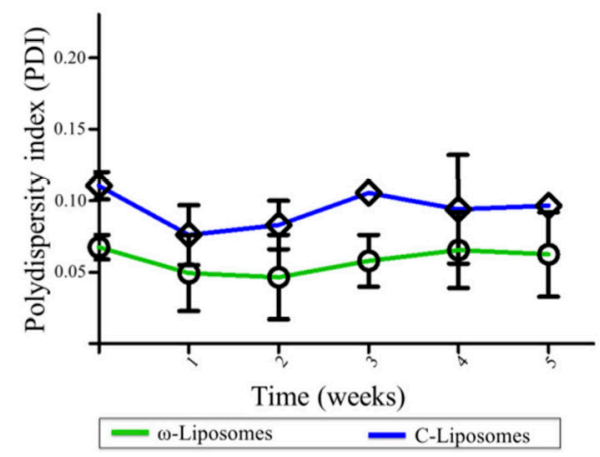

Figure 2। Purity and colloidal stability of docosahexaenoic acid (DHA)-loaded liposomes ( $\omega$-liposomes) and control liposomes (C-liposomes). (A)The absence of free DHA in $\omega$-liposomes was confirmed by size-exclusion chromatography. (B and C) Colloidal stability of $\omega$-liposomes and C-liposomes were investigated by dynamic light scattering measuring the changes in (B) mean size and (C) polydispersity index upon storage for over 30 days at $4^{\circ} \mathrm{C}$. 


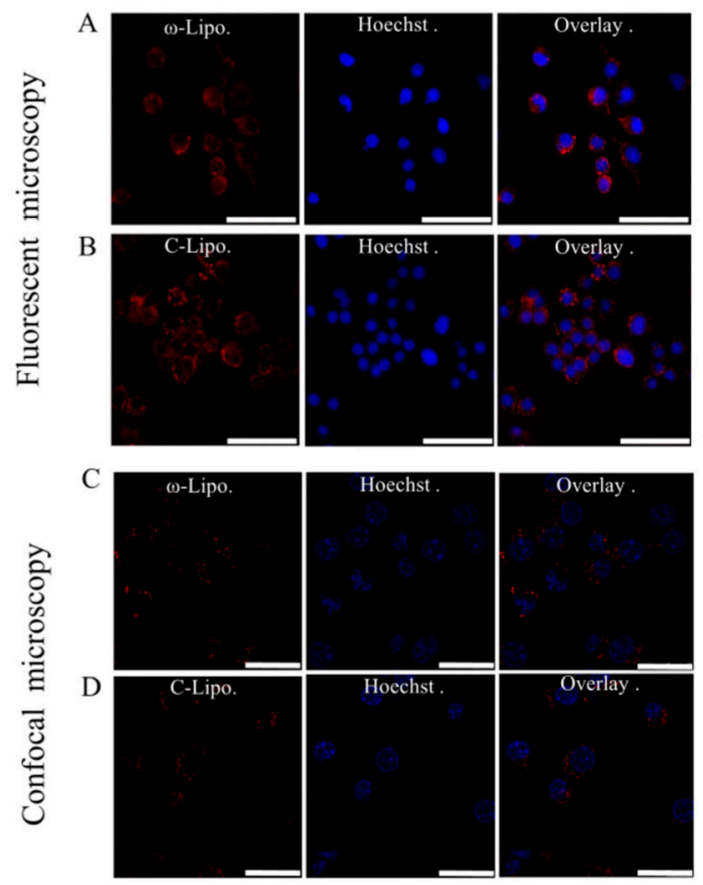

Figure 3 | Cell-interaction and uptake studies of rhodamine-labeled docosahexaenoic acid-loaded liposomes ( $\omega$ - Lipo) and control liposomes (C-Lipo) in RAW264.7 macrophages. RAW264.7 murine macrophages were incubated for 4 hours at $37^{\circ} \mathrm{C}$ with rhodamine-labeled liposomes $(0.125 \mathrm{mM}$ total lipid; red). Cells were visualized with fluorescent microscopy (A and B) or by confocal microscopy (C and D). Blue staining represents cell nuclei. Scale bars $=20 \mu \mathrm{m}$. 


\subsection{Effect of $\omega$-Liposomes in Activated Immune Cells}

Unbalanced reactive nitrogen species (RNS) and ROS are often generated by stimulated immune cells and play a crucial role in the development and progression of multiple inflammatory disorders [23-25]. The effect of $\omega$-liposomes on the production of nitric oxide $(\mathrm{NO})$ and $\mathrm{ROS}$ was evaluated in murine macrophages and human PMNs, respectively. NO is an important mediator in inflammation that reacts with superoxide to form peroxynitrite, a powerful oxidizing and tissue-damaging moiety. LPS induced a significant increase in NO production by RAW macrophages, and $\omega$-liposomes reduced LPS-induced NO production significantly, with up to $80 \%$ reduction at the highest tested concentration. Remarkably, C-liposomes also inhibited LPS-induced NO production by RAW cells dose-dependently, although less strongly than $\omega$-liposomes (50\% vs $80 \%$ at $0.5 \mathrm{mM}$ TL and $35 \%$ vs $60 \%$ at $0.25 \mathrm{mM} \mathrm{TL}$ ) (Figure 4A). In a different experimental setup in which cells had been prestimulated with LPS and then treated with liposomes, $\omega$-liposomes showed similar potent anti-inflammatory activity (Figure S5A-C). Since nuclear factor kappaB (NFkB) activation plays a crucial role in inducing NO production via inducible nitric oxide synthase, we sought to determine the effect of liposomes on RAW264.7 cells stably transfected with an NFkB-reporter gene, which expresses firefly luciferase upon activation. Both formulations showed inhibition of NFkB activation similarly to the observed inhibitory effects on NO production (Figure 4B). Of note, the liposomal formulations were not toxic to either of the cell lines at the tested concentration range and exposure time (Figure $4 \mathrm{C}$ and $\mathrm{D}$ ). 

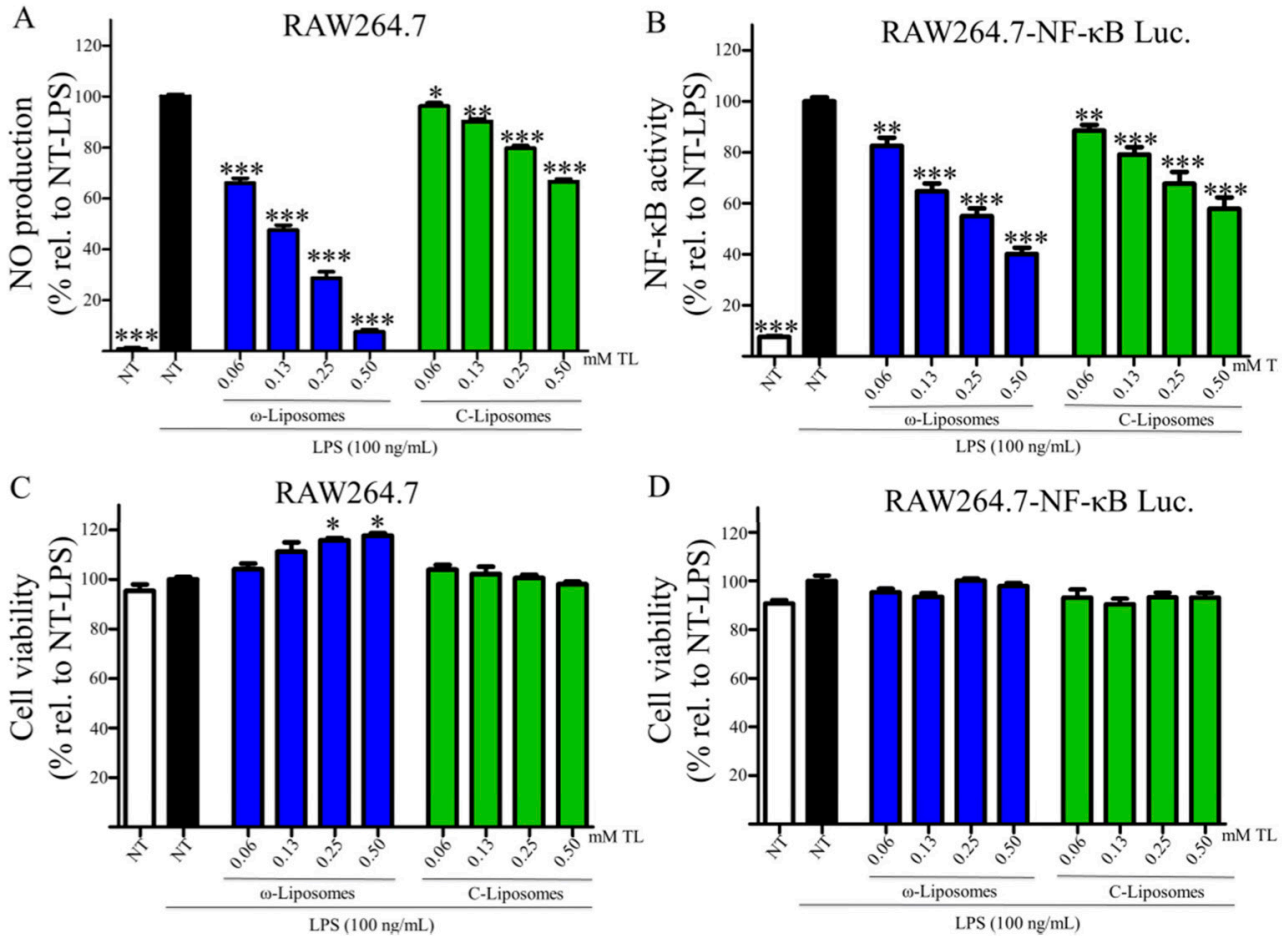

Figure 4 I Effect of docosahexaenoic acid-loaded liposomes ( $\omega$-liposomes) and control liposomes (C-liposomes) on lipopolysaccharide (LPS)-induced nitric oxide production and NFkB activation in murine macrophages. (A) RAW264.7 cells were treated with the liposomes and stimulated with LPS for 24 hours. NO production was measured in the supernatant with Griess reagent. (B) RAW264.7 NFkB Luc cells were treated with liposomes for 4 hours, washed twice with medium, then stimulated with LPS for another 2 hours. Luciferase activity was determined using the One-Glo luciferase assay. (C and D). Cell viability was assessed by MTS assay under the same experimental conditions. Data presented as mean \pm standard error of mean from three independent experiments (each $n \geq 4$ ). ${ }^{*} P<0.05,{ }^{* *} P<0.01,{ }^{* * *} P<0.001$ compared to control nontreated (NT) LPS-stimulated cells (Student's t-test). White bars indicate nontreated, nonstimulated control cells; black bars indicate stimulated control cells not treated with liposomal formulations. Luc, luciferase; MTS, (3-(4,5-dimethylthiazol-2-yl)-5-(3 carboxymethoxyphenyl)2-(4-sulfophenyl)-2H-tetrazolium); $\mathrm{NO}$, nitric oxide; TL, total lipid. 
The effects of $\omega$-liposomes on the production of two proinflammatory cytokines $\mathrm{MCP} 1$ and TNF $\alpha$ - were also studied, as they are key regulators in the recruitment of immune cells to inflammatory lesions and exacerbation of the inflammation [26,27]. THP1 macrophages were stimulated with LPS $(100 \mathrm{ng} / \mathrm{mL})$ and then treated with either $\omega$-liposomes or C-liposomes. $\omega$-Liposomes reduced the production of both cytokines, while C-liposomes had only minor effects (Figure 5A and B). The highest concentration of $\omega$-liposomes, $0.5 \mathrm{mM} \mathrm{TL}$, resulted in a minor reduction in THP1 cell viability of less than $30 \%$ compared to the untreated control cells (Figure 5C).

A

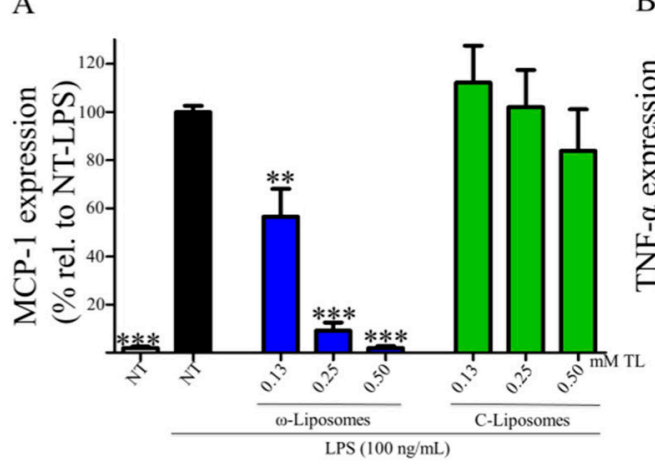

B

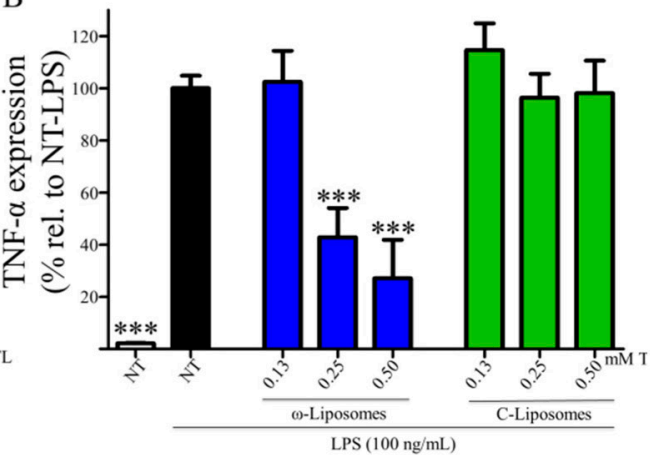

$\mathrm{C}$

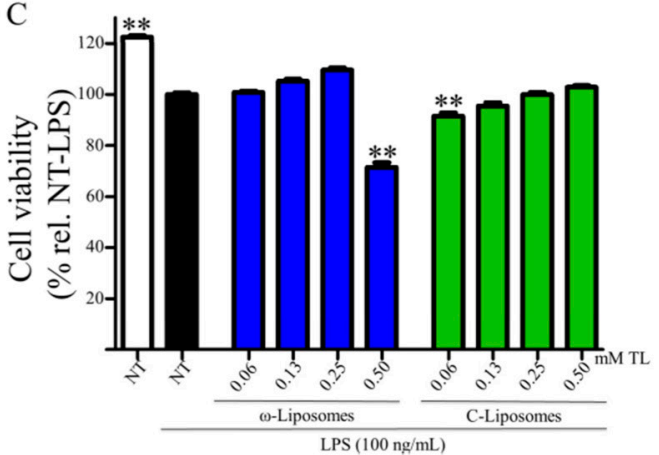

Figure 5| Effect of docosahexaenoic acid-loaded liposomes ( $\omega$-liposomes) and control liposomes (C-liposomes) on lipopolysaccharide (LPS)-induced proinflammatory cytokine production in macrophages. Human THP1 macrophages were treated with the liposomes and stimulated with LPS for 24 hours. (A) MCP1 and (B) TNF $\alpha$ released in the supernatant were analyzed by enzyme-linked immunosorbent assay. Data presented as mean \pm standard deviation of one representative experiment performed in triplicate. (C) Cell viability was assessed by MTS assay under the same experimental conditions. ${ }^{* *} P<0.01,{ }^{* *} P<0.001$ compared to control nontreated (NT) LPS-stimulated cells (Student's t-test). White bars indicate nontreated, nonstimulated control cells; black bars indicate stimulated control cells not treated with liposomal formulations. TL, total lipid. 
In addition to macrophages, neutrophils (PMNs) are a key source of ROS [28]. Therefore, the effect of liposomes in an oxidative burst setup was assessed, where zymosan-induced an increase in ROS production by neutrophils. ROS was remarkably reduced with $\omega$-liposome treatment by $60 \%$ and $90 \%$ at $0.06 \mathrm{mM}$ and $0.13 \mathrm{mM}$ TL, respectively. C-liposomes had no effect on zymosan-induced ROS production at any tested concentration (Figure 6).

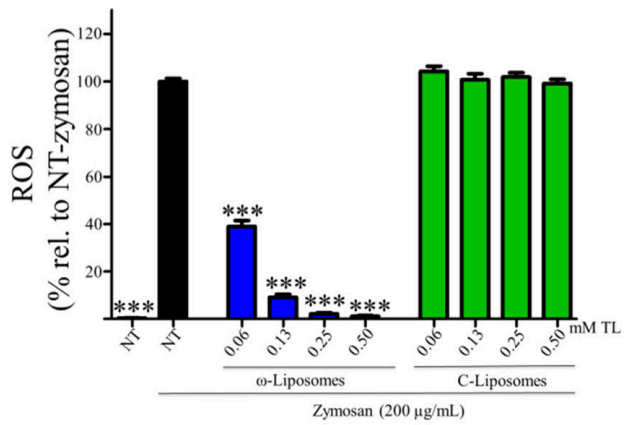

Figure 61 Effect of docosahexaenoic acid-loaded liposomes ( $\omega$-liposomes) and control liposomes (C-liposomes) on zymosan-induced neutrophil oxidative burst. Polymorphonuclear neutrophils freshly isolated from a buffy coat were treated immediately with liposomes and stimulated with zymosan. Reactive oxygen species (ROS) generation was measured by using a luminol-amplified chemiluminescence assay. Data presented as mean \pm standard error of mean from one representative experiment performed in triplicate (each $n \geq 4$ ). ${ }^{* *} P<0.001$ compared to control nontreated (NT) zymosan-stimulated cells (Student's t-test). White bar indicates nontreated, nonstimulated control cells; black bar indicates stimulated control cells not treated with liposomal formulations. TL, total lipid.

The effects of $\omega$-liposomes were further examined on LPS-induced TLR4 activation. TLR4 is a key receptor recognizing damage-associated molecular patterns and pathogen-associated molecular patterns. As such, it plays a key role in atherosclerosis, cancer, and other chronic inflammatory diseases [29-32]. Using HEK-blue TLR4 cells, the induction of TLR4-controlled expression of the reporter molecule secreted embryonic alkaline phosphatase (SEAP) was monitored. LPS triggered a fivefold increase in SEAP activity, which was reduced by $\omega$-liposomes in a dose-dependent manner, whereas C-liposomes had no effect (Figure 7A). $\omega$-Liposomes at $0.5 \mathrm{mM}$ TL (highest concentration) resulted in a minor reduction in cell viability of less than 30\% compared to the untreated control cells (Figure 7B).

A number of studies have investigated the anti-inflammatory effects of PUFAs in general, and DHA specifically. These studies focused mainly on increasing the dietary intake of PUFAs, in in vivo and clinical studies, or by the use of DHA in its free form in vitro $[3,5,31,33,34]$. DHA significantly decreased the expression of cytokineinduced leukocyte adhesion molecule expression in human endothelial cells, while EPA, another member of PUFAs, did not [33]. In a genomic study, Bouwens et al. demonstrated that a high oral dose of DHA and EPA decreased the expression of 
genes involved in inflammatory pathways, such as eicosanoid synthesis, interleukin signaling, NFkB, and a decrease in oxidative stress and cell adhesion on the whole genome of peripheral blood mononuclear cells [12].

A

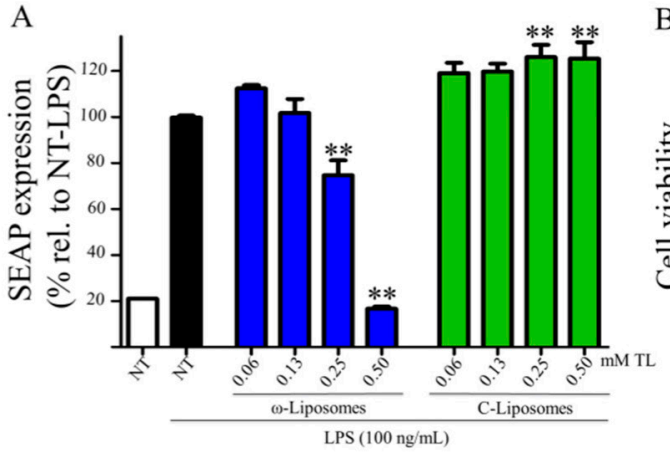

B

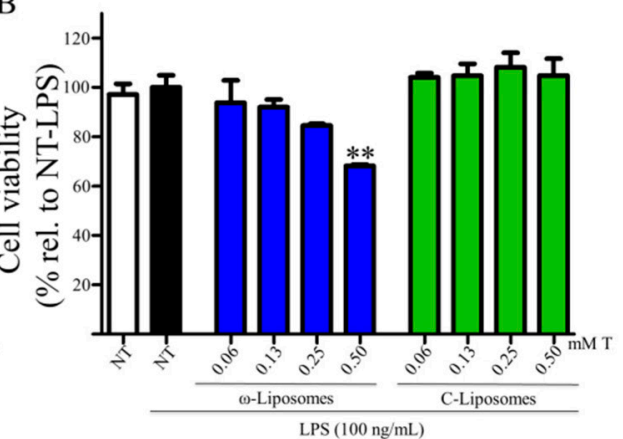

Figure $7 \mid$ Effect of docosahexaenoic acid-loaded liposomes ( $\omega$-liposomes) and control liposomes (C-liposomes) on lipopolysaccharide (LPS)-induced TLR4 activation. Human HEK-Blue TLR4 cells were treated with the liposomes and stimulated with LPS for 18 hours. (A) TLR4 activation was monitored by measuring the secreted embryonic alkaline phosphatase (SEAP) in the medium calorimetrically. (B) Cell viability was assessed by MTS assay under the same experimental conditions. Data presented as mean \pm standard error of mean from two independent experiments (each $n \geq 4$ ). ${ }^{* *} P<0.01$ compared to control nontreated (NT) LPS-stimulated cells (Student's t-test). White bars indicate nontreated, nonstimulated control cells; black bars indicate stimulated control cells not treated with liposomal formulations. TL, total lipid.

\subsection{Effect of $\omega$-Liposomes in Cancer Cell Lines}

Aberrantcell proliferation is the hallmark of cancer and otherchronic inflammatory diseases. Although targeting inflammation in cancer may reduce the invasive features of tumors, anti-inflammatory therapies are seen as an adjunct therapy [35]. Studies have demonstrated the effects of dietary PUFAs on the prevention and inhibition of cancers [2,4]. Therefore, we investigated if $\omega$-liposomes could reduce aberrant cell proliferation. Bromodeoxyuridine (BrdU)-incorporation assays were performed, in which a thymidine analog incorporates only into the DNA of proliferating cells. $\omega$-Liposomes significantly reduced the proliferation of two fast-growing tumor models - human FaDu squamous carcinoma and murine 4T1 breast cancer - in vitro, while C-liposomes did not affect cell proliferation (Figure 8). $\omega$-Liposomes did not reduce the proliferation of control cells, as tested on normal HUVECs (Figure S6).

The exact mechanisms of action of PUFAs in general and DHA specifically are not well known. Possibly, the incorporation of PUFAs into the cell membrane may affect membrane fluidity, ligand recognition, and downstream signaling [36]. Furthermore, GPR120, a novel G-protein-coupled receptor, was identified as a sensor for PUFAs [37]. Also, PUFAs may act as precursors for specialized lipid mediators, such as resolvins and protectins, which possess potent anti-inflammatory effects and assist in the resolution of inflammation [38]. 

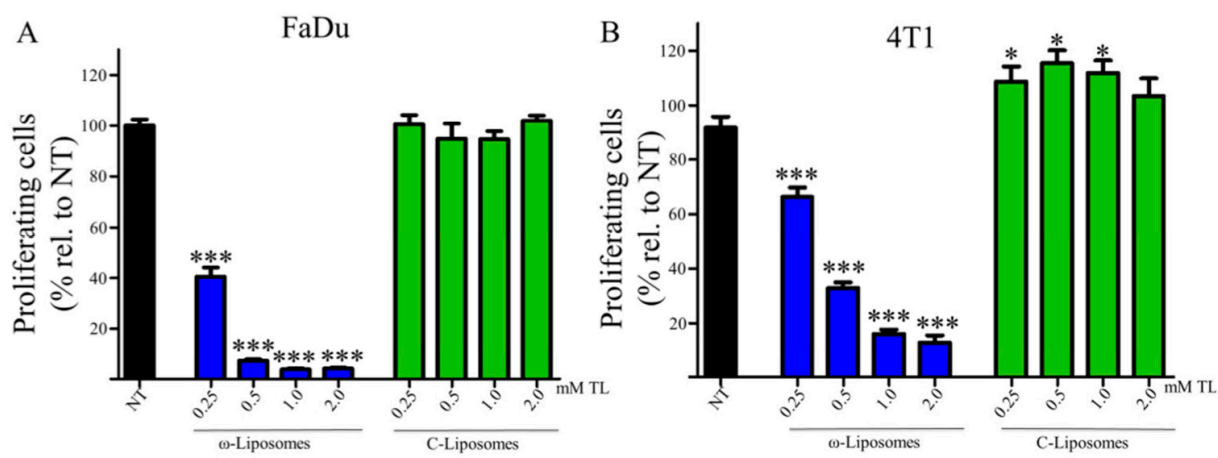

Figure 8| Effect of docosahexaenoic acid-loaded liposomes ( $\omega$-liposomes) and control liposomes (C-liposomes) on tumor-cell proliferation. (A) FaDu cells and (B) 4T1 cells were exposed for 24 hours to $\omega$-liposomes and C-liposomes, after which the medium was replaced with medium containing bromodeoxyuridine (BrdU) and cells incubated for additional 4-6 hours. Afterward, BrdU incorporation was determined by enzyme-linked immunosorbent assay. Data presented as mean \pm standard error of mean from three independent experiments (each $n \geq 4$ ). ${ }^{*} P<0.05$, ${ }^{* * *} P<0.001$ compared to control nontreated (NT) cells (Student's t-test). TL, total lipid.

In this study, a simple nanonutraceutical-based strategy was adopted to deliver effective doses of PUFAs, which dampen the inflammatory phenotype of inflammatory immune cells involved in chronic inflammation. Their antiproliferative activities against cancer cells were also demonstrated. The developed $\omega$-liposomes possess several advantages over the classical dietary intake of DHA, which requires a long duration of intake at high doses. First, formulation into liposomes allows the solubilization of DHA in aqueous solutions at high concentrations, which is required for injection in a low volume. Next, PEGylated liposomes circulate longer and accumulate to a higher extent in inflamed tissues.

Most nanoparticles for in vivo use, which have a particle size of 10-300 nm, nonspecifically accumulate in the reticuloendothelial system (RES), eg, in the liver and spleen, and other tissues. Nanoparticles are also however accumulated in pathological lesions by the EPR effect, as demonstrated for atherosclerotic plaques, inflamed joints in rheumatoid arthritis, and tumors. Such lesions are rich in immune cells, mainly macrophages, which drive the pathophysiological inflammation and contribute to the leaky vasculature $[13,15,16]$. These macrophages have the highest uptake activity compared to other professional phagocytes and other cells [13]. PEGylation will prolong the circulation time of liposomes, and hence increases the chance for extravasation by EPR to the inflamed tissue.

Therefore, this approach can improve the pharmacokinetic profile of DHA and will boost its activities. Of note, a clinically approved total parenteral nutrition (eg, Omegaven), which is rich in DHA and EPA, is prescribed at a dose up to $2.5 \mathrm{~g} /$ day for 4 weeks to reduce risks of liver damage [39]. Such non-PEGylated emulsion is cleared more quickly (half-life 54 minutes, as described in the manufacturer's manual) when compared to long-circulating PEGylated liposomes, such as Doxil (half-life 20-30 
hours) [40], and hence will have less chance to accumulate in inflamed tissues by EPR extravasation. In addition to the longer circulation, liposomes can improve the chemical stability of DHA, which is susceptible to fast oxidation [41-43]. Moreover, $\omega$-liposomes are a tuneable drug-delivery platform in which other drugs can be loaded into the liposomal aqueous core, for synergism, or a targeting ligand can be attached to the surface, for an active targeting approach toward specific cell types [44].

\section{CONCLUSION}

DHA was successfully incorporated in long-circulating PEGylated liposomes to form $\omega$-liposomes. The formulation remained stable and active upon storage over a month. Furthermore, $\omega$-liposomes possess strong antioxidant and antiinflammatory effects in vitro, as demonstrated by the inhibition of the production of ROS, NO, MCP1, and TNF $\alpha$, and the inhibition of NFkB activation, in activated immune cells. They also induce strong inhibition of tumor cell proliferation. This nanonutraceutical represents a nanomedicine-based approach for delivery of relatively safe nutraceutical components that can be applied for the prevention or management of chronic inflammatory diseases and cancer. 


\section{REFERENCES}

[1] T. Yoshihara, K. Shimada, K. Fukao, E. Sai, Y. Sato-Okabayashi, R. Matsumori, T. Shiozawa, H. Alshahi, T. Miyazaki, N. Tada, H. Daida, Omega 3 Polyunsaturated Fatty Acids Suppress the Development of Aortic Aneurysms Through the Inhibition of MacrophageMediated Inflammation, Circ. J. 79 (2015) 1470-1478. doi:10.1253/ circj.CJ-14-0471.

[2] C.M. Barnes, D. Prox, E.A. Christison-Lagay, H.D. Le, S. Short, F. Cassiola, D. Panigrahy, D. Chaponis, C. Butterfield, D. Nehra, E.M. Fallon, M. Kieran, J. Folkman, M. Puder, Inhibition of neuroblastoma cell proliferation with omega-3 fatty acids and treatment of a murine model of human neuroblastoma using a diet enriched with omega-3 fatty acids in combination with sunitinib, Pediatr. Res. 71 (2012) 168-178. doi:10.1038/pr.2011.28.

[3] P. Saravanan, N.C. Davidson, E.B. Schmidt, P.C. Calder, Cardiovascular effects of marine omega-3 fatty acids, Lancet (London, England). 376 (2010) 540-550. doi:10.1016/S01406736(10)60445-X.

[4] P. Terry, P. Lichtenstein, M. Feychting, A. Ahlbom, A. Wolk, Fatty fish consumption and risk of prostate cancer, Lancet (London, England). 357 (2001) 1764-1766. doi:S0140-6736(00)04889-3.

[5] P.C. Calder, Long-chain fatty acids and inflammation, Proc. Nutr. Soc. 71 (2012) 284-289. doi:10.1017/S0029665112000067.

[6] M.J. Pittet, F.K. Swirski, Monocytes link atherosclerosis and cancer, Eur. J. Immunol. 41 (2011) 2519-2522. doi:10.1002/ eji.201141727.

[7] Q. Li, S. Withoff, I.M. Verma, Inflammation-associated cancer: NF-kappaB is the lynchpin, Trends Immunol. 26 (2005) 318-325. doi:S1471-4906(05)00102-X.

[8] D. Richard, K. Kefi, U. Barbe, P. Bausero, F. Visioli, Polyunsaturated fatty acids as antioxidants, Pharmacol. Res. 57 (2008) 451-455. doi:10.1016/j.phrs.2008.05.002.

[9] J.S. Ross, N.E. Stagliano, M.J. Donovan, R.E. Breitbart, G.S. Ginsburg, Atherosclerosis and cancer: common molecular pathways of disease development and progression, Ann. N. Y. Acad. Sci. 947 (2001) 271-273.

[10] E.B. Schmidt, K. Varming, J.O. Pedersen, H.H. Lervang, N. Grunnet, C. Jersild, J. Dyerberg, Long-term supplementation with n-3 fatty acids, II: Effect on neutrophil and monocyte chemotaxis, Scand. J. Clin. Lab. Invest. 52 (1992) 229-236.

[11] P. Geusens, C. Wouters, J. Nijs, Y. Jiang, J. Dequeker, Long-term effect of omega-3 fatty acid supplementation in active rheumatoid arthritis. A 12-month, double-blind, controlled study, Arthritis Rheum. 37 (1994) 824-829.

[12] M. Bouwens, O. van de Rest, N. Dellschaft, M.G. Bromhaar, L.C. de Groot, J.M. Geleijnse, M. Muller, L.A. Afman, Fish-oil supplementation induces antiinflammatory gene expression profiles in human blood mononuclear cells, Am. J. Clin. Nutr. 90 (2009) 415424. doi:10.3945/ajcn.2009.27680.

[13] R. Weissleder, M. Nahrendorf, M.J. Pittet, Imaging macrophages with nanoparticles, Nat. Mater. 13 (2014) 125-138. doi:10.1038/ nmat3780.

[14] H. Maeda, J. Wu, T. Sawa, Y. Matsumura, K. Hori, Tumor vascular permeability and the EPR effect in macromolecular therapeutics: a review, J. Control. Release. 65 (2000) 271-284. doi:S0168-3659(99)00248-5.

[15] J.M. van den Hoven, S.R. Van Tomme, J.M. Metselaar, B. Nuijen, J.H. Beijnen, G. Storm, Liposomal drug formulations in the treatment of rheumatoid arthritis, Mol. Pharm. 8 (2011) 1002-1015. doi:10.1021/mp2000742.

[16] M.E. Lobatto, C. Calcagno, A. Millon, M.L. Senders, F. Fay, P.M. Robson, S. Ramachandran, T. Binderup, M.P.M. Paridaans, S. Sensarn, S. Rogalla, R.E. Gordon, L. Cardoso, G. Storm, J.M. Metselaar, C.H. Contag, E.S.G. Stroes, Z.A. Fayad, W.J.M. Mulder, Atherosclerotic 
plaque targeting mechanism of long-circulating nanoparticles established by multimodal imaging, ACS Nano. 9 (2015) 1837-1847. doi:10.1021/nn506750r.

[17] T.M. Allen, P.R. Cullis, Liposomal drug delivery systems: from concept to clinical applications, Adv. Drug Deliv. Rev. 65 (2013) 36-48. doi:10.1016/j.addr.2012.09.037.

[18] T. Lammers, F. Kiessling, W.E. Hennink, G. Storm, Drug targeting to tumors: principles, pitfalls and (pre-) clinical progress, J. Control. Release. 161 (2012) 175-187. doi:10.1016/j. jconrel.2011.09.063.

[19] G.P. Eckert, S. Chang, J. Eckmann, E. Copanaki, S. Hagl, U. Hener, W.E. Muller, D. Kogel, Liposome-incorporated DHA increases neuronal survival by enhancing nonamyloidogenic APP processing, Biochim. Biophys. Acta. 1808 (2011) 236-243. doi:10.1016/j. bbamem.2010.10.014.

[20] G. Rouser, S. Fleischer, A. Yamamoto, S. Fkeischer, A. Yamamoto, Two dimensional thin layer chromatographic separation of polar lipids and determination of phospholipids by phosphorus analysis of spots, Lipids. 5 (1970) 494-496. doi:10.1007/BF02531316.

[21] Z. Qin, The use of THP-1 cells as a model for mimicking the function and regulation of monocytes and macrophages in the vasculature, Atherosclerosis. 221 (2012) 2-11. doi:10.1016/j.atherosclerosis.2011.09.003.

[22] M.A. Miller, Y.R. Zheng, S. Gadde, C. Pfirschke, H. Zope, C. Engblom, R.H. Kohler, Y. Iwamoto, K.S. Yang, B. Askevold, N. Kolishetti, M. Pittet, S.J. Lippard, O.C. Farokhzad, R. Weissleder, Tumour-associated macrophages act as a slow-release reservoir of nanotherapeutic Pt(IV) pro-drug, Nat. Commun. 6 (2015) 8692. doi:10.1038/ncomms9692.

[23] R. Franco, O. Schoneveld, A.G. Georgakilas, M.I. Panayiotidis, Oxidative stress, DNA methylation and carcinogenesis, Cancer Lett. 266 (2008) 6-11. doi:10.1016/j. canlet.2008.02.026.

[24] S. Reuter, S.C. Gupta, M.M. Chaturvedi, B.B. Aggarwal, Oxidative stress, inflammation, and cancer: how are they linked?, Free Radic. Biol. Med. 49 (2010) 1603-1616. doi:10.1016/j. freeradbiomed.2010.09.006.

[25] H. Li, S. Horke, U. Forstermann, Vascular oxidative stress, nitric oxide and atherosclerosis, Atherosclerosis. 237 (2014) 208-219. doi:10.1016/j.atherosclerosis.2014.09.001.

[26] G.D. Kalliolias, L.B. Ivashkiv, TNF biology, pathogenic mechanisms and emerging therapeutic strategies, Nat. Rev. 12 (2016) 49-62. doi:10.1038/nrrheum.2015.169.

[27] S.L. Deshmane, S. Kremlev, S. Amini, B.E. Sawaya, Monocyte Chemoattractant Protein-1 (MCP-1): An Overview, J. Interf. Cytokine Res. 29 (2009) 313-326. doi:10.1089/jir.2008.0027.

[28] H.L. Wright, R.J. Moots, R.C. Bucknall, S.W. Edwards, Neutrophil function in inflammation and inflammatory diseases, Rheumatology (Oxford). 49 (2010) 1618-1631. doi:10.1093/ rheumatology/ keq045.

[29] K. Brand, S. Page, G. Rogler, A. Bartsch, R. Brandl, R. Knuechel, M. Page, C. Kaltschmidt, P.A. Baeuerle, D. Neumeier, Activated transcription factor nuclear factor-kappa B is present in the atherosclerotic lesion, J. Clin. Invest. 97 (1996) 1715-1722. doi:10.1172/JCI118598.

[30] E. Pikarsky, R.M. Porat, I. Stein, R. Abramovitch, S. Amit, S. Kasem, E. Gutkovich-Pyest, S. Urieli-Shoval, E. Galun, Y. Ben-Neriah, NF-kappaB functions as a tumour promoter in inflammation-associated cancer, Nature. 431 (2004) 461-466. doi:10.1038/ nature02924.

[31] H. Li, X.Z. Ruan, S.H. Powis, R. Fernando, W.Y. Mon, D.C. Wheeler, J.F. Moorhead, Z. Varghese, EPA and DHA reduce LPS-induced inflammation responses in HK-2 cells: evidence for a PPAR-gamma-dependent mechanism, Kidney Int. 67 (2005) 867-874. doi:KID151.

[32] S.W. Wong, M.J. Kwon, A.M. Choi, H.P. Kim, K. Nakahira, D.H. Hwang, Fatty acids modulate Toll-like receptor 4 activation through regulation of receptor dimerization and recruitment into lipid rafts in a reactive oxygen species-dependent manner, J. Biol. Chem. 284 (2009) 27384-27392. doi:10.1074/ jbc.M109.044065. 
[33] R. De Caterina, M.I. Cybulsky, S.K. Clinton, M. a Gimbrone, P. Libby, M.A. Gimbrone Jr, P. Libby, The omega-3 fatty acid docosahexaenoate reduces cytokine-induced expression of proatherogenic and proinflammatory proteins in human endothelial cells, Arterioscler. Thromb. 14 (1994) 1829-1836. doi:10.1161/01.ATV.14.11.1829.

[34] M.M. Rahman, J.M. Veigas, P.J. Williams, G. Fernandes, DHA is a more potent inhibitor of breast cancer metastasis to bone and related osteolysis than EPA, Breast Cancer Res. Treat. 141 (2013) 341-352. doi:10.1007/ s10549-013-2703-y.

[35] E.R. Rayburn, S.J. Ezell, R. Zhang, Anti-Inflammatory Agents for Cancer Therapy, Mol. Cell. Pharmacol. 1 (2009) 29-43. doi:10.4255/mcpharmacol.09.05.

[36] D.W. Ma, J. Seo, L.A. Davidson, E.S. Callaway, Y.Y. Fan, J.R. Lupton, R.S. Chapkin, n-3 PUFA alter caveolae lipid composition and resident protein localization in mouse colon, FASEB J. 18 (2004) 1040-1042. doi:10.1096/ fj.03-1430fje.

[37] D.Y. Oh, S. Talukdar, E.J. Bae, T. Imamura, H. Morinaga, W. Fan, P. Li, W.J. Lu, S.M. Watkins, J.M. Olefsky, GPR120 Is an Omega-3 Fatty Acid Receptor Mediating Potent Anti-inflammatory and Insulin-Sensitizing Effects, Cell. 142 (2010) 687-698. doi:10.1016/j. cell.2010.07.041.

[38] B.T. Kalish, H.D. Le, J.M. Fitzgerald, S. Wang, K. Seamon, K.M. Gura, K. Gronert, M. Puder, Intravenous fish oil lipid emulsion promotes a shift toward anti-inflammatory proresolving lipid mediators, Am. J. Physiol. Liver Physiol. 305 (2013) G818-28. doi:10.1152/ajpgi.00106.2013.

[39] K.T. Park, C. Nespor, J. Kerner Jr, The use of Omegaven in treating parenteral nutritionassociated liver disease, J. Perinatol. 31 Suppl 1 (2011) S57-60. doi:10.1038/jp.2010.182.

[40] A. Gabizon, H. Shmeeda, Y. Barenholz, Pharmacokinetics of pegylated liposomal Doxorubicin: review of animal and human studies, Clin. Pharmacokinet. 42 (2003) 419436. doi:4252.

[41] J.H. Song, Y. Inoue, T. Miyazawa, Oxidative stability of docosahexaenoic acid-containing oils in the form of phospholipids, triacylglycerols, and ethyl esters, Biosci. Biotechnol. Biochem. 61 (1997) 2085-2088. doi:10.1271/bbb.61.2085.

[42] K. Kubo, S. Sekine, M. Saito, Docosahexaenoic acid-containing phosphatidylethanolamine in the external layer of liposomes protects docosahexaenoic acid from 2,2'-azobis(2aminopropane)dihydrochloride-mediated lipid peroxidation, Arch. Biochem. Biophys. 410 (2003) 141-148. doi:S0003986102006793.

[43] F.S. Lu, N.S. Nielsen, M. Timm-Heinrich, C. Jacobsen, Oxidative stability of marine phospholipids in the liposomal form and their applications, Lipids. 46 (2011) 3-23. doi:10.1007/ s11745-010-3496-y.

[44] R. van der Meel, S. Oliveira, I. Altintas, R. Heukers, E.H. Pieters, P.M. van Bergen en Henegouwen, G. Storm, W.E. Hennink, R.J. Kok, R.M. Schiffelers, Inhibition of tumor growth by targeted anti-EGFR/IGF-1R nanobullets depends on efficient blocking of cell survival pathways, Mol. Pharm. 10 (2013) 3717-3727. doi:10.1021 / mp400212v. 


\section{APPENDIX: SUPPLEMENTARY DATA}

A

Before extrusion

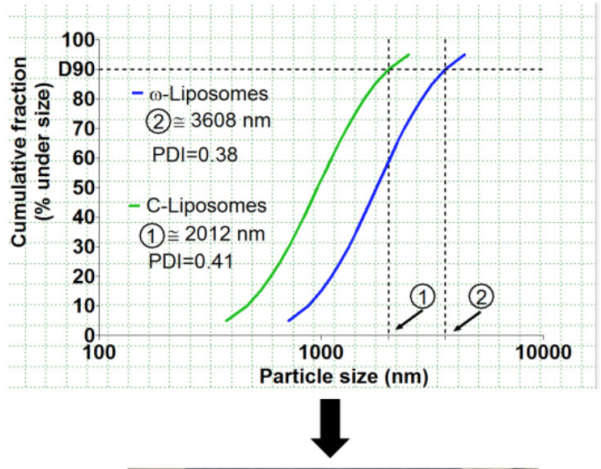

B

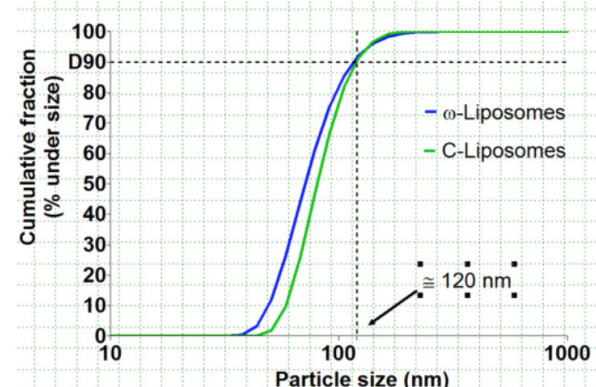

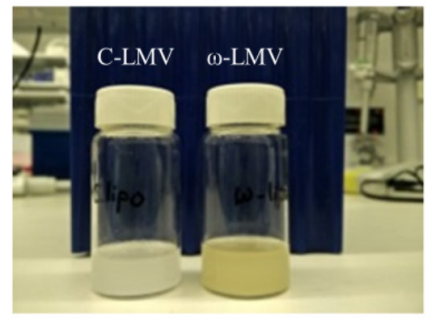

\section{Extrusion}

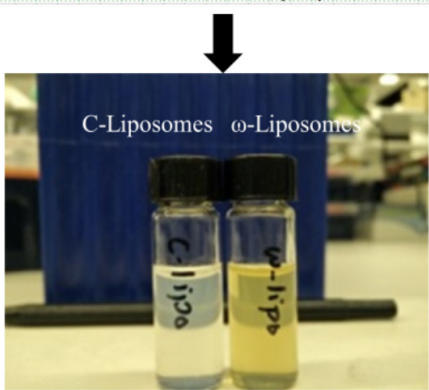

Figure S1| Physical appearance and calculation of D90 of $\omega$-liposomes and control liposomes (C-liposomes) before (A) and after (B) extrusion. Abbreviations: C-LMV, control large, multilamellar vesicles; D90, size distribution at 90\%; PDI, polydispersity index; $\omega$-liposomes, docosahexaenoic acidloaded liposomes; $\omega$-LMV, docosahexaenoic acid-loaded large, multilamellar vesicles.

A

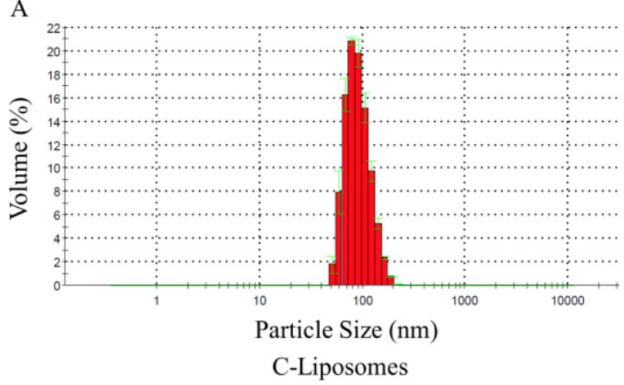

B

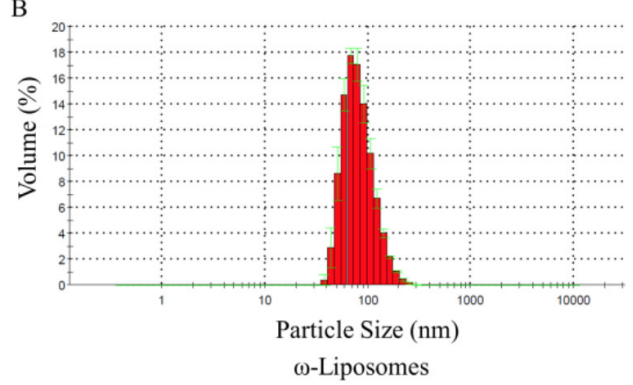

Figure S2 | Dynamic light scattering generated histograms demonstrating the particle-size distribution of $\omega$-liposomes (B) and control liposomes (C-liposomes) (A) after extrusion. $\omega$-liposomes, docosahexaenoic acid-loaded liposomes. 
A

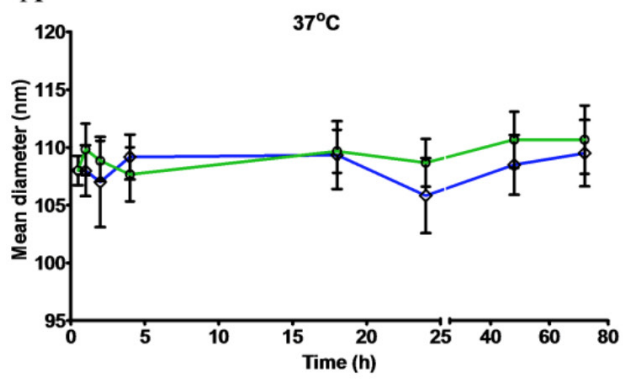

$\mathrm{C}$

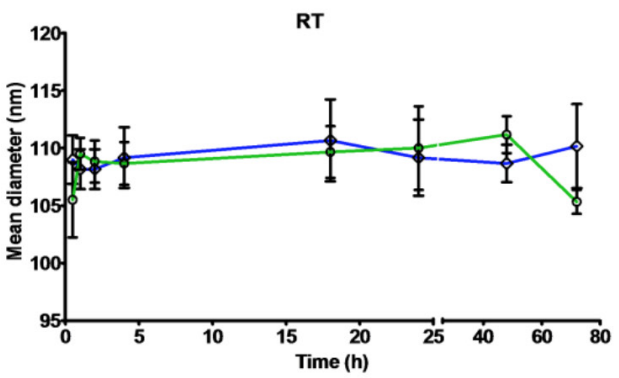

B

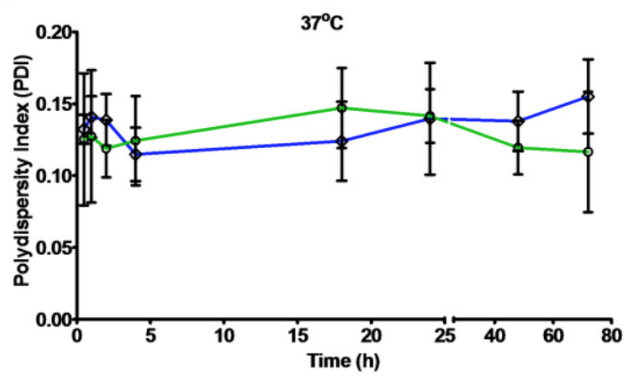

$\mathrm{D}$

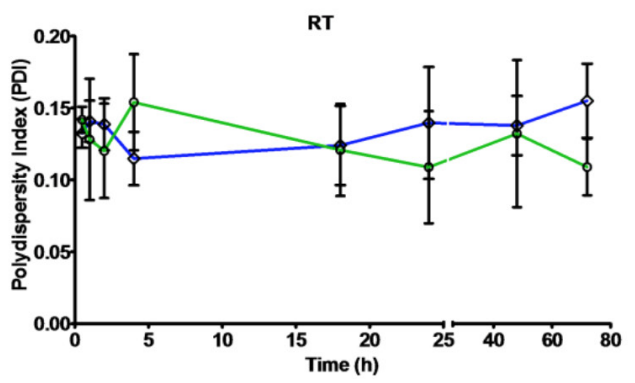

- C-Liposomes

Figure S3 | Colloidal stability of $\omega$-liposomes and control liposomes (C-liposomes) at room temperature (RT) and $37^{\circ} \mathrm{C}$. Particle size (A and C) and polydispersity index (B and D) were monitored with dynamic light scattering over 72 hours. $\omega$-liposomes, docosahexaenoic acid-loaded liposomes.

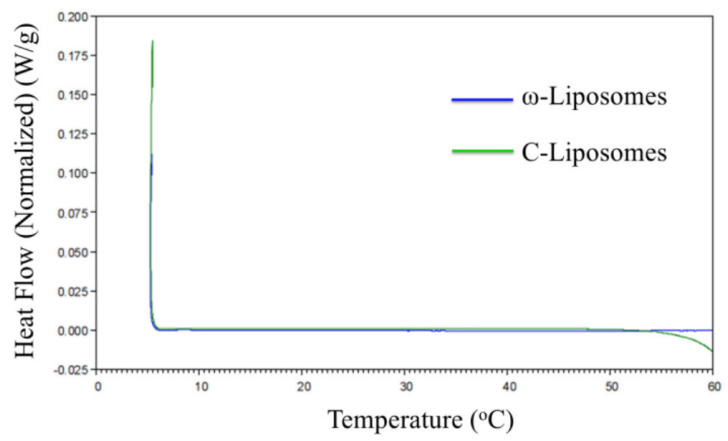

Figure S4| Thermotropic stability of liposomes. The thermotropic stability of the liposomes was analyzed using differential scanning calorimetry and HEPES buffered saline as a reference. The thermal behavior of the liposomes was monitored between $4{ }^{\circ} \mathrm{C}$ and $60{ }^{\circ} \mathrm{C}$, with increasing steps of $0.5^{\circ} \mathrm{C}$ per minute. C-liposomes, control liposomes; HEPES, 4-(2-hydroxyethyl)-1-piperazineethanesulfonic acid; $\omega$-liposomes, docosahexaenoic acid-loaded liposomes. 

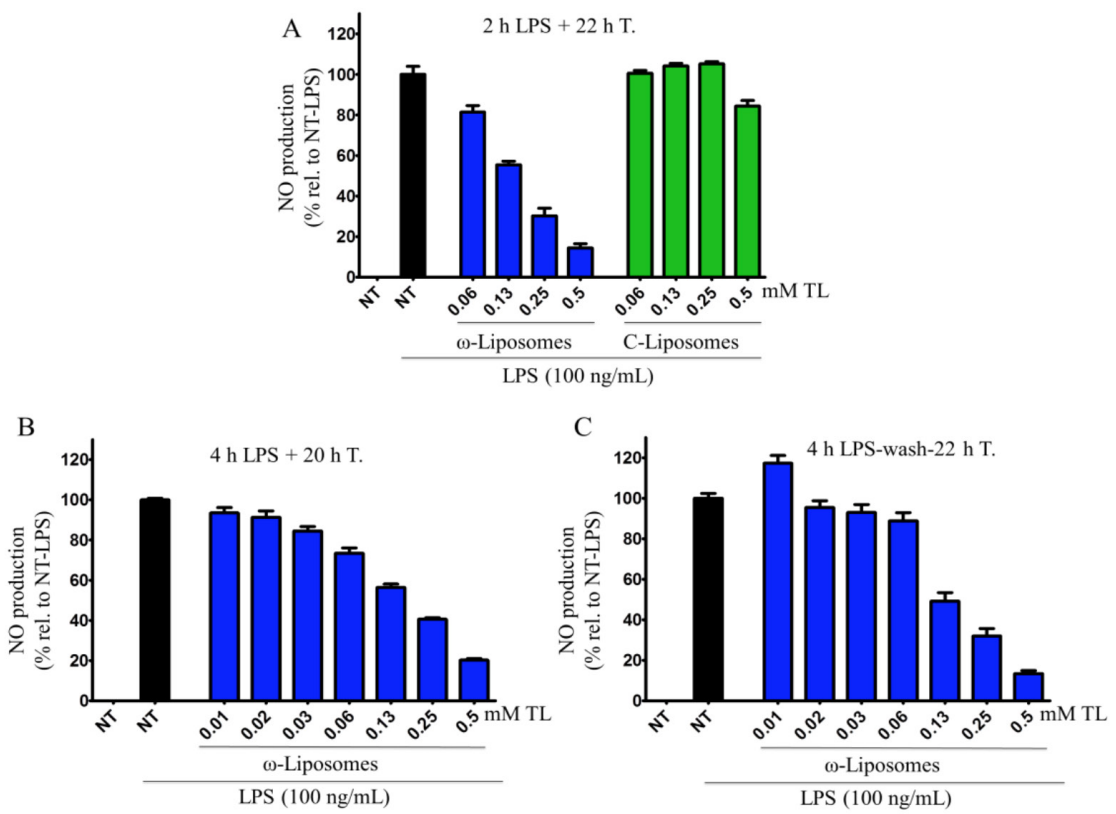

Figure S5 | Effect of $\omega$-liposomes and control liposomes (C-liposomes) on lipopolysaccharide (LPS)induced nitric oxide production in prestimulated murine macrophages. (A) RAW264.7 cells were stimulated with LPS for 2 or 4 hours (B, C). Afterward, LPSwas washed (C) or not (A and B), and cells were treated with $\omega$-liposomes or C-liposomes. NO production was measured in the supernatant with Griess reagent. Data presented as mean \pm standard error of mean from a representative experiment $(n=4)$. NT, nontreated; TL, total lipid; $\omega$-liposomes, docosahexaenoic acid-loaded liposomes.

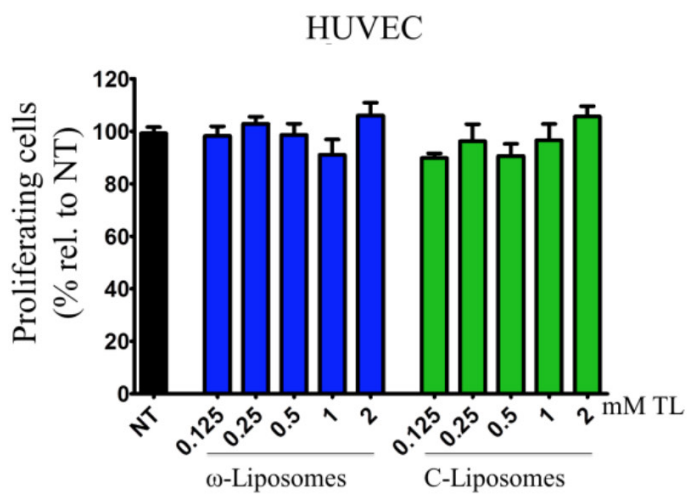

Figure S6 | Effect of $\omega$-liposomes and control liposomes (C-liposomes) on the proliferation of human umbilical vein endothelial cells (HUVECs). HUVECs were seeded at 4,000 cells/well in a 96-well plate. Cells were exposed for 24 hours to $\omega$-liposomes and C-liposomes, after which the medium was replaced with medium containing bromodeoxyuridine (BrdU) and cells incubated for an additional 4-6 hours. Afterward, BrdU incorporation was determined by enzyme-linked immunosorbent assay. Data presented as mean \pm standard error of mean from a representative experiment $(n=6)$. NT, nontreated; TL, total lipid; $\omega$-liposomes, docosahexaenoic acid-loaded liposomes. 




\section{CHAPTER 4}

\section{Multiple Pathway Assessment to Predict Anti-Atherogenic Efficacy of Drugs Targeting Macrophages in Atherosclerotic Plaques*}

\section{ABSTRACT}

$\mathrm{M}$ acrophages play a central role in atherosclerosis development and progression, hence, targeting macrophage activity is considered an

of the anti-inflammatory compound prednisolone to atherosclerotic plaque macrophages in patients, which did however not translate into therapeutic efficacy. This unanticipated finding calls for in-depth screening of drugs intended for targeting plaque macrophages. We evaluated the effect of several candidate drugs on macrophage activity, rating overall performance with respect to changes in cytokine release, oxidative stress, lipid handling, endoplasmic reticulum (ER) stress, and proliferation of macrophages. Using this in vitro approach, we observed that the anti-inflammatory effect of prednisolone was counterbalanced by multiple adverse effects on other key pathways. Conversely, pterostilbene, T0901317, and simvastatin had an overall anti-atherogenic effect on multiple pathways, suggesting their potential for liposomal delivery. Conclusion: This dedicated assay setup provides a framework for high- throughput assessment. Further, in vivo studies are warranted to determine the predictive value of this macrophage-based screening approach and its potential value in nanomedicinal drug development for cardiovascular patients. 


\section{INTRODUCTION}

Atherosclerosis is a multifaceted disease of the arterial wall, underlying the vast majority of cardiovascular diseases [1]. Triggered by endothelial cell dysfunction, circulating lipids accumulate in the arterial wall and become modified through oxidation. Recruited macrophages become foam cells when taking up these oxidized lipids, which is a hallmark of initial atherosclerotic lesions. Over time, a complex interplay of maladaptive responses contributes to atherosclerosis progression, including, amongst others, chronic local inflammation, oxidative stress, impaired cholesterol efflux, and excessive cell proliferation [2].

Past decades, the widespread use of statin-based lipid lowering strategies has revolutionized cardiovascular disease management, reducing the risk of an acute event by $25-35 \%$ [3]. Nonetheless, a considerable residual risk remains,[4] driving the pursuit for novel anti-atherosclerotic strategies. Since plaque macrophages are crucial in atherogenesis, main mechanisms related to macrophage activity, including inflammation, oxidative stress, lipid metabolism, and proliferation are considered potential therapeutic targets [5]. Nanomedicine offers an attractive strategy to locally target macrophage activity within an atherosclerotic plaque [6]. In addition to promising results in experimental models, $[7,8]$ we recently reported successful targeting of plaque macrophages in patients with atherosclerosis using a liposomal delivery platform for prednisolone [9]. However, the unexpected lack of antiinflammatory efficacy strongly argued for a more in-depth characterization of drug effects on plaque macrophages [9].

Therefore, we set up a dedicated series of in vitro assays to rapidly screen drug compounds for their effects on multiple key pathways of macrophage activity. Seven compounds recognized for their beneficial modulating effect on one of these pathways were selected to evaluate their effect on all other aforementioned macrophage pathways (Table 1). To facilitate potential nanomedicinal development, we aimed to screen drugs and compounds that have a good safety profile in humans and are suitable for liposomal encapsulation. We demonstrate here that we can rapidly assess the overall performance of drug candidates to identify those likely to exert anti-atherogenic effects on lesional macrophages. 
Table 1 I Selected drug compounds for multi-pathway screening

\begin{tabular}{|c|c|c|c|}
\hline Drug & Mode of action & Clinical use & Status for atherosclerosis \\
\hline $\begin{array}{l}\text { Prednisolone (Anti- } \\
\text { inflammatory) }\end{array}$ & $\begin{array}{l}\text { Glucocorticoid } \\
\text { receptor agonist }\end{array}$ & $\begin{array}{l}\text { Inflammatory, } \\
\text { oncological and } \\
\text { hematological } \\
\text { disorders }\end{array}$ & $\begin{array}{l}\text { Phase I/II: Liposomal } \\
\text { formulation of } \\
\text { prednisolone phosphate } \\
\text { showed no efficacy[9] }\end{array}$ \\
\hline $\begin{array}{l}\text { Methotrexate (MTX) } \\
\text { (Anti-inflammatory) }\end{array}$ & $\begin{array}{l}\text { Folic acid } \\
\text { antagonist }\end{array}$ & $\begin{array}{l}\text { Neoplastic diseases, } \\
\text { rheumatoid arthritis, } \\
\text { psoriasis }\end{array}$ & $\begin{array}{l}\text { Phase III: Systemic } \\
\text { low-dose MTX trial in } \\
\text { progress (CIRT)[47] }\end{array}$ \\
\hline $\begin{array}{l}\text { T0901317 (T09) } \\
\text { (Cellular cholesterol } \\
\text { efflux stimulator) }\end{array}$ & $\begin{array}{l}\text { Liver } \mathrm{X} \text { receptor } \\
\text { (LXR) agonist }\end{array}$ & Only preclinical use & $\begin{array}{l}\text { Preclinical: Systemic } \\
\text { dosing reduces } \\
\text { atherosclerosis in animal } \\
\text { models, but promotes } \\
\text { hepatic lipogenesis[25-30] }\end{array}$ \\
\hline $\begin{array}{l}\text { Pterostilbene (Anti- } \\
\text { oxidant) }\end{array}$ & $\begin{array}{l}\text { Free radical } \\
\text { scavenging }\end{array}$ & $\begin{array}{l}\text { No clinical } \\
\text { indications; } \\
\text { available as dietary } \\
\text { supplement }\end{array}$ & $\begin{array}{l}\text { Preclinical: Long-term } \\
\text { oral dosing of resveratrol } \\
\text { (analog) reduces } \\
\text { atherosclerosis in mice } \\
\text { and rabbits [32-36] }\end{array}$ \\
\hline $\begin{array}{l}\text { Mercaptopurine } \\
(6-\mathrm{MP}) \text { (Anti- } \\
\text { proliferative) }\end{array}$ & $\begin{array}{l}\text { Purine } \\
\text { antagonist }\end{array}$ & $\begin{array}{l}\text { Organ } \\
\text { transplantation, } \\
\text { leukemia, auto- } \\
\text { immune disorders }\end{array}$ & $\begin{array}{l}\text { Preclinical: Drug- } \\
\text { eluting cuff reduces } \\
\text { atherosclerosis in mice[43] }\end{array}$ \\
\hline $\begin{array}{l}\text { Simvastatin } \\
\text { (Lipid lowering / } \\
\text { Anti-inflammatory) }\end{array}$ & $\begin{array}{l}\text { HMG-CoA } \\
\text { reductase } \\
\text { inhibitor }\end{array}$ & $\begin{array}{l}\text { Primary and } \\
\text { secondary } \\
\text { prevention of } \\
\text { atherosclerosis }\end{array}$ & $\begin{array}{l}\text { Preclinical: rHDL- } \\
\text { vehicle delivery reduced } \\
\text { atherosclerosis in mice[7] }\end{array}$ \\
\hline $\begin{array}{l}\text { Rapamycin (Anti- } \\
\text { inflammatory) }\end{array}$ & mTOR inhibitor & $\begin{array}{l}\text { Organ } \\
\text { transplantation, } \\
\text { drug-eluting stents }\end{array}$ & $\begin{array}{l}\text { Preclinical: Oral dosing } \\
\text { reduces atherosclerosis } \\
\text { in mice[50-57]; local } \\
\text { delivery strategies are } \\
\text { being developed }[48,49]\end{array}$ \\
\hline
\end{tabular}

Abbreviations: $\mathrm{MTX}=$ methotrexate; CIRT $=$ cardiovascular inflammation reduction trial; HMG-CoA = 3- hydroxy-3-methyl-glutaryl-coenzyme A; rHDL = recombinant high-density lipoprotein; $\mathrm{mTOR}=$ mammalian target of rapamycin. 


\section{MATERIALS AND METHODS}

\subsection{Materials}

All chemicals were purchased from Sigma unless mentioned otherwise. T0901317 (T09) was purchased from Cayman Chemical. The compounds were dissolved in dimethyl sulfoxide (DMSO), yet ensuring that in all experiments the final DMSO fraction in culture wells was below $0.05 \%(\mathrm{v} / \mathrm{v})$. Lipoprotein depleted serum (LPDS) was prepared from fetal bovine serum by ultracentrifugation in $\mathrm{KBr}$ at a density of $1.21 \mathrm{~g} / \mathrm{mL}$. After centrifugation at 50,000 RPM and $4{ }^{\circ} \mathrm{C}$ for 50,000 RPM, the lipoprotein layer was removed by aspiration. The bottom fraction was dialyzed against phosphate buffered saline (PBS) and sterile filtered. The purity is determined via HPLC.

\subsection{Cell culture}

Human monocytic THP-1 cells [10], RAW264.7 murine macrophages [11] and murine bone marrow-derived macrophages (BMDM) are widespread models to study macrophage function in atherosclerosis. THP-1 cells and RAW264.7 macrophages were obtained from the American Type Culture Collection. RAW264.7 cells stably transfected with the $3 \times-\mathrm{NF}-\kappa \mathrm{B}$-luc plasmid were kindly provided by Prof. M.P.J. de Winther [11].

THP-1 cells and RAW264.7 were cultured in RPMI-1640 and DMEM high glucose, respectively. Both media were supplemented with penicillin $(100 \mathrm{U} / \mathrm{mL})$, streptomycin $(100 \mu \mathrm{g} / \mathrm{mL})$ and $10 \%$ fetal bovine serum (FBS; GIBCO Invitrogen). THP-1 cells were differentiated into macrophages with $50 \mathrm{ng} / \mathrm{mL}$ phorbol-12myristate-13-acetate (PMA) for $24 \mathrm{~h}$, after which cells were washed and left in the PMA-free medium for another $24 \mathrm{~h}$ before adding the compounds.

Bone marrow cells were isolated from both femurs and tibiae of wild-type mice (C57BL/6). Cells were cultured in RPMI-1640 with penicillin $(100 \mathrm{U} / \mathrm{mL})$, streptomycin $(100 \mu \mathrm{g} / \mathrm{mL})$ and 10\% FBS and 15\% L929 conditioned medium for 8 days to generate BMDM according to a method previously described [12].

For the oxidative burst assay, polymorphonuclear neutrophils (PMNs) were isolated by Ficoll centrifugation of buffy coats purchased from Sanquin (Amsterdam) blood supply. 


\subsection{Cell viability}

THP-1, RAW264.7, and BMDM cells were seeded in 96-well plates $(5 \times 104$ cells/well). The next day, cells were treated with the compounds in concentrations ranging from 0.3 to $30 \mu \mathrm{M}$ for $24 \mathrm{~h}$. The toxicity of compounds was determined by colorimetric MTT cell viability assay as described previously [13].

\subsection{NF- $\kappa B$ transcriptional activity}

RAW264.7 NF-kB-luc macrophages were seeded in 96-well plates $(7 \times 104$ cells/ well). After $24 \mathrm{~h}$, cells were washed and treated with the compound for $2 \mathrm{~h}$ after which cells were stimulated with lipopolysaccharide (LPS) $(100 \mathrm{ng} / \mathrm{mL})$ for another $18 \mathrm{~h}$. NF- $\kappa \mathrm{B}$ luciferase activity was determined by the ONE-GloTM Luciferase Assay System (Promega).

\subsection{Pro-inflammatory cytokine production}

Quantitation of secreted cytokine concentrations of tumor necrosis factor alpha (TNF- $\alpha$ ) and interleukin (IL)-6 was performed by using the Cytometric Bead Array Human Inflammation Kit (BD Biosciences). THP-1 and BMDM cells were seeded in 96-well plates $(5 \times 104$ cells / well). After differentiation with PMA, cells were treated with each compound for $2 \mathrm{~h}$. Thereafter, LPS was added at final concentration of $100 \mathrm{ng} / \mathrm{mL}$ for another $22 \mathrm{~h}$. Before analysis, cellular debris was removed from the supernatants of treated cells by centrifugation ( $5 \mathrm{~min}$ at $500 \mathrm{~g}$ ).

\subsection{RNA isolation, cDNA synthesis, and qPCR}

THP- 1 cells were seeded into 12 -well plates $(5 \times 105$ cells / well). After differentiation with PMA, cells were first incubated with oxidized low-density lipoprotein (oxLDL; $50 \mu \mathrm{g} / \mathrm{mL}$; Alfa Aesar) for $24 \mathrm{~h}$, then treated with the compounds for an additional 18 $\mathrm{h}$. Total RNA was extracted using Trizol and cDNA was synthesized from $1 \mu \mathrm{g}$ RNA with the iScript cDNA Synthesis kit (BioRad). Quantitative real-time PCR (qPCR) was performed using SensiFASTTM SYBR ${ }^{\circledR}$ (BC Biotech) and measured with the CFX384 system (BioRad). Specific primers for human CD36, ATP-binding cassette transporter $\mathrm{A} 1$ (ABCA1), fatty acid binding protein 4 (FABP4), C/EBP homologous protein $(\mathrm{CHOP})$, inositol-requiring transmembrane kinase/ endonuclease 1 (IRE1) and ribosomal protein 36B4 were designed (Supplementary Table S1). 


\subsection{Nitric oxide (NO) production}

In vitro evaluation of NO production of THP-1 macrophages using the Griess assay is not achievable.[14] RAW264.7 macrophages and BMDM were seeded in 96-well plates $(1 \times 105$ cells / well $)$ and next day washed and pre-treated with each compound for $2 \mathrm{~h}$ followed by $100 \mathrm{ng} / \mathrm{mL}$ LPS for another $22 \mathrm{~h}$. Nitrite $\left(\mathrm{NO}_{2}{ }^{-}\right)$ concentrations in the supernatants were measured by adding $100 \mu \mathrm{l}$ freshly made Griess reagent ((0.1\% N-(1 Naphtyl)ethylene-diamine dihydrochloride (Merck), 2.5\% phosphoric acid (Merck), $1 \%$ sulfanilamide (Sigma))) to $100 \mu \mathrm{L}$ culture supernatant. Serial dilutions of nitrite standard solution were used to generate a standard curve ranging from 0 to $100 \mu \mathrm{M}$ of nitrite. The absorbance was measured at $550 \mathrm{~nm}$ with a microplate reader (SPECTROstar Nano).

\subsection{Production reactive oxygen species (ROS)}

PMNs were suspended in Hanks' Buffered Saline Solution (HBSS) substituted with $1 \%$ gelatine solution in deionized $\mathrm{H}_{2} \mathrm{O}$ (HBSS-gel.). Cells were counted and diluted in the HBSS-gel to a concentration of $1 \times 106$ cells $/ \mathrm{mL}$. Zymosan A from Saccharomyces cerevisiae was used as ROS inducer. Luminol, dissolved in DMSO and diluted in HBSS resulting in a final DMSO concentration lower than $0.1 \%(\mathrm{v} / \mathrm{v})$, was used as luminescence enhancer and was added to the PMNs in a 1:1 volume ratio in white 96-well plates. Compounds were added to the previously indicated final concentrations and zymosan was added to final concentration of $0.2 \mathrm{mg} / \mathrm{mL}$. Luminescence was measured using Titertek Luminoskan (TechGen International). The assay was repeated using buffy coats from different donors.

\subsection{OxLDL uptake}

Human oxLDL (Alfa Aesar) was labeled with DyLight 488 NHS Ester (\#46402 Thermo Scientific). The manufacturer has oxidized the LDL via copper sulfate oxidation and the degree of oxidation was 99\% (determined via the TBARS assay). Briefly, $0.43 \mu \mathrm{L}$ Dylight 488 was added per $250 \mu \mathrm{L}$ of $2 \mathrm{mg} / \mathrm{mL}$ oxLDL. The solution was protected from light and incubated on a shaker at RT for $1 \mathrm{~h}$. Subsequently, unbound dye was washed away by dialysis at $4{ }^{\circ} \mathrm{C}$ overnight. THP- 1 complete medium was replaced by medium containing 10\% LPDS and pre-treated overnight with each compound. Subsequently, $25 \mu \mathrm{g} / \mathrm{mL}$ oxLDL-Dylight 488 was added to treated or non-treated cells for 6 or $24 \mathrm{~h}$. Finally, cells were washed once with icecold phosphate-buffered saline (PBS) containing 5\% FBS, twice with ice-cold PBS and lysed using radioimmunoprecipitation assay buffer (TEKnova). Fluorescence was measured with a Typhoon scanner (GE Healthcare). 


\subsection{Cholesterol efflux}

THP-1 cells were seeded in 24-wells plates at a density of $5 \times 105$ cells $/ \mathrm{mL}$. Macrophages were treated with compounds for $8 \mathrm{~h}$ and loaded with cholesterol overnight using $30 \mu \mathrm{g} / \mathrm{mL}$ cholesterol mixed with $0.5 \mu \mathrm{Ci} / \mathrm{mL}$ tritium-labelled cholesterol (Amersham) in RPMI with penicillin-streptomycin (100 U/ml-100 $\mu \mathrm{g}$ / $\mathrm{mL}$ ) and $0.2 \%$ free fatty acid (FFA)-free bovine serum albumin (BSA). After washing with PBS containing $0.2 \%$ FFA-free BSA, cells were incubated for $4 \mathrm{~h}$ with $20 \mu \mathrm{g}$ / $\mathrm{mL}$ apolipoprotein-A1 (apoA- 1; Calbiochem) in RPMI (without FBS) containing penicillin-streptomycin $(100 \mathrm{U} / \mathrm{mL}-100 \mu \mathrm{g} / \mathrm{mL})$ and $0.2 \%$ FFA-free BSA (Sigma). Cells were lysed using isopropanol 100\%. Tritium was measured for both cell culture medium and cell lysates using a scintillation counter.

\subsection{BrdU incorporation}

Proliferation was primarily evaluated in RAW264.7 cells since differentiation of THP-1 cells with PMA is known to halt proliferation.[15] RAW264.7 and THP-1 cells were seeded in 96-well plates $(3 \times 103$ cells/well), washed the next day with serum free medium and subsequently serum starved for $24 \mathrm{~h}$. Thereafter, serum free medium was replaced with complete medium reconstituted with each compound except for the control, in which only complete medium was added. After $24 \mathrm{~h}$, proliferation was measured using the 5-bromo-2'- deoxyuridine (BrdU) ELISA kit (Roche) according to the manufacturer's recommendations.

\subsection{Overall performance score}

The experiments provided outcomes known to have either an atheroprotective or atherogenic effect, which was expressed in fold change compared to the untreated control. A scoring system was applied where a statistically significant change compared to control was scored as +1 or -1 point depending on whether the effect was atheroprotective or atherogenic, respectively. A two-fold change or more was scored by adding or subtracting 2 points. The total score was tallied and compounds were ranked accordingly. Subsequently, a summarizing heat map was generated in Multiple Experiment Viewer (MeV 4.9.0., Microarray Software Suite, www.tm4.org).

\subsection{Statistical analysis}

All assays were at least performed in three independent experiments. Statistical analysis was performed using GraphPad Prism 5 software. Statistical significance was calculated using the unpaired Student's t-test (Welch corrected when necessary). Values are represented as mean \pm SEM. The significance level was set at $p<0.05$. 


\section{RESULTS}

\subsection{Cell viability}

Prior to evaluating the chosen macrophage pathways relevant to atherosclerosis, cell viability was assessed after treatment with drug concentrations ranging from 0.3 $\mu \mathrm{M}$ to $30 \mu \mathrm{M}$ to assess the non-toxic dose to be used for the other assays. MTT cell viability assays showed that none of the compounds affected THP-1 cell viability, apart from rapamycin, which was toxic at $30 \mu \mathrm{M}$. In addition to human cells, viability assays of murine RAW264.7 cells and BMDM showed comparable results (Supplemental Figure S1). Thus, for each compound, a concentration of $30 \mu \mathrm{M}$ was applied, whereas for rapamycin a concentration of $3 \mu \mathrm{M}$ was used in the screening assays.

\subsection{Anti-inflammatory potency}

Anti-inflammatory effects of the selected compounds were first evaluated with LPS- induced NF- $\kappa$ B activation in RAW264.7 NF- $\kappa$ B-luc macrophages (Figure 1A). A strong $(>50 \%)$ inhibition of LPS-induced NF- $\kappa$ B activity was observed after prednisolone, T09 and 6-MP treatment compared to LPS stimulation only (all p < $0.001)$. MTX, pterostilbene and simvastatin showed a modest $(25 \%)$ reduction on NF$\kappa B$ activity (all $p<0.01)$, whereas rapamycin increased NF- $\kappa B$ activity $(p=0.006)$. The subsequent release of pro- inflammatory cytokines following LPS was determined in THP-1 macrophages. Prednisolone, T09, and pterostilbene strongly (> 50\%) reduced the production of TNF $\alpha$ and IL-6. Simvastatin did repress TNF $\alpha$ but had no statistically significant effect on IL-6 (Figure 1B + C). MTX, 6-MP, and rapamycin did not significantly influence cytokine release, apart from a reduction ( $>50 \%$ ) in IL-6 by rapamycin $(p=0.046)$. Generally similar effects for cytokine release were observed for BMDM (Supplemental Figure S2). 
A

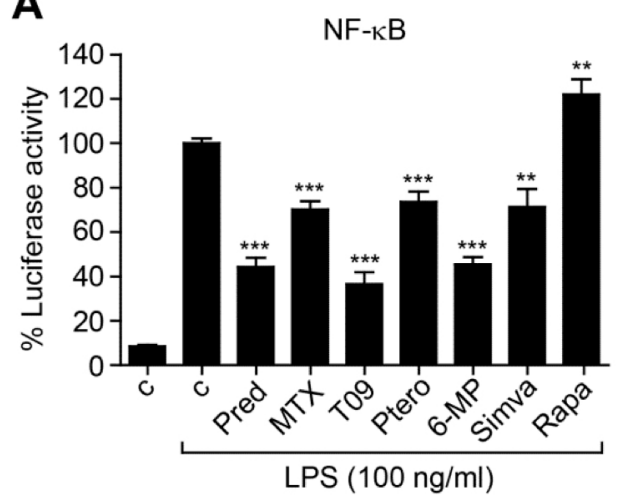

C

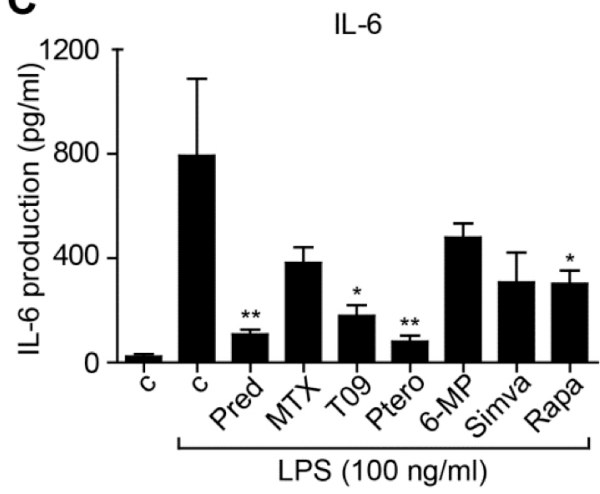

B

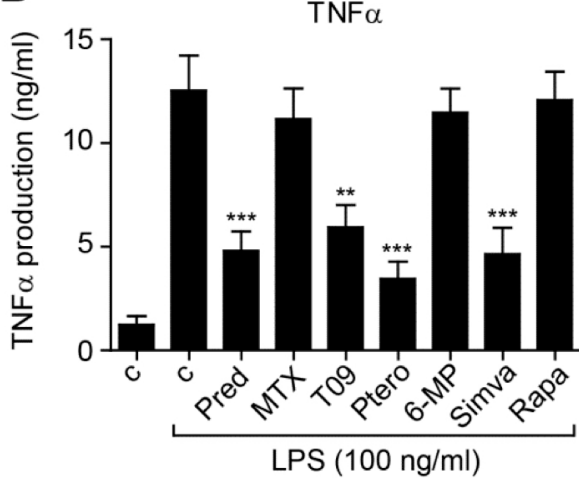

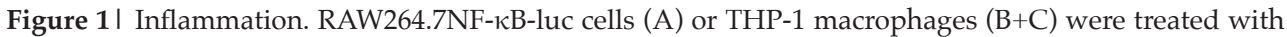
the compounds and stimulated with LPS (100 ng/mL) for $24 \mathrm{~h}$. (A) NF- $\mathrm{kB}$ activity was determined by luciferase assay and fold change was calculated relative to LPS stimulation only by combining data from three independent experiments ( $\mathrm{n}=6$ / experiment). $(\mathrm{B}+\mathrm{C}) \mathrm{TNF} \alpha$ and IL-6 production was measured using a cytometric bead array. Data are presented as mean $\pm S E M$; ${ }^{*} P<0.05,{ }^{* *} P<0.01,{ }^{* * *} P<0.001$ compared to LPS stimulation only. $\mathrm{c}=$ control; Pred $=$ prednisolone; MTX = methotrexate; T09= T0901317; Ptero= pterostilbene; $6-\mathrm{MP}=6$ - mercaptopurine; Simva $=$ simvastatin; $\mathrm{Rapa}=$ rapamycin .

\subsection{Generation of reactive oxygen species}

Potential anti-oxidative effects were studied using LPS-induced NO production and zymosan-induced PMN ROS generation. After treatment with prednisolone and rapamycin, a substantial reduction in NO production by $>40 \%$ compared to LPS stimulation only (both $\mathrm{p}<0.001$ ) was observed, while pterostilbene and T09 reduced NO production in the order of 25 to $30 \%$ (both $\mathrm{p}<0.001$ ). MTX, simvastatin, and $6-\mathrm{MP}$ did not affect NO production at all (Figure. 2A). In BMDM, NO production was reduced the most $(>50 \%)$ by prednisolone, pterostilbene and T09 (Supplemental Figure. S3). With respect to ROS, pterostilbene was the strongest inhibitor of ROS production (8 fold change). T09, 6-MP, and simvastatin diminished ROS production to a less extent, while prednisolone, MTX, and rapamycin did not affect ROS production (Figure 2B) 


\subsection{Lipid handling by macrophages}

Important changes in macrophage lipid handling with regard to atherosclerosis comprise the uptake, efflux and intracellular trafficking of lipids. First, the uptake of fluorescently labeled oxLDL was assessed in THP-1 macrophages. Correcting for background fluorescence measured in control cells without oxLDL, T09 and pterostilbene reduced oxLDL uptake by $45 \%$ (both $\mathrm{p}<0.001$ ) compared to oxLDL stimulation only (Figure 3A), whereas MTX, simvastatin, and rapamycin decreased oxLDL uptake to a lesser extent (all $\mathrm{p}<0.001)$. Similar results were observed when oxLDL treatment was extended to $24 \mathrm{~h}$ (Supplementary Figure S4). In line with the diminished oxLDL uptake, both T09 and pterostilbene markedly downregulated the expression of the lipid uptake receptor CD36 (both $p<0.001$; Figure 3B).

Next, treatment effects on cholesterol efflux from THP-1 cells to apoA-1 were assessed. As expected, the LXR-agonist T09 greatly increased cholesterol efflux by 2.5 -fold $(p<0.001)$, in respect to untreated control cells. Simvastatin $(p=0.005)$ and rapamycin ( $\mathrm{p}=0.017$ ) also augmented cholesterol efflux, whereas the other compounds did not affect this process (Figure 3C). mRNA expression of the cholesterol efflux transporter ABCA1 was significantly (>2.5-fold) elevated by T09 and pterostilbene, while it was reduced in response to prednisolone (45\%) and simvastatin (20\%) (all p $<0.001$ compared to oxLDL stimulation only) (Figure 3D).

\subsection{Endoplasmic reticulum (ER) stress}

To examine whether the compounds affected macrophage ER stress, changes in mRNA expression of ER stress related proteins in THP-1 macrophages pre-exposed to oxLDL were assessed. As regulator of intracellular lipid trafficking [16-18] and lipid-induced ER stress [16,19], mRNA expression of lipid chaperone FABP4 was found to be significantly increased by 6-MP $(\mathrm{p}=0.002)$ and up to almost 2 -fold by prednisolone $(\mathrm{p}<0.001)$ treatment compared to oxLDL stimulation only, whereas T09 and pterostilbene decreased FABP4 expression by 30-70\% (both $\mathrm{p}<0.001$ ) (Figure 4A).

Prolonged or unresolved ER stress can induce apoptosis mediated through CHOP and IRE1 [20]. Compared to oxLDL treated control, mRNA expression of CHOP was most notably increased by treatment with T09 $(>1000 \%)$, pterostilbene $(>200 \%), 6-\mathrm{MP}(75 \%)$ (all p < 0.001); whereas prednisolone modestly decreased CHOP by $20 \%(p=0.002)$. MTX and simvastatin had no significant effects on CHOP expression (Figure 4B). IRE1 mRNA expression was upregulated (50\%) in response to prednisolone, T09, pterostilbene and rapamycin compared to oxLDL stimulation only, while modestly upregulated by MTX and 6-MP. Simvastatin treatment did not change IRE1 levels. (Figure 4C). 


\subsection{Macrophage proliferation}

The proliferative capacity of macrophages after treatment was assessed using BrdU incorporation in RAW264.7 cells (Fig. 4D). Simvastatin and pterostilbene demonstrated the strongest anti-proliferative effect by reducing the BrdU incorporation with $75 \%$ and 55\% (both $\mathrm{p}<0.001$ ), respectively. T09, 6-MP, and rapamycin also significantly inhibited cell proliferation, though to a less extent. MTX did not affect macrophage proliferation. In THP-1 cells fluorescence intensity was generally lower, as may be expected with this cell line. Still, a reduction pattern ( $\geq 50 \%$ ) similar to RAW264.7 was observed for T09, pterostilbene and 6-MP (Supplemental Figure S5). 
A
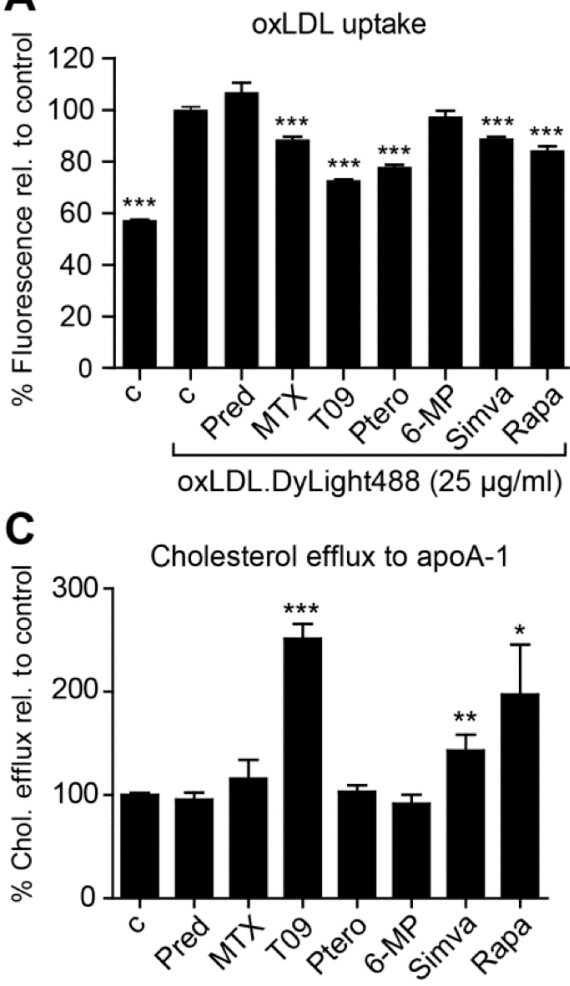

B
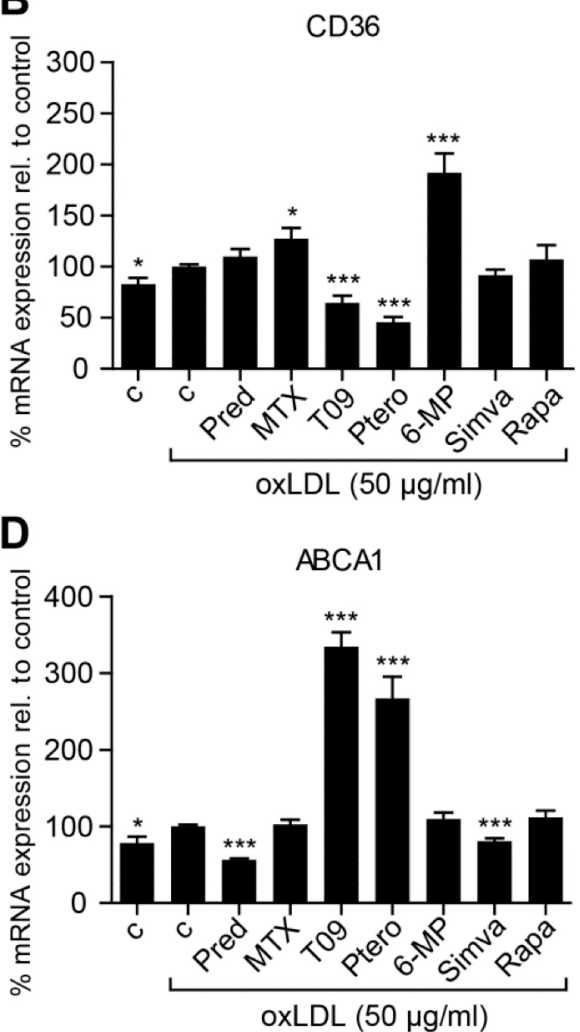

Figure 3| Macrophage lipid handling. THP-1 macrophages were treated with compounds and oxLDLDyLight 488 for $6 \mathrm{~h}$ (A) or oxLDL for $24 \mathrm{~h}$ (B, D). (A) OxLDL uptake was determined in protein lysates and by (B) mRNA expression of CD36 using qPCR. (C) Cholesterol efflux was measured by Tritium-labeled cholesterol loading and using apoA1 as acceptor. (D) mRNA expression of ABCA1 was determined using qPCR. Total mRNA input was corrected for the housekeeping gene 36B4. Fold change was calculated relative to oxLDL stimulation only by combining data from three independent experiments $(n=3 /$ experiment). Data are presented as mean $\pm \mathrm{SEM}$; ${ }^{*} P<0.05,{ }^{* *} P<0.01,{ }^{* * *} P<0.001$ compared to oxLDL stimulation (A, B, D) or untreated cells (C). c = control; Pred = prednisolone; MTX = methotrexate; T09 = T0901317; Ptero = pterostilbene; 6-MP = 6-mercaptopurine; Simva = simvastatin; Rapa = rapamycin; rel.= relative. 

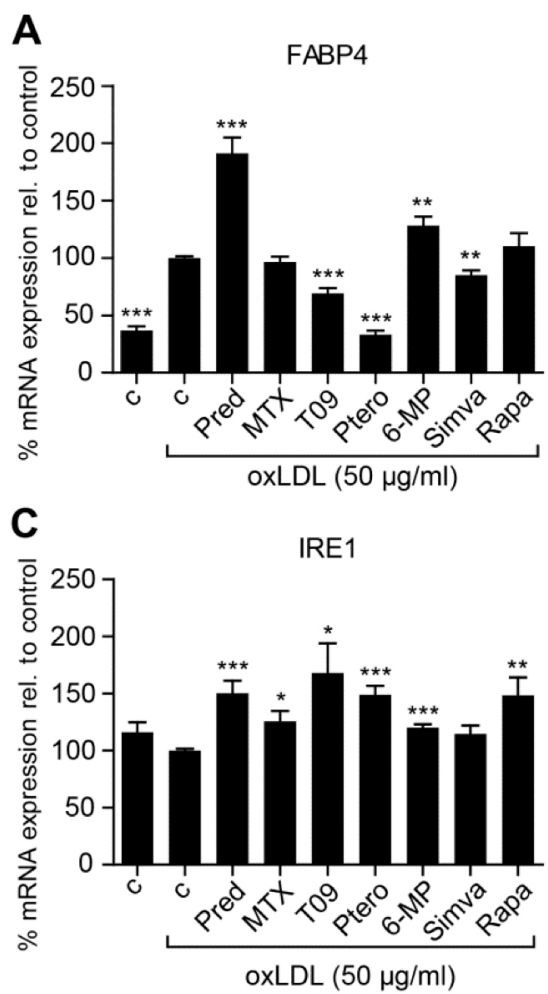

B

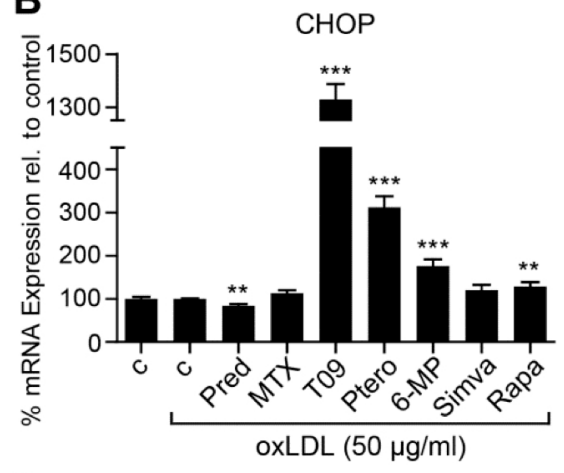

D

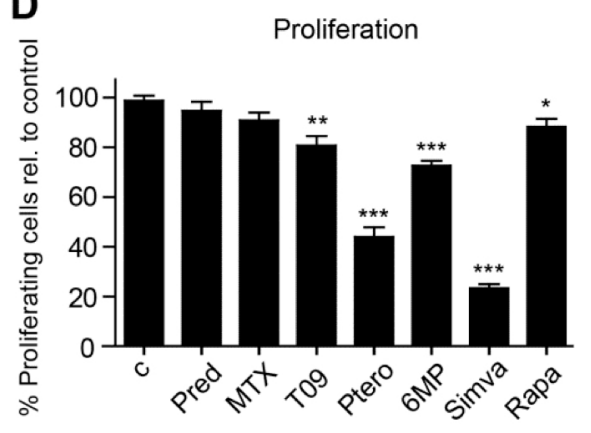

Figure 4| Macrophage oxLDL-induced ER-stress and macrophage proliferation. (A-C) To measure ER stress, THP-1 macrophages were treated with compounds and oxLDL for $24 \mathrm{~h}$. mRNA was isolated, cDNA was made and qPCR was performed for FABP4 (A), CHOP (B) and IRE1 (C). Total mRNA input was corrected for the house keeping gene 36B4. (D) Macrophage proliferation was determined in RAW264.7 cells by BrdU-incorporation after an initial serum starvation. Proliferating cells were expressed as a percentage relative to untreated controls. Three independent experiments $(n=6 /$ experiment $)$ were performed and data were combined. Data are presented as mean \pm SEM (A-D); ${ }^{*} P<0.05,{ }^{* *} P<0.01,{ }^{* * *} P<0.001$ compared to oxLDL stimulation $(\mathrm{A}-\mathrm{C})$ or untreated control (D). $\mathrm{c}=$ control; Pred = prednisolone; MTX = methotrexate; $\mathrm{T} 09$ = T0901317; Ptero = pterostilbene; 6-MP = 6- mercaptopurine; Simva = simvastatin; Rapa $=$ rapamycin; rel.= relative.

Finally, the outcomes of the assays were expressed in an atheroprotective or atherogenic score. The overall performance of each compound was assessed, with heterogeneous results between the different compounds and assays, largely in concordance with their mode of action. The constructed heat map illustrates that T09, pterostilbene, and simvastatin were identified as highest scoring compounds for anti-atherosclerotic impact on plaque macrophages (Figure 5). 


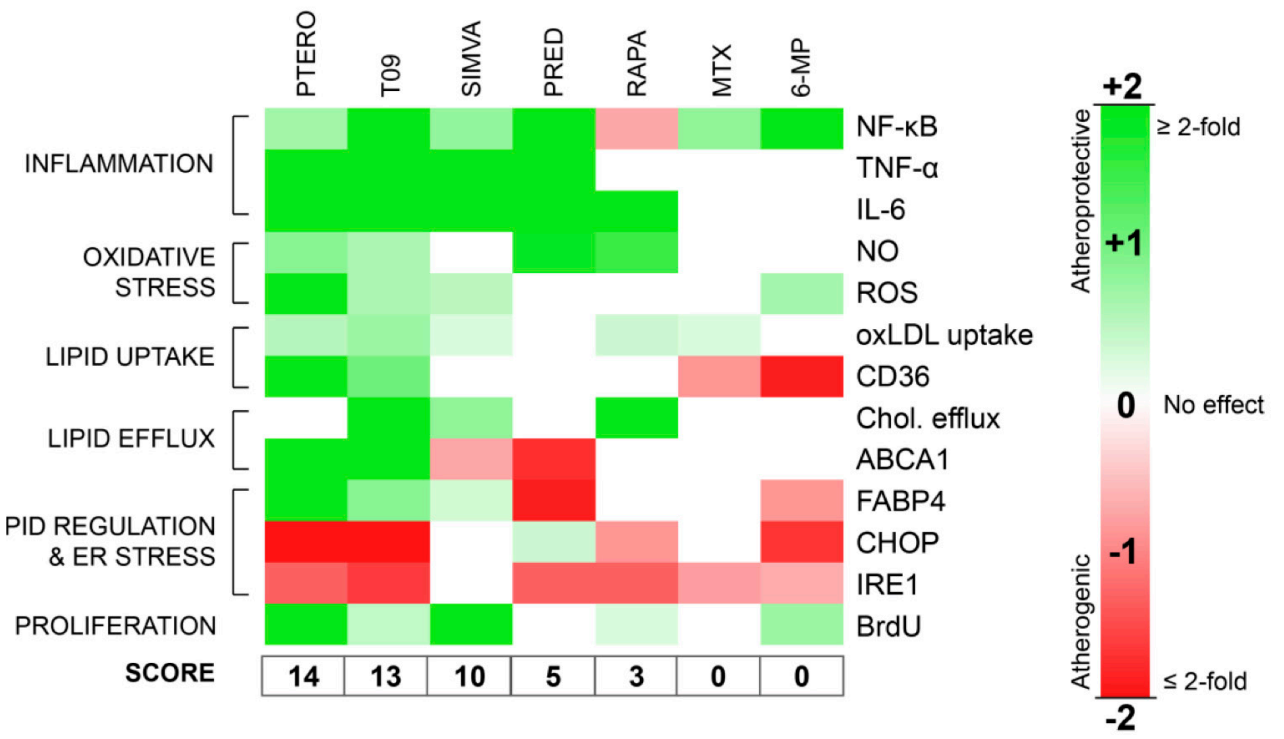

Figure 5 | Summary. Results from each macrophage assay were expressed in fold change compared to stimulated controls to reflect an atheroprotective or atherogenic effect. A heat map was generated in MultiArray viewer (MeV4.9.0). Ptero=pterostilbene; T09=T0901317; Simva=simvastatin; Pred=prednisolone; Rapa=rapamycin; MTX=methotrexate; 6-MP=6-mercaptopurine.

\section{DISCUSSION}

In this study, we assessed multiple macrophage-related pathways to evaluate the overall anti-atherogenic impact of several drug candidates. We focused on key mechanisms of macrophage activity in atherosclerotic plaques: inflammation, oxidative stress, lipid handling, ER stress, and proliferation. Using a dedicated in vitro approach, we rated the overall performance of the 7 candidate drugs known to interfere in one or more of these pathways. The overall performance of the well-known anti-inflammatory corticosteroid prednisolone was found to be counterbalanced by pro-atherogenic effects, leading to a low anti-atherogenic score. Conversely, pterostilbene, T09 and simvastatin exhibited a strong overall antiatherogenic performance in macrophages, exerting beneficial effects in multiple pathways at the same time, suggesting their potential for plaque macrophagetargeted liposomal delivery. In vivo validation studies are warranted to corroborate the predictive value of this macrophage-based screening approach and its potential value in compound selection for nanomedicinal delivery in cardiovascular patients. 


\subsection{Screening multiple pathways to estimate the anti-atherogenic impact}

We recently demonstrated successful liposomal delivery of prednisolone to plaque macrophages in patients with the advanced atherosclerotic disease [9]. Prednisolone is a potent, anti-inflammatory compound, exerting pleiotropic effects on many signaling pathways via the glucocorticoid receptor, which is widely used to reduce inflammatory activity in both acute and chronic inflammatory diseases. [21] In patients with atherosclerosis, we were, however, unable to show a reduction in overall plaque inflammatory activity with even a small increase in FDG-PET/CT signal, despite successful plaque delivery of the liposomal payload [9]. In our in vitro assays, we corroborate that prednisolone indeed potently inhibits inflammatory activity, including NF- $\kappa \mathrm{B}$ activity and attenuation of inflammatory cytokines. Concomitantly, prednisolone has a strong adverse impact on lipid handling, as it reduced expression of ABCA1 and upregulated the lipotoxicity mediator FABP4. Moreover, prednisolone activated the ER stress pathways by increasing IRE1 levels. These findings imply unsuitability of prednisolone to exert an overall antiatherogenic effect on macrophages in an atherosclerotic, lipid-rich environment.

\subsection{Promising anti-atherogenic candidates}

From the selected variety of drug candidates, simvastatin, T09 and pterostilbene were identified to have a broad anti-atherogenic effect in our assays, attributed to their beneficial impact on inflammation, oxidative stress, lipid handling, and proliferation. Simvastatin has been widely acknowledged for its marked LDL-c lowering effect [22]. Previous studies have emphasized non-lipid pleiotropic effects of statins [23]. In the present study, we corroborate a spectrum of anti-atherogenic effects on macrophages. The potential relevance of these macrophage-related effects is supported by our recent findings using targeted delivery of statins to macrophages. Namely, statins packaged within reconstituted HDL were found to be delivered effectively to plaque macrophages,[7] where statins were shown to exert potent anti-inflammatory [7] as well as anti-proliferative effects [24].

The LXR-agonist T09 has been previously shown to have strong anti-atherogenic effects mediated by both enhanced cholesterol efflux and suppression of inflammation [25-30]. Here, we indeed establish these beneficial effects on both lipid handling and inflammation, as attested by the decrease in NF- $\kappa \mathrm{B}$ activity, reduction of inflammatory cytokines, decrease in oxLDL uptake and increase in cholesterol efflux. The anti- atherogenic effect of T09 is further favored by the observed reduction of oxidative stress and a decrease in macrophage proliferation. However, clinical development has been hampered by adverse effects on hepatic lipogenesis, leading to hepatic steatosis and dyslipidemia. Local delivery to macrophages and/ or development of novel LXR-agonists may overcome these drawbacks. 
The evaluation of pterostilbene (and its analog resveratrol) in atherogenesis has so far been limited. Pterostilbene has been attributed a wide range of beneficial effects in various medical settings, including cancer prevention and therapy, neurological decline, and metabolic syndrome [31]. We here show the anti-atherogenic potential of pterostilbene on sever-al macrophage activities. Pterostilbene suppresses inflammation by decreasing NF- $\kappa \mathrm{B}$ activity strongly inhibiting the release of inflammatory cytokines, decreases oxidative stress by potently lowering the production of NO and ROS, beneficially affects lipid handling, and decreases macrophage proliferation. Together with some preliminary experimental studies showing that oral supplementation of resveratrol may reduce atherosclerosis, [3236] this favorable in vitro profile paves the way for further exploration of pterostilbene in a macrophage-targeted therapy approach.

Remarkably, we observed discrepancies between ABCA1 expression and cholesterol efflux after simvastatin and pterostilbene treatment. Although reduced ABCA1 expression may be expected based on statin-mediated inhibitory effects on LXR and SREBP-2 pathways, [37] the increase in cholesterol efflux to apoA-1 was not expected. Previous studies do not corroborate such a divergent effect [3739]. These results underline the complexities of lipid metabolism in macrophages and that mRNA expression does not equal downstream functionality. In addition, pterostilbene treatment resulted in up- regulation of ABCA1, although the cholesterol efflux assay unexpectedly did not show an increase. While data on pterostilbene in current literature is lacking, its analog resveratrol has shown similar effects on ABCA1 expression,[40] however, this was indeed coupled with an increase in cholesterol efflux [41,42]. Further investigations are warranted to assess whether pterostilbene has inherently different effects on cholesterol efflux than resveratrol, or that this is due to differences in experimental setup.

\subsection{Compounds potentially lacking anti-atherogenic impact}

Despite demonstrating clear prednisolone-like anti-inflammatory effects, we find that 6- MP and MTX fail to convince in our in vitro screening as anti-atherogenic compounds, considering their lack of efficacy in non-inflammatory pathways. Preclinical work for 6-MP in atherosclerosis has so far been scarce [43] and reports from clinical use in inflammatory disorders lean towards an increased cardiovascular risk [44-46]. In contrast, MTX is seen as a viable compound for atherosclerosis. Currently, a low-dose of MTX is being evaluated as add-on therapy in cardiovascular patients in the Cardiovascular Inflammation Reduction Trial (CIRT) [47]. Even though MTX did not cumulate any points in our heat map, overall it appears to be a relatively neutral compound without any excessive atherogenic pathway effects. With this in mind, we await with interest the results of CIRT; although not specifically plaque targeted, 
these results will add to our understanding of the value and relative contribution of the separate pathways in our assays.

Similar to MTX, rapamycin also exhibited a generally neutral effect in our assays with a low total score. While already applied for over a decade in drug eluting coronary stents, targeted delivery strategies seem to be underway $[48,49]$ following anti-atherogenic efficacy in mice models of atherosclerosis [50-57].

\subsection{Limitations}

Our study has several limitations. First, we assess the effects of the compounds only on macrophage activity, thereby disregarding potentially beneficial or harmful effects on other relevant plaque cell types, such as endothelial cells, smooth muscle cells and immune cells other than macrophages [2]. However, since we intend to employ a targeted drug delivery system based on endothelial permeability and phagocytosis, it is reasonable to expect the largest and most relevant impact on plaque macrophages. In addition, we used compounds in their free (unencapsulated) form in our macrophage assays. As a next step, experiments using encapsulated formulations of promising compounds are to be performed to assess any additional effects of the delivery vehicle itself. Lastly, we use only in vitro tests, mimicking an atherogenic environment by adding atherogenic factors such as oxLDL to monocyte/ macrophage cell lines. However, the extrapolation of in vitro tests towards clinical impact remains to be established. Hence, in vivo validation testing is warranted to determine the value of our screening assays as a translational tool in atherosclerotic disease. Thus far, earlier in vivo experiments with nano-delivery of simvastatin [7] and prednisolone [9] appear to be in agreement with our in vitro data, hinting at the feasibility of the currently employed in vitro testing strategy.

\subsection{Future perspectives}

Nanomedicinal drug delivery has the potential to take cardiovascular disease management to the next level. Transitioning from previous work in which we focused on targeting inflammation as the main driver behind atherosclerosis, the current study clearly underlines the need to broaden our focus and take a wide array of macrophage-related processes into account for nanomedicinal drug development. Out of a variety of drug candidates, we identified T09, pterostilbene and simvastatin as potential anti-atherogenic compounds for plaque macrophage-targeted therapy. Our current assay setup provides a promising framework for relatively high-throughput screening, as it offers a rapid and comprehensive method to simultaneously screen multiple drug candidates or genuine unknown putative candidates for their antiatherogenic potency. We envision our work will facilitate the process of bringing novel cardiovascular treatment strategies from bench to bedside. 


\section{REFERENCES}

[1] Mozaffarian D, Benjamin EJ, Go AS, Arnett DK, Blaha MJ, Cushman M, et al. Heart Disease and Stroke Statistics-2015 Update: A Report From the American Heart Association. Circulation 2014;131:e29-322. doi:10.1161 / CIR.0000000000000152.

[2] Libby P. Inflammation in atherosclerosis. Nature 2012;420:868-74. doi:10.1038 / nature01323.

[3] Efficacy and safety of LDL-lowering therapy among men and women: meta-analysis of individual data from 174000 participants in 27 randomised trials. Lancet 2015. doi:10.1016/ S0140-6736(14)61368-4.

[4] Libby P. The forgotten majority: unfinished business in cardiovascular risk reduction. J Am Coll Cardiol 2005;46:1225-8. doi:10.1016/j.jacc.2005.07.006.

[5] Tiwari RL, Singh V , Barthwal MK. Macrophages: an elusive yet emerging therapeutic target of atherosclerosis. Med Res Rev 2008;28:483-544. doi:10.1002/med.20118.

[6] Lobatto ME, Fuster V, Fayad ZA, Mulder WJM. Perspectives and opportunities for nanomedicine in the management of atherosclerosis. Nat Rev Drug Discov 2011;10:83552. doi:10.1038/nrd3578.

[7] Duivenvoorden R, Tang J, Cormode DP, Mieszawska AJ, Izquierdo-Garcia D, Ozcan C, et al. Astatin-loaded reconstituted high-density lipoprotein nanoparticle inhibits atherosclerotic plaque inflammation. Nat Commun 2014;5:3065. doi:10.1038/ncomms4065.

[8] Lobatto ME, Fayad ZA, Silvera S, Vucic E, Calcagno C, Mani V, et al. Multimodal clinical imaging to longitudinally assess a nanomedical anti-inflammatory treatment in experimental atherosclerosis. Mol Pharm 2010;7:2020-9. doi:10.1021/mp100309y.

[9] Der Valk FM van, van Wijk DF, Lobatto ME, Verberne HJ, Storm G, Willems MCM, et al. Prednisolone- containing liposomes accumulate in human atherosclerotic macrophages upon intravenous administration. Nanomedicine 2015. doi:10.1016/j.nano.2015.02.021.

[10] Qin Z. The use of THP-1 cells as a model for mimicking the function and regulation of monocytes and macrophages in the vasculature. Atherosclerosis 2012;221:2-11. doi:10.1016/j.atherosclerosis.2011.09.003.

[11] Groeneweg M, Kanters E, Vergouwe MN, Duerink H, Kraal G, Hofker MH, et al. Lipopolysaccharide- induced gene expression in murine macrophages is enhanced by prior exposure to oxLDL. J Lipid Res 2006;47:2259-67. doi:10.1194/jlr.M600181-JLR200.

[12] Hamers AAJ, Vos M, Rassam F, Marinković G, Marincovic G, Kurakula K, et al. Bone marrow-specific deficiency of nuclear receptor Nur77 enhances atherosclerosis. Circ Res 2012;110:428-38. doi:10.1161/CIRCRESAHA.111.260760.

[13] Sylvester PW. Optimization of the tetrazolium dye (MTT) colorimetric assay for cellular growth and viability. Methods Mol Biol 2011;716:157-68. doi:10.1007/978-1-61779-012-6-9.

[14] Fang FC, Vazquez-Torres A. Nitric oxide production by human macrophages: there's NO doubt about it. Am J Physiol Lung Cell Mol Physiol 2002;282:L941-3. doi:10.1152/ ajplung.00017.2002.

[15] Auwerx J, Staels B, Van Vaeck F, Ceuppens JL. Changes in IgG Fc receptor expression induced by phorbol 12-myristate 13-acetate treatment of THP-1 monocytic leukemia cells. Leuk Res 1992;16:317-27. doi:10.1016/0145-2126(92)90070-N.

[16] Saksi J, Ijäs P, Mäyränpää MI, Nuotio K, Isoviita PM, Tuimala J, et al. Low-expression variant of fatty acid- binding protein 4 favors reduced manifestations of atherosclerotic disease and increased plaque stability. Circ Cardiovasc Genet 2014;7:588-98. doi:10.1161/ CIRCGENETICS.113.000499.

[17] Makowski L, Boord JB, Maeda K, Babaev VR, Uysal KT, Morgan MA, et al. Lack of macrophage fatty-acid- binding protein aP2 protects mice deficient in apolipoprotein $\mathrm{E}$ 
against atherosclerosis. Nat Med 2001;7:699-705. doi:10.1038/89076.

[18] Layne MD, Patel A, Chen YH, Rebel VI, Carvajal IM, Pellacani A, et al. Role of macrophageexpressed adipocyte fatty acid binding protein in the development of accelerated atherosclerosis in hypercholesterolemic mice. FASEB J 2001;15:2733-5. doi:10.1096/fj.010374fje.

[19] Erbay E, Babaev VR, Mayers JR, Makowski L, Charles KN, Snitow ME, et al. Reducing endoplasmic reticulum stress through a macrophage lipid chaperone alleviates atherosclerosis. Nat Med 2009;15:1383-91. doi:10.1038/nm.2067.

[20] Scull CM, Tabas I. Mechanisms of ER stress-induced apoptosis in atherosclerosis. Arterioscler Thromb Vasc Biol 2011;31:2792-7. doi:10.1161 / ATVBAHA.111.224881.

[21] Rhen T, Cidlowski JA. Antiinflammatory action of glucocorticoids--new mechanisms for old drugs. N Engl J Med 2005;353:1711-23. doi:10.1056/NEJMra050541.

[22] Baigent C, Keech A, Kearney PM, Blackwell L, Buck G, Pollicino C, et al. Efficacy and safety of cholesterol- lowering treatment: prospective meta-analysis of data from 90,056 participants in 14 randomised trials of statins. Lancet 2005;366:1267-78. doi:10.1016/ S0140-6736(05)67394-1.

[23] Liao JK, Laufs U. Pleiotropic effects of statins. Annu Rev Pharmacol Toxicol 2005;45:89118. doi:10.1146/ annurev.pharmtox.45.120403.095748.

[24] Tang J, Lobatto ME, Hassing L, van der Staay S, van Rijs SM, Calcagno C, et al. Inhibiting macrophage proliferation suppresses atherosclerotic plaque inflammation. Sci Adv 2015;1:e1400223-e1400223. doi:10.1126/sciadv.1400223.

[25] Grefhorst A, Elzinga BM, Voshol PJ, Plösch T, Kok T, Bloks VW, et al. Stimulation of lipogenesis by pharmacological activation of the liver $\mathrm{X}$ receptor leads to production of large, triglyceride-rich very low density lipoprotein particles. J Biol Chem 2002;277:3418290. doi:10.1074/jbc.M204887200.

[26] Terasaka N, Hiroshima A, Koieyama T, Ubukata N, Morikawa Y, Nakai D, et al. T-0901317, a synthetic liver $\mathrm{X}$ receptor ligand, inhibits development of atherosclerosis in LDL receptordeficient mice. FEBS Lett 2003;536:6- 11. doi:10.1016/S0014-5793(02)03578-0.

[27] Verschuren L, de Vries-van der Weij J, Zadelaar S, Kleemann R, Kooistra T. LXR agonist suppresses atherosclerotic lesion growth and promotes lesion regression in apoE*3Leiden mice: time course and mechanisms. J Lipid Res 2009;50:301-11. doi:10.1194/jlr.M800374JLR200.

[28] Honzumi S, Shima A, Hiroshima A, Koieyama T, Terasaka N. Synthetic LXR agonist inhibits the development of atherosclerosis in New Zealand White rabbits. Biochim Biophys Acta 2011;1811:1136-45. doi:10.1016/j.bbalip.2011.08.009.

[29] Chen J, Zhao L, Sun D, Narsinh K, Li C, Zhang Z, et al. Liver X receptor activation attenuates plaque formation and improves vasomotor function of the aortic artery in atherosclerotic ApoE ${ }^{-1-}$ mice. Inflamm Res 2012;61:1299-307. doi:10.1007/ s00011-012-0529-4.

[30] Srivastava RAK. Evaluation of anti-atherosclerotic activities of PPAR- $\alpha$, PPAR- $\gamma$, and LXR agonists in hyperlipidemic atherosclerosis-susceptible $\mathrm{F}(1) \mathrm{B}$ hamsters. Atherosclerosis 2011;214:86-93. doi:10.1016/j.atherosclerosis.2010.10.033.

[31] Estrela JM, Ortega A, Mena S, Rodriguez ML, Asensi M. Pterostilbene: Biomedical applications. Crit Rev Clin Lab Sci 2013;50:65-78. doi:10.3109/10408363.2013.805182.

[32] Wang Z, Zou J, Cao K, Hsieh T-C, Huang Y, Wu JM. Dealcoholized red wine containing known amounts of resveratrol suppresses atherosclerosis in hypercholesterolemic rabbits without affecting plasma lipid levels. Int J Mol Med 2005;16:533-40.

[33] Do G-M, Kwon E-Y, Kim H-J, Jeon S-M, Ha T-Y, Park T, et al. Long-term effects of resveratrol supplementation on suppression of atherogenic lesion formation and cholesterol synthesis in apoE-deficient mice. Biochem Biophys Res Commun 2008;374:55-9. doi:10.1016/j. bbrc.2008.06.113. 
[34] Matos RS, Baroncini LAV, Précoma LB, Winter G, Lambach PH, Caron EY, et al. Resveratrol causes antiatherogenic effects in an animal model of atherosclerosis. Arq Bras Cardiol 2012;98:136-42.

[35] Berbée JFP, Wong MC, Wang Y, van der Hoorn JWA, Khedoe PPSJ, van Klinken JB, et al. Resveratrol protects against atherosclerosis, but does not add to the antiatherogenic effect of atorvastatin, in APOE*3- Leiden.CETP mice. J Nutr Biochem 2013;24:1423-30. doi:10.1016/j.jnutbio.2012.11.009.

[36] Tomayko EJ, Cachia AJ, Chung HR, Wilund KR. Resveratrol supplementation reduces aortic atherosclerosis and calcification and attenuates loss of aerobic capacity in a mouse model of uremia. J Med Food 2014;17:278-83. doi:10.1089/jmf.2012.0219.

[37] Niesor EJ, Schwartz GG, Perez A, Stauffer A, Durrwell A, Bucklar-Suchankova G, et al. Statin-induced decrease in ATP-binding cassette transporter A1 expression via microRNA33 induction may counteract cholesterol efflux to high-density lipoprotein. Cardiovasc Drugs Ther 2015;29:7-14. doi:10.1007/s10557-015- 6570-0.

[38] Qiu G, Hill JS. Atorvastatin inhibits ABCA1 expression and cholesterol efflux in THP1 macrophages by an LXR-dependent pathway. J Cardiovasc Pharmacol 2008;51:388-95. doi:10.1097 / FJC.0b013e318167141f.

[39] Wong J, Quinn CM, Brown AJ. Statins inhibit synthesis of an oxysterol ligand for the liver $x$ receptor in human macrophages with consequences for cholesterol flux. Arterioscler Thromb Vasc Biol 2004;24:2365-71. doi:10.1161/01.A TV .0000148707.93054.7d.

[40] Sevov M, Elfineh L, Cavelier LB. Resveratrol regulates the expression of LXR-alpha in human macrophages. Biochem Biophys Res Commun 2006;348:1047-54. doi:10.1016/j. bbrc.2006.07.155.

[41] Allen AM, Graham A. Mitochondrial function is involved in regulation of cholesterol efflux to apolipoprotein (apo)A-I from murine RAW 264.7 macrophages. Lipids Health Dis 2012;11:169. doi:10.1186/1476-511X-11-169.

[42] Voloshyna I, Hai O, Littlefield MJ, Carsons S, Reiss AB. Resveratrol mediates antiatherogenic effects on cholesterol flux in human macrophages and endothelium via PP AR $\gamma$ and adenosine. Eur J Pharmacol 2013;698:299-309. doi:10.1016/j.ejphar.2012.08.024.

[43] Pols TWH, Bonta PI, Pires NMM, Otermin I, Vos M, de Vries MR, et al. 6-mercaptopurine inhibits atherosclerosis in apolipoprotein $\mathrm{e}^{*} 3$-leiden transgenic mice through atheroprotective actions on monocytes and macrophages. Arterioscler Thromb Vasc Biol 2010;30:1591-7. doi:10.1161/ ATVBAHA.110.205674.

[44] Solomon DH, Avorn J, Katz JN, Weinblatt ME, Setoguchi S, Levin R, et al. Immunosuppressive medications and hospitalization for cardiovascular events in patients with rheumatoid arthritis. Arthritis Rheum 2006;54:3790- 8. doi:10.1002 / art.22255.

[45] Vanrenterghem YFC, Claes K, Montagnino G, Fieuws S, Maes B, Villa M, et al. Risk factors for cardiovascular events after successful renal transplantation. Transplantation 2008;85:209-16. doi:10.1097/TP .0b013e318160254f.

[46] Haque S, Gordon C, Isenberg D, Rahman A, Lanyon P, Bell A, et al. Risk factors for clinical coronary heart disease in systemic lupus erythematosus: the lupus and atherosclerosis evaluation of risk (LASER) study. J Rheumatol 2010;37:322-9. doi:10.3899/jrheum.090306.

[47] Everett BM, Pradhan AD, Solomon DH, Paynter N, Macfadyen J, Zaharris E, et al. Rationale and design of the Cardiovascular Inflammation Reduction Trial: a test of the inflammatory hypothesis of atherothrombosis. Am Heart J 2013;166:199-207.e15. doi:10.1016/j.ahj.2013.03.018.

[48] Miao Z-L, Deng Y-J, DU H-Y, Suo X-B, Wang X-Y, Wang X, et al. Preparation of a liposomal delivery system and its in vitro release of rapamycin. Exp Ther Med 2015;9:941-6. doi:10.3892/ etm.2015.2201.

[49] Kilroy JP, Dhanaliwala AH, Klibanov AL, Bowles DK, Wamhoff BR, Hossack JA. 
Reducing neointima formation in a swine model with IVUS and sirolimus microbubbles. Ann Biomed Eng 2015. doi:10.1007/s10439- 015-1315-6.

[50] Beutner F, Brendel D, Teupser D, Sass K, Baber R, Mueller M, et al. Effect of everolimus on pre-existing atherosclerosis in LDL-receptor deficient mice. Atherosclerosis 2012;222:33743. doi:10.1016/j.atherosclerosis.2012.03.003.

[51] Waksman R, Pakala R, Burnett MS, Gulick CP, Leborgne L, Fournadjiev J, et al. Oral rapamycin inhibits growth of atherosclerotic plaque in apoE knock-out mice. Cardiovasc Radiat Med 2003;4:34-8.

[52] Zhao L, Ding T, Cyrus T, Cheng Y, Tian H, Ma M, et al. Low-dose oral sirolimus reduces atherogenesis, vascular inflammation and modulates plaque composition in mice lacking the LDL receptor. Br J Pharmacol 2009;156:774-85. doi:10.1111/j.1476-5381.2008.00080.x.

[53] Mueller MA, Beutner F, Teupser D, Ceglarek U, Thiery J. Prevention of atherosclerosis by the mTOR inhibitor everolimus in LDLR-/ - mice despite severe hypercholesterolemia. Atherosclerosis 2008;198:39-48. doi:10.1016/j.atherosclerosis.2007.09.019.

[54] Pakala R, Stabile E, Jang GJ, Clavijo L, Waksman R. Rapamycin attenuates atherosclerotic plaque progression in apolipoprotein $\mathrm{E}$ knockout mice: inhibitory effect on monocyte chemotaxis. J Cardiovasc Pharmacol 2005;46:481-6.

[55] Castro C, Campistol JM, Sancho D, Sánchez-Madrid F, Casals E, Andrés V. Rapamycin attenuates atherosclerosis induced by dietary cholesterol in apolipoprotein-deficient mice through a p27 Kip1 -independent pathway. Atherosclerosis 2004;172:31-8.

[56] Basso MD, Nambi P, Adelman SJ. Effect of sirolimus on the cholesterol content of aortic arch in ApoE knockout mice. Transplant Proc 2003;35:3136-8.

[57] Elloso MM, Azrolan N, Sehgal SN, Hsu P-L, Phiel KL, Kopec CA, et al. Protective effect of the immunosuppressant sirolimus against aortic atherosclerosis in apo E-deficient mice. Am J Transplant 2003;3:562-9. 


\section{CHAPTER 4 Multiple Pathway Assessment to Predict Anti-Atherogenic Efficacy of Drugs}

\section{APPENDIX: SUPPLEMENTARY DATA}

Supplementary Table S1 I Human primer sequences used for semi-quantitative real-time PCR

\begin{tabular}{lll}
\hline Gene & Forward primer sequence (5`3`) & Reverse primer sequence (5`3`) \\
\hline 36 B4 & ACGGGTACAAACGAGTCCTG & GCCTTGACCTTTTCAGCAAG \\
CD36 & TGCAAAATCCACAGGAAGTG & CAGCGTCCTGGGTTACATTT \\
ABCA1 & ATGAGGACAACAACTACAAAGCC & GGGAAAGAGGACTAGACTCCAAA \\
ABCG1 & TCCTATGTCAGGTATGGGTTCG & CGTCTCGTCGATGTCACAGT \\
FABP4 & AGCACCATAACCTTAGATGGGG & CGTGGAAGTGACGCCTTTCA \\
CHOP & TTGCCTTTCTCCTTCGGGAC & GA TTCTTCCTCTTCA TTTCCAGGAG \\
PERK & GGA TGAA TGGACGA TGT A & A T A TGTTGGA TGGCTTGA \\
IRE1 & GAAGCA TGTGCTCAAACACC & TCTGTCGCTCACGTCCTG \\
ATF6 & CCTGTCCTACAAAGTACCATGAG & CCTTTAATCTCGCCTCTAACCC \\
\hline
\end{tabular}



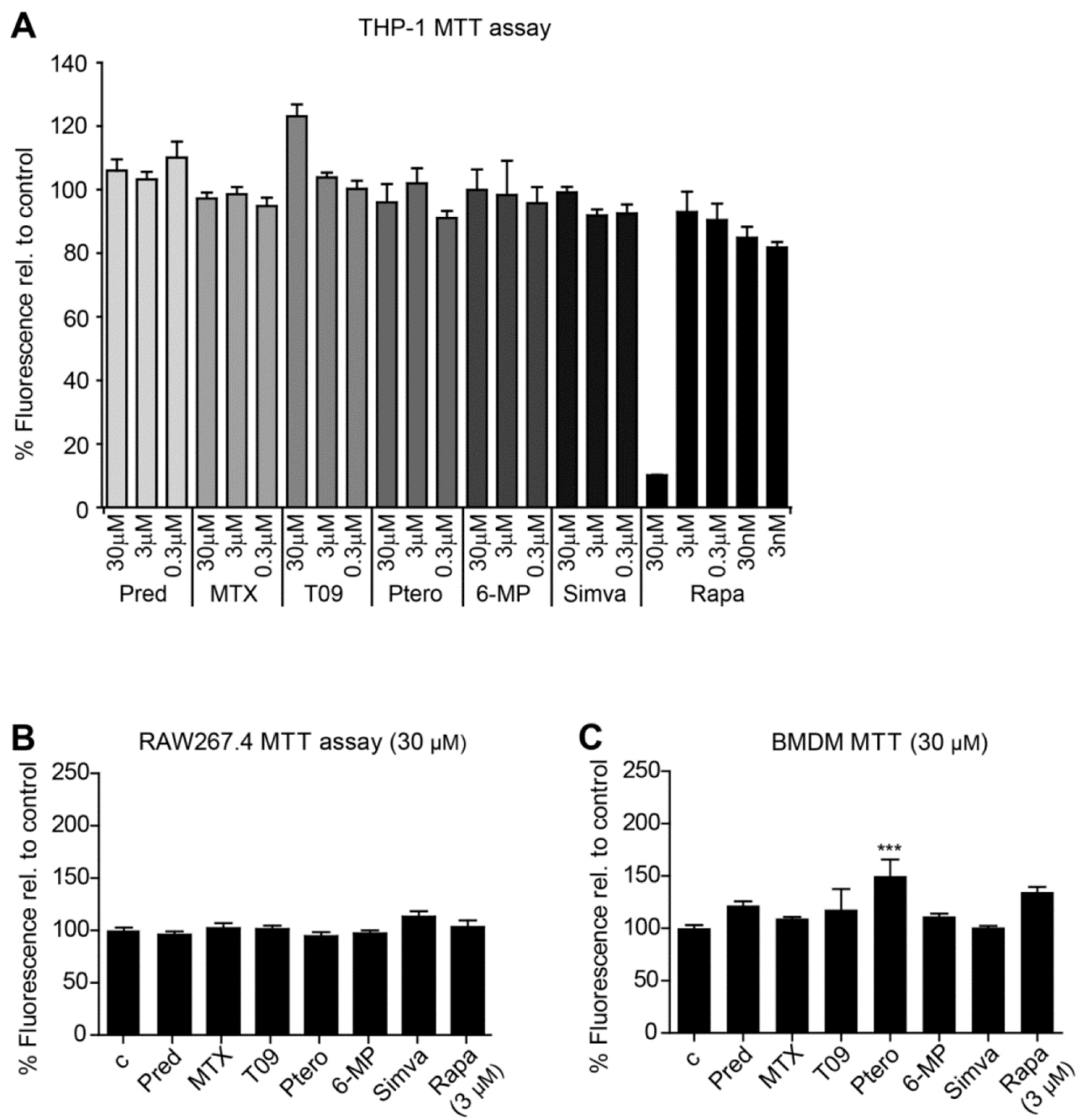

Supplemental Figure S1 I Cell viability was determined by measuring active mitochondria using MTT. Cell viability was calculated as a percentage from untreated cells. A 50\% decrease in fluorescence was considered toxic. Data was combined from two independent experiments $(n=5)$ and are presented as mean \pm SEM; ${ }^{*} \mathrm{P}<0.05,{ }^{* *} \mathrm{P}<0.01,{ }^{* *} \mathrm{P}<0.001$ compared to control. 

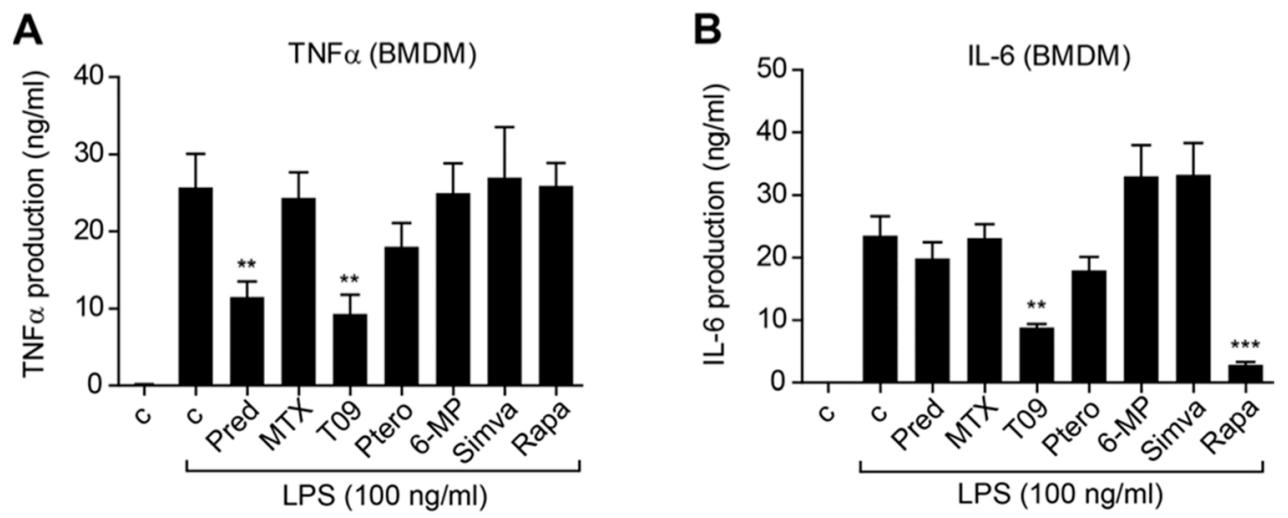

Supplemental Figure S2 | Pro-inflammatory cytokines. Bone marrow-derived macrophages (BMDM) were treated with the compounds and stimulated with LPS (100 ng/mL) for $24 \mathrm{~h}$. TNF $\alpha$ and IL-6 production were measured using a cytometric bead array. Data was combined from three independent experiments ( $\mathrm{n}=6$ / experiment) and are presented as mean \pm SEM; ${ }^{*} P<0.05,{ }^{* *} P<0.01,{ }^{* * *} P<0.001$ compared to LPS stimulation only.

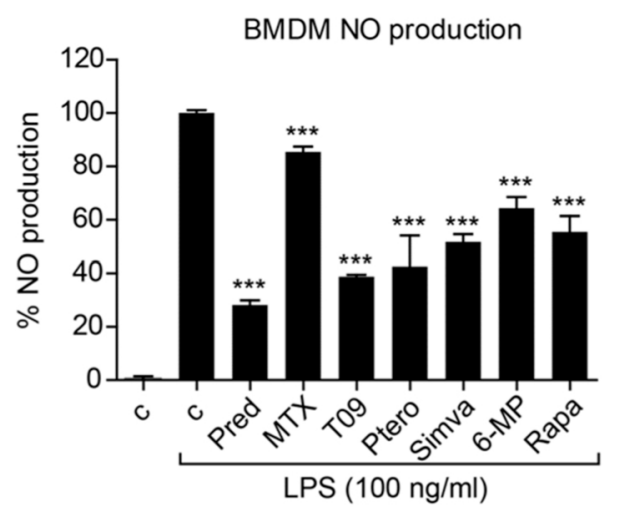

Supplemental Figure S3 I BMDM were treated with the compounds and stimulated with LPS (100 ng/ $\mathrm{mL}$ ) for $24 \mathrm{~h}$ after which nitric oxide (NO) production was determined with Griess reagent. Fold change was calculated relative to LPS stimulation only (c) by combining data from three independent experiments ( $\mathrm{n}=3$ / experiment). Data are presented as mean $\pm \mathrm{SEM}$; ${ }^{*} P<0.05,{ }^{* *} P<0.01,{ }^{* * *} P<0.001$ compared to LPS only. $\mathrm{c}$ = control; Pred = prednisolone; MTX = methotrexate; T09 = T0901317; Ptero = pterostilbene; 6-MP = 6-mercaptopurine; Simva $=$ simvastatin; Rapa $=$ rapamycin . 


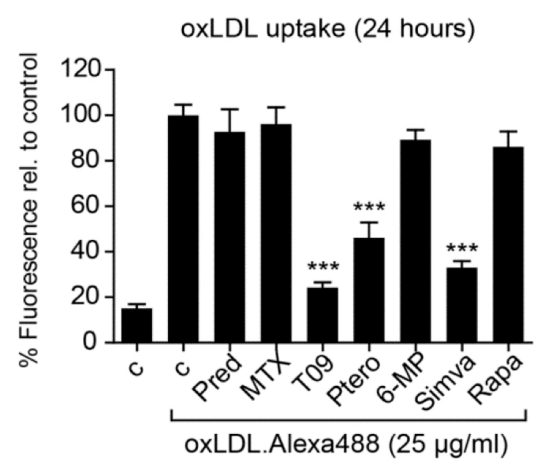

Supplemental Figure S4I THP-1 macrophages were treated with compounds and ox-LDL-DyLight488 for $24 \mathrm{~h}$ and oxLDL uptake was determined in protein lysates. Fold change was calculated relative to oxLDL stimulation only by combining data from three independent experiments ( $\mathrm{n}=3$ / experiment). Data are presented as mean $\pm \mathrm{SEM} ;{ }^{*} P<0.05,{ }^{* *} P<0.01$, ${ }^{* * *} P<0.001$ compared to oxLDL stimulation only.

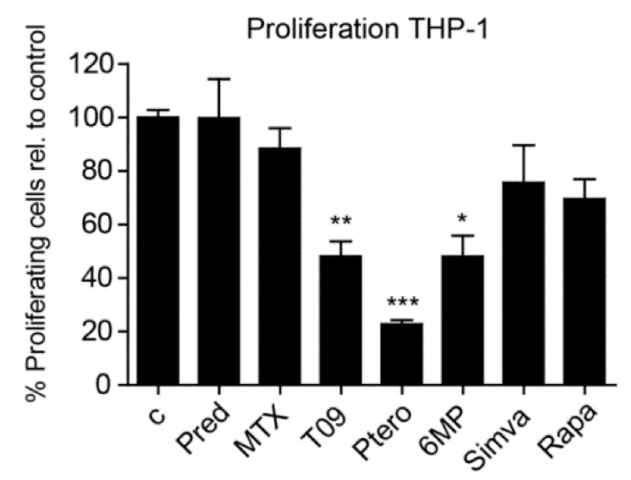

Supplemental Figure S5I Macrophage proliferation was determined in THP-1 cells by BrdUincorporation after an initial serum starvation. Proliferating cells were expressed as a percentage relative to untreated controls. Data are presented as mean $\pm S E M ;{ }^{*} P<0.05,{ }^{* *} P<0.01,{ }^{* *} P<0.001$. 

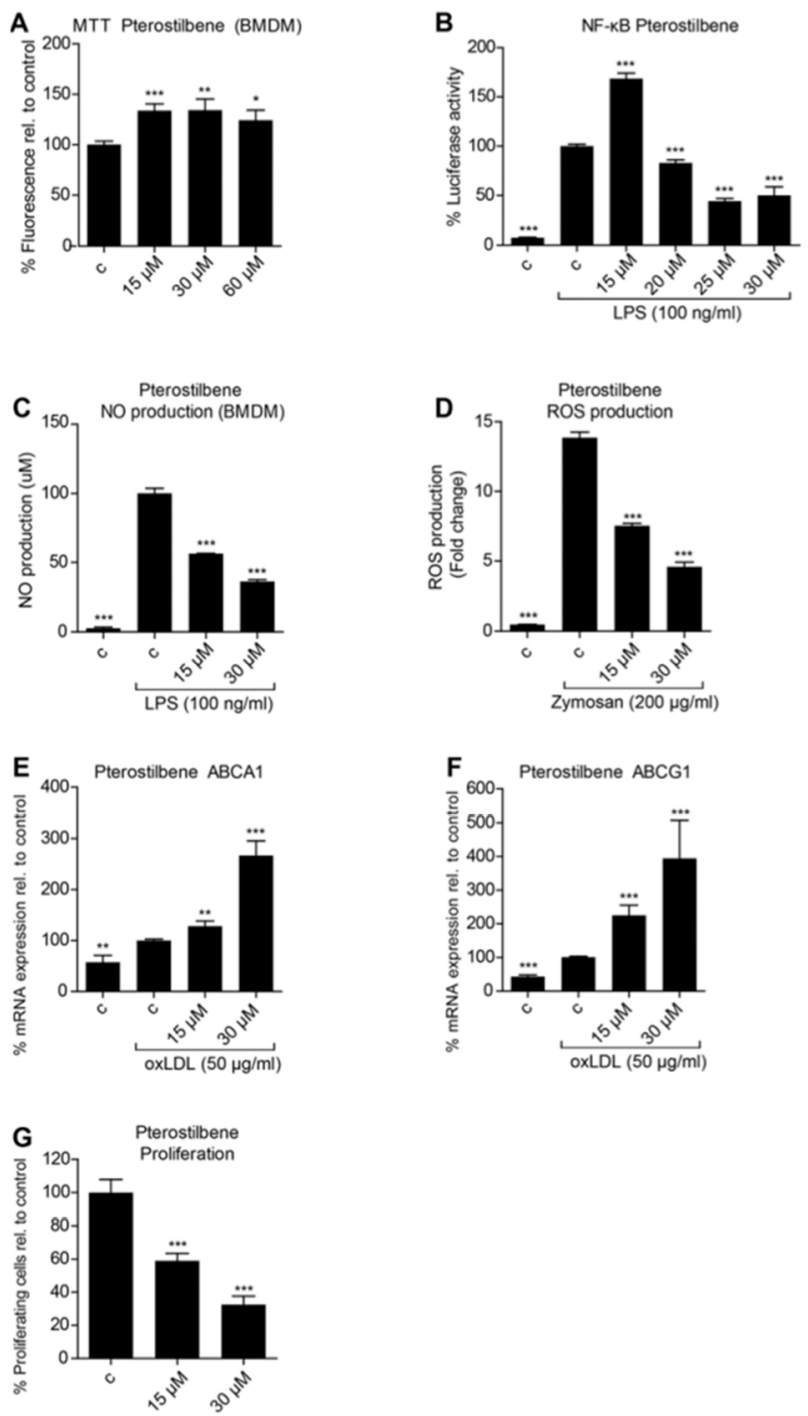

Supplemental Figure S6 I Pterostilbene: dose-dependent effects. The macrophage modulating effects of pterostilbene were further investigated. (A) Cell viability was determined by measuring active mitochondria using MTT in bone-marrow derived macrophages. (B) NF-kB activity after treatment with different concentrations of pterostilbene was determined by luciferase assay. (C) BMDM were treated with pterostilbene and stimulated with LPS $(100 \mathrm{ng} / \mathrm{mL})$ for $24 \mathrm{~h}$ after which nitric oxide (NO) production was determined with Griess reagent. (D) PMNs were treated with pterostilbene and stimulated with zymosan to measure the inhibition of reactive oxygen species. $(\mathrm{E}+\mathrm{F})$ mRNA expression of ABCA1 and ABCG1 was determined using qPCR. Total mRNA input was corrected for the housekeeping gene 36B4. (G) Macrophage proliferation was determined in RAW264.7 cells by BrdU-incorporation after an initial serum starvation. Data from each experiment was combined from three independent experiments $(n=3)$ and are presented as mean $\pm \mathrm{SEM}$; ${ }^{*} P<0.05,{ }^{* *} P<0.01,{ }^{* * *} P<0.001$ compared to control. 




\section{CHAPTER 5}

\section{Immune Cell Screening of a Nanoparticle Library Improves Atherosclerosis Therapy*}

\section{ABSTRACT}

I

mmunological complexity in atherosclerosis warrants targeted treatment of specific inflammatory cells that aggravate the disease. With the initiation of large phase III trials investigating immunomodulatory drugs for atherosclerosis, cardiovascular disease treatment enters a new era. We here propose a radically different approach: implementing and evaluating in vivo a combinatorial library of nanoparticles with distinct physiochemical properties and differential immune cell specificities. The library's nanoparticles are based on endogenous high-density lipoprotein, which can preferentially deliver therapeutic compounds to pathological macrophages in atherosclerosis. Using the apolipoprotein E-deficient $\left(\right.$ Apoe $\left.e^{-1-}\right)$ mouse model of atherosclerosis, we quantitatively evaluated the library's immune cell specificity by combining immunological techniques and in vivo positron emission tomography imaging. Based on this screen, we formulated a liver X receptor agonist (GW3965) and abolished its liver toxicity while still preserving its therapeutic function. Screening the immune cell specificity of nanoparticles can be used to develop tailored therapies for atherosclerosis and other inflammatory diseases. 


\section{INTRODUCTION}

Research in the past decades has revealed the immune system's central role in the pathophysiology of cancer,[1] diabetes,[2] and atherosclerosis [3, 4]. Because macrophages drive pathological progression of these diseases, immunomodulatory small-molecule compounds modulating macrophage function are promising therapeutic candidates for treating these maladies. However, these compounds' lack of cellular specificity necessitates a strategy for targeted delivery to harmful immune cells without negatively affecting beneficial immune cells. Despite numerous studies of nanoparticle-based delivery, rational attempts to screen meticulously designed nanoparticles for immune cell specificity in vivo have never been reported.

We created a combinatorial library of hybrid lipoprotein-inspired nanoparticles with distinct physiochemical properties (particularly size and chemical composition) with differential immune cell specificity. We then chose atherosclerosis, a lipiddriven inflammatory process of the large arteries, as a model disease to evaluate our nanoparticle library. Atherosclerosis accounts for the majority of cardiovascular deaths worldwide [5], and macrophages are the major immune cells to drive the pathological inflammation and the progression of atherosclerotic plaques [6, 7]. These plaques, which are present throughout the vasculature, have a complicated cellular composition [8]. To improve the therapeutic index of small-molecule immunomodulatory compounds, plaque macrophage-specific delivery, with minimal delivery to nonpathological cells in healthy tissues, is essential.

We used the apolipoprotein E-deficient $\left(\mathrm{Apoe}^{-1-}\right)$ mouse model of atherosclerosis to evaluate our nanoparticle library because it accurately recapitulates some important immunological aspects of human atherosclerosis [9]. Using a combination of optical methods, immunological techniques, and in vivo positron emission tomography (PET) imaging, we carefully selected candidate nanoparticles from the library for subsequent atherosclerosis drug delivery studies. As a proof of concept, we incorporated the liver X receptor agonist GW3965, a therapeutic compound that did not reach clinical application due to its serious adverse effects on the liver,[10, 11] in two nanoparticles-one with favorable organ distribution and immune cell specificity and one without. In $A p o e^{-1-}$ mice with the advanced disease, the favorable nanoparticle from the library screen was shown to abolish GW3965's liver toxicity while remaining effective on atherosclerotic plaque macrophages. 


\section{RESULTS}

\subsection{Study Design}

We created a combinatorial library comprising 15 high-density lipoproteinmimicking nanoparticles and two extensively studied nanoparticles, a PEGylated micellar and a long-circulating liposomal nanoparticle. We then combined in vitro assays and in vivo experiments in atherosclerotic Apoe ${ }^{-1-}$ mice to study the nanoparticle library's biological behavior by using near-infrared fluorescence imaging (NIRF), flow cytometry, immunofluorescence, and radiolabeling. Based on the results of this library screening, we formulated two GW3965-loaded HDL nanoparticles with distinctly different immune cell specificity and organ distribution. Finally, we quantitatively studied the pharmacokinetics, immune cell specificity, liver toxicity, and therapeutic effects of these drug-loaded nanoparticles in $A p o e^{-I-}$ mice with advanced atherosclerosis (Figure 1A).

\subsection{HDL Nanoparticle Library}

Previous studies indicate that the size, phospholipid composition, ratio of phospholipid to apolipoprotein A-1 (APOA1), or the inclusion of payloads can affect HDL-mimicking nanoparticles' in vivo performance [12-14]. In our current study, we created a library containing HDL-mimicking nanoparticles that differ in size, shape, composition, and payload, all of which have been reported to affect nanoparticles' in vivo targeting efficiency [15]. To fine-tune nanoparticle size and morphology, we added either triglyceride or polymers [polylactic-co-glycolic acid (PLGA) or polylactic acid (PLA)] to the HDL core (Table S1); this allowed us to modulate nanoparticle size from about $10 \mathrm{~nm}$ (NP1, NP2, NP3, and NP9) or $30 \mathrm{~nm}$ (NP6, NP7, NP10, and NP11) to over $100 \mathrm{~nm}$ (NP12) (Figure 1B and C). We observed that the inclusion of a core component, namely triglycerides or polymers, results in a nanoparticle shape change from discoidal to spherical, as can be clearly appreciated when comparing NP5 to NP7 and NP15. In addition to size and shape, we also varied phospholipid composition (Table S1). Because oxidization greatly affects HDL function,[16] we sought to test if this modification also changed the HDL-mimicking nanoparticles' drug delivery capability. We oxidized the phospholipids and APOA1 of NP1 to produce NP2 (Figure S1B). The nanoparticle sizes were measured by dynamic light scattering, and their morphologies were visualized by transmission electron microscopy (TEM) 
a

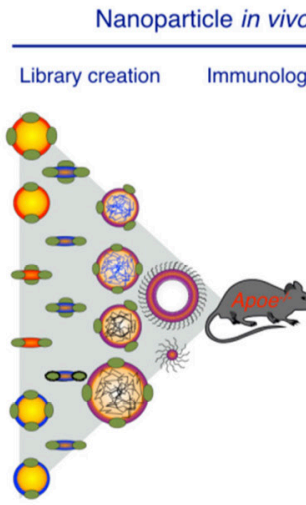

mune cell screen
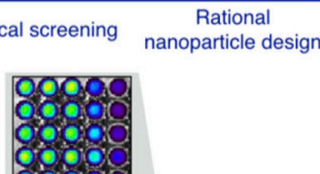
0000
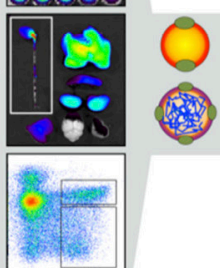

Improving drug toxicity profile

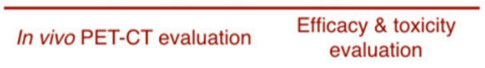

evaluation

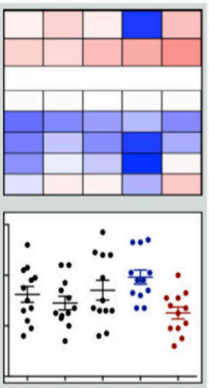

b
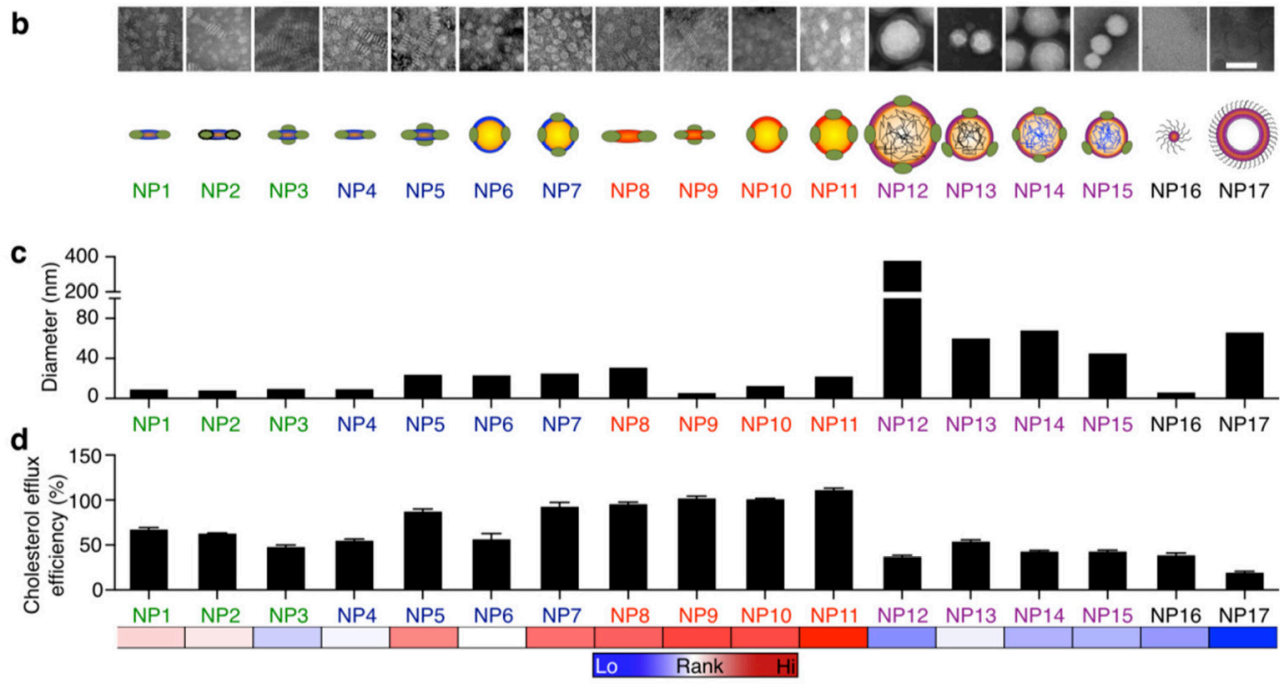

Figure 1 I Study design and in vitro characterization of the nanoparticle library. (A) In the in vivo immune cell screen study (left), we first created a combinatorial nanoparticle library and then evaluated the library in atherosclerotic Apoe $e^{-/-}$mice by using blood half-life determination, NIRF, and flow cytometry. The library screening data lead to rational design of one GW3965-loaded nanoparticle with favorable characteristics and one without. In the second study, the two nanoparticles were radio- and fluorescencelabeled, and they were quantitatively and therapeutically evaluated by using PET-CT imaging, mRNA profiling, flow cytometry, and liver toxicity assays (right). (B) Representative high magnification TEM images of negatively stained nanoparticles. Low-magnification images and a discussion of the different structures are presented in Figure S1. (Scale bar, $50 \mathrm{~nm}$.) (C) The size of nanoparticles as measured by dynamic laser scattering (DLS). (D) Cholesterol efflux capacity of the nanoparticles in primary macrophages normalized to natural human HDL $(n=6)$. Error bars are SDs. The color-coded bar at the bottom shows the relative rank of each nanoparticle, with the red indicating high cholesterol efflux efficiency and the blue indicating low efficiency. 


\subsection{HDL Nanoparticle Library's Cholesterol Efflux Efficiency}

Natural HDL facilitates reverse cholesterol transport, the intrinsic mechanism that removes cholesterol from macrophages in atherosclerotic plaques and protects against atherosclerosis $[19,20]$. Cholesterol efflux capacity indicates the nanoparticles' biological similarity to native HDL. Therefore, we measured the 17 nanoparticles' ability to induce cholesterol efflux from cholesterol-laden primary macrophages. We found 1-palmitoyl-2- oleoyl-sn-glycero-3-phosphocholine (POPC)-based nanoparticles (NP8, NP9, NP10, and NP11) to be the most efficient in extracting cholesterol from macrophages; in contrast, polymer-core HDL nanoparticles produced the least cholesterol efflux (NP12, NP13, NP14, and NP15). Liposomal and micellar nanoparticles, without APOA1 on their surface to bind the cholesterol efflux receptors Abca1 and Abcg1, performed similarly to polymer-core HDL nanoparticles (Figure 1D). These results demonstrate the essential role of APOA1, as well as the impact of phospholipid and core composition, in promoting cholesterol efflux. It has been proposed that APOA1 changes conformation when interacting with its specific receptors to extract cholesterol [19]. The artificial polymeric cores of NP12, NP13, NP14, and NP15 might limit the conformational flexibility of APOA1, leading to impaired cholesterol efflux. On the other hand, POPC-based HDL nanoparticles display less rigidity, are more similar to natural HDL, and therefore result in more efficient cholesterol efflux.

\subsection{Nanoparticles' Physiochemical Properties Affect Their in vivo Behavior}

We i.v. injected the library's 1,1'-dioctadecyl-3,3,3',3'- tetramethylindotricarbocyanine iodide (DiR)-labeled nanoparticles into atherosclerotic Apoe ${ }^{-1-}$ mice. We found that NP1 and NP10, with diameters between $7 \mathrm{~nm}$ and $30 \mathrm{~nm}$, exhibited the longest blood half-lives of $5.0 \mathrm{~h}$ and $6.3 \mathrm{~h}$, respectively. NP12 and NP14, with diameters larger than $70 \mathrm{~nm}$, had the shortest blood half-lives of $0.71 \mathrm{~h}$ and $0.67 \mathrm{~h}$, respectively (Figure 2A). The difference between the longest and the shortest blood half-life was almost 10-fold (Table S2).

Using NIRF, we investigated nanoparticle accumulation in the heart, aorta, lung, liver, spleen, kidney, brain, and muscle $24 \mathrm{~h}$ after i.v. administration (Figure 2B and Figure S2A). Among all nanoparticles, liver accumulation was generally the highest, followed by spleen, kidney, and lung accumulation (Figure S2B). Because the aorta is the primary target tissue whereas the liver and spleen are clearance organs, we determined accumulation in the aorta relative to these two organs [21]. Our measurements showed a 3.4-fold difference between the highest and lowest aorta-to-liver accumulation ratios (NP5 vs. NP12, $P<0.01$, Figure S2D), and a 4.7-fold difference between the aorta-to-spleen accumulation ratios (NP5 vs. NP12, $P<0.01$, 
Figure S2E). For the nanoparticles' relative performance, see Figure 2B. Distribution of DiR-labeled nanoparticle in certain tissues is difficult to quantify in vivo due to the limited penetrating depth of light and varying absorbance rates among tissues. [22] Radiolabeled nanoparticles' biodistribution and pharmacokinetics can be quantitatively measured. From our initial optical imaging screen, we selected NP10, NP14, NP15, and NP17 to be labeled with Zr through the hydrophobic chelator deferoxamine (DFO)-C34, which serves as a surrogate for hydrophobic payloads. In line with the optical imaging results, we observed that the smaller nanoparticles (NP10 and NP15) had longer blood half- lives than the larger ones (NP14 and NP17, Figure 2C). We also observed that the HDL- based nanoparticles (NP10, NP14, and NP15), particularly NP10, accumulated more efficiently in atherosclerotic plaques than the liposomal nanoparticle (Figure 2D and E). Overall, NP10 displayed the most favorable performance among the four selected nanoparticles (Figure 2C-F). 

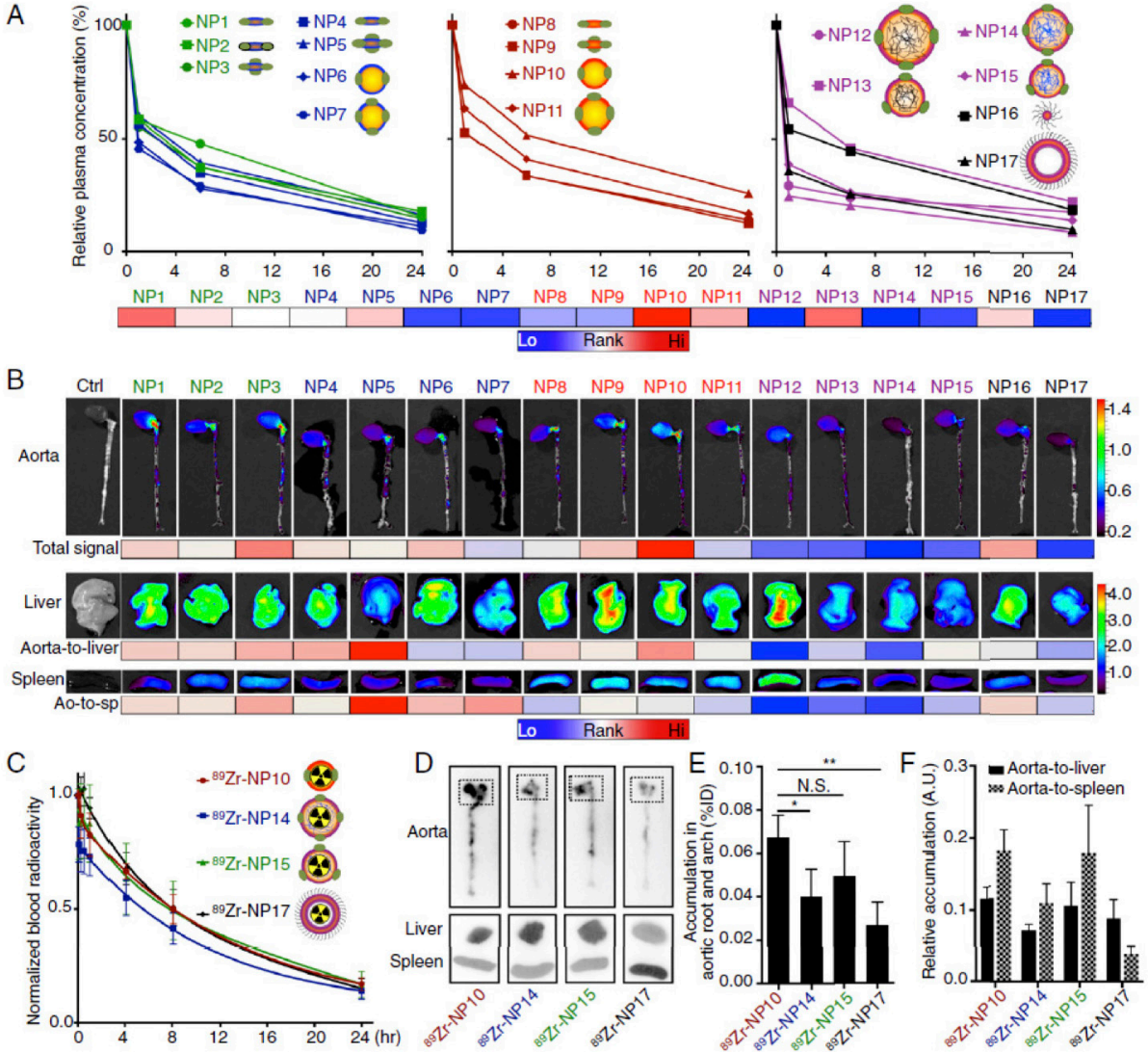

Figure 2I In vivo evaluation of the nanoparticle library. (A) Relative nanoparticle plasma concentration in $A p o e^{-I-}$ mice that were fed a 12-wk high-cholesterol diet. The values were derived from the nearinfrared dye DiR incorporated in the nanoparticles. The heat map below the graphs ranks the blood half-lives, with the red indicating a long and the blue a short blood half-life ( $\mathrm{n}=5$ per nanoparticle). The values of blood half-lives are provided in Table S2. (B) Representative near-infrared fluorescence images of nanoparticle accumulation in aorta, liver, and spleen. The heat map below the aorta images ranks the mean total fluorescent tissue signal, the heat map below the liver images ranks the total aorta-to-liver signal, and the heat map below the spleen images ranks the mean aorta-to-spleen accumulation (Ao-to$\mathrm{sp}$ ) ratio, with the red indicating a high and the blue a low ratio ( $n=5$ for each nanoparticle). Bar graphs are provided in Figure S2. (C) Blood half-lives of four selected nanoparticles radiolabeled with ${ }^{89} \mathrm{Zr}$ ( $\mathrm{n}=$ 3 per nanoparticle) were $7.0 \mathrm{~h}$ for ${ }^{89} \mathrm{Zr}-\mathrm{NP} 10,5.7 \mathrm{~h}$ for ${ }^{89} \mathrm{Zr}-\mathrm{NP} 14,7.1 \mathrm{~h}$ for ${ }^{89} \mathrm{Zr}-\mathrm{NP} 15$, and $6.6 \mathrm{~h}$ for ${ }^{89} \mathrm{Zr}$ NP17. Blood radioactivity (percentage injected dose per gram of tissue) of all time points is normalized to that of the first time point-2 min after injection. (D) Representative autoradiography images of aortas, livers, and spleens $24 \mathrm{~h}$ after nanoparticle injection. Dashed windows indicate the aortic root and arch area analyzed in E. (E) Nanoparticle accumulation in aortic roots and arches as measured by the percentage of injected dose ( $\% \mathrm{ID})(\mathrm{n}=3$ per nanoparticle). (F) Relative accumulation of nanoparticles between aortas and liver or spleen. Arbitrary units (A.U.) were defined by the aortic accumulation (percentage injected dose per gram of tissue) divided by the hepatic or splenic accumulation (percentage injected dose per gram of tissue). Error bars are SDs. Statistics was calculated with nonparametric two-tailed Student's t-test. N.S., not statistically significant; ${ }^{*} P<0.05$; ${ }^{* *} P<0.01$. 


\subsection{Distinct Immune Cell Targeting Patterns within the Nanoparticle Library}

Macrophages and monocytes are the key immune cells that drive atherosclerosis progression [4]. In Apoe $e^{-1-}$ atherosclerotic mice, these cells mainly reside in atherosclerotic plaques, spleen, blood, and bone marrow.[4] Using a robust flow cytometry procedure adapted from previous studies, $[23,24]$ we were able to identify macrophages, monocytes, and nonmyeloid immune cells ( $\mathrm{Lin}^{+}$) in the aortas; macrophages, Ly-6Chi monocytes, dendritic cells (DCs), and neutrophils in the spleens; and Ly-6Chi monocytes, Ly-6Clo monocytes, DCs, and neutrophil in the blood (Figure 3A). In the aortas, all HDL-mimicking nanoparticles efficiently targeted macrophages and monocytes. The difference between the highest and lowest nanoparticle accumulations was 5.7-fold in macrophages (NP3 vs. NP17, $P<$ 0.01 ) and 2.7-fold in monocytes (NP3 vs. NP7, $P<0.001$; Figure 3B). In the spleens, all nanoparticles had the highest accumulation in macrophages, which do the bulk of nanoparticle clearance, with a 3.8-fold difference between the highest and lowest accumulation levels (NP12 vs. NP17, $P<0.01$, Figure 3B). In the blood, DCs and Ly-6Chi monocytes displayed the highest nanoparticle association, with a 3.8-fold difference between the highest and lowest association levels (NP10 vs. NP5, $P<$ 0.01 ) in DCs and a 3.79-fold difference in Ly-6Chi monocytes (NP3 vs. NP14, $P<$ 0.0001 , Figure 3B). In addition, the nanoparticles were far less effective in targeting Ly-6Clo monocytes, the patrolling monocytes in the blood, compared with Ly-6Chi monocytes (Figure 3B).

Although aortic macrophages are the main target of immunomodulatory nanoparticles, splenic macrophages, which clear nanoparticles from the blood and reduce their bioavailability to aortic macrophages, [25] need to be avoided. We evaluated the ratios of nanoparticle accumulation in aortic macrophages versus splenic macrophages, and we found a 3.8-fold difference between the highest and lowest aortic-to-splenic ratios (NP16 vs. NP12, $P<0.0001$, Figure S3A). This differential targeting specificity to atherosclerotic macrophages was confirmed by immunofluorescence in the aortic roots (Figure S3B). Altogether, the flow cytometry data reveal that the distinct physiochemical nanoparticle properties within the library lead to drastically different immune cell targeting patterns (Figure 3C). 


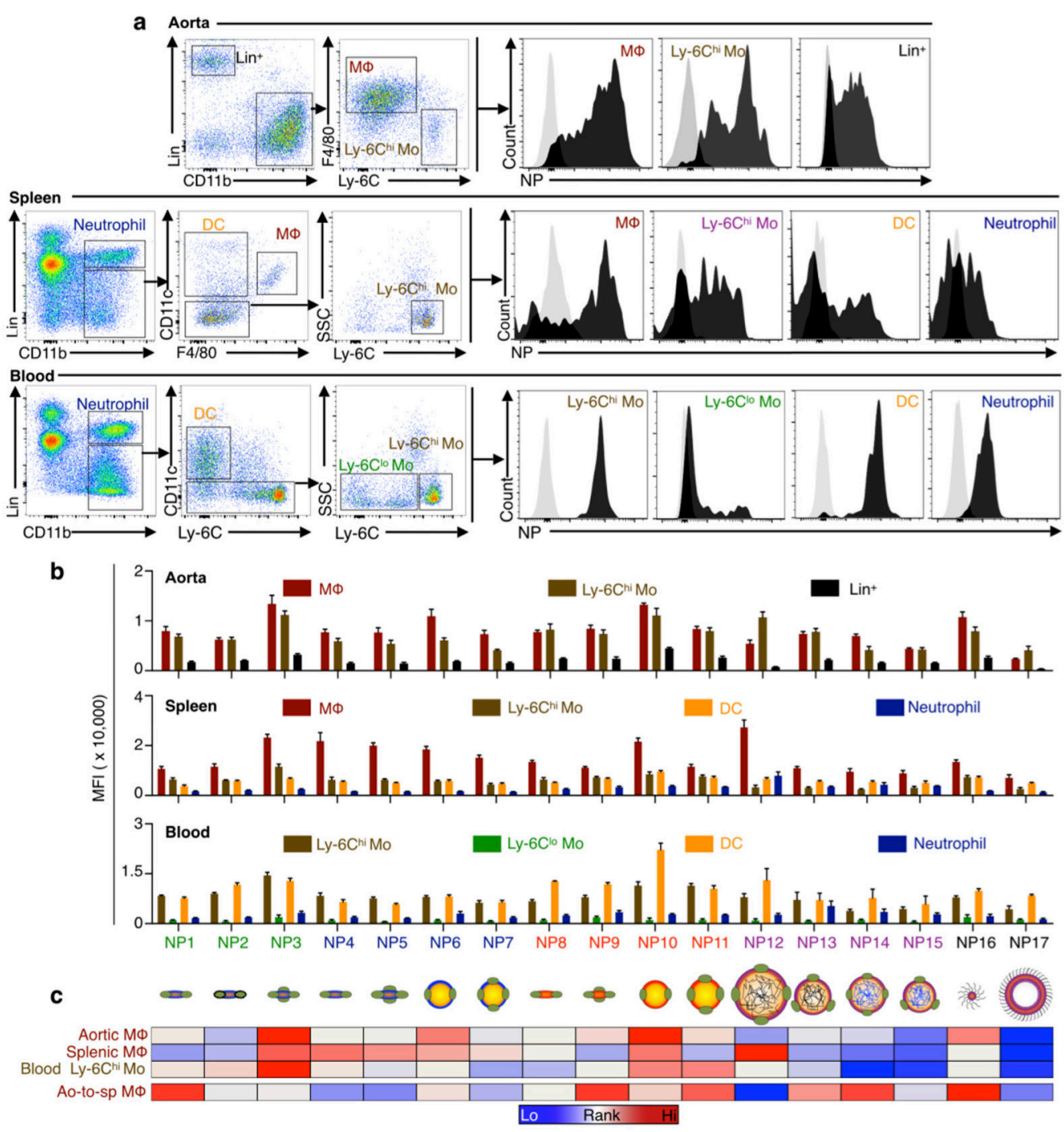

Figure 3| Nanoparticle immune cell specificity. (A) The flow cytometry gating procedures to identify relevant immune cells in aorta, spleen, and blood. Black histograms on the right show representative signal distribution of different immune cells in the mice injected with nanoparticles compared with the cells from control animals injected with PBS (gray histogram on the left in each graph). (B) Quantification of mean fluorescence intensity (MFI) of each immune cell type in different tissues; $n=5$ for each nanoparticle, and error bars are SEMs. (C) Heat map ranks targeting efficiency in key immune cells (aortic macrophages, spleen macrophages, and blood Ly-6Chi monocytes), with red indicating a high and blue indicating a low MFI in the first three rows. The last row shows the aortic-to-splenic macrophage MFI ratio (Ao-to-sp $\mathrm{M} \Phi)$, and its quantitative values are provided in Figure S3A. 


\subsection{GW3965-Loaded Nanoparticle Development}

Liver X receptor (LXR) agonists promote cholesterol efflux from macrophages in atherosclerotic plaques, and they have been proposed as novel immunomodulatory drugs for the disease [26]. However, most experimental LXR agonists fail clinical translation or early-stage clinical trials due to poor safety profiles. For example, GW3965, an effective LXR agonist promoting cholesterol efflux from atherosclerotic macrophages, $[27,28]$ did not reach the clinical phase, because of its liver toxicity in hamsters and monkeys, [10] as well as in human hepatocytes [11].

In our nanoparticle library studies, NP10 was found to have high cholesterol efflux promotion efficiency, a long blood half-life, high relative aorta-to-liver accumulation, and a high relative aortic-to splenic macrophage association ratio (Figure S4A). These features make NP10 a promising candidate for avoiding GW3965's liver toxicity and enhancing its efficacy on atherosclerotic plaque macrophages. Therefore, we replaced NP10's hydrophobic triglyceride cargo with hydrophobic GW3965 and created a GW3965-loaded nanoparticle (Rx-HDL) that was morphologically similar but not identical, due to the different nanoparticle composition, to NP10 in size ( 30 $\mathrm{nm}$ ), phospholipid composition (POPC-dominant), and morphology (Figure S4B). Further, we identified NP14 as an unfavorable nanoparticle for GW3965 delivery due to the nanoparticle's poor cholesterol efflux efficiency, short blood half-life, and low relative aorta-to-liver accumulation (Fig. S4A). By loading GW3965 into the PLGA matrix of NP14, we created a PLGA-core GW3965-loaded nanoparticle (RxPLGA-HDL) with similar size, phospholipid composition, and morphology to NP14 (Figure S4B). Notably, the size and cholesterol efflux capability of the two drugloaded nanoparticles were drastically different (Figure S4B and C).

\subsection{Quantitative Evaluation of GW3965-Loaded Nanoparticles}

Nanoparticles that are $89 \mathrm{Zr}$-labeled can be quantitatively characterized by in vivo PET imaging as well as by ex vivo radioactivity counting [29]. To accurately understand the in vivo performance of the two nanoparticles, we loaded the hydrophobic ${ }^{89} \mathrm{Zr}$-DFO-C34 into Rx-HDL and Rx-PLGA-HDL (Figure 4A), and radioactive high-performance chromatography showed both Rx-HDL and RxPLGA-HDL to be efficiently radiolabeled (Figure S4D).

In $A$ poe $e^{-1-}$ atherosclerotic mice, Rx-HDL circulated in the blood much longer (weighted $t_{1 / 2}=10.5 \mathrm{~h}, \mathrm{n}=3$ ) than Rx-PLGA-HDL (weighted $\mathrm{t}_{1 / 2}=5.0 \mathrm{~h}, \mathrm{n}=3$, Figure $4 \mathrm{~B})$ or its precursor NP10 $\left(\mathrm{t}_{1 / 2}=7.0 \mathrm{~h}\right.$, Figure $\left.2 \mathrm{C}\right)$, demonstrating its favorable features. We then used PET-computed tomography hybrid imaging (PET-CT) to measure the nanoparticle dynamic accumulation in the cardiac blood pool, the liver, and the spleen $30 \mathrm{~min}$ and $24 \mathrm{~h}$ after i.v. administration ( $\mathrm{n}=5$ per nanoparticle, 
Figure 4C; also see Movies S1-S4). After $30 \mathrm{~min}$, Rx-HDL had higher accumulation in the cardiac blood pool (29.9 maximum percentage injected dose per gram of tissue (Max \% ID/g) vs. $24.5 \mathrm{Max} \% \mathrm{ID} / \mathrm{g}, P<0.05)$ but lower accumulation in the liver than Rx-PLGA-HDL (21.7 Max \%ID/g vs. 29.7 Max \%ID/g, P<0.05). After 24 h, RxHDL liver accumulation was still 36\% lower than Rx- PLGA-HDL (26.5 Max \%ID/g vs. $41.7 \mathrm{Max} \% \mathrm{ID} / \mathrm{g}, P<0.05$, Figure $4 \mathrm{C}$ and D). Autoradiography revealed that both nanoparticles displayed patchy aorta accumulation, in accordance with the heterogeneous distribution of atherosclerotic plaques in this tissue [30] (Figure 4E). Additionally, autoradiography confirmed the highest nanoparticle accumulation to be in the liver and spleen (Figure 4E), a result that was corroborated by an extensive biodistribution analysis (Figure S4E).

Having labeled both nanoparticles with DiR (Figure 4A), we used our flow cytometry protocol (Figure 3A) to quantify immune cell targeting specificity (Figure S4F). Rx-HDL predominantly targeted macrophages in the aortas, and its accumulation was twofold higher than Rx-PLGA-HDL $(P<0.01)$. In the spleen, Rx-HDL had 33\% less accumulation in splenic macrophages than Rx-PLGAHDL $(P<0.05)$. In the blood, Rx-HDL targeted Ly-6Chi monocytes 2.4 -fold more efficiently than Rx-PLGA-HDL $(P<0.01$, Figure $4 \mathrm{~F}$ and $\mathrm{G})$. Collectively, these data show that, compared with Rx-PLGA-HDL, Rx-HDL has a longer blood half-life, lower accumulation in the liver, higher accumulation in atherosclerotic plaque macrophages, and lower accumulation in splenic macrophages. 


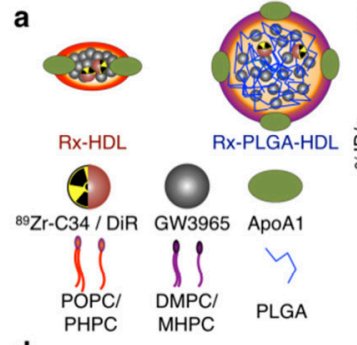

d

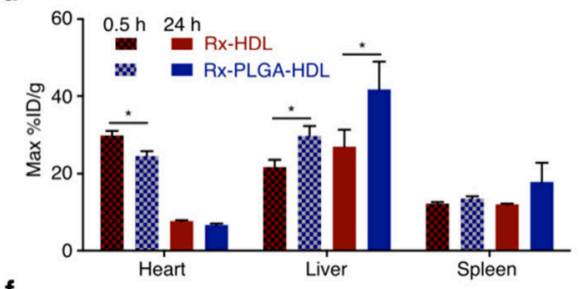

f

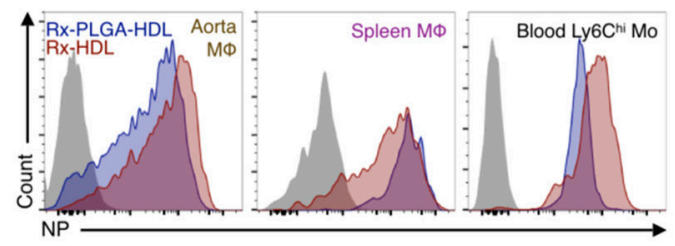

e
C

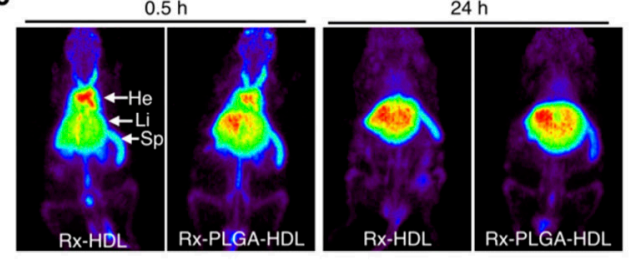

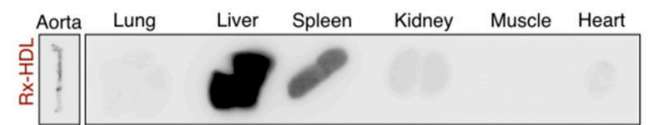
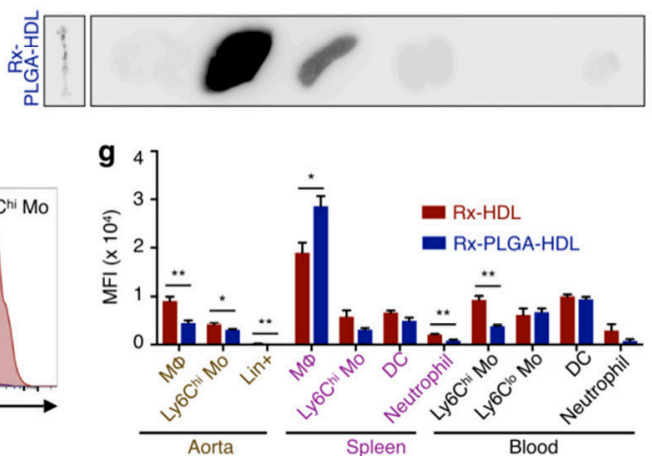

Figure 4 I In vivo quantitative evaluation of GW3965-loaded nanoparticles. (A) Schematic depictions of small Rx-HDL plaque macrophage-targeting and large polymer-hybrid Rx-PLGA-HDL nanoparticles. The two nanoparticles were either radiolabeled with ${ }^{89} \mathrm{Zr}$ or labeled with the near-infrared fluorescent dye DiR. (B) Blood half- lives were determined in three mice per nanoparticle. (C) Representative PET images of mice that received either the small or large nanoparticle at $0.5 \mathrm{~h}$ and $24 \mathrm{~h}$ after i.v. administration. The 3D-rendered images are provided as Movies S1-S4 ( $n=5$ per nanoparticle). (D) Quantification of radioactivity in the heart, liver, and spleen. (E) Representative autoradiographic images of key organs. Full biodistribution of all organs is provided in Fig. S4E. (F) Representative histograms of selected immune cell targeting specificity in the aorta, spleen, and blood. (G)MFI quantification of relevant immune cells in the tissues ( $\mathrm{n}=4$ per nanoparticle). Error bars are SEM. Statistics was calculated with nonparametric two-tailed Student's t test. ${ }^{*} P<0.05$; ${ }^{* *} P<0.01$.

\subsection{Nanoparticle Abolishes Liver Toxicity and Preserves Efficacy of GW3965}

To test if Rx-HDL's optimal in vivo performance reduced GW3965s liver toxicity, we gave four i.v. injections (one injection every $2 \mathrm{~d}$, at a dose of $10 \mathrm{mg} / \mathrm{kg} \mathrm{GW3865)}$ of Rx- HDL, its vehicle control (HDL), Rx-PLGA-HDL, its vehicle control (PLGAHDL), and PBS to Apoe $e^{-/-}$atherosclerotic mice ( $\mathrm{n}=12$ per group).

In the liver, Rx-PLGA-HDL increased the expression of two of the three major GW3965-related toxicity genes, and Rx-HDL increased the expression of one gene (Figure 5A). Importantly, we measured hepatic triglyceride and cholesterol levels, 
which are the major biomarkers of GW3965-induced hepatic steatosis [10]. The Rx-PLGA-HDL group had 35.4\% more hepatic triglyceride than its control vehicle PLGA-HDL group $(P=0.014)$ and $21 \%$ more than the PBS group $(P=0.12)$. For hepatic cholesterol, the Rx-PLGAHDL group had 31\% higher levels than the PLGAHDL group $(P=0.018)$ and $17 \%$ higher levels than the PBS group $(P=0.014)$. These results suggest that the high liver accumulation of Rx-PLGA-HDL caused severe liver toxicity (Figure 5B and C). On the other hand, the Rx- HDL group had 26.5\% lower hepatic triglyceride levels than its vehicle HDL control group $(P=0.06)$ and $22.7 \%$ lower levels than the PBS group $(P=0.076)$. The Rx-HDL group also had $20 \%$ lower hepatic cholesterol levels than the HDL group $(P=0.06)$ and $33.3 \%$ lower levels than the PBS group $(P=0.00043)$. A recent study suggested that high HDL levels are associated with a lower degree of steatosis, which might explain the reduced hepatic triglyceride and cholesterol levels in mice treated with vehicle HDL nanoparticles [31]. Furthermore, GW3965 has been reported to increase HDL levels in mice [32], likely explaining the additional hepatic benefits in Rx-HDL-treated mice. Most importantly, compared with the Rx-PLGA-HDL group, the Rx-HDL group had $36.1 \%$ lower hepatic triglyceride $(P=0.00083)$ and $43.0 \%$ lower hepatic cholesterol levels $(P<0.0001)$. These results demonstrate that the two GW3965loaded nanoparticles' distinct liver accumulations resulted in differential liver toxicity profiles (Figure $5 \mathrm{~A}-\mathrm{C}$ ).

To measure the treatments' efficacies on aortic macrophages, we isolated the cells from aortic roots by laser capture microdissection and measured their mRNA expression levels by quantitative real-time PCR (qPCR). We found that Rx-HDL increased expression of five GW3965 target genes compared with the vehicle HDL control, whereas Rx-PLGA-HDL increased only four genes compared with the vehicle PLGA-HDL control (Figure 5D). Furthermore, Rx-HDL produced elevated expression of the five target genes compared with Rx-PLGA-HDL (Figure S5A and B). These results suggest successful GW3965 delivery to macrophages in atherosclerotic plaques. We did not observe clear effects of either nanoparticle on genes related to macrophage inflammation, as the number of genes with increased expression was almost equal to the number with decreased expression (Figure 5E and Figure S5A and B). Similarly, the macrophage levels in aortic roots were the same among all groups (Figure S5D and E).

Based on the favorable properties of Rx-HDL, we further evaluated its therapeutic effects in a 6-wk, long-term treatment regimen focusing on cellular lipid levels in aortas. Working from a BODIPY (4,4-difluoro-1,3,5,7,8-pentamethyl-4-bora-3a,4adiaza- sindacene)- based flow cytometry protocol [33], we developed a procedure to quantify the cellular lipid levels of macrophages, monocytes, and CD45 negative nonimmune cells $\left(\mathrm{CD}_{4} 5^{-}\right)$in the aortas (Figure S6). Importantly, we found that the 6-wk treatment of Rx- HDL (10 mg/kg GW3965, two i.v. injections per week) resulted in $28 \%$ less total lipid in aortic macrophages $(P=0.224$; Figure 5F). Further, the treatment reduced total lipid levels in monocytes by $43.0 \%(P=0.011$, Figure $5 \mathrm{G})$ and in all $\mathrm{CD}_{4} 5^{-}$nonimmune cells by $40.0 \%(\mathrm{P}=0.021$, Figure $5 \mathrm{H})$. Furthermore, 
no therapeutic effects were observed when the compound was given orally with the same 6-wk treatment regimen (10 $\mathrm{mg} / \mathrm{kg}$ GW3965, two gavages per week) compared with the placebo-treated group (Figure S6C-H).

Consistent with the liver toxicity results after the short-term Rx-HDL treatment, blood cholesterol and triglyceride levels were similar to those in mice receiving PBS treatment (Figure S7A and B). In addition, the long-term treatment of Rx-HDL did not cause any observable toxicity in the liver, kidney, heart, and blood cells (Figure S7C-L). Taken together, the results show that long-term Rx-HDL treatment produces significant therapeutic benefits without causing toxicity in major organs, most notably the liver, whereas long-term oral treatment at the same dose did not produce any therapeutic benefits.

a

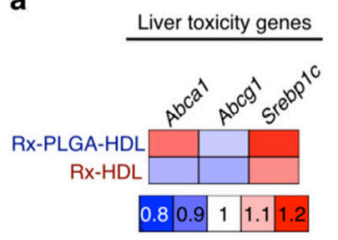

b

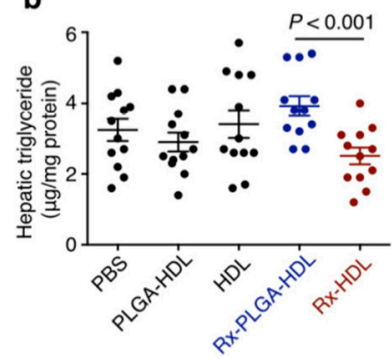

c

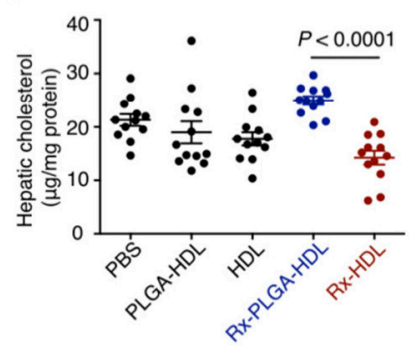

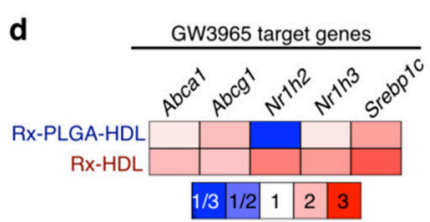

e

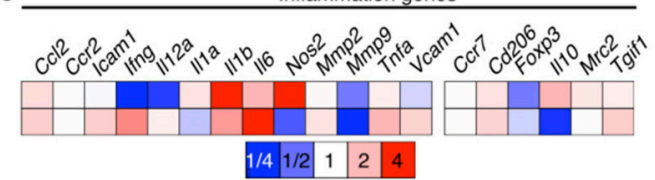

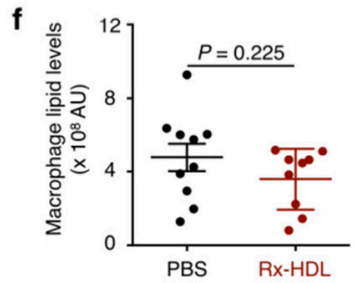
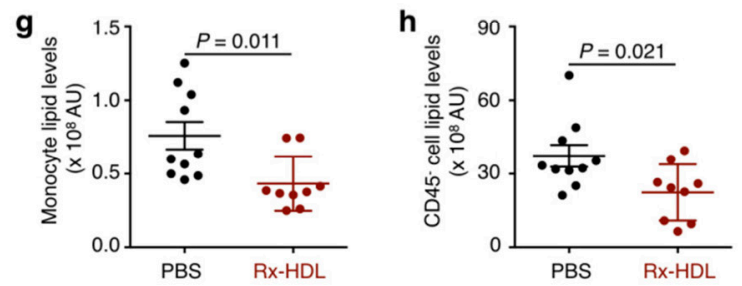

Figure 5| Evaluation of the toxicity and efficacy of GW3965 (Rx)-loaded nanoparticles. In A-E, Apoe $e^{-/-}$ mice received four i.v. administrations of nanoparticles or PBS on every other day $(n=12$ per group). (A) The mRNA expression levels of three GW3965 target genes in liver homogenates. (B) Triglyceride and (C) cholesterol levels in the liver, the primary organ suffering from the toxic effects of GW3965. (D) The mRNA expression levels of five GW3965 target genes in aortic macrophages. (E) The mRNA expression levels of 19 inflammation-related genes in aortic macrophages. All gene expression was normalized to housekeeping gene Hprt1. The bar graph presentation of the heat maps is in Fig. S5 ( $\mathrm{n}=12$ per group). In $\mathrm{F}-\mathrm{H}, \mathrm{Apoe}^{-/-}$mice ( $\mathrm{n}=10$ in PBS; $\mathrm{n}=9$ in Rx- HDL) received 12 i.v. injections in $6 \mathrm{wk}$. Lipid levels of aortic cells were analyzed by flow cytometry. Cellular lipid levels were calculated by multiplying the mean fluorescence intensity of a cell type by the number of the cells per aorta (AU $=$ MFI $\times$ number of cells). The total lipid levels of aortic (F) macrophages, $(\mathrm{G})$ monocytes, or $(\mathrm{H})$ nonimmune $\mathrm{CD} 45^{-}$cells per aorta in the mice are presented here. Gating procedure is provided in Fig. S6 A and B. Error bars in all graphs are SEM. Statistics was calculated with nonparametric two-tailed Student's t-test. 
In summary, we developed a rational library screen strategy to identify nanoparticles with favorable immune cell specificity and biodistribution in an atherosclerosis mouse model. On the basis of this nanoparticle screen, we optimized GW3965 delivery to plaque macrophages and, while preserving its efficacy on atherosclerotic plaques (Figure 5 D-H), abolished GW3965 liver toxicity (Figure 5A-C and Figure S7), a well-known adverse effect of LXR agonists.

\section{DISCUSSION}

By fine-tuning the components and synthesis procedures, we created a combinatorial library of 17 nanoparticles with distinct composition, size, and morphology. These distinct physiochemical properties resulted in an approximate sixfold difference in promoting cholesterol efflux from macrophages, 10-fold difference among blood half-lives, 3.4-fold difference in relative aorta-to-liver accumulation, and 3.8-fold difference in relative aortic- to-splenic macrophage accumulation. From this library screening, we identified the favorable lipid composition (POPC-dominant), pharmacokinetics (long blood half-life), size (around $30 \mathrm{~nm}$ ), and morphology (spherical) to achieve optimal plaque macrophagespecific drug delivery. We hypothesize that the combination of long blood half-life and small size allow efficient and prolonged atherosclerotic plaque penetration and subsequent macrophage accumulation. A favorable nanoparticle lipid composition and morphology increases stability and promotes delivery of the encapsulated small molecules to the targeted cells, as suggested by our recent study [34]. In a proof-ofconcept application, we used these guidelines to identify two nanoparticles from the library as the most and least favorable nanoparticles for delivering the liver-toxic compound GW3965. Although the unfavorable nanoparticle Rx-PLGA-HDL caused severe GW3965-induced liver toxicity, the favorable nanoparticle Rx-HDL did not cause observable liver toxicity in treated animals but did preserve the compound's therapeutic efficacy on the atherosclerotic plaques.

Despite the clinical introduction of antibody immunotherapies for atherosclerosis,[35] such biological drugs can only modulate a limited number of extracellular targets, such as PCSK9 $[36,37]$ or receptors on the cell surface. [38] Intracellular entities present many more immunological targets that can be effectively controlled by immunomodulatory small molecules. Most experimental small molecules for atherosclerosis, however, failed clinical trials due to their unfavorable toxicity profiles, generally caused by high accumulation in nontargeted tissues or in nontargeted cells within the targeted tissues [39]. To convert these immunomodulatory small molecules into precision medicines for atherosclerosis, organ specific and cell-specific delivery is highly desirable.

Our approach allows the creation and immunological screening of a combinatorial nanoparticle library with distinct organ biodistribution and immune cell targeting 
specificities. By using PET imaging, NIRF, and flow cytometry to generate an extensive database detailing the in vivo performance of the library nanoparticles, we were able to rationally design a strategy that avoids the specific limitations of an immunomodulatory compound. Through this process, we successfully converted a liver-toxic immunomodulatory compound into a precision nanomedicine for atherosclerotic plaque macrophage treatment.

A polymer-core nanoparticle (NP13) in the library has similarly optimal performance to the best-performing NP10 (Fig. S4A). Polymer-based nanoparticles have been formulated with chemical compounds,[40] peptides,[41] and nucleic acids [42]. This variety suggests that NP13 may be able to deliver a wide range of therapeutic molecules. In addition, a few nanoparticles (NP3, NP9, and NP10) in the library show high targeting specificity to Ly-6Chi monocytes and DCs, which are attractive targets in certain types of cancer,[43] asthma, [44] and diabetes [45]. It would be interesting to apply the same library screening strategy to develop nanoparticle-based specific drug delivery to these immune cells in relevant diseases.

This nanoparticle library screening strategy in immune cells allowed us to improve the therapeutic index of an immunomodulatory molecule that causes hepatic toxicity and has failed clinical translation. Our precision nanomedicine strategy is radically different from the current clinical therapeutics as well as those in experimental phases. Moreover, the approach's potential to deliver various compounds preferentially to other immune cells would expand its application to numerous immunologically implicated diseases, such as myocardial infarction, diabetes, and cancer.

\section{MATERIALS AND METHODS}

\subsection{Synthesis of Library Nanoparticles}

The compositions of all NP synthesis materials are listed in Table S1. The synthesis procedure for NP1 through NP11 was similar to a previous method [12]. Briefly, phospholipids, DiR, and triglyceride were dissolved in a chloroform/ methanol solvent, dried to form a thin film, and then hydrated with a human APOA1 solution. The homogenized solution was sonicated with a tip sonicator, and the aggregates and free lipids were removed by passing through a series of filters. The synthesis procedure for NP12 through NP15 was adapted from a previous microfluidics-based method [14]. Briefly, a solution containing $0.79 \mathrm{~mL}$ of a PLGA or PLA solution in acetonitrile $(100 \mathrm{mg} / \mathrm{mL}), 1.58 \mathrm{~mL}$ of a 3:1 molar ratio of 1,2-dimyristoyl-sn-glycero-3- phosphocholine/1-myristoyl-2-hydroxy-sn-glycerophosphocholine (DMPC/MHPC) in ethanol $(5 \mathrm{mg} / \mathrm{mL}), 0.36 \mathrm{mg}$ of DiR, $7.9 \mathrm{~mL}$ of ethanol, and $14.5 \mathrm{~mL}$ of acetonitrile was prepared. The aforementioned solution was 
injected in the middle channel of the microfluidic device at a rate of $2 \mathrm{~mL} / \mathrm{min}$, and a solution of APOA1 $(0.01 \mathrm{mg} / \mathrm{mL}$ in PBS) was injected into the outer channels at a rate of $10 \mathrm{~mL} / \mathrm{min}$. The product was collected, washed with PBS, and concentrated using tangential filtration [100,000-Da molecular mass cutoff (MMCO)] to remove acetonitrile, ethanol, and lipid-free APOA1. For micelle NP16, $2 \mathrm{~mL}$ of chloroform solution containing $211 \mathrm{mg}$ of 1,2-distearoyl-sn-glycero-3-phosphoethanolamine$\mathrm{N}$-[amino(polyethylene glycol)-2000] (DSPE-PEG2000) and $0.4 \mathrm{mg}$ of DIR $(0.5 \% \mathrm{~mol})$ was slowly dripped into $10 \mathrm{~mL}$ of PBS solution heated at $85{ }^{\circ} \mathrm{C}$ under vigorous stirring. After dripping, the solution was kept at $85^{\circ} \mathrm{C}$ until all of the chloroform was totally evaporated. The micelle solution was washed and concentrated in PBS using Millipore centrifugal filter (50,0000 Da MMCO). For liposome NP17, a lipid film was first prepared by evaporating a chloroform solution containing $35.4 \mathrm{mg}$ of 1,2-dipalmitoyl-sn-glycero-3-phosphocholine (DPPC), $11.1 \mathrm{mg}$ of DSPE-PEG2000, $10.24 \mathrm{mg}$ of cholesterol, and $0.4 \mathrm{mg}$ of $\mathrm{DiR}(61.1 \%, 2 \%, 33.4 \%, 0.5 \%$ in molar percentage). The residue of chloroform was removed by blowing nitrogen gas. The resulting film was hydrated with $10 \mathrm{~mL}$ PBS, vortexed, and subsequently sonicated for $25 \mathrm{~min}$. After centrifugation at $18000 \times \mathrm{g}$ for $10 \mathrm{~min}$ to remove aggregates, the liposome solution was washed and concentrated in PBS using Millipore centrifugal filter (100,000 Da MMCO). To oxidize NP1, a PBS solution containing NP1 was stirred at $37^{\circ} \mathrm{C}$ in the presence of EDTA, $\alpha$-tocopherol, and 2,2'-Azo-bis(2- amidinopropane) dihydrochloride for $20 \mathrm{~h}$. The resulting oxidized nanoparticle (NP2) was washed thoroughly with PBS.

\subsection{Synthesis of GW3965-Loaded Nanoparticles.}

The synthesis procedure for Rx-HDL was similar to that used for NP10. In addition to phospholipids [POPC and 1-palmitoyl-2-hexadecyl-sn-glycero-3-phosphocholine (PHPC), 3:1 by weight] and DiR, GW3965 was added to make up $26 \%$ by weight of the total starting materials. For Rx-PLGA-HDL, a solution containing $1 \mathrm{~mL}$ of GW3965 in DMSO (50 mg/mL), $2 \mathrm{~mL}$ of PLGA $(100 \mathrm{mg} / \mathrm{mL}), 2 \mathrm{~mL}$ of lipids (10 $\mathrm{mg} / \mathrm{mL}$ ), $53 \mathrm{~mL}$ of acetonitrile, and $22 \mathrm{~mL}$ of ethanol was prepared. The organic solution was injected in the middle channel of the microfluidic device at a rate of 2 $\mathrm{mL} / \mathrm{min}$, while a solution of APOA $10.01 \mathrm{mg} / \mathrm{mL}$ in $1 \times \mathrm{PBS})$ was injected into the outer channels at a rate of $10 \mathrm{~mL} / \mathrm{min}$. The product was collected, washed with PBS, and concentrated to $\sim 2 \mathrm{mg} / \mathrm{mL}$ using tangential and centrifugal filtration $(100,000$ Da MMCO and 300,000 Da MMCO, respectively). After synthesis, GW9365 was extracted from the final nanoparticles by acetonitrile, and the compound amount was measured by HPLC. The incorporation rate of Rx-HDL was above $90 \%$, and that for Rx-PLGA-HDL was about 50\%. The GW3965 injecting dose was adjusted on the basis of the measured GW3965 concentration in the nanoparticle solution. 


\subsection{Animals and Treatment Procedure}

All procedures and experiments were approved by the Institutional Animal Care and Use Committee of Icahn School of Medicine at Mount Sinai. About 300 female

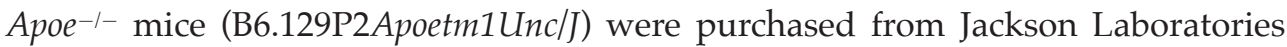
and then fed a high-fat diet (Harlan Teklad TD.88137, 42\% calories from fat) for 16 wk. These mice developed advanced atherosclerosis in their aortic roots and aortas after $10 \mathrm{wk}$ of a high-fat diet and mimicked advanced disease in humans after $16 \mathrm{wk}$ of the diet. Female C57BL / 6 mice were fed on regular chow. All nanoparticles were i.v. injected into the lateral tail veins. Care was taken to ensure that each injection was less than $150 \mu \mathrm{L}$ in volume. For the in vivo characterization study of the 17 nanoparticles, Rx-HDL, and Rx-PLGA-HDL, one i.v. injection was performed. No obvious toxicity or side effects were observed after the injections, except for NP12. After NP12 injection, mice had slow movement, and one out of five died within 24 $\mathrm{h}$ after the injection. For short-term toxicity study of Rx-HDL and Rx- PLGA-HDL, four injections in $8 \mathrm{~d}$ were given using a GW3965 dose of $10 \mathrm{mg} / \mathrm{kg}$ per injection. For the long-term treatment study, the mice received two i.v. injections of Rx- HDL $(\mathrm{n}=12)$ per week for $6 \mathrm{wk}$ at the dose of $10 \mathrm{mg} / \mathrm{kg}$ GW3965 per injection or an equal volume of PBS $(n=12)$. A separate cohort of female Apoe ${ }^{-1-}$ mice with the same length of high-fat diet received 6 wk of oral GW3965 ( $=6,10 \mathrm{mg} / \mathrm{kg}$ GW3965, two times per week) or PBS $(n=5)$. No abnormal activities or deaths occurred during the treatment regimen.

\subsection{Micro-PET/CT Imaging}

Apoe $e^{-1-}$ atherosclerotic mice were injected with either 89 Zr-labeled Rx-HDL or RxPLGA-HDL at about $200 \mu \mathrm{Ci}$ per mouse $(\mathrm{n}=5)$. At $30 \mathrm{~min}$ and $24 \mathrm{~h}$ after the injection, the mice were imaged on an Inveon PET / CT scanner (Siemens Healthcare Global) under isoflurane-induced (Baxter Healthcare) anesthesia. Whole-body static PET scans recorded a minimum of 50 million coincident events in $\sim 15 \mathrm{~min}$. The energy and coincidence timing windows were $350 \mathrm{keV}$ to $700 \mathrm{keV}$ and $6 \mathrm{~ms}$, respectively. The image data were normalized to correct for nonuniform PET response, dead-time count losses, positron branching ratio, and physical decay to the time of injection, but no attenuation, scatter, or partial-volume averaging correction was applied. The counting rates in the reconstructed images were converted to activity concentrations (percentage injected dose per gram of tissue) via a system calibration factor derived from imaging a mouse-sized water-equivalent phantom containing 89Zr. Images were analyzed using ASIPro VMTM software (Concorde Microsystems). Activity concentration was quantified by averaging the maximal values of at least 10 regions of interest (ROIs) drawn on consecutive slices of the chosen organs. 


\subsection{Liver mRNA Expression Measurement.}

Total RNA was obtained from snap frozen liver tissue and homogenized in TRIzol reagent (Ambion). The homogenate was spun down to pellet tissue, and the aqueous TRIzol supernatant was collected and processed using the Direct-Zol. RNA miniprep kit (Zymo Research Corporation) was used for RNA purification. The RNA was then reverse- transcribed using the Verso cDNA kit (Thermo Scientific) and diluted using RNase/ DNase- free water. Quantitative real-time PCR was performed with Taqman Gene Expression Master Mix (Applied Biosystems), and with Taqman primer/probe mixes for Abca1, Abcg1, or Srebp1c. Gene expression was normalized to $18 \mathrm{~S}$ ribosomal RNA (rRNA) expression.

\subsection{Aortic Macrophage mRNA Expression Measurement}

The quality of RNA extracts from atherosclerotic plaques was measured by Agilent 2100 bioanalyzer (Agilent Technologies). High-quality [RNA integrity number (RIN) > 7] RNA samples were amplified with a WT-Ovation Pico RNA amplification system (NuGen). The cDNA from the amplification reactions were used to run a microfluidics- based mRNA profiling chip (BioMark; Fluidigm), which had high reproducibility. Hprt1 was used as the housekeeping gene. The following 24 genes were measured: Abca1, Abcg1, Srebp1c, Nr1h2, Hr1h3, Ccl2, Ccr2, Icam1, Ifng, Il12a, Il1a, Il1b, Il6, Nos2, Mmp2, Mmp9, Tnfa, Vcam1, Ccr7, Cd206, Foxp3, Il10, Mrc2, and Tgif1. Twelve biological repeats were included for each gene.

\subsection{Cellular Lipid Measurement with Flow Cytometry}

Single cells were prepared from aortas from either Apoe ${ }^{-1-}$ mice or wild-type C57BL / 6 mice and then stained with antibody mixtures as described in SI Materials and Methods, Flow Cytometry. On the basis of a previous procedure [33], the stained cells were washed with PBS two times at room temperature and then incubated with $250 \mu \mathrm{L}$ PBS containing $0.5 \mu \mathrm{g} / \mathrm{mL}$ BODIPY (D-3922; Molecular Probes) for 15 min. The cells were washed with flow cytometry buffer two times and analyzed in a Becton Dickinson LSRII flow cytometer.

\subsection{Statistics}

Data are presented as mean \pm SE of the mean (SEM) unless otherwise noted. Twotailed Student's t-test was used to calculate statistical significance. GraphPad Prism 5.0 for PC (GraphPad Software Inc.) was used for statistical analysis. $P<0.05$ was regarded as significant; ${ }^{*}$ denotes $P$ value $<0.05$, and ${ }^{* *}$ denotes $P$ value $<0.01$ in all figures in the chapter. 


\section{REFERENCES}

[1] W.H. Fridman, F. Pages, C. Sautes-Fridman, J. Galon, The immune contexture in human tumours: impact on clinical outcome., Nat. Rev. Cancer. 12 (2012) 298-306. doi:10.1038/ nrc3245.

[2] A. Lehuen, J. Diana, P. Zaccone, A. Cooke, Immune cell crosstalk in type 1 diabetes., Nat. Rev. Immunol. 10 (2010) 501-513. doi:10.1038/ nri2787.

[3] K.J. Moore, F.J. Sheedy, E.A. Fisher, Macrophages in atherosclerosis: a dynamic balance, Nat. Rev. 13 (2013) 709-721. doi:10.1038/nri3520.

[4] F.K. Swirski, M. Nahrendorf, Leukocyte behavior in atherosclerosis, myocardial infarction, and heart failure., Science. 339 (2013) 161-166. doi:10.1126/ science.1230719.

[5] P. Libby, Inflammation in atherosclerosis., Nature. 420 (2002) 868-874. doi:10.1038/ nature01323.

[6] T. Heidt, H.B. Sager, G. Courties, P. Dutta, Y. Iwamoto, A. Zaltsman, C. von Zur Muhlen, C. Bode, G.L. Fricchione, J. Denninger, C.P. Lin, C. Vinegoni, P. Libby, F.K. Swirski, R. Weissleder, M. Nahrendorf, Chronic variable stress activates hematopoietic stem cells, Nat. Med. 20 (2014) 754-758. doi:10.1038/nm.3589.

[7] C.S. Robbins, I. Hilgendorf, G.F. Weber, I. Theurl, Y. Iwamoto, J.-L.L. Figueiredo, R. Gorbatov, G.K. Sukhova, L.M.S. Gerhardt, D. Smyth, C.C.J. Zavitz, E.A. Shikatani, M. Parsons, N. van Rooijen, H.Y. Lin, M. Husain, P. Libby, M. Nahrendorf, R. Weissleder, F.K. Swirski, Local proliferation dominates lesional macrophage accumulation in atherosclerosis, Nat. Med. 19 (2013) 1166-1172. doi:10.1038/nm.3258.

[8] E. Galkina, K. Ley, Immune and inflammatory mechanisms of atherosclerosis (*)., Annu. Rev. Immunol. 27 (2009) 165-197. doi:10.1146/ annurev.immunol.021908.132620.

[9] P. Dutta, G. Courties, Y. Wei, F. Leuschner, R. Gorbatov, C.S. Robbins, Y. Iwamoto, B. Thompson, A.L. Carlson, T. Heidt, M.D. Majmudar, F. Lasitschka, M. Etzrodt, P. Waterman, M.T. Waring, A.T. Chicoine, A.M. van der Laan, H.W.M. Niessen, J.J. Piek, B.B. Rubin, J. Butany, J.R. Stone, H.A. Katus, S.A. Murphy, D.A. Morrow, M.S. Sabatine, C. Vinegoni, M.A. Moskowitz, M.J. Pittet, P. Libby, C.P. Lin, F.K. Swirski, R. Weissleder, M. Nahrendorf, Myocardial infarction accelerates atherosclerosis., Nature. 487 (2012) 325-9. doi:10.1038/ nature11260.

[10] P.H.E. Groot, N.J. Pearce, J.W. Yates, C. Stocker, C. Sauermelch, C.P. Doe, R.N. Willette, A. Olzinski, T. Peters, D. d'Epagnier, K.O. Morasco, J.A. Krawiec, C.L. Webb, K. Aravindhan, B. Jucker, M. Burgert, C. Ma, J.P. Marino, J.L. Collins, C.H. Macphee, S.K. Thompson, M. Jaye, Synthetic LXR agonists increase LDL in CETP species., J. Lipid Res. 46 (2005) 21822191. doi:10.1194/jlr.M500116-JLR200.

[11] P. Kotokorpi, E. Ellis, P. Parini, L.-M. Nilsson, S. Strom, K.R. Steffensen, J.-A. Gustafsson, A. Mode, Physiological differences between human and rat primary hepatocytes in response to liver $X$ receptor activation by 3-[3-[N-(2-chloro-3-trifluoromethylbenzyl)(2,2-diphenylethyl)amino]propyloxy]phe nylacetic acid hydrochloride (GW3965)., Mol. Pharmacol. 72 (2007) 947-955. doi:10.1124/mol.107.037358.

[12] J. Tang, M.E. Lobatto, L. Hassing, S. van der Staay, S.M. van Rijs, C. Calcagno, M.S. Braza, S. Baxter, F. Fay, B.L. Sanchez-Gaytan, R. Duivenvoorden, H.B. Sager, Y.M. Astudillo, W. Leong, S. Ramachandran, G. Storm, C. Perez-Medina, T. Reiner, D.P. Cormode, G.J. Strijkers, E.S.G. Stroes, F.K.Swirski, M. Nahrendorf, E.A. Fisher, Z.A. Fayad, W.J.M. Mulder, Inhibiting macrophage proliferation suppresses atherosclerotic plaque inflammation, Sci. Adv. 1 (2015) e1400223. doi:10.1126/ sciadv.1400223.

[13] R. Duivenvoorden, J. Tang, D.P. Cormode, A.J. Mieszawska, D. Izquierdo-Garcia, C. Ozcan, M.J. Otten, N. Zaidi, M.E. Lobatto, S.M. van Rijs, B. Priem, E.L. Kuan, C. Martel, B. Hewing, H. Sager, M. Nahrendorf, G.J. Randolph, E.S.G. Stroes, V. Fuster, E.A. Fisher, Z.A. 
Fayad, W.J.M. Mulder, A statin-loaded reconstituted high-density lipoprotein nanoparticle inhibits atherosclerotic plaque inflammation., Nat. Commun. 5 (2014) 3065. doi:10.1038/ ncomms4065.

[14] B.L. Sanchez-Gaytan, F. Fay, M.E. Lobatto, J. Tang, M. Ouimet, Y. Kim, S.E.M. van der Staay, S.M. van Rijs, B. Priem, L. Zhang, E.A. Fisher, K.J. Moore, R. Langer, Z.A. Fayad, W.J.M. Mulder, HDL-Mimetic PLGA Nanoparticle To Target Atherosclerosis Plaque Macrophages, Bioconjug. Chem. 26 (2015) 443-451. doi:10.1021/bc500517k.

[15] D.P. Cormode, J.C. Frias, Y. Ma, W. Chen, T. Skajaa, K. Briley-Saebo, A. Barazza, K.J. Williams, W.J. Mulder, Z.A. Fayad, E.A. Fisher, HDL as a contrast agent for medical imaging., Clin. Lipidol. 4 (2009) 493-500. doi:10.2217/ clp.09.38.

[16] Y. Nagano, H. Arai, T. Kita, High density lipoprotein loses its effect to stimulate efflux of cholesterol from foam cells after oxidative modification., Proc. Natl. Acad. Sci. U. S. A. 88 (1991) 6457-6461.

[17] Y. Matsumura, Poly (amino acid) micelle nanocarriers in preclinical and clinical studies., Adv. Drug Deliv. Rev. 60 (2008) 899-914. doi:10.1016/j.addr.2007.11.010.

[18] V.P. Torchilin, Recent advances with liposomes as pharmaceutical carriers., Nat. Rev. Drug Discov. 4 (2005) 145-160. doi:10.1038/nrd1632.

[19] A.R. Tall, L. Yvan-Charvet, N. Terasaka, T. Pagler, N. Wang, HDL, ABC transporters, and cholesterol efflux: implications for the treatment of atherosclerosis., Cell Metab. 7 (2008) 365-375. doi:10.1016/j.cmet.2008.03.001.

[20] A.R. Tall, L. Yvan-Charvet, Cholesterol, inflammation and innate immunity, Nat. Rev. 15 (2015) 104-116. doi:10.1038/nri3793.

[21] M. Schiener, M. Hossann, J.R. Viola, A. Ortega-Gomez, C. Weber, K. Lauber, L.H. Lindner, O. Soehnlein, Nanomedicine-based strategies for treatment of atherosclerosis, Trends Mol. Med. 20 (2014) 271-281. doi:10.1016/j.molmed.2013.12.001.

[22] R. Weissleder, V. Ntziachristos, Shedding light onto live molecular targets., Nat. Med. 9 (2003) 123-128. doi:10.1038/nm0103-123.

[23] E. Galkina, B.L. Harry, A. Ludwig, E.A. Liehn, J.M. Sanders, A. Bruce, C. Weber, K. Ley, CXCR6 promotes atherosclerosis by supporting T-cell homing, interferon-gamma production, and macrophage accumulation in the aortic wall., Circulation. 116 (2007) 1801-1811. doi:10.1161/CIRCULATIONAHA.106.678474.

[24] E. Galkina, A. Kadl, J. Sanders, D. Varughese, I.J. Sarembock, K. Ley, Lymphocyte recruitment into the aortic wall before and during development of atherosclerosis is partially L-selectin dependent., J. Exp. Med. 203 (2006) 1273-1282. doi:10.1084/ jem.20052205.

[25] R. Weissleder, M. Nahrendorf, M.J. Pittet, Imaging macrophages with nanoparticles, Nat. Mater. 13 (2014) 125-138. doi:10.1038/ nmat3780.

[26] C. Hong, P. Tontonoz, Liver X receptors in lipid metabolism: opportunities for drug discovery, Nat. Rev. Drug Discov. 13 (2014) 433-444. doi:10.1038/nrd4280.

[27] S.B. Joseph, E. McKilligin, L. Pei, M.A. Watson, A.R. Collins, B.A. Laffitte, M. Chen, G. Noh, J. Goodman, G.N. Hagger, J. Tran, T.K. Tippin, X. Wang, A.J. Lusis, W.A. Hsueh, R.E. Law, J.L. Collins, T.M. Willson, P. Tontonoz, Synthetic LXR ligand inhibits the development of atherosclerosis in mice., Proc. Natl. Acad. Sci. U. S. A. 99 (2002) 7604-7609. doi:10.1073/ pnas.112059299.

[28] S.U. Naik, X. Wang, J.S. Da Silva, M. Jaye, C.H. Macphee, M.P. Reilly, J.T. Billheimer, G.H. Rothblat, D.J. Rader, Pharmacological activation of liver X receptors promotes reverse cholesterol transport in vivo., Circulation. 113 (2006) 90-97. doi:10.1161/ CIRCULATIONAHA.105.560177.

[29] M.A. Deri, B.M. Zeglis, L.C. Francesconi, J.S. Lewis, PET imaging with (8)(9)Zr: from radiochemistry to the clinic., Nucl. Med. Biol. 40 (2013) 3-14. doi:10.1016/j. nucmedbio.2012.08.004. 
[30] S.H. Zhang, R.L. Reddick, J.A. Piedrahita, N. Maeda, Spontaneous hypercholesterolemia and arterial lesions in mice lacking apolipoprotein E., Science. 258 (1992) 468-471.

[31] K.-T. Wu, P.-L. Kuo, S.-B. Su, Y.-Y. Chen, M.-L. Yeh, C.-I. Huang, J.-F. Yang, C.-I. Lin, M.-H. Hsieh, M.-Y. Hsieh, C.-F. Huang, W.-Y. Lin, M.-L. Yu, C.-Y. Dai, H.-Y. Wang, Nonalcoholic fatty liver disease severity is associated with the ratios of total cholesterol and triglycerides to high-density lipoprotein cholesterol., J. Clin. Lipidol. 10 (2016) 420-5.e1. doi:10.1016/j. jacl.2015.12.026.

[32] L.R. Brunham, J.K. Kruit, T.D. Pape, J.S. Parks, F. Kuipers, M.R. Hayden, Tissue-specific induction of intestinal ABCA1 expression with a liver $X$ receptor agonist raises plasma HDL cholesterol levels., Circ. Res. 99 (2006) 672-674. doi:10.1161/01.RES.0000244014.19589.8e.

[33] D.L. Herber, W. Cao, Y. Nefedova, S. V Novitskiy, S. Nagaraj, V.A. Tyurin, A. Corzo, H.-I. Cho, E. Celis, B. Lennox, S.C. Knight, T. Padhya, T. V McCaffrey, J.C. McCaffrey, S. Antonia, M. Fishman, R.L. Ferris, V.E. Kagan, D.I. Gabrilovich, Lipid accumulation and dendritic cell dysfunction in cancer., Nat. Med. 16 (2010) 880-886. doi:10.1038/nm.2172.

[34] Y. Zhao, F. Fay, S. Hak, J. Manuel Perez-Aguilar, B.L. Sanchez-Gaytan, B. Goode, R. Duivenvoorden, C. de Lange Davies, A. Bjorkoy, H. Weinstein, Z.A. Fayad, C. PerezMedina, W.J. Mulder, Augmenting drug-carrier compatibility improves tumour nanotherapy efficacy, Nat. Commun. 7 (2016) 11221. doi:10.1038/ncomms11221.

[35] A. Mullard, PCSK9 inhibitors are go., Nat. Rev. Drug Discov. 14 (2015) 593. doi:10.1038/ nrd4730.

[36] M.S. Sabatine, R.P. Giugliano, S.D. Wiviott, F.J. Raal, D.J. Blom, J. Robinson, C.M. Ballantyne, R. Somaratne, J. Legg, S.M. Wasserman, R. Scott, M.J. Koren, E.A. Stein, Efficacy and safety of evolocumab in reducing lipids and cardiovascular events., N. Engl. J. Med. 372 (2015) 1500-1509. doi:10.1056 / NEJMoa1500858.

[37] J.G. Robinson, M. Farnier, M. Krempf, J. Bergeron, G. Luc, M. Averna, E.S. Stroes, G. Langslet, F.J. Raal, M. El Shahawy, M.J. Koren, N.E. Lepor, C. Lorenzato, R. Pordy, U. Chaudhari, J.J.P. Kastelein, Efficacy and safety of alirocumab in reducing lipids and cardiovascular events., N. Engl. J. Med. 372 (2015) 1489-1499. doi:10.1056/ NEJMoa1501031.

[38] P. Chames, M. Van Regenmortel, E. Weiss, D. Baty, Therapeutic antibodies: successes, limitations and hopes for the future., Br. J. Pharmacol. 157 (2009) 220-233. doi:10.1111/ j.1476-5381.2009.00190.x.

[39] D. Cook, D. Brown, R. Alexander, R. March, P. Morgan, G. Satterthwaite, M.N. Pangalos, Lessons learned from the fate of AstraZeneca's drug pipeline: a five-dimensional framework., Nat. Rev. Drug Discov. 13 (2014) 419-431. doi:10.1038/nrd4309.

[40] A.J. Mieszawska, Y. Kim, A. Gianella, I. van Rooy, B. Priem, M.P. Labarre, C. Ozcan, D.P. Cormode, A. Petrov, R. Langer, O.C. Farokhzad, Z.A. Fayad, W.J.M. Mulder, Synthesis of polymer-lipid nanoparticles for image-guided delivery of dual modality therapy., Bioconjug. Chem. 24 (2013) 1429-1434. doi:10.1021 / bc400166j.

[41] G. Fredman, N. Kamaly, S. Spolitu, J. Milton, D. Ghorpade, R. Chiasson, G. Kuriakose, M. Perretti, O. Farokhzad, O. Farokzhad, I. Tabas, Targeted nanoparticles containing the proresolving peptide Ac2-26 protect against advanced atherosclerosis in hypercholesterolemic mice., Sci. Transl. Med. 7 (2015) 275ra20. doi:10.1126/scitranslmed. aaa1065.

[42] E. Choleris, S.R. Little, J.A. Mong, S. V Puram, R. Langer, D.W. Pfaff, Microparticlebased delivery of oxytocin receptor antisense DNA in the medial amygdala blocks social recognition in female mice., Proc. Natl. Acad. Sci. U. S. A. 104 (2007) 4670-4675. doi:10.1073/pnas.0700670104.

[43] K. Palucka, J. Banchereau, Cancer immunotherapy via dendritic cells., Nat. Rev. Cancer. 12 (2012) 265-277. doi:10.1038/nrc3258.

[44] H. Hammad, B.N. Lambrecht, Dendritic cells and epithelial cells: linking innate and 
adaptive immunity in asthma., Nat. Rev. Immunol. 8 (2008) 193-204. doi:10.1038/nri2275. [45] G. Mukherjee, T.P. Dilorenzo, The immunotherapeutic potential of dendritic cells in type 1 diabetes., Clin. Exp. Immunol. 161 (2010) 197-207. doi:10.1111/j.1365-2249.2010.04157.x.

[46] C. Pérez-Medina, J. Tang, D. Abdel-Atti, B. Hogstad, M. Merad, E.A. Fisher, Z.A. Fayad, J.S. Lewis, W.J.M. Mulder, T. Reiner, PET Imaging of Tumor-Associated Macrophages with 89Zr-Labeled High-Density Lipoprotein Nanoparticles, J. Nucl. Med. 56 (2015) 1272-1277. doi:10.2967/jnumed.115.158956. 


\section{APPENDIX: SUPPLEMENTARY DATA}

\section{SI Materials and Methods}

Radiochemistry. The $89 \mathrm{Zr}$ was produced at Memorial Sloan Kettering Cancer Center on an Ebco TR19/9 variable-beam energy cyclotron (Ebco Industries Inc.) via the ${ }^{89} \mathrm{Y}(\mathrm{p}, \mathrm{n}){ }^{89} \mathrm{Zr}$ reaction and purified with a method previously reported [46]. Radioactivity was measured with a Capintec CRC-15R dose Calibrator (Capintec).

Synthesis of C34-DFO. The 2-Hexadecyl-octanoic acid (30 mg, $59 \mu \mathrm{mol}$ ), (Benzotriazol-1-yloxy) Tris(dimethylamino)phosphonium hexafluorophosphate (29 mg, $66 \mu \mathrm{mol}$ ), and N,N-diisopropylethylamine (DIEA, $10 \mu \mathrm{L}$ ) were dissolved in anhydrous dichloromethane $(2 \mathrm{~mL})$ and stirred at $40{ }^{\circ} \mathrm{C}$ for $10 \mathrm{~min}$ under nitrogen atmosphere. Next, a solution of deferoxamine mesylate $(30 \mathrm{mg}, 46 \mu \mathrm{mol})$ and DIEA $(10 \mu \mathrm{L})$ in anhydrous DMSO $(0.7 \mathrm{~mL})$ was added, and the resulting mixture was stirred for $4 \mathrm{~h}$ at $40{ }^{\circ} \mathrm{C}$ under nitrogen. The cloudy suspension was allowed to cool to room temperature, and dichloromethane was removed under reduced pressure. Hydrochloric acid $(0.1 \mathrm{M}, 1 \mathrm{~mL})$ was added, and the mixture was stirred for 10 min at room temperature. The solid was filtered and washed with $0.1 \mathrm{M} \mathrm{HCl}(3 \times 1$ $\mathrm{mL})$, DMSO $(3 \times 1 \mathrm{~mL})$, water $(3 \times 1 \mathrm{~mL})$, and, finally, dichloromethane $(3 \times 1 \mathrm{~mL})$ and dried to yield a white solid $(31 \mathrm{mg}, 64 \%)$. Mass cytometry analysis generated the following data: MS-ES+: 1,074, (M+Na)+; MS-ES-: 1,050 (M-H)-, 1,086 (M+Cl)-. Proton NMR analysis provided the subsequent values: $1 \mathrm{H}-\mathrm{NMR}$ (CDCl3/CD3OD), $\delta$ in parts per million: 0.55 (triplet, 6H); 0.92 [broad signal (br), 60H]; $1.06(\mathrm{br}, 6 \mathrm{H})$; 1.27 multiplet $(\mathrm{m}), 6 \mathrm{H}] ; 1.32(\mathrm{~m}, 6 \mathrm{H}) ; 1.76(\mathrm{~m}, 1 \mathrm{H}) ; 1.80$ singlet $(\mathrm{s}), 3 \mathrm{H}] ; 2.15(\mathrm{t}, 4 \mathrm{H})$; $2.46(\mathrm{t}, 4 \mathrm{H}) ; 2.87(\mathrm{~m}, 6 \mathrm{H}) ; 3.29(\mathrm{~m}, 6 \mathrm{H})$. All chemicals were purchased from SigmaAldrich.

Radiolabeling of Library Nanoparticles and GW3965-Loaded Nanoparticles. Nanoparticles were labeled using a slightly modified version of the synthesis procedure for regular screening nanoparticles or GW3965-loaded nanoparticles. Briefly, DiR of the library nanoparticles or $0.5 \%$ of GW3965 payload was replaced with C34-DFO while synthesizing radiolabeled nanoparticles. After synthesis and purification, the C34-DFO- labeled nanoparticles were incubated with $89 \mathrm{Zr}$-oxalate in PBS ( $\mathrm{pH} \mathrm{7.1} \mathrm{7.4)} \mathrm{at} 37{ }^{\circ} \mathrm{C}$ for $2 \mathrm{~h}$ at an activity-to-APOA1 ratio of $\sim 1 \mathrm{mCi} / \mathrm{mg}$ (or $\sim 1 \mathrm{mCi} / 10 \mathrm{mg}$ lipids for NP17). The radiolabeled nanoparticles were purified by spin filtration by using 10,000 Da MMCO filter tubes. Radiochemical yields were in excess of $80 \%$, and radiochemical purities, as determined by size exclusion chromatography, were greater than $95 \%$ for all nanoparticles.

Blood Half-Life Determination. In the library study, all nanoparticles were injected at a fixed DiR dose of $1 \mathrm{mg} / \mathrm{kg}$. The theoretical 0 time point was calculated by assuming that a mouse's blood accounts for $6 \%$ of its body weight and the density of mouse blood was $1.06 \mathrm{~g} / \mathrm{mL}$. The mice were bled $1 \mathrm{~h}, 6 \mathrm{~h}$, and $24 \mathrm{~h}$ after the injection. 
The DiR concentration was determined by comparing DiR fluorescence signal to a DiR standard curve. DiR signal was measured in an IVIS200 system (PerkinElmer) at a 750-nm excitation wavelength and an 820-nm emission wavelength. Blood halflife was calculated by finding the $x$ coordinate from the curve's intersection with a horizontal line at 50\% signal of time 0 . Five mice were used for each nanoparticle. Regarding the radiolabeled nanoparticles, animals were injected with $\sim 20 \mu \mathrm{Ci}$ to $\sim 30 \mu \mathrm{Ci}$ of the corresponding nanoparticles, and blood samples ( 10 $\mu \mathrm{L}$ each) were collected $2 \mathrm{~min}, 30 \mathrm{~min}, 1 \mathrm{~h}, 2 \mathrm{~h}, 4 \mathrm{~h}, 8 \mathrm{~h}$, and $24 \mathrm{~h}$ after the i.v. injection. The blood was weighted, and its radioactivity was determined using a gamma counter (PerkinElmer). Blood half-life was calculated via two-phase decay. Three mice were used for each nanoparticle.

Autoradiography and Biodistribution. Upon sacrifice, animals were perfused with $20 \mathrm{~mL}$ of PBS through cardiac puncture. Lung, liver, aorta, spleen, kidney, femur muscle, and heart were collected, and their radiotracer distribution was determined by placing the tissues in a film cassette against a phosphorimaging plate (BASMSM- 2325, Fujifilm) for either $14 \mathrm{~h}$ (mouse aortas) or $4 \mathrm{~h}$ (all other organs). Phosphorimaging plates were read in a Typhoon 7000IP plate reader (GEHealthcare). Biodistribution in aorta, heart, liver, spleen, blood, lung, skin, brain, pancreas, stomach, small intestine, large intestine, kidney, muscle, and bone was determined by first weighing the tissues and then measuring their radioactivity in a gamma counter (PerkinElmer). The relative activity per tissue is presented as percentage of injected dose per gram of tissue.

Nanoparticles Characterization. The synthesized nanoparticles' size was measured by DLS. To measure the DiR concentration in nanoparticles, we first extracted DiR from the nanoparticles with acetonitrile, and the amount of DiR was determined by measuring the signature absorbance of DiR at $750 \mathrm{~nm}$. Nanoparticle morphology was determined by transition emission microscopy. Briefly, the original PBS solvent was replaced with an ammonium acetate buffer and then mixed with $2 \%$ (wt/vol) sodium phosphotungstate $(\mathrm{pH}=7.4)$ to negatively stain the nanoparticles. The solution was then added to a TEM grid and imaged with a Hitachi H7650 system linked to a Scientific Instruments and Applications digital camera controlled by Maxim CCD software. To measure nanoparticle APOA1 concentration, APOA1 was separated from the other nanoparticle components by acetonitrile precipitation. The pellets' protein component was then measured with a standard BCA assay (Thermo Scientific).

HPLC and Radio-HPLC. HPLC was performed on a Shimadzu HPLC system equipped with two LC-10AT pumps and an SPD-M10AVP photodiode array detector. Radio-HPLC was performed using a Lablogic Scan-RAM Radio-TLC/ HPLC detector. Size exclusion chromatography was performed on a Superdex 10/300 column (GEHealthcare Life Sciences) using PBS as an eluent at a flow rate of $1 \mathrm{~mL} / \mathrm{min}$. GW3865 detection was performed on a C18 column (Shimadzu) with an isocratic flow of water and acetonitrile at a flow rate of $1 \mathrm{~mL} / \mathrm{min}$. 
Cholesterol Efflux Assay. Bone marrow cells were flushed from the tibia and femurs of 6- to 8-wk-old C57BL/ 6 mice and differentiated into macrophages by incubation in DMEM media supplemented with 10\% FBS, 1\% P/S, and 15\% L929conditioned media for $7 \mathrm{~d}$. Bone marrow-derived macrophages were subsequently incubated with media containing $0.5 \mu \mathrm{Ci} / \mathrm{mL}[3 \mathrm{H}]$-cholesterol and acLDL (50 $\mu \mathrm{g} / \mathrm{mL}$ ) for $30 \mathrm{~h}$. Cells were washed twice with PBS and equilibrated overnight in media containing $2 \mathrm{mg} / \mathrm{mL}$ fatty acid- free albumin. Efflux to APOA1 (50 $\mu \mathrm{g} /$ $\mathrm{mL}$ ) or various nanoparticles $(50 \mu \mathrm{g} / \mathrm{mL}$ normalized to APOA1 concentration) was carried out for $8 \mathrm{~h}$. Cell media were removed, and the cells were lysed in $0.1 \mathrm{M} \mathrm{NaOH}$ solution. The $[3 \mathrm{H}]$-cholesterol contents of media and cell lysates were measured by liquid scintillation counting. Efflux is expressed as a percentage of $3 \mathrm{H}$ - cholesterol in medium by using the following formula: [3H-cholesterol in medium $/(3 \mathrm{H}-$ cholesterol in medium $+3 \mathrm{H}$-cholesterol in cells) $] \times 100 \%$.

NIRF. Twenty-four hours after i.v. injection of DiR-containing nanoparticles, the mice were perfused with $20 \mathrm{~mL}$ of PBS, and brain, lung, heart, aorta, spleen, liver, kidney, and femur muscle were collected. Their total DiR fluorescent signal was measured in an IVIS 200 at a 750-nm excitation wavelength and an 820-nm emission wavelength. The radiant efficiency was calculated using Live Imaging (PerkinElmer). For each nanoparticle, five mice were used.

Flow Cytometry. We used a protocol similar to one previously reported [12]. Briefly, at $24 \mathrm{~h}$ after i.v. injection of DiR-loaded nanoparticles, blood was collected and the animals were perfused. Afterward, aortas and spleens were collected, diced, and digested with a mixture of enzymes, including liberase TH (Roche), hyaluronidase (Sigma-Aldrich), and DNase (Sigma-Aldrich), in a $37{ }^{\circ} \mathrm{C}$ oven for 1 h. A single-cell suspension was made by removing tissue aggregates, extracellular matrix, and cell debris from the solution. Red blood cells were removed from the blood sample by lysis buffer. DiR was detected on the APC-Cy7 channel. To identify macrophages, monocytes, DCs, neutrophils, and other immune cells, a lineage of antibodies (Lin) recognizing CD90 (clone 53.2.1), B220 (clone RA3-6B2), CD49b (clone DX5), NK1.1 (clone PK136), Ly-6G (clone 1A8), and Ter-119 (clone TER-119) and antibodies recognizing Ly-6C (clone AL21), F4/80 (BM8), and CD11c (N418) were used. Antibodies were purchased from eBioscience, BD Biosciences, and Biolegend. Due to the large number of samples, two to three nanoparticles were measured per batch, each of which included 12 to 17 mice (10 to 15 from tested nanoparticles, 1 for reference DiR-nanoparticles, and 1 non injected control to set up compensation). The APC-Cy7 channel of the flow cytometer was calibrated using APC-Cy7 calibration beads (Cat\# ECFP-F7; Spherotech). Signal variation among batches was corrected by normalizing to the beads' signal. All samples were measured on an LSRII (BD Biosciences) flow cytometer. Results were analyzed with FlowJo (Ashland), and statistics were calculated with Prism (GraphPad). 
Hepatic Triglyceride and Cholesterol Measurement. Lipids were extracted from the livers, which were snap-frozen in $-80{ }^{\circ} \mathrm{C}$ immediately after sacrifice. Portions of liver tissue (generally $<100 \mathrm{mg}$ ) were weighed, suspended in $2 \times$ weight volume of PBS, and homogenized. To analyze protein content, $10 \mu \mathrm{L}$ of the total homogenate underwent a standard Lowry assay (BioRad); $50 \mu \mathrm{L}$ of the total homogenate was suspended in $3 \mathrm{~mL}$ of isopropanol and incubated overnight at $4{ }^{\circ} \mathrm{C}$ while rocking to extract lipids. The solvent/lipid mixture was then centrifuged at 1,000 $\times \mathrm{g}$ for $10 \mathrm{~min}$, and supernatant was collected in glass tubes and dried under a stream of nitrogen. Following complete evaporation of the extraction isopropanol, lipids were stored at $-20{ }^{\circ} \mathrm{C}$ until analysis. For quantification, dried lipids were resuspended in $1 \mathrm{~mL}$ of isopropanol, and analysis of triglycerides and total cholesterol was performed on diluted lipid solutions using standard colorimetric assays (Wako) as per manufacturer's protocol. The cholesterol and triglyceride concentrations were normalized to the measured protein concentration per sample.

Immunostaining and Laser Capture Microdissection. As previously described [2], 6- $\mu \mathrm{m}$-thick frozen sections were made from the aortic roots of 60 mice. Six evenly distributed sections were stained with CD68 (clone MCA1957; Serotec). The stained slides were digitally scanned using a slide scanner (3DHistech Panoramic Scanner). When CD68- positive area was quantified, the positively stained area was calculated using a MATLAB procedure we had developed previously (2). A total of 360 sections were analyzed. For laser capture microdissection (LCM), 36 aortic roots sections per animal were used to extract atherosclerotic macrophages using Leica LDM6500. All LCM reagents were maintained, and procedures were done under RNase-free conditions. The sections were fixed in $70 \%$ ethanol for $1 \mathrm{~min}$, washed in $\mathrm{H}_{2} \mathrm{O}$, stained with Mayer's hematoxylin (VWR Scientific) for $1 \mathrm{~min}$, washed in $\mathrm{H}_{2} \mathrm{O}$, incubated in PBS (to develop blue color) for $15 \mathrm{~s}$, washed in $\mathrm{H}_{2} \mathrm{O}$, partially dehydrated in $70 \%$ followed by $95 \%$ ethanol, stained in eosin Y (VWR Scientific) for 5 s, washed in 95\% ethanol, and then completely dehydrated in 100\% ethanol (30 s), xylene (30 s), and xylene $(5 \mathrm{~min})$. The sections were then air-dried for $10 \mathrm{~min}$. Macrophages were identified under a microscope and verified by anti-CD68 staining on the guiding slides. Isolated macrophages were immediately lysed, and their RNA were extracted and stored in a $-80^{\circ} \mathrm{C}$ freezer. For LCM, 2,160 sections were used. 
a
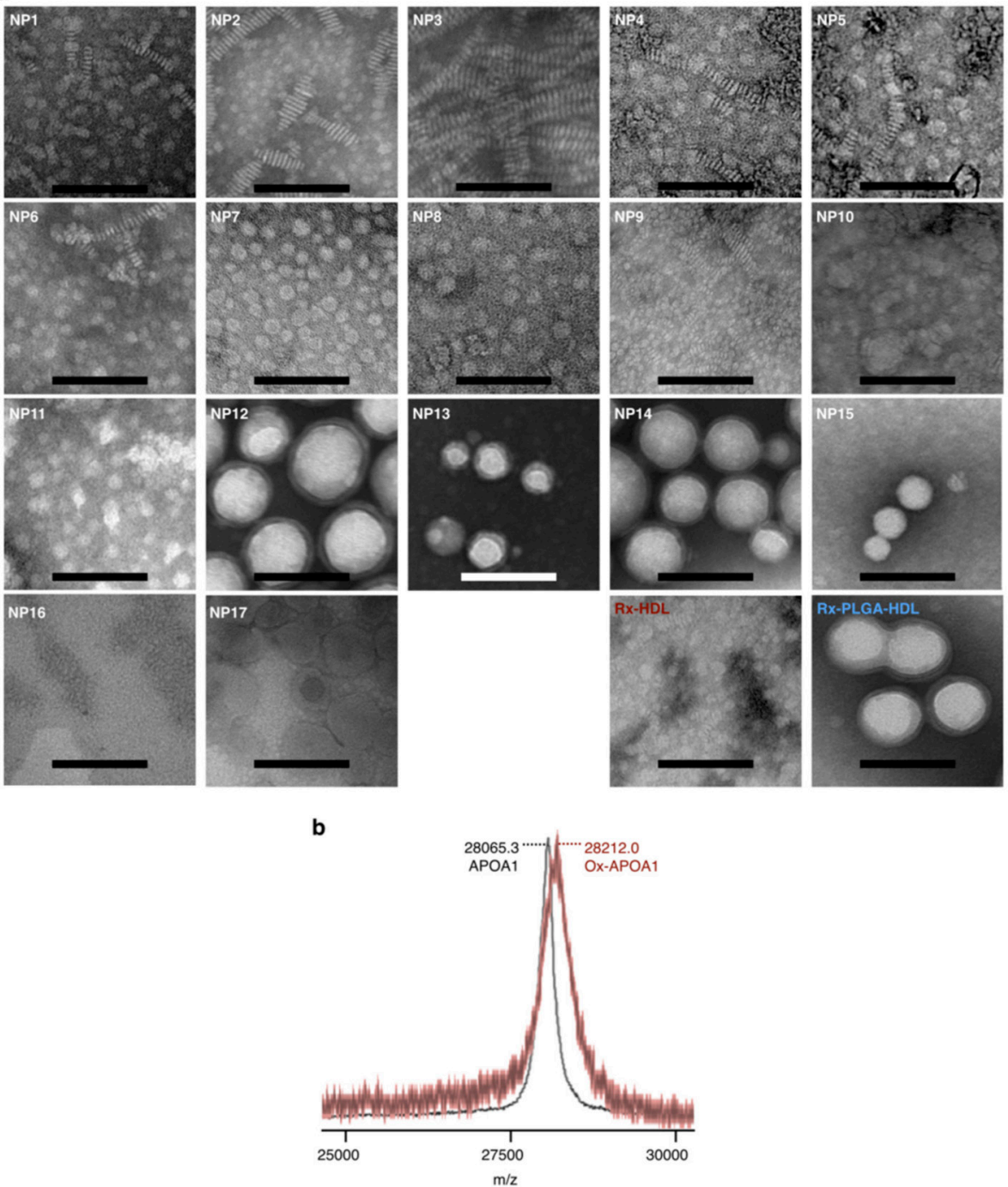

Figure S1 I Chemical characterization of the nanoparticle library. (A) Low-magnification TEM images of the 17 nanoparticles, as well as the two therapeutic nanoparticles-Rx-HDL and Rx-PLGA-HDL. NP1-5 have a discoidal shape, as is typical for HDL nanoparticles reconstituted from phospholipids and APOA1. POPC-based nanoparticles require a higher amount of APOA1 to force disk formation, as can be observed when comparing NP8 and NP9. The inclusion of triglycerides as a core material results in nanoparticles with $20 \mathrm{~nm}$ (NP6, NP7, NP10, and NP11), whereas the inclusion of PLGA or PLA polymers allows fine tuning of nanoparticle sizes, ranging from $40 \mathrm{~nm}$ for NP15 to over $100 \mathrm{~nm}$ for NP12. (Scale bar, $100 \mathrm{~nm}$.) (B) Mass spectrometry of oxidized nanoparticle. An HDL-mimicking nanoparticle made mainly of DMPC and APOA1 (NP1) was oxidized to generate NP2, which was analyzed by mass spectrometry. Mass spectrometry shows the increase of APOA1 M.W. by about 146.7 on average, indicating the oxidization of APOA1 (Ox-APOA1). 
a
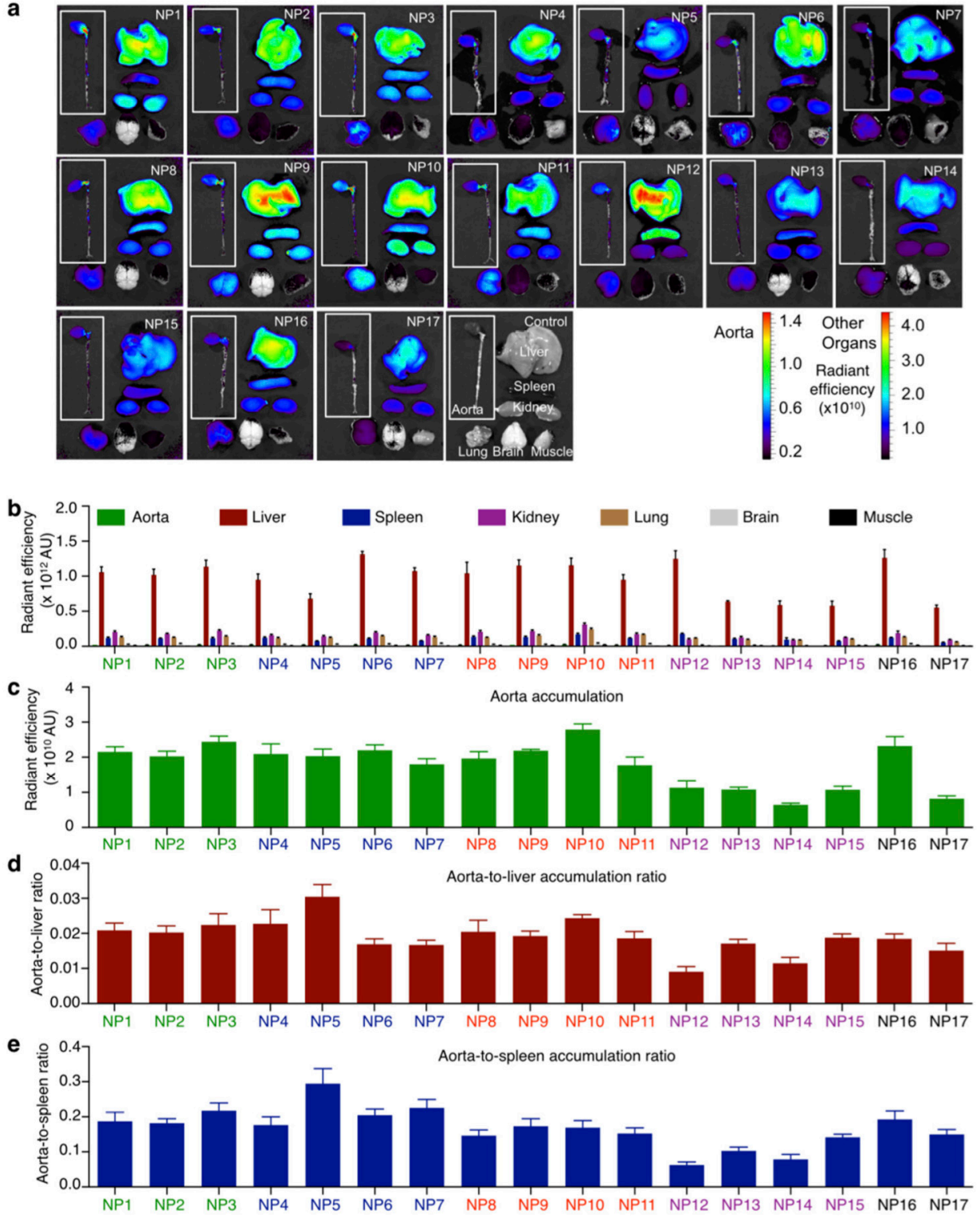

Figure S2 I Representative and quantitative NIRF data. (A) Representative NIRF images of key organs. Quantification of total signal of (B) all organs and (C) aortas. (D) The aortic-to-liver accumulation ratio is calculated by dividing the total signal of aorta with that of liver. (E) The aorta-to-spleen accumulation ratio is calculated by dividing the total signal of aorta with that of spleen. For B-E, $\mathrm{n}=5$ for per nanoparticle, and the error bars are SEM. 

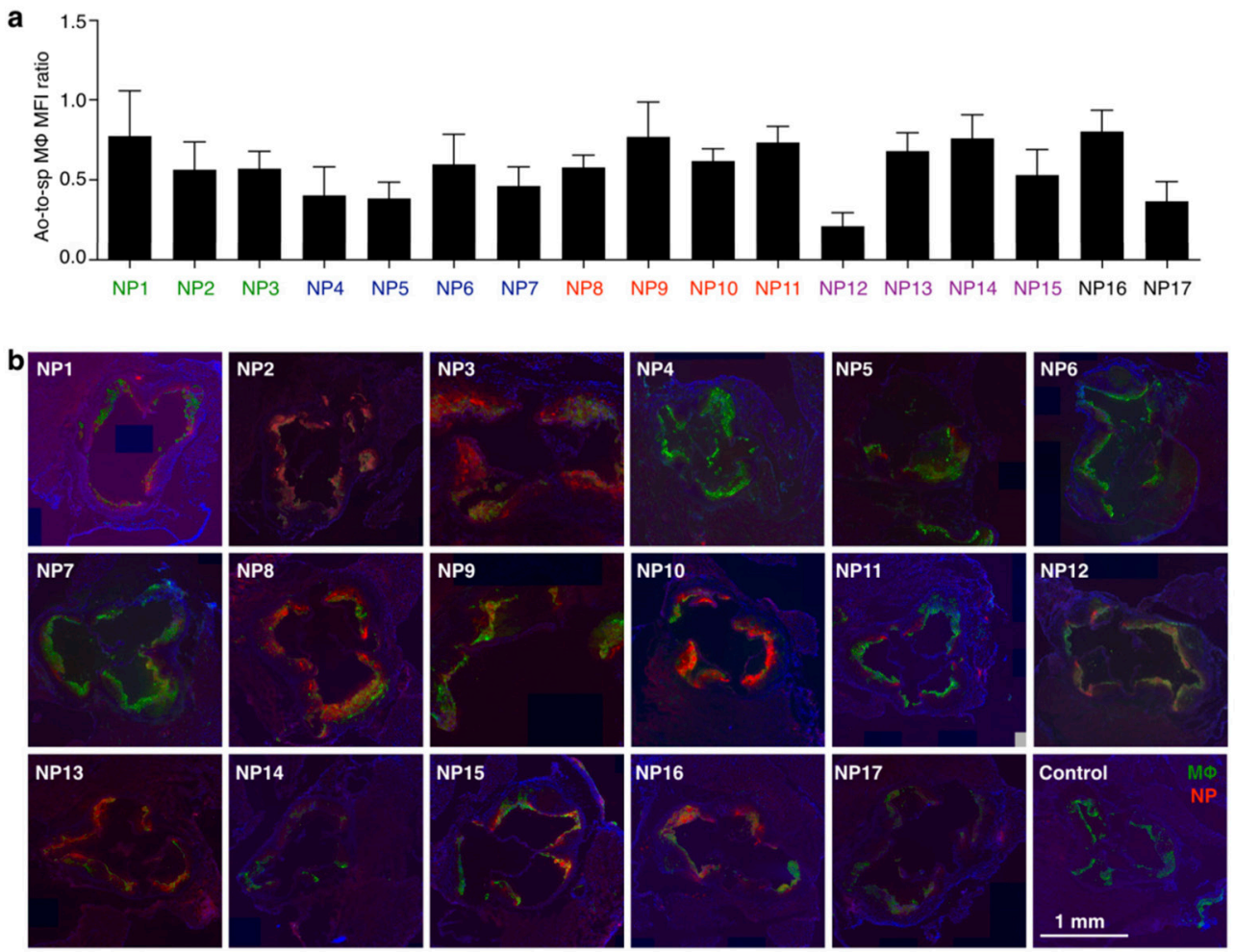

Figure S3 I Macrophage targeting efficiency of the nanoparticle library. (A) Aortic-to-splenic (Ao-to-sp) macrophage MFI $(M \Phi)$ ratio is calculated by dividing the MFI of aortic macrophages by that of splenic macrophages ( $n=5$ per nanoparticle). Error bars are SEM. (B) Representative immunostaining images of aortic roots from the animals injected with the nanoparticles. CD68 was used to identify macrophages in the frozen section. Nanoparticles were identified by detecting their fluorescent label DiR.. 

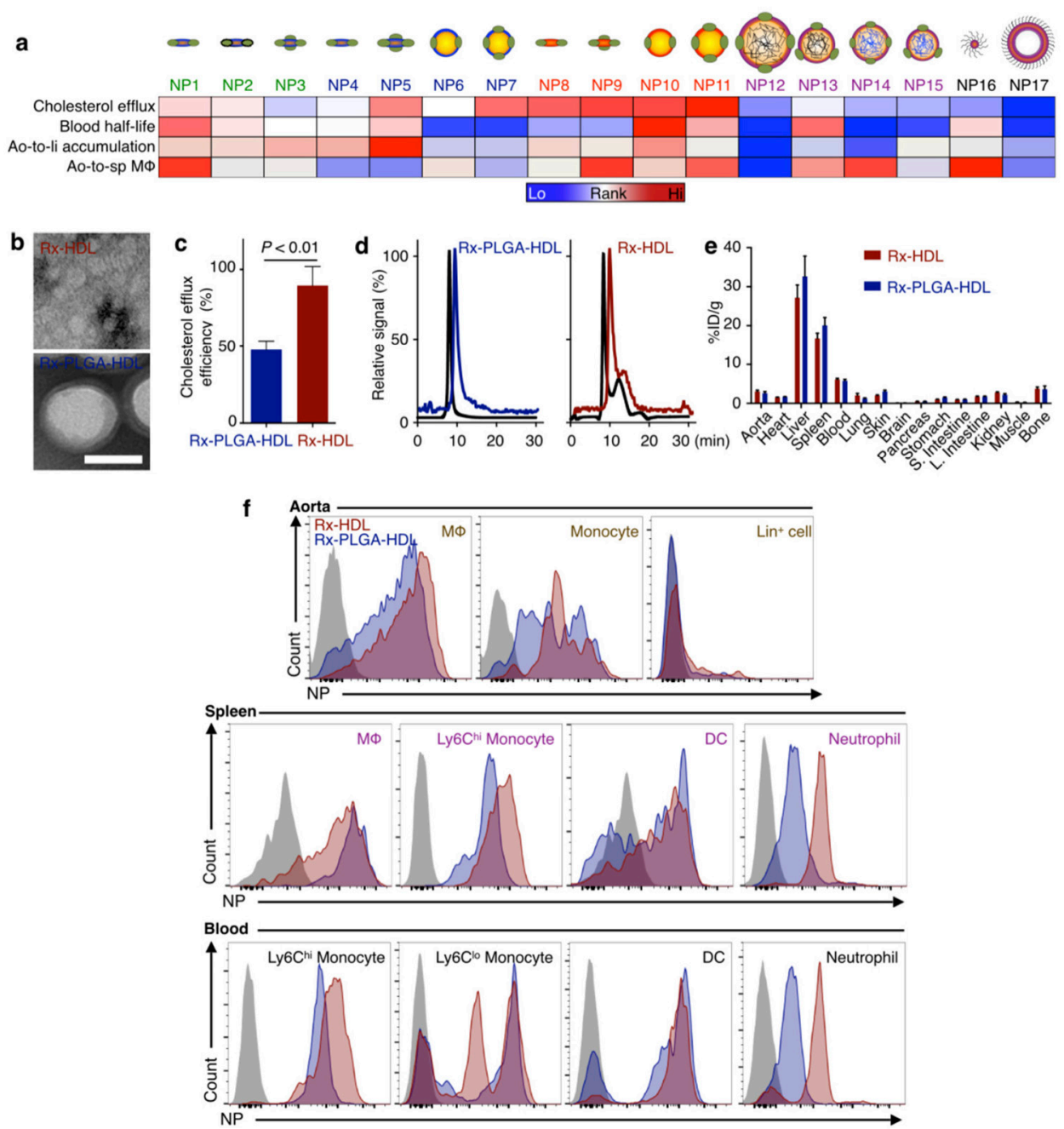

Figure S4 I In vivo evaluation of drug-loaded nanoparticles. (A) The summary of the performance of the nanoparticle library in the immunological screening study, with the red showing the favorable and the blue showing the unfavorable performance according to the specific limitations of GW3965. Highrank nanoparticles show high cholesterol efflux capacity, long blood half-life, high relative aorta-toliver (Ao-to-li) accumulation ration, and high relative aortic-to splenic macrophage MFI ratio. NP10 was found to have the highest overall rank. (B) Representative TEM images of negatively stained drugloaded nanoparticles. Overview of the images can be found in Fig. S1A. (Scale bar, $50 \mathrm{~nm}$.) (C) Relative cholesterol efflux capacity of drug-loaded nanoparticles $(n=6)$. Nonparametric Student's t test was used for statistics. (D) Radioactive HPLC eluting profiles of drug-loaded nanoparticles. The black profile is the UV absorbance, which indicates the nanoparticle itself. The blue and red profiles are the ${ }^{89} \mathrm{Zr}$ radioactive signal. A 2.5-min delay between UV and radioactive profiles is added on purpose to better present the profiles. (E) Extensive biodistribution of radio-labeled and GW3965-loaded nanoparticles in relevant organs ( $\mathrm{n}=5$ per nanoparticle). (F) Representative flow cytometry histograms of relevant immune cells in different tissues. All error bars are SEM. 

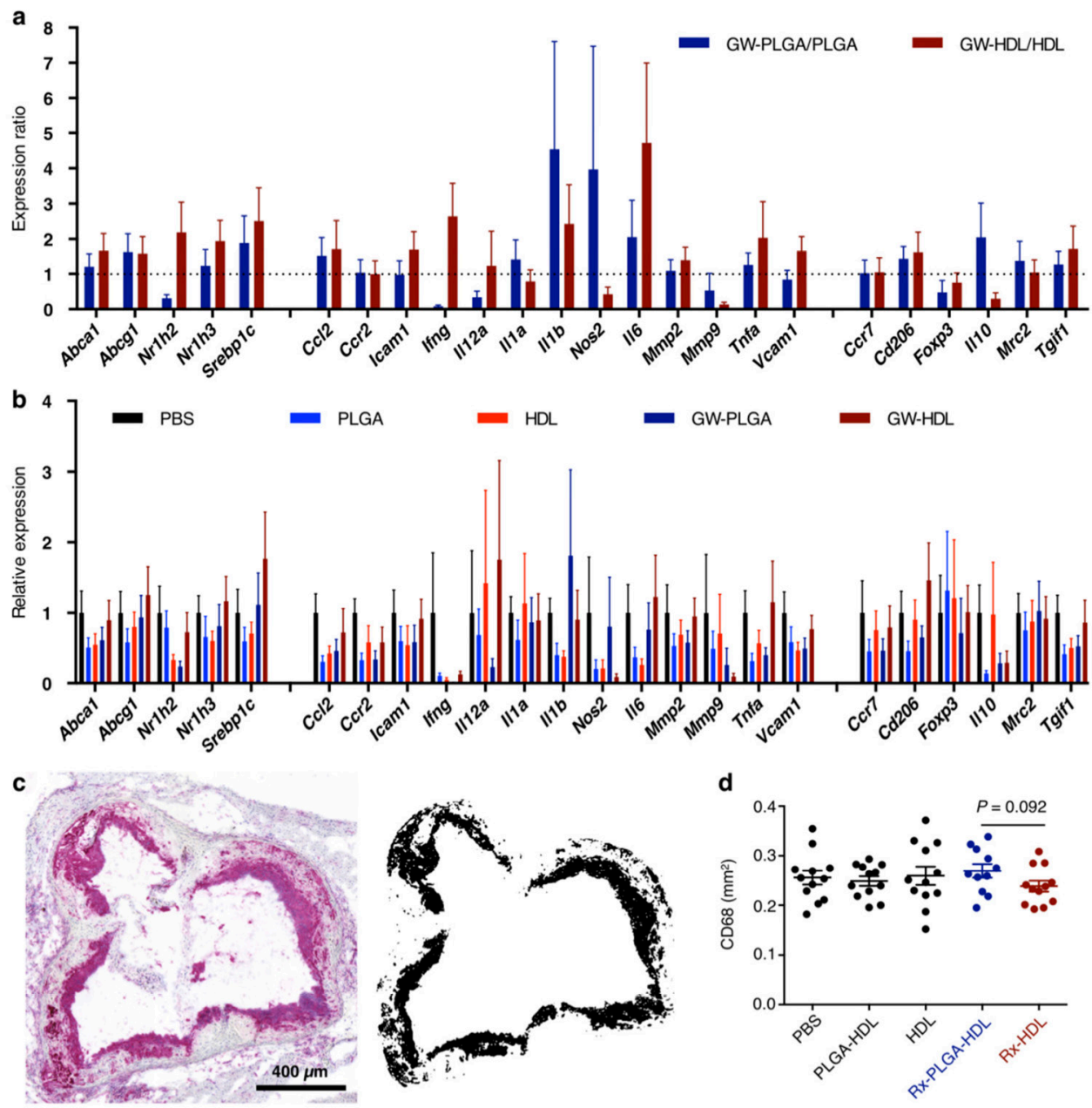

Figure S5 I Efficacy of drug-loaded nanoparticles on aortic macrophages. (A) Quantification of mRNA expression of the selected genes in atherosclerotic plaque macrophages. The relative expression of a gene is calculated by following these formulas: relative gene expression of gene $\mathrm{X}$ of Rx-HDL group $=($ expression of gene $X$ in the Rx- HDL group/ expression of gene $X$ in the HDL group); relative gene expression of gene $X$ of Rx-PLGA-HDL group $=$ (expression of gene $X$ in the Rx-PLGA-HDL group/expression of gene $X$ in the PLGA-HDL group). (B) Relative gene expression normalized to average expression of both Rx-PLGAHDL and Rx-HDL groups by following a formula: gene $X$ expression in one group = (expression of gene $X$ in the given group/average expression of gene $X$ in both Rx-PLGA-HDL and Rx-HDL groups). (C) Relative gene expression normalized to PBS group by following the following formula: gene $\mathrm{X}$ expression of a given group $=$ (expression of gene $X$ in the given group/expression of gene $X$ in the PBS group) . (D) Representative CD68 immunostaining of aortic roots. CD68 was used as the macrophage marker in this experiment. (Left) The original image and (Right) the mask generated by a MATLAB procedure. The dark pixels in the mask were calculated and used to calculate the CD68 positive area in the original immunostaining image. (E) Quantification of macrophage levels in all treatment group. $\mathrm{n}=12$ per group (A-C and E). Nonparametric Student's t test was used to calculate statistics, and all error bars are SEM. 


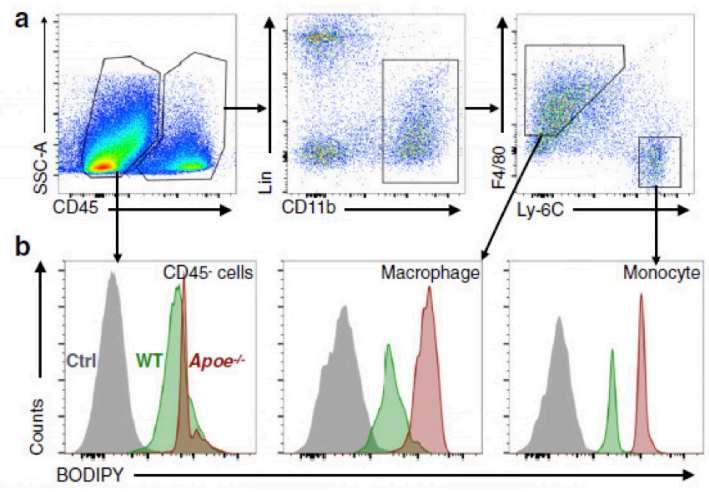

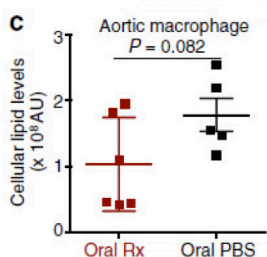

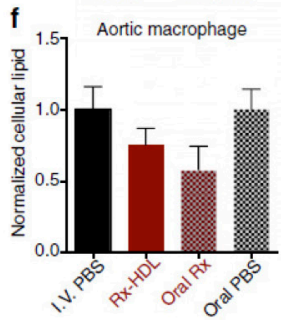

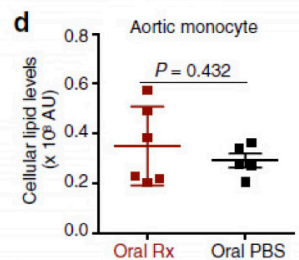

g

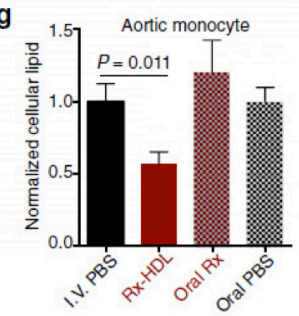

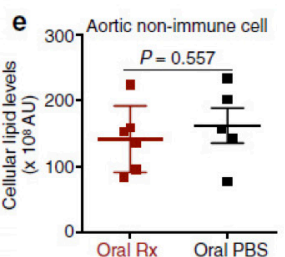

h

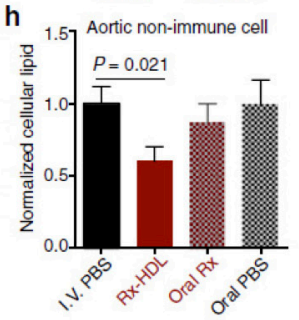

Figure S6 I Cellular lipid measurement flow cytometry protocol and the efficacy evaluation of 6-wk treatment. Aortic cells from 1-y-old C57BL/ 6 wild-type $(\mathrm{WT})$ mice $(\mathrm{n}=2)$ and Apoe ${ }^{-/-}$mice $(\mathrm{n}=2)$ with 10 mo of high- cholesterol diet were stained with BODIPY. Intracellular BODIPY signal was measured by a flow cytometry procedure used in the previous experiments (Fig. 3A). (A) Representative gating procedure shows the identification of CD45 negative $\left(\mathrm{CD} 45^{-}\right)$nonimmune cells, macrophages, and monocytes in the aortas. (B) Representative BODIPY signal levels in (Left) nonimmune cells, (Middle) macrophages, and (Right) monocytes are shown in the histogram graphs. Gray histograms represent the non-BODIPY-stained cells; green histograms represent cellular BODIPY levels of the cells from wildtype mice; red histograms (Ctrl) represent cellular BODIPY levels of the cells from Apoe ${ }^{-1-}$ mice. Cells from $A$ poe $^{-1-}$ mice show much higher BODIPY levels than those from wild-type mice, due to their higher cellular lipid levels than those in wild-type mice. (C-E) Apoe ${ }^{-1-}$ mice received oral treatment of either GW3965 (Oral Rx, n=6) or PBS (Oral PBS, $n=5$ ) twice per week for 6 wk. Cellular lipid levels were calculated by multiplying the mean fluorescence intensity of a cell type with the number of the cells per aorta (AU = MFI X number of cells). Cellular lipids levels of the two groups were compared in aortic macrophages (C), aortic monocytes (D), and aortic nonimmune cells (E). (F-H) Two batches of female $A_{p o e^{-I-}}$ mice with the same age and length of high-cholesterol diet were given either i.v. treatments (I.V. PBS or Rx-HDL; Fig. 5 F-H) or oral treatments (Oral Rx or Oral PBS). Due to the batch and measurement difference, the cellular lipid levels of aortic cell populations treated with either Rx-HDL or Oral Rx were normalized to those from mice treated with either i.v. PBS or oral PBS, respectively. Cellular lipid levels from the two batches of treated mice were compared with their corresponding control-treated mice in aortic macrophages $(\mathrm{F})$, aortic monocytes $(\mathrm{G})$, and aortic nonimmune cells $(\mathrm{H})$. Error bars in all graphs are SEM. Statistics was calculated with nonparametric two-tailed Student's t test. 

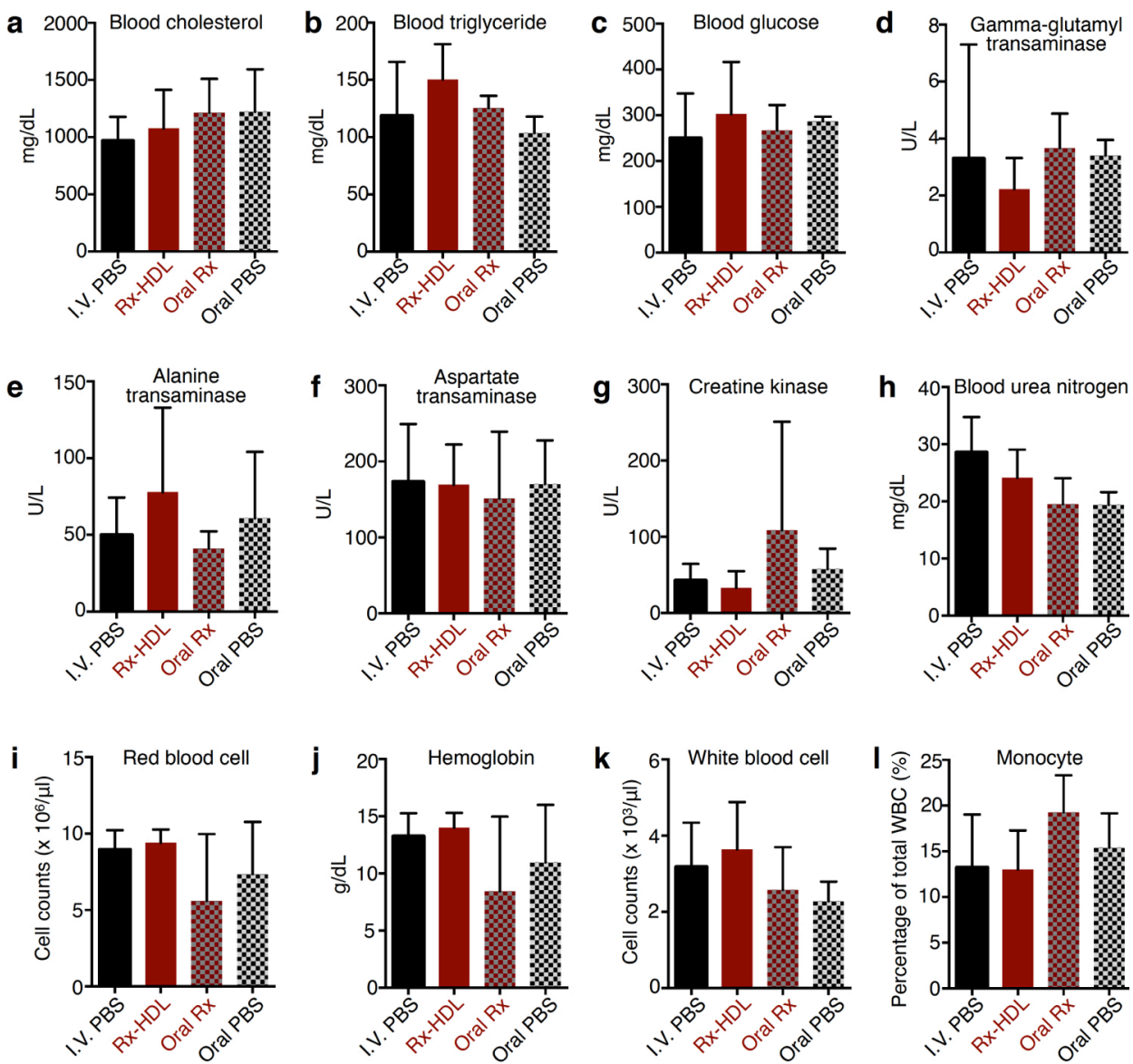

Figure S7 | A long-term Rx-HDL treatment does not cause toxic effects. Mice were treated with i.v. PBS (I.V. PBS, $n=10$ ), i.v. Rx-HDL (Rx-HDL, $n=9$ ), oral GW3965 (Oral $R x, n=6$ ), or oral PBS (Oral PBS, $\mathrm{n}=5$ ) two times per week for $6 \mathrm{wk}$, and key toxicity markers were measured. (A) Blood cholesterol, (B) triglyceride, and (C) glucose levels are metabolism markers. (D) Gamma-glutamyl transaminase, (E) alanine transaminase, and (F) aspartate transaminase are liver damage markers. (G) Creatine kinase is a cardiac toxicity marker. $(\mathrm{H})$ Blood urea nitrogen is a kidney damage marker. The (I) red blood cell counts, (J) hemoglobin levels, (K) white blood cell counts, and (L) percentage of monocytes in blood white cells are shown here, too. No statistical significance was found in any measurements above. Nonparametric two-tailed Student's t test was used to calculate statistics between treatment and PBS control, and all error bars are SD. 


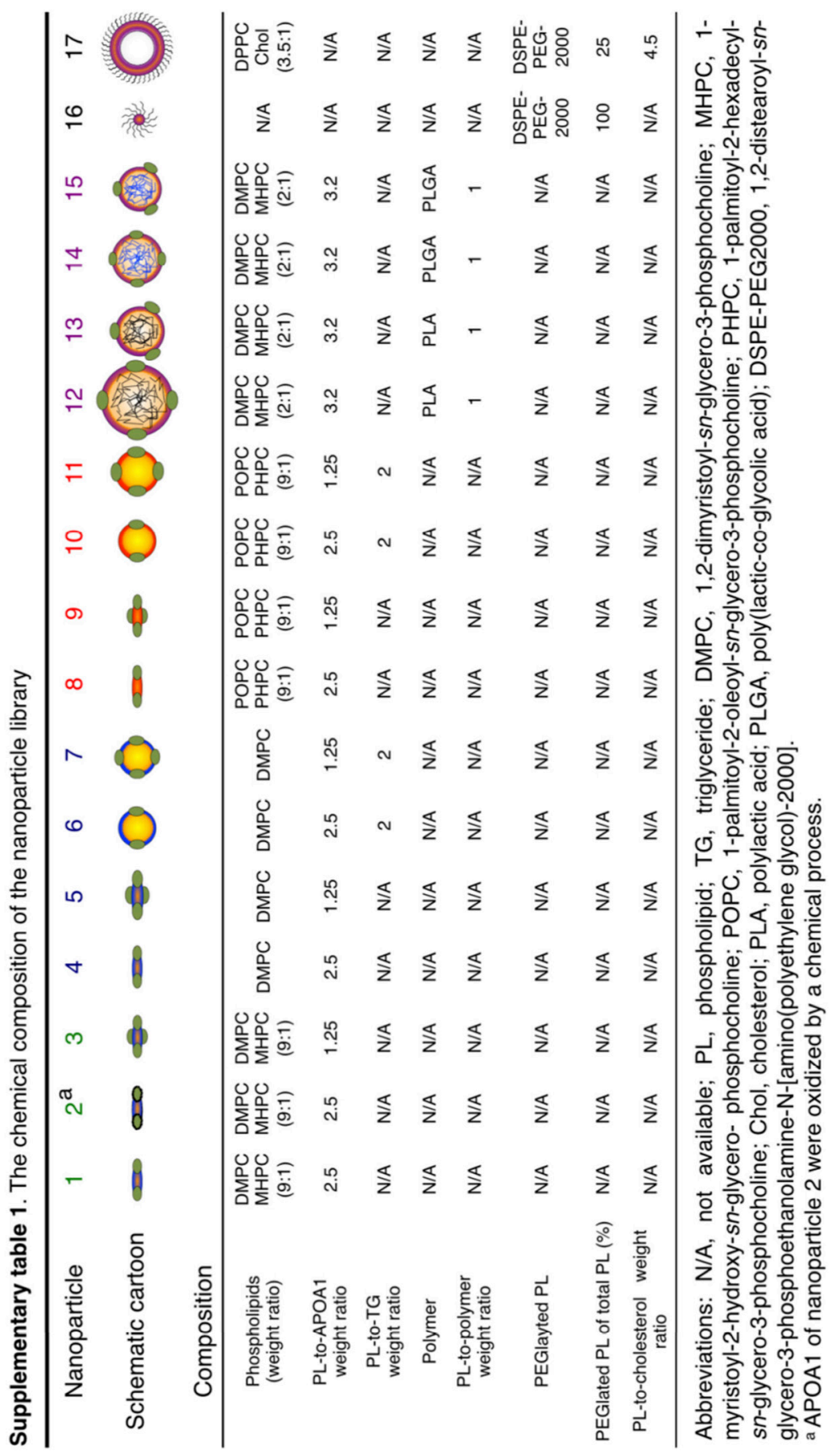


152 CHAPTER 5 Immune Cell Screening of a Nanoparticle Library Improves Atherosclerosis Therapy

Supplementary table 2 I Blood half-lives of nanoparticle library

\begin{tabular}{llllllllllllllllll}
\hline Nanoparticle & 1 & 2 & 3 & 4 & 5 & 6 & 7 & 8 & 9 & 10 & 11 & 12 & 13 & 14 & 15 & 16 & 17
\end{tabular}

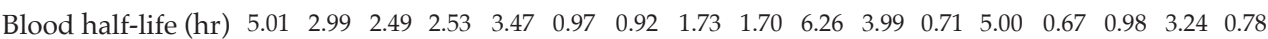

Blood half-lives values were determined from 5 mice per nanoparticle. 




\section{CHAPTER 6}

\section{A Systematic Comparison of Clinically Viable Nanomedicines Targeting HMG-CoA Reductase in Inflammatory Atherosclerosis*}

\section{ABSTRACT}

A therosclerosis is a leading cause of worldwide morbidity and mortality whose management could benefit from novel targeted therapeutics. Nanoparticles are emerging as targeted drug delivery systems in chronic inflammatory disorders. To optimally exploit nanomedicines, understanding their biological behavior is crucial for further development of clinically relevant and efficacious nanotherapeutics intended to reduce plaque inflammation. Here, three clinically relevant nanomedicines, i.e., high-density lipoprotein ([S]-HDL), polymeric micelles ([S]-PM), and liposomes ([S]-LIP), that are loaded with the HMG-CoA reductase inhibitor simvastatin [S], were evaluated in the apolipoprotein E-deficient $\left(\right.$ Apoe $\left.^{-1-}\right)$ mouse model of atherosclerosis. We systematically employed quantitative techniques, including in vivo positron emission tomography imaging, gamma counting, and flow cytometry to evaluate the biodistribution, nanomedicines' uptake by plaque-associated macrophages/ monocytes, and their efficacy to reduce macrophage burden in atherosclerotic plaques. The three formulations demonstrated distinct biological behavior in $A p o e^{-/-}$mice. While [S]-PM and [S]-LIP possessed longer circulation half-lives, the three platforms accumulated to similar levels in atherosclerotic plaques. Moreover, [S]-HDL and [S]PM showed higher uptake by plaque macrophages in comparison to [S]-LIP, while [S]-PM demonstrated the highest uptake by Ly6Chigh monocytes. Among the three formulations, [S]-PM displayed the highest efficacy in reducing macrophage burden in advanced atherosclerotic plaques. In conclusion, our data demonstrate that [S]-PM is a promising targeted drug delivery system, which can be advanced for the treatment of atherosclerosis and other inflammatory disorders in the clinical settings. Our results also emphasize the importance of a thorough understanding of nanomedicines' biological performance, ranging from the whole body to the target cells, as well drug retention in the nanoparticles. Such systematic investigations would allow rational applications of nanomaterials', beyond cancer, facilitating the expansion of the nanomedicine horizon. 


\section{INTRODUCTION}

Atherosclerosis is a chronic, systemic inflammatory disease of the large and medium-sized arteries, which can lead to life-threating events such as myocardial infarction and stroke[1]. According to the World Health Organization, 17.5 million deaths per year, an estimated $31 \%$ of all deaths worldwide, can be attributed to atherosclerotic cardiovascular diseases[2]. The initiation and progression of atherosclerotic lesions are currently understood to have a central inflammatory component in which immune cells, including inflammatory monocytes and macrophages, play key roles[3,4]. At early stages, elevated levels of circulating apolipoprotein B-containing lipoproteins induce focal expression of endothelial adhesion molecules[5], which promote the recruitment of inflammatory Ly6Chigh monocytes to the arterial wall[6]. Once adhered, monocytes transmigrate into the subendothelial space and differentiate into resident macrophages[4]. The continued accumulation of lipoproteins and immune cells, including macrophages, accelerates the development of focal lesions known as atherosclerotic plaques[7]. In advanced atherosclerosis, plaque-associated macrophages proliferate and secrete proinflammatory mediators, reactive oxygen species, and proteases which destabilize the plaque and aggravate the disease[8,9]. Additionally, recent preclinical[10] and clinical[11] work have identified cardiovascular events as key contributors to the aggravation of plaque inflammation, increasing secondary event's risk[12]. Thus, silencing plaque inflammation by targeting monocyte/ macrophage burden is a compelling disease management strategy.

Over the last few decades, extensive research has been conducted to explore the potential use of nanomaterials as novel drug delivery systems in cancer[13-17] and other inflammatory disorders[18,19]. While liposomes[20] have always been frontrunners, the nanomedicine field has also witnessed an exponential increase in the number of new nanomaterials[21,22], including polymeric micelles[23,24] and high-density lipoproteins (HDL)[25] as systems which have already matured up to the level of clinical application[23,26,27]. However, the majority of studies explore and focus on only one specific nanomaterial, mainly for tumor targeting[28], without comparing performance against other existing nanocarriers. Beyond cancer, more comprehensive investigations of these nanomaterials, covering drugnanocarrier compatibility (i.e., loading efficiency, and drug retention and release) and in vivo performance (i.e., pharmacokinetics, tissue distribution, atherosclerotic plaque targeting and efficacy), are necessary. Understanding the factors that govern nanomedicines' in vivo performance is critical to ensure rational optimization, adequate quality control, and potential early stage clinical translation[29,30].

3-hydroxy-3-methylglutaryl coenzyme A reductase (HMG-CoA) inhibitors, also known as statins, are the standard of care for patients with atherosclerotic cardiovascular diseases. Their oral application leads to reduced low-density lipoprotein (LDL) cholesterol blood levels, primarily by modulating hepatic LDL 
receptor expression[31]. Also, HMG-CoA inhibitors display pleiotropic effects at high doses, including anti-inflammatory and anti-proliferative activities[32,33]. In a clinical trial, high dose oral statin therapy was shown to reduce atherosclerotic inflammation, using 18F-fluorodeoxyglucose-positron emission tomography / computed tomographic imaging (FDG-PET/CT) in patients[34]. Moreover, in an atherosclerotic mouse model, Sparrow et al. showed that oral simvastatin, a frequently used HMG-CoA inhibitor, has anti-inflammatory and anti-atherosclerotic effects at a dose of $100 \mathrm{mg} / \mathrm{kg}$, independent of its cholesterol-lowering properties [35]. However, increasing the oral statin standard dose in human is not possible because of the dose-dependent adverse effects such as myopathy and hepatoxicity[36]. At the same time, orally administered statins undergo hepatic metabolism, which results in a very poor plaque bioavailibility[37]. Therefore, intravenous nanocarrier-mediated targeting of HMG-CoA inhibitors to atherosclerotic plaques is an attractive approach to achieve enhanced anti-inflammatory and anti-atherosclerotic effects, as we have shown for simvastatin-loaded HDL ([S]-HDL)[38,39].

In this paper, we present a comprehensive comparative analysis of three established and clinically viable nanomedicines targeting the HMG-CoA inhibitor simvastatin [S] to atherosclerotic plaques. The nanomedicines include [S]-HDL, [S]-N-(2-hydroxypropyl)-methacrylamide benzyl mPEG-b-p(HPMAm-Bz))-based PEGylated polymeric micelles (mPEG-b-p(HPMAm-Bz); [S]-PM), and [S]-PEGylated liposomes ([S]-LIP) (Figure 1). We aimed to systematically investigate and compare the performance of the three [S]-nanomedicines in Apoe ${ }^{-1-}$ mice with advanced atherosclerosis. To enable this, we implemented a quantitative and comparative approach, combining sensitive techniques, including in vivo positron emission tomography (PET), gamma counting, and multicolor flow cytometry, acquiring critical performance parameters of these three distinct nanomedicine platforms. The head-to-head comparison disclosed marked differences between the platforms, underscoring the significance of using quantitative and sensitive techniques, such as PET and flow cytometry, to relate organ distribution and immune cell specificity to nanomedicines' efficacy. 


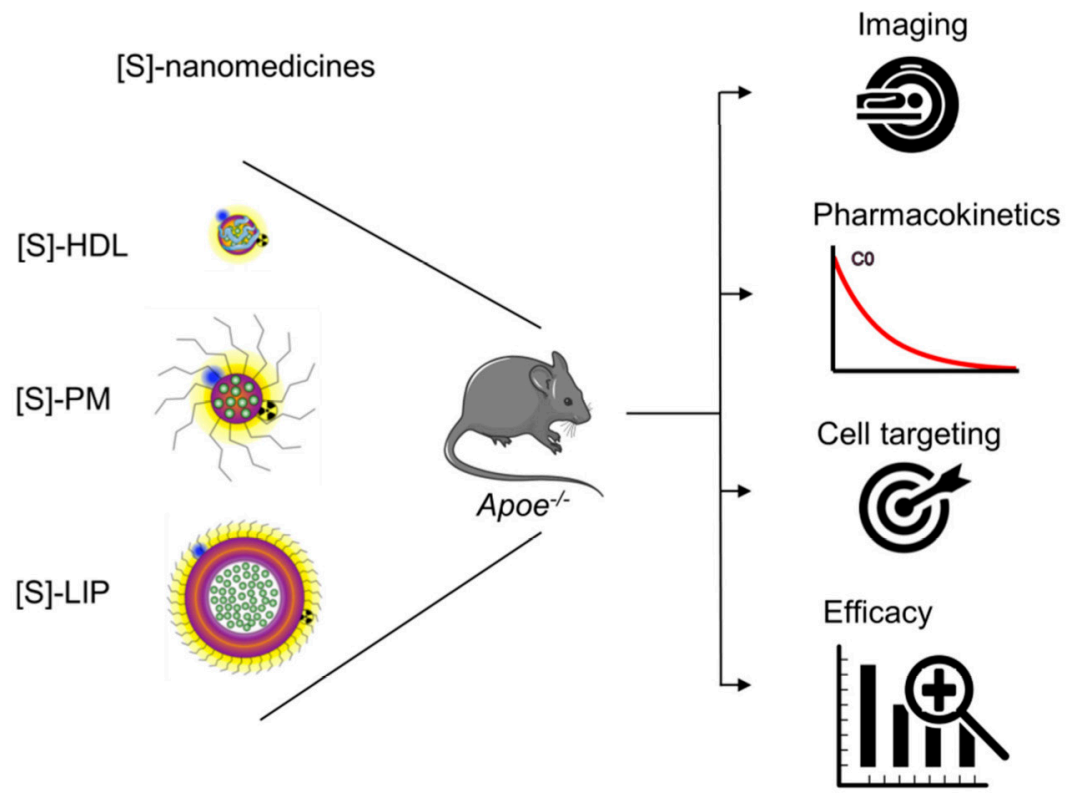

Figure 1 | Schematic of the [S]-nanomedicines and study design. [S]-HDL, [S]-PM, and [S]-LIP were evaluated in $A \mathrm{poe}^{-1-}$ mice with advanced atherosclerosis. To attain a thorough understanding of the in vivo behavior of the three platforms, we dual-labeled them with Zirconium- $\left.89{ }^{89} \mathrm{Zr}\right)$ and the near-infrared dye Cy5.5. The ${ }^{89} \mathrm{Zr}$ signal was exploited to provide a sensitive and quantitative assessment of their biodistribution and pharmacokinetics using noninvasive PET/CT imaging and gamma counting. The Cy5.5 signal was used mainly to evaluate immune cell targeting in atherosclerotic plaques, blood, and spleen using flow cytometry. The efficacy of the three [S]-nanomedicines in $A p o e^{-1-}$ was quantitatively assessed by flow cytometry.[S]: simvastatin; HDL: high density lipoprotein; PM: polymeric micelles; LIP: liposomes; $\mathrm{Apoe}^{-/-}$: apolipoprotein E-deficient.

\section{MATERIALS AND METHODS}

\subsection{Materials and radiochemistry}

1,2-Dipalmitoyl-sn-glycero-3-phosphocholine (DPPC), N-(carbonyl-methoxyPEG2000)-1,2-distearoyl-sn-glycero-3-phosphoethanolamine (DSPE-PEG2000), cholesterol, 1-myristoyl-2-hydroxy-sn-glycerophosphocholine (MHPC), and 1,2-dimyristoyl-sn-glycero-3-phosphatidyl-choline (DMPC) were purchased from Avanti Polar Lipids. The copolymers (methoxy poly(ethylene glycol)-b-(N-(2benzoyloxypropyl) methacrylamide) (mPEG-b-p(HPMAm-Bz) and the aminofunctionalized version, mPEG-b-p(HPMA-Bz-co-AEMAm), were synthesized by free radical polymerization, using $\mathrm{MPEG} 2 \mathrm{ABCPA}$ as macroinitiator and HPMA$\mathrm{Bz}$ as a monomer, as previously reported[40]. Briefly, the monomer and the macroinitiator were dissolved at a molar ratio of 200:1 in dried (over $4 \AA \AA$ molecular 
sieves) acetonitrile (Biosolve Ltd) at a final concentration of $300 \mathrm{mg} / \mathrm{mL}$. The solution was flushed with nitrogen gas for $30 \mathrm{~min}$ and then immersed in a silicone bath at $70{ }^{\circ} \mathrm{C}$ for at least $17 \mathrm{~h}$. Afterward, the solution was placed in cold diethyl ether to precipitate the formed polymer and centrifuged at 2600x $\mathrm{g}$ for $15 \mathrm{~min}$. The supernatant was discarded, and the pellet was re-dissolved in acetonitrile. This procedure was repeated three times, and the final product was dried under vacuum overnight at room temperature (RT) to remove the remaining organic solvent. For the synthesis of the amine-functionalized copolymer, 2\% (moles) of AEMAm, N-(2aminoethyl) methacrylamide hydrochloride, (relative to HPMA-Bz) was added to the reaction mixture and the reaction was conducted as described above. DSPECyanine5.5 (Cy5.5) was purchased from SyMO-Chem BV while synthesis and Cy5.5 labeling of mPEG-b-p(HPMAm-Bz) were done according to a method reported before[40]. Simvastatin and simvastatin sodium salt were obtained from AK Scientific and Cayman Chemical, respectively. All other materials were purchased from Sigma-Aldrich unless otherwise stated.

Zirconium-89 ( $\left.{ }^{89} \mathrm{Zr}\right)$ was produced at Memorial Sloan Kettering Cancer Center on a TR19/9 variable-beam energy cyclotron (Ebco Industries Inc.) via the ${ }^{89} \mathrm{Y}(\mathrm{p}, \mathrm{n})$ ${ }^{89} \mathrm{Zr}$ reaction and purified in accordance with the previously reported method[41]. Activity was measured using a CRC 15R dose calibrator (Capintec).

\subsection{Characterization of mPEG-b-p(HPMA-Bz) Copolymer}

Gel Permeation Chromatography (GPC) was performed to determine the number average molecular weight $(\mathrm{Mn})$, weight average molecular weight $(\mathrm{Mw})$ and polydispersity $(\mathrm{Mw} / \mathrm{Mn})$ of the synthesized polymers. Two serial PLgel $5 \mu \mathrm{m}$ MIXED-D columns (Polymer Laboratories) were used, and the run was performed in $10 \mathrm{mM} \mathrm{LiCl} \mathrm{DMF}$, at a flow rate of $1 \mathrm{~mL} / \mathrm{min}$ and a column temperature of 65 ${ }^{\circ} \mathrm{C}$. PEGs of narrow molecular weight distribution were used as standards. Samples were prepared at a concentration of $5 \mathrm{mg} / \mathrm{mL}$ in $10 \mathrm{mM} \mathrm{LiCl} \mathrm{DMF}$ and allowed to dissolve for $30 \mathrm{~min}$ at $37^{\circ} \mathrm{C}$.

1H-NMR spectrum of mPEG-b-p(HPMAm-Bz) was recorded using a Gemini 300 $\mathrm{MHz}$ spectrometer (Varian Associates Inc. NMR Instruments), using DMSO-d6 as a solvent and the DMSO peak at $2.50 \mathrm{ppm}$ as a reference. Chemical shifts of PEGHPMA-Bz were assigned as described elsewhere[40]. The ratio between HPMA-Bz and $\mathrm{mPEG}$ was determined by the integral value of aromatic protons of HPMA-Bz (8.0 ppm, $2 \mathrm{H}$, aromatic $\mathrm{CH}$ ) divided by two, and the integral value of the methylene protons of mPEG (3.40-3.60 ppm, 448H, O-CH2-CH2) divided by 448 (average number of protons per PEG chain, $\mathrm{Mn}=5000 \mathrm{~g} / \mathrm{mol}$ ). The average number molecular weight $(\mathrm{Mn})$ of the block copolymer was determined as follows:

$$
\mathrm{M}_{\mathrm{n}}=\frac{\text { (integral at } 8.0 \mathrm{ppm} \text { ) } \mathrm{x} \text { molar mass of HMPA-Bz }}{\text { integral at } 3.40-3.60 \mathrm{ppm} / 448}+5000 \mathrm{~g} / \mathrm{mol}
$$


The copolymers used to prepare the [S]-PM, i.e. mPEG-b-p(HPMAm-Bz) and the amine-functionalized, mPEG-b-p(HPMA-Bzco-AEMAm), were synthesized by free radical polymerization via a macroinitiator route[40]. Both copolymers show similar number-average molecular weight $(\mathrm{Mn}) \sim 22 \mathrm{KDa}$ and PDI $\sim 1.7$ based on GPC and $1 \mathrm{H}-\mathrm{NMR}$ analyses (Supporting Figure 1 and 2 and Supporting Table 1).

\subsection{Assessment of simvastatin and simvastatin sodium salt activity}

To assure that both simvastatin, used in [S]-HDL and [S]-PM, and simvastatin sodium salt, used in [S]-LIP, have equipotent pharmacological activity, we assessed their dose-dependent effects on macrophage proliferation in vitro. RAW264.7 macrophages, obtained from ATCC, were cultured in Dulbecco's Modified Eagle's Medium (DMEM) high glucose (Corning) supplemented with $10 \%$ (v/v) fetal bovine serum, penicillin (100 IU / mL), streptomycin $(100 \mu \mathrm{g} / \mathrm{mL})$, and amphotericin B $(0.25$ $\mu \mathrm{g} / \mathrm{mL}$ ), and incubated at $37{ }^{\circ} \mathrm{C}$ under a $5 \% \mathrm{CO}_{2}$ atmosphere. Cells were seeded at a density of 5000 cells per well in a 96-well plate. After $24 \mathrm{~h}$, cells were treated with simvastatin or simvastatin sodium salt at the indicated concentrations for $24 \mathrm{~h}$. To determine the number of proliferating cells, bromodeoxyuridine (BrdU) reagent was added to the cells for $4 \mathrm{~h}$, and an ELISA BrdU colorimetric immunoassay (HoffmanLa Roche Ltd) was performed, according to the manufacturer's protocol. The BrdU assay showed that both forms of simvastatin possess comparable dose-dependent inhibitory effects on macrophage proliferation (Supporting Figure 3).

\subsection{Formulation of simvastatin nanomedicines}

Formulation of the HMG-CoA inhibitor simvastatin HDL ([S]-HDL) was done according to a method reported before[38,39]. In brief, simvastatin, DMPC, and MHPC, in a weight \% ratio of 14:78:8, respectively, were dissolved in a chloroform/ methanol mixture (4:1 by volume) and a lipid film was prepared by rotary evaporation (Büchi Labortechnik) and dried under a nitrogen stream for $1 \mathrm{~h}$ until complete dryness. The lipid film was then hydrated with human apolipoprotein A1 (APOA1, isolated from human plasma) in PBS (APOA1 and phospholipids in a 1:9 weight ratio). The mixture was incubated at $37^{\circ} \mathrm{C}$ until the film was completely hydrated. The lipid dispersion was subjected to ultrasonication to form [S]-HDL nanomedicine formulation.

To formulate HMG-CoA inhibitor liposomes ([S]-LIP), we used simvastatin provided as sodium salt, a water-soluble derivative that allows its entrapment in liposomes. Liposome preparation was modified from a method described previously[42]. Briefly, DPPC, cholesterol, and DSPE-PEG2000, in a weight $\%$ ratio of 61.7:33.3:5, respectively, were dissolved a chloroform/methanol mixture (4:1 
by volume) in a round-bottom flask. A lipid film was prepared by evaporation of the solvents and subsequently hydrated with a solution of $5 \mathrm{mg} / \mathrm{mL}$ simvastatin (sodium salt) in PBS (5 mL). The mixture was then heated at $60{ }^{\circ} \mathrm{C}$ until the film was completely hydrated. To downsize the lipid dispersion, the lipid particles were subjected to multiple extrusion steps through polycarbonate membranes (Whatman, Nuclepore) with a final pore size of $100 \mathrm{~nm}$ using a Lipex extruder (Northern Lipids).

Preparation of HMG-CoA inhibitor HPMA-based polymeric micelles ([S]PM) was done as described by Shi et al.[40]. Briefly, mPEG-b-p(HPMAm-Bz) copolymer $(10 \mathrm{mg})$ and simvastatin $(2 \mathrm{mg})$ were both completely dissolved in 0.5 $\mathrm{mL}$ tetrahydrofuran (THF). Then the polymer/drug mixture was slowly added dropwise to $2 \mathrm{~mL}$ of Milli-Q water while stirring to form [S]-PM. The mixture was then incubated for $48 \mathrm{~h}$ at RT to allow evaporation of THF.

[S]-HDL was washed with PBS at least three times using ultrafiltration units with a $50 \mathrm{kDa}$ molecular weight cutoff (MWCO) (Sartorius Stedim Biotech SA). [S]-PM or [S]-LIP were purified with PBS using VivaFlow crossflow cassettes filtration units with a $100 \mathrm{kDa}$ MWCO (Sartorius Stedim Biotech SA). All the three formulations were centrifuged at $4000 \mathrm{x} g$ for $30 \mathrm{~min}$ to remove any aggregates. Finally, each formulation was filtered through a $0.22 \mu \mathrm{m}$ nylon membrane filter (CellTreat Scientific Products).

\subsection{Dual labeling of simvastatin nanomedicines}

The [S]-nanomedicines were labeled with Cy5.5 to allow near-infrared fluorescence (NIRF) imaging and flow cytometry studies. Briefly, $0.5 \%$ of DSPECy5.5 was incorporated into the lipid film of either [S]-HDL or [S]-LIP during the lipid film formation (modified from[43]). For [S]-PM, 0.5\% of Cy5.5-labeled mPEG$\mathrm{b}-\mathrm{p}(\mathrm{HPMAm}-\mathrm{Bz})$ was added to the simvastatin/polymer mixture in THF before adding to Milli-Q water (modified from Shi et al.[40]).

To additionally label the nanomedicines with ${ }^{89} \mathrm{Zr}$ for PET imaging and quantitative biodistribution assessment, a similar approach was followed as we previously reported[41,44]. Briefly, 0.5\% of DSPE-desferrioxamine (DSPE-DFO)[41] was incorporated in the lipid film of [S]-HDL or [S]-LIP. For the polymeric micelles, to functionalize the polymer with $\mathrm{DFO}$, a solution of the amine-functionalized copolymer mPEG-b-p(HPMA-Bz-co-AEMAm) $(2.0 \mathrm{mg})$, p-isothiocyanatobenzylDFO (Macrocyclics) $(40 \mu \mathrm{g}, \sim 1 \mathrm{eq})$ and $\mathrm{N}, \mathrm{N}$-diisopropylethylamine $(1.0 \mu \mathrm{L})$ in dimethylsulfoxide $(0.21 \mathrm{~mL})$ was heated at $40{ }^{\circ} \mathrm{C}$ for $8 \mathrm{~h}$. The mixture was then allowed to cool down to RT. Water $(1.8 \mathrm{~mL})$ was added, and the resulting milky solution was concentrated by centrifugal filtration using a $10 \mathrm{kDa}$ MWCO tube and washed three times with Milli-Q water $(2 \mathrm{~mL})$. The final retentate was dried under vacuum to yield the DFO-modified polymer as a white solid $(2.0 \mathrm{mg})$. The DFOmodified mPEG-b-p(HPMAm-Bz) was added at $5 \%$ of the total polymer weight to the formulation mix at the expense of non-modified mPEG-b-p(HPMAm-Bz). 
Subsequently, a PBS dispersion of the corresponding DFO-bearing nanomedicine formulation was reacted with $\left[{ }^{89} \mathrm{Zr}\right] \mathrm{Zr} 2(\mathrm{C} 2 \mathrm{O} 4) 2$ at $\mathrm{pH} 7.1-7.4$ for $1 \mathrm{~h}$ at $37{ }^{\circ} \mathrm{C}$. The dispersion was allowed to cool down to RT, and the radiolabeled nanocarriers were purified by centrifugal filtration using $10 \mathrm{kDa}$ MWCO filter tubes for [S]-HDL, and $100 \mathrm{kDa}$ MWCO filter tubes for [S]-LIP and [S]-PM. The radiochemical yield was 80 $\%$ with radiochemical purities more than $95 \%$ for the three formulations.

\subsection{Characterization}

The mean particle size and polydispersity index (PDI) of the different [S]nanomedicines used in this study were determined by dynamic light scattering using

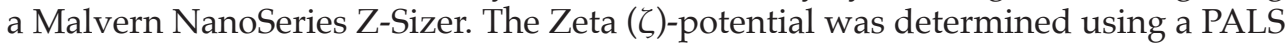
analyzer (Brookhaven Instruments Corp.) where [S]-nanomedicine samples were diluted in $10 \mathrm{mM}$ HEPES buffer ( $\mathrm{pH}$ 7.4) before measurements. The morphology of the nanomedicines was determined by transition emission microscopy (TEM). Briefly, the original PBS solvent was replaced by an ammonium acetate buffer and then mixed with $2 \%(\mathrm{wt} / \mathrm{vol})$ sodium phosphotungstate $(\mathrm{pH}=7.4)$ to negatively stain the [S]-nanomedicine. The solution was then cast dried on a nickel grid and imaged with a Hitachi H7650 TEM.

\subsection{HPLC and radio-HPLC}

Simvastatin concentrations in the different samples were determined as previously reported[38]. The simvastatin content of the nanocarriers or samples from drug release studies (described in the following section) was determined by HPLC, using a Prominence LC-20AB system (Shimadzu) equipped with a C18 column at a flow rate of $0.5 \mathrm{~mL} / \mathrm{min}$, utilizing an isocratic mobile phase composed of $80 \%$ acetonitrile, $20 \% \mathrm{H}_{2} \mathrm{O}$, and $0.1 \%$ trifluoroacetic acid. Simvastatin was detected at a wavelength of $238 \mathrm{~nm}$ using SPD-M10AVP photodiode array detector.

Radio-HPLC was performed using a similar Shimadzu system additionally equipped with a Lablogic Scan-RAM Radio-TLC/HPLC detector. Size-exclusion chromatography was performed on a Superdex 10/300 column (GE Healthcare Life Sciences) using PBS as eluent at a flow rate of $1 \mathrm{~mL} / \mathrm{min}$.

\subsection{Drug release in serum}

The release of simvastatin from the nanocarriers was studied as described previously [45,46], with some slight modifications. Briefly, $0.1 \mathrm{~mL}$ of [S]-HDL, [S]PM, or [S]-LIP (1 mg/mL simvastatin), was mixed with $0.9 \mathrm{~mL}$ fetal bovine serum (FBS) and samples were incubated at $37^{\circ} \mathrm{C}$. for $1,2,4,8$, and $24 \mathrm{~h}$. At these time points, samples were subjected to size-exclusion high-performance liquid chromatography 
(SEC) for nanocarrier-protein separation and fractionation using a Prominence LC-20AB HPLC system (Shimadzu) equipped with a fraction collector, FRC-10A (Shimadzu) and a Superose 6 10/300 GL Fast protein liquid chromatography column (GE Healthcare Life Sciences). PBS was used as eluent at a flow rate of $0.6 \mathrm{~mL} /$ min. The nanocarrier fractions (containing simvastatin retained in the nanocarrier) and serum fractions (protein-associated simvastatin) were collected, and their corresponding simvastatin content was extracted with acetonitrile and analyzed by HPLC as detailed above.

\subsection{Animals and treatment procedure}

Female Apoe ${ }^{-/-}$mice (B6.129P2-Apoetm1Unc/J, 6 weeks old) were purchased from The Jackson Laboratory and were fed a high-fat diet (HFD) (Harlan Teklad TD.88137, $42 \%$ calories from fat) for 18 weeks (average weight: $27.5 \pm 3.3 \mathrm{~g}$ ). Under these conditions, the animals develop atherosclerotic lesions because of the high LDL cholesterol concentrations in the blood resulting from the lack of apolipoprotein E [47]. All animal experiments were performed in accordance with protocols approved by the Institutional Animal Care and User Committees of Mount Sinai and Memorial Sloan Kettering Cancer Center. All experiments adhered to National Institutes of Health guidelines for animal welfare. All animals were injected intravenously with the corresponding nanomedicines in a 150-200 $\mu \mathrm{L}$ of PBS solution via the lateral tail vein.

\subsection{Micro-PET/CT imaging}

After $24 \mathrm{~h}$ of injecting the ${ }^{80} \mathrm{Zr}$-[S]-nanomedicines $(0.18 \pm 0.03 \mathrm{mCi} /$ animal $)$, the animals ( $\mathrm{n}=3$ per treatment type) were anesthetized with a mixture of isoflurane (Baxter Healthcare) and oxygen gas (2\% for induction and $1 \%$ for maintenance), and scans were then obtained using an Inveon PET/CT scanner (Siemens Healthcare Global). Whole-body PET static scans recording a minimum of 50 million coincident events were performed with duration of 10-20 min. Whole body standard lowmagnification CT scans were obtained with the x-ray tube set at a voltage of $80 \mathrm{kV}$ and current of $500 \mathrm{~mA}$. The CT scan was acquired using 120 rotational steps for a total of $220 \mathrm{o}$, yielding an estimated scan time of $120 \mathrm{~s}$ with an exposure of $145 \mathrm{~ms} /$ frame. The counting rates in the reconstructed images were converted to activity concentrations $(\% \mathrm{ID} / \mathrm{g})$ by use of a system calibration factor derived from the imaging of a mousesized water-equivalent phantom containing the radionuclide. Images were analyzed using Inveon Research Workplace software (Siemens Healthcare Global). Activity concentration was quantified by averaging the maximum values in at least 5 ROIs drawn on adjacent slices of the tissue of interest. 


\subsection{Pharmacokinetics and biodistribution}

Apoe ${ }^{-/-}$mice ( $\mathrm{n} \geq$ per [S]-nanomedicine type) were administered $26 \pm 5 \mu \mathrm{Ci}{ }^{80} \mathrm{Zr} /$ animal of the corresponding dual labeled ${ }^{89} \mathrm{Zr} / \mathrm{Cy} 5.5$ [S]-nanomedicines at doses of $0.8 \mathrm{mg}$ Cy5.5/ $\mathrm{kg}$ and $60 \mathrm{mg}$ simvastatin $/ \mathrm{kg}$ (as in the therapeutic study). Blood was sampled ( 10-20 $\mu \mathrm{L}$ / each sample) at predetermined time points ( $30 \mathrm{~min}, 1,2,4,8$, and $24 \mathrm{~h}$ ) after injection. After $24 \mathrm{~h}$, the mice were euthanized and perfused with $30 \mathrm{~mL}$ PBS/mouse through cardiac puncture, and organs of interest (aortas, liver, spleen, kidneys, lungs, heart, and brain) were excised. The blood samples (at the different time points) and samples of other tissues were weighed, and their radioactivity content was quantified using Wizard 2470 Automatic Gamma Counter (Perkin Elmer). The values were corrected for ${ }^{89} \mathrm{Zr}$ decay and radioactivity concentration was calculated as a percentage of injected dose per gram of tissue (\% ID /g).

[S]-nanomedicines biodistribution was also qualitatively assessed by NIRF imaging and autoradiography. Perfused tissue samples were placed on a thick black paper and imaged on a Xenogen IVIS Spectrum Preclinical Imaging System (Perkin Elmer). Fluorescence images were acquired with selected excitation and emission band-pass filters: for Cy5.5 $\lambda \mathrm{Exc}=640 \pm 18 \mathrm{~nm}, \lambda \mathrm{Em}=720 \pm 10 \mathrm{~nm}$. Exposure time for each image was $2 \mathrm{~s}$. To assess the radiotracer distribution, tissues were placed on a film cassette against a phosphorimaging plate (BASMSM-2325, Fujifilm) for either $24 \mathrm{~h}$ (aortas) or $18 \mathrm{~h}$ (all other organs) at $-20^{\circ} \mathrm{C}$. The plates were then read at a pixel resolution of $25 \mu \mathrm{m}$ in a Typhoon 7000IP plate reader (GE Healthcare).

\subsection{Flow cytometry}

To study the uptake of [S]-nanomedicines by immune cells, we used flow cytometry protocols similar to what was previously reported[38,47]. Briefly, Apoe ${ }^{-1-}$ mice ( $\mathrm{n}=4$ per group, 18 weeks on HFD) were injected with Cy5.5-labeled [S]-nanomedicines at $0.8 \mathrm{mg}$ Cy5.5/ $\mathrm{kg}$ (at a dose of $60 \mathrm{mg} / \mathrm{kg}$ simvastatin). After $24 \mathrm{~h}$, to prepare cells for flow cytometry, blood was collected by cardiac puncture into tubes with a $50 \mathrm{mM}$ EDTA solution as an anticoagulant. Red blood cells (RBCs) were lysed using RBC lysis buffer (BD Biosciences). Afterward, animals were perfused with $30 \mathrm{~mL}$ PBS/ mouse. The whole aortas, from the aortic root to the iliac bifurcation, and spleens were harvested. Single-cell suspensions were obtained as follows: aortas were diced and digested with a cocktail of enzymes, including $4.5 \mathrm{U} / \mathrm{mL}$ liberase $\mathrm{TH}$ (Roche), $60 \mathrm{U} / \mathrm{mL}$ hyaluronidase (Sigma-Aldrich), and $40 \mathrm{U} / \mathrm{mL}$ DNase (Sigma-Aldrich), in a $37{ }^{\circ} \mathrm{C}$ oven for $1 \mathrm{~h}$ while shaking. Then, tissue aggregates, extracellular matrix, and cell debris were removed by filtration. Spleens were also diced, and RBCs were removed using the same lysis buffer described above. To identify macrophages, monocytes, dendritic cells, neutrophils, and other immune cells, a cocktail of antibodies was used (antibodies, clones, and suppliers shown in Table S.2). Cy5.5 signal was detected on the Alexa700 channel. 
In the efficacy study, we quantified the number of macrophages and Ly6Chigh monocytes in atherosclerotic plaques. Complete blood count was also performed to assess the number of immune cells in the blood. Mice ( $n=8-10 /$ group, 18 weeks on HFD) were administered the corresponding treatments ([S]-nanomedicines, $60 \mathrm{mg} /$ $\mathrm{kg}$ simvastatin; PBS, or oral simvastatin, $60 \mathrm{mg} / \mathrm{kg}$ ) once every $72 \mathrm{~h}$ for a week (three injections in total). Mice were sacrificed $24 \mathrm{~h}$ after the last injection, and immune cells from aorta and blood were isolated and quantified. For both the targeting study and the efficacy study, we adopted a strict flow cytometry protocol by running 'fluorescence minus one' (FMO) controls to precisely gate the right cell population. In an FMO control stain sample, all reagents used in a given multicolor sample are included except the reagent with which the exact cell population is to be determined. All samples were measured on an LSRII (BD Biosciences) flow cytometer, and results were analyzed with FlowJo (Tree Star) software.

Serum from animals in the efficacy study was subjected to a biochemical analysis (ALX Laboratories, NY) to determine typical blood biochemistry constituents, including cholesterol and triglycerides, and toxicity markers like alanine transaminase.

\subsection{Statistical analysis}

Statistical analysis was performed using Prism (GraphPad), and data are expressed as a mean \pm standard error of the mean. Differences between groups were assessed by one-way analysis of variance (ANOVA) followed by a post hoc test (Bonferroni's test) for multiple comparisons. ${ }^{*} \mathrm{P} \leq 0.05$; ${ }^{* *} \mathrm{P} \leq 0.01$; ${ }^{* *} \mathrm{P} \leq 0.001 \mathrm{~A}$ value of $\mathrm{P}<0.05$ was considered statistically significant.

\section{RESULTS AND DISCUSSION}

The aim of this study was to target HMG-CoA in atherosclerotic plaques by simvastatin-loaded nanomedicines and to understand the parameters that control their in vivo performance. Liposomes were included as they are often regarded as the gold standard nanomedicine drug delivery system and were the first to make clinical translation[20,43]. Additionally, liposomes have been shown to successfully target atherosclerotic plaques in rabbits[43,48] and humans[49]. HDL, a natural selfassembled nanocarrier, has an intrinsic affinity to plaque macrophages in different atherosclerosis animal models[44,50]. The natural targeting ability of HDL and the possibility to incorporate hydrophobic compounds in its core makes it an attractive platform for drug delivery[51]. HPMA block copolymer-based micelles were shown to improve the retention of hydrophobic chemotherapeutic drugs in their matrix[52-54], offering effective targeted drug delivery to tumors and inflammatory sites[53,55,56]. However, they have not yet been evaluated in atherosclerosis. 
Here, we explored their ability to target atherosclerotic plaques for the first time and compared their performance with previously assessed HDL and liposomal formulations.

\subsection{Preparation and characterization of simvastatin nanomedicines}

[S]-HDL was prepared by ultrasonication, a well-established method to encapsulate hydrophobic drugs in HDL nanoparticles[57,58]. [S]-LIP was prepared using a more water-soluble derivative, i.e., simvastatin (sodium salt). [S]-PM was formulated by dropping a THF solution of the polymer and simvastatin into water followed by evaporation of THF, a method that was previously used to encapsulate hydrophobic drugs[52,59].

DLS analysis showed that the mean particle sizes (Z-average) of the three [S]nanomedicines were different and none of them was larger than $100 \mathrm{~nm}$. [S]-HDL possessed the smallest mean diameter $(\sim 50 \mathrm{~nm})$ with a PDI $\sim 0.22$ (Figure $2 \mathrm{~A}$ and Table 1). Analysis of [S]-HDL by TEM showed the typical discoidal shape of HDL nanoparticles as reported before[38] (Figure 2B). DLS analysis showed that [S]-PM and [S]-LIP had larger mean diameters of $\sim 80$ and $\sim 95 \mathrm{~nm}$, respectively (Figure 2A and Table 1). Both formulations showed a low PDI $\sim 0.1$ (Table 1). TEM images of [S]-PM and [S]-LIP showed spherical particles (Figure 2B). The zeta $(\zeta)$ potential of the three [S]-nanomedicines was negative in $10 \mathrm{mM}$ HEPES buffer ( $\mathrm{pH}$ 7.4), ranging from $-5 \mathrm{mV}$ for [S]-PM to $\sim-15 \mathrm{mV}$ for both the liposomal and HDL formulations (Table 1). All the three [S]-nanomedicines had high simvastatin entrapment efficiency $(>60 \%)$ as shown in Table 1.

Table 1 | Characteristics of [S]-nanomedicines.

\begin{tabular}{llll}
\hline & {$[S]-H D L$} & {$[S]-P M$} & {$[S]-L I P$} \\
\hline$Z_{\text {ave. }}$ & $50 \pm 6 \mathrm{~nm}$ & $80 \pm 7 \mathrm{~nm}$ & $94 \pm 10 \mathrm{~nm}$ \\
PDI & $0.22 \pm 0.03$ & $0.11 \pm 0.01$ & $0.10 \pm 0.06$ \\
Zeta $(\zeta)$ & $-15.8 \pm 3.5$ & $-5.1 \pm 0.9$ & $-15.1 \pm 0.7$ \\
potential $(\mathrm{mV})$ & & $65 \pm 8 \%$ & $71 \pm 3 \%$ \\
EE $(\%)$ & $60 \pm 7 \%$ & &
\end{tabular}

Data are presented as mean \pm SD of three separately prepared batches of [S]-HDL, [S]-PM, or [S]LIP.[S]: simvastatin; HDL: high density lipoprotein; PM: polymeric micelles; LIP: liposomes; $\mathrm{Z}_{\text {ave: }}$ : $\mathrm{Z}$ average; mV: millivolt; PDI; polydispersity index, EE; entrapment efficiency. 


\subsection{Drug release kinetics in serum}

The extent and rate of drug release while nanocarriers circulate in the bloodstream is an important factor which can strongly affect a drug-loaded nanocarrier's targeting efficiency and resulting therapeutic efficacy. The release kinetics of the three simvastatin nanoformulations in $90 \%$ serum at $37^{\circ} \mathrm{C}$, mimicking physiological conditions, was analyzed. Using size exclusion chromatography, we separated the nanocarrier from the serum proteins, and then analyzed the simvastatin content in each fraction (Supporting Figure 4). The release kinetics of simvastatin from [S]-HDL was similar to that of [S]-LIP but much more rapid than [S]-PM (Figure $2 \mathrm{C})$. By the end of the $24 \mathrm{~h}$ incubation period, $~ 50 \%$ of the simvastatin from [S]PM was released compared to $\sim 80 \%$ and $90 \%$ in the case of [S]-LIP and [S]-HDL, respectively. The higher drug retention in case of [S]-PM can be explained by purposely designed physicochemical features that enhance hydrophobic and $\pi-\pi$ stacking interactions[40,56]. In the case of [S]-LIP, simvastatin sodium salt was used to increase drug entrapment in the liposomal formulation. However, according to the manufacturer (Cayman), simvastatin (sodium salt) is sparingly soluble in aqueous solutions and probably also possess an affinity for the lipid bilayer, which may have resulted in drug leakage from the [S]-LIP upon interaction with serum proteins. Similarly, HDL has a dynamic structure, which could result in the exchange of lipid components upon interaction with other serum proteins, resulting in drug leakage[45]. 
[S]-HDL

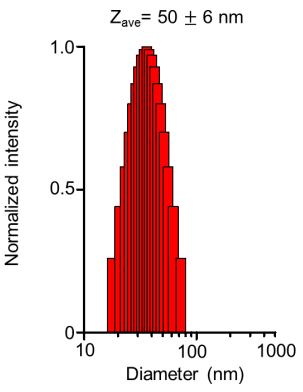

[S]-HDL
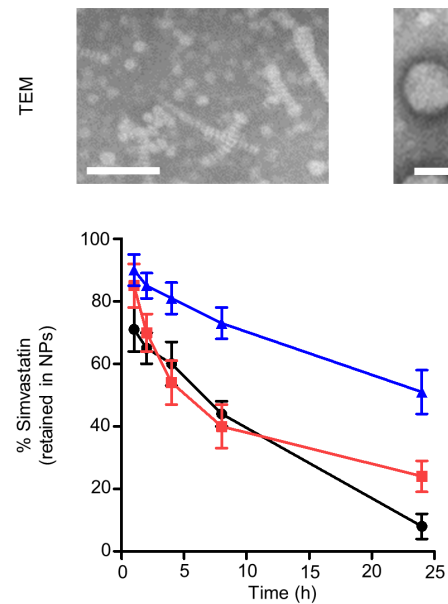

[S]-PM

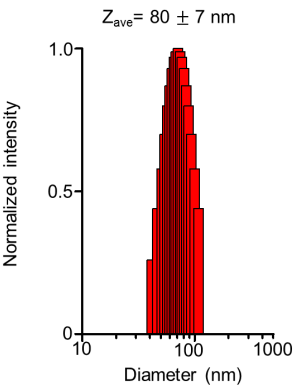

[S]-PM

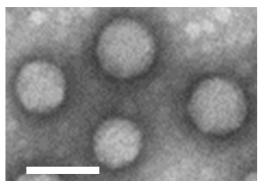

[S]-LIP

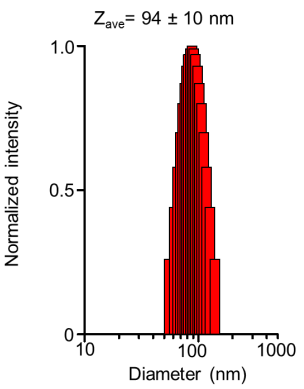

[S]-LIP
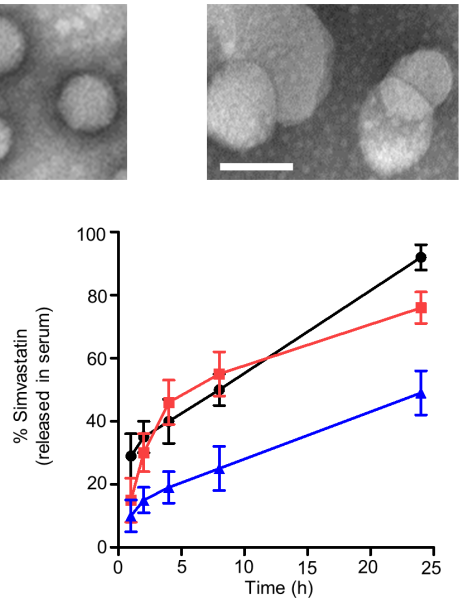

[S]-LIP -

Figure 2 । Characterization and drug release kinetics of the [S]-nanomedicines. (A) Particle size distribution of [S]-HDL, [S]-PM, and [S]-LIP was evaluated by dynamic light scattering. (B) Negative staining TEM images of the aforementioned nanomedicines. Scale bar $=100 \mathrm{~nm}$. (C) The release of simvastatin from the three nanomedicines in fetal bovine serum (FBS) was assessed using size-exclusion chromatography and high-performance liquid chromatography (HPLC) as described in Supporting Figure 3. [S]: simvastatin; HDL: high density lipoprotein; PM: polymeric micelles; LIP: liposomes; TEM: transmission electron microscopy; NPs: nanoparticles; h: hours; nm: nanometer.

\subsection{Dual labeling of simvastatin nanomedicines}

To better understand [S]-nanomedicines' biodistribution and uptake by immune cells, we employed a dual-labeling strategy using ${ }^{89} \mathrm{Zr}$ as a radioactive tag for PET imaging and gamma counting, and Cy5.5 for NIRF imaging and flow cytometry. ${ }^{89} \mathrm{Zr}$ has a physical half-life of $78.4 \mathrm{~h}$, making it suitable for long-circulating nanoparticles[41] and antibodies[60]. Additionally, PET imaging and gamma counting are sensitive, quantitative methods for evaluating [S]-nanomedicines' pharmacokinetics, whole-body, and organ biodistribution, while flow cytometry 
allows investigating immune cell specificity in atherosclerotic plaques and other tissues in a quantitative fashion. Both labels (i.e. ${ }^{89} \mathrm{Zr}$ and Cy5.5) on [S]-HDL, [S]PM, and [S]-LIP eluted at the same time on size-exclusion chromatography columns, indicative of proper label stability.

\subsection{Pharmacokinetics, in vivo imaging and biodistribution}

In vivo evaluation of the three [S]-nanomedicines started with an assessment of their circulation kinetics in $A p o e^{-1-}$ mice using gamma counting to quantify blood radioactivity levels in time (Figure 3B). After $24 \mathrm{~h}$, [S]-PM and [S]-LIP showed longer circulation times in blood in comparison with [S]-HDL $(\sim 10 \%$ ID/g remaining in blood vs $\sim 1 \%$ ID / $g$ for [S]-HDL). The longer circulation time of the former platforms likely relates to their PEGylated surface and relatively large size as compared to [S]HDL.
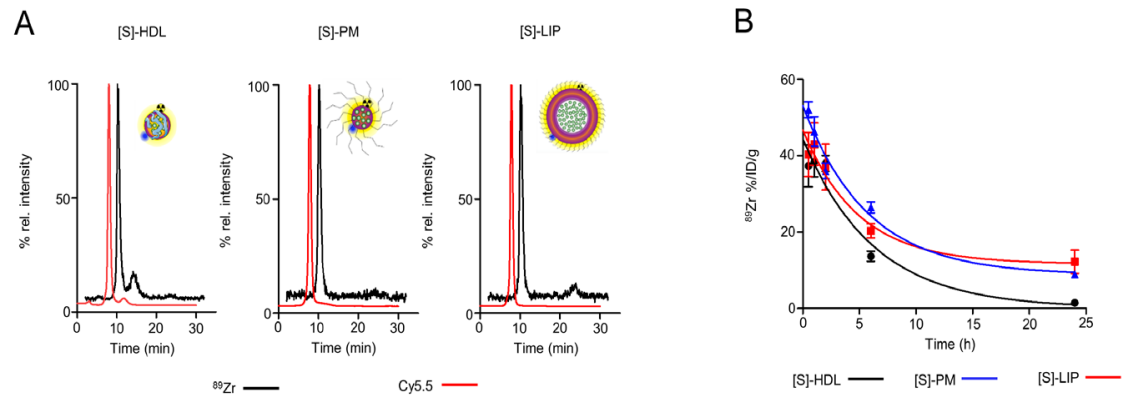

C

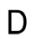

\section{$E$}
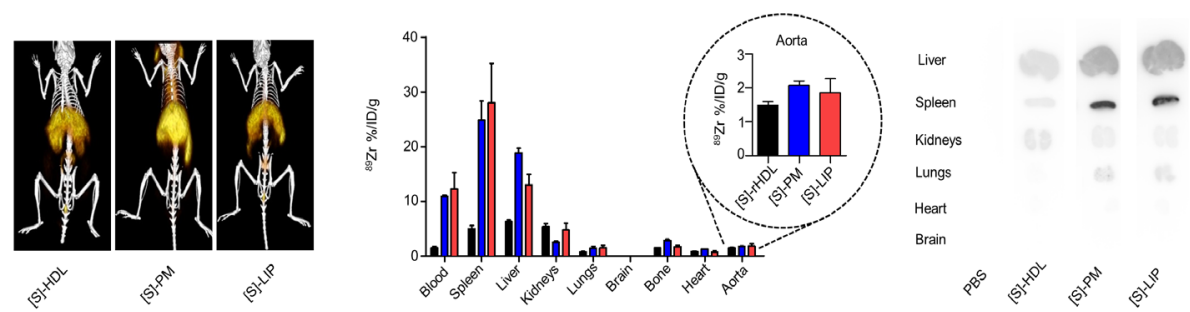

Figure 3 | Dual labeling of [S]-nanomedicines, and their pharmacokinetics and biodistribution evaluation in $A p o e^{-1-}$ mice with advanced atherosclerosis. (A) Size exclusion chromatograms showing coelution of Cy5.5 ( $\lambda=675 \mathrm{~nm}$, red trace) and ${ }^{89} \mathrm{Zr}$ (black, radioactive trace) on the corresponding nanomedicines (The radioactive trace was nudged by $5 \%$ to prevent visual overlapping). (B) Blood time-activity curves for the different ${ }^{89} \mathrm{Zr}$-labeled [S]-nanomedicines, as determined by gamma counting ( $\mathrm{n} \geq 3$ per condition). (C) Three-dimensional rendering of PET/CT fusion images at $24 \mathrm{~h}$ after injection ( $\mathrm{n}=3$ per condition).

(D) Quantitative assessment of radioactivity distribution in selected tissues using gamma counting 24 $\mathrm{h}$ after injection ( $\mathrm{n} \geq 3$ per condition). (E) Autoradiography images of selected tissues $24 \mathrm{~h}$ after injection. [S]: simvastatin; HDL: high density lipoprotein; PM: polymeric micelles; LIP: liposomes; \% rel. intensity: percentage relative intensity; ${ }^{89} \mathrm{Zr}$ : Zirconium-89; Cy5.5: Cyanine5.5 ; \%ID/g: percentage injected dose per gram of tissue; min: minutes; h: hours. 
To study the tissue distribution of the [S]-nanomedicines non-invasively, we employed PET/CT imaging (Figure 3C). PET imaging offers high sensitivity with deep tissue penetration[50,61]. At $24 \mathrm{~h}$ after injection, PET/CT imaging showed predominant accumulation in liver and spleen. This is in line with the dominant role of mononuclear phagocytic system' (MPS) macrophages in the removal of intravenously administered nanosystems[62]. [S]-HDL showed a relatively high signal in the kidneys, which is in line with a previously reported[50,63,64] renal clearance of comparatively smaller nanoparticles. The total body signal detected in mice which received [S]-PM or [S]-LIP was higher than that of [S]-HDL, implying faster body clearance of the latter (Supporting Figure 5).

We subsequently excised the tissues of interest from $A \mathrm{Ape}^{-1-}$ mice and analyzed their radioactivity content $24 \mathrm{~h}$ after i.v. administration. Ex vivo analysis of the radioactivity distribution corroborated the observations from pharmacokinetic and PET imaging studies (Figure 3D and E). [S]-PM and [S]-LIP showed the highest accumulation in spleen and liver, $\sim 25-30 \% \mathrm{ID} / \mathrm{g}$ and $10-15 \% \mathrm{ID} / \mathrm{g}$, respectively (Figure 3D), while [S]-HDL showed relatively high kidney accumulation. These results were in line with the qualitative autoradiographic analysis (Figure 3E) and NIRF imaging (Supporting Figure 6).

\subsection{Targeting to atherosclerotic plaques and lesion- associated macrophages and monocytes}

We investigated the plaque targeting ability of the [S]-nanomedicines Apoe ${ }^{-/-}$ mice aortas. Interestingly, using gamma counting, the radioactivity concentrations in the excised aortas were similar for the three formulations ( 1.5-2\% ID/g) with no statistically significant differences (Figure 3D, right). These findings are especially striking given the difference in blood circulation half-lives. We also evaluated regional distribution within the aorta using autoradiography and NIRF imaging for the three formulations (Figure 4A). We found co-localization between radioactivity disposition and NIRF signal in the focal atherosclerotic lesions, especially in aortic roots, an area which is known to reproducibly develop atherosclerotic plaque[65-67].

Sincemacrophagesand monocytes are the key immunecellsthat driveatherosclerosis progression[9], we sought to study the uptake of the three [S]-nanomedicine types by these key cells once localized in plaques in aortas of $A p o e^{-1-}$ mice with advanced atherosclerosis. We applied a flow cytometry protocol and gating procedures adapted from previous studies[8,47] (Figure 4B). After $24 \mathrm{~h}$ i.v. administration, the aortas were excised, and the uptake of Cy5.5 labeled-[S]-nanomedicines by macrophages and monocytes was quantified. Interestingly, distinct uptake patterns for the three [S]-nanomedicines by aortic macrophages and Ly6Chigh monocytes were found (Figures 4C and 4D). The HDL formulation showed relatively high uptake by aortic macrophages, in line with what was previously reported in atherosclerosis and 
cancer models[44,47]. Interestingly, [S]-PM showed a similar degree of high uptake, not only by aortic macrophages but also by Ly6Chigh monocytes. On the other hand, [S]-LIP demonstrated the lowest macrophage/monocyte targeting efficiency in comparison to the other two formulations (Figure 4C and D).

A

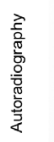

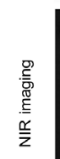
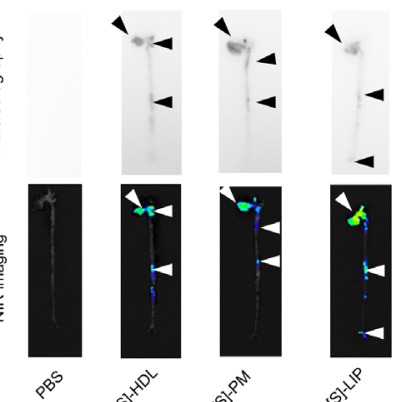

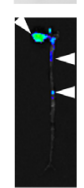

Sक्ष

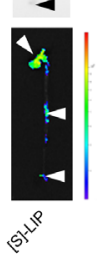

C F $4 / 80 \mathrm{M} \Phi /$ aorta

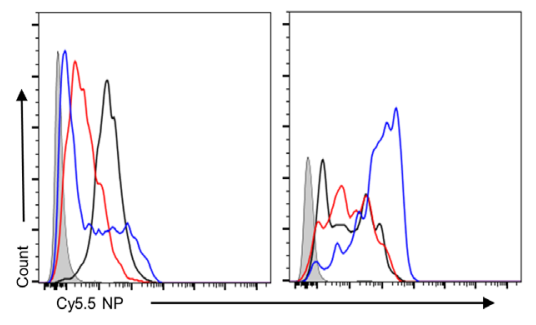

B

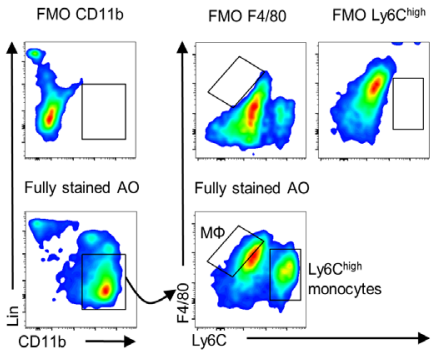

D

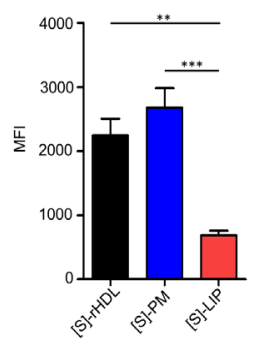

Ly6C high monocyte/aorta

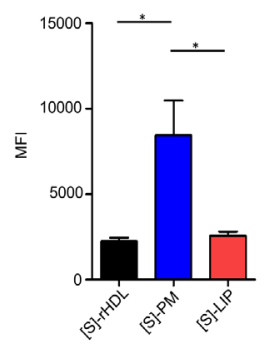

Figure 4 I Plaque targeting and uptake of [S]-nanomedicines by macrophages and monocytes in $A_{p o e^{-/-}}$mice with advanced atherosclerosis. (A) Autoradiography (upper panel) and NIRF(lower panel) images of excised aortas from $A p o e^{-1-}$ mice $24 \mathrm{~h}$ after injection. (B) Flow cytometry gating procedures and fluorescence minus one (FMO) control samples to identify aortic macrophages (M $\Phi)$ and Ly6Chigh monocytes. (C) Black ([S]-HDL), blue ([S]-PM), and red ([S]-LIP) histograms showing representative signal distribution of aortic macrophages and Ly6Chigh monocytes in mice injected with the [S]nanomedicines compared with the cells from control animals injected with PBS (gray histogram on the left in each graph). (D) Quantification of mean fluorescence intensity (MFI) of macrophages and monocytes in the atherosclerotic aortas ( $\mathrm{n}=4$ per condition). [S]: simvastatin; HDL: high density lipoprotein; PM: polymeric micelles; LIP: liposomes; NIR: near-infrared; FMO: fluorescence minus one; MФ: macrophages; lin: lineage; Cy5.5 NP: cyanine 5.5 labeled nanoparticles. Data presented as mean \pm SEM. ${ }^{*} P \leq 0.05 ;{ }^{* *} P$ $\leq 0.01{ }^{* * *} P \leq 0.001$. 
Although our efficacy readout parameter for [S]-nanomedicine treatment was reduction in aortic plaque macrophage/monocyte content, uptake by other immune cell types present in the blood pool and spleen may affect treatment outcome. Similar to our findings in the aorta, [S]-PM showed a much higher affinity for blood Ly6Chigh monocytes in comparison to [S]-HDL and [S]-LIP ( $\sim 10$ fold and $\sim 5$ fold, respectively, Supporting Figure 7). Similarly, the association of [S]-PM with blood dendritic cells (DC) was also much higher than for [S]-HDL and [S]-LIP $(\sim 29$ fold and 8 fold, respectively, Supporting Figure 7). Also, in the spleen (Supporting Figure 8), [S]-PM showed superior targeting efficiency towards the phagocytic target cells.

\subsection{Therapeutic efficacy and safety}

Reducing the number of plaque macrophages has been shown to decrease vessel wall inflammation and improve therapeutic outcomes in several key studies[8,10,39]. Also, blocking the recruitment of pro-inflammatory monocytes is a rational strategy to reduce inflammation in atherosclerotic plaques[68] and other diseases[69,70]. Here, we quantitatively assessed the efficacy of [S]-nanomedicine treatment by evaluating plaque macrophage/monocyte content using flow cytometry. Apoe ${ }^{-/-}$mice with advanced atherosclerosis were administered three doses of [S]-HDL, [S]-PM, [S]LIP (60 mg/Kg simvastatin per dose), equivalent oral simvastatin (as control), or PBS i.v. (as placebo) (Figure 5A). All mice ( $\mathrm{n}=8-10 /$ group) were age-matched and received the assigned treatments within a single week. Aortic macrophages and pro-inflammatory Ly6Chigh monocytes were gated as described before in Figure $4 \mathrm{~B}$. As compared to the controls saline and oral simvastatin, a significant decrease in plaque macrophage content was observed in the [S]-PM and [S]-HDL treatment groups (Figure 5B). [S]-LIP and oral [S] treatment groups did not yield significant reductions in plaque macrophage content (vs. saline treatment). Interestingly, none of the nanomedicine treatments had a statistically significant impact on plaque Ly6Chigh monocyte content, indicating that the different treatments do not affect monocyte recruitment, in line with what we observed previously [38,39]. 
A

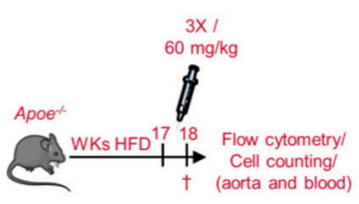

C
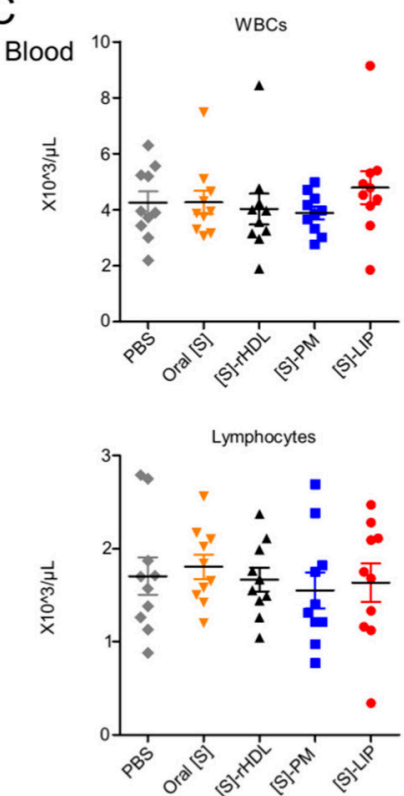

B
Aorta
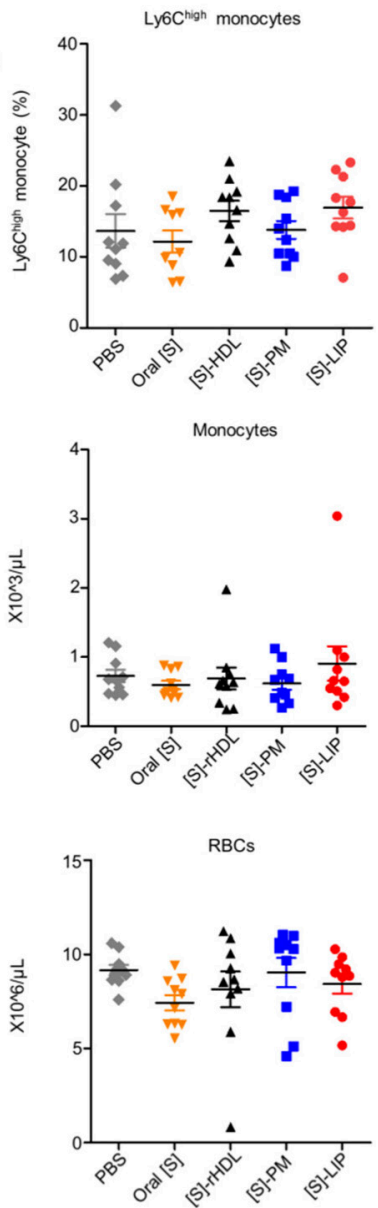
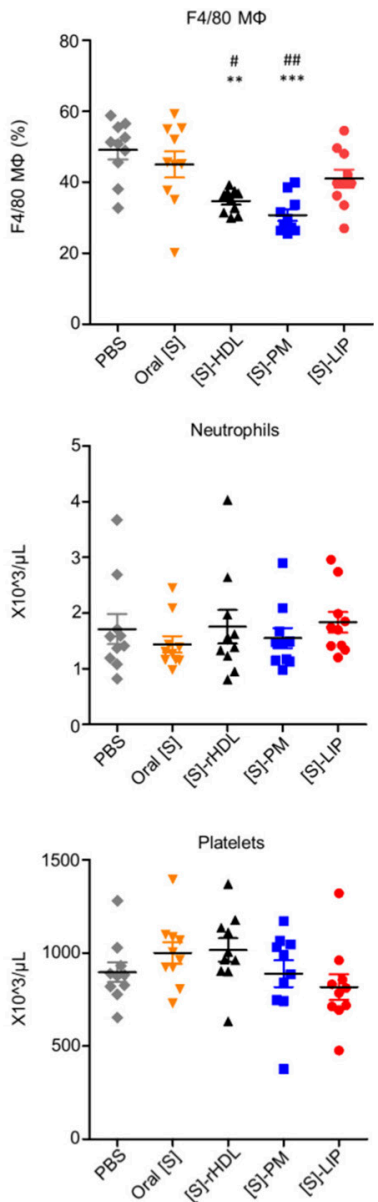

Figure 5 I Therapeutic Efficacy of [S]-nanomedicines. (A) A schematic depiction of the efficacy study, in which $\mathrm{Apoe}^{-1-}$ mice were administered $60 \mathrm{mg} / \mathrm{kg}$ simvastatin i.v., for [S]-nanomedicines, oral gavage (control group), or PBS (placebo) for one week. (B) Quantification of aortic Ly6C high monocytes and macrophages using flow cytometry (percentage of $\mathrm{CD} 45^{+}$total aortic leukocytes). (C) Complete blood count analysis. (n=8-10 per condition). Apoe $e^{-1-}$ : apolipoprotein E-deficient; PBS: phosphate buffered saline; [S]: simvastatin; HDL: high density lipoprotein; PM: polymeric micelles; LIP: liposomes; Wks: weeks; HFD: high fat diet; MФ: macrophages; WBCs; white blood cells, RBCs; red blood cells. Data presented as mean \pm SEM. ${ }^{*} P \leq 0.05 ;{ }^{* *} P \leq 0.01 ;{ }^{* *} P \leq 0.001$. ${ }^{*}$ relative to the PBS group. \# relative to Oral [S]. 
We further analyzed blood as the direct recruitment pool for monocytes and other immune cells. None of the treatment groups showed a significant change in the number of total circulating leukocytes, monocytes, neutrophils, lymphocytes, red blood cells (RBCs), or platelets (vs. saline) (Figure 5C). Additionally, the [S]nanomedicine treatments did not significantly alter the concentration of serum triglycerides, total cholesterol, and glucose (vs. oral [S] treatment or placebo) (Supporting Figure 9). Similarly, no significant concentration changes were found in toxicity blood markers (Supporting Figure 9).

Nanomedicines are developed to ultimately achieve local, targeted drug delivery to pathological lesions (atherosclerotic plaques in our study) upon intravenous injection, aiming to improve the therapeutic efficacy and safety profile of the free drug. Nanocarrier characteristics such as composition, size, and morphology, which likely affect their circulation kinetics, drug release kinetics, extravasation, penetration, and importantly, specificity to the target cell(s), will have a direct impact on the therapeutic efficacy of nanomedicines. In our study, all treatments contained the same drug, the HMG-CoA reductase inhibitor simvastatin, which was administered at the same dose, and all were evaluated in age/diet matched Apoe $e^{-/-}$ mice, a well-established model of atherosclerosis[71].

In our head-to-head study, we found the [S]-LIP yielded the least efficacy in reducing macrophage burden in atherosclerotic plaques. Although [S]-LIP possessed a long blood half-life, its poor plaque macrophage targeting efficiency, in addition to leakage of simvastatin from liposomes in circulation, might have contributed to this low efficacy. On the contrary, despite its comparatively shorter circulation half-life, [S]HDL demonstrated better efficacy than [S]-LIP. This noticeable difference is probably due to HDL's high specificity for plaque macrophages. These results also suggest that HDL can be an ideal imaging probe for lesion-associated macrophages [44,50]. Among the three platforms, [S]-PM demonstrated the most favorable features of a targeted drug delivery approach, including long circulation times, increased drug-nanocarrier stability in serum, and improved cellular targeting efficiency. These features resulted in the most prominent reduction in plaque macrophage burden. Remarkably, although the three nanomedicines showed uptake by pro-inflammatory Ly6Chigh monocytes, especially [S]-PM, none of the treatments altered monocyte content in atherosclerotic plaques. These different treatment effects on plaque macrophages and monocytes are in line with the new paradigm that considers atherosclerosis as a multiphasic process in which local macrophage proliferation, rather than monocyte recruitment, governs advanced atherosclerotic plaque progression[8,72]. Thus, tackling monocyte recruitment in advanced atherosclerosis with (nano-) medicines may not be an ideal approach for atheroprotection as demonstrated by Lindau et al.[73]. However, blocking the recruitment of pro-inflammatory monocytes may be imperative in conditions / diseases in which active monocytosis and recruitment can exacerbate the inflammatory response. An example of these conditions is the rapid monocyte kinetics triggered by acute myocardial infarction[68]. 
Additionally, the three [S]-nanomedicines did not change the metabolic parameters in plasma nor significantly alter major biomarkers of HMG-CoA reductase inhibitor-induced myo- or hepatotoxicity[37]. Moreover, HDL - one of our body's own nanoparticles - and liposomes are well known for their biocompatibility and biodegradability. PM composed of pHPMA polymers are also known to be nonimmunogenic and biocompatible [74-76]; they have been extensively investigated for drug delivery (reviewed by Talelli et al.[56]) Moreover, several pHPMA-based therapies have been clinically evaluated (reviewed by Duncan and Vicent [77]). Our data indicate the safety of our treatments, an important step for the potential clinical translation of the nanomedicines under investigation. However, more detailed safety studies will need to be executed before any clinical application. Furthermore, factors such manufacturability under good manufacture practice (GMP) conditions, cost, patient selection, and best therapeutic approach (e.g. high dose short-term injections with and without oral therapy) also need to be carefully considered for clinical translation[78].

\section{CONCLUSIONS}

In this systematic head-to-head study, we have adopted sensitive, quantitative techniques to evaluate three clinically applicable nanomedicines targeting HMGCoA reductase, in regards to their physicochemical characteristics and in vivo performance in $A \mathrm{Apoe}^{-1-}$ mice with advanced atherosclerosis. It was found that [S]PM possesses superior efficacy, in comparison to [S]-HDL and [S]-LIP, to locally reduce the macrophage burden in advanced atherosclerotic plaques. This is likely due to the higher targeted drug delivery efficiency, as a result of the combination of the enhanced retention of simvastatin in the polymeric micelles and the high cellular targeting efficiency to plaque macrophages. These findings are crucial for further development and clinical application of nanomedicines to reduce inflammation in advanced atherosclerosis. Furthermore, the work presented in this paper suggests that the systematic investigation of nanomaterials, beyond cancer, is crucial for the future of nanomedicine design, optimization, and clinical applicability in several disease contexts. 


\section{REFERENCES}

[1] E. Falk, Pathogenesis of Atherosclerosis, J. Am. Coll. Cardiol. 47 (2006) C7-C12. doi:10.1016/j.jacc.2005.09.068.

[2] P. Libby, History of Discovery : Inflammation in Atherosclerosis, Arter. Thromb Vasc Biol. 32 (2012) 2045-2051. doi:10.1161 / ATVBAHA.108.179705.History.

[3] G.K. Hansson, A. Hermansson, The immune system in atherosclerosis, Nat. Immunol. 12 (2011) 204-212. doi:10.1038/ni.2001.

[4] K.J. Moore, F.J. Sheedy, E.A. Fisher, Macrophages in atherosclerosis: a dynamic balance, Nat. Rev. 13 (2013) 709-721. doi:10.1038/nri3520.

[5] S. Blankenberg, S. Barbaux, L. Tiret, Adhesion molecules and atherosclerosis, Atherosclerosis. 170 (2003) 191-203. doi:S0021-9150(03)00097-2.

[6] K.J. Woollard, F. Geissmann, Monocytes in atherosclerosis: subsets and functions., Nat. Rev. Cardiol. 7 (2010) 77-86. doi:10.1038/nrcardio.2009.228.

[7] J.F. Bentzon, F. Otsuka, R. Virmani, E. Falk, Mechanisms of Plaque Formation and Rupture, Circ. Res. 114 (2014).

[8] C.S. Robbins, I. Hilgendorf, G.F. Weber, I. Theurl, Y. Iwamoto, J.-L.L. Figueiredo, R. Gorbatov, G.K. Sukhova, L.M.S. Gerhardt, D. Smyth, C.C.J. Zavitz, E.A. Shikatani, M. Parsons, N. van Rooijen, H.Y. Lin, M. Husain, P. Libby, M. Nahrendorf, R. Weissleder, F.K. Swirski, Local proliferation dominates lesional macrophage accumulation in atherosclerosis, Nat. Med. 19 (2013) 1166-1172. doi:10.1038/nm.3258.

[9] K.J. Moore, I. Tabas, Macrophages in the pathogenesis of atherosclerosis, Cell. 145 (2011) 341-355. doi:10.1016/j.cell.2011.04.005.

[10] P. Dutta, G. Courties, Y. Wei, F. Leuschner, R. Gorbatov, C.S. Robbins, Y. Iwamoto, B. Thompson, A.L. Carlson, T. Heidt, M.D. Majmudar, F. Lasitschka, M. Etzrodt, P. Waterman, M.T. Waring, A.T. Chicoine, A.M. van der Laan, H.W.M. Niessen, J.J. Piek, B.B. Rubin, J. Butany, J.R. Stone, H.A. Katus, S.A. Murphy, D.A. Morrow, M.S. Sabatine, C. Vinegoni, M.A. Moskowitz, M.J. Pittet, P. Libby, C.P. Lin, F.K. Swirski, R. Weissleder, M. Nahrendorf, Myocardial infarction accelerates atherosclerosis., Nature. 487 (2012) 325-9. doi:10.1038/ nature11260.

[11] H. Emami, P. Singh, M. MacNabb, E. Vucic, Z. Lavender, J.H. Rudd, Z.A. Fayad, J. LehrerGraiwer, M. Korsgren, A.L. Figueroa, J. Fredrickson, B. Rubin, U. Hoffmann, Q.A. Truong, J.K. Min, A. Baruch, K. Nasir, M. Nahrendorf, A. Tawakol, Splenic metabolic activity predicts risk of future cardiovascular events: demonstration of a cardiosplenic axis in humans, JACC.Cardiovascular Imaging. 8 (2015) 121-130. doi:10.1016/j.jcmg.2014.10.009.

[12] G.W. Stone, A. Maehara, A.J. Lansky, B. de Bruyne, E. Cristea, G.S. Mintz, R. Mehran, J. McPherson, N. Farhat, S.P. Marso, H. Parise, B. Templin, R. White, Z. Zhang, P.W. Serruys, A Prospective Natural-History Study of Coronary Atherosclerosis, N. Engl. J. Med. 364 (2011) 226-235. doi:10.1056/ NEJMoa1002358.

[13] T. Lammers, F. Kiessling, W.E. Hennink, G. Storm, Drug targeting to tumors: principles, pitfalls and (pre-) clinical progress, J. Control. Release. 161 (2012) 175-187. doi:10.1016/j. jconrel.2011.09.063.

[14] Y.H. Bae, K. Park, Targeted drug delivery to tumors: Myths, reality and possibility, J. Control. Release. 153 (2011) 198-205. doi:10.1016/j.jconrel.2011.06.001.

[15] D. Peer, J.M. Karp, S. Hong, O.C. Farokhzad, R. Margalit, R. Langer, Nanocarriers as an emerging platform for cancer therapy., Nat. Nanotechnol. 2 (2007) 751-760. doi:10.1038/ nnano.2007.387.

[16] V.P. Torchilin, Targeted pharmaceutical nanocarriers for cancer therapy and imaging., AAPS J. 9 (2007) E128-47. doi:10.1208/aapsj0902015. 
[17] B.J. Crielaard, T. Lammers, R.M. Schiffelers, G. Storm, Drug targeting systems for inflammatory disease: one for all, all for one, J. Control. Release. 161 (2012) 225-234. doi:10.1016/j.jconrel.2011.12.014.

[18] B. Ozbakir, B.J. Crielaard, J.M. Metselaar, G. Storm, T. Lammers, Liposomal corticosteroids for the treatment of inflammatory disorders and cancer, J. Control. Release. 190 (2014) 624-636. doi:10.1016/j.jconrel.2014.05.039 .

[19] T.M. Allen, P.R. Cullis, Liposomal drug delivery systems: from concept to clinical applications, Adv. Drug Deliv. Rev. 65 (2013) 36-48. doi:10.1016/j.addr.2012.09.037.

[20] R. Weissleder, M. Nahrendorf, M.J. Pittet, Imaging macrophages with nanoparticles, Nat. Mater. 13 (2014) 125-138. doi:10.1038/nmat3780.

[21] J.A. Hubbell, A. Chilkoti, Nanomaterials for Drug Delivery, Science (80-. ). 337 (2012).

[22] C. Oerlemans, W. Bult, M. Bos, G. Storm, J.F.W. Nijsen, W.E. Hennink, Polymeric micelles in anticancer therapy: Targeting, imaging and triggered release, Pharm. Res. 27 (2010) 2569-2589. doi:10.1007/s11095-010- 0233-4.

[23] C. Deng, Y. Jiang, R. Cheng, F. Meng, Z. Zhong, Biodegradable polymeric micelles for targeted and controlled anticancer drug delivery: Promises, progress and prospects, Nano Today. 7 (2012) 467-480. doi:10.1016/j.nantod.2012.08.005.

[24] K.K. Ng, J.F. Lovell, G. Zheng, Lipoprotein-inspired nanoparticles for cancer theranostics, Acc. Chem. Res. 44 (2011) 1105-1113. doi:10.1021 / ar200017e.

[25] B.A. Kingwell, M.J. Chapman, A. Kontush, N.E. Miller, HDL-targeted therapies: progress, failures and future, Nat. Rev. Drug Discov. 13 (2014) 445-464. doi:10.1038/nrd4279.

[26] H. Cabral, K. Kataoka, Progress of drug-loaded polymeric micelles into clinical studies, J. Control. Release. 190 (2014) 465-476. doi:10.1016/j.jconrel.2014.06.042.

[27] T. Lammers, F. Kiessling, M. Ashford, W. Hennink, D. Crommelin, G. Strom, Cancer nanomedicine: is targeting our target?, Nat. Rev. Mater. 1 (2016) 16069. doi:10.1038/ natrevmats.2016.69.

[28] R. van der Meel, S. Oliveira, I. Altintas, R. Heukers, E.H.E. Pieters, P.M.P. van Bergen en Henegouwen, G. Storm, W.E. Hennink, R.J. Kok, R.M. Schiffelers, Inhibition of Tumor Growth by Targeted Anti-EGFR/IGF-1R Nanobullets Depends on Efficient Blocking of Cell Survival Pathways, Mol. Pharm. 10 (2013) 3717-3727. doi:10.1021 / mp400212v.

[29] Y. Min, J.M. Caster, M.J. Eblan, A.Z. Wang, Clinical Translation of Nanomedicine, Chem. Rev. 115 (2015) 11147-11190. doi:10.1021 / acs.chemrev.5b00116.

[30] J.L. Goldstein, M.S. Brown, Regulation of the mevalonate pathway, Nature. 343 (1990) 425-430. doi:10.1038/343425a0.

[31] J.K. Liao, U. Laufs, Pleiotropic effects of statins., Annu. Rev. Pharmacol. Toxicol. 45 (2005) 89-118. doi:10.1146/annurev.pharmtox.45.120403.095748.

[32] A. Alaarg, K.H.K.H. Zheng, F.M.F.M. van der Valk, A.E.A.E. da Silva, M. Versloot, L.C.Q.L.C.Q. van Ufford, D.M. Schulte, G. Storm, J.M.J.M. Metselaar, E.S.G.E.S.G. Stroes, A.A.A.J.J. Hamers, Multiple pathway assessment to predict anti-atherogenic efficacy of drugs targeting macrophages in atherosclerotic plaques, Vascul. Pharmacol. 82 (2015). doi:10.1016/j.vph.2016.04.006.

[33] A. Tawakol, Z.A. Fayad, R. Mogg, A. Alon, M.T. Klimas, H. Dansky, S.S. Subramanian, A. Abdelbaky, J.H.F. Rudd, M.E. Farkouh, I.O. Nunes, C.R. Beals, S.S. Shankar, Intensification of Statin Therapy Results in a Rapid Reduction in Atherosclerotic Inflammation: Results of a Multicenter Fluorodeoxyglucose-Positron Emission Tomography/Computed Tomography FeasibilityStudy, J. Am. Coll. Cardiol. 62 (2013) 909-917. doi:10.1016/j. jacc.2013.04.066.

[34] C.P. Sparrow, C.A. Burton, M. Hernandez, S. Mundt, H. Hassing, S. Patel, R. Rosa, A. Hermanowski- Vosatka, P.-R. Wang, D. Zhang, L. Peterson, P.A. Detmers, Y.-S. Chao, S.D. Wright, Simvastatin Has Anti- Inflammatory and Antiatherosclerotic Activities 
Independent of Plasma Cholesterol Lowering, Arterioscler. Thromb. Vasc. Biol. 21 (2001).

[35] J. Armitage, The safety of statins in clinical practice, Lancet. 370 (2007) 1781-1790. doi:10.1016/S0140- 6736(07)60716-8.

[36] S. Bellosta, R. Paoletti, A. Corsini, Safety of Statins: Focus on Clinical Pharmacokinetics and Drug Interactions, Circulation. 109 (2004) III-50-III-57. doi:10.1161/01. CIR.0000131519.15067.1f.

[37] R. Duivenvoorden, J. Tang, D.P. Cormode, A.J. Mieszawska, D. Izquierdo-Garcia, C. Ozcan, M.J. Otten, N. Zaidi, M.E. Lobatto, S.M. van Rijs, B. Priem, E.L. Kuan, C. Martel, B. Hewing, H. Sager, M. Nahrendorf, G.J. Randolph, E.S.G. Stroes, V. Fuster, E.A. Fisher, Z.A. Fayad, W.J.M. Mulder. A statin-loaded reconstituted high- density lipoprotein nanoparticle inhibits atherosclerotic plaque inflammation, Nat. Commun. 5 (2014) 227239. doi:10.1038/ncomms4065.

[38] J. Tang, M.E. Lobatto, L. Hassing, S. van der Staay, S.M. van Rijs, C. Calcagno, M.S. Braza, S. Baxter, F. Fay, B.L. Sanchez-Gaytan, R. Duivenvoorden, H.B. Sager, Y.M. Astudillo, W. Leong, S. Ramachandran, G. Storm, C. Perez-Medina, T. Reiner, D.P. Cormode, G.J. Strijkers, E.S.G. Stroes, F.K.Swirski, M. Nahrendorf, E.A. Fisher, Z.A. Fayad, W.J.M. Mulder, Inhibiting macrophage proliferation suppresses atherosclerotic plaque inflammation, Sci. Adv. 1 (2015) e1400223. doi:e1400223.

[39] Y. Shi, R. Van Der Meel, B. Theek, E. Oude Blenke, E.H.E. Pieters, M.H.A.M. Fens, J. Ehling, R.M. Schiffelers, G. Storm, C.F. Van Nostrum, T. Lammers, W.E. Hennink, Complete regression of xenograft tumors upon targeted delivery of paclitaxel via $\Pi-\Pi$ stacking stabilized polymeric micelles, ACS Nano. 9 (2015) 3740- 3752. doi:10.1021/ acsnano.5b00929.

[40] C. Pérez-Medina, D. Abdel-Atti, Y. Zhang, V. a Longo, C.P. Irwin, T. Binderup, J. RuizCabello, Z. a Fayad, J.S. Lewis, W.J.M. Mulder, T. Reiner, A Modular Labeling Strategy for In Vivo PET and Near-Infrared Fluorescence Imaging of Nanoparticle Tumor Targeting., J. Nucl. Med. 55 (2014) 1706-1712. doi:10.2967/jnumed.114.141861.

[41] M. Banciu, J.M. Metselaar, R.M. Schiffelers, G. Storm, Antitumor activity of liposomal prednisolone phosphate depends on the presence of functional tumor-associated macrophages in tumor tissue, Neoplasia. 10 (2008) 108-117. doi:10.1593/ neo.07913.

[42] M.E. Lobatto, C. Calcagno, A. Millon, M.L. Senders, F. Fay, P.M. Robson, S. Ramachandran, T. Binderup, M.P. Paridaans, S. Sensarn, S. Rogalla, R.E. Gordon, L. Cardoso, G. Storm, J.M. Metselaar, C.H. Contag, E.S. Stroes, Z.A. Fayad, W.J. Mulder, Atherosclerotic plaque targeting mechanism of long-circulating nanoparticles established by multimodal imaging, ACS Nano. 9 (2015) 1837-1847. doi:10.1021/nn506750r.

[43] C. Pérez-Medina, J. Tang, D. Abdel-Atti, B. Hogstad, M. Merad, E.A. Fisher, Z.A. Fayad, J.S. Lewis, W.J.M. Mulder, T. Reiner, C. Perez-Medina, J. Tang, D. Abdel-Atti, B. Hogstad, M. Merad, E.A. Fisher, Z.A. Fayad, J.S. Lewis, W.J.M. Mulder, T. Reiner, PET Imaging of Tumor-Associated Macrophages with ${ }^{89} \mathrm{Zr}$-Labeled High- Density Lipoprotein Nanoparticles, J. Nucl. Med. 56 (2015) 1272-1277. doi:10.2967/jnumed.115.158956.

[44] Y. Zhao, F. Fay, S. Hak, J. Manuel Perez-Aguilar, B.L. Sanchez-Gaytan, B. Goode, R. Duivenvoorden, C. de Lange Davies, A. Bjorkoy, H. Weinstein, Z.A. Fayad, C. PerezMedina, W.J.M. Mulder, A. Bjørkøy, H. Weinstein, Z.A. Fayad, C. Pérez-Medina, W.J.M. Mulder, Augmenting drug-carrier compatibility improves tumour nanotherapy efficacy, Nat. Commun. 7 (2016) 11221. doi:10.1038/ncomms11221.

[45] J.Z. Nordin, Y. Lee, P. Vader, I. Mäger, H.J. Johansson, W. Heusermann, O.P.B. Wiklander, M. Hällbrink, Y. Seow, J.J. Bultema, J. Gilthorpe, T. Davies, P.J. Fairchild, S. Gabrielsson, N.C. Meisner-Kober, J. Lehtiö, C.I.E. Smith, M.J.A. Wood, S. EL Andaloussi, Ultrafiltration with size-exclusion liquid chromatography for high yield isolation of extracellular vesicles preserving intact biophysical and functional properties, Nanomedicine Nanotechnology, 
Biol. Med. 11 (2015) 879-883. doi:10.1016/j.nano.2015.01.003.

[46] J. Tang, S. Baxter, A. Menon, A. Alaarg, B.L. Sanchez-Gaytan, F. Fay, Y. Zhao, M. Ouimet, M.S. Braza, V.A. Longo, D. Abdel-Atti, R. Duivenvoorden, C. Calcagno, G. Storm, S. Tsimikas, K.J. Moore, F.K. Swirski, M. Nahrendorf, E.A. Fisher, C. Pérez-Medina, Z.A. Fayad, T. Reiner, W.J.M. Mulder, Immune cell screening of a nanoparticle library improves atherosclerosis therapy, Proc. Natl. Acad. Sci. U. S. A. 113 (2016). doi:10.1073/ pnas.1609629113.

[47] M.E. Lobatto, Z.A. Fayad, S. Silvera, E. Vucic, C. Calcagno, V. Mani, S.D. Dickson, K. Nicolay, M. Banciu, R.M. Schiffelers, J.M. Metselaar, L. van Bloois, H.-S.S. Wu, J.T. Fallon, J.H. Rudd, V. Fuster, E.A. Fisher, G. Storm, W.J.M. Mulder, M.A. Wiener, Multimodal clinical imaging to longitudinally assess a nanomedical anti- inflammatory treatment in experimental atherosclerosis, Mol. Pharm. 7 (2010) 2020-2029. doi:10.1021/mp100309y.

[48] F.M. van der Valk, D.F. van Wijk, M.E. Lobatto, H.J. Verberne, G. Storm, M.C.M. Willems, D.A. Legemate, A.J. Nederveen, C. Calcagno, V. Mani, S. Ramachandran, M.P.M. Paridaans, M.J. Otten, G.M. Dallinga-Thie, Z.A. Fayad, M. Nieuwdorp, D.M. Schulte, J.M. Metselaar, W.J.M. Mulder, E.S. Stroes, Prednisolone-containing liposomes accumulate in human atherosclerotic macrophages upon intravenous administration., Nanomedicine. 11 (2015) 1039-46. doi:10.1016/j.nano.2015.02.021.

[49] C. Pérez-Medina, T. Binderup, M.E. Lobatto, J. Tang, C. Calcagno, L. Giesen, C.H. Wessel, J. Witjes, S. Ishino, S. Baxter, Y. Zhao, S. Ramachandran, M. Eldib, B.L. Sánchez-Gaytán, P.M. Robson, J. Bini, J.F. Granada, K.M. Fish, E.S.G. Stroes, R. Duivenvoorden, S. Tsimikas, J.S. Lewis, T. Reiner, V. Fuster, A. Kjær, E.A. Fisher, Z.A. Fayad, W.J.M. Mulder, In Vivo PET Imaging of HDL in Multiple Atherosclerosis Models, JACC Cardiovasc. Imaging. 9 (2016) 950-961. doi:10.1016/j.jcmg.2016.01.020.

[50] B.L. Sanchez-Gaytan, F. Fay, M.E. Lobatto, J. Tang, M. Ouimet, Y. Kim, S.E.M. van der Staay, S.M. van Rijs, B. Priem, L. Zhang, E.A. Fisher, K.J. Moore, R. Langer, Z.A. Fayad, W.J.M. Mulder, HDL-Mimetic PLGA Nanoparticle To Target Atherosclerosis Plaque Macrophages, Bioconjug. Chem. 26 (2015) 443-451. doi:10.1021/bc500517k.

[51] O. Naksuriya, Y. Shi, C.F. Van Nostrum, S. Anuchapreeda, W.E. Hennink, S. Okonogi, HPMA-based polymeric micelles for curcumin solubilization and inhibition of cancer cell growth, Eur. J. Pharm. Biopharm. 94 (2015) 501-512. doi:10.1016/j.ejpb.2015.06.010.

[52] Y. Shi, T. Lammers, G. Storm, W.E. Hennink, Physico-Chemical Strategies to Enhance Stability and Drug Retention of Polymeric Micelles for Tumor-Targeted Drug Delivery, Macromol. Biosci. 17 (2017) 1600160. doi:10.1002/mabi.201600160.

[53] L. Quan, Y. Zhang, B.J. Crielaard, A. Dusad, S.M. Lele, C.J. Rijcken, J.M. Metselaar, H. Kostkova, T. Etrych, K. Ulbrich, F. Kiessling, T.R. Mikuls, W.E. Hennink, G. Storm, T. Lammers, D. Wang, Nanomedicines for inflammatory arthritis: head-to-head comparison of glucocorticoid-containing polymers, micelles, and liposomes, ACS Nano. 8 (2014) 458466. doi:10.1021/nn4048205.

[54] M. Talelli, C.J.F. Rijcken, C.F. van Nostrum, G. Storm, W.E. Hennink, Micelles based on HPMA copolymers , Adv. Drug Deliv. Rev. 62 (2010) 231-239. doi:10.1016/j. addr.2009.11.029.

[55] A.G. Lacko, N.A. Sabnis, B. Nagarajan, W.J. McConathy, HDL as a drug and nucleic acid delivery vehicle., Front. Pharmacol. 6 (2015) 247. doi:10.3389/ fphar.2015.00247.

[56] B.L. Sanchez-Gaytan, F. Fay, S. Hak, A. Alaarg, Z.A. Fayad, C. Pérez-Medina, W.J.M. Mulder, Y. Zhao, Real-Time Monitoring of Nanoparticle Formation by FRET Imaging, Angew. Chemie Int. Ed. (2017). doi:10.1002/anie.201611288.

[57] Y. Shi, S. Kunjachan, Z. Wu, F. Gremse, D. Moeckel, M. van Zandvoort, F. Kiessling, G. Storm, C.F. van Nostrum, W.E. Hennink, T. Lammers, Fluorophore labeling of corecrosslinked polymeric micelles for multimodal in vivo and ex vivo optical imaging, 
Nanomedicine. 10 (2015) 1111-1125. doi:10.2217/nnm.14.170.

[58] B.M. Zeglis, P. Mohindra, G.I. Weissmann, V. Divilov, S.A. Hilderbrand, R. Weissleder, J.S. Lewis, Modular Strategy for the Construction of Radiometalated Antibodies for Positron Emission Tomography Based on Inverse Electron Demand Diels-Alder Click Chemistry, Bioconjug. Chem. 22 (2011) 2048-2059. doi:10.1021/bc200288d.

[59] S. Kunjachan, J. Ehling, G. Storm, F. Kiessling, T. Lammers, Noninvasive Imaging of Nanomedicines and Nanotheranostics: Principles, Progress, and Prospects, Chem. Rev. 115 (2015) 10907-10937. doi:10.1021/ cr500314d.

[60] T. Lammers, SMART drug delivery systems: Back to the future vs. clinical reality, Int. J. Pharm. 454 (2013) 527-529. doi:10.1016/j.ijpharm.2013.02.046.

[61] H.S. Choi, W. Liu, P. Misra, E. Tanaka, J.P. Zimmer, B. Itty Ipe, M.G. Bawendi, J. V Frangioni, Renal clearance of quantum dots., Nat. Biotechnol. 25 (2007) 1165-70. doi:10.1038/nbt1340.

[62] E.J. Keliher, Y.-X. Ye, G.R. Wojtkiewicz, A.D. Aguirre, B. Tricot, M.L. Senders, H. Groenen, F. Fay, C. Perez-Medina, C. Calcagno, G. Carlucci, T. Reiner, Y. Sun, G. Courties, Y. Iwamoto, H.-Y. Kim, C. Wang, J.W. Chen, F.K. Swirski, H.-Y. Wey, J. Hooker, Z.A. Fayad, W.J.M. Mulder, R. Weissleder, M. Nahrendorf, Polyglucose nanoparticles with renal elimination and macrophage avidity facilitate PET imaging in ischaemic heart disease, Nat. Commun. 8 (2017) 14064. doi:10.1038/ncomms14064.

[63] V.V. Itskovich, R.P. Choudhury, J.G.S. Aguinaldo, J.T. Fallon, S. Omerhodzic, E.A. Fisher, Z.A. Fayad, Characterization of aortic root atherosclerosis in ApoE knockout mice: Highresolution in vivo and ex vivo MRM with histological correlation, Magn. Reson. Med. 49 (2003) 381-385. doi:10.1002/mrm.10360.

[64] J. Baglione, J.D. Smith, Quantitative Assay for Mouse Atherosclerosis in the Aortic Root, in: Cardiovasc. Dis. Vol. 2 Mol. Med., Humana Press, New Jersey, 2006: pp. 83-96. doi:10.1385/1-59745-213-0:83.

[65] G.S. Getz, C.A. Reardon, Animal models of atherosclerosis., Arterioscler. Thromb. Vasc. Biol. 32 (2012) 1104-15. doi:10.1161 / ATVBAHA.111.237693.

[66] F. Leuschner, P. Dutta, R. Gorbatov, T.I. Novobrantseva, J.S. Donahoe, G. Courties, K.M. Lee, J.I. Kim, J.F. Markmann, B. Marinelli, P. Panizzi, W.W. Lee, Y. Iwamoto, S. Milstein, H. Epstein-Barash, W. Cantley, J. Wong, V. Cortez-Retamozo, A. Newton, K. Love, P. Libby, M.J. Pittet, F.K. Swirski, V. Koteliansky, R. Langer, R. Weissleder, D.G. Anderson, M. Nahrendorf, Therapeutic siRNA silencing in inflammatory monocytes in mice, Nat. Biotechnol. 29 (2011) 1005-1010. doi:10.1038/nbt.1989.

[67] E. Zigmond, C. Varol, J. Farache, E. Elmaliah, A.T. Satpathy, G. Friedlander, M. Mack, N. Shpigel, I.G. Boneca, K.M. Murphy, G. Shakhar, Z. Halpern, S. Jung, Ly6Chi Monocytes in the Inflamed Colon Give Rise to Proinflammatory Effector Cells and Migratory AntigenPresenting Cells, Immunity. 37 (2012) 1076-1090. doi:10.1016/j.immuni.2012.08.026.

[68] H.-J. Anders, V. Ninichuk, D. Schlöndorff, Progression of kidney disease: blocking leukocyte recruitment with chemokine receptor CCR1 antagonists., Kidney Int. 69 (2006) 29-32. doi:10.1038/sj.ki.5000053.

[69] K.S. Meir, E. Leitersdorf, Atherosclerosis in the Apolipoprotein E-Deficient Mouse, Arterioscler. Thromb. Vasc. Biol. 24 (2004).

[70] F.K. Swirski, I. Hilgendorf, C.S. Robbins, From proliferation to proliferation: monocyte lineage comes full circle, Semin. Immunopathol. 36 (2014) 137-148. doi:10.1007/s00281013-0409-1.

[71] A. Lindau, C. Härdtner, S.P. Hergeth, K.D. Blanz, B. Dufner, N. Hoppe, N. Anto-Michel, J. Kornemann, J. Zou, L.M.S. Gerhardt, T. Heidt, F. Willecke, S. Geis, P. Stachon, D. Wolf, P. Libby, F.K. Swirski, C.S. Robbins, W. McPheat, S. Hawley, M. Braddock, R. Gilsbach, L. Hein, C. von zur Mühlen, C. Bode, A. Zirlik, I. Hilgendorf, C. Hardtner, S.P. Hergeth, K.D. Blanz, B. Dufner, N. Hoppe, N. Anto-Michel, J. Kornemann, J. Zou, L.M.S. Gerhardt, 
T. Heidt, F. Willecke, S. Geis, P. Stachon, D. Wolf, P. Libby, F.K. Swirski, C.S. Robbins, W. McPheat, S. Hawley, M. Braddock, R. Gilsbach, L. Hein, C. von zur Muhlen, C. Bode, A. Zirlik, I. Hilgendorf, Atheroprotection through SYK inhibition fails in established disease when local macrophage proliferation dominates lesion progression, Basic Res. Cardiol. 111 (2016) 20-016-0535-8. Epub 2016 Feb 18. doi:10.1007/ s00395-016-0535-8.

[72] J.I. Hare, T. Lammers, M.B. Ashford, S. Puri, G. Storm, S.T. Barry, Challenges and strategies in anti-cancer nanomedicine development: An industry perspective, Adv. Drug Deliv. Rev. 108 (2017) 25-38. doi:10.1016/j.addr.2016.04.025. 


\section{APPENDIX: SUPPLEMENTARY DATA}

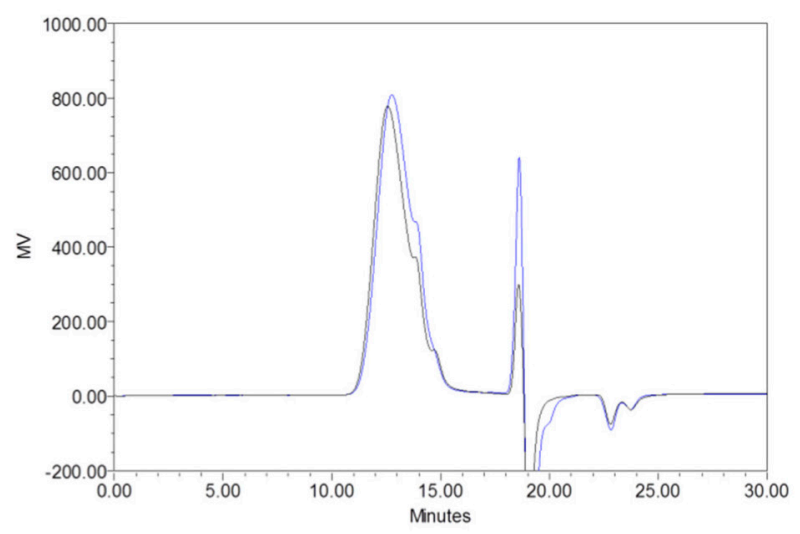

Supporting Figure 1। GPC chromatogram of mPEG-b-p(HPMA-Bz) copolymer (black) and the aminefunctionalized mPEG-b-p(HPMA-Bz-co-AEMAm) copolymer (blue).

Supporting Table 1। Characteristics of mPEG-b-p(HPMA-Bz) copolymer and mPEG-bp(HPMA-Bz- co-AEMAm) copolymer

\begin{tabular}{lccccc}
\hline $\begin{array}{l}\text { Block } \\
\text { copolymer } \\
\text { batch }\end{array}$ & $\mathrm{M}_{\mathrm{n}}{ }^{\mathrm{a}}$ & $\begin{array}{c}\text { Ratio } \\
\text { monomer } \\
\mathrm{M}^{\mathrm{a}}\end{array}$ & $\mathrm{M}_{\mathrm{n}}{ }^{\mathrm{b}}$ & $\mathrm{M}_{\mathrm{w}} \mathrm{b}^{\mathrm{b}}$ & PDI $^{\mathrm{b}}$ \\
\hline $\begin{array}{l}\text { mPEG- } b- \\
\mathrm{p} \text { (HPMA-Bz) }\end{array}$ & 16660 & 47 & 22170 & 36960 & 1.67 \\
$\begin{array}{l}\text { mPEG- } b- \\
\begin{array}{l}\text { p(HPMA-Bz- } \\
\text { co-AEMAm) }\end{array}\end{array}$ & 20930 & 65 & 21515 & 36270 & 1.69 \\
\hline
\end{tabular}

${ }^{\mathrm{a} C}$ Calculated from ${ }^{1} \mathrm{H}-\mathrm{NMR}$ spectra. ${ }^{\mathrm{b}}$ Calculated from GPC analysis.

$\mathrm{M}_{\mathrm{n}}$; number average molecular weight, $\mathrm{M}_{\mathrm{w}}$; weight average molecular weight, PDI; polydispersity index. 

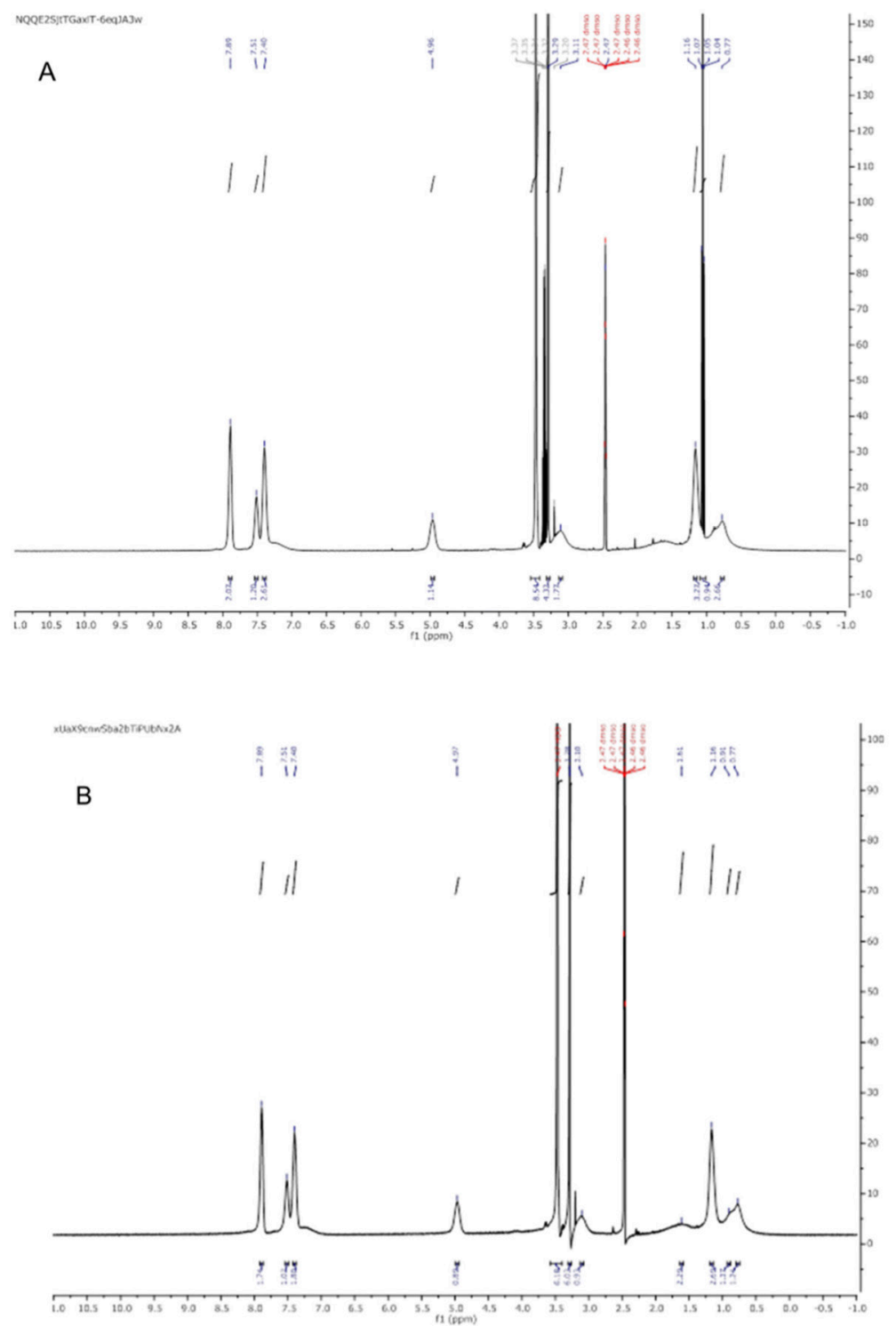

Supporting Figure $2 \mid{ }^{1}$ H-NMR spectra of (A) mPEG- $b$-p(HPMA-Bz) and (B) mPEG- $b$-p(HPMA-Bz-coAEMA, recorded in DMSO- $d_{6}$. 


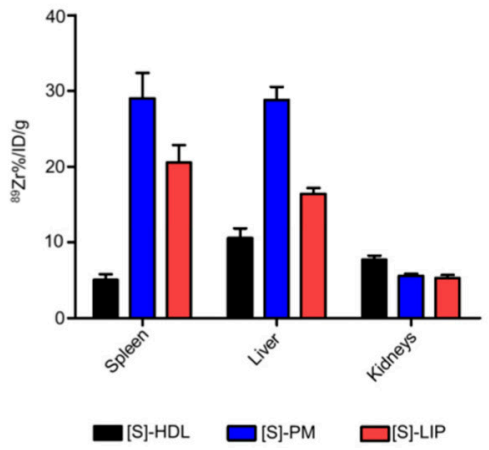

Supporting Figure $5 \mathrm{I}$ In vivo PET/CT quantification of [S]-nanomedicines in major organs $24 \mathrm{~h}$ after injection ( $\mathrm{n}=3$ per condition).

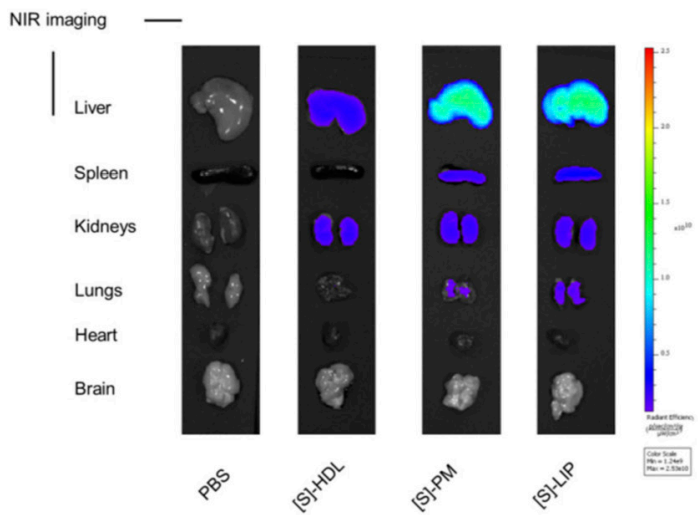

Supporting Figure 6| Near-infrared fluorescence (NIRF) images of Cy5.5 labeled [S]-nanomedicines accumulation, compared to PBS, in selected tissues $24 \mathrm{~h}$ after injection. [S]: simvastatin; HDL: high density lipoprotein; PM: polymeric micelles; LIP: liposomes. 
Supporting Table $2 \mid$ Antibodies used for flow cytometry and gating immune cells

\begin{tabular}{ccc}
\hline Antibody & Clone & Source \\
\hline FC Block (CD16/CD32) & $2.4 \mathrm{G} 2$ & BD Biosciences \\
CD45 & $30-\mathrm{F} 11$ & BioLegend \\
CD90.2 & $53-2.1$ & eBioscience \\
CD49b & DX5 & eBioscience \\
CD45R & RA3-6B2 & eBioscience \\
NK1.1 & PK136 & eBioscience \\
Ly-6G & 1 A8 & eBioscience \\
Ter-119 & TER-119 & eBioscience \\
CD11c & N418 & BioLegend \\
CD11b & M1/70 & BioLegend \\
Ly-6C & AL-21 & BD Biosciences \\
F4/80 & BM8 & BioLegend \\
\hline
\end{tabular}




\section{Blood}

A

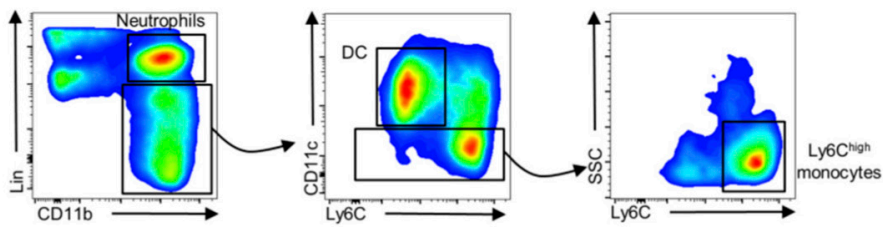

B

Neutrophils

DC

Ly6C ${ }^{\text {high }}$ monocytes
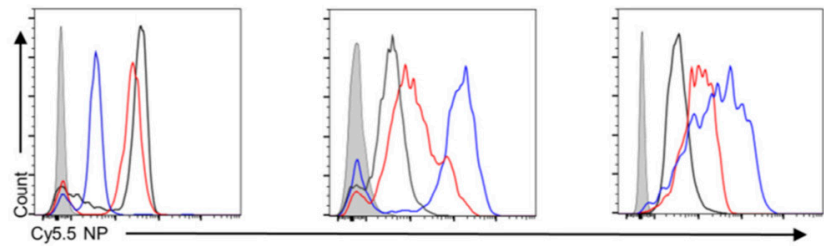

C Neutrophils

DC

Ly6C high monocytes
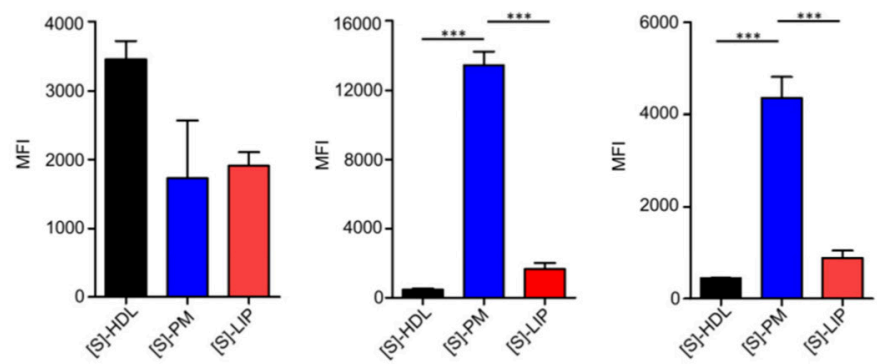

Supporting Figure 7/ Uptake of [S]-nanomedicines by myeloid immune cell cells in the blood of Apoe $e^{-1-}$ mice with advanced atherosclerosis. (A) The flow cytometry gating procedures to identify relevant immune cells in the blood. (B) Black ([S]-HDL), blue ([S]-PM), and red ([S]-LIP) histograms show the representative signal distribution of blood neutrophils, dendritic cells (DC), and Ly6C ${ }^{\text {high }}$ monocytes in the mice injected with nanomedicines compared with the cells from control animals injected with PBS (gray histogram on the left in each graph) (C) Quantification of mean fluorescence intensity (MFI) of each immune cells in the blood ( $\mathrm{n}=4$ per condition). Lin: lineage; DC: dendritic cells; Cy5.5: Cyanine5.5; [S]: simvastatin; HDL: high density lipoprotein; PM: polymeric micelles; LIP: liposomes. Data presented as mean \pm SEM. * $P \leq 0.05 ;{ }^{* *} P \leq 0.01 ;{ }^{* *} P \leq 0.001$. 
Spleen

A

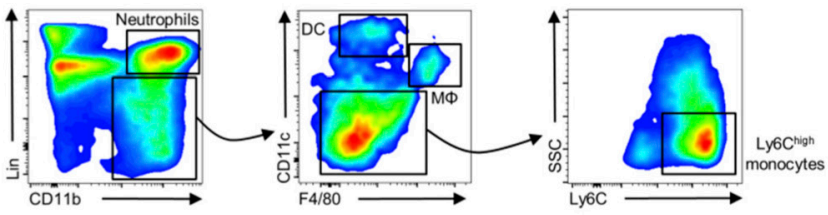

B

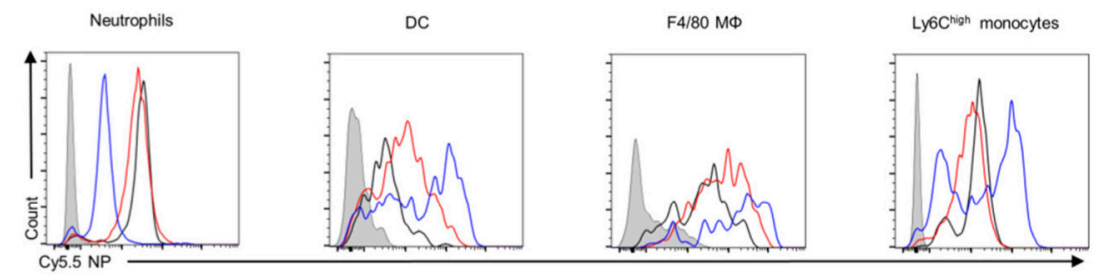

C

Neutrophils

DC

$\mathrm{F} 4 / 80 \mathrm{M} \Phi$

Ly6C high monocytes
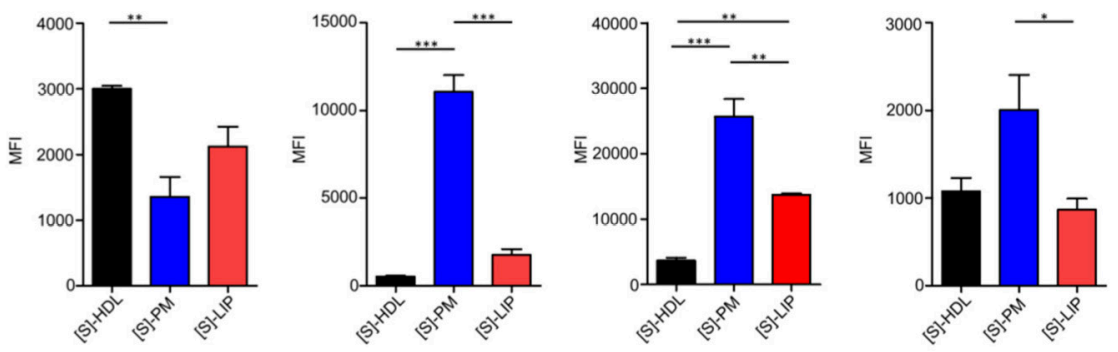

Supporting Figure 8| Uptake of [S]-nanomedicines by myeloid immune cell cells in the spleen of Apoe $e^{-I-}$ mice with advanced atherosclerosis. (A) The flow cytometry gating procedures to identify relevant immune cells in the spleen (B) Black ([S]-HDL), blue ([S]-PM), and red ([S]-LIP) histograms show the representative signal distribution of splenic neutrophils, dendritic cells (DC), macrophages $(\mathrm{M} \Phi)$, and Ly6 $\mathrm{C}^{\text {high }}$ monocytes in the mice injected with nanomedicines compared with the cells from control animals injected with PBS (gray histogram on the left in each graph) (C) Quantification of mean fluorescence intensity (MFI) of each immune cells in the spleen $(\mathrm{n}=4$ per condition). Lin: lineage; DC: dendritic cells; $(\mathrm{M} \Phi)$ : macrophages; [S]: simvastatin; HDL: high density lipoprotein; PM: polymeric micelles; LIP: liposomes. Data presented as mean \pm SEM. * $P \leq 0.05$; ${ }^{* *} P \leq 0.01$; ${ }^{* * *} P \leq 0.001$. 
A

Cholesterol

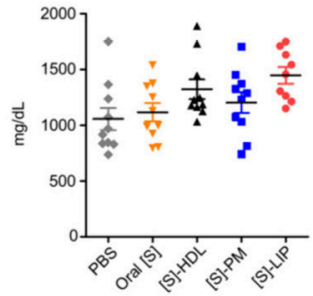

E

Alanine Transaminase

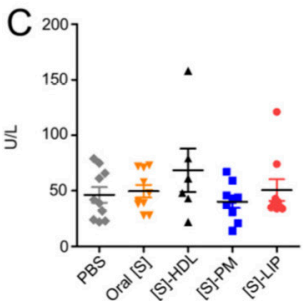

B Triglycerides

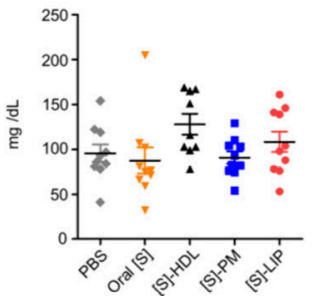

F Aspartate Transaminase

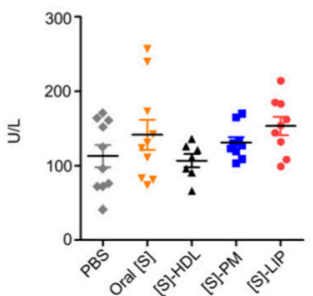

C

Glucose

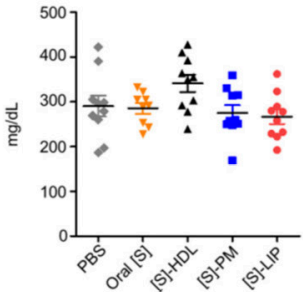

G

Blood Urea Nitrogen

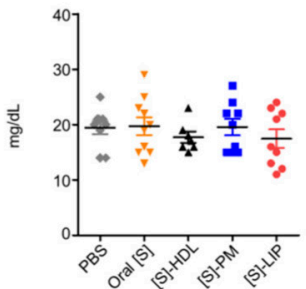

D Creatine Kinase

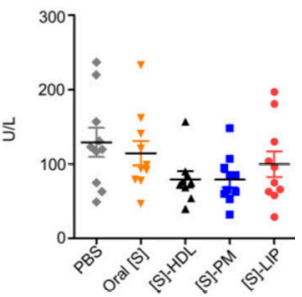

$\mathrm{H}$ Total Protein

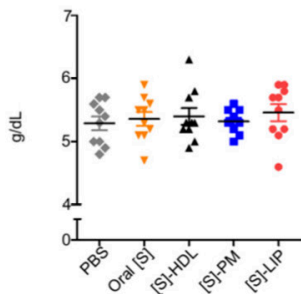

Supporting Figure 9| [S]-nanomedicine treatments do not cause metabolic or toxic effects. Mice treated with [S]-nanomedicines ( $\mathrm{n}=8-10$ treatment group; in Figure 5) did not develop any signs toxicity. Key biomarkers were measured in the plasma of the treated mice. Key metabolic markers: A) Cholesterol, B) Triglycerides, and C) Glucose. Key toxicity markers: D) Creatine Kinase, E) Alanine Transaminase (ALT), F) Aspartate Transaminase (AST), G) Blood Urea Nitrogen, and H) Total Protein. PBS: phosphatebuffered saline; [S]: simvastatin; HDL: high density lipoprotein; PM: polymeric micelles; LIP: liposomes Data presented as mean \pm SEM. No significant differences among the treatment groups were found. 



\section{CHAPTER 7}

\section{Hyaluronan Nanoparticles Selectively Target Plaque-Associated Macrophages and Improve Plaque Phenotype in Atherosclerosis*}

\section{ABSTRACT}

$\mathrm{H}$ yaluronan is a biologically active polymer, which can be formulated into nanoparticles. In our study, we aimed to probe atherosclerosis-associated they can ameiorate atherosleross. Hyaluronan nanoparticles (HA-NP) were prepared by reacting amine-functionalized oligomeric hyaluronan (HA) with cholanic ester, and labeled with a fluorescent or radioactive label. HA-NPs were characterized in vitro by several advanced microscopy methods. The targeting properties and biodistribution of HA-NPs were studied in Apoe $e^{-1-}$ mice, which received either fluorescent or radiolabeled HA-NPs and examined ex vivo by flow cytometry or nuclear techniques. Furthermore, three atherosclerotic rabbits received ${ }^{89} \mathrm{Zr}$-HA-NPs and were imaged by PET/MRI. The therapeutic effects of HA-NPs were studied in $A p o e^{-1-}$ mice, which received weekly doses of $50 \mathrm{mg} / \mathrm{kg}$ HA-NPs during a 12 week high-fat diet feeding period. Hydrated HA-NPs were ca. $90 \mathrm{~nm}$ in diameter and displayed very stable morphology under hydrolysis conditions. Flow cytometry revealed 6 to 40 -fold higher uptake of Cy7-HA-NPs by aortic macrophages compared to normal tissue macrophages. Interestingly, both local and systemic HANP-immune cell interactions significantly decreased over the disease progression. ${ }^{89} \mathrm{Zr}$-HA-NPs-induced radioactivity in atherosclerotic aortas was $30 \%$ higher than in wild-type controls. PET imaging of rabbits revealed 6-fold higher standardized uptake values compared to the muscle. The plaques of HA-NP-treated mice contained $30 \%$ less macrophages compared to control and free HA-treated group. In conclusion, we show favorable targeting properties of HA-NPs, which can be exploited for PET imaging of atherosclerosis-associated inflammation. Furthermore, we demonstrate the anti- inflammatory effects of HA-NPs in atherosclerosis. 


\section{INTRODUCTION}

Hyaluronan is a linear polymer consisting of $\mathrm{N}$-acetyl-glucosamine and a $\beta$-glucuronic acid, which displays distinctive physicochemical and biological properties [1]. As a key component of the extracellular matrix, it effectively lubricates and binds water, and - by interacting with several hyaluronan receptors - hyaluronan regulates cell adhesion, migration and proliferation [2]. During inflammation, the hyaluronan lining on vascular endothelium mediates immune cell rolling and extravasation, and - at the site of inflammation - the hyaluronic-rich microenvironment stimulates the tissue penetration by and division of immune cells [3]. In oncology, hyaluronan-CD44 receptor interactions have been proposed as key players in tumor progression and metastasis [4-7]. Notably, the biological activity of hyaluronan depends strongly on its degree of polymerization. Low- molecular weight hyaluronan oligomers have been shown to stimulate inflammation and angiogenesis,[8-12] whereas highly polymerized (multi) megadalton (MDa) hyaluronan acts as a suppressor of these processes [8-10,13-16].

The aforementioned properties, non-immunogenicity and cost-effective production of hyaluronan have propelled its application in biomedicine. At present, hyaluronan's local administration is applied in arthrology, ophthalmology and esthetic medicine,[17,18] but its systemic application is highly challenging due to rapid blood clearance, [19-22] and susceptibility to hydrolysis. In this respect, hyaluronan-based therapeutics might benefit from nanomedicine-based formulation strategies, which have shown their tunability for cellular interactions [23] and profound impact on the pharmacokinetic properties of various biomaterials $[24,25]$. Although hyaluronan has been deposited on the surface of either lipid or polymeric nanoparticles, studies exploiting the polymeric backbones of hyaluronan for nanoparticle assembly, which can be achieved by chemical modification of hyaluronan carboxyl groups, are scarce [26-32].

In our study, we exploit hyaluronan suprachemistry to formulate nanoparticles, which we propose for targeting of atherosclerosis-associated inflammation [33-36]. This is motivated by the critical role of hyaluronan-immune cell interactions during the inflammatory processes, [3] which drive the atherosclerosis progression and severe complications [37,38]. The diagnosis and treatment of high-risk patients still remains an unresolved problem [39,40]. Macrophages, the key propagators of atherosclerosis and primary phagocytes in atherosclerotic plaques, express several hyaluronanbinding receptors, including CD44, ICAM-1, LYVE-1, RHAMM and TLR-4 [41-44]. Notably, macrophages are highly dynamic cell population, which molecular and functional profile is strongly influenced by the tissue microenvironment [45,46]. The assessment of these phenotypic alternations may be of important predictive and prognostic value in atherosclerosis. 
In view of the rapid hyaluronan degradability, we extensively studied the morphological and chemical stability of nanoparticle-formulated hyaluronan. To this aim, we employed several advanced microscopy methods, including atomic force microscopy, cryo-scanning electron microscopy and super-resolution fluorescence microscopy. Flow cytometry allowed us to study the nature of the interactions between nanoparticulate hyaluronan and immune cells, and how the atherosclerotic plaque progression affects these interplays. Furthermore, we performed radiolabeling of HA-NPs with ${ }^{89} \mathrm{Zr}$ for quantitative evaluation of plaque targeting efficacy, clearance kinetics and biodistribution in atherosclerotic Apoe $e^{-1-}$ mice. The translational impact of ${ }^{89} \mathrm{Zr}$-HA-NPs was investigated by non-invasive imaging of atherosclerotic rabbits using fully integrated positron emission tomography and magnetic resonance imaging (PET/MRI) system. We also investigated the impact of hyaluronan assembly into nanoparticles on its biological activity, by probing the atheroprotective effects of HA-NPs.

\section{RESULTS AND DISCUSSION}

\subsection{Morphology of hyaluronan-based nanoparticles and the impact of hydrolysis}

To determine the morphological characteristics of hyaluronan-based nanoparticles (HA- NPs) and their stability under hydrolytic conditions, we applied several advanced microscopy methods (Figure 1A). Atomic force microscopy (AFM) revealed that the air- dried HA-NPs were spherical structures with a mean diameter of $32 \pm 0.5 \mathrm{~nm}$ (Figure 1AB, first panel). Interestingly, after hyaluronidase (HYAL) treatment, the nanoparticles were still highly abundant and their size changed only marginally, i.e., we observed them to be $26 \pm 5.1 \mathrm{~nm}$ in diameter. Cryo-scanning electron microscopy (cryo-SEM) of a snap-frozen sample of HA-NPs in buffered saline showed larger nanoparticles of $c a .80 \mathrm{~nm}$ in diameter (Figure1A, second panel). In the HYAL-treated samples, the nanoparticles were also readily present. Wet-mode environmental SEM (ESEM) showed larger aggregates of HA-NPs compared to all other methods, which was likely induced by the surface drying (Figure 1A, third panel). Interestingly, hydrolyzed HA-NPs appeared to retain more water, as deduced from their lower image contrast at higher vacuum. dSTORM analysis of surface-immobilized Cy5.5-labeled HA-NPs in aqueous conditions revealed the presence of fluorescent nanostructures with a median diameter of 90 nm (Figure 1A,B). HYAL treatment did not affect the nanoparticle size considerably. Notably, however, Cy5.5 blinking events appeared to be more disperse for HYALtreated nanoparticles. In contrast, dSTORM images of disulfo Cy5-COOH, which was used as a small-molecular weight control, showed diffusely distributed Cy5 molecules and only a small degree of molecule aggregation (Figure S1, supporting information). 
The hydrolytic stability of HA-NPs was established by dynamic light scattering (DLS). Interestingly, the mean hydrodynamic diameter increased from 100 to $125 \mathrm{~nm}$ after HYAL treatment (intensity-based) (Figure S2A, supporting information). Under both neutral and hydrolytic conditions, there was a small peak ( 10-20\%) of larger aggregates of $600-800 \mathrm{~nm}$. The zeta potential marginally changed from $-31.3 \pm 2.6$ to $-33.3 \pm 2.2 \mathrm{mV}$, indicative of a stable nanosuspension. In comparison, the hydrolysis of high-molecular weight HA resulted in a zeta potential drop from $-19.0 \pm 0.5$ to $-8.5 \pm 1.2 \mathrm{mV}$. The summary of quantitative parameters obtained for HA-NPs and HYAL-treated counterparts by different methods is displayed in Figure S2B.

HA-NPs and their hydrolysis products were further analyzed by the size exclusion chromatography (Figure S3, supporting information). The median retention time $\left(t_{R}\right)$ of HA-NPs was ca. 9 min, which indicates MDa structures. Hydrolyzed HANPs displayed rather small shift towards longer $t_{R^{\prime}}$ i.e, the median $t_{R}$ of about 12 min, $300 \mathrm{kDa}$. The size distribution was broader but, to large extent, overlapping with that of HA-NPs. In the case of free HA, our data indicate its aggregation into large structures, i.e, above the exclusion limit of 1.3 MDa. However, HA hydrolysis resulted in the formation of smaller HA products, ranging between $50 \mathrm{kDa}$ and several hundreds of Da. The aforementioned results show therefore different effects of hydrolysis on the NP-formulated HA compared to the linear counterpart. At the same time, we observed the intrinsic aggregation of oligomeric HA under the investigated conditions.

Moreover, to determine the morphology of in vivo-administered HA-NPs, we applied super resolution microscopy (dSTORM). In the Figure 1E, we show representative widefield and ASTORM images of aortic endothelium with the engulfed HA-NPs, which could be visualized and spatially resolved exclusively by the dSTORM technique. The observed NP morphology corresponded to that of the in vitro visualized counterparts (Figure $1 \mathrm{~F}$ ), indicative of HA-NP's stability under in vivo conditions. 

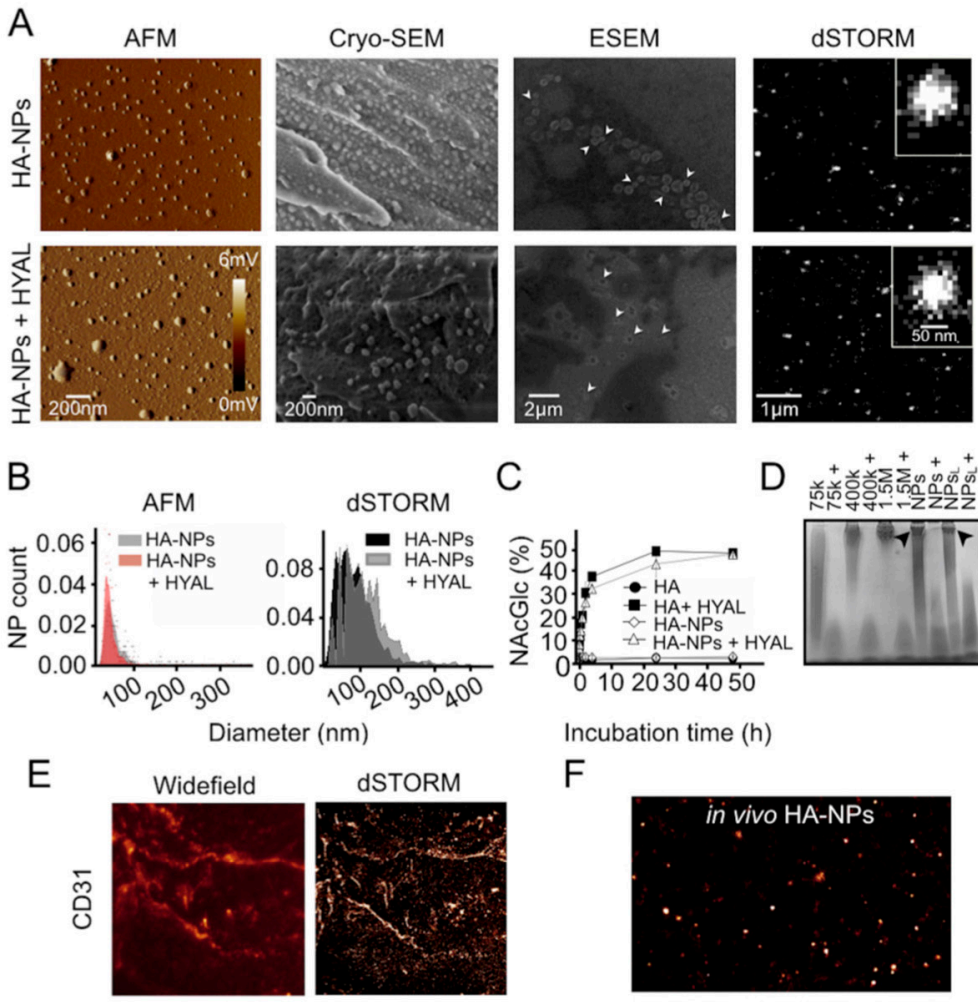

$\mathrm{F}$
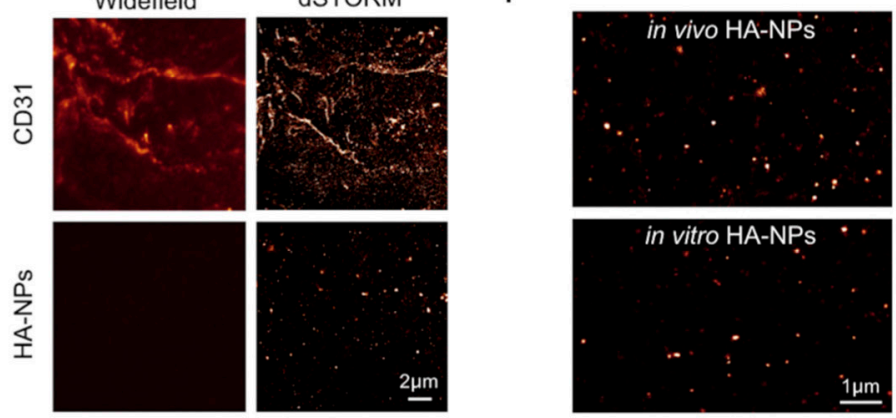

Figure 1| A) Hyaluronan nanoparticles (HA-NPs) (upper panel) and HA-NPs after hyaluronidase (HYAL) treatment (lower panel) visualized by atomic force microscopy (AFM) (first panel), cryo-scanning electron microscopy (Cryo-SEM) (second panel), environmental scanning electron microscopy (ESEM) (third panel) and direct stochastic optical reconstruction microscopy (dSTORM) (fourth panel). The scale bars shown in lower images refer also to the upper images. White arrowheads in ESEM images indicate the location of nanoparticles. The image insets in fourth panel show representative dSTORM images of a single nanoparticle at higher magnification. B) The normalized distribution of nanoparticle diameter obtained from AFM (left panel) and dSTORM (right panel) data. In the left panel, the size distribution of HA-NPs and hydrolyzed HA-NPs (HA-NPs + HYAL) is shown in grey and red, respectively, and, in the right panel, in black and grey, respectively. C) The amount of terminal N-acetlyglucosamine (NAcGlc) released from hyaluronan (HA) and HA-NPs during hydrolysis with HYAL, which is expressed as the percentage of total NAcGlc of substrate hyaluronan. D) Transmitted light image of stained polyacrylamide gel showing the migration spots of different molecular weight hyaluronan, i.e. $\sim 75 \mathrm{kDa}(75 \mathrm{k}), \sim 400 \mathrm{kDa}$ (400k) and $1.5 \mathrm{MDa}(1.5 \mathrm{M})$, non-labeled hyaluronan nanoparticles (NPs), fluorescently-labeled HANPs (NPsL), and their hydrolysis products. Symbol "+" indicates the hydrolyzed samples. Two black arrowheads point at the poorly migrating high-molecular weight hyaluronan in HA-NP samples. E) Widefiled (left panel) and two-color dSTORM (right panel) microscopy images of aortic endothelium of a mouse that received an injection of Cy5.5-HA-NPs $2 \mathrm{~h}$ before the sacrifice. Endothelial cells were stained with CD31-Alexa Fluor 555. F) Comparison of HA-NP morphology in a mouse aortic endothelium after the in vivo administration of Cy5.5-HA-NPs and on a coverglass after the in vitro seeding of Cy5.5-HANPs, assessed by dSTORM. Scale bars in lower images refer to those in the upper panel. 
The visualization of HA supramolecular structure in solution is very challenging due to its high hydrophilicity [47]. We demonstrated that HA-NPs can be successfully visualized in the hydrated state by cryo- and environmental SEM, and indirectly by dSTORM (Figure 1A). The 3 to 4 -fold higher NP size in aqueous conditions compared to the AFM-assessed dried form reflects the high water-binding properties of HA-NPs. Importantly, all the applied methods showed the limited impact of hydrolysis on the nanoparticle morphology and size distribution. Intriguingly, the release of terminal N-acetyl-glucosamine, which is a consequence of glycoside bond breakdown by HYAL, was only slightly lower for HA-NPs compared to free HA (Figure 1C). It needs to be stressed that both the HA crosslinking and conjugation of cholanic ester, which occur during NP assembly, do not affect the glycosidic bonds. This is in line with the unaltered HYAL activity. At the same time, the electrophoresis experiment revealed the presence of larger oligomeric products in HYAL- treated HA-NPs (NPs+ and NPsL + ) compared to free HA formulations (75k $+, 400 \mathrm{k}+$ and $1.5 \mathrm{M}+)$ (Figure 1D). This implies the presence of stable bonds in HANPs, which resisted the HYAL digestion. In the same experiment, the untreated HANPs displayed poor gel migration, corresponding to that of high-molecular weight hyaluronan $(\geq 1.5 \mathrm{MDa})$, but also contained some oligomeric HA. NPs and NPs+ therefore displayed a different migration profile, which is in contrast to the results by microscopy or DLS. Possibly, a high voltage used for electrophoresis (300V) was able to dissociate oligomeric components of NPs+, which does not occur under neutral conditions.

From the aforementioned experiments, we can conclude that NP-incorporated HA undergoes hydrolysis, which does not lead to NP disintegration. We believe that the breakdown of glycoside bonds leads to a decreased HA packing in HA-NPs, as suggested by DLS and dSTORM measurements. At the same time, HA cross-linking by enthylenediamine bridges as well as its covalent and amphiphilic interactions [48] with hydrophobic cholanic acid molecules prevented the nanoparticles from disintegrating.

\subsection{Selectivity of hyaluronan nanoparticles towards pro-inflammatory macrophage phenotype}

The targeting properties of HA-NPs were studied in vitro in bone marrowderived macrophages (BMDM), which were differentiated into several macrophage phenotypes using oxidized low-density lipoprotein (oxLDL), interleukin-4 (IL-4) or lipopolysaccharide (LPS) and interferon- $\gamma$ (INF $\gamma)$. The cellular uptake of HA-NPs was measured by flow cytometry, which is displayed in Figure 2A (upper panel). The LPS-stimulated macrophages, which represent the pro-inflammatory macrophage phenotype, displayed the highest uptake of HA-NPs. It was ca. three fold higher compared to the naive and anti- inflammatory (IL-4) macrophages. Interestingly, oxLDL, which is an important pro- atherogenic factor[49], significantly decreased 
the HA-NP uptake efficacy in pro- inflammatory macrophages by approximately $30 \%(p=0.0043)$. Similar experiments were performed for Cy5-labeled free HA and Cy5.5-labeled dextran-NPs (Figure S4A). In contrast to HA-NPs, relative differences between the investigated macrophage phenotypes were much less apparent after incubation with free HA or dextran-NPs. Interestingly, and in line with the HANP findings, oxLDL decreased the uptake of HA by $50 \%$. Although oxLDL is a recognized pro-atherogenic factor, its in vitro effects on macrophages are unclear, particularly in LPS-stimulated pro-inflammatory macrophages [50]. As oxLDL is a poor inducer of foam cell formation under in vitro conditions, the lipid loading of macrophages cannot underlay the observed drop in HA-NP uptake efficacy. Alternatively, oxLDL can act as a competitor for the scavenger receptors CD36 or SR-A, which might lead to a decreased HA-NP uptake.

Phagocytosis is the primary mechanism driving nanoparticle engulfment by macrophages [51,52]. However, we also considered an additive effect of HA-NP interaction with hyaluronan receptors, expressed by macrophages [41-44]. We therefore studied the effects of different stimuli on the expression of hyaluronan receptors in BMDM and how it relates to HA-NP-receptor interactions and overall HA-NP uptake. Flow cytometry analysis revealed the overexpression of almost all hyaluronan receptors, except for ICAM-1, by IL-4 stimulated (anti-inflammatory) macrophages (Figure S4A, right panel) (supporting information). This correlated well with the enhanced cell membrane association of HA-NP at $4{ }^{\circ} \mathrm{C}$, as visualized by confocal microscopy (Figure S4B). Notably, however, this enhanced hyaluronan receptor expression did not lead to more efficient HA-NP engulfment, as described above (Figure 4A, left panel). Furthermore, we performed competition experiments with free HA and CD44 antibody. Interestingly, only in the case of LPS- treated (pro-inflammatory) macrophages, the competition with free HA led to a significant decrease in the HA-NP uptake (Figure S4C). Anti-CD44 antibody had no or positive impact on HA-NP uptake (Figure S4C). It needs to be stressed that previous studies have shown that hyaluronan and CD44 antibodies can bind to different sites on the CD44 receptor.[53] Furthermore, CD44-mediated hyaluronan binding to macrophages requires activation of the receptor $[54,55]$. We also cannot exclude the interference of LPS contamination of anti-CD44 antibody in the latter experiment.

\subsection{Hyaluronan nanoparticles target plaque-associated macrophages in atherosclerotic mice}

The in vivo targeting efficacy and selectivity of HA-NPs was studied in Apoe ${ }^{-/-}$ mice, in which either early or advanced atherosclerosis was induced by a six (6w HFD) or twelve weeks high-fat diet (12w HFD), respectively. Flow cytometry on digested atherosclerotic aortas enabled single-cell analysis of immune cell populations and a quantitative comparison of the HA-NP uptake in these cells (Figure 2B). In both groups, aortic macrophages displayed the highest median fluorescence intensity 
per cell (MFI) generated by Cy7-HA-NPs compared to both splenic and bone marrow-resident macrophages (Figure 2B). These differences were however the most pronounced in the 6w HFD group, i.e. the uptake of HA-NPs by the aortic macrophages was 6- and 40-fold higher compared to splenic and bone marrowresident macrophages, respectively. In contrast, fluorescent poly(lactic-co-glycolic acid (PLGA)-NPs, used as a control nanoformulation, displayed considerably higher uptake in splenic macrophages compared to aortic counterparts (Figure S5A, supporting information). Among the aortic immune cell populations, macrophages were found to engulf some PLGA-NPs (Figure S5B), albeit at lower levels than HANPs, indicative of the latter platform's favorable atherosclerotic plaque inflammation targeting features. Interestingly, we found dramatic differences in HA-NP uptake between macrophages in early and advanced aortic lesions, i.e. MFI was $3060 \pm 836$ and $697 \pm 142$ a.u. (arbitrary units), respectively $(P=0.00003)$. These results coincide with our in vitro findings on the negative impact of oxLDL (Figure 2A) and suggest the occurrence of phenotypic changes in the macrophage population during disease progression. Early lesions are primarily composed of viable and freshly recruited macrophages, which display a high phagocytic activity. In contrast, the prolonged exposure to high cholesterol/lipid levels induces the macrophage lipid overload, and eventually cell apoptosis and necrosis. Other microenvironmental factors, such as oxLDL or reactive oxygen species, which concentrations are elevated in complex plaques, might have also negative effect on the macrophage activity [56]. A similar trend was observed for other immune cell populations in the aorta (Figure S6, supporting information) and, interestingly, in other tissues, including blood monocytes and splenic macrophages, which suggest a systemic effect of prolonged HFD. In line with our findings, the systemic immune effects of HFD have been described in the context of enhanced lipid loading of innate immune cells and their increased production [57]. We excluded the impact of instrumental error, by monitoring MFI for unstained and stained control samples (Figure S7, supporting information).

Furthermore, we investigated the relation between HA-NP uptake in aortic immune cell populations, the efficacy to bind free HA and the expression of CD44 receptor. In the early atherosclerosis group, we found a significant correlation between HA-NP uptake and both HA-binding efficacy and CD44 expression, with Spearman's correlation coefficients of 0.62 and 0.74 , respectively (both $P=0.0001$ ) (Figure 2C). In mice with advanced lesions, the aforementioned parameters did not correlate. This suggests that initially the hyaluronan receptor-HA-NP interactions might play a role in the overall HA-NP uptake, which deprives during the disease progression. 
A

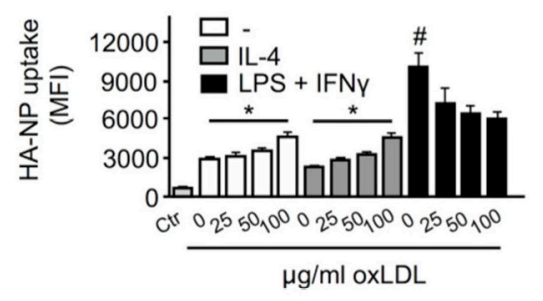

B

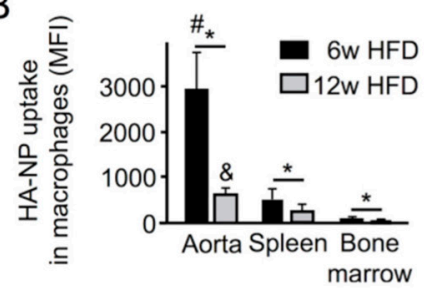

C
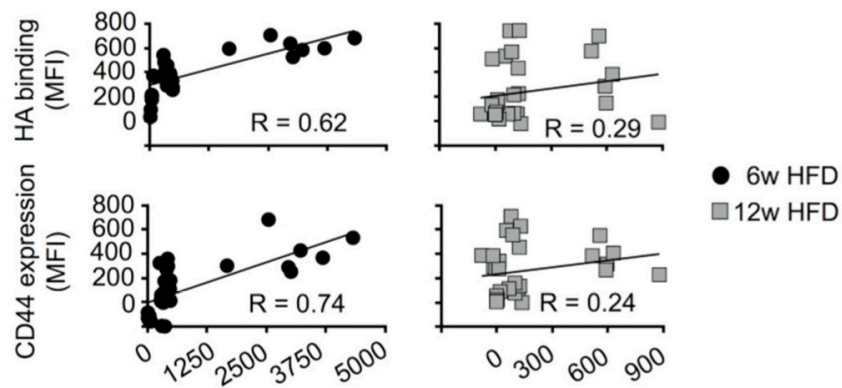

HA-NP uptake (MFI)

D

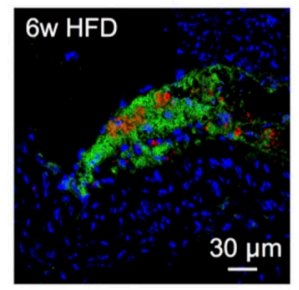

E

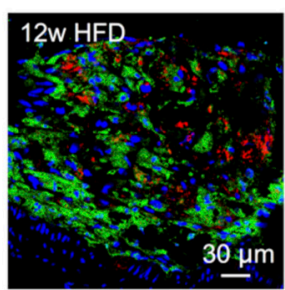

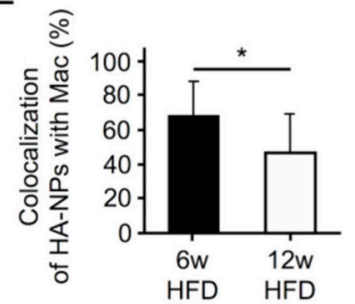

Figure 2 | A) Flow cytometry analysis of the cellular uptake of Cy5.5-labeled hyaluronan nanoparticles (HA-NPs) in different phenotypes of bone marrow-derived macrophages. The macrophages are divided into three main phenotypic groups: naive (-) (white bars), interleukin 4 (IL-4)-stimulated (grey bars), and lipopolysaccharide (LPS) and interferon $\gamma(\mathrm{INF} \gamma)$-stimulated (black bars). Moreover, three concentrations of oxidized low-density lipoprotein (oxLDL) were used for co-stimulation, i.e. 25, 50 and $100 \mu \mathrm{g} / \mathrm{ml}$. The cellular uptake of HA-NPs is expressed as the median fluorescence intensity per cell (MFI). Bars represent mean MFI/condition \pm SD (ns = 3). Symbol "*" indicates a significant difference and "\#" indicates significantly higher MFI compared to all other conditions at $\mathrm{p}<0.05$. B) The uptake efficacy of Cy7-HANPs in aortic, splenic and bone marrow macrophages measured by flow cytometry. Black and grey bars represent the data obtained for mice fed with a high fat diet for 6 (6w HFD) and 12 weeks (12w HFD), respectively. Symbol "*" indicates inter-group differences, whereas "\#" and " $\$$ " indicate significantly higher MFI compared to all other macrophage populations within 6w HFD and 12w HFD group, respectively, and at $p<0.05$. C) Scatter plots showing the relation between the uptake efficacy of HA-NPs and free hyaluronan (HA)-binding (upper panel), and expression of CD44 receptor (lower panel). All data were obtained by flow cytometry and are expressed as the median fluorescence intensity per cell (MFI). The correlations were investigated for pooled aortic leukocyte populations, i.e, macrophages, Ly $6 \mathrm{c}^{\text {high }}$ and Ly $6 \mathrm{c}^{\text {low }}$ monocytes and neutrophils, in 6w HFD (left panel, black circles) and 12w HFD mice (right panel, grey squares). The black lines serve as guides for the eye. The correlation coefficient, $R$, was obtained from the non-parametric Spearman's correlation test. D) Representative confocal microcopy images of aortic lesions detected in 6w HFD (left image) and 12wHFD group (right image). The Cy5.5-HA-NPs are shown in red, CD68 staining of macrophages is shown in green and cell nuclei are blue. E) Selectivity of HA-NPs towards plaque-associated macrophages expressed as the percentage of HA-NP-positive area that colocalizes with CD68-positive macrophage area. Bars represent mean \pm SD. Symbol "*» represent the significant difference at $\mathrm{p}<0.05$. 
Confocal microscopy analysis of mouse aortic arches confirmed the efficient uptake of HA-NPs in both early and advanced lesions (Figure 2D). However, based on the fraction of HA-NP-positive plaque area that colocalized with macrophage staining, we found a higher selectivity of HA-NPs towards macrophages in early atherosclerotic plaques, which was $67 \pm 19 \%$ (Figure 2E). In advanced lesions, the macrophage selectivity was $46 \pm 20 \%$, which means that only this fraction of the total accumulated HA-NPs was engulfed by plaque macrophages, and implies a considerably higher off-target accumulation of HA-NPs. We believe that this might due to the impaired phagocytic activity of macrophages in advanced lesions (Figure2B) and the presence of necrotic sites, which may facilitate passive accumulation of NPs [58,59].

$\mathrm{NP}$ accumulation mechanisms in atherosclerotic plaques are rather scarcely explored [60]. Considering very limited neovascularization in mouse lesions, i.e, the microvascular fraction of $c a .1 \%$ based on our histological evaluation, the dysfunctional host endothelium seems to be a major player in NP plaque retention. This can occur via leaky endothelial junctions, which are formed in the response to pro-atherogenic stimuli and a high tension induced by the subendothelial plaque deposition $[49,61,62]$. The leaky endothelial junctions have been proposed as a primary pathway of LDL accumulation in the vessel wall [40]. Alternatively, NPs might enter the plaques via the transcellular migration pathway, by exploiting intracellular vesicles [63]. As shown in Figure 1E,F, we could detect diffusely distributed HA-NPs in aortic endothelial cells. The subendothelial matrix is known to undergo degradation by the infiltrating macrophages and therefore it does not create a physical barrier for NPs.

\subsection{Atherosclerosis-targeting, circulation kinetics, and biodistribution of ${ }^{89} \mathrm{Zr}-\mathrm{HA}-\mathrm{NPs}$ in mice}

The accumulation of ${ }^{89} \mathrm{Zr}$-HA-NPsin mouse atheroscleroticlesions was determined by autoradiography and gamma counting $24 \mathrm{~h}$ post-administration (Figure 3A,B). Both early and advanced atherosclerotic aortas displayed focal accumulation of radioactive material at typical sites of atherosclerotic plaque formation, i.e. aortic root, arch and renal artery branching (Figure 3A). In contrast, the aortas of wild-type mice displayed low and homogeneously distributed radioactivity. Radioactivity quantification of excised aortas by gamma counting revealed significantly higher percentage of injected dose (\%ID) of ${ }^{89} \mathrm{Zr}$ - HA-NPs in atherosclerotic mice compared to their normal counterparts of approximately $30 \%$ (Figure 3B). Interestingly, despite a dramatic difference in disease stage, early lesions were equally well detectable as advanced plaques, and displayed similar radioactivity concentrations. This was confirmed by ex vivo fluorescence imaging of aortas after injection of Cy5.5-HA-NPs (Figure S8, supporting information). This may be an important finding in view of the present challenges in the detection of early atherosclerosis.[64] In comparison, the 
previous studies on 18F-FDG-PET, a method that probes cell metabolism, have shown an increasing metabolic activity associated with the atherosclerosis progression, which suggests an increasing inflammatory burden.[65,66] We believe that these discrepancies originate from the fact of assessing different aspects of macrophage activity. While macrophage glycolysis was shown to increase significantly in advanced lesions[67], their phagocytic activity seems to undergo regression, as demonstrated by our FACS data (Figure 2A,B).

Furthermore, we studied the clearance kinetics of ${ }^{89} \mathrm{Zr}$-HA-NPs by gamma counting of blood samples. In contrast to the previously reported fast clearance of radiolabeled hyaluronan [19-21], ${ }^{89} \mathrm{Zr}-\mathrm{HA}-\mathrm{NPs}$ displayed biexpotential decay kinetics with a short blood half-life $\left(t_{1 / 2}, \alpha\right)$ of approximately $0.5 \mathrm{~h}$ and long $t_{1 / 2}, \beta$ of $9 \mathrm{~h}$ (Figure $3 \mathrm{C}$ ). This might be due to the limited sensitivity of HA-NPs to the blood serum HYAL and/or decreased recognition by the hyaluronic acid receptor for endocytosis (HARE) in the liver, which mediates systemic clearance of hyaluronan from the vascular and lymphatic circulation [68]. Nevertheless, we should also not rule out the impact of a relatively high HA-NP dose, which might lead to clearance saturation [20]. The quantitative determination of ${ }^{89} \mathrm{Zr}-\mathrm{HA}-\mathrm{NP}$ biodistribution in mice showed that the liver and spleen are the primary clearance organs of HA-NPs, containing between 10 and $20 \% \mathrm{ID} / \mathrm{g}$ (Figure S9, supporting information). The heart, which is an important organ in context of human coronary atherosclerosis, displayed significantly lower $\% \mathrm{ID} / \mathrm{g}$ compared to the aorta. This suggests a potentially high target-to-background signal for PET imaging of coronary disease, which cannot be achieved by the clinically applied 18F-FDG-PET since fluorodeoxyglucose is avidly taken up by myocardial cells [69]. In our study, we assume the in vivo integrity of HA-NPs and a fluorescent or radioactive label, which is based on the covalent conjugation and strong in vitro data on the nanoparticle stability. Nevertheless, we cannot reject the possibility of partial nanoparticle disintegration, which can occur under the in vivo conditions [70].

\subsection{PET imaging of rabbit atherosclerosis with ${ }^{89} \mathrm{Zr}$ - HA-NPs}

Subsequently, we investigated the performance of ${ }^{89} \mathrm{Zr}$-HA-NP for PET imaging of atherosclerosis. Three rabbits were dynamically scanned on a PET/MRI scanner for $2 \mathrm{~h}$ after ${ }^{89} \mathrm{Zr}$-HA-NP injection, as well as statically at $12 \mathrm{~h}$ and $24 \mathrm{~h}$ postadministration. Representative PET/MRI fusion images are displayed in Figure 3D, on which we non- invasively monitored the circulation kinetics (Figure 3E) and time-dependent biodistribution (Figure $3 \mathrm{~F}$ ) of ${ }^{89} \mathrm{Zr}$-HA-NPs. The initially high aortic PET signal, which was generated by the circulating ${ }^{89} \mathrm{Zr}-\mathrm{HA}-\mathrm{NPs}$, decreased over time (Figure 3D,E). At $12 \mathrm{~h}$ post-administration, the aorta showed a relatively low but detectable PET signal, with some hot spots localized in the abdominal aorta (Figure 3G). The maximum standardized uptake values (SUVmax) were six fold 
higher for the aorta compared to the skeletal muscle $(p=0.03)$ (Figure 3G). The signal remained constant until $24 \mathrm{~h}$ post-administration. The clearance kinetics derived from PET images afforded a $t_{1 / 2}$ of approximately $3 \mathrm{~h}$ (Figure $3 \mathrm{~F}$ ). With respect to the biodistribution, assessed at $24 \mathrm{~h}$ post-administration by gamma counting, ${ }^{89} \mathrm{Zr}$ HA-NPs reached the highest concentration of $c a .0 .05 \% \mathrm{ID} / \mathrm{g}$ in the liver, spleen, kidney and lungs, but considering the total organ mass, the liver and spleen were the primary clearance organs (Figure $3 \mathrm{H}$ ). Similar to the mouse data, the heart tissue displayed very low radioactivity $(\sim 0.002 \% \mathrm{ID} / \mathrm{g})$, which is desirable in context of the coronary atherosclerosis imaging. The confocal microscopy analysis of rabbit aortas confirmed the accumulation of Cy5.5-HA-NPs (showed in red) in the abdominal aorta, which underwent the balloon injury, and no detectable NP fluorescence in the untreated thoracic aorta (Figure 3I). In the abdominal aorta, HA-NPs were localized in macrophages-rich areas and were internalized by aortic macrophages (Figure 3I, lower image panel).

The aforementioned results underline the translational aspect of our study. We applied ${ }^{89} \mathrm{Zr}$-deferoxamine (DFO)-labeling strategy, which assures an excellent match between nanoparticle biological half-life and ${ }^{89} \mathrm{Zr}$ physical half-life[71], and which we previously demonstrated in lipid- and polymer-based nanoparticle systems.[72,73] We showed that HA-NPs can be effectively labeled with ${ }^{89} \mathrm{Zr}$-DFO and enable the detection of atherosclerosis in two animal models, also at the early disease stage. The imaging studies that exploited hyaluronan-mediated targeting of atherosclerosis are scarce.[74-76] Previously, only Lee et al.[76] based their nanoformulation on HA and proposed for the fluorescence imaging of atherosclerosis. We, however, provide in-depth analysis of the underlying HA-NP-immune cell interactions and a strong translational aspect by employing non-invasive PET/MRI.

Our study shows also some interspecies differences between the mice and rabbits regarding HA-NP kinetics and biodistribution. In mice, ${ }^{89} \mathrm{Zr}-\mathrm{HA}-\mathrm{NPs}$ displayed biexponential decay kinetics, which has been previously attributed to the nanoparticle size heterogeneity, i.e., larger nanoparticles are more rapidly cleared from the circulation compared to smaller counterparts [77]. Moreover, a different clearance mechanism needs to be considered. Our FACS data indicate a growing involvement of blood phagocytes in the clearance of HA-NPs over time post-administration, as shown in the Figure S10A (supporting information) in blood samples taken $15 \mathrm{~min}$ and $2 \mathrm{~h}$ post-injection. Furthermore, we observed a shift in the monocyte population towards Ly6 $\mathrm{c}^{\text {high }}$ phenotype (Figure S10B). Possibly, the fast clearance phase is driven by the nanoparticle filtration by reticuloendothelial system, while in the slow phase, HA-NPs might be predominantly associated with the blood circulating phagocytes. In rabbits, displaying monoexponential clearance kinetics of ${ }^{89} \mathrm{Zr}-\mathrm{HA}-\mathrm{NPs}$, these mechanisms were not investigated. 
A

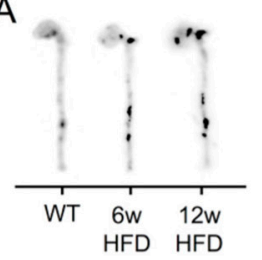

D

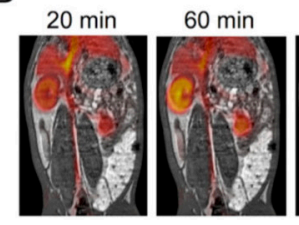

F

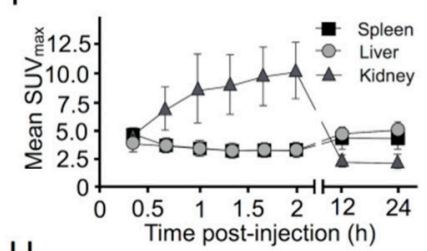

$\mathrm{H}$

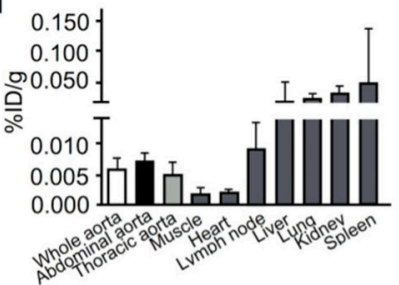

B

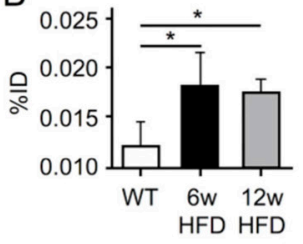

C

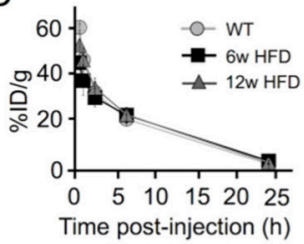

E

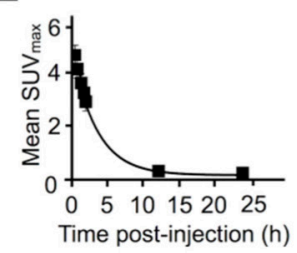

G
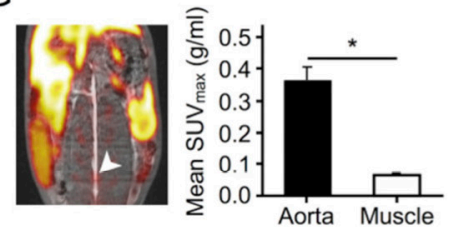

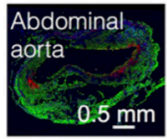

Thoracic aorta
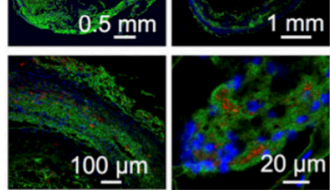

Figure $3 \mid$ A) Autoradiography images of aortas excised from a wild-type mouse (WT, left) and two atherosclerotic mice that were on a high fat diet for either 6 weeks ( $6 \mathrm{w} \mathrm{HFD,} \mathrm{middle)} \mathrm{or} 12$ weeks (12w HFD, right). $24 \mathrm{~h}$ before the sacrifice, the mice received intravenous injection of ${ }^{89} \mathrm{Zr}$-HA-NPs. In the images, dark spots indicate higher radioactivity deposition. B) The comparison between the radioactivity levels in healthy aortas (WT, white bar) and aortas with early (6w HFD, black bars) or advanced atherosclerosis (12w HFD, grey bars). The radioactivity was measured for the entire aorta by gamma counting $24 \mathrm{~h}$ after i.v. administration of ${ }^{89} \mathrm{Zr}$-HA-NPs and it is expressed as the percentage of injected dose (\%ID). C) Blood clearance kinetics of ${ }^{89} \mathrm{Zr}$-HA-NPs determined by gamma counting in blood samples probed at different timepoints after NP injection. The data were obtained for the abovementioned mouse groups and are presented as the mean $\pm \mathrm{SD}$ of $\% \mathrm{ID}$ per gram of blood $(\% \mathrm{ID} / \mathrm{g})$ over time. D) Coronal PET/ MRI fusion images of an atherosclerotic rabbit, showing the organ radioactivity distribution at different timepoints after intravenous injection of ${ }^{89} \mathrm{Zr}$-HA-NPs. E) Clearance kinetics of ${ }^{89} \mathrm{Zr}$-HA-NPs determined non-invasively in rabbits by measuring standardized uptake values (SUV) in the aortic blood. F) The time- dependent biodistribution of ${ }^{89} \mathrm{Zr}-\mathrm{HA}-\mathrm{NPs}$ determined by dynamic $(20 \mathrm{~min}-2 \mathrm{~h}$ ) and static (12 $\mathrm{h}$ and $24 \mathrm{~h}$ ) PET imaging in the spleen (black squares), liver (grey circle) and kidney (grey triangles). G) Left panel displays a representative PET/MRI fusion image of an atherosclerotic rabbit $12 \mathrm{~h}$ after the administration of ${ }^{89} \mathrm{Zr}$-HA-NPs. PET signal hot spot can be observed in the abdominal aorta (white arrowhead). In right panel, the bar chart shows the difference between SUV of the aorta and skeletal muscle at $12 \mathrm{~h}$ post-injection. H) Biodistribution of ${ }^{89} \mathrm{Zr}-\mathrm{HA}-\mathrm{NPs}$ in different rabbit organs quantified ex vivo by gamma counting and presented as $\% \mathrm{ID} / \mathrm{g}$ of tissue. I) Confocal microscopy images of the abdominal (upper left) and thoracic aorta (upper right) from a rabbit that was co-injected with both ${ }^{89} \mathrm{Zr}$ HA-NPs and Cy5.5-HA-NPs. The Cy5.5-HA-NPs are displayed in red, RAM-11 staining of macrophages is shown in green and cell nuclei are blue. In the lower panel, higher magnification images of aortic aorta show the engulfed HA-NPs by macrophages. In all bar charts, bars represent mean \pm SD and symbol "** indicates the significant difference at $p<0.05$. 
With respect to the biodistribution, we report very different retention values of a total dose per gram of mouse and rabbit organ. These differences originate from the fact that we normalize the organ radioactivity to the total injected dose and organ weight. However, the organ weights of a mouse and New Zealand White rabbit differ dramatically e.g., for the liver, it is $\sim 1-1.5 \mathrm{~g}$ and $\sim 140-150 \mathrm{~g}$, respectively. Therefore, $10 \% \mathrm{ID} / \mathrm{g}$ of a mouse tissue represents practically a full organ retention, while 0.05 $\% \mathrm{ID} / \mathrm{g}$ of a rabbit liver represents a dose retention in only small fraction of this organ. Please note that the relative differences between the organs' radioactivity are rather similar in both mice and rabbits.

\subsection{Hyaluronan nanoparticles improve the atherosclerotic plaque phenotype in atherosclerotic mice}

In view of the previously reported anti-inflammatory activity of high-molecular weight HA [10], we hypothesized that HA-NPs, composed of mega Dalton HA aggregates, may also exert anti-inflammatory effects. To test this hypothesis, we studied the effects of HA- NPs on atherosclerosis in Apoe $e^{-1-}$ mice, which received weekly injections of HA-NPs for 12 weeks. The effects were compared to free $75 \mathrm{kDa}$ HA (free HA), which served as a substrate for the nanoparticle formulation, and PBS (control).

Histological evaluation of aortic roots involved the assessment of mean plaque area and plaque composition (macrophages and collagen) (Figure 4A). The HANP-treated mice displayed the most favorable plaque phenotype regarding all the aforementioned parameters. The lesions were significantly smaller compared to the control group and contained approximately $30 \%$ fewer macrophages compared to both the control and free HA-treated mice. Moreover, they displayed 30-40\% higher collagen content, which is an important factor for plaque stability [78]. The anti-inflammatory effects of HA-NPs were confirmed by flow cytometry analysis of aortic arches (Figure 4B) and by CD45 staining of aortic roots (Figure S11, supporting information). Both methods revealed $\sim 30-50 \%$ lower number of immune cells in the HA-NP-treated mice compared to both free HA-treated and control mice. At the same time, we found no systemic immune effects of HA-NPs, as deduced from the analysis of blood, spleen and bone marrow (Figure S12, supporting information). 
A
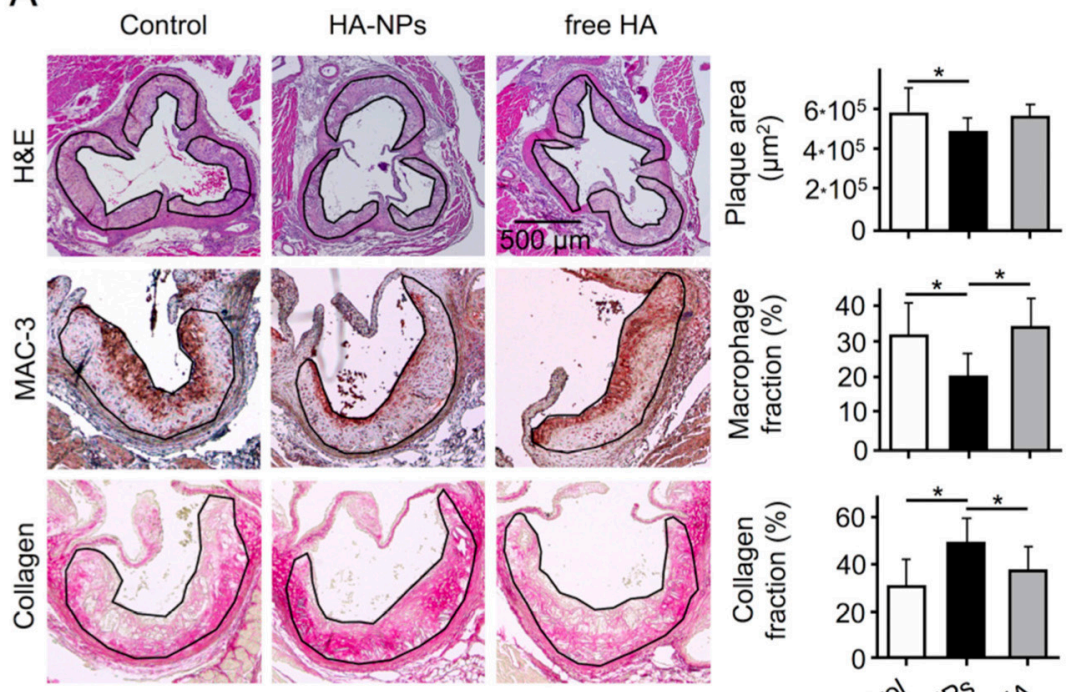

B
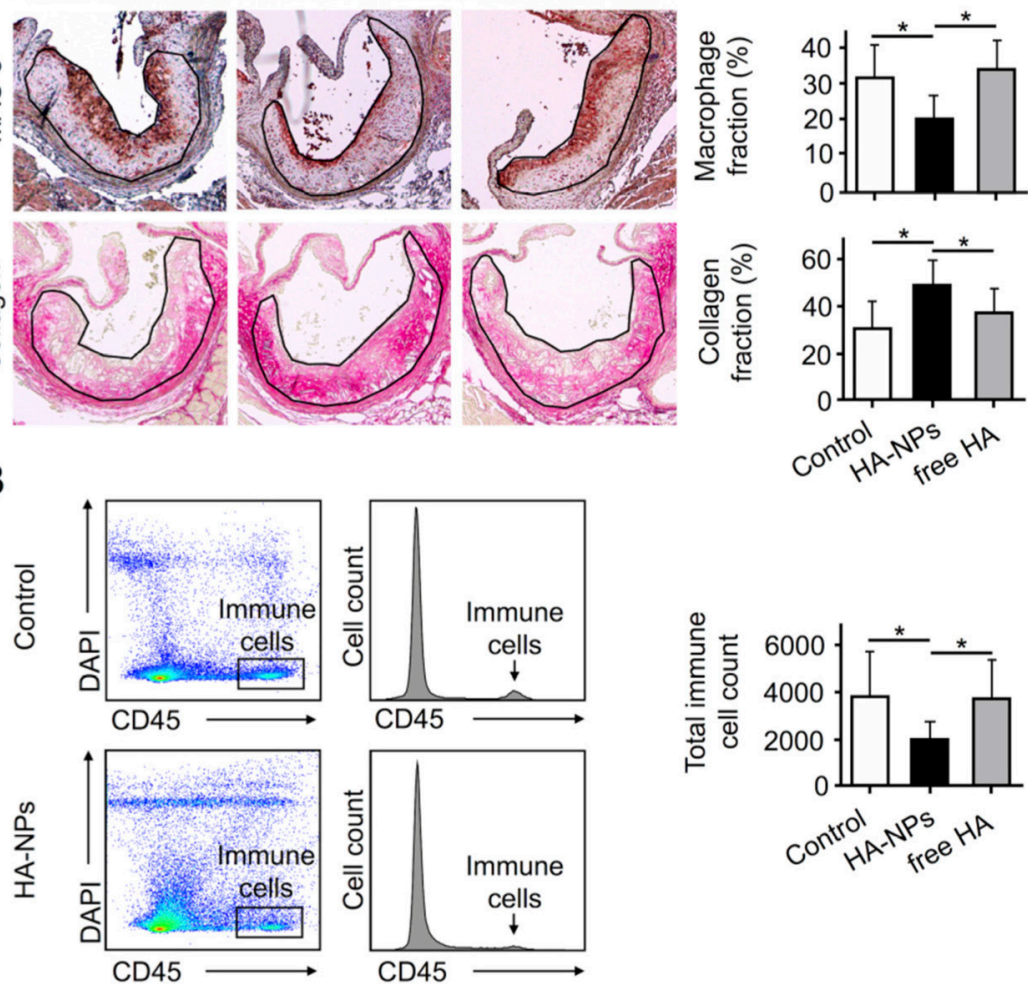

Figure 4 I A) Representative images of aortic roots from mice that received either PBS (Control, left image panel), HA-NPs (middle image panel) or free HA (right image panel) during a 12-week high-fat feeding period. The sections were stained with hematoxylin and eosin (H\&E), macrophage-specific antibody (MAC-3) or sirius red (collagen). Scale bar in upper right image refers to all H\&E-stained sections. Bar charts display the mean plaque area (top), percentage of plaque area containing macrophages (middle) and collagen (bottom) in the aforementioned treatment groups. B) Flow cytometry analysis of aortic arches of the treated mice. Left panel shows representative cell scatter plots and histograms obtained for a Control (upper panel) and HA-NPs-treated mouse (lower panel). The immune cells are defined as CD45-positive cells. Bar chart compares the total immune cell count in the Control, HA-NPs- and free HA-treated mice. In all bar charts, bars represent mean \pm SD and symbol "*" indicates the significant difference at $\mathrm{p}<0.05$. 
We show that nanoparticle-formulated hyaluronan exerted anti-inflammatory activity, which was previously attributed exclusively to high-molecular weight linear hyaluronan (HMW-HA) [10]. While the application of high viscosity HMW-HA in treatment of atherosclerosis is limited by the necessity of local administration[79], our formulation displays favorable blood circulation kinetics (Figure 3C,E) and selectivity towards pro- inflammatory/plaque-associated macrophages (Figure 2A,B). Previously developed HA- containing NPs induced therapeutic effects via other key-acting therapeutic agents, while exploiting HA as a targeting moiety $[29,80]$. A possible mechanism of HA-NP atheroprotective activity involves a direct interaction of HA-NPs with macrophages / monocytes, which might lead to the downregulation of inflammatory response. This has been previously described for HMW-HA [10] and, in our study, it is implied by the reduced immune cell infiltrates and higher collagen content (Figure 4). In line with this hypothesis, our in vitro study in bone marrow-derived macrophages (BMDMs) showed that a high concentration of HA-NPs significantly inhibited production of nitric oxide (NO) and tumor necrosis factor (TNF), the key pro-inflammatory mediators (Figure S13, supporting information). Similar trend was observed for the pro-inflammatory interleukins IL-6 and IL-12. In contrast, oligomeric HA had no significant effect on the expression on NO or TNF, while it significantly increased the IL-6 and IL-12 production. Interestingly, we also observed a significant stimulatory effect of HA-NPs on the expression of arginase-1 gene (Arg-1), which is considered as an important marker of pro-fibrotic macrophages and its overexpression may contribute to the atherosclerotic plaque stability. Concurrently, we anticipate a potential impact of long circulating HA-NPs on the vascular glycocalyx, which is predominantly composed of hyaluronan [81]. HA-NPs might serve as a circuiting reservoir of hyaluronan and be "built into" the glycocalyx, which, in consequence, might limit the immune cell infiltration into the lesion site.

\section{CONCLUSIONS}

We have developed highly biocompatible HA-NPs as a platform for imaging and therapy of atherosclerosis. In our nanoformulation, hyaluronan serves as both the primary building block and the targeting/bioactive molecule, which yields high morphological stability of HA-NPs under hydrolytic conditions. HA-NPs are preferentially taken up by pro-inflammatory macrophages in vitro and display high selectivity towards atherosclerotic plaque-associated macrophages in mice. Interestingly, we found that the efficacy of macrophage-HA-NP interactions is strongly dependent on the disease stage, which we attribute to phenotypic dynamics of macrophage population. Furthermore, we showed that radiolabeled HA-NPs target mouse atherosclerotic lesions and enable PET imaging of atherosclerosis in rabbits. Finally, we demonstrated that HA-NPs exert atheroprotective effects by decreasing the immune cell infiltration in aortic lesions. 


\section{MATERIALS AND METHODS}

\subsection{Hyaluronan nanoparticle preparation}

Hyaluronan (HA) (66-99 kDa) was purchased from Lifecore Biomedical (Chaska, MN USA). $200 \mathrm{mg}$ of HA was dissolved in $10 \mathrm{ml}$ of 2-(N-morpholino) ethanesulfonic acid (MES) buffer (Sigma-Aldrich, Zwijndrecht, Netherlands) pH 6 and activated with $92 \mathrm{mg}$ of 1-ethyl-3-(3-dimethylaminopropyl)carbodiimide hydrochloride (EDC) (Thermo Fisher Scientific, Ermelo, Netherlands) and $104 \mathrm{mg}$ of N-hydroxysulfosuccinimide (sulfo NHS) (Thermo Fisher Scientific). EDC and sulfo NHS were dissolved in $200 \mu$ l of MES buffer $\mathrm{pH} 6$ and added sequentially to HA solution during stirring, with 15 min interval. Subsequently, $\mathrm{pH}$ was adjusted up to 7 using $5 \mathrm{M} \mathrm{NaOH}, 128 \mu \mathrm{l}$ of ethylenediamine (Sigma-Aldrich) was added and $\mathrm{pH}$ was rapidly adjusted down to 7 using $5 \mathrm{M} \mathrm{HCl}$. The reaction mixture was stirred at room temperature overnight. The amine-functionalized HA (HA-NH2) was purified by dialysis against water (Spectra/Por 2 membrane, 12-14kDa cut- off, Spectrum Labs, Breda, The Netherlands) and 4 series of ethanol precipitation (3 volumes ethanol: 1 volume water). To induce HA precipitation, $\mathrm{NaCl}$ (Sigma-Aldrich) was added to the post-dialysis water solution of HA to obtain a final concentration of 5 $\% \mathrm{NaCl}$. Subsequently, 3 parts of ethanol was added and the mixture was shaken vigorously until white precipitate appeared, which was pelleted by centrifugation at $4000 \mathrm{rpm}$ for $20 \mathrm{~min}$. The supernatant was removed by careful pipetting and HANH2-containing pellet was redissolved in ultrapure water. For subsequent series of ethanol precipitation, $\mathrm{NaCl}$ was added and process was repeated. After the fourth ethanol precipitation, the HA pellet was dissolved in ultrapure water, dialyzed against water for overnight and freeze-dried (Zirbus VaCo2-II, Zirbus, Bad Grund Germany). Dry lyophilizate was weighted (the average yield was 70-80\%) and analyzed with respect to the amine content. The number of primary amines per HA molecule was 6-12, as determined by the 2,4,6-trinitrobenzene sulfonic acid assay (Thermo Fisher Scientific).

In the second step, cholanic NHS ester was prepared by reacting $30 \mathrm{mg}$ of cholanic acid with $17 \mathrm{mg}$ of $\mathrm{N}, \mathrm{N}^{\prime}$-dicyclohexylcarbodiimide (i.e, $83 \mu \mathrm{l}$ of $1 \mathrm{M}$ solution) (Thermo Fisher Scientific) and $13 \mathrm{mg}$ of $\mathrm{N}$-hydroxysuccinimide in dry dimethylformamide (DMF) (all aforementioned chemicals were from Sigma-Aldrich). First, cholanlic acid was dissolved in $400 \mu \mathrm{l}$ of DMF and $83 \mu \mathrm{l}$ of $\mathrm{N}, \mathrm{N}^{\prime}$-dicyclohexylcarbodiimide solution was added, which was followed by vortexing. Next, N-hydroxysuccinimide was dissolved in $\sim 50 \mu \mathrm{l}$ of DMF and added to the reaction mixture and vortexed again. The reaction was allowed overnight at room temperature without stirring. The precipitate of urea derivative, which is a by- product of the reaction, was removed by centrifugation at $10000 \mathrm{rpm}$ for $10 \mathrm{~min}$. The supernatant was aspired carefully from above the pellet and was used for further reaction. The supernatant containing 6 mol excess of cholanic ester, was added drop by drop to the solution of HA-NH2 
in $0.1 \mathrm{~N} \mathrm{NaHCO} 3 \mathrm{pH} 9(20 \mathrm{mg} / \mathrm{ml})$. The reaction was stirred overnight at room temperature and its product was purified by dialysis against water and filtration. Subsequently, the material was freeze-dried, weighted and stored at -20 0C.

\subsection{Fluorescence labeling of hyaluronan nanoparticles}

Fluorescent hyaluronan nanoparticles (HA-NPs) were prepared by conjugating an NHS- derivative of a fluorescent dye to the residual primary amino groups on the nanoparticles. First, HA-NPs were dispersed in $0.1 \mathrm{~N} \mathrm{NaHCO} 3 \mathrm{pH} 8.5$ at the concentration of $10 \mathrm{mg} / \mathrm{ml}$ and added to a DMF solution of either Cy5.5- or Cy7NHS ester (Lumiprobe GmbH, Hannover, Germany) (60\% water-based buffer: 40 $\% \mathrm{DMF}$ ), and stirred for $4 \mathrm{~h}$ at room temperature. 8 mol dye was added per $1 \mathrm{~mol}$ of residual $\mathrm{NH} 2$ group. The unreacted dye was removed by ethanol precipitation ( 4 times or until the supernatant was clear) and dialyzed against water for $24 \mathrm{~h}$. The labeling resulted in approximately $0.2 \mathrm{~mol}$ dye $/ \mathrm{mol}$ of HA, based on absorbance measurements.

\subsection{Radiolabeling of hyaluronan nanoparticles}

HA-NPs nanoparticles were modified with deferoxamine B (DFO) to enable radiolabeling with Zirconium-89 ( $\left.{ }^{89} \mathrm{Zr}\right)$. p-SCN-Bn-DFO (Macrocyclix, Plano, TX, USA) in DMSO (5 mg/ml) was added to a nanosuspension of HA-NPs in $0.1 \mathrm{M}$ carbonate buffer ( $\mathrm{pH}$ 8.9) $(10 \mathrm{mg} / \mathrm{ml})$ in steps of $5 \mu \mathrm{l}$ until a 2-fold molar excess over residual amine groups of NP-incorporated HA. The reaction mixture was vortex-mixed after each addition and then shaken at $37{ }^{\circ} \mathrm{C}$ for $4 \mathrm{~h}$. The DFOmodified nanoparticles (HA-NPs-DFO) were purified by spin filtration using $10 \mathrm{kDa}$ molecular weight cut-off (MWCO) (Sartorius Stedim Biotech $\mathrm{GmbH}$, Goettingen, Germany) and washing 4 times with fresh PBS. The final retentate was diluted with PBS to achieve a final HA concentration of $5 \mathrm{mg} / \mathrm{ml}$. The HA-DFO nanoparticles were reacted with ${ }^{89} \mathrm{Zr}$ oxalate in PBS ( $\mathrm{pH} 7.4$ ) for $2 \mathrm{~h}$ at $37{ }^{\circ} \mathrm{C}$. Radiolabeled HA nanoparticles ( ${ }^{89} \mathrm{Zr}$-HA-NPs) were separated from unreacted ${ }^{89} \mathrm{Zr}$ by centrifugal filtration using $10 \mathrm{kDa}$ MWCO and washing with sterile PBS. The radiochemical yield was $94 \pm 1 \%(\mathrm{n}=3)$, and the radiochemical purity $>98 \%$ as determined by size exclusion chromatography. The number of DFO labels per HA molecule was measured to be $\sim 1$ by the isotope dilution method. 


\subsection{Animal experiments}

\subsubsection{Mice}

Eight weeks old Apoe ${ }^{-/-}$mice (Charles River Laboratories) were fed with a highfat diet (TD.88137, Envigo, Alconbury Huntington, UK) for either 6 or 12 weeks to induce early and advanced atherosclerotic lesions, respectively. The wild-type mice served as negative controls. After the diet period, the mice received an injection of HA-NPs $(25 \mathrm{mg} / \mathrm{kg})$, which were labeled with either a fluorescent dye or radiotracer, via the tail vein. $24 \mathrm{~h}$ after injection, the mice were sacrificed, perfused with PBS and aortas with other organs were removed and underwent different examinations. The entire aorta with aortic roots, spleen, blood and bone marrow from mice that received Cy7-HA-NPs $(n=7)$ were analyzed by flow cytometry. The immunohistochemical analysis of aortic arches was performed in mice that were administered with Cy.5.5HA-NPs $(n=5)$. The organs from ${ }^{89} \mathrm{Zr}$-HA-NPs- injected mice were analyzed by autoradiography and gamma counting $(\mathrm{n}=4)$.

In the therapeutic study, 8 weeks-old $A p o e^{--}$-mice on a high-fat diet received once a week for 12 weeks an intravenous injection of either HA-NPs, free HA (both 50 $\mathrm{mg} / \mathrm{kg} /$ week) or PBS (ns = 10). After 12 weeks of treatment, the mice were sacrificed and aortas perfused with cold PBS. The excised aortic arch and descending aorta including the renal arterial branching were analyzed by flow cytometry, whereas aortic roots were analyzed by histology. All mouse experiments were performed in accordance with protocols approved by the Animal Experiment Committee of Academic Medical Center in Amsterdam or Institutional Animal Care and Use Committee of Mount Sinai School of Medicine (MSSM) in New Y ork.

\subsubsection{Rabbits}

Three male New Zealand White rabbits (2.5-3.0 months old) were purchased from Charles River Laboratories (Wilmington, MA, USA). To induce the formation of atherosclerotic plaques, endothelial denudation of the aorta was performed by angioplasty. Animals were anesthetized after an intramuscular (i.m.) administration of ketamine $(20 \mathrm{mg} / \mathrm{kg}$ ) (Fort Dodge Animal Health, Overland Park, Kansas, USA) and xylazine ( $5 \mathrm{mg} / \mathrm{kg}$ ) (Bayer AG, Leverkusen, Germany). A 4F-Fogarty embolectomy catheter (Edwards Lifesciences, Irvine, CA) was introduced in the femoral artery and ascended up to the level of the left subclavian artery and inflated until a pressure of $2 \mathrm{~atm}$ was established. Next, the balloon was slowly deflated while retracting until the iliac bifurcation was reached, all performed under X-ray guidance using a clinical cardiovascular intervention Philips system (Allura Xper FD20 / 10, Philips Healthcare, Best, The Netherlands). Procedure was repeated using the contralateral femoral artery as point of entry, 4 weeks after first procedure and 6 weeks after the initiation of a high cholesterol diet, composed of regular chow diet 
enriched with $0.3 \%$ cholesterol and $4.7 \%$ coconut oil (Research diets, Inc. Brunswick, New Jersey, USA). After 8 weeks, diet was changed to $0.15 \%$ enriched cholesterol with $4.7 \%$ coconut oil diet and continued for the remainder of the experiment. The rabbit experiments were performed in accordance with protocols approved by the Institutional Animal Care and Use Committees of MSSM, and followed National Institutes of Health guidelines for animal welfare.

\subsection{Flow cytometry of mouse tissues}

Flow cytometry (FACS) measurements were performed to determine the targeting properties and immune effects of HA-NPs in Apoe ${ }^{-1-}$ mice. $24 \mathrm{~h}$ after Cy7-HA-NP administration (targeting study) or 12 weeks after HA-NP therapy (therapeutic study), the mice were sacrificed and blood was collected by cardiac puncture. Subsequently, the mice were perfused with cold PBS and the entire aorta, including roots, was excised. After $1 \mathrm{~h}$ digestion in $4 \mathrm{U} / \mathrm{ml}$ of Liberase TM (Roche Life Sciences, Almere Netherlands), $60 \mathrm{U} / \mathrm{ml}$ of hyluronidase (Sigma-Aldrich), $40 \mathrm{U} / \mathrm{ml}$ DNase I (Sigma-Aldrich) of PBS solution at $37^{\circ} \mathrm{C}$, the aorta digests were resuspended, filtered through the cell strainer (Fisher Scientific) and washed 3 times with FACS buffer (1 $\%$ BSA in PBS). The spleen was homogenized manually and strained through the cell strainer, whereas bone marrow was flushed out from the femur bone by using cold PBS and resuspended into single cell suspension by using 26G needle. Subsequently, the blood, spleen and bone marrow underwent erythrocyte lysis (eBioscience, San Diego, CA, USA) and washed 3 times with FACS buffer.

The cell suspensions from all the organs were stained for FACS analysis. First, the mouse Fc block (BD Pharmingen, Breda, Netherlands) was added. 10 minutes thereafter, the antibody cocktail was introduced. The following antimouse antibodies were used for FACS staining of mice injected with Cy7-HA-NPs: Brilliant Violet 510TM-CD45, Brilliant Violet 711TM-CD11b (both BioLegend, San Diego, CA, USA), FITC-F4/80 (CI:A3-1) (Bio-Rad, Oxford, UK), PE-CF594-Ly6c (AL-21) (BD Pharmingen), Brilliant Violet 650TM-CD44 (IM7). All the antibodies were diluted to 1:200 with FACS buffer. DAPI (ThermoFisher Scientific) was used at the concentration of $2 \mu \mathrm{g} / \mathrm{ml}$ as dead cell staining. Moreover, we prepared fluorescently labelled hyaluronan to determine the hylaronan- binding efficacy by different immune cell populations. In short, HA (60-90 kDa) was dissolved in PBS buffer $\mathrm{pH} 7.2$ and reacted with EDC and sulfo NHS (both ThermoFisher Scientific) at room temperature, while stirring (quantities are given in the section on HA- NP preparation). After $0.5 \mathrm{~h}, 8$ molar excess of disulfo Cy5-hydrazide (Cyandye, Sunny Isles Beach, FL, US) in PBS pH 7.2 was added to the EDC- and sulfoNHS-reacted HA and stirred for $4 \mathrm{~h}$ at room temperature. The reaction product, Cy5-HA, was purified by dialysis and ethanol precipitation (4 times). For FACS staining, $50 \mu \mathrm{g} / \mathrm{ml}$ of Cy5HA was used in a cocktail with the aforementioned antibodies. The cell suspensions were incubated with antibodies and Cy5-hyaluronan for $30 \mathrm{~min}$ and washed 3 times with FACS buffer. 
The FACS staining of mouse material from the therapeutic study included the following antibodies: Pacific Blue-conjugated NK-1.1, CD90.2, Ly-6G, TER-119, CD49b and CD45R/B220, Brilliant Violet 510TM-CD45, PE-CD11b, FITC- Ly6c (AL21), PE- Cy7-F4/ 80 (CI:A3-1) and Cy5-HA (custom made). All the antibodies where purchased from the BioLegend and used at the dilution of 1:200 excluding FITCLy6c, which was diluted 1:100.

FACS measurements were performed on either BD LSRFortessa (BD Biosciences) (targeting study) or BD LSR II (BD Biosciences) (therapeutic study). The obtained data were analyzed with FlowJo V10 software (FLOWJO, Ashland, OR, USA). The macrophages, monocytes and neutrophils were defined according to the gating strategy presented in Figure S6A (supporting information). For these cell populations, the uptake of Cy7-HA-NPs was determined and expressed as median fluorescence intensity per cell (MFI). Furthermore, MFI of Brilliant Violet 650TMCD44 and Cy5-HA were used as quantifiers of CD44 expression and HA-binding efficacy, respectively. The analysis of FACS data obtained from mice that underwent 12 week-treatment was limited to the assessment of total immune cell population (CD45-positive), which was due to overall low cell numbers obtained from aortic arches.

\subsection{Autoradiography of mouse aortas}

The perfused and excised aortas were placed in a film cassette against a phosphorimaging plate (BASMS-2325, Fujifilm, Valhalla, NY) for $72 \mathrm{~h}$ at $-20{ }^{\circ} \mathrm{C}$ to determine the radiotracer distribution. Phosphorimaging plates were read at a pixel resolution of $25 \mu \mathrm{m}$ with a Typhoon 7000IP plate reader (GE Healthcare, Pittsburgh, $\mathrm{PA})$. The images were post-processed using ImageJ software.

\subsection{Pharmacokinetics and biodistribution in mice}

Gamma counting was used to assess the biodistribution and clearance kinetics of ${ }^{89} \mathrm{Zr}$ - HA-NPs and performed on a Wizard2 2470 Automatic Gamma Counter (Perkin Elmer, Waltham, MA). First, the injection samples of ${ }^{89} \mathrm{Zr}-\mathrm{HA}-\mathrm{NP}$ were measured to determine the total injected dose of radioactivity (ID). Two $\mathrm{min}, 30 \mathrm{~min}, 2 \mathrm{~h}, 6 \mathrm{~h}$ and $24 \mathrm{~h}$ post- administration, small blood samples were taken from the lateral tail vein. At $24 \mathrm{~h}$ after injection of ${ }^{89} \mathrm{Zr}$ - HA-NPs, the mice were sacrificed and the aorta, liver, spleen, kidneys, lung, heart and a part of the femoral muscle were collected. The radioactivity of each sample was measured and normalized to the ID and sample weight, and expressed as the percentage of ID that was retrieved in one gram of tissue (\% ID/g). For statistical comparison of aortas, the aortic radioactivity was normalized to the ID only and expressed as the percentage of injected dose (\%ID).

To determine the blood clearance parameters, the blood radioactivity data were fitted by a mono- and bi-exponential decay model. The best fitting model, as 
concluded from the correlation coefficient, r2, was used for reporting. The same strategy was used for the rabbit data.

\subsection{Immunohistochemistry of mouse tissues}

The mouse aortic arches were embedded in Tissue-Tek O.C.T. (Sakura; Alphen aan den Rijn, Netherlands) and frozen in 2-methylbutane (Sigma) on dry ice. Subsequently, was cut into $7 \mu \mathrm{m}$-thick sections and fixed with ice-cold acetone for 5 min. Before staining, they were blocked with $5 \%$ fetal calf serum (FCS) in PBS for 60 min. Next, the sections were incubated overnight at $4{ }^{\circ} \mathrm{C}$ with rat anti-mouse CD68 primary antibody (AbD Serotec; 1:200 dilution; Clone: FA-11). After washing, Alexa Fluor 488-conjugated donkey anti-rat IgG secondary antibody (Thermo Fischer Scientific; 1:500 dilution) was applied for 1 hour. Nuclei were stained with DAPI.

The images of stained aortic arches were acquired using Leica TCS SP8 confocal microscope (Leica Microsystems) at $\times 400$ magnification. On average, we imaged 4 different plaque areas in each arch section, while imaging 6 tissue sections per mouse. The obtained images were analyzed using Leica LAS-X software. First, we defined the plaque area, which was used as normalizing parameter. Furthermore, we determined the area positive for Alexa Fluor 488-CD68 (macrophage staining) or HA-NPs-Cy5.5. This was done by applying threshold to the appropriate fluorescence channel and creating mask, which was used to calculate the fluorescence-positive area. Furthermore, the area of colocalization of Alexa Fluor 488-CD68 with HANPs-Cy5.5 was determined. Subsequently, we calculated the percentage of HANP-positive area that colocalizes with macrophage staining, which was used as an indicative of the HA-NP selectivity towards plaque-associated macrophages.

To determine the effects of HA-NP treatment on the atherosclerotic plaque development, the mouse hearts with aortic roots were fixed in $4 \%$ paraformaldehyde (Sigma-Aldrich) and embedded in paraffin, after the process of dehydration. The aortic roots were cut into $7 \mu \mathrm{m}$-thick sections and stained with 1) hematoxyline and eosin (H\&E); 2) rat anti-mouse MAC-3 antibody (clone M3/84; BD Pharmingen; 1:30 dilution; overnight incubation), followed by rabbit anti-rat biotinylated secondary antibody (Vector Laboratories, Burlingame, CA; 1:300 dilution, 30 min incubation) and avidin-peroxidase (Vectastatin Elite ABC HRP Kit, Vector Laboratories), color was induced with ImmPact AMEC red peroxidase substrate (Vector Laboratories); and 3) sirius red staining (Sigma- Aldrich) (collagen staining). The stained tissues were analyzed with light microscope (Leica) at $\times 50$ magnification. The mean plaque area was assessed from H\&E-stained sections. This was done by delineating plaque areas in 5-6 root sections / mouse. The selected sections had to contain all three aortic valves. The average of all measurements was used as the mean plaque area. The fractions of macrophage and collagen area were assessed in a similar manner. First, the total plaque area was delineated and calculated. The further image analysis included only the plaque area. Color images were transformed into grey-scale 
images. Subsequently, a threshold was set to select only high-intensity pixels and create binary image. From the latter one, the threshold-limited area was calculated and normalized to the total plaque area. All the aforementioned image analyses were performed using Fiji software [82].

\subsection{PET/MR imaging in rabbits}

Rabbits ( $\mathrm{n}=3$, weight: $3.6 \pm 0.4 \mathrm{~kg}$ ) received a $24 \mathrm{G}$-catheter in the right marginal ear vein for injection with ${ }^{89} \mathrm{Zr}-\mathrm{HA}-\mathrm{NPs}$ and Cy5.5-HA-NPs $(1.40 \pm 0.02 \mathrm{mCi}$; 25 $\mathrm{mg}$ total HA). Anesthesia was induced by an intramuscular injection of ketamine (20 mg/kg) (Fort Dodge Animal Health, Overland Park, Kansas, USA), combined with xylazine $(0.5 \mathrm{mg} / \mathrm{kg}$ ) (Bayer, Shawnee Mission, Kansas, USA). Before scanning, all rabbits received a urine- catheter to prevent any disruptions from signal in the bladder and were placed in a body matrix coil. During scanning, rabbits received isoflurane anesthesia at $1.5 \%$ by inhalation and were oxygenated for the remaining of the PET/MR imaging experiment, while vital parameters were monitored.

Shortly after ${ }^{89} \mathrm{Zr}-\mathrm{HA}-\mathrm{NPs}$ injection, images were acquired in a dynamic fashion for the duration of $2 \mathrm{~h}$ using a clinical 3 Tesla PET/MRI Biograph mMR (Siemens, München, Germany). Attenuation correction of PET images was done using the built-in two-point Dixon MR-based attenuation correction (MR-AC) map and images reconstructed using the OP-OSEM algorithm. After scout scans, the PET scan was initiated and acquired simultaneously with bright-blood time-of-flight (TOF) noncontrast enhanced angiography was performed for localization of arterial anatomical landmarks (renal arteries and iliac bifurcation). Imaging parameters were: TR, 23 ms; TE, $2.8 \mathrm{~ms}$; flip angle, 20 degrees; spatial resolution, $0.35 \mathrm{~mm} 2$ (interpolated); slice thickness, $1 \mathrm{~mm}$. Simultaneously a black blood 3D T2 weighted Sampling Perfection with Application optimized Contrasts using different flip angle Evolution (SPACE) sequence was used for vessel wall delinetation. Imaging parameters were: TR, 1600 ms; TE, 115 ms; flip angle, 120 degrees; echo train length (ETL), 83; spatial resolution, $0.63 \mathrm{~mm} 2$; slice thickness, $0.63 \mathrm{~mm}$. Static PET scans were performed at $12 \pm 1.8$ and at $24 \pm 0.1 \mathrm{~h}$ after injection, using a TOF and MR-AC as described above.

Analysis of PET images was performed after all data were processed and divided in different time frames using a custom made program written in Matlab (MathWorks, Natick, MA, USA). All data was subsequently processed OsiriX imaging software (Pixmeo SARL, Bernex, Switzerland) by drawing regions of interest (ROIs) on the infrarenal abdominal aorta, and major organs (liver, spleen and kidneys). By averaging all acquired ROIs per organ ( $\geq 10$ per organ), mean standardized uptake values (mean SUVmax) $(\mathrm{g} / \mathrm{ml}$ ) values for each target tissues were obtained. 


\subsection{Pharmacokinetics and biodistribution in rabbits}

Radioactivity half-lives were determined by drawing blood from the ear arteries at 1 and $30 \mathrm{~min}$, and at 1, 2, 12, and $24 \mathrm{~h}$ post-injection. All rabbits were sacrificed by an i.v. injected overdose of $100 \mathrm{mg} / \mathrm{kg}$ sodium pentobarbital and flushed afterwards with a bolus of heparinized saline. All rabbits were pinched in the feet to check for reaction prior to systemic perfusion with at least $500 \mathrm{ml}$ of saline solution. Aortas were excised and divided in thoracic and abdominal aorta, the latter with celiac trunk and renal arteries attached, serving as landmarks. The following organs were harvested: heart, lungs, liver, spleen, kidneys, para-aortic lymph node, and muscle. All tissues were weighted before counting with a Wizard2 2480 automatic gamma counter (Perkin Elmer).

\subsection{Immunohistochemistry of rabbit aorta}

From rabbits that were coinjected with ${ }^{89} \mathrm{Zr}$ - and Cy5.5-labeled HA-NPs, several samples of abdominal and thoracic aorta were collected. The samples were frozen in Tissue-Tek O.C.T. (Sakura) and cut into $7 \mu \mathrm{m}$-thick sections. Before staining, they were fixed for $5 \mathrm{~min}$ in ice-cold acetone. After blocking with $4 \%$ FCS, the aortic sections were stained with a mouse anti-rabbit macrophage antibody (RAM-11, Dako, Heverlee, Belgium). After overnight incubation and washing with Trisbuffered saline, Alexa Fluor 594-conjugated secondary antibody was added and incubated for $0.5 \mathrm{~h}$. DAPI was used as a nuclear staining. The images were acquired using confocal microscope Leica TCS SP8 at $\times 200$ magnification or higher.

\subsection{Statistical analysis}

The normality of data distribution was tested by using Shapiro-Wilk test.[83] The in vitro FACS data on the macrophage uptake efficacy of Cy5.5-HA-NPs were analyzed with one-way ANOVA with Tukey post hoc test. The same test was used to study the differences in the Cy7-HA-NP uptake efficacy between the aortic, splenic and bone marrow macrophages. This comparison was done within the same mouse group, i.e, 6w HFD or 12w HFD. The inter-group differences were studied for each macrophage population separately with the independent samples t-test. To study the correlation between the HA-NP uptake and CD44 expression, and HA-binding in aortic leucocyte populations, we performed Spearman's correlation test for non-normally distributed data. Furthermore, a t- test was performed for the following parameters: the fraction of Cy5.5-HA-NP-positive area that colocalized with macrophage staining and SUVmax in rabbits. The \%ID in mouse aortas of two atherosclerotic mouse groups and wild-type mice were compared with one- way ANOVA with Tukey post hoc test. The same analysis method was used to determine the effects of weekly dosing of HA-NPs, free HA and PBS. All the analyses were performed using IBM SPSS Statistics 23 and setting the significance level at $\mathrm{p}<0.05$. 


\section{REFERENCES}

[1] T.C. Laurent, U.B. Laurent, J.R. Fraser, The structure and function of hyaluronan: An overview., Immunol. Cell Biol. 74 (1996) A1-7. doi:10.1038/icb.1996.32.

[2] B.P. Toole, Hyaluronan: from extracellular glue to pericellular cue, Nat. Rev. Cancer. 4 (2004) 528-539. doi:10.1038/nrc1391.

[3] A.C. Petrey, C.A. de la Motte, Hyaluronan, a Crucial Regulator of Inflammation, Front. Immunol. 5 (2014) 101. doi:10.3389/ fimmu.2014.00101.

[4] B.P. Toole, Hyaluronan-CD44 interactions in cancer: Paradoxes and possibilities, Clin. Cancer Res. 15 (2009) 7462-7468. doi:10.1158/1078-0432.CCR-09-0479.

[5] M. Zöller, CD44: can a cancer-initiating cell profit from an abundantly expressed molecule?, Nat. Rev. Cancer. 11 (2011) 254-267. doi:10.1038/nrc3023.

[6] S. Misra, P. Heldin, V.C. Hascall, N.K. Karamanos, S.S. Skandalis, R.R. Markwald, S. Ghatak, Hyaluronan-CD44 interactions as potential targets for cancer therapy, FEBS J. 278 (2011) 1429-1443. doi:10.1111/j.1742-4658.2011.08071.x.

[7] W.H. McBride, J.B. Bard, Hyaluronidase-sensitive halos around adherent cells. Their role in blocking lymphocyte-mediated cytolysis., J. Exp. Med. 149 (1979) 507-15. doi:10.1084/ jem.149.2.507.

[8] M. Slevin, S. Kumar, J. Gaffney, Angiogenic oligosaccharides of hyaluronan induce multiple signaling pathways affecting vascular endothelial cell mitogenic and wound healing responses, J. Biol. Chem. 277 (2002) 41046-41059. doi:10.1074/ jbc.M109443200.

[9] P. Rooney, S. Kumar, J. Ponting, M. Wang, The role of hyaluronan in tumour neovascularization (review), Int. J. Cancer. 60 (1995) 632-636. doi:10.1002 / ijc.2910600511.

[10] R. Stern, A.A. Asari, K.N. Sugahara, Hyaluronan fragments: An information-rich system, Eur. J. Cell Biol. 85 (2006) 699-715. doi:10.1016/j.ejcb.2006.05.009.

[11] E.J. Oh, K. Park, K.S. Kim, J. Kim, J.A. Yang, J.H. Kong, M.Y. Lee, A.S. Hoffman, S.K. Hahn, Target specific and long-acting delivery of protein, peptide, and nucleotide therapeutics using hyaluronic acid derivatives, J. Control. Release. 141 (2010) 2-12. doi:10.1016/j. jconrel.2009.09.010.

[12] K.R. Taylor, J.M. Trowbridge, J.A. Rudisill, C.C. Termeer, J.C. Simon, R.L. Gallo, Hyaluronan Fragments Stimulate Endothelial Recognition of Injury through TLR4, J. Biol. Chem. 279 (2004) 17079-17084. doi:10.1074/jbc.M310859200.

[13] P.L. Bollyky, J.D. Lord, S.A. Masewicz, S.P. Evanko, J.H. Buckner, T.N. Wight, G.T. Nepom, Cutting edge: high molecular weight hyaluronan promotes the suppressive effects of CD4+CD25+ regulatory T cells., J. Immunol. 179 (2007) 744-7. doi:10.4049/ JIMMUNOL.179.2.744.

[14] K. Masuko, M. Murata, K. Yudoh, T. Kato, H. Nakamura, Anti-inflammatory effects of hyaluronan in arthritis therapy: Not just for viscosity., Int. J. Gen. Med. 2 (2009) 77-81.

[15] J. V Forrester, E.A. Balazs, Inhibition of phagocytosis by high molecular weight hyaluronate., Immunology. 40 (1980) 435-46.

[16] X. Tian, J. Azpurua, C. Hine, A. Vaidya, M. Myakishev-Rempel, J. Ablaeva, Z. Mao, E. Nevo, V. Gorbunova, A. Seluanov, High-molecular-mass hyaluronan mediates the cancer resistance of the naked mole rat., Nature. 499 (2013) 346-9. doi:10.1038/ nature12234.

[17] J.A. Burdick, G.D. Prestwich, Hyaluronic acid hydrogels for biomedical applications, Adv. Mater. 23 (2011) H41-H56. doi:10.1002/adma.201003963.

[18] G.H. Lo, M. LaValley, T. McAlindon, D.T. Felson, Intra-articular hyaluronic acid in treatment of knee osteoarthritis: a meta-analysis., JAMA. 290 (2003) 3115-21. doi:10.1001/ jama.290.23.3115.

[19] J.R. Fraser, T.C. Laurent, H. Pertoft, E. Baxter, Plasma clearance, tissue distribution and 
metabolism of hyaluronic acid injected intravenously in the rabbit., Biochem. J. 200 (1981) 415-24. http: / / www.ncbi.nlm.nih.gov / pubmed / 7340841 (accessed April 25, 2017).

[20] L. Jadin, L.H. Bookbinder, G.I. Frost, A comprehensive model of hyaluronan turnover in the mouse, Matrix Biol. 31 (2012) 81-89. doi:10.1016/j.matbio.2011.11.002.

[21] M. Laznicek, A. Laznickova, D. Cozikova, V. Velebny, Preclinical pharmacokinetics of radiolabelled hyaluronan, Pharmacol. Reports. 64 (2012) 428-437.

[22] J.R. Fraser, T.C. Laurent, A. Engström-Laurent, U.G. Laurent, Elimination of hyaluronic acid from the blood stream in the human., Clin. Exp. Pharmacol. Physiol. 11 (1984) 17-25. doi:10.1111/j.1440-1681.1984.tb00235.x.

[23] P. Del Pino, F. Yang, B. Pelaz, Q. Zhang, K. Kantner, R. Hartmann, N. Martinez De Baroja, M. Gallego, M. Möller, B.B. Manshian, S.J. Soenen, R. Riedel, N. Hampp, W.J. Parak, Basic Physicochemical Properties of Polyethylene Glycol Coated Gold Nanoparticles that Determine Their Interaction with Cells, Angew. Chemie - Int. Ed. 55 (2016) 5483-5487. doi:10.1002/ anie.201511733.

[24] M.E. Davis, Z. (Georgia) Chen, D.M. Shin, Nanoparticle therapeutics: an emerging treatment modality for cancer., Nat. Rev. Drug Discov. 7 (2008) 771-782. doi:10.1038/ nrd2614.

[25] S.D. Li, L. Huang, Pharmacokinetics and biodistribution of nanoparticles, Mol. Pharm. 5 (2008) 496-504. doi:10.1021/mp800049w.

[26] O.P. Oommen, C. Duehrkop, B. Nilsson, J. Hilborn, O.P. Varghese, Multifunctional Hyaluronic Acid and Chondroitin Sulfate Nanoparticles: Impact of Glycosaminoglycan Presentation on Receptor Mediated Cellular Uptake and Immune Activation., ACS Appl. Mater. Interfaces. 8 (2016) 20614-24. doi:10.1021/acsami.6b06823.

[27] S. Lee, K.Y. Choi, H. Chung, J.H. Ryu, A. Lee, H. Koo, I.C. Youn, J.H. Park, I.S. Kim, S.Y. Kim, X. Chen, S.Y. Jeong, I.C. Kwon, K. Kim, K. Choi, Real time, high resolution video imaging of apoptosis in single cells with a polymeric nanoprobe, Bioconjug. Chem. 22 (2011) 125-131. doi:10.1021/bc1004119.

[28] H.Y. Yoon, H. Koo, K.Y. Choi, S.J. Lee, K. Kim, I.C. Kwon, J.F. Leary, K. Park, S.H. Yuk, J.H. Park, K. Choi, Tumor-targeting hyaluronic acid nanoparticles for photodynamic imaging and therapy, Biomaterials. 33 (2012) 3980-3989. doi:10.1016/j.biomaterials.2012.02.016.

[29] R.G. Thomas, M.J. Moon, J.H. Kim, J.H. Lee, Y.Y. Jeong, Effectiveness of Losartan-Loaded Hyaluronic Acid (HA) Micelles for the Reduction of Advanced Hepatic Fibrosis in C3H/ HeN Mice Model., PLoS One. 10 (2015) e0145512. doi:10.1371/journal.pone.0145512.

[30] K.Y. Choi, H.Y. Yoon, J.H. Kim, S.M. Bae, R.W. Park, Y.M. Kang, I.S. Kim, I.C. Kwon, K. Choi, S.Y. Jeong, K. Kim, J.H. Park, Smart nanocarrier based on PEGylated hyaluronic acid for cancer therapy, ACS Nano. 5 (2011) 8591-8599. doi:10.1021/nn202070n.

[31] S.J. Lee, S.C. Ghosh, H.D. Han, R.L. Stone, J. Bottsford-Miller, D.Y. Shen, E.J. Auzenne, A. Lopez-Araujo, C. Lu, M. Nishimura, C. V Pecot, B. Zand, D. Thanapprapasr, N.B. Jennings, Y. Kang, J. Huang, W. Hu, J. Klostergaard, A.K. Sood, Metronomic activity of CD44-targeted hyaluronic acid-paclitaxel in ovarian carcinoma, Clin. Cancer Res. 18 (2012) 4114-4121. doi:10.1158/1078-0432.CCR-11-3250.

[32] M. Bodnár, L. Daróczi, G. Batta, J. Bakó, J.F. Hartmann, J. Borbély, Preparation and characterization of cross-linked hyaluronan nanoparticles, Colloid Polym. Sci. 287 (2009) 991-1000. doi:10.1007/s00396-009-2061-9.

[33] M.E. Lobatto, V. Fuster, Z.A. Fayad, W.J.M. Mulder, Perspectives and opportunities for nanomedicine in the management of atherosclerosis., Nat. Rev. Drug Discov. 10 (2011) 835-52. doi:10.1038/nrd3578.

[34] J. Tang, M.E. Lobatto, L. Hassing, S. van der Staay, S.M. van Rijs, C. Calcagno, M.S. Braza, S. Baxter, F. Fay, B.L. Sanchez-Gaytan, R. Duivenvoorden, H.B. Sager, Y.M. Astudillo, W. Leong, S. Ramachandran, G. Storm, C. Perez-Medina, T. Reiner, D.P. Cormode, G.J. 
Strijkers, E.S.G. Stroes, F.K.Swirski, M. Nahrendorf, E.A. Fisher, Z.A. Fayad, W.J.M. Mulder, Inhibiting macrophage proliferation suppresses atherosclerotic plaque inflammation, Sci. Adv. 1 (2015) e1400223. doi:10.1126/ sciadv.1400223.

[35] R. Duivenvoorden, J. Tang, D.P. Cormode, A.J. Mieszawska, D. Izquierdo-Garcia, C. Ozcan, M.J. Otten, N. Zaidi, M.E. Lobatto, S.M. van Rijs, B. Priem, E.L. Kuan, C. Martel, B. Hewing, H. Sager, M. Nahrendorf, G.J. Randolph, E.S.G. Stroes, V. Fuster, E.A. Fisher, Z.A. Fayad, W.J.M. Mulder, A statin-loaded reconstituted high-density lipoprotein nanoparticle inhibits atherosclerotic plaque inflammation., Nat. Commun. 5 (2014) 3065. doi:10.1038/ ncomms4065.

[36] G. Fredman, N. Kamaly, S. Spolitu, J. Milton, D. Ghorpade, R. Chiasson, G. Kuriakose, M. Perretti, O. Farokhzad, O. Farokzhad, I. Tabas, Targeted nanoparticles containing the proresolving peptide Ac2-26 protect against advanced atherosclerosis in hypercholesterolemic mice., Sci. Transl. Med. 7 (2015) 275ra20. doi:10.1126/ scitranslmed. aaa1065.

[37] B.P. Toole, T.N. Wight, M.I. Tammi, Hyaluronan-cell interactions in cancer and vascular disease., J. Biol. Chem. 277 (2002) 4593-6. doi:10.1074/jbc.R100039200.

[38] S. Chai, Q. Chai, C.C. Danielsen, P. Hjorth, J.R. Nyengaard, T. Ledet, Y. Yamaguchi, L.M. Rasmussen, L. Wogensen, Overexpression of hyaluronan in the tunica media promotes the development of atherosclerosis, Circ. Res. 96 (2005) 583-591. doi:10.1161/01. RES.0000158963.37132.8b.

[39] J.M. Tarkin, M.R. Dweck, N.R. Evans, R.A.P. Takx, A.J. Brown, A. Tawakol, Z.A. Fayad, J.H.F. Rudd, Imaging Atherosclerosis., Circ. Res. 118 (2016) 750-69. doi:10.1161/ CIRCRESAHA.115.306247.

[40] C. Weber, H. Noels, Atherosclerosis: current pathogenesis and therapeutic options., Nat. Med. 17 (2011) 1410-22. doi:10.1038/nm.2538.

[41] J.P. Foley, D. Lam, H. Jiang, J. Liao, N. Cheong, T.M. McDevitt, A. Zaman, J.R. Wright, R.C. Savani, Toll-like Receptor 2 (TLR2), Transforming Growth Factor- $\beta$, Hyaluronan (HA), and Receptor for HA-mediated Motility (RHAMM) Are Required for Surfactant Protein A-stimulated Macrophage Chemotaxis, J. Biol. Chem. 287 (2012) 37406-37419. doi:10.1074/jbc.M112.360982.

[42] P.W. Noble, F.R. Lake, P.M. Henson, D.W.H. Riches, Hyaluronate activation of CD44 induces insulin-like growth factor-1 expression by a tumor necrosis factor-alphadependent mechanism in murine macrophages, J. Clin. Invest. 91 (1993) 2368-2377. doi:10.1172/ JCI116469.

[43] K. Schledzewski, M. Falkowski, G. Moldenhauer, P. Metharom, J. Kzhyshkowska, R. Ganss, A. Demory, B. Falkowska-Hansen, H. Kurzen, S. Ugurel, G. Geginat, B. Arnold, S. Goerdt, Lymphatic endothelium-specific hyaluronan receptor LYVE-1 is expressed by stabilin-1+, F4/80+, CD11b+ macrophages in malignant tumours and wound healing tissue in vivo and in bone marrow cultures in vitro: implications for the assessment of lymphangiogene, J. Pathol. 209 (2006) 67-77.

[44] X.H. Xu, P.K. Shah, E. Faure, O. Equils, L. Thomas, M.C. Fishbein, D. Luthringer, X.P. $\mathrm{Xu}$, T.B. Rajavashisth, J. Yano, S. Kaul, M. Arditi, Toll-like receptor-4 is expressed by macrophages in murine and human lipid-rich atherosclerotic plaques and upregulated by oxidized LDL, Circulation. 104 (2001) 3103-3108. doi:10.1161/hc5001.100631.

[45] K. Ley, Y.I. Miller, C.C.Hedrick, Monocyte and macrophage dynamics during atherogenesis, Arterioscler. Thromb. Vasc. Biol. 31 (2011) 1506-1516. doi:10.1161/ ATVBAHA.110.221127.

[46] A. Mantovani, Macrophage diversity and polarization : in vivo veritas Time to restore individual rights for IL-2 and, Immunobiology. 108 (2004) 408-409. doi:10.1182/ blood-2006-05-019430.

[47] J.E. Scott, Y. Chen, A. Brass, Secondary and tertiary structures involving chondroitin and 
chondroitin sulphates in solution, investigated by rotary shadowing/electron microscopy and computer simulation, Eur. J. Biochem. 209 (1992) 675-680. doi:10.1111/j.1432-1033.1992. tb17335.x.

[48] J.E. Scott, Supramolecular Organization of Extracellular-Matrix Glycosaminoglycans, Invitro and in the Tissues, Faseb J. 6 (1992) 2639-2645.

[49] S. Sitia, L. Tomasoni, F. Atzeni, G. Ambrosio, C. Cordiano, A. Catapano, S. Tramontana, F. Perticone, P. Naccarato, P. Camici, E. Picano, L. Cortigiani, M. Bevilacqua, L. Milazzo, D. Cusi, C. Barlassina, P. Sarzi-Puttini, M. Turiel, From endothelial dysfunction to atherosclerosis, Autoimmun. Rev. 9 (2010) 830-834. doi:10.1016/j.autrev.2010.07.016.

[50] L.J.H. Van Tits, R. Stienstra, P.L. van Lent, M.G. Netea, L.A.B. Joosten, A.F.H. Stalenhoef, Oxidized LDL enhances pro-inflammatory responses of alternatively activated M2 macrophages: A crucial role for Krüppel-like factor 2, Atherosclerosis. 214 (2011) 345-349. doi:10.1016/j.atherosclerosis.2010.11.018.

[51] S.M. Moghimi, A.C. Hunter, J.C. Murray, Long-circulating and target-specific nanoparticles: theory to practice., Pharmacol. Rev. 53 (2001) 283-318. doi:VL - 53.

[52] F. Ahsan, I.P. Rivas, M.A. Khan, A.I. Torres Suárez, Targeting to macrophages: role of physicochemical properties of particulate carriers - liposomes and microspheres-on the phagocytosis by macrophages, J. Control. Release. 79 (2002) 29-40. doi:10.1016/S01683659(01)00549-1.

[53] Z. Zheng, S. Katoh, Q. He, K. Oritani, K. Miyake, J. Lesley, R. Hyman, A. Hamik, R.M. Parkhouse, A.G. Farr, P.W. Kincade, Monoclonal antibodies to CD44 and their influence on hyaluronan recognition., J. Cell Biol. 130 (1995) 485-95.

[54] J. Lesley, R. Schulte, R. Hyman, Binding of hyaluronic acid to lymphoid cell lines is inhibited by monoclonal antibodies against Pgp-1, Exp. Cell Res. 187 (1990) 224-233. doi:10.1016/0014-4827(90)90085-O.

[55] J. Lesley, R. Hyman, CD44 can be activated to function as an hyaluronic acid receptor in normal murine T cells, Eur. J. Immunol. 22 (1992) 2719-2723. doi:10.1002/ eji.1830221036.

[56] S. Tavakoli, R. Asmis, Reactive oxygen species and thiol redox signaling in the macrophage biology of atherosclerosis., Antioxid. Redox Signal. 17 (2012) 1785-95. doi:10.1089/ ars.2012.4638.

[57] A.R. Tall, L. Yvancharvet, Cholesterol, inflammation and innate immunity, Nat. Rev. Immunol. 15 (2015) 104-116. doi:10.1038/nri3793.

[58] E.A. Sykes, J. Chen, G. Zheng, W.C.W. Chan, Investigating the impact of nanoparticle size on active and passive tumor targeting efficiency, ACS Nano. 8 (2014) 5696-5706. doi:10.1021/nn500299p.

[59] A. Albanese, P.S. Tang, W.C.W. Chan, The Effect of Nanoparticle Size, Shape, and Surface Chemistry on Biological Systems, Annu. Rev. Biomed. Eng. 14 (2012) 1-16. doi:10.1146/ annurev-bioeng-071811-150124.

[60] M.E. Lobatto, C. Calcagno, A. Millon, M.L. Senders, F. Fay, P.M. Robson, S. Ramachandran, T. Binderup, M.P.M. Paridaans, S. Sensarn, S. Rogalla, R.E. Gordon, L. Cardoso, G. Storm, J.M. Metselaar, C.H. Contag, E.S.G. Stroes, Z.A. Fayad, W.J.M. Mulder, Atherosclerotic plaque targeting mechanism of long-circulating nanoparticles established by multimodal imaging, ACS Nano. 9 (2015) 1837-1847. doi:10.1021/nn506750r.

[61] C.S. Stancu, L. Toma, A. V. Sima, Dual role of lipoproteins in endothelial cell dysfunction in atherosclerosis, Cell Tissue Res. 349 (2012) 433-446. doi:10.1007/ s00441-012-1437-1.

[62] E. Falk, Pathogenesis of Atherosclerosis, J. Am. Coll. Cardiol. 47 (2006) C7-C12. doi:10.1016/j.jacc.2005.09.068.

[63] A. von Eckardstein, L. Rohrer, Transendothelial lipoprotein transport and regulation of endothelial permeability and integrity by lipoproteins., Curr. Opin. Lipidol. 20 (2009) 197205. doi:10.1097/MOL.0b013e32832afd63. 
[64] J. Sanz, Z.A. Fayad, Imaging of atherosclerotic cardiovascular disease., Nature. 451 (2008) 953-957. doi:10.1038/nature06803.

[65] A.M.F. Hag, S.F. Pedersen, C. Christoffersen, T. Binderup, M.M. Jensen, J.T. Jørgensen, D. Skovgaard, R.S. Ripa, A. Kjaer, 18F-FDG PET Imaging of Murine Atherosclerosis: Association with Gene Expression of Key Molecular Markers, PLoS One. 7 (2012) e50908. doi:10.1371/journal.pone.0050908.

[66] Y. Zhao, Y. Kuge, S. Zhao, H.W. Strauss, F.G. Blankenberg, N. Tamaki, Prolonged highfat feeding enhances aortic 18F-FDG and 99mTc-annexin A5 uptake in apolipoprotein E-deficient and wild-type C57BL / 6J mice., J. Nucl. Med. 49 (2008) 1707-14. doi:10.2967/ jnumed.108.051847.

[67] A. Tawakol, P. Singh, M. Mojena, M. Pimentel-Santillana, H. Emami, M. Macnabb, J.H.F. Rudd, J. Narula, J.A. Enriquez, P.G. Través, M. Fernández-Velasco, R. Bartrons, P. MartínSanz, Z.A. Fayad, A. Tejedor, L. Boscá, HIF-1 $\alpha$ and PFKFB3 Mediate a Tight Relationship Between Proinflammatory Activation and Anerobic Metabolism in Atherosclerotic Macrophages., Arterioscler. Thromb. Vasc. Biol. 35 (2015) 1463-1471. doi:10.1161/ ATVBAHA.115.305551.

[68] J.A. Weigel, R.C. Raymond, C. McGary, A. Singh, P.H. Weigel, A blocking antibody to the hyaluronan receptor for endocytosis (HARE) inhibits hyaluronan clearance by perfused liver, J. Biol. Chem. 278 (2003) 9808-9812. doi:10.1074/jbc.M211462200.

[69] Y. Sheikine, K. Akram, FDG-PET imaging of atherosclerosis: Do we know what we see?, Atherosclerosis. 211 (2010) 371-380. doi:10.1016/j.atherosclerosis.2010.01.002.

[70] N. Feliu, D. Docter, M. Heine, P. del Pino, S. Ashraf, J. Kolosnjaj-Tabi, P. Macchiarini, P. Nielsen, D. Alloyeau, F. Gazeau, R.H. Stauber, W.J. Parak, In vivo degeneration and the fate of inorganic nanoparticles, Chem. Soc. Rev. 45 (2016) 2440-2457. doi:10.1039 / C5CS00699F.

[71] C. Pérez-Medina, D. Abdel-Atti, Y. Zhang, V. a Longo, C.P. Irwin, T. Binderup, J. RuizCabello, Z. a Fayad, J.S. Lewis, W.J.M. Mulder, T. Reiner, A Modular Labeling Strategy for In Vivo PET and Near-Infrared Fluorescence Imaging of Nanoparticle Tumor Targeting., J. Nucl. Med. 55 (2014) 1706-1712. doi:10.2967/jnumed.114.141861.

[72] C. Pérez-Medina, T. Binderup, M.E. Lobatto, J. Tang, C. Calcagno, L. Giesen, C.H. Wessel, J. Witjes, S. Ishino, S. Baxter, Y. Zhao, S. Ramachandran, M. Eldib, B.L. Sánchez-Gaytán, P.M. Robson, J. Bini, J.F. Granada, K.M. Fish, E.S.G. Stroes, R. Duivenvoorden, S. Tsimikas, J.S. Lewis, T. Reiner, V. Fuster, A. Kjær, E.A. Fisher, Z.A. Fayad, W.J.M. Mulder, In Vivo PET Imaging of HDL in Multiple Atherosclerosis Models, JACC Cardiovasc. Imaging. 9 (2016) 950-961. doi:10.1016/j.jcmg.2016.01.020.

[73] C. Pérez-Medina, D. Abdel-Atti, J. Tang, Y. Zhao, Z.A. Fayad, J.S. Lewis, W.J.M. Mulder, T. Reiner, C. Perez-Medina, D. Abdel-Atti, J. Tang, Y. Zhao, Z.A. Fayad, J.S. Lewis, W.J.M. Mulder, T. Reiner, Nanoreporter PET predicts the efficacy of anti-cancer nanotherapy, Nat. Commun. 7 (2016) 11838. doi:10.1038/ncomms11838 [doi].

[74] M.H. El-Dakdouki, K. El-Boubbou, M. Kamat, R. Huang, G.S. Abela, M. Kiupel, D.C. Zhu, X. Huang, CD44 targeting magnetic glyconanoparticles for atherosclerotic plaque imaging, Pharm. Res. 31 (2014) 1426-1437. doi:10.1007/s11095-013-1021-8.

[75] M. Kamat, K. El-Boubbou, D.C. Zhu, T. Lansdell, X. Lu, W. Li, X. Huang, Hyaluronic acid immobilized magnetic nanoparticles for active targeting and imaging of macrophages., Bioconjug. Chem. 21 (2010) 2128-35. doi:10.1021/bc100354m.

[76] G.Y. Lee, J.-H. Kim, K.Y. Choi, H.Y. Yoon, K. Kim, I.C. Kwon, K. Choi, B.-H. Lee, J.H. Park, I.-S. Kim, Hyaluronic acid nanoparticles for active targeting atherosclerosis., Biomaterials. 53 (2015) 341-8. doi:10.1016/j.biomaterials.2015.02.089.

[77] R.L. Juliano, D. Stamp, The effect of particle size and charge on the clearance rates of liposomes and liposome encapsulated drugs, Biochem. Biophys. Res. Commun. 63 (1975) 651-658. doi:10.1016/S0006-291X(75)80433-5. 
[78] K.J. Moore, I. Tabas, Macrophages in the pathogenesis of atherosclerosis, Cell. 145 (2011) 341-355. doi:10.1016/j.cell.2011.04.005.

[79] J. Gaffney, S. Matou-Nasri, M. Grau-Olivares, M. Slevin, Therapeutic applications of hyaluronan., Mol. Biosyst. 6 (2010) 437-43. doi:10.1039/b910552m.

[80] R.G. Thomas, M. Moon, S. Lee, Y.Y. Jeong, Paclitaxel loaded hyaluronic acid nanoparticles for targeted cancer therapy: In vitro and in vivo analysis, Int. J. Biol. Macromol. 72 (2014) 510-518. doi:10.1016/j.ijbiomac.2014.08.054.

[81] S. Reitsma, D.W. Slaaf, H. Vink, M.A.M.J. Van Zandvoort, M.G.A. Oude Egbrink, The endothelial glycocalyx: Composition, functions, and visualization, Pflugers Arch. Eur. J. Physiol. 454 (2007) 345-359. doi:10.1007/ s00424-007-0212-8.

[82] J. Schindelin, I. Arganda-Carreras, E. Frise, V. Kaynig, M. Longair, T. Pietzsch, S. Preibisch, C. Rueden, S. Saalfeld, B. Schmid, J.-Y. Tinevez, D.J. White, V. Hartenstein, K. Eliceiri, P. Tomancak, A. Cardona, Fiji: an open-source platform for biological-image analysis., Nat. Methods. 9 (2012) 676-82. doi:10.1038/nmeth.2019.

[83] S.S. Shapiro, M.B. Wilk, An Analysis of Variance Test for Normality (Complete Samples), Biometrika. 52 (1965) 591. doi:10.2307/ 2333709. 


\section{APPENDIX I: SUPPLEMENTARY DATA}

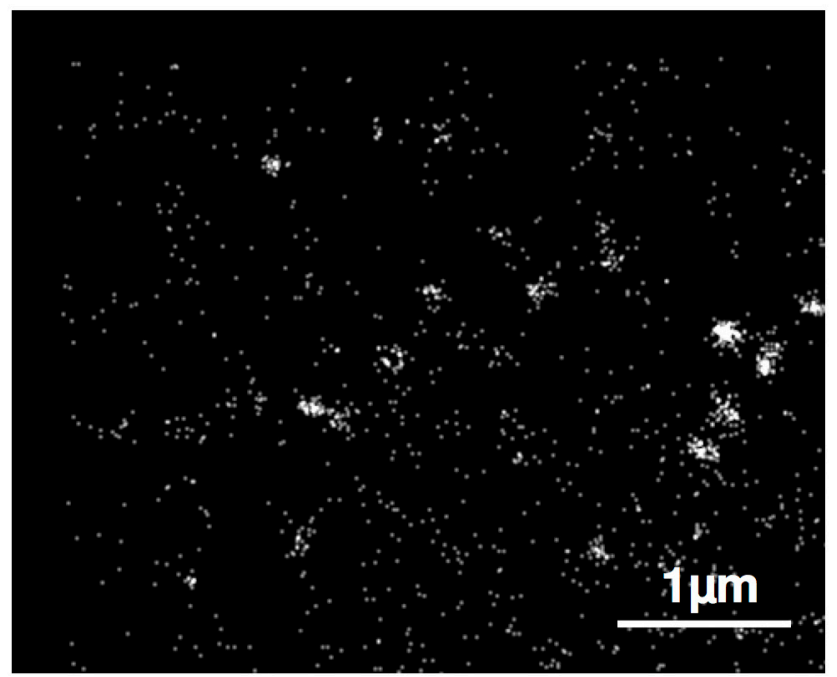

Figure S1 I Direct stochastic optical reconstruction microscopy (dSTORM) image of surface immobilized carboxylic acid-derivative of Cy5. 

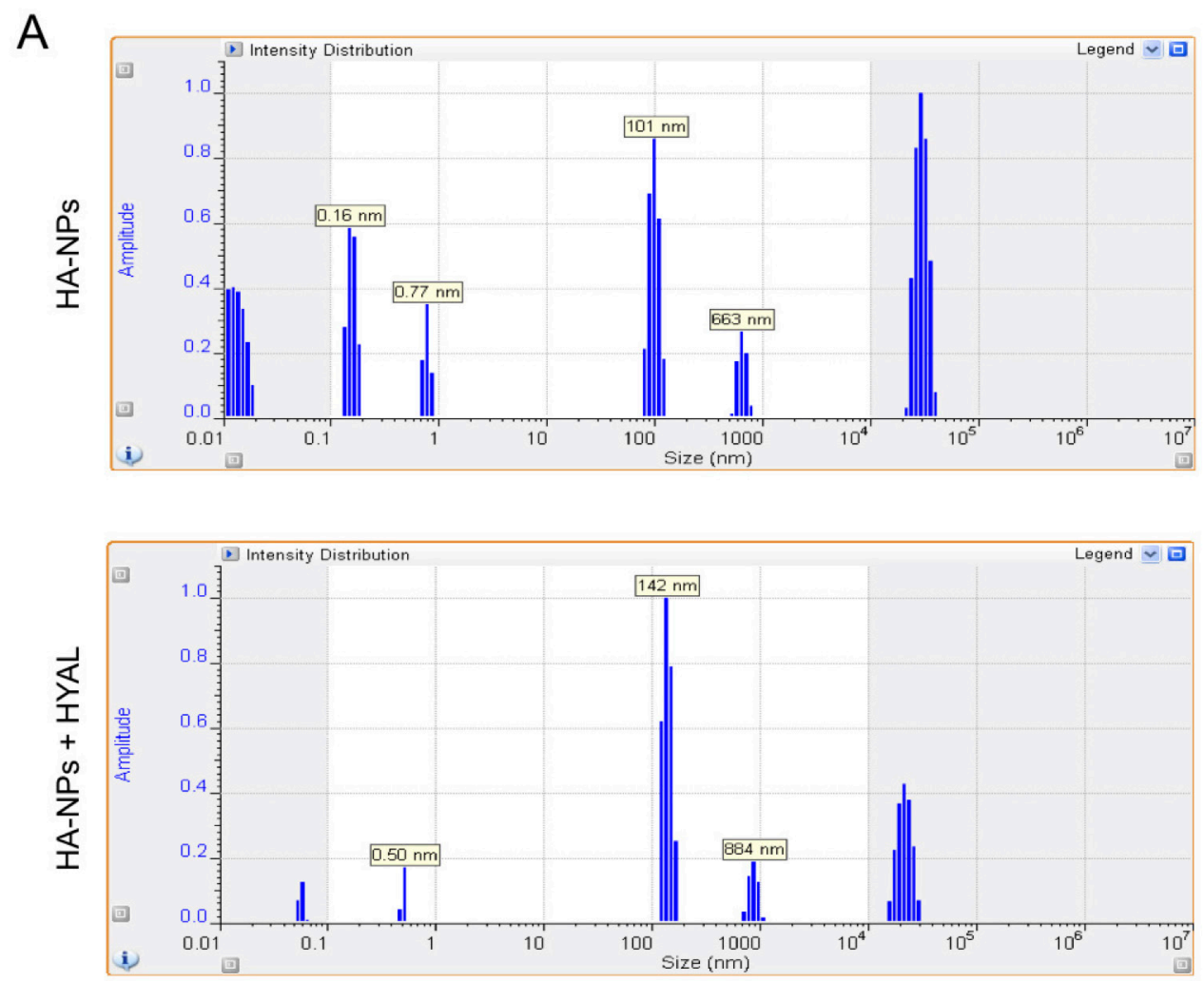

B

\begin{tabular}{l|c:c} 
& HA-NPs & HA-NPs + HYAL \\
\hline AFM & $32.0+/-0.5 \mathrm{~nm}$ & $26.0+/-5.1 \mathrm{~nm}$ \\
\hdashline dSTORM & $104+/-60 \mathrm{~nm}$ & $102+/-61 \mathrm{~nm}$ \\
\hdashline DLS & $100+/-17 \mathrm{~nm}$ & $125+/-27 \mathrm{~nm}$ \\
\hdashline Zeta sizer & $31.3+/-2.6 \mathrm{mV}$ & $33.3+/-2.2 \mathrm{mV}$
\end{tabular}

Figure S2 | A) Representative intensity histograms of hyaluronan nanoparticles (HA-NPs, upper panel) and HA- NPs after treatment with hyaluronidase (HYAL) (HA-NPs + HYAL, lower panel), measured by dynamic light scattering (DLS). (B) The results obtained by atomic force microscopy (AFM), dSTORM, DLS and zeta sizer, displayed as the mean parameter value \pm SD. 


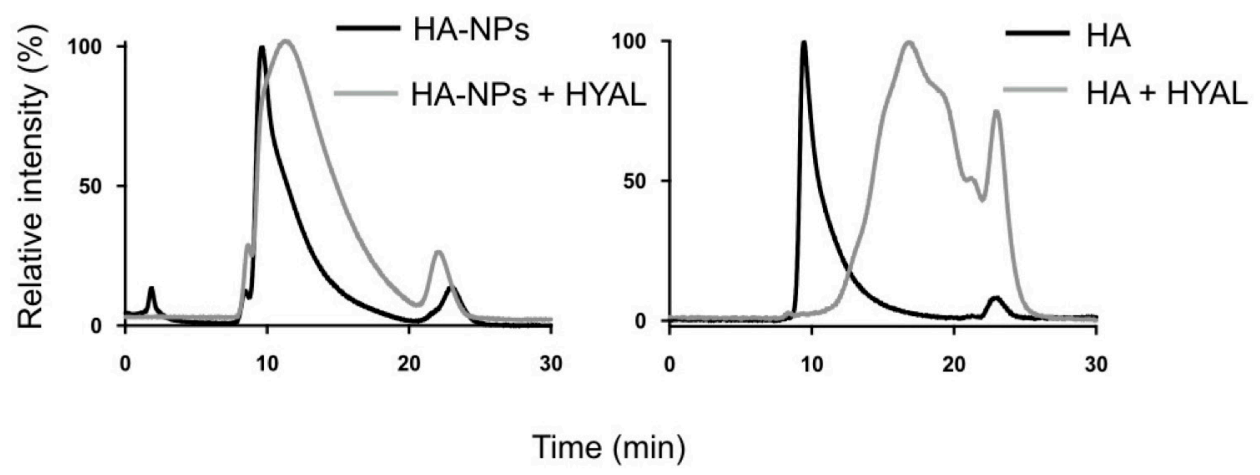

Figure S3 | Retention time histograms obtained by the size exclusion chromatography for HA-NPs (left panel) and linear HA (right panel) at the baseline state (black lines) and after hydrolysis with hyaluronidase (HYAL) (grey lines). 
A
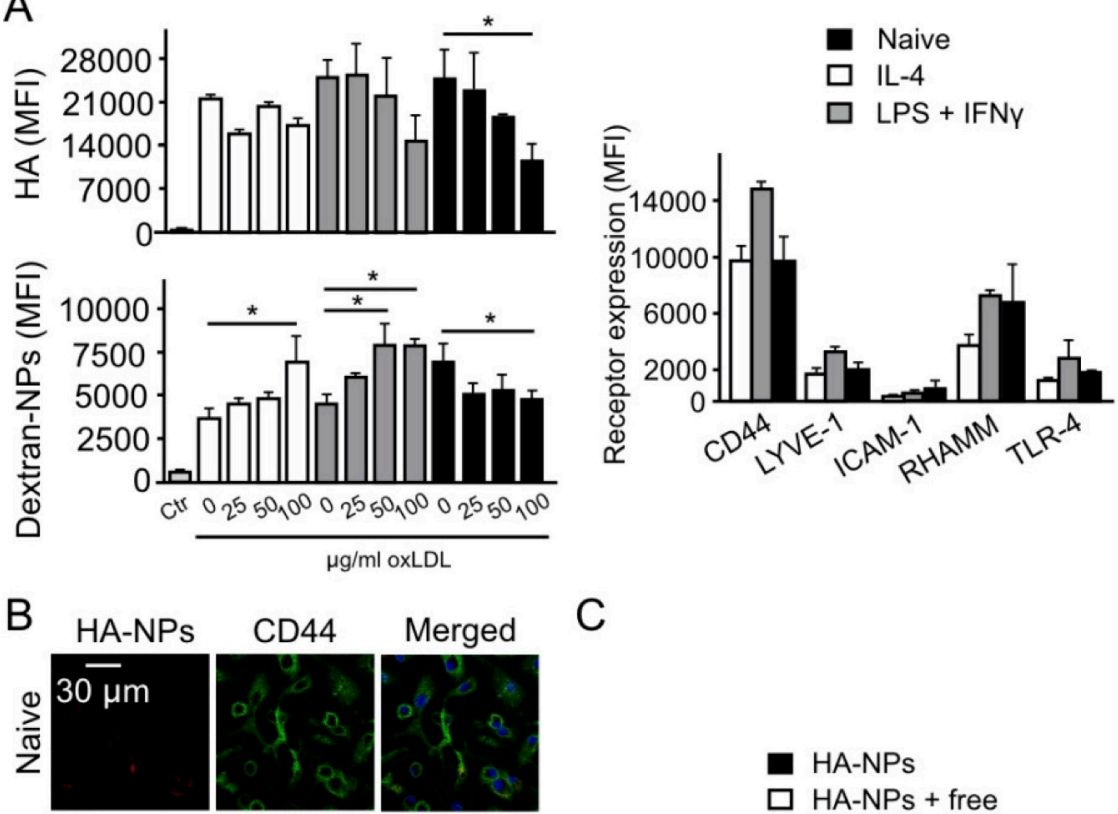

C
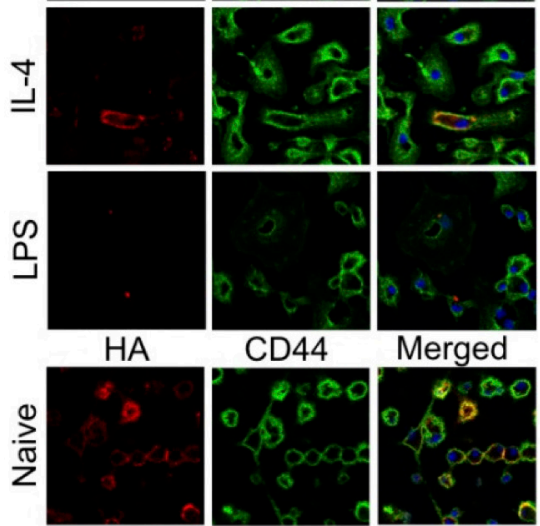
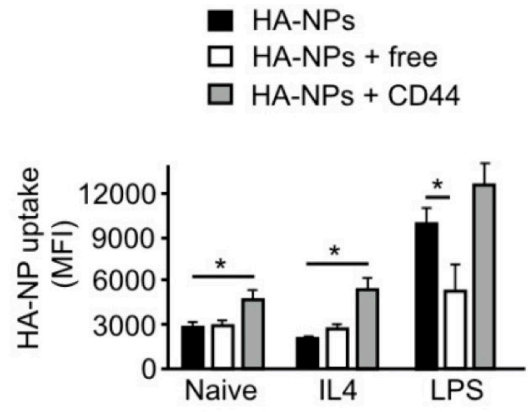

Figure S4 | A) Flow cytometry analysis of the cellular uptake of Cy5-labeled hyaluronan (HA) (middle panel) and Cy5.5-labeled dextran nanoparticles (Dextran-NPs) (lower panel) in different phenotypes of bone marrow-derived macrophages. The macrophages are divided into three main phenotypic groups: naive (-) (white bars), interleukin 4 (IL-4)-stimulated (grey bars), and lipopolysaccharide (LPS) and interferon $\gamma$ (INF $\gamma$ )-stimulated (black bars). Moreover, three concentrations of oxidized low-density lipoprotein (oxLDL) were used for co-stimulation, i.e. 25, 50 and $100 \mu \mathrm{g} / \mathrm{ml}$. The cellular uptake of fluorescently labeled materials is expressed as the median fluorescence intensity per cell (MFI). Bars represent mean \pm SD. Symbol "*" indicates the significant difference at $p<0.05$. B) Confocal microscopy images of bone marrow-derived macrophages that were either non-stimulated (naive) or stimulated with either IL4 or LPS + interferon $\gamma$, and incubated with $50 \mu \mathrm{g} / \mathrm{ml}$ of Cy5.5-HA-NPs (red, left panel) for 5 hours at $4{ }^{\circ} \mathrm{C}$. The cells were stained with anti-CD44 antibody (green, middle panel) and cell nuclei with DAPI (blue in merged images). In the lower panel, the images of naive macrophages incubated with Cy5HA at the same conditions as mentioned above. Scale bar in upper left image refers to all other images. C) The level of HA-NP uptake in differently stimulated macrophages (described above) incubated for $24 \mathrm{~h}$ with HA-NPs only (black bars), HA-NPs in competition with free HA, added at equal concentrations of $50 \mu \mathrm{g} / \mathrm{ml}$ (white bars), and HA-NPs and anti-CD44 antibody (grey bars). 

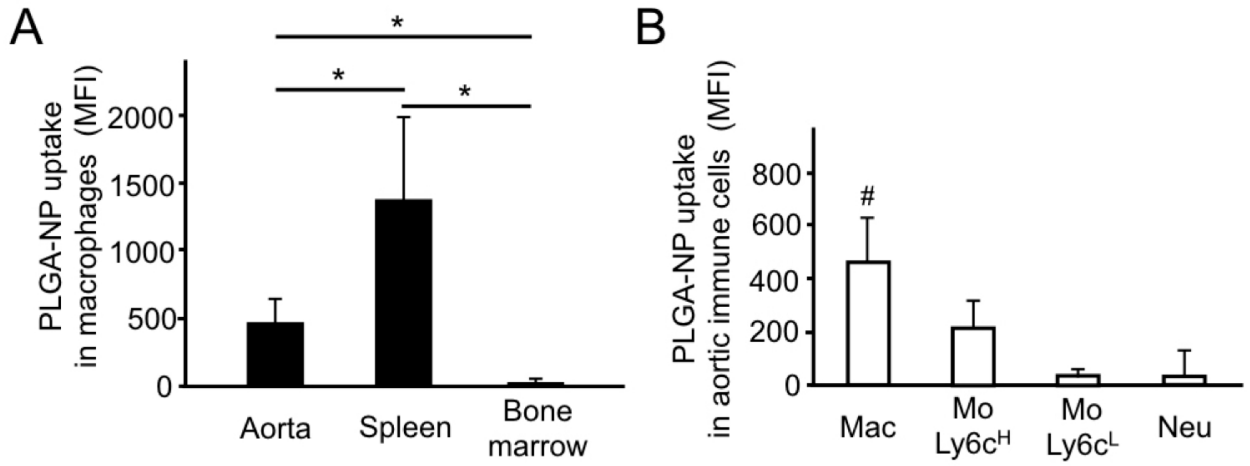

Figure S5I Median fluorescence intensity (MFI) per cell generated by the uptake of Cy5.5-PLGANPs in A) aortic, splenic and bone marrow resident macrophages, and B) different aortic immune cell populations, including macrophages (Mac), monocytes with a high and low expression of Ly6c (Ly6c ${ }^{\mathrm{H}}$ and $\mathrm{Ly}_{6} \mathrm{c}^{\mathrm{L}}$, respectively) and neutrophils (Neu). Bars represent mean $\pm \mathrm{SD}$. Symbol "** represent the significant difference at $\mathrm{p}<0.05$. Symbol "\#" indicates significantly higher values compared to all other conditions at $\mathrm{p}<0.05$.

A
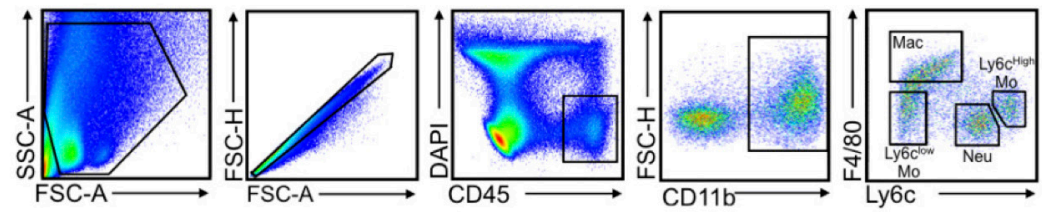

$\mathrm{B}$

Aorta

Blood
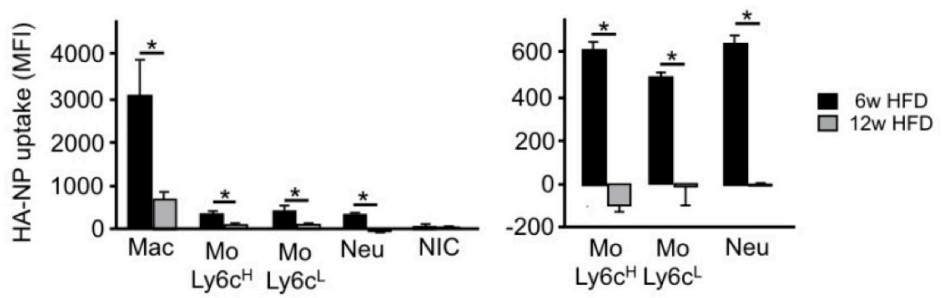

Figure S6 I A) Representative scatter plots of aortic cells displaying gates that ware used to define the macrophages (Mac), Ly6 $\mathrm{c}^{\text {low }}$ and Ly6 $\mathrm{c}^{\text {high }}$ monocytes (Mo) and neutrophils (Neu). B) Median fluorescence intensity (MFI) per cell generated by the uptake of Cy5.5-HA-NPs in aortic (left panel) and blood (right panel) immune cell populations, including macrophages (Mac), monocytes with high and low expression of Ly6c $\left(\mathrm{Ly} 6 \mathrm{c}^{\mathrm{H}}\right.$ and Ly6c $\mathrm{c}^{\mathrm{L}}$, respectively), neutrophils and non-immune cells (CD45 negative) (NIC). The black and grey bars represent the data obtained for mice fed with a high fat diet for 6 (6w HFD) and 12 weeks (12w HFD), respectively. Bars represent mean \pm SD. Symbol " "*" represent the significant difference at $\mathrm{p}<0.05$. 


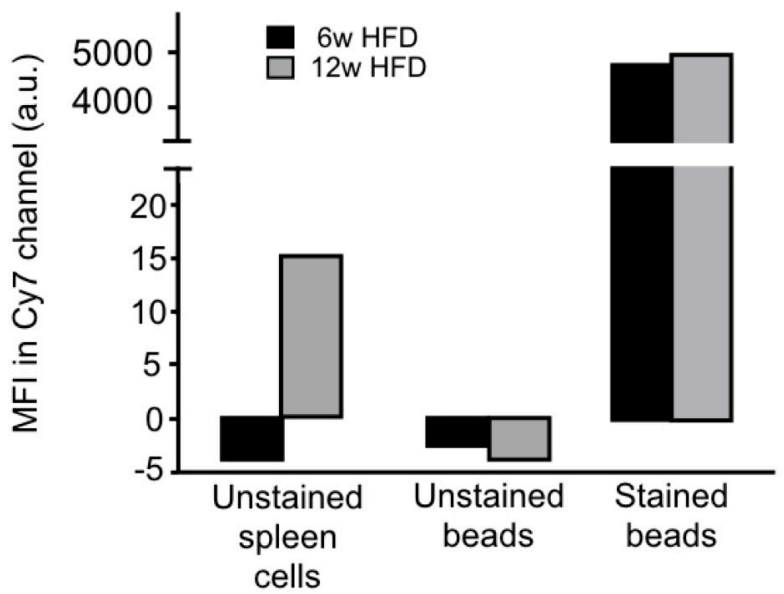

Figure S7 I Median fluorescence intensity (MFI) in Cy7 channel of unstained and Cy7-stained control samples, which show minor inter-measurement variability. The samples were measured at the same session as one of the mouse groups, i.e., 6w HFD (black bars) or 12w HFD (grey bars).

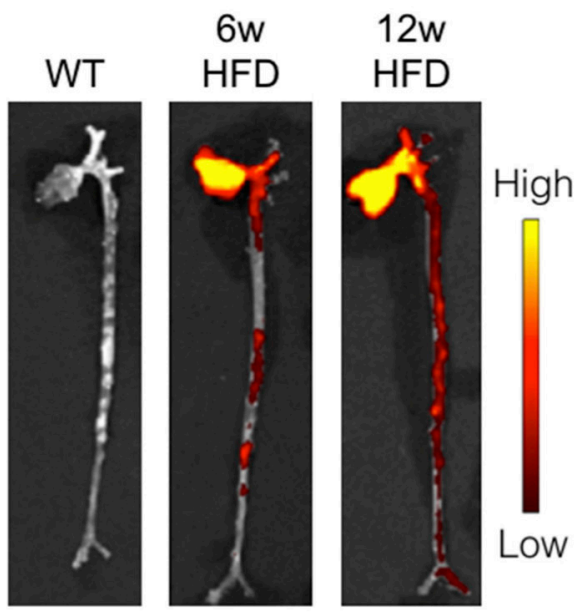

Figure S8 I Representative fluorescence images of mouse aortas without atherosclerosis (WT, left panel), with early atherosclerotic lesions induced by 6 weeks high fat diet (6w HFD, middle panel) and with advanced atherosclerotic lesions induced by 12 weeks high fat diet (12w HFD, right panel). The mice were injected intravenously with Cy5.5-labeled hyaluronan nanoparticles (HA-NPs) $24 \mathrm{~h}$ before the image acquisition on excised aortas. The images were obtained using IVIS Imaging System and radiance efficiency is displayed in a hot color scale. 


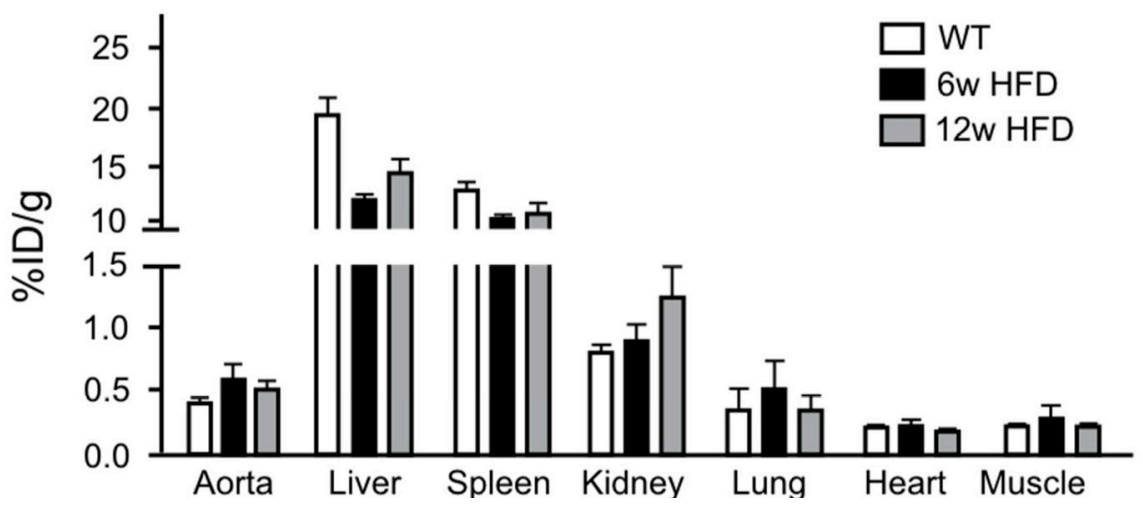

Figure S9| Biodistribution of ${ }^{89} \mathrm{Zr}-\mathrm{HA}-\mathrm{NPs}$ in wild-type mice (WT, white bars), mice with early atherosclerosis (6w HFD, black bars) and mice with advanced atherosclerosis (12w HFD, grey bars). The organs were collected $24 \mathrm{~h}$ after injection of ${ }^{89} \mathrm{Zr}-\mathrm{HA}-\mathrm{NPs}$ and measured by gamma counter. The results were expressed as the percentage of total injected dose (ID) per gram of tissue.

\section{A}

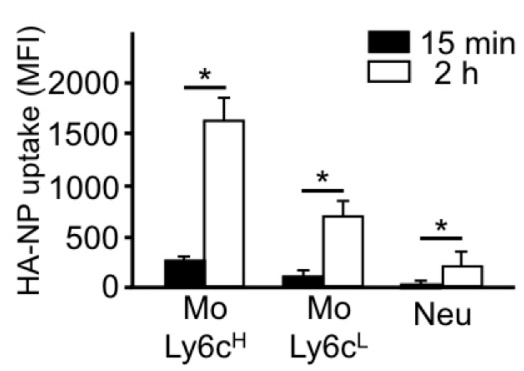

B

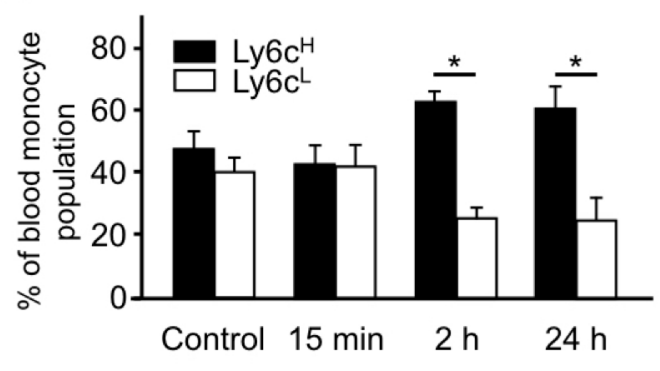

Figure S10 I A) Median fluorescence intensity generated by Cy5.5-HA-NPs in blood circulating Ly6chigh $\left(\mathrm{Ly} 6 \mathrm{c}^{\mathrm{H}}\right)$ and -low $\left(\mathrm{Ly} 6 \mathrm{c}^{\mathrm{L}}\right)$ monocytes and neutrophils (Neu) $15 \mathrm{~min}$ (black bars) and $2 \mathrm{~h}$ (white bars) after intravenous administration of NPs. B) The percentages of Ly6c $\mathrm{c}^{\mathrm{H}}$ (black bars) and Ly6c ${ }^{\mathrm{L}}$ (white bars) monocytes in the total monocyte population in blood of non-injected mice (Control) or mice that received Cy5.5-HA-NPs $15 \mathrm{~min}, 2 \mathrm{~h}$ and $24 \mathrm{~h}$ before the sacrifice. In all graphs, bars represent mean \pm SD. "*” indicate significant difference at $\mathrm{p}<0.05$. 

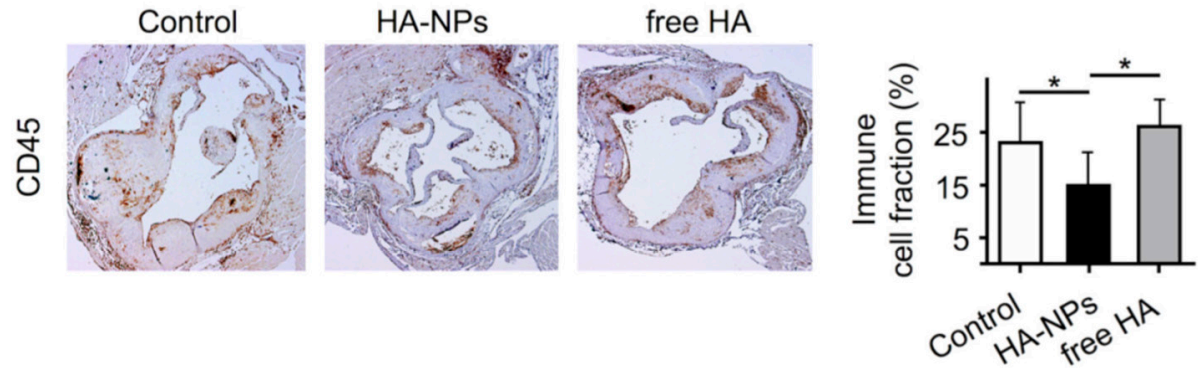

Figure S11 I Representative images of aortic roots from mice that received either PBS (Control, left image panel), HA-NPs (middle image panel) or free HA (right image panel) for 12 weeks of a high fat diet. The sections were stained with immune cell-specific CD45 antibody. The bar chart displays the percentage of plaque area that was positive for staining and represent mean \pm SD. Symbol " $"$ " indicates the significant difference at $\mathrm{p}<0.05$.
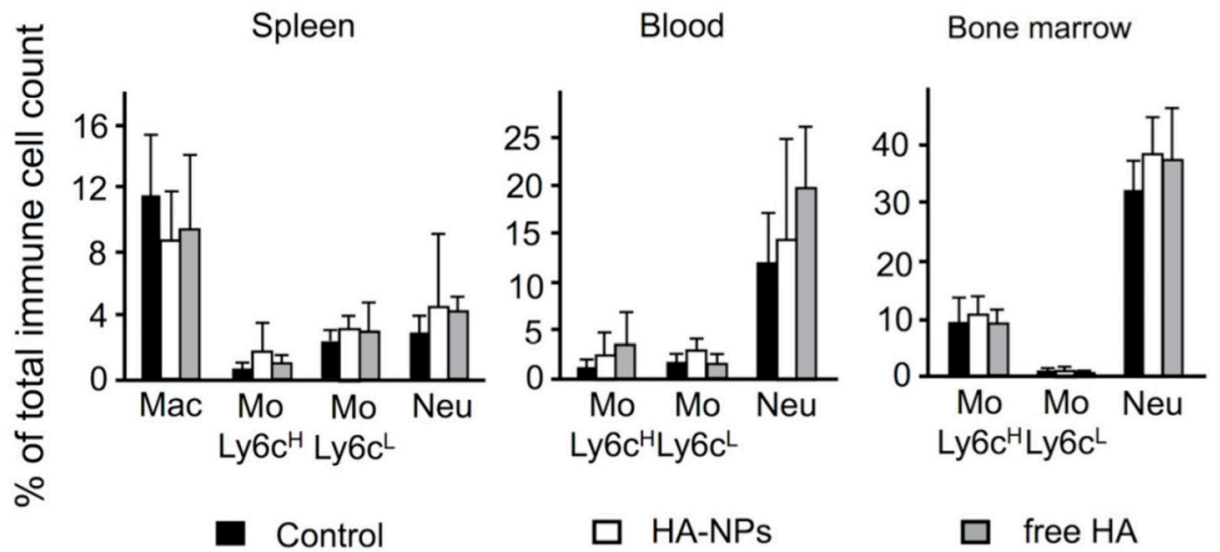

Figure S12 | Flow cytometry measurements of different immune cell population, i.e., macrophages (Mac), Ly6c cow $\left(\mathrm{Ly} 6 \mathrm{c}^{\mathrm{L}}\right)$ and Ly6chigh $\left(\mathrm{Ly} 6 \mathrm{c}^{\mathrm{H}}\right)$ monocytes $(\mathrm{Mo})$ and neutrophils $(\mathrm{Neu})$ in the spleen, blood and bone marrow of the PBS- (black bars), HA-NP- (white bars) and free HA-treated mice (grey bars). Bars represent the mean percentage of immune cell population \pm SD. 

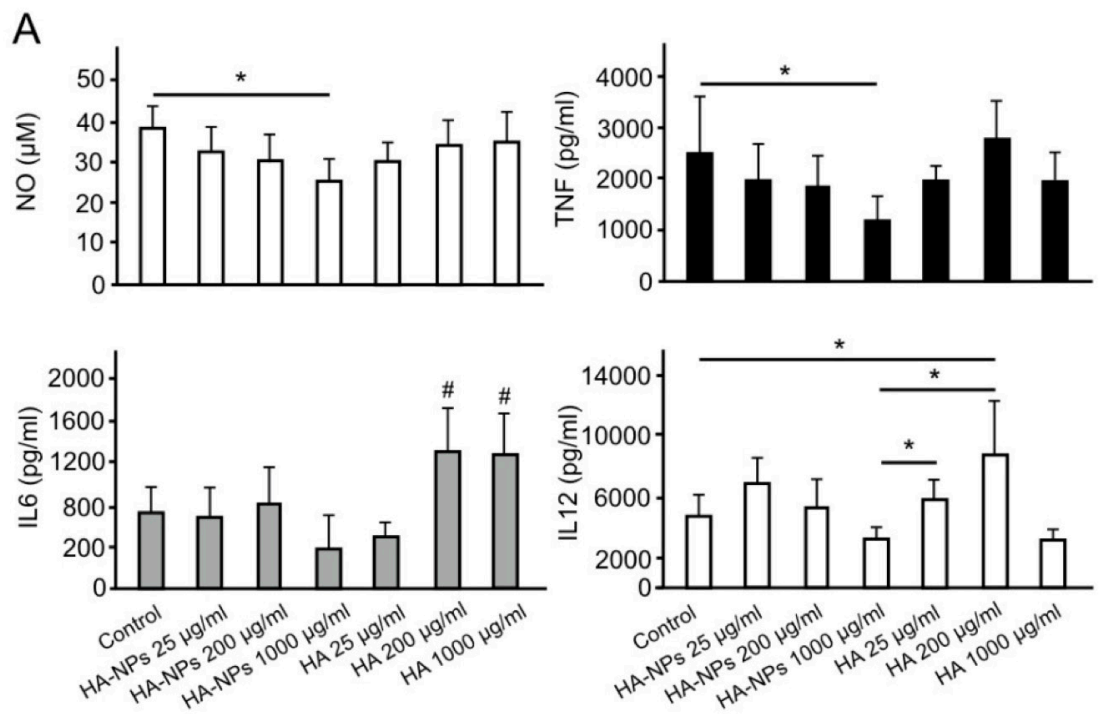

B

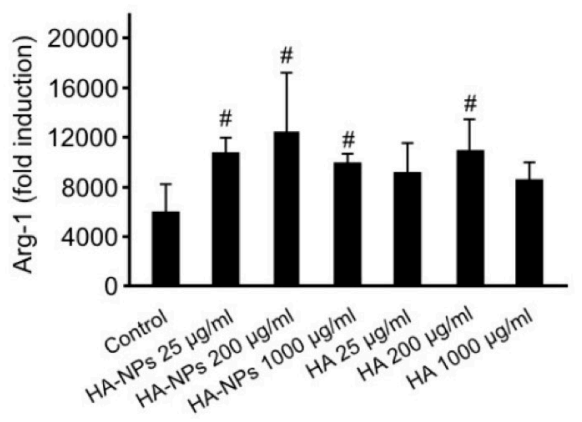

Figure S13 I Anti-inflammatory effects of HA-NPs in bone-marrow-derived macrophages (BMDMs). A) The concentrations of pro-inflammatory markers: nitric oxide (NO) (upper left), tumor necrosis factor (TNF) (upper right), interleukin-6 (IL-6) (lower left) and interleukin-12 (IL-12) (lower right), excreted by BMDMs that were stimulated with lipopolysaccharide (LPS) and interferon- $\gamma$ (INF- $\gamma$ ) for $24 \mathrm{~h}$ (Control) or first pre-stimulated for $24 \mathrm{~h}$ with HA-NPs at three different concentrations i.e., $25 \mu \mathrm{g} / \mathrm{ml}, 200 \mu \mathrm{g} /$ $\mathrm{ml}$ or $1000 \mu \mathrm{g} / \mathrm{ml}$, and subsequently stimulated with LPS and INF- $\gamma$. Free oligomeric HA was used at the same concentrations and using the same protocol as mentioned above. In the lower left panel, "\#" indicates significantly higher concentration compared to Control, HA-NPs (all concentrations) and HA at $25 \mu \mathrm{g} / \mathrm{ml}$. In the lower right panel, significant differences "*" are shown only for Control and HANPs $1000 \mu \mathrm{g} / \mathrm{ml}$. B) The expression of arginase-1 gene (Arg-1) (relative induction compared to naive macrophages), which is associated with pro-fibrotic macrophage phenotype. BMDMs were stimulated either with interleukin-4 (IL-4) for 24h (Control) or first pre-stimulated with HA-NPs or HA for $24 \mathrm{~h}$ at the concentrations mentioned above and subsequently stimulated with IL-4. "\#" indicates significantly higher values compared to Control at $\mathrm{p}<0.05$. In all graphs, bars represent mean \pm SD. All data were tested by one-way ANOVA with LSD post-hoc test. 


\section{APPENDIX II: SUPPLEMENTARY METHODS}

\section{Preparation of poly(lactic-co-glycolic acid)-based nanoparticles (PLGA-NPs)}

First, $6 \mathrm{mg}$ of carboxyl-modified Cy5.5 (Lumiprobe, Hannover, Germany) was dissolved in $893.4 \mu \mathrm{l}$ of dichlorometane (DCM) (Sigma-Aldrich, St Louis, MO, USA) and mixed with $10.66 \mu \mathrm{l}$ of $1 \mathrm{M} \mathrm{N}, \mathrm{N}^{\prime}$-dicyclohexylcarbodiimide (DCC) (SigmaAldrich) for $5 \mathrm{~min}$. At the same time, $1.3 \mathrm{mg}$ of 4-dimethylaminopyridine (DMAP) and $320 \mathrm{mg}$ of PLGA (lactide:glycolide, 50:50; molecular weight: 30-60 kDa; SigmaAldrich) was dissolved in $6.5 \mathrm{ml}$ of DCM. Subsequently, DMAP-PLGA solution was added to the Cy5.5 solution and left in the dark under gentile stirring for 16 hours. After this time, the reaction mixture was dried in a desiccator at room temperature. The dried product was dissolved in $5 \mathrm{ml}$ of acetonitrile (ACN) (Sigma-Aldrich) and precipitated using $20 \mathrm{ml}$ ice-cold methanol. After centrifugation at $6000 \mathrm{~g}$ for $15 \mathrm{~min}$, the supernatant, containing unconjugated Cy5.5, was discarded and the precipitation was repeated 2 more times. The conjugation efficiency of Cy5.5 was determined from the fluorescence emission of the product measured against a Cy5.5 calibration curve. The labeling efficacy was $~ 50 \%$.

$20 \mathrm{~g}$ PLGA-PEG and $4.2 \mathrm{mg}$ of Cy5.5-PLGA was dissolved in $2.42 \mathrm{ml}$ of ACN $(10 \mathrm{mg} / \mathrm{ml})$. To form nanoparticles, ACN solution was dripped into $20 \mathrm{ml}$ of PBS at a rate of $0.2 \mathrm{ml} / \mathrm{min}$ at room temperature under vigorous stirring. The solution was stirred continuously for $1 \mathrm{~h}$ after dripping to induce evaporation of the organic solvent. Subsequently, the sample was centrifuged at $4000 \mathrm{rpm}$ for $10 \mathrm{~min}$ to remove possible aggregates. This was followed by the sample wash with PBS (3 times) using a 100kDa vivaspin (Sartorius, Goettingen, Germany). The nanoparticles were stored at $4{ }^{\circ} \mathrm{C}$ protected from light. They were approximately $50 \mathrm{~nm}$ in diameter, according to DLS measurements, and their blood half-life was around $3 \mathrm{~h}$ (Zhao, Y.et al. Nat. Commun., 2016), which is similar to the HA-NP parameters. 


\section{Determination of hyaluronan nanoparticle morphology}

\subsection{Atomic force microscopy (AFM)}

$50 \mu \mathrm{l}$ of HA-NPs in ultrapure water $(1 \mu \mathrm{g} / \mathrm{ml})$ were spotted onto freshly cleaved mica plate (Ted Pella, Inc., Redding, CA) and incubated at room temperature for 2 min. Subsequently, the liquid was carefully removed with a paper wipe and the sample was air- dried for 7 days. At least five different regions on the mica surface were examined. Images were collected using a MultiMode AFM with a Nanoscope V controller (Veeco Metrology LLC, Santa Barbara, CA) equipped with an E-scanner in tapping mode at $22-24{ }^{\circ} \mathrm{C}$. All images were recorded using silicon microcantilevers (OMCL-AC160TS-W2, Olympus) withaspringconstantof 2N/m(manufacturerspecified)andatascanrateof1-3Hz.The target amplitude was $300 \mathrm{mV}$ with a set point of $230 \mathrm{mV}$ for all measurements. Images were acquired in different scan directions and at different scales to verify the consistency of the evaluated structures. The diameter of HA-NPs was determined from the AFM images, normalized, binned, and plotted using Matlab (MathWorks, Inc., Natick, MA).

\subsection{Cryo-scanning electron microscopy}

$10 \mu \mathrm{l}$ of the HA nanoparticles solution was sandwiched between two metal discs ( $3 \mathrm{~mm}$ diameter, $0.1 \mathrm{~mm}$ cavities) and cryoimmobilized in a high-pressure freezing device (HPM10; Bal-Tec). The frozen samples were mounted on a holder under liquid nitrogen and transferred to a freeze fracture device (BAF 60; Bal-Tec) by using a vacuum cryotransfer device (VCT 100; Bal-Tec). Samples were fractured at a temperature of $-140{ }^{\circ} \mathrm{C}$ and etched for $5 \mathrm{~min}$ at $-105^{\circ} \mathrm{C}$ at a vacuum higher than $5 \times$ 107 mbar. Samples were observed uncoated in an Ultra 55 SEM (Zeiss, Oberkochen, Germany) by using a secondary electron in-lens detector and a backscattered electron in-lens detector (operating at $1.7 \mathrm{kV}-1 \mathrm{kV}$ ) in the frozen-hydrated state by use of a cryostage at a temperature of $-120^{\circ} \mathrm{C}$.

\subsection{Environmental scanning electron microscopy}

An XL30 ESEM-FEG (FEI) was used to study the HA nanoparticles under the gradual dehydration by changing relative humidity (rh). This was achieved by controlling vapor pressure in the observation chamber, while keeping the other conditions stationary at $5 \mathrm{C}$ and at $25 \mathrm{kV}$, making use of the factory settings that have been programmed into the ESEM using an isobar that associates the pressure, temperature and relative humidity.74 The samples were first imaged wet at 7 Torr (referred to as wet). They were then dried at $0 \% \mathrm{rh}$ ( 0.1 Torr) and imaged at different humidity, at the water pressure less than 6 Torr samples was observed as totally dry. The best results were obtained at the water pressure between 6.5 Torr and 6.3 Torr. 


\subsection{Super-resolution fluorescence microscopy}

Super-resolution microscopy images of HA-NPs were acquired using Leica SR GSD system (Leica Microsystems, Son, Netherlands), using the direct stochastic optical reconstruction microscopy (dSTORM) technique. For sample preparation, round cover slide glasses (24 mm diameter, thickness $1.5(0.16-0.19 \mathrm{~mm})$, Thermo Fisher Scientific) were cleaned with $1 \mathrm{M} \mathrm{KOH} \mathrm{(1h} \mathrm{incubation)} \mathrm{and} \mathrm{PBS} \mathrm{(4} \mathrm{washes).}$ Subsequently, they were coated with $100 \mathrm{mg} / \mathrm{ml}$ of poly-D-lysine (Merck Millipore, Darmstadt, Germany) for $0.5 \mathrm{~h}$ and washed extensively with PBS. Cy5.5-HA-NPs were diluted down to approximately $1 \mathrm{ng} \mathrm{HA} / \mathrm{ml}$ of PBS and administered on the coated glass surface. After few seconds of incubation, the glasses were washed extensively with PBS. The sample was imaged using a buffer, which contained 20 $\mathrm{mM}$ cysteamine (Sigma-Aldrich), 1\% (v/v) mercaptoethanol (Sigma-Aldrich), $170 \mathrm{U}$ of glucose oxidase type seven from Aspergillius (Sigma-Aldrich), $1400 \mathrm{U}$ of catalase from bovine liver (Sigma-Aldrich) and 10\% (w/v) of glucose in $50 \mathrm{mM}$ Tris- $\mathrm{HCl}$ and $10 \mathrm{mM} \mathrm{NaCl}$ solution.

The 2D images were acquired using $642 / 500 \mathrm{~mW}$ laser and $160 \times$ oil immersion objective. $18 \times 18 \mu \mathrm{m} 2$ images were acquired using the exposure time of $10 \mathrm{~ms}$ and by collecting 40,000 frames. The detection threshold was 20 photons and in-plane resolution was $10 \mathrm{~nm}$. Data analysis was performed in Fiji using the ThunderSTORM plugin software [75].

The HA-NP morphology was also investigated after the in vivo administration of Cy5.5- labeled nanomaterial in atherosclerotic mice. $2 \mathrm{~h}$ after intravenous injection of Cy5.5-HA- NPs, the mice were sacrificed, the aortae were excised and fixed using $4 \%$ PFA for $10 \mathrm{~min}$. The vessel samples were subsequently cut-open longitudinally to expose the endothelial surface. The endothelial lining was stained with rat antimouse CD31 primary antibody and goat anti-rat Alexa Fluor 555 secondary antibody. Just before imaging, the vessel samples were incubated in an imaging buffer $(100 \mu \mathrm{l}$ of sodium DL-lactate, $50 \mu \mathrm{l}$ of $0.5 \mathrm{M} \beta$-mercaptoethylamine hydrochloride (MEA) ( $\mathrm{pH}$ 8-8.5), $3 \mu \mathrm{l}$ of $5 \mathrm{M} \mathrm{NaOH}, 15 \mu \mathrm{l}$ of $3 \%$ (v/v) OxyFluorTM (Oxyrase Inc., Mansfield, Ohio, USA) and $350 \mu \mathrm{l}$ of PBS) and mounted in a drop of the same buffer between a histological glass and a coverglass (\#1.5).

Samples were images on a Leica SR-GSD microscope using 532/500mW and $642 / 500 \mathrm{~mW}$ lasers, and 160× oil immersion objective. First, Cy5.5 (HA-NP label) was imaged, followed by Alexa Fluor 555 (CD31). Around 10,000 frames were collected with an image size of $18 \times 18 \mu \mathrm{m} 2$. The images were reconstructed with a threshold of 50 photons and a pixel size of $10 \mathrm{~nm}$ by Leica image software. 


\section{Determination of hyaluronan breakdown products after hyaluronidase treatment}

\subsection{Colorimetric assessment of terminal $\mathrm{N}$-acetylglucosamine}

The determination of N-acetyl-D-glucosamine, which becomes exposed at terminal ends of hyaluronan as a result of hyaluronidase (HYAL) activity, was performed using the colorimetric method described previously by Reissig et al.76 First, HA and HA-NPs were diluted in PBS pH 6 to the concentration of $0.75 \mathrm{mg} /$ ml. Subsequently, HYAL (Sigma- Aldrich) was added at the final concentration of $100 \mathrm{U} / \mathrm{ml}$ and incubated in water bath at $37^{\circ} \mathrm{C}$. The samples have been probed at several time points during incubation with HYAL i.e., at $0 \mathrm{~min}$ (before adding enzyme), $2 \mathrm{~min}, 5 \mathrm{~min}, 15 \mathrm{~min}, 30 \mathrm{~min}, 45 \mathrm{~min}, 1 \mathrm{~h}, 2 \mathrm{~h}, 4 \mathrm{~h}, 6 \mathrm{~h}, 24 \mathrm{~h}$ and $48 \mathrm{~h}$ after adding HYAL. HA and HA-NPs without HYAL addition were used as negative controls. N-acethyloglucosamine (Sigma-Aldrich) served as positive control. At each timepoint, $100 \mu \mathrm{l}$ of sample was aspired and diluted up to $0.5 \mathrm{ml}$ in PBS in a $13 \times 100 \mathrm{~mm}$ test-tube, and heated for $5 \mathrm{~min}$ at $100{ }^{\circ} \mathrm{C}$ to stop the enzyme reaction. Subsequently, $0.1 \mathrm{ml}$ of $0.8 \mathrm{M}$ potassium tetraborate $(\mathrm{pH} 9)$ was added. The mixture was heated in a heating block at $100{ }^{\circ} \mathrm{C}$ for exactly 3 minutes and cooled in tap water. $3 \mathrm{ml}$ of $\mathrm{p}$ - dimethylaminobenzaldehyde (DMAB) reagent (10 g of DMAB dissolved in $100 \mathrm{ml}$ of glacial acetic acid containing $12.5 \%$ (v/v) of $10 \mathrm{~N} \mathrm{HCl}$; shortly before use it was diluted with 9 volumes of glacial acetic acid) are then added, and, immediately after mixing, the tubes are placed in a water bath at $37^{\circ} \mathrm{C}$. After 20 minutes the tubes were cooled under tap water and the absorbance was measured at $544 \mathrm{~nm}$ wavelength. The experiment was performed in triplicate and using three different batches of HA-NPs.

\subsection{Determination of hyaluronan polymerization by gel electrophoresis}

The mobility of HA-NPs and their breakdown products was investigated by gel electrophoresis. Free hyaluronan of different molecular weights (Lifecore Biomedical), i.e., $\sim 75 \mathrm{kDa}(66-99 \mathrm{kDa}), \sim 400 \mathrm{kDa}(300-450 \mathrm{kDa})$ and $\sim 1.5 \mathrm{MDa}$ (1.0-1.8 MDa), was used as a reference. The electrophoresis was performed using polyacrylamide gels with a concentration gradient of $4-20 \% 8 \times 8 \mathrm{~cm}$ (Invitrogen), TBE buffer (Invitrogen) and X-cell SureLock Mini Cell apparatus. Hyaluronan samples were prepared at the concentration of $1 \mathrm{mg} / \mathrm{ml}$ in PBS $\mathrm{pH}$. The samples were incubated for $2 \mathrm{~h}$ at $37^{\circ} \mathrm{C}$ either without or with HYAL, which was added at the final concentration of $100 \mathrm{U} / \mathrm{ml}$. Subsequently, the samples were heated for $5 \mathrm{~min}$ at $100{ }^{\circ} \mathrm{C}$ to deactivate the enzyme. $20 \mu \mathrm{l}$ of each sample was mixed with $5 \mu \mathrm{l}$ of highdensity loading buffer and pippeted into to gel wells. Electrophoresis was carried out at room temperature at the constant voltage of $300 \mathrm{~V}$ for $\sim 40 \mathrm{~min}$. After the run, 
gels were removed from the cassette and stained with $0.005 \%$ Stains-All solution in $50 \%$ ethanol for $1 \mathrm{~h}$. Subsequently, the gel was destained in $10 \%$ ethanol solution and scanned in transmission mode using GenoSmart gel system (GenoSmart Ltd, Hod Hasharon, Israel).

\subsection{Size exclusion analysis of HA-NPs and their hydrolysis products}

Fast protein liquid chromatography (FPLC) measurements of HA-NPs and their hydrolysis products (treatment as described above) were performed on a Shimadzu HPLC system equipped with two LC-10AT pumps and an SPD-M10AVP photodiode array detector, and using a Superdex 75 10/300 GL column (GE Healthcare Life Sciences, Pittsburgh, PA). PBS was used as an eluent at a flow rate of $1.0 \mathrm{~mL} / \mathrm{min}$. Oligomeric HA (60-90kDa) and its hydrolysis products were used as comparison for HA-NP samples. The exclusion limit was 1.3 MDa, which corresponded to the retention time $\left(t_{R}\right)$ of $9 \mathrm{~min}$. The following size standards were used: thyroglobulin $670 \mathrm{kDa}, \mathrm{t}_{\mathrm{R}}$ : 9.8 min; y-globulin $158 \mathrm{kDa}, \mathrm{t}_{\mathrm{R}}$ : $13.4 \mathrm{~min}$, ovoalbumin $44 \mathrm{kDa}, \mathrm{t}_{\mathrm{R}}: 16.6$ min, myoglobulin $17 \mathrm{kDa}, \mathrm{t}_{\mathrm{R}}: 19.1 \mathrm{~min}$, vitamin $\mathrm{B} 121.35 \mathrm{kDa}, \mathrm{t}_{\mathrm{R}}: 22.8 \mathrm{~min}$. Albumin's $(67 \mathrm{kDa}) \mathrm{t}_{\mathrm{R}}$ was $14.7 \mathrm{~min}$.

\section{Bone marrow-derived macrophage experiments}

Bone marrow cells were isolated from mouse femur and cultured in RPMI medium supplemented with L929-conditioned medium to generate bone marrow-derived macrophages, as described by Peiser et al [77]. To induce the pro-inflammatory macrophage phenotype, cells were stimulated with $10 \mathrm{ng} / \mathrm{ml}$ of lipopolysaccharide (LPS) (Sigma-Aldrich) and $10 \mathrm{U} / \mathrm{ml}$ interferon gamma (IFN- $\gamma$ ) (PeproTech, London, UK) for $72 \mathrm{~h}$. To stimulate macrophages towards the anti-inflammatory phenotype, cells were treated with $20 \mathrm{ng} / \mathrm{ml}$ of interleukin 4 (IL-4) (PeproTech) for $72 \mathrm{~h}$. Furthermore, cells were co-stimulated with oxidized low-density lipoprotein(oxLDL) (Alfa Aesar, Massachusetts, USA) at three different concentrations: $25 \mu \mathrm{g} / \mathrm{ml}, 50 \mu \mathrm{g} /$ $\mathrm{ml}$ or $100 \mu \mathrm{g} / \mathrm{ml}$, or contained no oxLDL. The naive macrophages were either nonstimulated or stimulated with oxLDL. After $48 \mathrm{~h}$ from the start of stimulation, Cy5.5HA-NPs were added at $200 \mu \mathrm{g}$ HA/ ml. $24 \mathrm{~h}$ thereafter, the cells were washed 3 times with PBS, detached and analyzed by flow cytometry (FACSCanto; BD Biosciences, San Jose, CA, USA). The median flourescence intensity/ cell was used as a quantifier of the Cy5.5-HA-NP uptake. 




\section{CHAPTER 8}

\section{Summary and Perspectives}

\section{SUMMARY}

Atherosclerosis, a chronic inflammatory vascular disease, which has been recently identified in 5000-year mummies [1] remains undefeated. It is the common underlying cause of deadly cardiovascular diseases (CVD), including heart attacks, strokes, peripheral vascular diseases. Collectively, CVD is the number one killer among the non- communicable diseases globally (17.7 million people annually), far more deadly than cancer ( 8.8 million) [2,3]. This tremendous socioeconomic burden calls for further investigation and investment to develop effective, innovative, and clinically viable interventions for the treatment of atherosclerosis. One important initiative in this direction is NanoAthero, a European Consortium that funded the research work presented in this thesis. This program aims to demonstrate the preliminary clinical feasibility of the use of nanosystems for targeted imaging and treatment of advanced atherosclerosis [4]. The enthusiasm generated for the use of nanocarrier drug delivery systems in atherosclerosis is mainly driven by the significant progress made in the field of oncological nanomedicine [5]. Capitalizing on the achievements in the nanomedicine field, the main aim of this thesis is to contribute to the development and use of targeted nanomedicines in atherosclerosis. To this end, we adopted a 'disease first' approach to develop efficient targeted nanomedicines, in which particular attention is paid to the underlying pathophysiological processes in atherosclerosis. Macrophages are key players in these processes that affect atherosclerotic plaque inflammation and vulnerability to rupture. Moreover, their phagocytic capacity makes macrophages ideal targets for nanomedicine-based approaches. Understanding the role of plaque-associated macrophages and their interactions with the different nanocarriers is crucial for the successful development of efficacious, clinically relevant nanotherapeutics for atherosclerotic cardiovascular diseases.

Chapter 1 represents a general introduction to the subject and an outline of this thesis. Chapter 2 provides an overview of the interplay between the advances in nanomedicine and our understanding of chronic inflammatory disorders, including atherosclerosis. Although atherosclerosis and other chronic inflammatory diseases may represent different phenotypical outcomes, they possess a common denominator, inflammation, and share interactive pathophysiological features in which monocyte recruitment, macrophage polarization, and enhanced vascular permeability play critical roles. Understanding and exploiting the commonalities in 
monocyte/macrophage dynamics and functions in chronic inflammatory diseases can facilitate efficient nanomedicine development for such diseases.

The research dealing with the application of nanomedicines for targeting inflammatory disorders starts in Chapter 3, which describes the use of the omega-3 ( $\omega 3$ ) polyunsaturated fatty acid docosahexaenoic acid (DHA) as a nature-inspired, biologically active component incorporated into PEGylated liposomes. $\omega 3$ polyunsaturated fatty acids (PUFAs) are widely used orally by patients suffering from atherosclerotic cardiovascular diseases and cancer, which share common key pathophysiological features, including inflammation, oxidative stress, and uncontrolled cell proliferation [6,7]. $\omega 3$ PUFAs can be used to resolve the underlying inflammation via their anti-inflammatory effects and as precursors of pro-resolving lipid mediators [8]. However, the beneficial effects of $\omega 3$ fatty acids cannot be fully exploited unless diets are enriched with high concentrations and used over long periods of time (ranging from 0.4 to $4 \mathrm{~g}$ / day for at least four months, Clinical Trial Identifiers: NCT01078909 and NCT01865448). Chapter 3 introduces a nanomedicinebased approach based on DHA liposomes to achieve enhanced delivery of PUFAs to inflammatory cells [9]. Liposomes were chosen as they are the front runner platform in the field of targeted nanomedicine, and are suitable for parenteral administration. We found that DHA liposomes ( $\omega$-liposomes) displayed similar physicochemical characteristics to control liposomes. The mean diameter and size distribution of the $\omega$-liposomes remained unchanged compared to control liposomes, and retained their activity upon storage for more than five weeks. $\omega$-liposomes showed antioxidant and anti-inflammatory effects in vitro, as demonstrated by the inhibition of the production of reactive nitrogen/oxygen species, monocyte chemotactic protein 1 (MCP1), and Tumor necrosis factor alpha (TNF $\alpha)$, and reducing $\mathrm{NF} \kappa \mathrm{B}$ activation in activated immune cells. Moreover, the treatment with the $\omega$-liposomal formulation inhibited tumor cell proliferation. We concluded that this nanonutraceutical formulation has promising potential and deserves further research attention.

The majority of nanopharmaceutical applications are targeted delivery systems containing small molecule drugs. The selection of an appropriate drug candidate is critical for successful targeted drug delivery strategy in atherosclerosis. Chapter 4 describes multiple pathway screening assays to select small molecule drug candidate(s). We evaluated the effects of these compounds on the primary pathways that involve macrophages in atherosclerosis, including lipid metabolism, endoplasmic reticulum (ER) stress, macrophage cell proliferation, the release of proinflammatory cytokines, and unbalanced oxidative stress. We found that the anti-inflammatory effect of prednisolone might be counterbalanced by adverse effects on the lipid efflux and ER stress pathways. Conversely, pterostilbene (a natural anti-oxidant molecule), liver $X$ receptor (LXR) agonists (lipid efflux stimulators), and statins (HMG-CoA reductase inhibitors) were identified to have an overall superior antiatherogenic effect, attributed to their beneficial impact on inflammation, oxidative stress, lipid handling, and macrophage proliferation. Since both LXR agonists and statins are small molecule drugs which have advanced to clinical studies/use, we 
further investigated these compounds for targeted drug delivery approaches in the following two chapters using animal models of atherosclerosis.

In Chapter 5, the LXR agonist GW3965, which failed in clinical trials due to liver toxicity [10], was investigated for a nanoparticle-based drug delivery approach in the apolipoprotein E-deficient $\left(A p o e^{-1-}\right)$ mouse model of atherosclerosis [11]. First, a combinatorial library of 17 nanoparticles (NP) with distinct physiochemical properties (mainly size and chemical composition) and different immune cell specificities was developed and evaluated in vivo. The library's nanoparticles are based on the natural high- density lipoprotein, which can preferentially deliver therapeutic compounds to pathological macrophages in atherosclerosis. To improve the therapeutic index of an LXR agonist, plaque macrophage-targeted delivery, with minimal delivery to nonpathological cells in other tissues, is essential. Using a combination of optical methods, immunological techniques, and in vivo positron emission tomography (PET) imaging, two nanoparticles from the library were carefully selected for subsequent safety and efficacy studies. The hydrophobic GW3965, referred to as Rx in the chapter, was loaded into the first nanocarrier candidate, HDL nanoparticles (NP 10, Rx-HDL), and found to have high cholesterol efflux efficiency, a relatively long blood half-life, high relative aorta-to-liver accumulation, and a high relative aortic-to splenic macrophage association ratio. The second candidate (Rx- PLGAHDL) was based on polylactic-co-glycolic acid (PLGA) core HDL nanoparticles (NP 14) and showed unfavorable characteristics for GW3965 delivery such as poor cholesterol efflux efficiency, relatively short blood half-life, and low relative aortato-liver accumulation. The Rx-PLGA-HDL increased the expression of two of the three major GW3965-related toxicity genes, while the Rx-HDL's optimal in vivo performance abolished liver toxicity. In the aortic macrophages, Rx-HDL increased expression of five GW3965 target genes compared with the vehicle HDL control, whereas Rx-PLGA-HDL increased expression of only four genes compared with the vehicle PLGA-HDL control. Treatment with Rx-HDL resulted in $28 \%$ less total lipid in aortic macrophages. Furthermore, the treatment reduced total lipid levels in monocytes by $43.0 \%$ and in all $\mathrm{CD} 45^{-}$cells by $40.0 \%$. In conclusion, targeted delivery of the LXR agonist GW3965 to atherosclerotic plaque macrophages using HDL nanoparticles improved the therapeutic efficacy of the drug while eliminating its hepatotoxicity. Therefore, screening nanoparticles' specificity to immune cells appears to be a viable strategy to develop tailored therapies for atherosclerosis and other inflammatory diseases.

Chapter 6 discusses a comprehensive head-to-head analysis of three clinically viable nanomedicines targeting the HMG-CoA reductase inhibitor simvastatin [S] to advanced atherosclerotic plaques in $A p o e^{-1-}$ mice. Intravenous nanocarriermediated targeting of HMG-CoA inhibitors to atherosclerotic plaques is an attractive approach to achieve enhanced anti-inflammatory and anti-atherosclerotic effects, beyond statins' lipid lowering effects [12]. In this study, we compared [S]HDL, [S]-N-(2-hydroxypropyl)- methacrylamide benzyl mPEG-b-p(HPMAmBz))-based PEGylated polymeric micelles (mPEG-b-p(HPMAm-Bz); [S]-PM), and 
[S]-PEGylated liposomes ([S]-LIP). Liposomes were included as they are often regarded as the standard nanomedicine for targeted drug delivery and were the first to advance to the clinical stage. Additionally, liposomes have been shown to successfully target atherosclerotic plaques in rabbits [13] and humans [14]. HDL, a natural self-assembled nanocarrier, has an intrinsic affinity for plaque macrophages in different atherosclerosis animal models, as we showed in Chapter 5. The natural targeting ability of HDL and the possibility to incorporate hydrophobic compounds in its core makes it an attractive platform for drug delivery. A new generation of HPMA block copolymer-based micelles was shown to improve the retention of hydrophobic chemotherapeutic drugs in their matrix by $\pi-\pi$ stacking [15], offering effective targeted drug delivery to tumors [16] and inflammatory sites [17]. However, they have not yet been appraised in atherosclerosis. Here, we explored their ability to target atherosclerotic plaques for the first time and compared their performance with previously assessed HDL and liposomal formulations. Our head-to-head comparison disclosed marked differences between the platforms:

- Superior drug retention in vitro in [S]-PM under physiological conditions,

- Comparable and longer circulation half-lives of [S]-PM and [S]-LIP

- Similar accumulation of the three [S]-nanoformulations in atherosclerotic plaques

- Distinct uptake of [S]-nanomedicines within the lesion by plaque-associated macrophages and monocytes

- Improved efficacy of [S]-PM in reducing plaque macrophage burden

These findings underscore the importance of studying the drug retention in nanoparticles while they are circulating in the bloodstream and their uptake by target immune cells. Moreover, our head-to-head approach demonstrates the value of applying or repurposing nanomaterials to deliver compounds in maladaptive inflammation-associated diseases, beyond cancer.

Chapter 7 presents a different innovative strategy involving hyaluronan nanoparticles (HA-NPs) to target plaque macrophages and to increase atherosclerotic plaque stability. Hyaluronan is a linear biologically active polymer that displays unique physicochemical and biological properties [18]. As a primary component of the extracellular matrix, and through interaction with several hyaluronan receptors, including CD44, ICAM-1, and TLR-4, hyaluronan regulates cell adhesion, migration, and proliferation [19]. At the inflammatory lesions, hyaluronan and hyaluronanbinding proteins on the vascular endothelium mediate rolling, extravasation, and proliferation of immune cells, which can drive the initiation and progression of chronic inflammatory diseases [20], including atherosclerosis. Such unique biological activities of hyaluronan, supplemented with non-immunogenicity and cost-effective production, have boosted its biomedical applications [21], mainly in arthrology, ophthalmology and aesthetic medicine. The systemic use of hyaluronan is challenging due to its rapid blood clearance and susceptibility to hydrolysis [22]. We exploited hyaluronan's supramolecular chemistry to formulate nanoparticles. Several advanced microscopy methods, including atomic force microscopy, cryo- 
scanning electron microscopy, and super-resolution fluorescence microscopy were used to assure the morphological and chemical stability of HA-NPs. Flow cytometry showed 6- to 40-fold higher uptake of Cy7-HA-NPs by aortic macrophages compared to normal tissue macrophages. Remarkably, both local and systemic HANP-immune cell interactions significantly decreased over the disease progression period. Additionally, ${ }^{89} \mathrm{Zr}$ - HA-NPs accumulated in atherosclerotic aortas of Apoe $e^{-1-}$ mice $30 \%$ higher than in wild-type controls. The translational potential of HA-NPs was also investigated in a rabbit model of atherosclerosis. PET imaging of rabbits revealed 6-fold higher standardized uptake values of HA-NPs in the atherosclerotic aorta compared to muscle tissue. To investigate HA-NPs therapeutic effects, Apoe $e^{-1-}$ mice were injected intravenously with $50 \mathrm{mg} / \mathrm{kg}$ / week of HA-NPs during a 12 week high-fat diet feeding period. The atherosclerotic lesions were significantly smaller and contained approximately $30 \%$ fewer macrophages compared to both the control and free HA-treated mice. Also, atherosclerotic plaques from mice treated with HA-NPs displayed 30-40\% higher collagen content, which is considered a marker of plaque stability. In conclusion, biocompatible HA-NPs were developed, which should be further investigated for the potential anti-inflammatory effects.

\section{PERSPECTIVE}

This thesis adopts a disease-based approach for selecting drug candidates and for developing targeted nanomedicines to treat inflammatory atherosclerosis. Although the in vitro and in vivo results obtained show that individual small molecule compounds and nanoplatforms are potential candidates for the treatment of atherosclerosis, further optimization is needed to facilitate clinical translation.

\subsection{Bioactive natural compounds and inflammation resolution}

Selection of the right combination of the therapeutic cargo and the nanocarrier is critical for developing efficient targeted nanomedicines for atherosclerosis. Two of the compounds (i.e. LXR agonist and simvastatin) that were rated highly as antiatherogenic in Chapter 4 were successfully developed into HDL- or polymeric micellebased nanomedicines and showed their potential in experimental atherosclerosis (Chapter 5 and 6). However, pterostilbene, a natural compound ranked the highest as anti-atherogenic in Chapter 4 due to its anti-inflammatory, antioxidant, and lipid efflux activity, needs further investigation. In an early pilot study, we conducted an experiment in atherosclerotic mice using pterostilbene phosphate containing liposomes (PPL). However, this study did not show any differences between control empty liposomes and the PPL formulation (unpublished data). The following extensive in vivo studies in Chapter 5 and 6 demonstrated that liposomes 
show limited uptake by plaque-associated macrophages and may not be an ideal nanocarrier for targeted drug delivery in mice with advanced atherosclerosis. Thus, further investigation is warranted to validate the effects of pterostilbene delivery by other carrier systems, herein HDL or HPMA polymeric micelles, in experimental atherosclerosis.

Promoting inflammation resolution in atherosclerotic plaques may lead to improved therapeutic outcomes. The small molecule drugs studied in this thesis demonstrate anti-inflammatory actions, through different pathways. Resolution and anti-inflammation are not synonymous. Resolution is an active process that results in cessation of proinflammatory signaling, removal of debris from inflamed lesions, and the return to homeostatic conditions (i.e. tissue repair) [23]. Investigating targeted delivery of pro-resolving molecules in vivo can result in such dual antiinflammatory and pro-resolving effects. Examples of precursors of pro-resolving molecules are eicosapentaenoic acid (EPA) and docosahexaenoic acid (DHA) (Chapter 3), their derivatives such as docosahexaenoic acid docosahexaenoic acidcontaining phospholipids and triglycerides, and their pro-resolving lipid mediators / metabolites such as resolvin D1, resolvin D2, and maresin 1 [24]. These bioactive lipids can be easily incorporated into lipid-based nanoparticles to formulate a bioactive carrier per se (work in progress). Also, small molecule drugs may be coincorporated to achieve multiple-targeted nanotherapeutics.

More examples of bioactive lipids are oxysterols, which are cholesterol metabolites. Such compounds (e.g. desmosterol) have a plethora of physiological effects, including regulation of cholesterol, lipid, and glucose homeostasis [25]. Additionally, oxysterols can activate nuclear receptors such as LXRs, cholesterol sensing nuclear receptors, and can modulate inflammatory response [26]. A high level of desmosterol was showed to inhibit pro-inflammatory gene expression in atherosclerotic lesions through both LXR-dependent and LXR-independent mechanisms [27]. These bioactive lipids share a similar structure with cholesterol (Figure 1), an important constituent of some lipid-based nanoparticles. Thus, oxysterol containing nanoparticles can be used to enrich oxysterol levels in atherosclerotic plaque, improving therapeutic outcome. 
A

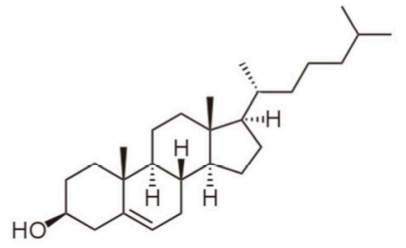

B

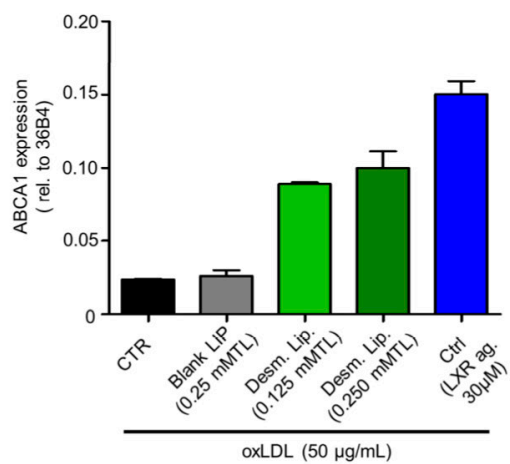

Desmosterol

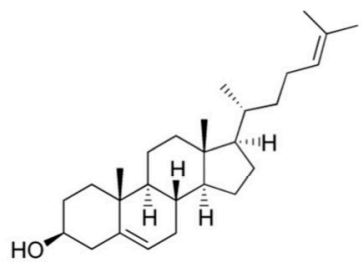

C

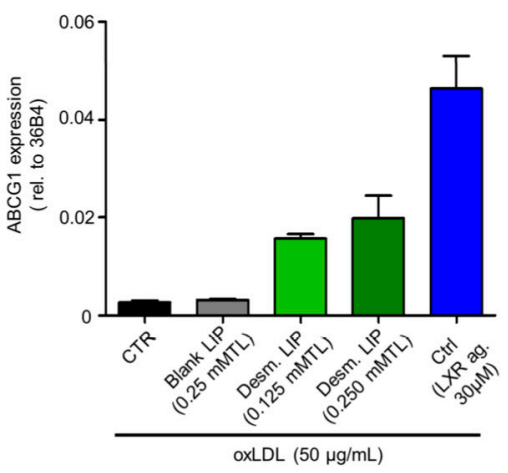

Figure 1| A) The chemical structure of cholesterol and desmosterol. Effect of blank liposomes (Blank LIP) and desmosterol liposomes (Desm. LIP) on gene expression of Cholesterol Transporters B) ABCA1 and C) ABCG1, relative to the housekeeping gene 36B4, in THP-1 macrophage. Desm.LIP (0.25 mM TL equivalent to $50 \mu \mathrm{g} / \mathrm{mL}$ desmosterol; $0.125 \mathrm{mM}$ TL equivalent to $25 \mu \mathrm{g} / \mathrm{mL}$ desmosterol). LXR ag. (LXR agonist) T0901317 served as a positive control. mM TL: millimolar total lipid.

\subsection{Tailoring disease-specific nanomedicines}

This thesis focuses on targeted drug delivery to innate immune cells, such as macrophages/monocytes in atherosclerosis, as they are the key effector cells and the most prone to take up nanomedicines. Our results demonstrated that $\omega$-liposomes (Chapter 5), LXR agonist-HDL (Chapter 5), simvastatin-loaded HDL and polymeric micelles (Chapter 6), and hyaluronan nanoparticles (Chapter 7) modulate the phenotype and number of plaque macrophages. However, other disease-related elements need to be investigated further as well. For example, the memory of monocyte/macrophages, a novel concept known as 'trained immunity', can be modulated by nanomedicines and may have long-term consequences on the progression of the disease [28]. Other aspects to consider are the effect of nanomedicines on the activation of adaptive immune cells, such as T and B cells, and the paracrine signaling between innate and adaptive immunity. The presence of activated $\mathrm{T}$ cells in atherosclerotic plaques indicates that they can affect disease progression, yet the mechanisms remain unclear [29]. Moreover, dissecting the impact

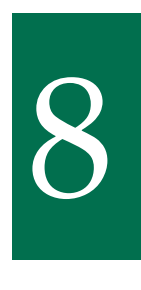


of targeted therapies on innate and adaptive immunity and a better understanding of the role of lesion-associated cells, such as smooth muscle cells and fibroblasts, will allow the design of better nanotherapeutics tailored to the characteristics and pathophysiology of the atherosclerotic disease [30].

In addition to targeting the immune cell compartment, plaque phenotype may change during the disease as shown in Chapter 7. Therefore, the strategies to develop targeted therapeutics to treat the early phases of the disease may not be suitable for late stages [31]. Finally, the most likely clinical scenario for nanomedicine application in atherosclerosis is after an acute event, such as myocardial infarction (MI). Validating the effects of nanomedicines in preclinical models that imitate this setting is essential for clinical translation.

\subsection{Imaging-guided revival of nanomedicine}

We employed PET/CT and PET/MR imaging modalities (Chapter 5,6 and 7), which demonstrated their potential to noninvasively investigate different aspects of nanomedicines' behavior in vivo (e.g. pharmacokinetics and biodistribution). However, these powerful imaging modalities can also be utilized to assess the effectiveness of nanomedicines noninvasively. In this context of image-guided therapy, PET/CT or PET/MR imaging of atherosclerotic plaques using 18F-sodium fluoride, a radiotracer used to evaluate plaque microcalcification [32], or 18F-FDG, for inflammation [33], can be employed. Additionally, nanobodies, which have faster blood clearance than antibodies, can be used for noninvasive nuclear imaging of relevant molecular targets of atherosclerotic plaques such as VCAM-1 [34].

Noninvasive imaging can also allow identifying subjects/patients who would benefit most from a nanotherapy. The majority of nanomedicine applications rely on the passive accumulation of nanoparticles in lesions due to the enhanced retention and permeability effect (so-called EPR effect), which may differ from patient to patient. Preselection of patients, for example by using a PET nanoreporter [35] approach, could improve the success rate of nanomedicine in the clinic.

\subsection{Pharmaceutical development of promising nanomedicines for clinical translation}

Finally, based on the favorable results presented in this thesis, we consider that HDL nanoparticles and HPMA polymeric micelles hold promise for future clinical applications in atherosclerosis. HDL nanoparticles show high affinity for plaqueassociated macrophages (in Chapter 5 and 6), despite the relatively short circulation kinetics. Such characteristics make HDL an ideal imaging platform for noninvasive monitoring of lesion-associated macrophages. On the other hand, HPMA polymeric micelles (in Chapter 6) possess longer circulation times, high stability/retention 
of hydrophobic drugs in their matrix, and high uptake by both pro-inflammatory monocytes and macrophages. Such features position HPMA polymeric micelles as a promising platform for targeted drug delivery to inflammatory lesions.

Scaling up the production of HDL and HPMA polymeric micelles may facilitate the clinical translation of these platforms. We reported a microfluidics-based approach for the large-scale production of HDL nanoparticles [36]. Through controlling mixing rates and lipid to protein ratios, we were able to tune the production of a variety of HDL nanoparticles that differ in shape and drug encapsulation efficiency. On the other hand, large-scale production of polymeric micelles is more established. Hrkach et al. reported the clinical-scale manufacturing of poly(lactide-co-glycolide) (PLGA) nanoparticles containing docetaxel, which were manufactured via a complicated nanoemulsion process, including several steps of solution preparation, high-energy emulsification and particle quench [37]. On the contrary, HPMA polymeric micelles are prepared in a simple, single step process of nanoprecipitation. Moreover, the drug loading and stabilization is via physical interaction, which eliminates the need for any chemical crosslinking/conjugation. Of note, a small fraction of the organic solvent is introduced during the process of producing HPMA polymeric micelles, which can subsequently be removed by simple evaporation or through the use of tangential flow filtration (TFF). Due to their small size, both HDL and HPMA polymeric micelles can be sterilized by filtration. Together, these simple steps can advance nanomedicines based on these two promising platforms to the clinic. 


\section{REFERENCES}

[1] A.H. Allam, R.C. Thompson, L.S. Wann, M.I. Miyamoto, A.-H. Nur El-Din, G.A. ElMaksoud, M. Al- Tohamy Soliman, I. Badr, H.A. El-Rahman Amer, M.L. Sutherland, J.D. Sutherland, G.S. Thomas, Atherosclerosis in ancient Egyptian mummies: the Horus study, JACC.Cardiovascular Imaging. 4 (2011) 315-327. doi:10.1016/j.jcmg.2011.02.002 [doi].

[2] P. Libby, Inflammation in atherosclerosis., Arterioscler. Thromb. Vasc. Biol. 32 (2012) 204551. doi:10.1161 / ATVBAHA.108.179705.

[3] The World Health Organization, Cardiovascular diseases (CVDs)-Fact sheet, (n.d.). http:/ / www.who.int/mediacentre/factsheets/fs317/en/.

[4] C. Chauvierre, D. Letourneur, The European project NanoAthero to fight cardiovascular diseases using nanotechnologies, Nanomedicine. 10 (2015) 3391-3400. doi:10.2217/ nnm.15.170.

[5] T. Lammers, L.Y. Rizzo, G. Storm, F. Kiessling, Personalized nanomedicine, Clin. Cancer Res. 18 (2012) 4889-4894. doi:10.1158/1078-0432.CCR-12-1414.

[6] J.S. Ross, N.E. Stagliano, M.J. Donovan, R.E. Breitbart, G.S. Ginsburg, Atherosclerosis: a cancer of the blood vessels?, Am. J. Clin. Pathol. 116 Suppl (2001) 97-107. doi:10.1309/ YNCK-9R19-5JA3-K2K9.

[7] J.S. Ross, N.E. Stagliano, M.J. Donovan, R.E. Breitbart, G.S. Ginsburg, Atherosclerosis and cancer: common molecular pathways of disease development and progression, Ann. N. Y. Acad. Sci. 947 (2001) 271-273.

[8] C.N. Serhan, Pro-resolving lipid mediators are leads for resolution physiology., Nature. 510 (2014) 92-101. doi:10.1038/ nature13479.

[9] A. Alaarg, N.Y. Jordan, J.J.F. Verhoef, J.M. Metselaar, G. Storm, R.J. Kok, Docosahexaenoic acid liposomes for targeting chronic inflammatory diseases and cancer: An in vitro assessment, Int. J. Nanomedicine. 11 (2016). doi:10.2147 / IJN.S115995.

[10] C. Hong, P. Tontonoz, Liver X receptors in lipid metabolism: opportunities for drug discovery, Nat. Rev. Drug Discov. 13 (2014) 433-444. doi:10.1038/nrd4280.

[11] J. Tang, S. Baxter, A. Menon, A. Alaarg, B.L. Sanchez-Gaytan, F. Fay, Y. Zhao, M. Ouimet, M.S. Braza, V.A. Longo, D. Abdel-Atti, R. Duivenvoorden, C. Calcagno, G. Storm, S. Tsimikas, K.J. Moore, F.K. Swirski, M. Nahrendorf, E.A. Fisher, C. Pérez-Medina, Z.A. Fayad, T. Reiner, W.J.M. Mulder, Immune cell screening of a nanoparticle library improves atherosclerosis therapy, Proc. Natl. Acad. Sci. U. S. A. 113 (2016). doi:10.1073/ pnas.1609629113.

[12] J. Tang, M.E. Lobatto, L. Hassing, S. van der Staay, S.M. van Rijs, C. Calcagno, M.S. Braza, S. Baxter, F. Fay, B.L. Sanchez-Gaytan, R. Duivenvoorden, H.B. Sager, Y.M. Astudillo, W. Leong, S. Ramachandran, G. Storm, C. Perez-Medina, T. Reiner, D.P. Cormode, G.J. Strijkers, E.S.G. Stroes, F.K.Swirski, M. Nahrendorf, E.A. Fisher, Z.A. Fayad, W.J.M. Mulder, Inhibiting macrophage proliferation suppresses atherosclerotic plaque inflammation, Sci. Adv. 1 (2015) e1400223. doi:e1400223 [pii].

[13] M.E. Lobatto, Z.A. Fayad, S. Silvera, E. Vucic, C. Calcagno, V. Mani, S.D. Dickson, K. Nicolay, M. Banciu, R.M. Schiffelers, J.M. Metselaar, L. van Bloois, H.S. Wu, J.T. Fallon, J.H. Rudd, V. Fuster, E.A. Fisher, G. Storm, W.J. Mulder, Multimodal clinical imaging to longitudinally assess a nanomedical anti-inflammatory treatment in experimental atherosclerosis, Mol. Pharm. 7 (2010) 2020-2029. doi:10.1021/mp100309y [doi].

[14] F.M. van der Valk, D.F. van Wijk, M.E. Lobatto, H.J. Verberne, G. Storm, M.C.M. Willems, D.A. Legemate, A.J. Nederveen, C. Calcagno, V. Mani, S. Ramachandran, M.P.M. Paridaans, M.J. Otten, G.M. Dallinga-Thie, Z.A. Fayad, M. Nieuwdorp, D.M. Schulte, J.M. Metselaar, W.J.M. Mulder, E.S. Stroes, Prednisolone-containing liposomes accumulate in 
human atherosclerotic macrophages upon intravenous administration., Nanomedicine. 11 (2015) 1039-46. doi:10.1016/j.nano.2015.02.021.

[15] Y. Shi, M.J. van Steenbergen, E.A. Teunissen, L. Novo, S. Gradmann, M. Baldus, C.F. van Nostrum, W.E. Hennink, П-П Stacking Increases the Stability and Loading Capacity of Thermosensitive Polymeric Micelles for Chemotherapeutic Drugs, Biomacromolecules. 14 (2013) 1826-1837. doi:10.1021/bm400234c.

[16] Y. Shi, R. Van Der Meel, B. Theek, E. Oude Blenke, E.H.E. Pieters, M.H.A.M. Fens, J. Ehling, R.M. Schiffelers, G. Storm, C.F. Van Nostrum, T. Lammers, W.E. Hennink, Complete regression of xenograft tumors upon targeted delivery of paclitaxel via ??? ??? stacking stabilized polymeric micelles, ACS Nano. 9 (2015) 3740-3752. doi:10.1021/ acsnano.5b00929.

[17] L. Quan, Y. Zhang, B.J. Crielaard, A. Dusad, S.M. Lele, C.J. Rijcken, J.M. Metselaar, H. Kostkova, T. Etrych, K. Ulbrich, F. Kiessling, T.R. Mikuls, W.E. Hennink, G. Storm, T. Lammers, D. Wang, Nanomedicines for inflammatory arthritis: head-to-head comparison of glucocorticoid-containing polymers, micelles, and liposomes, ACS Nano. 8 (2014) 458466. doi:10.1021/nn4048205 [doi].

[18] B.P. Toole, Hyaluronan: from extracellular glue to pericellular cue, Nat. Rev. Cancer. 4 (2004) 528-539. doi:10.1038/nrc1391.

[19] A.C. Petrey, C.A. de la Motte, Hyaluronan, a Crucial Regulator of Inflammation, Front. Immunol. 5 (2014) 101. doi:10.3389/ fimmu.2014.00101.

[20] B.P. Toole, Hyaluronan-CD44 Interactions in Cancer: Paradoxes and Possibilities, Clin. Cancer Res. 15 (2009) 7462-7468. doi:10.1158/1078-0432.CCR-09-0479.

[21] C.B. Highley, G.D. Prestwich, J.A. Burdick, Recent advances in hyaluronic acid hydrogels for biomedical applications, Curr. Opin. Biotechnol. 40 (2016) 35-40. doi:10.1016/j. copbio.2016.02.008.

[22] J.R. Fraser, T.C. Laurent, H. Pertoft, E. Baxter, Plasma clearance, tissue distribution and metabolism of hyaluronic acid injected intravenously in the rabbit., Biochem. J. 200 (1981) 415-24. http: / / www.ncbi.nlm.nih.gov/ pubmed / 7340841 (accessed April 25, 2017).

[23] C.N. Serhan, N. Chiang, T.E. Van Dyke, Resolving inflammation: dual anti-inflammatory and pro- resolution lipid mediators, Nat. Rev. 8 (2008) 349-361. doi:10.1038/nri2294 [doi].

[24] V. Chiurchiù, A. Leuti, J. Dalli, A. Jacobsson, L. Battistini, M. Maccarrone, C.N. Serhan, Proresolving lipid mediators resolvin D1, resolvin D2, and maresin 1 are critical in modulating T cell responses., Sci. Transl. Med. 8 (2016) 353ra111. doi:10.1126/ scitranslmed. aaf7483.

[25] G.J. Schroepfer, Oxysterols: modulators of cholesterol metabolism and other processes., Physiol. Rev. 80 (2000) 361-554. http://www.ncbi.nlm.nih.gov/pubmed/10617772 (accessed April 25, 2017).

[26] N.J. Spann, C.K. Glass, Sterols and oxysterols in immune cell function, Nat. Immunol. 14 (2013) 893-900. doi:10.1038/ni.2681.

[27] N.J. Spann, L.X. Garmire, J.G. McDonald, D.S. Myers, S.B. Milne, N. Shibata, D. Reichart, J.N. Fox, I. Shaked, D. Heudobler, C.R.H. Raetz, E.W. Wang, S.L. Kelly, M.C. Sullards, R.C. Murphy, A.H. Merrill, H.A. Brown, E.A. Dennis, A.C. Li, K. Ley, S. Tsimikas, E. Fahy, S. Subramaniam, O. Quehenberger, D.W. Russell, C.K. Glass, Regulated Accumulation of Desmosterol Integrates Macrophage Lipid Metabolism and Inflammatory Responses, Cell. 151 (2012) 138-152. doi:10.1016/j.cell.2012.06.054.

[28] S. Bekkering, L.A.B. Joosten, J.W.M. van der Meer, M.G. Netea, N.P. Riksen, Trained innate immunity and atherosclerosis, Curr. Opin. Lipidol. 24 (2013) 487-492. doi:10.1097/ MOL.0000000000000023.

[29] Z. Mallat, S. Taleb, H. Ait-Oufella, A. Tedgui, The role of adaptive T cell immunity in atherosclerosis., J. Lipid Res. 50 Suppl (2009) S364-9. doi:10.1194/jlr.R800092-JLR200.

[30] S.M. Evrard, L. Lecce, K.C. Michelis, A. Nomura-Kitabayashi, G. Pandey, K.-R. Purushothaman, V. d'Escamard, J.R. Li, L. Hadri, K. Fujitani, P.R. Moreno, L. Benard, P. 
Rimmele, A. Cohain, B. Mecham, G.J. Randolph, E.G. Nabel, R. Hajjar, V. Fuster, M. Boehm, J.C. Kovacic, Endothelial to mesenchymal transition is common in atherosclerotic lesions and is associated with plaque instability, Nat. Commun. 7 (2016) 11853. doi:10.1038/ ncomms 11853.

[31] A. Lindau, C. Härdtner, S.P. Hergeth, K.D. Blanz, B. Dufner, N. Hoppe, N. Anto-Michel, J. Kornemann, J. Zou, L.M.S. Gerhardt, T. Heidt, F. Willecke, S. Geis, P. Stachon, D. Wolf, P. Libby, F.K. Swirski, C.S. Robbins, W. McPheat, S. Hawley, M. Braddock, R. Gilsbach, L. Hein, C. von zur Mühlen, C. Bode, A. Zirlik, I. Hilgendorf, C. Hardtner, S.P. Hergeth, K.D. Blanz, B. Dufner, N. Hoppe, N. Anto- Michel, J. Kornemann, J. Zou, L.M.S. Gerhardt, T. Heidt, F. Willecke, S. Geis, P. Stachon, D. Wolf, P. Libby, F.K. Swirski, C.S. Robbins, W. McPheat, S. Hawley, M. Braddock, R. Gilsbach, L. Hein, C. von zur Muhlen, C. Bode, A. Zirlik, I. Hilgendorf, Atheroprotection through SYK inhibition fails in established disease when local macrophage proliferation dominates lesion progression, Basic Res. Cardiol. 111 (2016) 20-016-0535-8. Epub 2016 Feb 18. doi:10.1007/ s00395-016-0535-8 [doi].

[32] A. Irkle, A.T. Vesey, D.Y. Lewis, J.N. Skepper, J.L.E. Bird, M.R. Dweck, F.R. Joshi, F.A. Gallagher, E.A. Warburton, M.R. Bennett, K.M. Brindle, D.E. Newby, J.H. Rudd, A.P. Davenport, Identifying active vascular microcalcification by $18 \mathrm{~F}$-sodium fluoride positron emission tomography, Nat. Commun. 6 (2015) 7495. doi:10.1038/ncomms8495.

[33] H. Emami, P. Singh, M. MacNabb, E. Vucic, Z. Lavender, J.H.F. Rudd, Z.A. Fayad, J. Lehrer-Graiwer, M. Korsgren, A.L. Figueroa, J. Fredrickson, B. Rubin, U. Hoffmann, Q.A. Truong, J.K. Min, A. Baruch, K. Nasir, M. Nahrendorf, A. Tawakol, Splenic Metabolic Activity Predicts Risk ofFuture Cardiovascular Events, JACC Cardiovasc. Imaging. 8 (2015) 121-130. doi:10.1016/j.jcmg.2014.10.009.

[34] A. Broisat, S. Hernot, J. Toczek, J. De Vos, L.M. Riou, S. Martin, M. Ahmadi, N. Thielens, U. Wernery, V. Caveliers, S. Muyldermans, T. Lahoutte, D. Fagret, C. Ghezzi, N. Devoogdt, Nanobodies Targeting Mouse/Human VCAM1 for the Nuclear Imaging of Atherosclerotic Lesions, Circ. Res. 110 (2012) 927-937. doi:10.1161/CIRCRESAHA.112.265140.

[35] C. Pérez-Medina, D. Abdel-Atti, J. Tang, Y. Zhao, Z.A. Fayad, J.S. Lewis, W.J.M. Mulder, T. Reiner, C. Perez-Medina, D. Abdel-Atti, J. Tang, Y. Zhao, Z.A. Fayad, J.S. Lewis, W.J.M. Mulder, T. Reiner, Nanoreporter PET predicts the efficacy of anti-cancer nanotherapy, Nat. Commun. 7 (2016) 11838. doi:10.1038/ncomms11838 [doi].

[36] Y. Kim, F. Fay, D.P. Cormode, B.L. Sanchez-Gaytan, J. Tang, E.J. Hennessy, M. Ma, K. Moore, O.C. Farokhzad, E.A. Fisher, W.J.M. Mulder, R. Langer, Z.A. Fayad, Single step reconstitution of multifunctional high-density lipoprotein-derived nanomaterials using microfluidics, ACS Nano. 7 (2013) 9975-9983. doi:10.1021/nn4039063.

[37] J. Hrkach, D. Von Hoff, M.M. Ali, E. Andrianova, J. Auer, T. Campbell, D. De Witt, M. Figa, M. Figueiredo, A. Horhota, S. Low, K. McDonnell, E. Peeke, B. Retnarajan, A. Sabnis, E. Schnipper, J.J. Song, Y.H. Song, J. Summa, D. Tompsett, G. Troiano, T. Van Geen Hoven, J. Wright, P. LoRusso, P.W. Kantoff, N.H. Bander, C. Sweeney, O.C. Farokhzad, R. Langer, S. Zale, Preclinical Development and Clinical Translation of a PSMA-Targeted Docetaxel Nanoparticle with a Differentiated Pharmacological Profile, Sci. Transl. Med. 4 (2012). http: / / stm.sciencemag.org/ content/4/128/128ra39 (accessed May 27, 2017). 



\section{NEDERLANDSE SAMENVATTING}

Atherosclerose (slagaderverkalking maar beter bekend als 'aderverkalking') kan hartaanvallen, herseninfarcten en etalagebenen veroorzaken. Dit komt door afzetting van vettig materiaal, ook wel atherosclerotische plaques genoemd, op de binnenkant van slagaders, hetgeen de bloedstroom door die slagaders bemoeilijkt. Uiteindelijk kunnen dergelijke plaques de slagader ernstig blokkeren en zelfs geheel afsluiten. Ook kan een plaque scheuren en daardoor plaatselijk trombose (bloedstolsels) vormen. Als die stolsels losraken kunnen ze elders een embolie (afsluiting van de slagader) veroorzaken. Atherosclerose is de belangrijkste oorzaak van morbiditeit en mortaliteit in de westerse wereld. In dit proefschrift worden er mogelijkheden onderzocht om behandeling van atherosclerose te verbeteren met behulp van nanomedicijnen, nieuwe geneesmiddelen gebaseerd op nanodeeltjes die in staat zijn zich preferentieel te lokaliseren (targeting) in ziektegebieden zoals atherosclerotische plaques. Deze vom van targeting wordt mogelijk gemaakt door de aanwezigheid van een inflammatoir pathologisch proces dat de permeabiliteit van wanden van lokale bloedvaten verhoogt en zo de influx van immuuncellen (bv. monocyten/macrofagen) mogelijk maakt maar tegelijk ook nanodeeltjes toegang tot het ontstekingsgebied verschaft.

Hoofdstuk 1 geeft een algemene inleiding tot het onderwerp van het proefschrift en een overzicht van de inhoud. Hoofdstuk 2 schetst uitgebreid hoe nanomedicijnen kunneninwerken op chronische ontstekingsziekten, waaronder atherosclerose, vooral op het nivo van de onderliggende pathofysiologische processen. Macrofagen spelen een belangrijke rol bij de vorming van ontstekingen in atherosclerotische plaques en vergroten de kans dat deze scheuren. Dankzij hun fagocyterende gedrag zijn macrofagen een ideale target voor nanomedicjnen die als doel hebben de inflammatie in de plaques te verminderen. Hoofdstuk 3 geeft een eerste voorbeeld van een dergelijk nanomedicijn. Liposomen, het bekendste voorbeeld van een nanomedicijn, worden beladen met docosahexaeenzuur (DHA), een natuurlijk meervoudig onverzadigd vetzuur. Omega3-meervoudig onverzadigde vetzuren (PUFA's, ook bekend als het gezondheidsbevorderende ingrediënt van visolie), worden normaal gesproken alleen oraal ingenomen door ondermeer patiënten met hart- en vaatziekten en kanker. Verwerkt in liposomen vertoonde het omega3-vetzuur sterke antioxidante en anti-inflammatoire effecten in vitro, en veroorzaakte bovendien een sterke remming van de groei van tumorcellen. Deze 'nanonutraceutical' vertegenwoordigt een rationele, nieuwe aanpak voor het targeten van relatief veilige zgn. nutraceutische componenten bij chronische inflammatoire aandoeningen (waaronder atherosclerose) en kanker. Hoofdstuk 4 beschrijft een onderzoek waarin diverse farmaca uitgebreid worden onderzocht op hun anti-inflammatoire activiteiten in vitro. Als beste komen LXR agonisten and statines uit de bus. Vervolgens worden voorbeelden uit deze klassen van farmaca verpakt in nanomedicijnen, wat leidde tot de in Hoofdstuk 5 beschreven nanoformulering van high-density-proteïne (HDL) met de LXR-agonist GW3965, dat faalde in klinische proeven vanwege levertoxiciteit, en de in Hoofdstuk 6 beschreven nanoformuleringen van simvastatine in polymere micellen, liposomen 
en HDL. In vitro en in vivo resultaten laten duidelijk zien dat targeting van deze antiinflammatoire farmaca naar macrofagen therapeutische voordelen kan opleveren. In het laatste onderzoekshoofdstuk (Hoofdstuk 7) worden nanodeeltjes, gemaakt van het biologisch actieve polymeer hyaluronan, onderzocht op anti-inflammatoire effecten. Deze laatste aanpak is vooral interessant omdat de resultaten laten zien dat inbouw van farmaca niet noodzakelijk is om een anti-atherosclerotische werking in vivo te verkrijgen. Hoofdstuk 8 sluit af met een samenvatting van de bereikte resultaten en een visie op toekomstige toepassingsmogelijkheden van plaquegetargete nanomedicijnen voor de behandeling van atheroclerose. 


\section{ACKNOWLEDGEMENTS}

People say "you have been all over the world"; I say "I am not through learning yet". Sometimes, we try to define events with dates, months, and years. However, I think that time is a very relative thing; the repercussions of an event that happened in the past, or a person you met can be felt now or in the future. Therefore, I would rather define myself with the many experiences I have gone through, especially since I came to the Netherlands to pursue my graduate studies. I met many people on this journey that have helped and supported me in reaching this stage in my Ph.D. More importantly, they left long-lasting experiences, for which I am in debt to them.

My Ph.D. would not have started if it was not for my promotor Professor Storm. Gert, my gratitude to you is limitless. I see you as an experienced sailor who can comfortably and confidently sail in a quiet and "stormy" sea. Working with you on an interdisciplinary project such as NanoAthero is a lifetime experience. Through your connections, you arranged for me to conduct my research in academic and clinical settings in New York, Amsterdam, Utrecht, and Enschede, which enriched my Ph.D. at multiple levels. On a personal level, I learned a lot from your universal experiences that you cheerfully shared during our walks in Utrecht or during a Broadway show in Manhattan. Thank you!

My gratefulness is extended to Professor Mulder. Dear Willem, my work in your laboratory at Mount Sinai, New York was a unique experience. The way you direct a relatively small group as an operations manager to maximize the productivity of the whole lab was exceptional. Indeed, the visit paid off; as you said that "I fit in the team/family". In less than 18 months, I became experienced in in vivo imaging and animal studies, which led to the completion of $8+$ projects and publications in high impact journals. Besides work, I appreciated your openness and talks during our walks through Harlem. Also, your aspirations to excel in anything you do and to be the best of yourself are quite inspiring. Thank you so much, Willem!

Bart, my dear co-promoter, I am privileged to have met you on this journey. You have always been encouraging and supportive, especially with all the requirements and the meetings of NanoAthero. You have also been an active supporter of me exploring new ideas, and giving me all the freedom to establish myself as an independent Pharmaceutical Scientist. Also, your industrial experiences and your entrepreneurship gave me a different, unique angle of the field of drug delivery and nanomedicine. Hartelijk bedankt!

Robbert Jan, my first supervisor in my masters with extended supervision/ mentoring in my Ph.D., Thank you! It has always been a pleasure to work and talk with you. You understood me without even talking. This kind of chemistry in communication was really valuable and unique, and very productive as demonstrated in my publication with you. I am deeply grateful for all your support during my masters and Ph.D. 
In addition to my "main" Ph.D. supervisors, I was humbled and honored to work in the Laboratory of Prof. Dr. Wim Hennink during my masters and doctoral studies. Dear Wim, thank you for giving me the opportunity to learn from you and work with the incredible group of Pharmaceutics in Utrecht. I am also grateful for your guidance whenever it was needed in the past six years. You are always sharp, critical and insightful when it comes to science and work. I learned a lot from your incredible work ethics. Besides work, you are also fun when it comes borrels, games, and lab uitjes $\odot$. My gratitude is also extended to Professor Schiffelers. Dear Raymond, thank you for your mentorship and for arranging my first internship abroad at the Laboratory of Matthew Wood in Oxford. I will always remember how witty our conversations were and how smooth my first publication was, thanks to you. Your spirit is always uplifting!

On my journey of research in my masters, I met my daily supervisors: Roy van der Meel and Pieter Vader, two brilliant, young scientists who immensely influenced my decision to pursue a Ph.D. degree. Roy and Pieter, I learned a lot from you, starting from how to pipette into more than $1000^{*} 96$-well plates to how I can formulate scientific questions and write down my ideas and grants. You have been supervisors, colleagues, and friends who helped me navigate my way through my first steps in science and life in the western culture, in the Netherland and the UK. You made me feel like I am a family member with Anna, Stella, and the young Joel. Thank you is not enough!!!

To my colleagues in the Department of Pharmaceutics, Utrecht University, and the BST group, University of Twente: I enjoyed every moment of working with you during my masters and Ph.D. Every one of you, I mentioned or not, left only joyful and memorable experiences.

Jan Jaap, you have been my very close buddy and colleague since the first week of my masters. We, weirdly enough, have always been on the same wavelength when it comes to science, politics, fun, and many other things $\odot$. We always stayed in touch even when we are abroad. Good luck my friend with all your plans. You can do it!

Rachel, my awesome paranymph, I am honored to have met you on this journey. You are a wonderful person who is always willing to help in any event, during my masters, in navigating my way at UT during my Ph.D., and with sharing your previous adventures in the Netherlands. I am lucky to have a wise, grounded, and fun friend like you. I wish you all the best at Cristal Therapeutics and in your future plans.

My second fantastic paranymph, Jeffrey, the father of Prince Noah, we met later on my journey, I cannot remember how $\odot$. Since then, you have been genuinely supportive of me on my journey. During my stay in New York, your help was even bigger to me. We also happen to have the same promotor which means a lot!! $\odot$. Thank you, buddy! 
Aida, I got to work with you in the last bit of my Ph.D. Your responsiveness and support with making so much polymer is one of the main reasons that made this project successful. Thank you so much!

Karin Hendriks, your dedication and hard work are phenomenal. You are always willing to help with all the logistics and formalities at UT. Even when I was in NY, you made everything smooth for me. Thanks for the follow ups and for making the last bit of my Ph.D. a lot easier and on time. A VERY BIG THANK YOU!

Mies, thanks for the trainings and also for showing me all the real Dutch festivities.

Barbara, thanks for all the help and support in the past six years.

Sima, your artistic soul, and the concerts we attended, have always made me feel there are more beautiful things in life than science. Thank you!

To Erik, Mohamed, Martin, Lies, Karina, Andyk, Bo, Kristel, Dan Dan, Yinan, Louann, Neda, Marzieh et al. at Utrecht University and Praneeth, Iris, Karin (P), Ruchi, and Jai at Twente University: it has always been a pleasure to lunch, travel, or engage in stimulating discussions with you. Thank you for sharing special moments with me.

Sophia, you were my first student that I had the privilege to supervise in the lab directly. I hope you enjoyed your internship and that I could teach you something as I was taught before. Thanks for all the hard work and your desire to learn, which paid off in a good publication while you were a bachelor student. All the best with your future plans.

I spent the last two years of my Ph.D. working with fantastic people in Willem's lab in New York. The group was rather a family than just work colleagues. Zahi: thank you so much for giving me the opportunity to be a member of TMII. Carlos, I genuinely enjoyed working with you on different projects. You are easy going, focused on solving issues, and helping others. Thank you for all the radiochemistry and imaging. I learned a lot from you. Max and Mandy, thanks for helping out with the long hours of in vivo studies. Thijs and Ewelina, it was so much fun working with you in the extensive HA project. Thank you for letting me part of your research. Brenda, thanks for all the TEM lessons. All the best to you, Pepe, and your two beautiful angels, little Brenda and Dani. Francois, you are fun to travel and work with. You always inspire me with your hard work, even with a broken leg. Also, thank you for introducing me to "Die Antwoord" $\odot$. Jun, when someone tells me 'you are good at the in vivo studies', I tell them 'I learned from the master'. You, sir, are the master $\odot$. Thanks man for all the moonlighting, energy bars and skype calls in the middle of the night to fix the flow cytometry. It all paid off. Also, many thanks for arranging the hiking trips on the Hudson. All the best to you and Kate. 
Finally, my dear Yiming: on my trip to New York, I gained you as a very smart daily supervisor, a colleague, a friend, and a brother. You, Summer, and Bruce became my family here in NY as well as Hera's best friends when she joined me. Thank you for letting me be part of your scientific research and a non-Chinese member of the family $\odot$. Best of luck to you and Summer, and as for Bruce, he knows his way to his uncle...stories to be continued.

I would also like to take this opportunity to thank some special friends. Ahmad Saeed, thank you so much for all of your efforts, the graphic design, and the time you put into the layout of this thesis. Noha Samara, thanks a lot for the leadership and ownership you showed during my work at P\&G. It has benefited me through my scientific journey. Ahmed Abbas, Nancy, Susan (van Dommelen), Matina, Klaas, Marta, Irina, Luisa, Matt, Jessica, Andrei, Natalia, Hedi, Jo, Kasia: thank you for your friendship and for the life you gave me outside of the lab. We have always stayed close, even when we were separated by the Atlantic.

To my family in Egypt, and also the one in Houston, I could not have reached this stage without your love, help, and support. You have always been there for me. I love you!

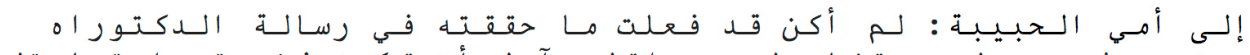

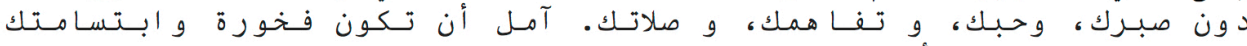

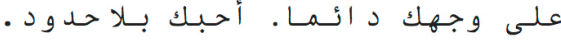

To my love, my wife, and my everything, Hera: you were completely unplanned in this journey, but the best part about it. I gained the world when I met you. Someone once said to me you have been all over the world, and now I can say, it was to meet you. You give me love, focus, and determination to succeed. Thank you for always supporting me. Thank you for being patient with me through endless long nights during experiments and with reading countless scientific papers. You are my best friend in the WHOLE WIDE WORLD. I love you to the moon and back $x$.

Amr

July 2017

On the Hudson, Yonkers, New York 


\section{LIST OF PUBLICATIONS}

\section{This thesis is based on the following publications}

1. Amr Alaarg, Carlos Pérez-Medina, Josbert M. Metselaar, Matthias Nahrendorf, Zahi A. Fayad, Gert Storm, Willem J.M. Mulder, Applying nanomedicine in maladaptive inflammation and angiogenesis, Advanced Drug Delivery Systems (2017), doi: 10.1016/j.addr.2017.05.009. (Chapter 2)

2. Amr Alaarg, Nan Yeun Jordan, Johan J.F. Verhoef, Josbert M. Metselaar, Gert Storm, Robbert J. Kok, Docosahexaenoic acid liposomes for targeting chronic inflammatory diseases and cancer: an in vitro assessment, International Journal of Nanomedicine (2016), doi: 10.2147 / IJN.S115995. (Chapter 3)

3. Amr Alaarg, Kang He Zheng, Fleur M. van der Valk, Acarilia Eduardo da Silva, Miranda Versloot, Linda C. Quarles van Ufford, Dominik M. Schulte, Gert Storm, Josbert M. Metselaar, Erik S.G. Stroes, Anouk A.J. Hamers, Multiple pathway assessment to predict anti-atherogenic efficacy of drugs targeting macrophages in atherosclerotic plaques, Vascular Pharmacology (2016), doi: 10.1016/j. vph.2016.04.006. (Chapter 4)

4. Jun Tang, Samantha Baxter, Arjun Menon, Amr Alaarg, Brenda L. SanchezGaytana, Francois Fay, Yiming Zhao, Mireille Ouimet, Mounia S. Braza, Valerie A. Longo, Dalya Abdel-Atti, Raphael Duivenvoorden, Claudia Calcagno, Gert Storm, Sotirios Tsimikas, Kathryn J. Moore, Filip K. Swirski, Matthias Nahrendorf, Edward A. Fisher, Carlos Pérez-Medina, Zahi A. Fayad, Thomas Reiner, Willem J.M. Mulder, Immune cell screening of a nanoparticle library improves atherosclerosis therapy, Proceedings of the National Academy of Sciences of the United States of America (2016), doi: 10.1073/ pnas.1609629113. (Chapter 5)

5. Amr Alaarg, Max L. Senders, Aida Varela-Moreira, Yiming Zhao, Jun Tang, Francois Fay, Thomas Reiner, Zahi A. Fayad, Carlos Pérez-Medina, Wim E. Hennink, Josbert M. Metselaar, Willem J.M. Mulder, Gert Storm. A systematic comparison of clinically viable nanomedicines targeting HMG-CoA reductase in inflammatory atherosclerosis, Journal of Controlled Release (2017), doi: 10.1016/j. jconrel.2017.07.013. (Chapter 6)

6. Thijs J. Beldman, Max L. Senders, Amr Alaarg, Carlos Perez-Medina, Yiming Zhao, Jun Tang, Francois Fay, Jacqueline Deichmöller, Benjamin Born, Nicole N. van der Wel, Ron A. Hoebe, Fortune Cohen, Elena Kartvelishvily, Michal Neeman, Thomas Reiner, Claudia Calcagno, Zahi A. Fayad, Menno P. J. de Winther, Ester Lutgens, Willem J.M. Mulder, Ewelina Kluza. Hyaluronan nanoparticles selectively target plaque-associated macrophages and improve plaque phenotype in atherosclerosis, ACS NANO (2017), doi: 10.1021 / acsnano.7b01385. (Chapter 7) 


\section{Other Publications}

7. Brenda L. Sanchez-Gaytan, Francois Fay, Sjoerd Hak, Amr Alaarg, Zahi A. Fayad, Carlos Pérez-Medina, Willem J. M. Mulder, Yiming Zhao, Real-time monitoring of nanoparticle formation by FRET imaging, Angewandte Chemie International Edition (2017), doi: 10.1002 / anie.201611288.

8. Max L. Senders, Sophie Hernot, Giuseppe Carlucci, Jan van de Voort, Francois Fay, Claudia Calcagno, Amr Alaarg, Yiming Zhao, Seigo Ishino, Anna Palmisano, Jun Tang, Samantha Baxter, Gilles Boeykens, Laura Zendman, Mark Lobatto, Nicolas Karakatsanis, Thomas Reiner, Jason S. Lewis, Zahi A. Fayad, Nick Devoogdt, Willem J. Mulder, Carlos Pérez-Medina, Nanobody-facilitated multiparametric PET/MRI phenotyping of experimental atherosclerosis, under peer-review.

9. Francois Fay, Line Hansen, Stefanie J.C.G. Hectors, Brenda Sanchez, Yiming Zhao, Jun Tang, Jazz Munitz, Amr Alaarg, Mounia S. Brazza, Anita Gianella, Stuart A. Aarson, Thomas Reiner, Jørgen Kjems, Robert Langer, Freek J.M. Hoeben, Henk M. Janssen, Claudia Calcagno, Gustav J. Strijkers, Zahi A. Fayad, Carlos Pérez-Medina, Willem J.M. Mulder, Investigating the cellular specificity in tumors of a surface converting nanoparticle by multimodal imaging, Bioconjugate Chemistry (2017), doi: 10.1021 /acs.bioconjchem.7b00086.

10. Eduard Willms, Henrik J. Johansson, Imre Mäger, Yi Lee, K. Emelie M. Blomberg, Mariam Sadik, Amr Alaarg, C.I. Edvard Smith, Janne Lehtiö, Samir EL Andaloussi, Matthew J.A. Wood, Pieter Vader, Cells release subpopulations of exosomes with distinct molecular and biological properties, Scientific Reports (2016), doi: 10.1038/ srep22519.

11. Fleur M. van der Valk, Dominik M. Schulte, Svenja Meiler, Jun Tang, Kang He Zheng, Jan Van den Bossche, Tom Seijkens, Matthias Laudes, Menno de Winther, Esther Lutgens, Amr Alaarg, Josbert M. Metselaar, Geesje M. Dallinga-Thie, Willem J.M. Mulder, Erik S.G. Stroes, Anouk A.J. Hamers. Liposomal prednisolone promotes macrophage lipotoxicity in experimental atherosclerosis, Nanomedicine: Nanotechnology, Biology, and Medicine (2016), doi: 10.1016/j.nano.2016.02.022.

12. Amr Alaarg, Raymond M. Schiffelers, Wouter W. van Solinge, Richard van Wijk. Red blood cell vesiculation in hereditary hemolytic anemia, Frontiers in Physiology (2013), doi: 10.3389/ fphys.2013.00365. 


\section{Conferences}

\section{The work in this thesis was presented at the following international conferences}

1. The Sino-American Pharmaceutical Professional Association Symposium (SAPA 2017), Revolution in Cancer Treatment-Immunotherapy and Beyond, New Jersey, USA. Poster Presentation.

2. The World Molecular Imaging Congress (WMIC 2016), New York, USA (September 2016). Poster Presentation.

3. The 43rd Controlled Release Society Meeting and Exposition (CRS 2016), Seattle, USA (July 2016). Poster and Oral Presentations.

4. The $6^{\text {th }}$ Annual Translational and Molecular Imaging Institute Symposium (TMII 2016), New York, USA (April 2016). Poster Presentation.

5. The $4^{\text {th }}$ Symposium on Phospholipids in Pharmaceutical Research, Heidelberg, Germany (September 2015). Poster Presentation.

6. The 83rd European Atherosclerosis Society Congress (EAS 2015), Glasgow, UK (March 2015). Poster Presentation and Young Investigator Fellowship Award. 


\section{Curriculum Vitae}

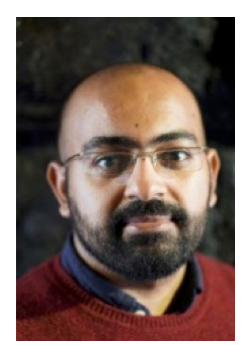

Amr was born to Egyptian parents on April 14, 1985, in Buraydah, Saudi Arabia. In 2002, he graduated from Toukh High School. In the same year, he started his higher education at Ain Shams University in Cairo, Egypt where he obtained his Bachelor degree in Pharmaceutical Sciences in 2007. During 2007 to 2009, he served in the Egyptian Army as a Pharmacist Soldier and was appointed as a Teaching Assistant at the Department of Industrial Pharmacy and Pharmaceutics, Ain Shams University, Cairo, Egypt. In 2009, he accepted an offer from the FMCG giant Procter \& Gamble $(P \& G)$ and started his assignment as a Supply Network Operation Planner for Baby Care products in the Middle East and Africa. In 2011, Amr was granted the Utrecht Excellence Scholarship (full scholarship) to enroll in the two-year Drug Innovation master's program at Utrecht University, the Netherlands. During this time, he performed his first research internship at the Department of Pharmaceutics (the Laboratory of Prof.Dr. Wim Hennink), Utrecht University developing and applying antiEGFR and anti-VEGFR nanobody-liposomes loaded with kinase inhibitor for cancer treatment. He commenced his second internship at the Laboratory of Prof.Dr. Matthew Wood at the University of Oxford to investigate the use of extracellular vesicles (microvesicles and exosomes) as bioinspired drug delivery systems. In 2013, Amr embarked on his Ph.D. journey under the supervision of Prof.Dr. Gert Storm in the European Consortium NanoAthero and at the Department of Biomaterials Science and Technology, the University of Twente, the Netherlands. During his Ph.D., Amr conducted his research at the Department of Pharmaceutics, Utrecht University, the Department of Vascular Medicine, Amsterdam Medical Center (both in the Netherlands), and the Translational and Molecular Imaging Institute, Mount Sinai School of Medicine, New York, USA (supervised by Prof.Dr. Willem Mulder). Through working with multidisciplinary teams, Amr investigated the development and application of novel targeted therapeutics for the treatment of atherosclerosis. The results of his research are described in this thesis, entitled 'Targeted Therapeutics in Inflammatory Atherosclerosis.' 